\title{
Report on \\ High-Rise Fireground Field Experiments
}
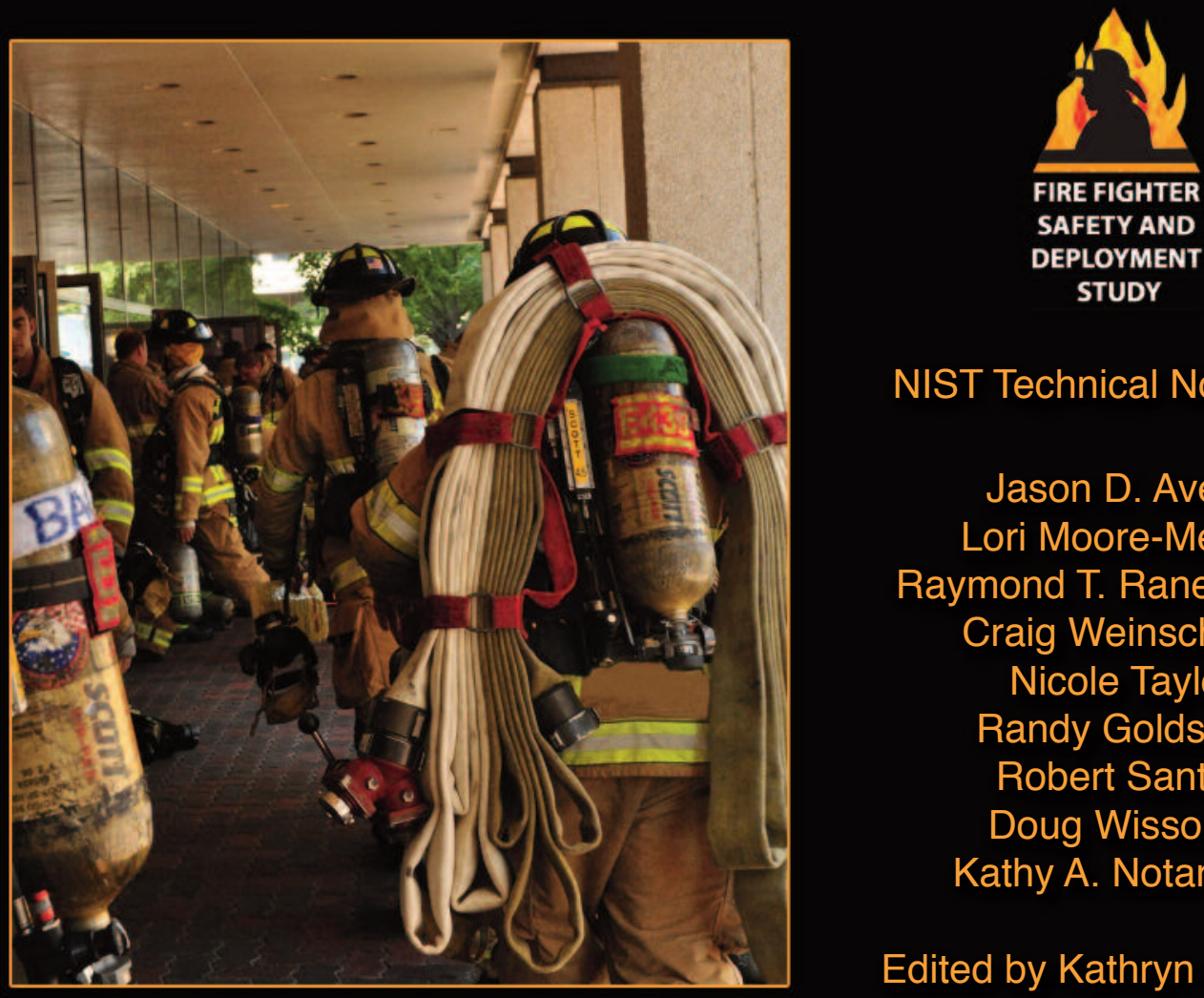

NIST Technical Note 1797

Jason D. Averill

Lori Moore-Merrell

Raymond T. Ranellone Jr.

Craig Weinschenk

Nicole Taylor

Randy Goldstein

Robert Santos

Doug Wissoker

Kathy A. Notarianni

Edited by Kathryn M. Butler

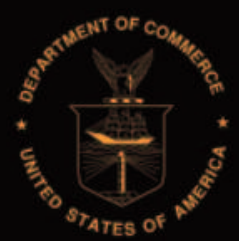

U.S. Department of Commerce

Rebecca Blank, Deputy Secretary

Patrick D. Gallagher, Under Secretary of Commerce for Standards and Technology and Director 


\section{Report on \\ High-Rise Fireground Field Experiments}

Jason D. Averill, Fire Research Division, National Institute of Standards and Technology Lori Moore-Merrell, International Association of Firefighters

Raymond T. Ranellone Jr., Fire Research Division, National Institute of Standards and Technology Craig Weinschenk, Fire Research Division, National Institute of Standards and Technology Nicole Taylor, International Association of Firefighters

Randy Goldstein, International Association of Firefighters

Robert Santos, Urban Institute

Doug Wissoker, Urban Institute

Kathy A. Notarianni, Worcester Polytechnic Institute

Edited by

Kathryn M. Butler, Fire Research Division, National Institute of Standards and Technology

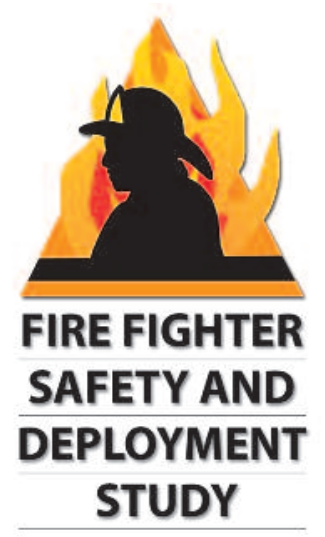


Certain commercial entities, equipment, or materials may be identified in this document in order to describe an experimental procedure or concept adequately. Such identification is not intended to imply recommendation or endorsement by the National Institute of Standards and Technology, nor is it intended to imply that the entities, materials, or equipment are necessarily the best available for the purpose.

National Institute of Standards and Technology Technical Note1797 Natl. Inst. Stand. Technol. Tech. Note 1797, 152 pages (April 2013) CODEN: NTNOEF 


\author{
Produced with the Cooperation of \\ Arlington County Fire Department \\ Alexandria Fire Department \\ Fairfax County Fire and Rescue \\ Prince William County Fire and Rescue \\ District of Columbia Fire Department \\ Montgomery County Fire and Rescue \\ Howard County Department of Fire and Rescue Services \\ Fairfax City Fire and Rescue \\ Prince George's County Fire/EMS Department \\ Loudoun County Fire and Rescue Department \\ Metropolitan Washington Airport Authority \\ Manassas City Fire and Rescue \\ Stafford County Fire and Rescue
}

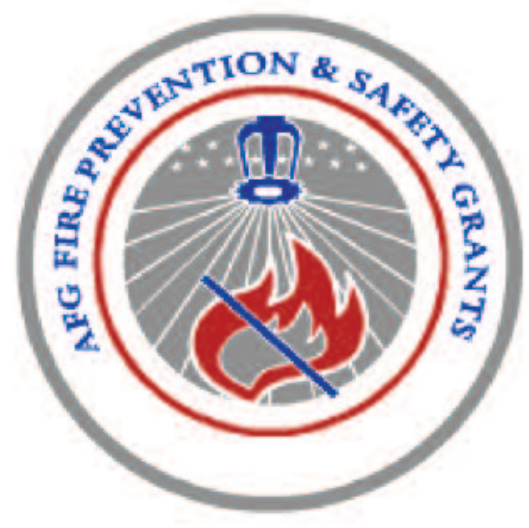

Funding provided through

DHS/FEMA's Grant Program Directorate for

FY 2010 and FY 2011 Assistance to Firefighters Grant Program

Fire Prevention and Safety Grants.

(EMW-2010-FP-01276) and (EMW-2011-FP-00588) 


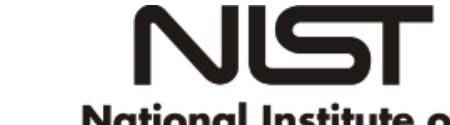

National Institute of

Standards and Technology

U.S. Department of Commerce
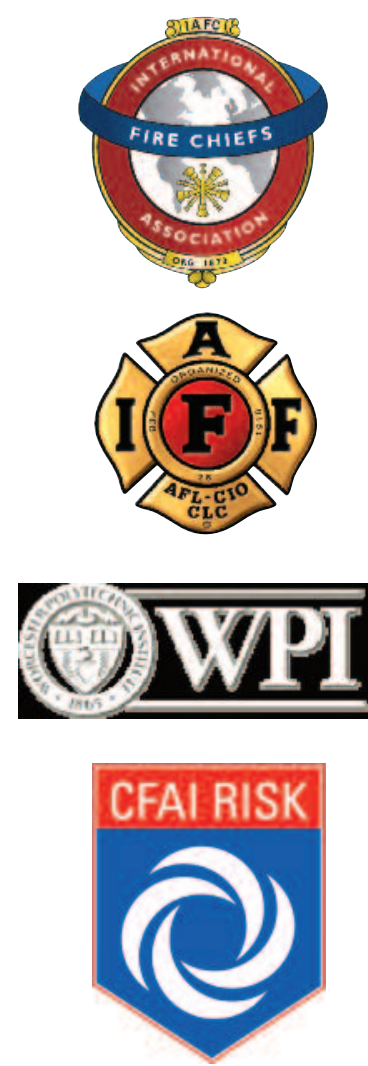

URBAN INSTITUTE 
O ver the past three decades, fire department response has expanded from fire prevention and fire suppression to include other community services such as emergency medical services, hazardous materials response, and special rescue. Today, service demands and public expectations placed upon local fire departments continue to rise as threats to communities have increased in consequences, affected greater populations and caused significant loss of life and property damage from both natural and man-made disasters. However, these expectations are currently being managed without an established technical basis for estimating the effects of deployment decisions on fulfillment of community expectations due to a lack of technical foundation. Therefore, in order to balance community expectations with limited resources, the fire service and community leaders require scientific data that quantifies the effects of changes in fire service deployment on the safety of the public and firefighters. This report, along with the companion Report on Residential

Fireground Experiments (Averill et al., 2010), establish a technical basis for deployment of resources to fireground events with varying levels of underlying hazards.

This report presents the results of 48 field experiments and 48 complementary fire modeling simulations that collectively quantify the impact of differing crew size deployments (3-person, 4-person, 5-person, and 6-person crews), different alarm assignments, and different vertical response modes on occupant survivability, firefighter safety, and property protection for four potential high-rise fire response scenarios.

For the high-rise fireground experiments, a 13 story vacant commercial building was used in Crystal City, Virginia. Props were built within the structure to closely resemble an occupied workplace including a mixture of employee cubicles and private offices. Each floor of the structure measured approximately $30,000 \mathrm{sq} \mathrm{ft}\left(2800 \mathrm{~m}^{2}\right)$. This is a modest high-rise building that represents a baseline best case scenario for high hazard ${ }^{1}$ environments.

Fire crews from 13 Metropolitan Washington D.C. area departments were deployed in response to simulated fires within this building. In addition to systematically controlling for the arrival times of the fire apparatus, crew size, alarm size, and vertical response mode were varied. Each resource deployment performed a series of 38 tasks that were timed.

Overall, the results of this study show that the number of fire service crew members in each company responding to a fire had a dramatic effect on the crew's ability to protect lives and property. When responding to a medium growth rate fire on the 10th floor of the high-rise structure, a 3-person crews ascending to the fire floor confronted an environment where the fire had released $60 \%$ more heat energy than the fire encountered by the 6-person crews. Larger fires expose firefighters to greater risks and are more challenging to extinguish.

In addition to the time-to-task portion of the study, fire modeling was used to correlate time-to-task completion by crew size, alarm size, and vertical response mode to the degree of toxicity of the environment in the structure for a range of fire growth rates.

Larger fires produce more risk exposure for firefighters and building occupants. In general, occupants being rescued by smaller crew sizes and by crews that used the stairs rather than the elevators were exposed to significantly greater dose of toxins from the fire. While the exact risk exposure for an occupant will depend on the fire growth rate, their proximity to the fire, and the floor on which the fire is located, it is clear that on-scene deployment decisions can have a dramatic effect in determining the fate of building occupants.

The study confirmed that a properly engineered and operational fire sprinkler system drastically reduces the risk exposure for both the building occupants and the firefighters. While this has been well understood for many years and most new high-rise buildings are constructed with fire sprinkler protection, The National Fire Protection Association (NFPA) estimates that 41 percent of U.S. high-rise office buildings, 45 percent of high-rise hotels, and 54 percent of high-rise apartment buildings are not equipped with sprinklers. Moreover, sprinkler systems fail in about one in 14 fires. Thus, fire departments should be prepared to manage the risks associated with unsprinklered high-rise building fires.

This study, like the Residential Fireground Experiments (Averill et al., 2010), is a unique scientific evaluation of the relationship between key fire service deployment variables and the resulting service delivery outcomes. The study includes input from and was reviewed by a comprehensive array of stakeholders, including many of the world's leading high-rise firefighting experts and experienced, professional firefighters from the Washington Metropolitan region. The results and conclusions will directly inform local fire chiefs and elected officials charged with matching fire risks in a community with a safe and effective fire department deployment configuration.

These research results will inform standards development organizations, such as the NFPA, and will allow for incorporation into consensus industry deployment standards such as NFPA 1710, Standard for the Organizational and Deployment of Fire Suppression Operations, Emergency Medical Operations, and Special Operations to the Public by Career Fire Departments. 


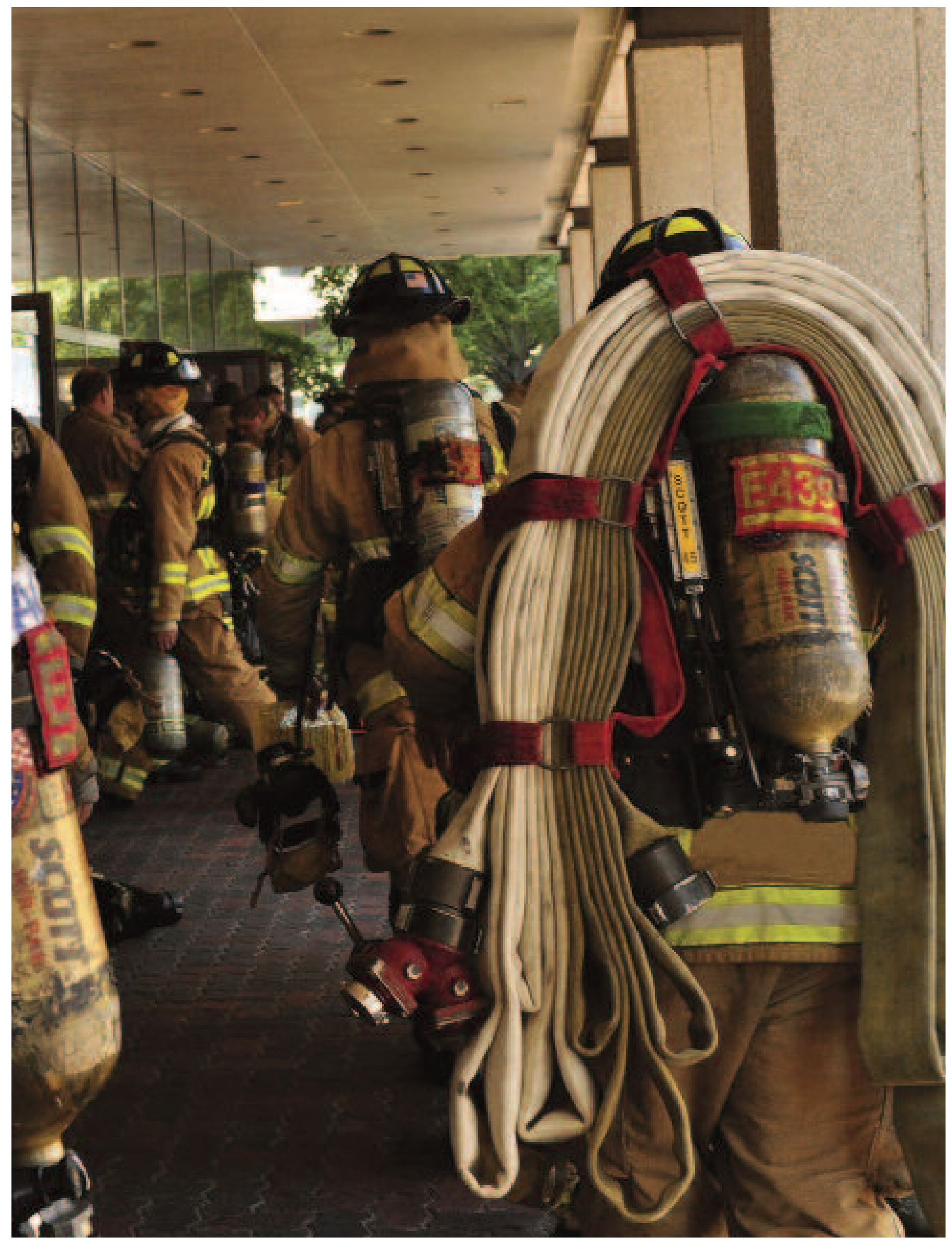




\begin{tabular}{|c|c|}
\hline & 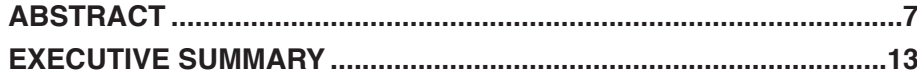 \\
\hline 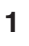 & BACKGROUND ................... \\
\hline & PROBLEM ............................... \\
\hline & REVIEW OF LITERATURE ..... \\
\hline & 3.1 Historic High-Rise Fires.. \\
\hline & 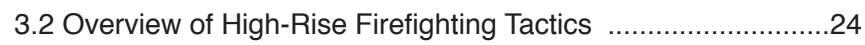 \\
\hline & PURPOSE AND SCOPE OF THE STUDY \\
\hline & $\begin{array}{l}\text { A BRIEF OVERVIEW OF THE EXPERIMENTAL } \\
\text { FIREGROUND OPERATIONS } \ldots \ldots \ldots \ldots \ldots \ldots \ldots \ldots \ldots \ldots\end{array}$ \\
\hline
\end{tabular}

5.1 The Relation of Time-to-Task Completion and Risk ................27

5.2 Standards of Response Cover ............................................28

6 EXPERIMENTAL METHODS .................................................29

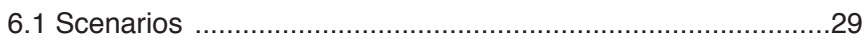

6.2 Participating Fire and Rescue Departments ..........................30

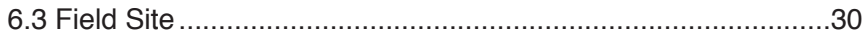

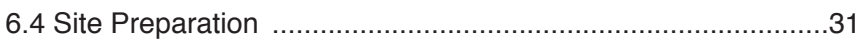

6.5 Instrumentation ............................................................... 33

6.6 Fire and Smoke Simulation ...............................................33

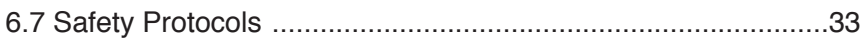

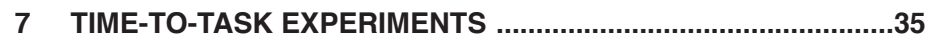

7.1 On-Scene Fire Department Tasks..........................................35

7.2 Determination of Full-Alarm Assignment.................................35

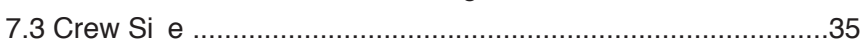

7.4 Number of Firefighters in the Experiments ..............................35

7.5 Department Participation .......................................................36

7.6 Officer and Crew Orientation ................................................37

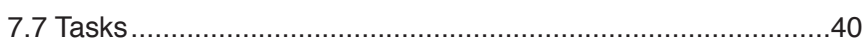

7.8 Data Collection: Standardi ed Control Measures .....................41

7.9 Task Flow Charts and Crew Cue Cards .................................41

7.10 Radio Communications..........................................................42

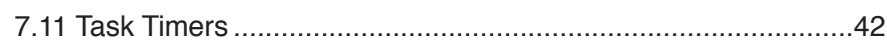

7.12 Search Buttons ................................................................... 43

7.13 Video Records .................................................................... 44

7.14 Crew Assignments ............................................................. 44

7.15 Response Time Assumptions ...............................................4

8 STAGES OF HIGH-RISE FIRE OPERATIONS ..........................48

8.1 Incident Command .............................................................. 48

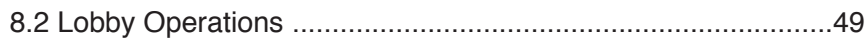

8.3 Staging and Rehabilitation on Floor 8 …...............................49

8.4 Logistics of Material Support (Stairs/Elevators) .....................50

8.5 Ambulance Transport for Fire Victims ....................................51

8.6 Floor 9, Floor 12, and Floor 13 Search and Rescue Operations ..52

8.7 Floor 10 Suppression Operations ........................................52

8.8 Floor 10 Search and Rescue Operations ...............................53

8.9 Floor 11 Suppression Operations .........................................59

8.10 Floor 11 Search and Rescue Operations ...............................59
9 ANALYSIS OF EXPERIMENTAL RESULTS ............................64

9.1 Time-to-Task Analysis ........................................................64

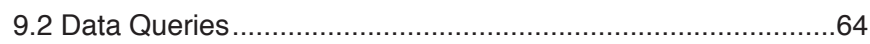

9.3 Statistical Methods - Time to Task ......................................64

9.4 Regression Analyses ..........................................................65

9.5 Search Buttons ...............................................................6

9.6 Measurement Uncertainty ...................................................68

10 TIME-TO-TASK RESULTS ......................................................69

10.1 How to Interpret Time-to-Task Graphs ....................................69

10.2 Overall Scene time and Crew Si e .......................................70

10.3 Advance Attack Line................................................................

10.4 Advance Second Line ....................................................... 72

10.5 Fire Out ........................................................................ 73

10.6 Search and Rescue 10th Floor ........................................73

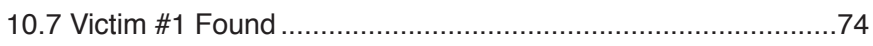

10.8 Victim \#1 Rescue .............................................................. 74

10.9 Victim \#1 Descent ................................................................ 75

10.10 Advance Line Above the Fire (11th Floor) ............................75

10.11 Search and Rescue 11th Floor.........................................76

10.12 Victim \#2 Found .................................................................. 76

10.13 Victim \#2 Rescued .............................................................

10.14 Victim \#2 Descent ........................................................... 77

10.15 Simultaneous Operations by Crew Si e ..............................78

10.16 Summary of Regression Results .................................... 80

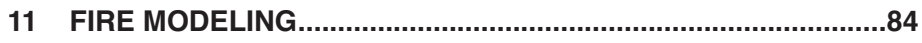

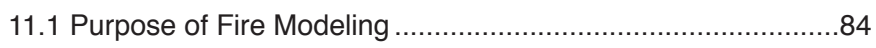

11.2 Research Question: Time to Untenable Conditions ................84

11.3 The NIST Fire Dynamics Simulator .....................................84

11.4 Development of the Design Fires for FDS Simulations ..........85

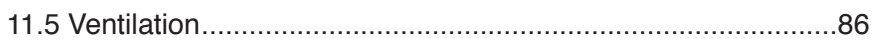

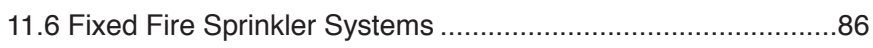

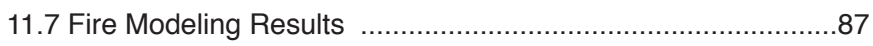

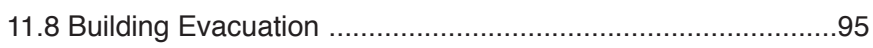

12 PHYSIOLOGICAL EFFECTS ON FIREFIGHTERS ...................96

13 STUDY LIMITATIONS ..........................................................97

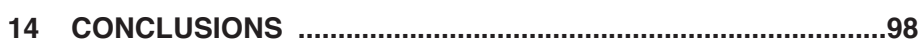

15 FUTURE RESEARCH ......................................................102

ACKNOWLEDGMENTS ….......................................................103

REFERENCES ….....................................................................104

APPENDICES

A DATA COLLECTION AND COMPANY PROTOCOLS FOR TIME-TO-TASK TESTS …...................................107

B REGRESSION COEFFICIENTS

C COMPARISONS USING REGRESSION RESULTS .........124

D DETAILED REGRESSION RESULTS ............................132

E CREW SIZE PERCENT COMPARISONS ........................138

F MEASUREMENT UNCERTAINTY …............................147

G DEVELOPMENT OF A DESIGN FIRE ..........................149 
Figure 1: High-rise building located at 223 23rd Street, Crystal City, VA............30 Figure 2: Office cubicle props on fire floor (10th) ...............................................31 Figure 3: Direction signage ........................................................................

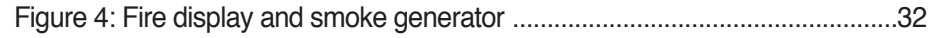
Figure 5: Fire /smoke conditions on 10th floor ..................................................32

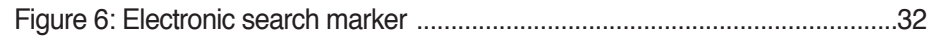

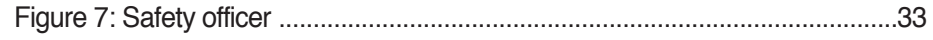
Figure 8: Crew rehab Crew hold area .....................................................34 Figure 9: Crew rehab Front of building .......................................................34

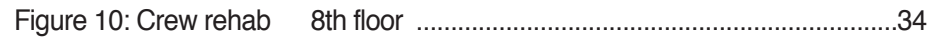

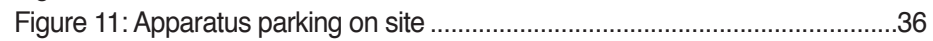

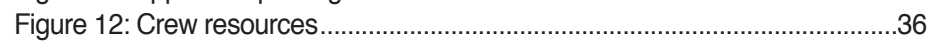

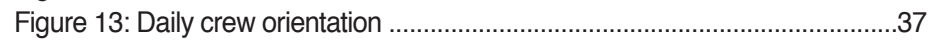

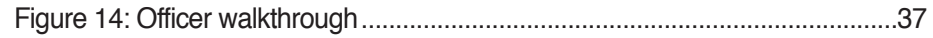

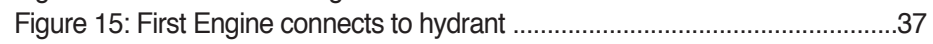
Figure 16: Driver connects to Siamese ….......................................................

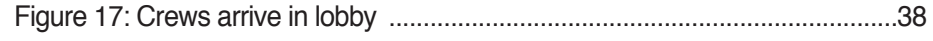

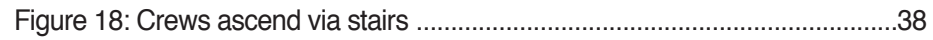

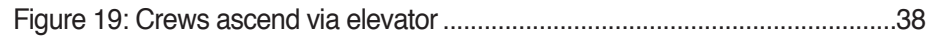
Figure 20: Firefighters assist in moving the attack line and second line ............38 Figure 21: Establish line above the fire (floor 11) ..............................................38 Figure 22: Search crew proceeds to fire floor .....................................................39 Figure 23: Search and rescue Fire floor ......................................................39 Figure 24: Search and rescue Floor above fire .............................................39 Figure 25: Victim located on floor above fire ....................................................39 Figure 26: Victim descends via stairs .........................................................39 Figure 27: Victim removed from building ........................................................39

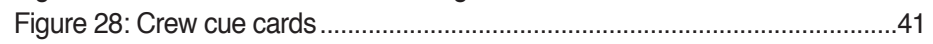
Figure 29: Task timers located throughout the high-rise structure .....................42 Figure 30: Task marker bands worn by firefighters during the experiments.......42 Figure 31: Search button locations on the fire floor. ...........................................43 Figure 32: Search button locations on the floor above the fire (floor 11). .........44 Figure 33: Timing for firefighter response and performance ..............................45 Figure 34: Route from each fire station to the fire location ...................................4

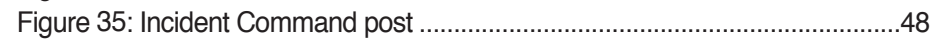

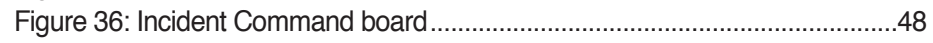
Figure 37: Lobby Control / Accountability ..........................................................49 Figure 38: Lobby Control / Elevator access ......................................................49

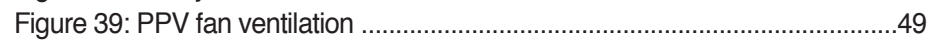

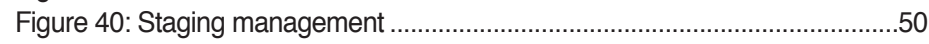

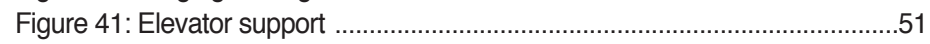
Figure 42: Stairwell support .......................................................................51 Figure 43: Ambulance standby at high-rise structure fire .................................51 Figure 44: EMS personnel exiting with victim rescued using a wheeled stretcher by elevators. . .51 Figure 45: EMS personnel carry victim down stairs using a stair chair device ..51 Figure 46: Pathway of hose lines from core through the fire area. The double dash lines represent the need for both hose lines to be present. ........................53 Figure 47: Schematic of the fire floor .............................................................54 Figure 48: Fire floor search by 3-person crew ................................................56

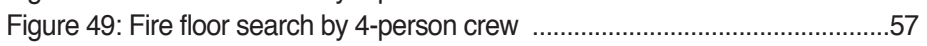
Figure 50: Fire floor search by 5- or 6-person crew .........................................58

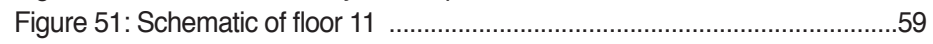

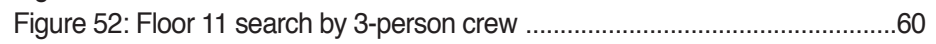

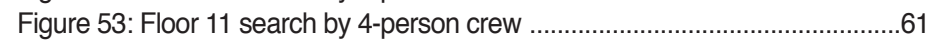
Figure 54: Floor 11 search by 5-person crew ..................................................62 Figure 55: Floor 11 search by 6-person crew .................................................62 Figure 56: Summary of time-to-task analysis ....................................................64 Figure 57: Time to complete all tasks by crew si e ..........................................65 Figure 58: Time to complete by crew si e and ascent mode ..............................65 Figure 59: Time to complete all tasks by crew si e and alarm si e ....................65 Figure 60: Start and end timing of critical tasks by crew si e.............................66 Figure 61: Button presses as a function of time on the fire floor for a 3-person crew using the stairs.
Figure 62: Button presses as a function of time on the fire floor for a 4-person crew using the stairs. .......................................................................67

Figure 63: Button presses as a function of time on the fire floor for a 5-person

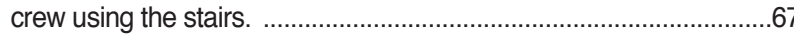

Figure 64: Button presses as a function of time on the fire floor for a 6-person crew using the stairs.

Figure 65: Example time-to-task graph .............................................................69 Figure 66: Average start and end times of critical tasks for a 3-person crew ....70 Figure 67: Average start and end times of critical tasks for a 4-person crew ....70 Figure 68: Average start and end times of critical tasks for a 5-person crew ....71 Figure 69: Average start and end times of critical tasks for a 6-person crew ....71 Figure 70: Advance Attack Line on Fire Floor .......................................................72 Figure 71: Advance Second Line on Fire Floor..................................................

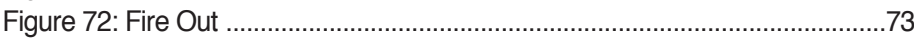
Figure 73: Search and Rescue on Fire Floor (10th) .........................................73

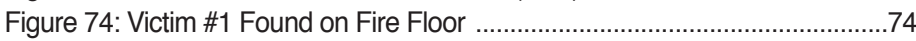
Figure 75: Victim \#1 Rescue Removed from IDLH atmosphere .....................74

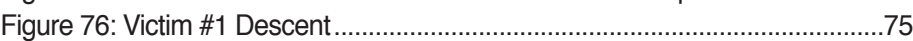
Figure 77: Advance Line on Floor Above the Fire (11th) ....................................75 Figure 78: Search and Rescue on Floor Above the Fire (11th) .........................76 Figure 79: Victim \#2 Found on Floor Above the Fire ........................................... Figure 80: Victim \#2 Rescued from Floor Above the Fire....................................77

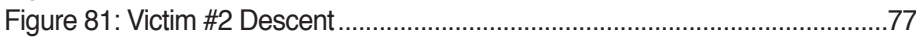

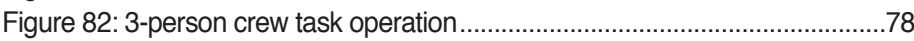

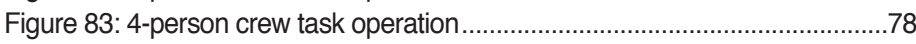

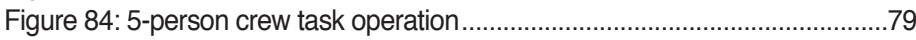
Figure 85: 6-person crew task operation ..........................................................79 Figure 86: All Tasks Complete comparing all three study variables....................80 Figure 87: Comparison of 3 high/4 low start, end, and duration times ..............82 Figure 88: Comparison of 4 high/5 low start, end, and duration times ...............82 Figure 89: Comparison of 5 high/ 6 low start, end, and duration times ..............82 Figure 90: Heat release rate versus time curve of a typical single cubicle fire from the Cook County Administration building compared to design curves of slow, medium, and fast growth (Madr ykowski et al. 2004).

Figure 91: Visuali ation of HRR for a medium growth fire for a 3-person crew using the stairs (left) and a 6-person crew using the elevators (right) at the time firefighters make entry to the floor.....................................87

Figure 92: HRR curves for the three growth fires for a 6-person crew taking the stairs. The vertical line represents the Water on Fire time and the start of firefighter suppression.

Figure 93: FED contours at an elevation of $3 \mathrm{ft}(0.9 \mathrm{~m})$ on the fire floor for a medium growth non-sprinklered fire at the time of firefighter entry. ..90

Figure 94: FED contours at an elevation of $3 \mathrm{ft}(0.9 \mathrm{~m})$ on the fire floor for a medium growth non-sprinklered fire at the time the search is complete.

Figure 95: FED area percentages of the fire floor at an altitude of $3 \mathrm{ft}(0.9 \mathrm{~m})$ at times of entry, victim rescue, and search complete for a

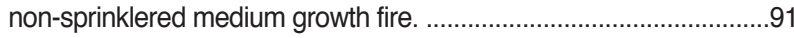

Figure 96: Crew si e of 3 using stairs ........................................................92

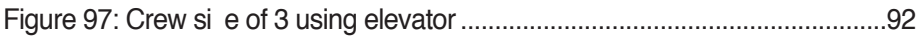

Figure 98: Crew si e of 4 using stairs .........................................................92

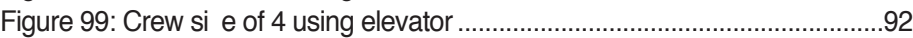

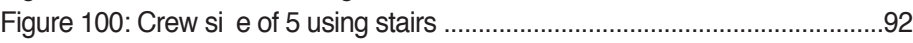

Figure 101: Crew si e of 5 using elevator .........................................................92

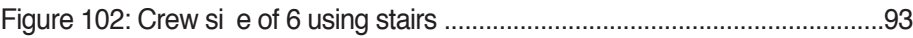

Figure 103: Crew si e of 6 using elevator .......................................................93

Figure 104: Heat release rate versus time of a typical single workstation fire from the Cook County Administration building (Madr ykowski et al. 2004).

Figure 105: Heat release rate versus time curve showing ignition of an adjacent cubicle from the Cook County Administration building experiments (Madr ykowski et al. 2004). 
Table 1: Sixteen unique experimental variations

Table 2: Actual crew si e for each experiment.

Table 3: Tasks and measurement parameters .....

Table 4: Go-to-work times calculated by GIS

Table 5: Critical tasks considered in analysis

Table 6: Average number of crews re . Average number of crews required to complete search and rescue on the fire floor for each crew si e...

.....67

Table 7: Average and standard deviation of time spent actively searching the fire floor as a function of crew si e. 68

Table 8: Relating FED values to percentage of population likely to be incapacitated and indicating coloring scheme for visuali ation.

Table 9: Time in minutes and seconds (MM:SS) for different growth rates to reach specified HRR values. . .85

Table 10: Comparison of the impact of crew si e and ascent method on average firefighter suppression time. ....86

Table 11: Sprinkler activation time and peak HRR value for slow, medium, and fast growth rate fires.

Table 12: Comparison of the impact of crew si e and ascent method on total heat release prior to suppression actions.

Table 13: FED at the time of victim rescue on the fire floor as a function of crew si e, ascent method, and fire growth rate.

. .89

Table 14: FED at $3 \mathrm{ft}(0.9 \mathrm{~m})$ elevation on the fire floor at the time of each button press, as a function of crew si e and ascent method for a medium growth rate fire without sprinklers.

. .93

Table 15: FED at $3 \mathrm{ft}(0.9 \mathrm{~m})$ elevation at the time of each fire floor button press, as a function of crew si e and ascent method for a medium growth rate fire with sprinklers.

Table 16: Total evacuation time as a function of occupant load and evacuation

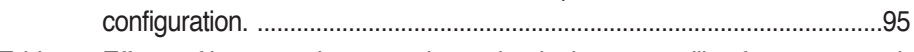

Table 17: Effects of incrementing crew si e on begin time, controlling for ascent mode and alarm si e; coefficients are in seconds. ...132

Table 18: Effects of incrementing crew si e on task duration time, controlling on ascent mode and alarm si e; coefficients are in seconds. 133

Table 19: Effects of incrementing crew si e on task end time, controlling for ascent mode and alarm si e; coefficients are in seconds.

Table 20: Contrasting high response to low response with smaller crew si es for begin times; coefficients are in seconds. ...135

Table 21: Contrasting high response to low response with smaller crew si es for duration times; coefficients are in seconds.

.136

Table 22: Contrasting high response to low response with smaller crew si es for end times; coefficients are in seconds. .........................................................137

Table 23 Times for each crew si e to complete a task...........................................138

Table 24 Table 25: Array of time differences. ............................................................138

Table 25 Calculations of time-to-task percentages .................................................138

Table 26 Sample time-to-task percentage table ......................................................139

Table 27 Percentage Table for Overall Time-to-Task Completion by Crew Si e ....139

Table 28 Percentage Table for Overall Time-to-Task Completion by Crew Si e and Stair/Elevator Ascent Mode 139

Table 29 Table for Overall Time-to-Task Completion by Crew Si e and High/Low Alarm Si e . 140

Table 30 Percentage Table for Advance Attack Line begin time .............................140

Table 31 Percentage Table for Advance Attack Line Duration.................................140

Table 32 Percentage Table for Advance Attack Line end time .................................140

Table 33 Percentage Table for Advance Second Line begin time ...........................141

Table 34 Percentage Table for Advance Second Line duration.................................141

Table 35 Percentage Table for Advance Second Line end time ..............................141

Table 36 Percentage Table for Fire Out begin time ..............................................141

Table 37 Percentage Table for Fire Out duration) ....................................................141

Table 38 Percentage Table for Fire Out end time ..................................................141
Table 39 Percentage Table for Search and Rescue 10th Floor begin time ............142

Table 40 Percentage Table for Search and Rescue 10th Floor duration ................142

Table 41 Percentage Table for Search and Rescue 10th Floor end time ................142

Table 42 Percentage Table for Victim \#1 Found begin time .....................................142

Table 43 Percentage Table for Victim \#1 Found duration .........................................142

Table 44 Percentage Table for Victim \#1 Found end time ......................................142

Table 45 Percentage Table for Victim \# 1 Rescue begin time ...................................143

Table 46: Percentage Table for Victim \# 1 Rescue duration ...................................143

Table 47: Percentage Table for Victim \# 1 Rescue end time ....................................143

Table 48: Percentage Table for Victim \#1 Descent begin time .............................143

Table 49: Percentage Table for Victim \#1 Descent duration ....................................143

Table 50: Percentage Table for Victim \#1 Descent end time .....................................143

Table 51: Percentage Table for Advance Line Above the Fire begin time..................144

Table 52: Percentage Table for Advance Line Above the Fire duration ...................144

Table 53: Percentage Table for Advance Line Above the Fire end time ..................144

Table 54: Percentage Table for Search and Rescue 11th Floor begin time ............144

Table 55: Percentage Table for Search and Rescue 11th Floor duration ................144

Table 56: Percentage Table for Search and Rescue 11th Floor end time.................144

Table 57: Percentage Table for Victim \#2 Found begin time .................................145

Table 58: Percentage Table for Victim \#2 Found duration ........................................145

Table 59: Percentage Table for Victim \#2 Found end time .......................................145

Table 60: Percentage Table for Victim \#2 Rescue begin time ...................................145

Table 61: Percentage Table for Victim \#2 Rescue duration .......................................145

Table 62: Percentage Table for Victim \#2 Rescue end time ....................................145

Table 63: Percentage Table for Victim \#2 Descent begin time .................................146

Table 64: Percentage Table for Victim \#2 Descent duration ..................................146

Table 65: Percentage Table for Victim \#2 Descent end time ....................................156

Table 66: Summary of Measurement Uncertainty ...............................................147

Table 67: Fuel properties and product yields for fuel components of design fire (Tewarson 2008). .149

Table 68: Peak heat release rate values from common objects ............................149 


\begin{tabular}{|c|c|}
\hline ALS & Advanced Life Support \\
\hline ASME & American Society of Mechanical Engineers \\
\hline $\mathrm{BC}$ & Battalion Chief \\
\hline BRAC & $\begin{array}{l}\text { Defense Base Closure and Realignment } \\
\text { Commission }\end{array}$ \\
\hline $\mathrm{DAQ}$ & Data Acquisition System \\
\hline ELVAC & Elevator evacuation model \\
\hline EMS & Emergency Medical Service \\
\hline FDNY & Fire Department of New York \\
\hline FDS & Fire Dynamics Simulator \\
\hline FED & Fractional effective Dose \\
\hline FF & Firefighter \\
\hline GJ & Gigajoules \\
\hline HAZMAT & $\mathrm{Ha}$ ardous materials \\
\hline HRR & Heat release rate \\
\hline IAFF & International Association of Fire Fighters \\
\hline IC & Incident Command \\
\hline ICS & Incident Command System \\
\hline IDLH & Immediately Dangerous to Life or Health \\
\hline IRIC & Initial Rapid Intervention Crew \\
\hline MW & Megawatts \\
\hline NFPA & National Fire Protection Association \\
\hline NFFF & National Fallen Firefighters Foundation \\
\hline NIST & National Institute of Standards and Technology \\
\hline PPV & Positive Pressure Ventilation \\
\hline Rehab & Rehabilitation area \\
\hline $\mathrm{RIC}$ & Rapid Intervention Crew \\
\hline RIT & Rapid Intervention Team \\
\hline SCBA & Self-Contained Breathing Apparatus \\
\hline SFPE & Society of Fire Protection Engineers \\
\hline SME & Subject Matter Experts \\
\hline
\end{tabular}


O verall, the results of this study show that the number of fire service crew members in each company responding to a fire in a 30,000 square foot, thirteen-story structure had a dramatic effect on the crew's ability to protect lives and property. This conclusion can be summarized in three principal parts.

First, when responding to a medium growth rate fire on the 10th floor, 3-person crews ascending to the fire floor confronted an environment where the fire had released $60 \%$ more heat energy than the fire encountered by the 6-person crews doing the same work. Unfortunately, larger fires expose firefighters to greater risks and are more challenging to suppress.

Second, larger fires produce more risk exposure for building occupants. In general, occupants being rescued by smaller crew sizes and by crews that used the stairs rather than the elevators were exposed to a significantly greater dose of toxins from the fire. While the exact risk exposure for an occupant will depend on the fire growth rate, their proximity to the fire, and the floor on which the fire is located, it is clear that on-scene deployment decisions can have a dramatic impact in determining the fate of building occupants.

Third, the study confirmed that a properly engineered and operational fire sprinkler system drastically reduces the risk exposure for both the building occupants and the firefighters. While this has been well understood for many years and most new high-rise buildings are constructed with fire sprinkler protection, NFPA estimates that 41 percent of U.S. high-rise office buildings, 45 percent of high-rise hotels, and 54 percent of high-rise apartment buildings are not equipped with sprinklers. Moreover, sprinkler systems fail in about one in 14 fires. Thus, fire departments should be prepared to manage the risks associated with unsprinklered high-rise building fires.

High-rise firefighting operations are considered high-hazard scenarios ${ }^{2}$ because of the potential for extremely large fires and the potentially large number of building occupants who may be exposed to the resulting heat and smoke. Fires that are not contained by sprinklers or other fire protection measures may grow to consume large portions of available floor area due to the significant time that it takes for firefighters to reach and suppress the fire, as well as the large quantities of fuel load typical of modern office spaces.

Additionally, high-rise buildings may have large floor areas and many floors at or above the fire that need to be searched for possible victims or occupants requiring assistance. Searching the fire floor is typically conducted in high heat and low visibility conditions due to the proximity of the fire. The remaining floors above the fire can take substantial resources and time to fully search.

Together, the tasks and hazards typical of the high-rise fireground combine to form a substantial operational challenge typical of the high-hazard class of response scenarios.

Firefighting continues to be a hazardous profession; the National Fire Protection Association (NFPA) reports over 70,000 firefighter injuries annually (Karter, 2012), with many occurring on the fireground. Residential fires, as examined in the NIST Report on Residential Fireground Experiments (Averill et al., 2010), typically dominate the fire loss statistics (property loss, civilian injuries and deaths, and firefighter injuries and deaths) due primarily to their frequency of occurrence. Independent of frequency, however, the residential fireground is considered a low hazard scenario in NFPA 1710, the national consensus standard for fire service deployment. High-rise fires, which are the subject of this report, pose unique operational challenges to fire service response, and represent a high hazard life safety scenario. Key challenges include the sheer scope and scale of conducting search and rescue operations, difficulty moving people and equipment vertically to the fire area, the size of the fire based on the time it takes to initiate firefighting operations, and logistical management of the significant number of firefighters and equipment required to complete critical tasks.

Despite the apparent hazards however, there are no scientifically-based tools available to community and fire service leaders to assess the effects of fixed sprinkler systems, fire suppression equipment or resource deployment and staffing decisions. Though community and fire service leaders have a qualitative understanding of the effect of certain resources allocation decisions, there is a universal lack of a sound basis for quantifying the total effects.

The purpose of conducting a series of high hazard, high-rise fireground experiments is to provide quantitative data on the effect of crew size, effective firefighting force assembly time, and vertical-response time on the intervention capability, effectiveness and safety of firefighters during a working high-rise, high risk building fire on an upper floor. The results of the project will inform the NFPA 1710 Technical Committee regarding the optimal crew size and total effective firefighting force for a first alarm assignment to a working high-rise or other high hazard fire. These high hazard response scenarios will also "bracket" the spectrum of fire response, acting as a complement to recently published low hazard Residential Fireground Deployment Study (Averill et al., 2010).

Satisfying several research objectives, this report focuses on the results of the high hazard high-rise fireground experiments. For these experiments, two stages of research were completed: (a) fireground time-to-task experiments in a 13 story high-rise building using simulated fire and smoke conditions, and (b) computer fire modeling to estimate the tenability conditions in the building as a function of the firefighter activities determined in part (a).

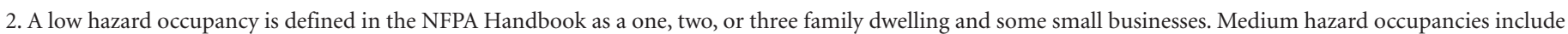
apartments, offices, mercantile and industrial occupancies not normally requiring extensive rescue or firefighting forces. High hazard occupancies include schools, hospitals, nursing homes, explosive plants, refineries, high-rise buildings, and other high life hazard or large fire potential occupancies. 
The following research question structured and guided the experimental design:

\section{In the event of a fire on an upper floor of a high rise building, what is the minimal fire service deployment configuration necessary to mitigate the event effectively and safely?}

More specifically, data were sought to answer the following questions about the time required to carry out tasks on the fireground under a variety of conditions.

\section{Time-to-Task Research Questions}

1) How do crew si e, ascent mode (stairs vs. elevator) and si e of full alarm assignment (i.e., alarm si e low versus high) affect overall (i.e., start to completion) response timing?

a. How do variations in crew si e affect overall response timing?

b. How much does ascent mode affect overall timing?

c. How much does the si e of a full alarm assignment affect overall response timing?

d. How do overall response times vary by combinations of crew si e, ascent, and alarm si e?

\section{Fire Modeling Research Questions}

1) How do performance times resulting from different combinations of crew si e, alarm si e, vertical ascent, and fixed fire sprinkler systems affect the development of standard fire growth scenarios?

2) How do crew si e, alarm si e, vertical ascent, and fixed fire sprinklers affect the resulting interior tenability on the fire floor?
Based upon the research questions, 16 unique scenarios were considered, as shown in the table below. Each of the scenarios assumes a fire on the 10th floor of a 13 story building with an open floor plan configuration measuring $100 \mathrm{ft}$ by $300 \mathrm{ft}(30 \mathrm{~m}$ by $91 \mathrm{~m})$, for an area of $30,000 \mathrm{sq} \mathrm{ft}\left(2800 \mathrm{~m}^{2}\right)$ per floor $)$. The fuel load is a standard cubicle configuration, with open-wall material, typical desk and drawer furniture, computers, printers and office chairs. Each scenario included two victims; one located on the fire floor and one located on the floor above the fire (Floor 11).
Scenario 1: High-alarm assignment ${ }^{3}$ with 6-person crews dispatched to the building. Two fire service access elevators are available for fire service use. (Best case)

Scenario 3: High-alarm assignment with 5-person crews dispatched to the building. Two fire service access elevators are available for fire service use.

Scenario 5: High-alarm assignment with 4-person crews dispatched to the building. Two fire service access elevators are available for fire service use.

Scenario 7: High-alarm assignment with 3 person crews dispatched to the building. Two fire service access elevators are available for fire service use.

Scenario 9: High-alarm assignment with 6-person crews dispatched to the building. Stairs are available for fire service use.

Scenario 11: High-alarm assignment with 5-person crews dispatched to the building. Stairs are available for fire service use.

Scenario 13: High-alarm assignment with 4-person crews dispatched to the building. Stairs are available for fire service use.

Scenario 15: High-alarm assignment with 3-person crews dispatched to the building. Stairs are available for fire service use.
Scenario 2: Low-alarm assignment ${ }^{3}$ with 6-person crews dispatched to the building. Two fire service access elevators are available for fire service use.

Scenario 4: Low-alarm assignment with 5-person crews dispatched to the building. Two fire service access elevators are available for fire service use.

Scenario 6: Low-alarm assignment with 4-person crews dispatched to the building. Two fire service access elevators are available for fire service use.

Scenario 8: Low-alarm assignment with 3-person crews dispatched to the building. Two fire service access elevators are available for fire service use.

Scenario 10: Low-alarm assignment with 6-person crews dispatched to the building. Stairs are available for fire service use.

Scenario 12: Low-alarm assignment with 5-person crews dispatched to the building. Stairs are available for fire service use.

Scenario 14: Low-alarm assignment with 4-person crews dispatched to the building. Stairs are available for fire service use.

Scenario 16: Low-alarm assignment with 3-person crews dispatched to the building. Stairs are available for fire service use. (Worst case)

3. Low Alarm Assignment is defined as 3 Engines, 3 Trucks, 2 Battalion Chiefs (with Aides), 2 Ambulances

High Alarm Assignment is defined as 4 Engines, 4 Trucks, 2 Battalion Chiefs (with Aides), 3 Ambulances 


\section{Primary Findings}

Of the 38 fireground tasks measured during the experiments, ${ }^{4}$ certain tasks were deemed critical, having he most significant impact on the success of firefighting operations. All differential outcomes described below are statistically significant at the $95 \%$ confidence level or better.

\section{Overall Time To Task Completion}

Overall scene time is the time that firefighters are actually engaged in tasks on the scene of a structure fire. During the experiments, this time included all operational tasks with the exception of overhaul ${ }^{5}$ and salvage ${ }^{6}$. The time to completion of all tasks decreases as crew size increases. On average, 3-person crews took nearly an hour to complete their fire response, while crews of 6 firefighters required a mean time of just under $40 \mathrm{~min}$ for completion. The performance of crews sized 4 and 5 were in-between, with crew size 5 taking about 2 min longer than crew size 6 , and crew size 4 taking about 9 min longer than crew size 5 but $12 \mathrm{~min}$ less than crew size 3 . Therefore, the time to complete all task times are substantially reduced for crew size of 6 compared to 5, 5 compared to 4 , and 4 compared to 3 .

\section{Advance Attack Line}

As firefighters engage on a fireground, putting water on the fire is one of the most important tasks. Extinguishing the fire is necessary to reduce the continuously escalating risks from fire and the toxic products of combustion. Before water can be put on a fire, however, a hose line must be stretched from the standpipe in the stairwell to the compartment where the fire is burning. In a more specific analysis comparing each crew size to a 3-person crew, the time differences increase as crew size increases. From the initiation of on-scene firefighting activities, a 3-person crew took $1 \mathrm{~min} 43 \mathrm{~s}$ (8.5\%) longer than a 4-person crew to stretch the hose line. A 3-person crew took 2 min $47 \mathrm{~s}(13.9 \%)$ longer than a 5-person crew to complete the same task. Finally, the most notable comparison was between a 3-person crew and a 6-person crew, with a $4 \min 28 \mathrm{~s} \mathrm{(22.3 \% )} \mathrm{difference} \mathrm{in} \mathrm{task} \mathrm{completion}$ time.

\section{Advance Second Line}

The size of the fire required two $2 \frac{1}{2}$ inch lines to fully suppress; therefore a second hose line had to be advanced from the standpipe in the stairwell to the fire. A 3-person crew took 4 min $4 \mathrm{~s}(17.4 \%)$ longer than a 5 -person crew to stretch the second line. A 4-person crew took 2 min $43 \mathrm{~s}$ (12.3\%) longer than a 5 -person crew to complete the same task. Finally, the most notable comparison was between a 3-person crew and a 6-person crew, with a 5 min $38 \mathrm{~s}(24.1 \%)$ difference in task completion time.

\section{Fire Out}

Extinguishing the fire out is critical to reducing risk to both firefighters entering the structure and to trapped occupants. Fire Out, in the study, was defined as having both the attack line and the second hose line in place. There was a $2 \mathrm{~min} 14 \mathrm{~s}$ difference $(8.1 \%)$ in the Fire Out time between the 3-and 4-person crews. There was an additional $1 \mathrm{~min} 15 \mathrm{~s}$ difference $(5.0 \%)$ in the Fire Out time between the 4 - and 5-person crews. (i.e., 5-person crews extinguished the fire $3 \mathrm{~min} 29 \mathrm{~s}$ faster than 3-person crews). Finally, there was a 7 min $2 \mathrm{~s}$ difference $(25.6 \%)$ in the Fire Out time between the 3 - and 6-person crews.

\section{Search and Rescue 10th Floor}

The fire floor was an open floor plan and contained 96 cubicles. In the high hazard high-rise commercial building, the 4-person crew started the search $1 \mathrm{~min} 23 \mathrm{~s}(7.8 \%)$ faster and completed the search and rescue $11 \mathrm{~min} 21 \mathrm{~s}(18.4 \%)$ faster than the 3 -person crews. In the same structure, the 5-person crews started the search 1 min $4 \mathrm{~s}(6.7 \%)$ faster than the 4-person crews and 2 $\min 27 \mathrm{~s}(14.1 \%)$ faster than the 3-person crew. Additionally, 5 -person crews completed the search faster than the 4- and 3 -person crews by $13 \min 34 \mathrm{~s}$ (29\%) and $24 \min 55 \mathrm{~s} \mathrm{(42 \% )}$ respectively. Six-person crews had the best times, starting the search $1 \mathrm{~min} 19 \mathrm{~s}$ faster and completing the search 2 min $57 \mathrm{~s}$ $(8.0 \%)$ faster than 5-person crews. The greatest difference in search times was between 6- and 3-person crews. Six-person crews started the search on the fire floor 3 min $46 \mathrm{~s}$ (22\%) faster and completed the search 27 min $51 \mathrm{~s}$ (47\%) faster than the 3-person crews.

\section{Victim \#1 Rescued}

There was a single victim located on the fire floor that was found and rescued by all crews. A 5-person crew located the victim on the fire floor $25 \mathrm{~min} 19 \mathrm{~s}$ (50.6\%) faster than a 3-person crew and $12 \min 7 \mathrm{~s}(32.9 \%)$ faster than a 4-person crew. Likewise, a 6-person crew located the victim on the fire floor $28 \mathrm{~min} 33 \mathrm{~s}$ (57.1 \%) faster than the 3-person crew, $15 \min 21 \mathrm{~s}(41.7 \%)$ faster than the 4-person crew, and $3 \mathrm{~min} 14 \mathrm{~s}$ (13.2\%) faster than a 5 -person crew.

Four-person crews also removed the victim from the IDLH ${ }^{7}$ environment and facilitated the victim's exit from the building 13 min $11 \mathrm{~s}(25.1 \%)$ faster than a 3-person crew. Likewise, 5-person crews were able to remove the victim from the fire environment and get them out of the building 11 min 39 s (29.7\%) faster than the 4-person crews, while 6-person crews removed the victim from the environment and got them out of the building $14 \mathrm{~min}$ $58 \mathrm{~s}(38.1 \%)$ faster than the 4-person crews and $3 \mathrm{~min} 19 \mathrm{~s}(12.0 \%)$ faster than the 5-person crews. Additionally, victim descent occurred 4 min 42 s more quickly for crews using elevator rather than stairs to get the victim out of the building.

\footnotetext{
4. In addition to the tasks denoted in this report, salvage and overhaul operations on the fireground are major tactical priorities that require significant time and resources in order to minimize loss. These tasks however, were not included in the study scenario.

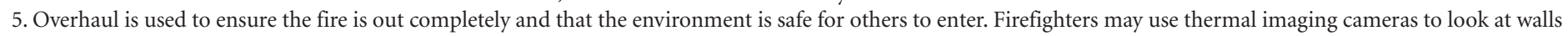
and ceilings to find hot spots, or they may tear out sections of walls and pull sections of ceilings to assure there has been no fire spread.

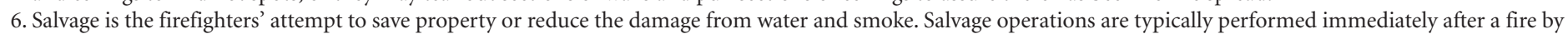
removing unharmed property from the fire area and covering it with canvas tarpaulin or other heavy protective material.

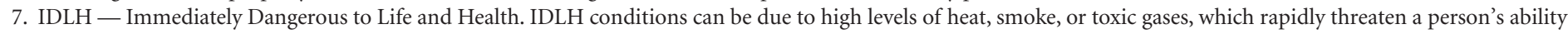
to effect their own escape.
} 


\section{Advance Line Above the Fire (11th Floor)}

In a high-rise structure, it is essential to place a hose line on the floor above the fire floor in the event of vertical fire spread. A 3 -person crew took 2 min $58 \mathrm{~s}(11.5 \%)$ longer than a 5-person crew to complete this task while, a 4-person crew took nearly 2 $\min (7.8 \%)$ longer than a 5-person crew. The most notable comparison was between a 3-person crew and a 6-person crew, with a $3 \mathrm{~min} 38 \mathrm{~s}(14.0 \%)$ difference in task completion time.

\section{Search and Rescue 11th Floor}

The floor above the fire was separated into a number of conference rooms and offices that had to be searched by each crew. During the experiments, the 4-person crews completed the search 9 min $31 \mathrm{~s}$ (18.6\%) faster than the 3-person crews. Meanwhile, the 5-person crews started a primary search/rescue 1 min $34 \mathrm{~s}(6.8 \%)$ faster than the 4-person crews and completed the search 2 min $37 \mathrm{~s}(6.3 \%)$ faster than the 4-person crews. In the same structure, the 6-person crews also started the search 1 min $30 \mathrm{~s}$ ( $6.6 \%)$ faster than the 4-person crews but completed the search $5 \min 8 \mathrm{~s}(12.3 \%)$ faster than the 4-person crews.

\section{Victim \#2 Rescued}

In addition to the victim on the fire floor, a second victim was located on the floor above the fire. Each crew operating on this floor was tasked with locating and rescuing the victim. The 5-person crews located the victim $17 \mathrm{~min} 23 \mathrm{~s}(34 \%)$ faster than the 3-person crews and 2 min $41 \mathrm{~s}(7.4 \%)$ faster than the 4-person crews. Likewise, 6-person crews located the victim on the floor above the fire $2 \min 48 \mathrm{~s}(7.7 \%)$ faster than the 4-person crews.

Four-person crews removed the victim from the IDLH environment and got them out of the building $14 \min 33 \mathrm{~s}(27.2 \%)$ faster than 3-person crews. Likewise, 5-person crews were able to remove the victim from the fire environment and get them out of the building $17 \min 9 \mathrm{~s}(32.1 \%)$ faster than 3-person crews and 2 $\min 36 \mathrm{~s}(6.7 \%)$ faster than the 4-person crews. Similarly, the 6-person crews rescued and removed the victim from the building 2 min 48 s (7.1\%) faster than 4-person crews. Additionally, victim descent occurred nearly 6 min more quickly for crews using elevator rather than stairs.

\section{Summary of Regression Analysis}

The effects of crew size, vertical ascent mode, and alarm size on the timing of critical firefighter tasks were studied using standard regression analysis. The analysis compared the times at which each task was started, the time to complete the task, and the time the task was completed. These timing values were given the labels begin time, duration, and end time, respectively.

\section{Crew Size}

Going from 3-person to 4-person crews had a large impact on advancing the attack line, advancing the second line, and begin times for search and rescue. Reductions in times to begin these tasks were in the range of $1 \mathrm{~min}$ to $2 \mathrm{~min}$. Going from 4 -person to 5 -person crews reduced the times to begin all critical tasks by 1 min to $2 \mathrm{~min}$. Increasing crew size from 5-person to 6-person crews showed significant reductions in begin time, just over 1 min, to advance the attack and second lines and for search and rescue on the fire floor (10th floor).

When assessing task end times and incrementally increasing crew size by a single firefighter (i.e., 3 to 4,4 to 5 , and 5 to 6 ), the largest time improvements are seen when going from crew size 3 to 4. As firefighter crews navigate the later tasks, the improvements cumulatively reach the $10 \mathrm{~min}$ to $15 \mathrm{~min}$ range. Very large time improvements are seen for the 10th Floor Search and Victim \#1 Rescue tasks (over $11 \mathrm{~min}$ ) when incrementing crew size from 4 to 5. The improvements in the times to complete all tasks are substantial ( $9 \mathrm{~min}$ to $12 \mathrm{~min}$ ) when incrementing crew size from 3 to 4 or from 4 to 5 .

\section{Fire Service Access Elevators}

All tasks were completed more than 4 min faster when the elevators were utilized compared to stairs. Begin times for nearly every critical task above ground level and nearly all end times were reduced compared to stair ascent. This is because using fire service access elevators dramatically reduced times associated with upward and downward transport of people or equipment. Using elevators to transport air bottles and other equipment from the lobby to Staging allowed completion of Establishment of Stairwell Support ${ }^{8}$ over 10 min more quickly than moving the equipment up the stairs. Additionally, the transport of both Victim \#1 and Victim \#2 from Staging to the outside of the building was faster when using the elevators (compared to the stairs), by $2 \min 41 \mathrm{~s}$ and $3 \mathrm{~min} 19 \mathrm{~s}$, respectively.

\section{Alarm Size}

Tasks assigned to engine 4 and truck 4, including Advancing the Line Above the Fire, Primary Search on Floor 11 and Rescuing Victim \#2, had begin time and end time reductions since those crews were dispatched in the first rather than the second alarm assignment.

\section{Combining Alarm Size and Crew Size}

Given the findings from the crew size analysis that adding one or two firefighters to a crew could generally achieve substantial task time decreases, a logical question is whether the meaningful benefits of a larger crew size could be realized by implementing a higher alarm response (additional engines and trucks) at a smaller crew size (e.g., high/4 compared to low/5). Another hypothesis is that a high response with lower crew size might yield similar results in task timing to that of a low response with higher crew size.

In summary, the analysis of the alarm response and crew size combinations suggests that the benefits of higher crew size exceed those of higher alarm assignment. Low alarm response with a higher crew size tends to be more favorable in critical task timings than the corresponding timings for a high alarm response with a crew size of one less firefighter.

\section{Combining Alarm Response and Ascent Mode}

In comparing different combinations of alarm response (high, low) and ascent mode (stairs, elevator), results contrasted several combinations of alarm size and ascent mode.

The alarm size had virtually no effect on critical task timings, with the exception of Primary Search of the Floor Above the Fire (Floor 11) and Victim \#2 Rescue. High alarm response realized a mean reduction in the range of $1 \mathrm{~min}$ to $4 \mathrm{~min}$ for these tasks. The Overall Time to Task Completion was also significantly smaller for high alarm response by $3 \mathrm{~min}$. No other task timing comparisons were statistically different.

In the elevator scenarios, high alarm response led to eight significantly lower timings than did a low alarm response. Results

8. Stairwell Support is also known as Ground Support, according to NFPA Standard 1561: Standard on Emergency Services Incident Management System. 
show 45 s reductions in begin time for Fire Out, Primary Search of Fire Floor 10, and Victim \#1 Found. Small reductions of just over a minute were noted in begin times for Search of the Floor Above the Fire (Floor 11) and Victim \#2 Found. Small reductions of $30 \mathrm{~s}$ to $2 \mathrm{~min}$ were also noted for times related to Advance the Line Above the Fire. No other task timing comparisons were statistically different.

\section{Fire Modeling Results}

In order to assess the hazard to occupants and firefighters as a consequence of different deployment configurations, computer fire modeling was performed. Three different 'standard' fires were simulated using the NIST Fire Dynamics Simulator (FDS) model. The three fires, characterized in the Handbook of the Society of Fire Protection Engineers (SFPE) (Hadjisophocleous and Mehaffey 2008) as slow, medium, and fast, ${ }^{9}$ grew non-linearly with time and had burning characteristics similar to the experimental results of typical office cubicle fires (Madrzykowski et al. 2004).

An Fractional Effective Dose (FED) $)^{10}$ value of 1.0 is defined as the toxic exposure at which $50 \%$ of the population would be incapacitated. The detailed probabilistic relationship between FED and the percentage of people incapacitated is unknown. However, an FED of 0.3 can be related qualitatively to a level that affects vulnerable members of the population, while an FED of 3.0 will incapacitate all but the least sensitive people.

Computer fire modeling using NIST's FDS demonstrated the effectiveness of a working fire sprinkler system for medium growth rate fires; the FED values remained well below a value of 0.3 for all crew sizes and ascent methods, while FED values for non-sprinkled structures typically exceeded 1.0 at some point during fire development. Thus, the overall hazard is greatly improved compared to the non-sprinklered fires for both firefighters and occupants. According to the NFPA, a working sprinkler system is $96 \%$ effective at controlling the growth and spread of fires in structures (NFPA 2006). Due to a number of high-profile fires in high-rise buildings, and considering their demonstrated effectiveness, sprinkler systems are often required in new high-rise buildings and many jurisdictions have required existing high-rise buildings to be retrofitted with sprinkler systems.

However, sprinkler systems are not installed or functional in all high-rise buildings. According to the NFPA (NFPA 2011), $41 \%$ of high-rise office buildings are not protected by sprinkler systems (compared to $25 \%$ of high-rise "care of sick" facilities, $45 \%$ of high-rise hotels and $54 \%$ of high-rise apartment buildings). Therefore, much of this report is focused on analysis of fire department deployment configurations responding to fires in an unsprinklered high-rise building.

Note, further, that sprinkler systems are designed to control fires, rather than suppress them. Fire department response is still required even in fully-sprinklered high-rises in order to extinguish the fire, to search for and rescue occupants requiring assistance, and to control the sprinklers (limiting water damage).
Additionally, NFPA estimates that sprinkler systems fail to operate in $7 \%$ of structure fires (one of every fourteen fires) primarily due to human error. "Two-thirds $(65 \%)$ of the sprinkler failures to operate were because the system had been shut off before the fire. Another one-sixth (16\%) occurred because manual intervention defeated the system, for example, by shutting off the sprinklers prematurely. Lack of maintenance accounted for $11 \%$ of the sprinkler failures and $5 \%$ occurred because the wrong type of system was present. Nearly all failures were therefore entirely or primarily problems of human action. Only $3 \%$ involved damage to system components." (NFPA 2006) Therefore, even when a large proportion of high-rise buildings within a jurisdiction are protected by sprinkler systems, the fire department should be prepared to deploy resources to hazards consistent with unsprinklered fires.

For unsprinklered scenarios, the time advantages gained by larger engine crew sizes and by using elevators versus stairs. allowing crews to complete tasks more quickly, improving the interior conditions, including temperature, visibility, and toxicity on the fire floor. For medium growth rate fires, firefighters entering the environment were found to encounter fires between $5 \mathrm{MW}$ to $11 \mathrm{MW}$ in size, depending on crew configuration and ascent method. This range in fire size can be visualized as the equivalent of two cubicles on fire for a 6-person crew versus five cubicles on fire for a 3-person crew.

Crew size and vertical ascent mode can significantly affect the likelihood of a successful rescue of victims on the fire floor. For victim rescue times discussed above, FED values in the cubicle where the victim was located ranged from 0.14 (6-person crew using the elevator) to 1.22 (3-person crew using the stairs). The FED, based on the biological effects of toxic gases, was used to assess the tenability of the fire environment. Consistently, smaller crew sizes resulted in greater exposure of victims and firefighters to combustion products compared to larger crew sizes.

Additionally, using the stairs delayed rescue and resulted in higher toxic exposures when compared to using the elevators.

\section{Limitations}

The scope of this study is limited to understanding the relative influence of deployment variables to the critical outcomes associated with a working high-rise structure fire. The applicability of the conclusions from this report to low hazard residential fires, outside fires, terrorism/natural disaster response, HAZMAT or other technical responses has not been assessed and should not be extrapolated from this report. Additionally, some important tasks, such as secondary search, property salvage, utility control, water mitigation, building overhaul, and returning firefighting equipment were not considered in these experiments. These tasks delay the return of units to service and should be considered in the design of fire department coverage. Other limitations that affect the interpretation of the data or conclusions are discussed in the report.

9. As defined in the SFPE Handbook, a fast fire grows to $1 \mathrm{MW}$ in $2 \min 30 \mathrm{~s}$; a medium fire grows to $1 \mathrm{MW}$ in $5 \mathrm{~min}$; a slow fire grows exponentially to $1 \mathrm{MW}$ in 10 min. A 1 MW fire can be thought of as a typical upholstered chair burning at its peak. A large sofa may produce a fire with a peak HRR value of 2 MW to 3 MW.

10. To characterize the accumulated hazard associated with inhalation of gases typical of combustion products, a time-integrated value known as the fractional effective dose (FED) was used. FED is an international standard, maintained by the International Standards Organization (ISO) and documented in ISO document 13571. FED is a probabilistic quantity used to estimate the impact of toxic gases on humans (ISO 2007). For this study, FED accounted for the effects of excess carbon monoxide and carbon dioxide inhalation and oxygen depletion. 


\section{Conclusions}

A total of 48 field experiments and complementary fire modeling simulations were conducted to determine the impact of crew size, alarm size and vertical response mode on firefighter safety and effectiveness at a high hazard high-rise commercial structure fire. This report quantifies the effects of changes to crew size, alarm size and/or vertical response mode for high hazard high-rise commercial firefighting operations in both sprinklered and non-sprinklered buildings. While resource deployment is addressed in the context of a high-rise structure type and high risk level, it is recognized that public policy decisions regarding the cost-benefit of specific deployment decisions are a function of many factors including geography, available resources and community expectations, as well as local hazards and risks. Though this report contributes significant knowledge to community and fire service leaders in regard to effective resource deployment for fire suppression, other factors contributing to policy decisions are not addressed.

The results provide a technical basis for the effectiveness of company crew size, alarm size and vertical response mode to be added to NFPA Standard 1710. The results also provide valid measures of total effective response force assembly on scene for high-rise fireground operations, as well as the expected performance of time-to-critical-task measures for high hazard high-rise commercial structure fires. Additionally, the results provide tenability measures associated with the occupant exposure rates to the range of fires considered by the fire model. The results of the project will also inform code provisions in the national model building codes which require fire service access elevators in new construction over $120 \mathrm{ft}(36 \mathrm{~m})$.

Future research should extend the findings of this report in order to quantify the effects of crew size and apparatus arrival times for moderate/medium hazard or other high hazard events, such as fires in mercantile establishments consisting of a row of stores and restaurants, warehouse facilities, responses to large-scale non-fire incidents, or technical rescue operations. 


\section{Background}

$\mathrm{H}$ igh-rise buildings present a unique threat to the fire service. Multi-floor fires such as the Interstate Building Fire, One Meridian Plaza Fire, World Trade Center collapse, Cook County Administration Building Fire, and Deutsche Bank Building Fire each represent serious challenges to operational capabilities of a modern fire department. Unfortunately, fire and city officials currently lack quantitative data to support specific resource deployment configurations or building code requirements germane to high-rise response effectiveness.

The National Fire Protection Association (NFPA ${ }^{\circledR}$ ) classifies high-rise buildings as high hazard occupancies in $\$$ A.3.3.28 of NFPA $1710^{\circledR 11}$, Standard for the Organization and Deployment of Fire Suppression Operations, Emergency Medical Operations, and Special Operations to the Public by Career Fire Departments. In \$5.2.3.1.2 of NFPA 1710, the standard further specifies a minimum staffing of "five or six on-duty personnel" per apparatus for high hazard responses. However, NFPA 1710 does not specify a minimum deployment configuration (either apparatus or total number of personnel) for high-rise building fires. Rather, the standard includes a performance statement in \$5.2.4.2.3: "Fire departments that respond to fires in high-, medium-, or low-hazard occupancies that present hazards greater than those found in the low-hazard occupancy described [above] shall deploy additional resources on the initial alarm."

According to NFPA (Hall 2011), an annual average of 15,700 structure fires were reported in high-rise buildings between 2005 and 2009. The annual loss associated with these fires included 53 civilian deaths, 546 civilian injuries, and more than \$235 million in direct property damage. Office buildings, hotels, apartment buildings, and health care facilities accounted for nearly half of these high-rise fires. During the same span of time, most high-rise building fires began no higher than the 6th floor, while approximately one-third of them began on the 7th floor or higher.

11. NFPA ${ }^{\circledR}$ is a registered trademark of the National Fire Protection Association, Quincy, MA 02169. National Fire Protection Standard 1710 Standard for the Organization and Deployment of Fire Suppression Operations, Emergency Medical Operations, and Special Operations to the Public by Career Fire Departments contains minimum requirements relating to the organization and deployment of fire suppression operations, emergency medical operations, and special operations to the public for career fire departments. The requirements address functions and objectives of fire department emergency service delivery, response capabilities, and resources. The purpose of this standard is to specify the minimum criteria addressing the effectiveness and efficiency of the career public fire suppression operations, emergency medical service, and special operations delivery in protecting the citizens of the jurisdiction and the occupational safety and health of fire department employees. At the time of the experiments, the 2010 edition of NFPA 1710 was the current edition. 
D espite the magnitude of the fire problem in the United States, there are no scientifically based tools available to community and fire service leaders to assess the effects of prevention, fixed sprinkler systems, firefighting equipment, or resource deployment and staffing decisions. Presently, community and fire service leaders have a qualitative understanding of the effect of certain resource allocation decisions. For example, a decision to double the number of firehouses, apparatus, and firefighters would likely result in a decrease in community fire losses, while cutting the number of firehouses, apparatus, and firefighters would likely yield an increase in the community fire losses, both human and property. However, decision makers lack a sound basis for quantifying the total economic impact of enhanced fire resources on the number of firefighter and civilian lives saved and injuries prevented.

Studies on adequate deployment of resources are needed to enable fire departments, cities, counties, and fire districts to design an acceptable level of resource deployment based upon community risks and service provision commitment. These studies will assist with strategic planning and municipal and state budget processes. Additionally, as resource studies refine data collection methods and measures, both subsequent research and improvements to resource deployment models will be afforded a sound scientific basis.

High-rise fires represent an extraordinary challenge to fire departments and are some of the most challenging incidents a fire department encounters. High-rise buildings may hold thousands of people above the reach of fire department aerial devices, and the chance of rescuing victims from the exterior is greatly reduced once a fire has reached flashover.

High-rise buildings were once located exclusively in larger cities, but today they are commonly found in small and mid-sized communities. Even if a department does not respond to a high-rise building at present, it may in the future as urban sprawl continues. The risk to firefighters and occupants increases in proportion to the height of the building and the height of the fire above grade level (Klaene, 2007). Once firefighters are operating above the reach of aerial devices, the only viable means of egress is the interior stairs; extra protection afforded by laddering the building is not possible. Therefore, a sound fire department deployment strategy, effective operational tactics, and engineered fire protection systems cannot be separated from firefighter safety. In attacking a fire in a high-rise building, as in any structure fire, engine company and truck company operations must be coordinated.

A critical variable in high-rise fire operations is the availability of reliable elevators. If firefighters can safely use the elevators to move people and equipment, fire-ground logistics may be significantly improved. When the fire is located several floors above ground level, there is a strong inclination to use the elevators. However, fire service access elevators (engineered to operate in a building during a fire emergency and complying with prescriptive building code requirements and the American Society of Mechanical Engineers (ASME) A 17.1 safety standard for elevators) may not be available in all buildings. Therefore, adequate stairways are necessary for firefighters to transport equipment and reach the fire floor for suppression. Moving supplies and staff up 10,20,30, or more stories is an arduous task. If it is not properly managed, firefighters may be exhausted and unable to fight the fire or rescue trapped occupants. Additionally, joint use of stairways by firefighters moving upward and occupants attempting to evacuate may increase the overall evacuation time of the occupants, as well as delay the firefighters' efforts to begin critical tasks such as fire suppression or search and rescue operations. 


\section{A Review of Important Literature}

$\mathrm{H}$ igh-rise buildings were once found exclusively in urban cities, however today they are commonly found in small and mid-sized suburban communities as well. In many cases high-rise buildings in suburban areas are newer, shorter, and protected by automatic sprinkler systems. Even if some fire departments do not respond to high-rise buildings at present, they likely will in the future in their own communities or as part of a mutual or automatic aid agreement.

The NFPA $101^{\circledR}$, Life Safety Code, 2012 edition $^{12}$ and the International Code Council-published International Building Code both define a high-rise structure as a building more than 75 $\mathrm{ft}(23 \mathrm{~m})$ in height, measured from the lowest level of fire department vehicle access to the bottom of the highest occupied floor. For the fire service, a high-rise structure may be defined as a building with sufficient height to exceed the fire department's exterior laddering capability (NFPA, 2012).

According to NFPA, there was an average of 15,700 reported structure fires in high-rise buildings every year between 2005 and 2009. The annual loss associated with these fires included 53 civilian deaths, 546 civilian injuries, and more than \$235 million in direct property damage (Hall, 2011).

A review of historic large loss fire incidents and the fire department response, as discussed below, revealed that the commercial office building occupancy is commonly subject to massive singular event losses. Consistent with the objective of the study, this report will investigate the low-frequency,

high-consequence office fire event that poses unusual hazards for building occupants and firefighters.

\subsection{Historic High-Rise Fires}

It is the intent of the study and this report to explore this low-frequency, high-consequence-type event that poses unusual hazards for both building occupants and firefighters. The paragraphs that follow describe a number of these large loss high-rise fires.

\section{Triangle Shirtwaist}

Near closing time on March 25, 1911, a fire broke out at the Triangle Waist Factory in New York City. In less than twenty minutes, 146 people were dead. Fire engulfed the 8th, 9th, and 10th floors of the Asch Building, occupied by the Triangle Waist Factory. The call to fire stations went out at 4:45 pm. Firefighters rushed to the scene but were unable to save workers trapped by locked doors and were unable to negotiate doors opening inward. Many occupants jumped to their deaths while others perished inside the burning building. The firefighters' ladders and hoses could not reach above the 6th floor. Rescue nets were insufficient to stop the fall of many victims jumping to flee the fire. Other occupants trying to escape perished in the collapse of the fire escape ladder, which did not reach the ground. The horror of the deaths led to numerous changes in occupational safety standards that currently ensure the safety of domestic workers today (Cornell, 2011).

\section{Winecoff Hotel}

In 1913, the Winecoff Hotel in Atlanta, Georgia was designed and built without sprinklers, fire escapes, or even an alarm system. The 15-story Winecoff Hotel had one central interior stairwell that extended from the lobby to the 15th floor, with one additional staircase that went from the 15th floor to the roof. Each floor had a corridor off the stairwell that led to guest rooms. On December 7, 1946, a fire was ignited in the hotel. Shortly after the start of the fire the only interior staircase was completely filled with smoke and intense heat. The hotel guests were trapped in their rooms with nowhere to go. Many guests never made it out of their rooms; they succumbed to smoke inhalation in their sleep or were killed by severe burns when the fire breached their doors. In total the fire killed 119 people as they leaped from windows, suffocated from smoke, or were burned alive. Several firefighters were injured after being knocked off their ladders by falling bodies.

The death toll in this fire was the impetus for national safety codes and strict enforcement. The response to this tragedy was so intense locally that public officials in several southern cities ordered all existing buildings be retrofitted and brought up to code within seven days or be shut down. Twenty-two engines and 12 ladder trucks eventually responded to the hotel fire (Winecoff Hotel, 2013).

\section{MGM Grand}

The 1980 fire in the MGM Grand started at the rear of the casino and eventually involved the entire casino floor measuring $150 \mathrm{ft} x$ $450 \mathrm{ft}$ (46 m x $140 \mathrm{~m}$ ). The MGM Grand, now Bally's in Clark County, Nevada, had 3400 registered guests at the time of the fire. Just after 7:00 on the morning of November 21, 1980, a fire broke out in a restaurant known as The Deli. The Clark County Fire Department was the first agency to respond. Other agencies that responded included the North Las Vegas Fire Department, Las Vegas Fire, and Rescue and the Henderson Fire Department.

Fire spread across the areas of the casino in which no fire sprinklers were installed. Smoke spread into the hotel tower. A total of 85 people were killed and 650 injured, including guests, employees, and 14 firefighters. While the primary damage was on the second floor casino and adjacent restaurants, most of the deaths were on the upper floors of the hotel, caused by smoke inhalation. Openings in vertical shafts (elevators and stairwells) and seismic joints allowed toxic smoke to spread to the top floor (Best, 2013).

The disaster led to the general publicizing of the fact that during a building fire, smoke inhalation is a more serious threat than flames. Seventy-five people died from smoke inhalation and carbon monoxide poisoning, four from smoke inhalation alone, three from burns and smoke inhalation; only one person died from burns alone, and one person died from massive skull trauma caused by jumping from a high window (Clark County FD).

The death toll, 85 people, made the MGM fire the second most deadly hotel fire in U.S. history (Sanders, 2007) — second only to the 119 who died in the Winecoff Hotel in Atlanta, Georgia, in 1946.

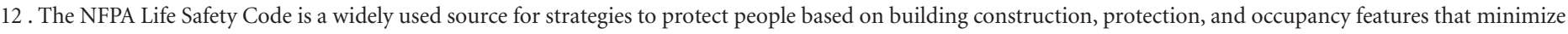
the effects of fire and related hazards in new and existing structures. 


\section{First Interstate}

A fire in the First Interstate Bank Building in Los Angeles on May 4, 1988 destroyed four floors of the 62-story building and heavily damaged a fifth floor. The First Interstate fire burned floors 12 to 15 and was stopped at the 16th floor. Fifty-one floors above the fire were exposed to the fire and smoke conditions (LAFD, 1988).

The fire required a total of 64 fire companies and 383 firefighters and took $31 / 2$ hours to control. Despite the magnitude of this fire, only a maintenance worker who took an elevator to the fire floor to investigate the alarm was killed. In addition to the 383 firefighters, 17 paramedic ambulances, 17 private ambulances, and 2 hospital disaster teams were on scene to provide emergency medical services (EMS)(Sanders, 2007).

In 1973, the year construction was completed on the building, sprinklers were not required for office towers. A sprinkler system was $90 \%$ installed at the time of the fire, but the system was inoperative, awaiting the installation of water flow alarms. The fire was eventually contained at 2:19 am and caused \$50 million in damages (USFA, 2010).

\section{East 50th Street New York - Apartment Building Fire}

On January 11th 1988, a fire started in an office on the first floor of a 10-story predominantly residential high-rise. The building was not sprinklered and had no smoke alarms, no fire alarm system, and no emergency lighting or illuminated exit signs, but it was still considered to be within fire code due to the age of the building. FDNY firefighters were on scene 4 min after a call to 9-1-1. Six minutes later at 8:29 pm, a second alarm was called. Fire spread was undeterred due to a stairwell door left open on the fire floor. Heavy smoke quickly filled the stairwell and the floors above the fire. Crews arriving on the second alarm were committed to rescue operations. A third alarm was requested at 8:47 pm and a fourth at 9:15 pm. All units responding in these assignments were committed to rescue operations on the 9 stories above the fire. Even though interior crews had extinguished the fire, smoke spread throughout the building, creating heavy demand on rescue operations. At 9:42 pm a fifth alarm was requested and brought the total firefighting force to 200 personnel and 38 pieces of apparatus. Searches were finally complete at 10:16 pm, nearly two hours after initial crews were dispatched to the scene. A total of 4 civilians perished and 2 civilians and 5 firefighters were injured (USFA, 1988).

\section{Peachtree}

On June 30, 1989 at 10:30 am, a small fire occurred on the 6th floor of a 10-story office building in Atlanta, Georgia. The Atlanta Bureau of Fire Services received a call at 10:29 am as an automatic alarm originating at 1720 Peachtree Street, Northwest and dispatched a normal assignment of three engines and two trucks, an EMS unit, and a battalion chief. However, they soon received nearly 20 phone calls in succession reporting a serious fire on the 6 th floor and then dispatched an additional rescue unit and a squad.

Extreme heat on the 6th floor required frequent rotation of crews. The initial crew on the fire floor extinguished a large portion of the fire but was forced to call for relief due to low air supply.

The search for victims and survivors was complicated by the confusing layout of the open plan offices and the fact that almost all of the doors were locked and required extensive forcible entry.

The building was occupied, and 5 of the 40 occupants on the sixth floor were killed, while 6 others were rescued by firefighters using aerial ladders (Sanders, 2007). The first victim of the fire was discovered in the hallway but was not removed immediately due to the extent of his injuries. Of the 5 fire victims who did not survive, 3 were removed from the building and resuscitation efforts were attempted. A total of 23 civilians and 6 firefighters were injured (USFA, 1989).

\section{One Meridian Plaza}

The One Meridian Plaza fire in Philadelphia, Pennsylvania, occurred at 8:40 pm on Saturday evening, February 23, 1991. The fire began on the 22nd floor after linseed oil-soaked rags ignited a blaze that raged out of control for hours (NY Times, 1991). Philadelphia firefighters fought the blaze but struggled due to a lack of power in the skyscraper and insufficient water pressure from the building's standpipes. More than 316 firefighters responded in the 12-alarm fire, and 3 firefighters died after becoming disoriented by heavy smoke (Chubb, 1991).

Firefighting efforts inside One Meridian Plaza were abandoned due to fears the structure would collapse. The fire was brought under control once it reached the 30th floor, which was one of the few floors that had automatic sprinklers installed. The blaze seriously damaged the building, destroyed eight floors, and damaged neighboring buildings (Chubb, 1991). Because the fire occurred outside normal working hours, the threat to occupants was likely lessened.

\section{World Trade Center Bombing}

At 12:18 pm on February 26th 1993 a vehicle bomb ripped through the sub-grade garage levels of the World Trade Center (WTC) in Manhattan. The blast severely damaged critical building emergency system infrastructure, including emergency generators, sprinkler systems, stand pipe systems, and public address systems. Electrical power was lost in many areas of the Vista Hotel, as well as in both Tower 1 and 2. FDNY Engine 10 and Ladder 10, stationed near the site, felt the blast and self-dispatched, believing it to be a transformer explosion. Upon receiving numerous 9-1-1 calls, dispatchers sent an initial deployment of 3 engines, 2 ladders, 1 Rescue Unit, and 1 Battalion Chief to the WTC complex. On arrival, Ladder 10 noted that the garage doors at the entrance to a garage were misshapen. Engine 10 requested a full box alarm for a working fire, which added 3 engines, 2 ladders, 1 Battalion Chief, and 1 Deputy Chief to the scene.

Multiple additional alarms were called to intervene in multiple location fires including Tower 1 , Tower 2, and the Vista hotel located between the towers. The magnitude of the event expanded quickly. Due to the size of the Towers and the number of potential victims, each building required a separate command sector. Each sector reported directly to the incident command post. At the height of the incident there were 156 fire companies and 31 chiefs on scene. The event resulted in 6 civilian fatalities, 1042 injuries and a total of 16 alarm assignments dispatched to the scene (USFA, 1993).

\section{Regis Tower}

Regis Tower, at 750 Adams Avenue in Memphis, Tennessee, is an 11-story concrete-and-steel high-rise building constructed in 1964. Its overall dimensions are $140 \mathrm{ft}(43 \mathrm{~m})$ long and $50 \mathrm{ft}(15 \mathrm{~m})$ deep. At 02:05 am on April 11, 1994, the central station monitoring service for the Regis Tower called the Memphis Fire Department to report an alarm indicating a trouble alarm on the ninth floor. The normal response to a possible high-rise fire was 3 engine companies, 2 ladder companies, and a battalion chief. The first Engine arrived followed shortly by a truck at 02:08 am (Chubb, 1995). 
Upon arrival, firefighters encountered heavy smoke and fire on the ninth floor. Fire companies made several attempts to rescue occupants and extinguish the fire from the interior. The fire self-vented, and a heavy volume of fire was visible from the exterior. An elevated master stream was directed into the apartment of origin to knock down any visible fire. Unfortunately, the door between the apartment of origin and the hallway was open, and the exterior stream pushed the fire into the 9th floor corridor where firefighters were advancing toward the fire. As heat and smoke conditions immediately became worse on the 9th floor, firefighters were forced to retreat. Two firefighters became disoriented and had to be rescued. Two firefighters and two civilian occupants were killed (Sanders, 2007).

\section{Cook County Administration Building}

On October 17, 2003, a fire on the 12th floor of the 37-story Cook County Administration Building in Chicago, Illinois resulted in 6 civilian fatalities. The fire was believed to have originated in a closet within a $2629 \mathrm{sq} f t\left(244 \mathrm{~m}^{2}\right)$ suite of offices on the east side of the 12th floor. Due to fire-resistive building features, fire damage was contained to the office suite, but smoke and fire traveled to the entire 12th floor. The fire self-vented through eight exterior windows on the east side of the suite, creating the potential for floor-to-floor fire extension on the exterior.

Upon arrival, firefighters were faced with an intense fire that they were unable to extinguish from an interior hallway position. Elevated master streams were used to knock down the fire from the exterior. Interior hose streams were then redeployed to achieve final extinguishment. Tight compartmentalization and closed office doors contained the fire to the suite where it originated (Sanders, 2007).

\section{Deutsche Bank}

On August 18, 2007 two fire fighters lost their lives while operating at a 7 alarm fire at a high rise in Manhattan, New York City. The 41-story Deutsche Bank was undergoing deconstruction and asbestos abatement due to the damage sustained after the collapse of the World Trade Center Buildings on September 11th, 2001. In the process of deconstruction, the building layout included maze-like partitions installed to prevent the spread of asbestos during abatement. The standpipe system was also being disassembled.

A fire broke out at 3:30 pm on the 17th floor, with an initial first alarm being sent at 3:37 pm. After confirmation of the fire, a full alarm response was sent along with additional units. This response included 4 engines, 3 ladders, 2 Battalion Chiefs, 1 Division Chief, 1 Squad, 1 Rescue, 1 HazMat, 1 HazMat Battalion Chief, and Chief aides, for a total of 65 firefighters. A second alarm was requested 5 min later, to which an additional 12 Engines, 7 Trucks, 10 Battalion Chiefs, 1 Division Chief, 3 Deputy Chiefs, 1 High Rise Engine, 1 Field Communication Unit, 1 Squad, 1 Rescue with Battalion Chief, and various other support apparatus responded. The second alarm brought an additional 160 personnel for a total of 225 firefighters on scene.

A construction worker reported that the fire was on the 17th floor, so initial crews took a construction elevator to the 15 th floor. An engine crew attempted to connect $2 \frac{1}{2}$ inch lines to the standpipe in the stairwell and found no connections on the 14th or 15 th floors. The crew then checked the 16 th floor to learn that it was blocked by a wooden hatch. The interior stairwells had hatches installed every two stories, which limited the movement of the firefighters between floors. Some hatches were opened by fire crews using saws, but even with open hatches only one firefighter could pass though at a time.

Engine $10 \mathrm{crew}$ eventually located a connection to a standpipe in a second stairwell and flaked out several lengths of hose then waited for water. Ultimately, it was discovered that the standpipe system was completely out of operation and was disconnected in the subterranean levels.

As the fire continued to spread uninhibited, Command ordered the crews back down to the 14th floor, as the fire was now burning on the 15th floor with heavy smoke and still no water in the standpipes.

At 4:14 pm a firefighter on the Engine 10 crew transmitted a Mayday after becoming disoriented. Firefighters responding to the Mayday found their way off the 15th floor though a breach in a plywood wall. Several crews were on the 15th floor either trying to find their way out, or searching for the lost firefighter. Eventually, all firefighters were ordered to exit the 15th floor as heat conditions were becoming more severe.

As conditions continued to worsen on the 14th through 17 th floor, engine crews began to drop hose lines on the exterior of the building. Once the exterior hose stretch was complete, additional lengths were attached to reach the seat of the fire. The hose line was charged with water at 4:44 pm, over an hour after the initial crews were dispatched.

Following a tenuous fire fight by firefighters from 45 Engine Companies, 30 Ladder Companies, 3 Rescue Companies, 19 Battalion Chief Units, 4 Division Chief's Units, and numerous other special and support units operating with little to no visibility, the fire was suppressed. As a result of the high risk environment, 115 firefighters were injured and two firefighters were killed (IFSTA, 2011).

\section{Wellesley Street, E., Toronto, Ontario, Canada}

On September 24, 2010, a six-alarm fire at a downtown Toronto high-rise building resulted in 14 people being sent to hospital and left an estimated 1,200 people temporarily homeless. Eight adult civilians, three firefighters and three children required medical care, while an additional 10 firefighters were treated for heat exhaustion (Allen, 2010).

The fire at 200 Wellesley Street, East started at 5:00pm on the 24 th floor and spread to two adjacent units. It grew into a six-alarm fire, with more than 120 firefighters and 27 pieces of fire apparatus at the scene. At 8:30 p.m., when it seemed progress has been made, bright orange flames could still be seen in the bedroom of one unit on the 23rd floor (Allen, 2010).

The growth of the fire was attributed to extreme fuel loading in one apartment. Ontario Fire Marshal described the fire fuel load as one of the worst hoarding fires in Canada. The tremendous growth and spread of the fire was a result of the excessive amount of combustible materials stored on the balcony and in the suite of origin. The extreme fuel load made the fire very difficult to fight, control and extinguish. Firefighters deemed the fire one of the hottest and most deep-seated fires they had ever fought and high winds made matters worse. Four hours after the blaze began, firefighters were still battling spot fires, created from falling debris. By 10 p.m., firefighters appeared to have knocked the flames down (CTV, 2010). 


\section{Shanghai, China}

On November 15, 2010, a fire destroyed a high-rise building in Shanghai, killing 58 people and injuring at least 90 others as the blaze tore through a 28-story block of flats in Shanghai. The building was being renovated when the fire started. The investigation of the fire made a preliminary conclusion that negligence by unlicensed welders on the tenth floor caused the bamboo scaffolding and attached nylon netting to ignite. Fire subsequently spread to the entire structure through ignition of the polyurethane foam insulation used on the building's outer walls.

Many people were trapped as fire engulfed the upper part of the building, beyond the reach of the fire hoses, while others were clinging to scaffolding surrounding the building as they waited to be rescued from the flames. Helicopters attempted to rescue some from the roof.

Firefighters battled for several hours to bring the blaze under control. More than 80 fire engines were called to battle the fire. By the time the fire was brought under control, more than 100 people had been rescued (BBC, 2010).

\section{Lakeview High Rise/Chicago}

NBC Chicago reported that a fire broke out on the 12th floor of the building around 2 am on October 25,2012. When a man and a woman living on the floor escaped with their dog they left their front door open, and the super-heated toxic gases from the fire spread into the hallway. The heat was near 1,500 degrees Fahrenheit at the ceiling, and a woman returning to her North Side apartment was killed when she took the elevator to her floor -- not knowing that a fire was raging in the hallway outside her apartment. When elevator doors opened on the 12th floor, the woman was standing in the elevator and likely exposed to direct fire spread. Nine other people, including two firefighters, were injured in the blaze (Chicago Tribune, 2012).

\subsection{Overview of High-Rise Firefighting Tactics}

In a high-rise fire, the risk to firefighters and occupants increases in proportion to the height of the building and the height of the fire above ground level. When firefighters are operating above the reach of aerial devices, the only viable means of egress is the interior stairs; extra protection afforded by laddering the building is not possible (Klaene, 2007). As the level of the fire floor gets higher, reliance on the standpipe also increases. Open-layout floor plans such as office buildings with cubicle farms can challenge both the standpipe's flow capacity and fire department resources.

A working fire in a high-rise building can threaten thousands of occupants and hundreds of responding firefighters. The most effective way to extinguish a high-rise fire is by mounting an offensive attack as early as possible, because in the vast majority of historic high-rise fires the best life safety tactic is extinguishing the fire. Good high-rise firefighting tactics and firefighter/occupant safety cannot be separated.

Tactics are a series of operational tasks that in combination accomplish an overall fireground strategy. Individual tactics are measurable and attainable intermediate outcomes that lead to the completion of incident objectives like extinguishing the fire. There are three tactical priorities in a high-rise fire; life safety, fire extinguishment, and property conservation (Klaene, 2007). After the fire is out, salvage and overhaul tactics are employed.

During firefighting, life safety tactics fall under the label of
Search and Rescue. Search is a fireground tactic that is a systematic approach to locating possible victims, and Rescue is the act of removing victims from known danger. Searches are normally conducted by an entire crew, supplemented by an attack or ventilation crew. The minimum number of firefighters for a search is two (IFSTA, 2010).

Fire extinguishment is a critical factor, since the intensity and size of the fire will determine the extent to which combustion gases are heated and how high they will rise inside the building. Building suppression systems, both active and passive, can impact fire growth, occupant safety, and firefighter safety and effectiveness. Such features include active fire detection and automatic sprinkler systems. Once firefighters are on scene, they will complete a series of fire confinement and extinguishment tasks. Firefighters access the structure, locate the fire, locate any avenues of spread, place hose lines, and establish a water supply. Once a water supply is established, water should be placed at the seat of the fire or in the compartment containing the fire to extinguish it.

Ventilation is a tactic that affects both life safety and fire extinguishment. Coordinated ventilation may be implemented at any time during the operation, but it should be coordinated with suppression and interior rescue activities. Ventilation is used to channel and remove heated air, smoke, fire gases, and other airborne contaminants. Applying proper ventilation at the right time and place is key to firefighter and occupant safety. Venting at the wrong time or place can draw active fire toward fresh air, which will injure or kill anyone in its path.

After the fire is extinguished and victims rescued, firefighters should move on to the task of salvage. Salvage is the firefighters' attempt to save property or reduce the damage from water and smoke. Salvage operations are typically performed immediately after a fire by removing unharmed property from the fire area and covering it with canvas tarpaulin or other heavy protective material. Salvage is a major tactical priority that requires significant time and resources in order to minimize loss. Salvage and overhaul operations (described below) were not included in the study scenario.

Overhaul is the completion of the extinguishment, which can result in what may appear to be additional damage to any property. The additional damage, however, is warranted to prevent more unexpected fire damage. Overhaul is used to ensure the fire is out completely and that the environment is safe for others, such as investigators or the property owner, to enter. Firefighters may use thermal imaging cameras to look at walls and ceilings to find hot spots, or they may tear out sections of walls and pull sections of ceilings to assure there has been no fire spread.

As indicated by the tasks that must be accomplished on a high-rise fireground, understanding the required resources is critical. The number of firefighters needed to safely and effectively combat a high-rise fire may be large. Although an offensive fire attack is the preferred strategy whenever conditions and resources permit, a defensive attack that limits operations to the outside of a building and generally results in more property damage must be considered when risks to firefighter safety are too great and benefits to building occupants are negligible. The offensive vs. defensive decision is based on a number of factors: fireground staffing available to conduct an interior attack, a sustained water supply, the ability to conduct ventilation, and risk vs. benefit analysis regarding firefighter and occupant safety. 


\section{Purpose and Scope of the Study}

T his project systematically studies the deployment of mobile and personnel resources and their affects on firefighter safety, civilian safety, and the protection of high-rise property. The purpose of the study is to enable fire departments and city/county managers to make sound decisions regarding optimal resource allocation using the results of scientifically based research.

Many variables may have an effect on the outcome of a high-rise fire. Due to limited resources, only variables considered likely to have the most significant effect on the outcome of the fire incident were included in the scope of the study. These variables included crew size, alarm size, and vertical response mode (stairs or elevators).

The results of the project will inform the NFPA 1710 Technical Committee regarding the optimal crew size and total effective firefighting force for a first alarm assignment to a working high-rise or other high hazard fire. The results of the project will provide valuable information for those developing national model building, fire, and life safety codes. Finally, these high hazard response scenarios will "bracket" the spectrum of fire response, acting as a complement to the recently published low hazard Residential Fireground Deployment Study (Averill et al., 2010).

This report describes the experiments that were conducted using on-duty fire service personnel and computational tools to simulate a response to a high-rise fire incident on an upper floor of a building. The experimental design and fire modeling methods are similar to those conducted in the Residential Fireground Deployment Study.
There were two parts of the experiments.

Field tests for critical time-to-task completion of key tasks in fire suppression and in occupant search-and-rescue

- Fire modeling to determine the effects of firefighter operations on fire growth and spread and on occupant survivability

The scope of this study is limited to understanding the relative influence of deployment variables on high hazard high-rise structure fires, similar in magnitude to the hazards described in NFPA 1710. This standard is limited in that it does not specify the precise nature of a high hazard high-rise structure fire. Therefore, a secondary objective of this study is to establish a technical basis for deployment of firefighters and equipment to a "typical" high-rise fire. While all fires and buildings have unique features and characteristics, it is believed that the building and operations used in these experiments are broadly representative of a high hazard fireground and will be useful in establishing the technical basis underlying the national standard for minimally safe and effective deployment.

When paired with the Residential Fireground Report (Averill et al., 2010), a significant benefit of this report is the ability to compare and contrast deployment outcomes for a range of hazard scenarios. A residential fire represents a low hazard, relatively high frequency scenario and a high-rise fire is a high hazard, relatively low frequency scenario. Note that the applicability of the conclusions from this report to outside fires, and response to hazardous material incidents, acts of terrorism, and natural disasters or other technical responses has not been assessed and should not be extrapolated from this report. 


\section{A Brief Overview of High-Rise Fireground Operations}

$\mathbf{R}$ egardless of the size of the burning structure, firefighting crews identify four priorities: life safety of occupants and firefighters, confinement of the fire, property conservation, and reduction of adverse environmental impact. Interdependent and coordinated activities of all firefighting personnel are required to meet the priority objectives.

High-rise fire response is a complex orchestration, often involving over 100 personnel operating over many floors in a building. While the exact sequence of operations may vary from one fire department to another and even from one fire to another depending upon the exact nature of the event, the following narrative describes the general sequence of activities during a high-rise fireground operation. Note that some activities, such as fire suppression or victim rescue, are considered milestone events because they are critical to the priority objectives noted above. The following overview is derrived largely from Klaene and Sanders (2007).

\section{The First Arriving Fire Crews:}

a The first engine positions the apparatus such that it can establish an uninterrupted water supply to critical building systems, including the standpipe and sprinkler system (if present) and operates the pump hydraulics at the apparatus as the remaining crew members enter the building.

- The first arriving officer establishes command (Incident Command) and conducts initial size-up by checking the alarm panel and consulting with building management. One firefighter or officer is assigned to the lobby to establish Lobby Control.

- Remaining firefighters and officers ascend the stairway to an area two floors below the fire via stairs when the elevator is unsafe for use or by elevator when the elevator is determined to be safe for use by firefighters. This crew sets up a staging area to hold firefighters preparing to fight the fire or to conduct search and rescue.

- Some crew members proceed to the next floor up (one floor below the fire). Upon arrival at the floor below the fire, one crew enters for the purpose of viewing the general floor layout in preporation for entering the fire floor. This crew also does a quick check for fire extension and connects a hose to the standpipe on that floor.

- Prior to being charged with water, a hose line $(200 \mathrm{ft}(61 \mathrm{~m})$ of 2 $1 / 2$ inch hose) is stretched from the standpipe on the floor below the fire to the stairwell on the floor above the fire. It is then looped back in the stairwell in preparation for entry to the fire floor.

- An Initial Rapid Intervention Crew (IRIC) is assembled on the staging floor prior to crews entering the fire floor. The NFPA and OSHA " 2 In/2 Out" 13 crew is designated prior to anyone entering an atmosphere that is immediately dangerous to life or health (IDLH) ${ }^{14}$. A full and sustained Rapid Intervention Crew (RIC) is assembled as soon as sufficient personnel arrive on scene to do so.
- Once fully charged with water, the hose line is advanced through the doorway into the fire floor hallway. Ceiling tiles (or ceiling if no tiles present) are pulled open to check for fire extension as the crew advances down the hallway toward the initial fire area. Checking the ceiling ensures that the crew will not become trapped due to a hidden fire in the overhead space.

- If fire extension has not progressed beyond the initial fire compartment, entry is forced into the fire compartment area.

- The hose line is extended into the fire compartment area and operated to extinguish the fire.

\section{As Other Fire Crews Arrive:}

n Upon arrival, a command officer (Battalion Chief 1) receives command transferred from the initial Incident Commander (IC).

- Upon arrival, Battalion Chief 2 is assigned as a Division supervisor on the fire floor and supervises all operations on that floor.

- After leaving a crew member in the lobby to place a high pressure mechanical fan in the stairwell in order to keep the stairwell free of smoke, a crew ascends to the fire floor to begin a search for any victims.

all arriving crews are directed to the staging area two floors below the fire floor, with the exception of later arriving larger crews that may first report to Base (staging outside the building) pending assignment.

- A second hose line, also $200 \mathrm{ft}(61 \mathrm{~m})$ of $21 / 2$ inch hose, is advanced from the standpipe on the fire floor to the fire area and is operated to extinguish the fire.

- One company of firefighters is assigned to Stairwell (or Elevator) Support to transport necessary supplies (Self Contained Breathing Apparatus (SCBA) air cylinders, water for hydrating firefighters, search ropes, etc.) to the staging floor for crews working in the building.

- A rehabilitation area is established two floors below the fire adjacent to the staging area in order to conduct a physical assessment of firefighters who have completed a unit of work (commonly the consumption of one air bottle by a firefighter or a predetermined time period).

A crew is assigned to check for fire extension on the floor above the fire with a $2 \frac{1}{2}$ inch hose line. A second hose line supplied by the floor above the fire standpipe is also deployed to that area.

- A crew is also assigned to conduct search and rescue operations on the floor above the fire.

- Crews are also assigned to upper floors (above the floor above the fire) to search the floors and both stairwells.

- Stairwells are ventilated using positive pressure ventilation (PPV), which includes opening a hatch at the top of the stairwell after the fire is reported to be out.

13. The "2 In/2 Out" policy is part of paragraph $(\mathrm{g})(4)$ of OSHA's revised respiratory protection standard, 29 CFR 1910.134. This paragraph applies to private sector workers engaged in interior structural firefighting and to Federal employees covered under Section 19 of the Occupational Safety and Health Act. States that have chosen to operate OSHA-approved occupational safety and health state plans are required to extend their jurisdiction to include employees of their state and local governments. OSHA's interpretation on requirements for the number of workers required being present when conducting operations in atmospheres that are immediately dangerous to life and health (IDLH) (OSHA 1995) covers the number of persons who must be on the scene before firefighting personnel may initiate an attack on a structural fire. An interior structural fire (an advanced fire that has spread inside of the building where high temperatures, heat, and dense smoke are normally occurring) would present an IDLH atmosphere and therefore require the use of respirators. In those cases, at least two standby persons, in addition to the minimum of two persons inside needed to fight the fire, must be present.

14. IDLH - Immediately Dangerous to Life and Health. IDLH conditions can be due to high levels of heat, smoke, or toxic gases which rapidly threaten a human's ability to affect their own escape. 
Later arriving Chief Officers are assigned to:

- Division Supervisor on floor above the fire.

- Staging

- Upper Division (upper floors)

- Safety

- Planning (formerly the IC as higher ranking chief officer assumes command)

- Operations

- Public Information

- Logistics

- Lobby Control (If the initial lobby control assignment was not an officer)

As shown by the assignments, the first tactical priorities are extinguishing, or at least controlling the fire and conducting a primary search and rescue in the immediate fire area; these task must include an IRIC team before suppression or search tasks can proceed. Successful fire control stops forward progress of the fire and protects crews conducting search and rescue activities, whereas extinguishment dramatically reduces the magnitude of the hazard.

The role of the Incident Commander is to develop an incident action plan to mitigate the incident and see that those actions are carried out in a safe, efficient, and effective manner. Command aides are responsible for assisting with situational assessment and communications with division, group, and crew officers to ensure personnel accountability.

The response time and interval between units arriving at the scene varies based on geographic distance from the station location of each company to the fire address (as well as any traffic or weather conditions). Companies dispatched from locations further from the incident address will generally take longer to arrive.

Throughout the entire incident, each crew officer is responsible for the safety and accountability of his or her personnel, including air management. The location and wellness of crews is tracked by the division supervisor's command aide through a system of personal accountability checks conducted at 20 min intervals. Overall incident accountability (resource status/situation status) is monitored by the Planning Chief and his or her resource status staff.

Air consumption and fatigue are major factors when a continuous attack or prolonged search and rescue are required. This is particularly true in buildings where firefighters must ascend to an upper floor via the stairway when the elevators are unsafe to use. When an extended operation is anticipated, a plan is required to relieve firefighters performing essential tasks in areas immediately dangerous to life and health before they deplete their air supply or become incapacitated due to exhaustion. All firefighters who complete a work assignment, who are exhausted, or are relieved due to low air supply are sent to a rehabilitation area, where they are medically evaluated, provided liquids, and taken out of service to rest. After a rest period of at least $15 \mathrm{~min}$, firefighters are moved forward to the staging area where they replace their air cylinder and continue to rehabilitate while awaiting reassignment. The rehab area and staging area are usually on the same floor (at least two floors below the fire floor), but separated from one another to avoid confusion and premature reassignment of fatigued firefighters.

SCBA air cylinders, medical supplies, water and other needed firefighting supplies are moved from the outside at ground level to the staging and rehab areas by stairwell support personnel.
Stairwell support provides a means of moving supplies from the first floor to the rehab/staging area by placing a firefighter on every other floor or so. Typically, firefighters are assigned to bring air cylinders and other equipment into the building using an elevator to transport equipment to the staging and rehab areas.

Once the fire is located and extinguished and occupants are rescued or evacuated, the IC reassesses the situation and develops a revised incident action plan. At this point, the Incident Commander either reassigns crews or calls additional companies to the scene to conduct a very thorough secondary search of the building, verify that the fire has not extended into void spaces, determine that the fire is fully extinguished, and conduct salvage and overhaul operations. These activities are important and should be completed as soon as possible since they can reduce the chance that the fire re-ignites and can significantly limit the extent of property damage. Personnel assigned to the secondary search should not be the same personnel who conducted the primary search. Due to limited resources, salvage, overhaul, and secondary search activities were not part of this high-rise experiment.

\subsection{The Relation of Time-to-Task Completion and Risk}

The goal of a properly designed fire department deployment strategy is to match the deployment of resources to the risks associated with different events. The community risk level is typically established through an overall profile of the community based on its unique mixture of demographics, socioeconomic factors, occupancy risk, fire management zones, and the level of services currently provided. Given that a particular hazard exists in a community, the consequences of an emergency event (e.g. fire) in such a hazard are ultimately determined by the mitigation efforts.

During any fire incident, the risks to firefighters and occupants are strongly correlated with how long it takes to implement mitigation strategies. This is particularly true in the high-rise scenario due to the scale of the response requirements and the potential number of building occupants who may be exposed to the risk. Critical Tasks for high-rise firefighting operations include fire suppression and completion of search and rescue operations, among others. Anything that delays the accomplishment of these critical tasks, whether it is due to the deployment of inadequate resources or to other factors such as delays in detecting the fire, delays in reporting the fire, or difficulty in accessing the building, reduces the likelihood of controlling the fire in time to prevent major damage, injuries, or possible loss of life, and increases the level of effort and danger to firefighters.

On any fireground, fighting larger fires means additional risks to both firefighters and occupants. During a significant building fire, smoke, heat, and fire are transported throughout the building. The rate and extent of travel depend on the nature and quantity of the fuel available to burn, the size of the fire compartment, available ventilation and other factors. The temperature of a compartment fire may rise to over $1,000^{\circ} \mathrm{F}\left(540^{\circ} \mathrm{C}\right)$, and toxic gases can accumulate to lethal levels rapidly. Therefore, the IC must prioritize the deployment of firefighting resources in order to accomplish priority tasks as quickly as possible, without compromising the safety of the firefighters or occupants in the process. Accomplishing these objectives requires strong situational awareness, communication with division, group, and company officers, and a shared understanding of the tactical priorities across the fireground. 


\subsection{Standards of Response Cover}

Developing a standard of response cover-the policies and procedures that determine the distribution, concentration, and reliability of fixed and mobile resources for response to fires (as well as other kinds of technical response)—related to service commitments to the community is a complex task. Fire and rescue departments must evaluate existing (or proposed) resources against identified risk levels in the community and against the tasks necessary to conduct safe, efficient and effective fire suppression at structures identified in these risk levels. Fires in high hazard/high-risk buildings, such as the high-rise building used for this experiment, require more fire department resources in a shorter period of time than do fires in a typical lower-risk residential building such as a single family dwelling. It is essential that the risks be evaluated based on the potential life safety, fuel load to be extinguished, and property conservation challenges.

Leaders must also evaluate geographic distribution and depth or concentration of resources deployed, based on time parameters. Recognition and reporting of a fire sets off a chain of events before firefighters arrive at the scene, such as call receipt and processing, dispatch of resources, donning of protective gear, and travel time. NFPA 1710 defines the overall time from dispatch to scene arrival as the total response time. This standard divides total response time into a number of discrete segments, of which travel time is defined as the time interval from the beginning of travel to the scene to the arrival at the scene.
Arrival of an effective firefighting response force must be immediately followed by organization of the resources into a logical, properly phased sequence of tasks, some of which need to be performed simultaneously. Knowing the approximate time it takes to accomplish each task with the allotted number of personnel and equipment is critical. Ideally, crews should arrive and intervene in sufficient time to prevent flashover or fire spread beyond the room of origin. Failure to prevent flashover affects the ability to safely remove occupants and to rescue persons who cannot self-evacuate. Decision-making regarding crew size, staffing levels and geographic distribution of resources must consider the times when simultaneous completion of tasks is required. There should be sufficient redundancy or overlap in the system to allow for simultaneous calls and high volume of near simultaneous responses without compromising the safety of the public or firefighters.

Policy makers have long lacked studies that quantify changes in fireground performance based on apparatus crew size and on-scene arrival time intervals. The experiments in this study were designed to observe the effect of these variables including company crew size, apparatus deployment (alarm size), the availability of stairs and elevators, and automatic sprinkler protection, on the time it takes to execute essential fireground tasks and on the tenability inside a high-rise building, particularly on the fire floor and floor above the fire. It is expected that the results of this study will be used to evaluate and develop high-rise related performance objectives in NFPA 1710. 


\section{Experimental Methods}

lanning for the field experiments was multifaceted. The resources required to perform field tests representative of a high-rise structure fire are numerous. Though grant funding was necessary, the experiments required resources in addition to the grant dollars available. Collaboration with subject matter experts as well as several fire and rescue departments was needed to assure the time-to-task portion of the experiments was completed.

Subject matter experts (SMEs) were identified to assist principal investigators in developing experiment protocols that would be broadly applicable to the U.S. fire service. Industry experts included metropolitan fire chiefs, metropolitan training officers, textbook authors, and nationally renowned fire service leaders with experience in high-rise firefighting. ${ }^{15}$

\subsection{Scenarios}

Based on SME input, including the fact that high-rise fires represent an extraordinary challenge to fire departments, scenarios were developed that included each variable in combination with the others. Variables included four levels of crew size (3-, 4-, 5-, and 6-person crews), two levels of alarm size (high $=4$ engines, 4 trucks, 2 battalion chiefs and 3 ambulances/low $=3$ engines, 3 trucks, 2 battalion chiefs and 2 ambulances), and two methods of vertical ascent (stairs/elevator). The various combinations of these variables resulted in 16 different scenarios, as shown in Table 1 below.

Each of the scenarios below involved a fire on the 10th floor of a 13 story building with an open floor plan configuration of $100 \mathrm{ft}$ by $300 \mathrm{ft}(30 \mathrm{~m}$ by $91 \mathrm{~m})$, for an area of $30,000 \mathrm{sq} \mathrm{ft}\left(2800 \mathrm{~m}^{2}\right)$. The primary fuel load is open-wall cubicle material with typical desk and drawers, computers, printers and office chairs. The scenarios included two victims (always in the same location); one located on the fire floor and one located on the floor above the fire (Floor 11).
Scenario 1: High-alarm assignment with 6-person crews dispatched to the building. Two fire service access elevators are available for fire service use. (Best case)

Scenario 3: High-alarm assignment with 5-person crews dispatched to the building. Two fire service access elevators are available for fire service use.

Scenario 5: High-alarm assignment with 4-person crews dispatched to the building. Two fire service access elevators are available for fire service use.

Scenario 7: High-alarm assignment with 3-person crews dispatched to the building. Two fire service access elevators are available for fire service use.

Scenario 9: High-alarm assignment with 6-person crews dispatched to the building. Stairs are available for fire service use.

Scenario 11: High-alarm assignment with 5- person crews dispatched to the building. Stairs are available for fire service use.

Scenario 13: High-alarm assignment with 4-person crews dispatched to the building. Stairs are available for fire service use.

Scenario 15: High-alarm assignment with 3-person crews dispatched to the building. Stairs are available for fire service use.
Scenario 2: Low-alarm assignment with 6-person crews dispatched to the building. Two fire service access elevators are available for fire service use.

Scenario 4: Low-alarm assignment with 5-person crews dispatched to the building. Two fire service access elevators are available for fire service use.

Scenario 6: Low-alarm assignment with 4-person crews dispatched to the building. Two fire service access elevators are available for fire service use.

Scenario 8: Low-alarm assignment with 3-person crews dispatched to the building. Two fire service access elevators are available for fire service use.

Scenario 10: Low-alarm assignment with 6-person crews dispatched to the building. Stairs are available for fire service use.

Scenario 12: Low-alarm assignment with 5-person crews dispatched to the building. Stairs are available for fire service use.

Scenario 14: Low-alarm assignment with 4-person crews dispatched to the building. Stairs are available for fire service use.

Scenario 16: Low-alarm assignment with 3-person crews dispatched to the building. Stairs are available for fire service use. (Worst case)

\section{Table 1: Sixteen unique experimental variations}

15. Subject matter experts included Russ Sanders (NFPA, Louisville KY Chief, Retired), Dennis Compton (IFSTA, NFFF, Mesa, AZ Chief, Retired), Peter Van Dorpe

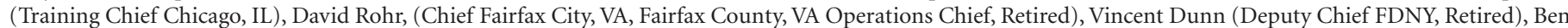

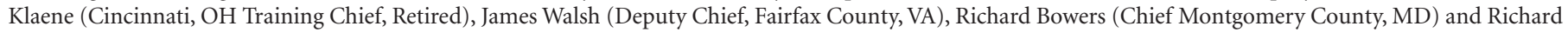
Travers (Deputy Chief FDNY, Retired). 
Experimental protocols, following the Incident Command System, were developed for each of the scenarios. Crew cue cards, denoting each task expected to be performed by participants, were prepared. (See Crew Cue Card examples in Appendix A.)

\subsection{Participating Fire and Rescue Departments}

In addition to the SME work, 13 Fire and Rescue Departments in the Washington D.C. metropolitan area committed resources to the experiments. The departments were all part of the Metropolitan Washington Council of Governments Region. ${ }^{16}$
- Arlington County Fire Department
- Alexandria Fire Department
- Fairfax County Fire and Rescue
- Prince William County Fire and Rescue
- District of Columbia Fire Department
Montgomery County Fire and Rescue
- Howard County Department of Fire and Rescue Services
- Fairfax City Fire and Rescue
- Prince George's County Fire/EMS Department
- Loudoun County Fire and Rescue Department
- Metropolitan Washington Airport Authority
- Manassas City Fire and Rescue
- Stafford County Fire and Rescue

Departments committed both engine and truck crews and equipment, as well as heavy rescue and Advanced Life Support (ALS) ambulance crews and equipment. The daily number of firefighters committed to the experiments varied from 72 to 136 . Participating departments used the experiments as intense multi-unit training exercises in high-rise firefighting. Every detailed task in the protocol used for the experiments provided an opportunity to engage in actual and accurate high-rise fire suppression and search and rescue operations.

\subsection{Field Site}

In addition to crew resources, a vacant high-rise building was necessary. The search for a building began in the D.C.

Metropolitan area. Each Fire and Rescue Department committed to the study searched their jurisdictions for available properties. Department Fire Inspectors were particularly knowledgeable of properties in their areas. The various property searches revealed potential vacant properties in the Crystal City (Arlington County), Virginia area. Due to $\mathrm{BRAC}^{17}$ activities, several buildings in the area were vacant. Once vacant buildings were identified, researchers worked with the local Fire and Rescue Department Chief Officers to contact building owners/managers. Following months of contacts and negotiation, a building was identified and secured. Vornado/Charles E. Smith ${ }^{18}$ committed a vacant 13 -story high-rise structure located at 223 23rd Street, Crystal City, Virginia to the experiments (Figure 1).
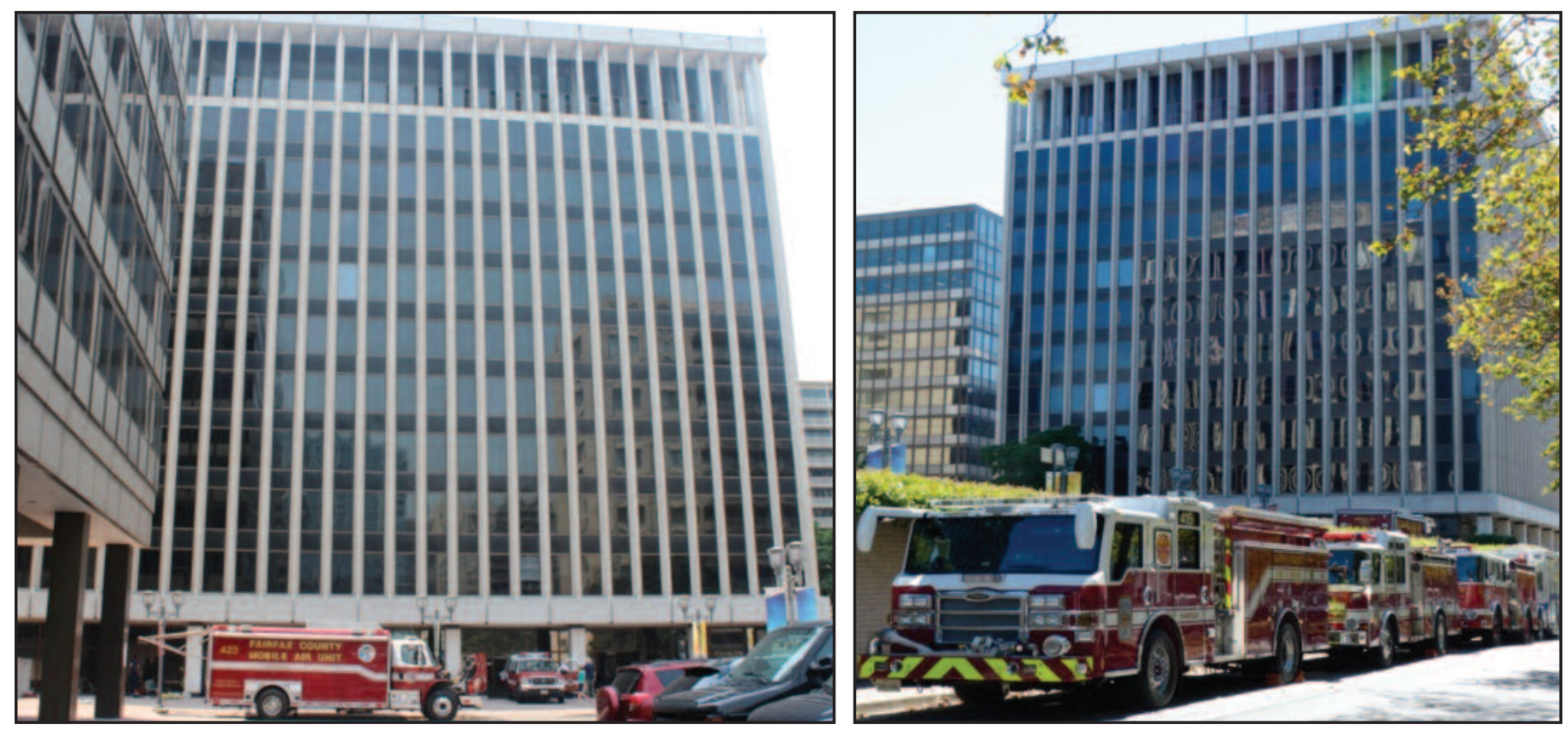

Figure 1: High-rise building located at 223 23rd Street, Crystal City, VA

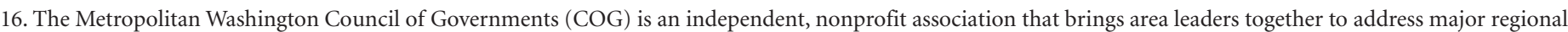

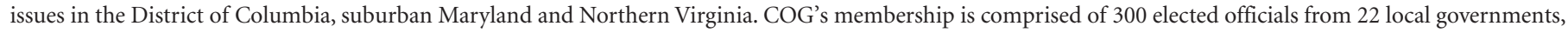
the Maryland and Virginia state legislatures, and U.S. Congress.

17. BRAC is the commonly used acronym for the formal name of the Defense Base Closure and Realignment Commission. The BRAC Commission was created to provide an objective, thorough, accurate, and non-partisan review and analysis, through a process determined by law, of the list of bases and military installations which the Department of Defense (DoD) has recommended be closed and/or realigned. The DoD list of recommendations was formally presented to the BRAC Commission on May 13th, 2005. http://www.brac.gov/About.html

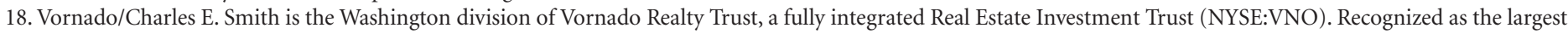
owner/manager of commercial properties in the Washington D.C. region, Vornado/Charles E. Smith owns and manages 74 office properties containing 18 million square feet and seven residential properties containing 2,424 units, primarily located in the District of Columbia and Northern Virginia. 


\subsection{Site Preparation}

Upon receiving access to the building, preparation began to build props, as well as to ensure that elevators, electricity, and other amenities were available for use.

Building elevators were evaluated, full weight-load tested, and certified to ASME standards ${ }^{19}$ to ensure that they were safe for use by study investigators and firefighters. Similarly, stairwells were cleaned and secured for use in the study.

Since the simulated fire was located on the 10th floor, cubicles were constructed to simulate the office environment of a typical Washington D.C. area commercial office building. Cubicles were built of plywood sheathing and $2 \times 4$ frames. The cubicle farm provided a common, though complex, search for firefighters on the fire floor (Figure 2).

In consultation with SME's, search patterns were established throughout both the 10th and 11th floor (the fire floor and floor

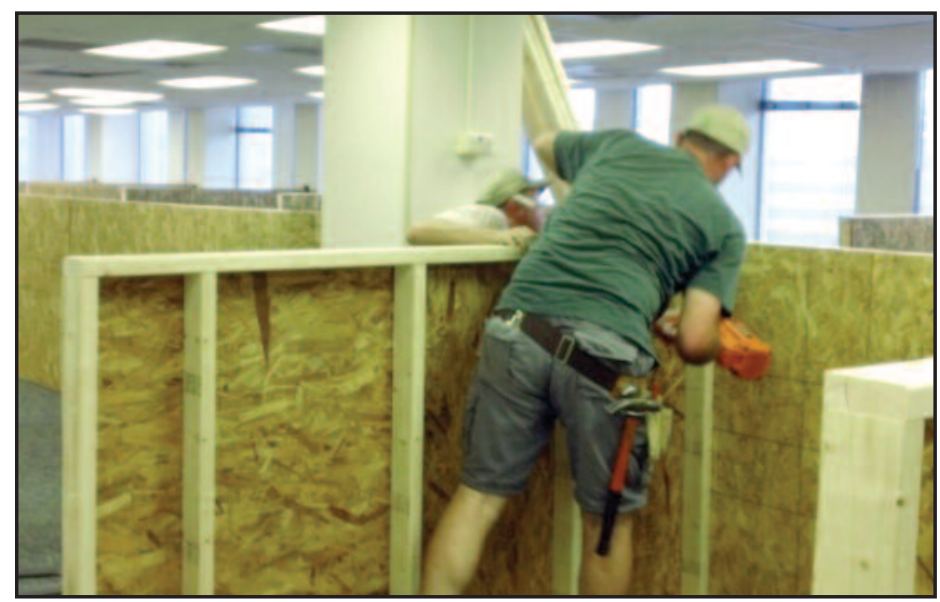

Figure 2: Office cubicle props on fire floor (10th)
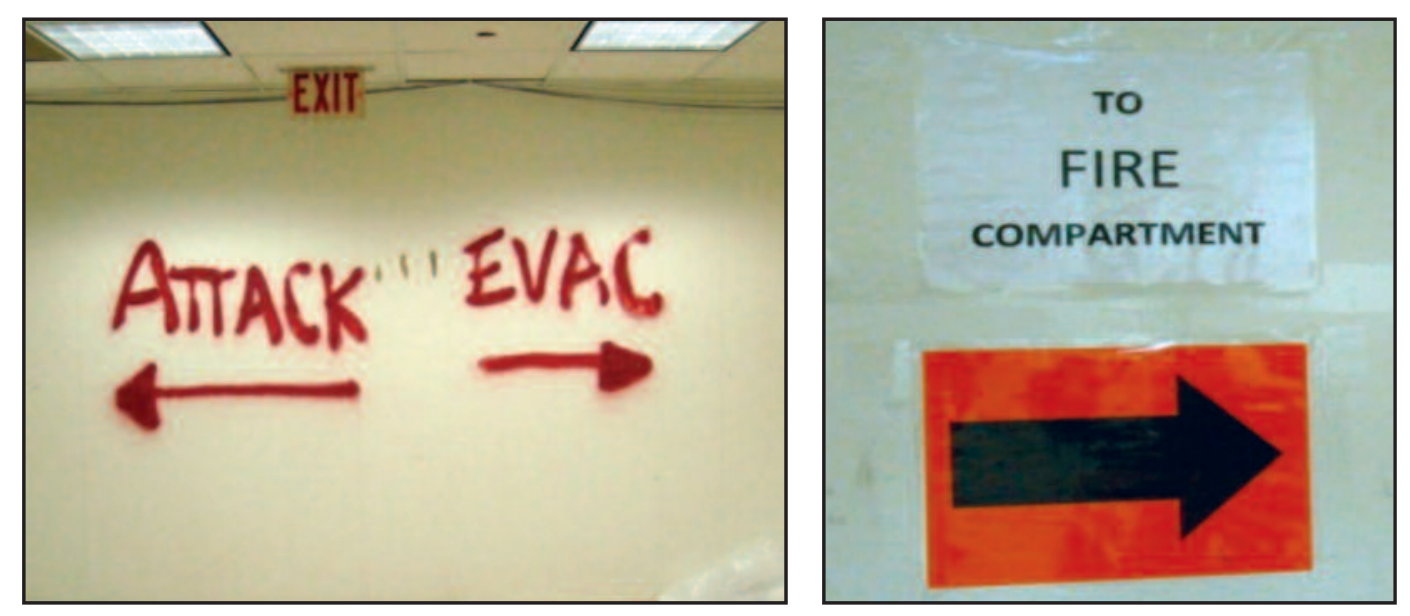

Figure 3: Direction signage above the fire). Directional signage was prepared and placed throughout the building (examples shown in Figure 3) to ensure crews moved in the same direction throughout the building while completing assigned tasks. These efforts assured the replication of each experiment in the same manner regardless of crew size, alarm level, or mode of vertical ascent (the study variables).

Fire and smoke simulators (a non-toxic suspension consisting primarily of propylene glycol) were acquired by the IAFF from BullEx Inc. ${ }^{20}$ to provide realistic visibility conditions on Floors 10 and 11. BullEx provided both digital fire displays and smoke generators necessary to simulate both heavy fire and smoke conditions on the fire floor and heavy smoke on the floor above the fire. The fire display and the smoke generator may be seen in Figure 4 and the visibility resulting from heavy smoke is shown in Figure 5. (See also Fire and Smoke Simulation, Section 6.6).

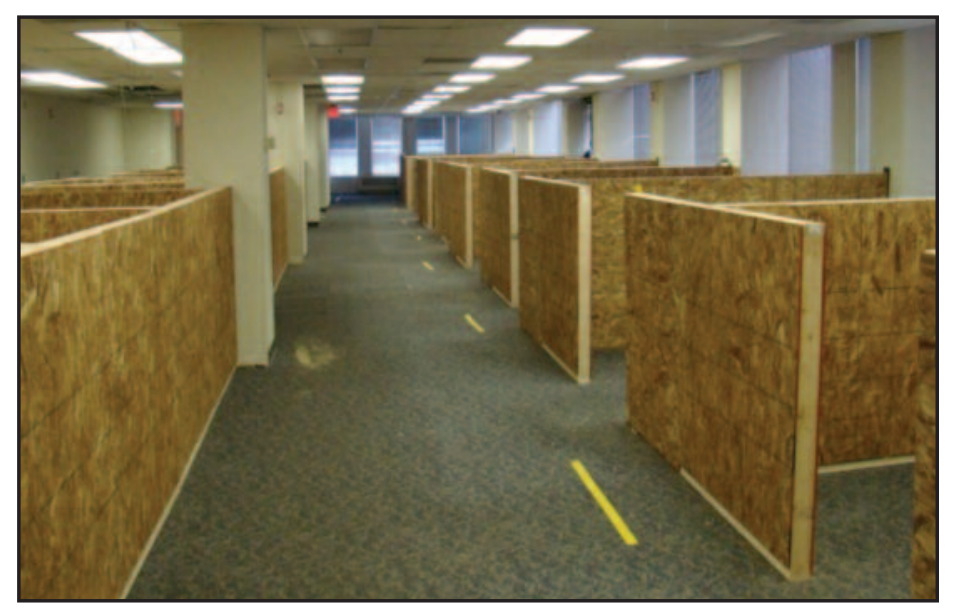

19. American Society of Mechanical Engineers (ASME) is a not-for-profit membership organization that enables collaboration, knowledge sharing, career enrichment, and skills development across all engineering disciplines. ASME has over 600 technical standards improving the safety and efficiency of boilers, elevators, cranes, nuclear energy, pipelines, and many other areas. ASME Standards are used in over 100 countries.

20. BullEx, Inc. is a technology company devoted to utilizing smart technology to develop life-saving products. 
Due to the potential for building damage, crews could not flow water in the high-rise structure, study investigators worked with participating department training academies to develop a substitute that would weigh and perform as closely as possible to a $2 \frac{1}{2}$ inch charged hose line. Montgomery County Training Academy instructors prepared more than $600 \mathrm{ft}(183 \mathrm{~m})$ of sand-filled $21 / 2$ inch hose lines. The hose lines were then separated into $200 \mathrm{ft}$ (61 $\mathrm{m})$ sections and capped on each end to contain the sand. The weight of the sand-filled lines closely approximated that of a charged line. The sand-filled lines also mimicked the movement of a charged line except at $90^{\circ}$ turns. Crews were instructed to be aware of the need for proper hose handling as they advanced the lines.

Unfortunately, ceilings in the building could not be pulled during each experiment to allow firefighters to check for fire

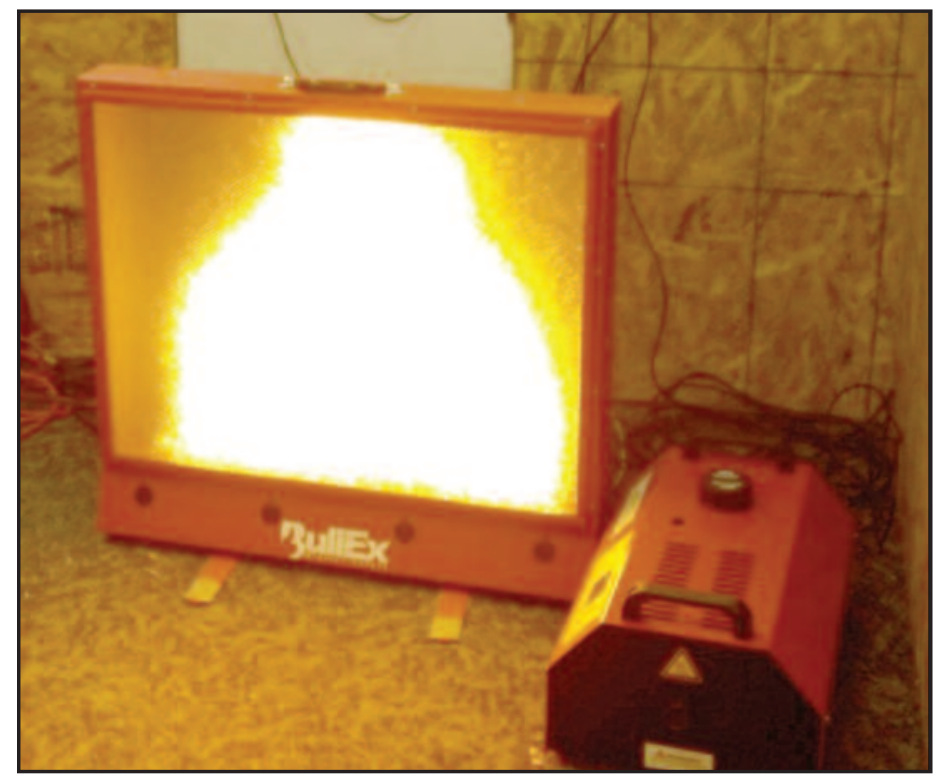

Figure 4: Fire display and smoke generator

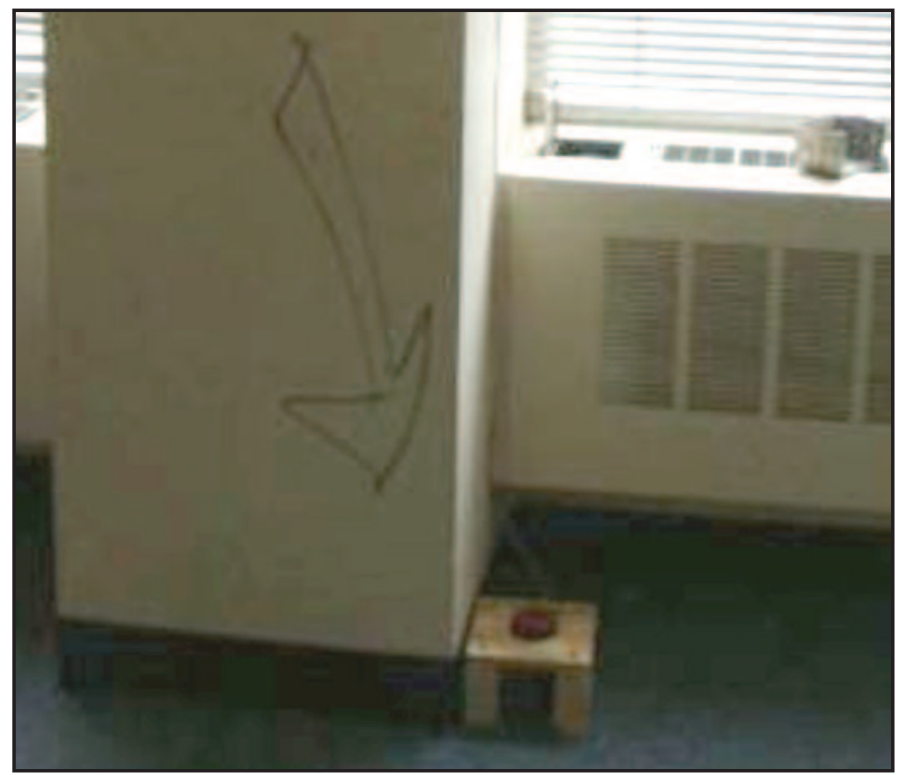

extension and then replaced for the next test. Therefore, pulley systems were built on the fire floor and the floor above the fire to simulate firefighters pulling ceiling tiles to check for fire extension. Weights were connected to one end of the pulley and a pike pole to the other end. Firefighters were instructed how many pulls on the pike pole were required to complete the assigned task.

Electronic search markers were created to track firefighters on the fire floor and the floor above the fire. NIST investigators developed an electronic push-button system (see Figure 6) that firefighters were to activate as they searched an area. These buttons allowed researchers to record the precise time at which firefighters reached various areas throughout these floors and are discussed in greater detail in Sections 6.5 and 7.12.

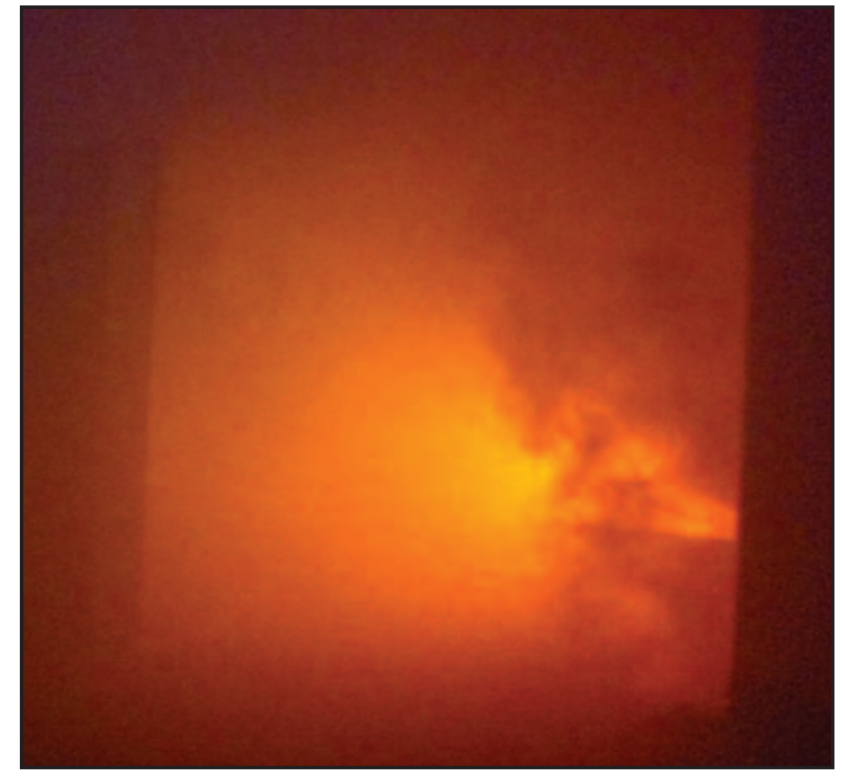

Figure 5: Fire /smoke conditions on 10th floor

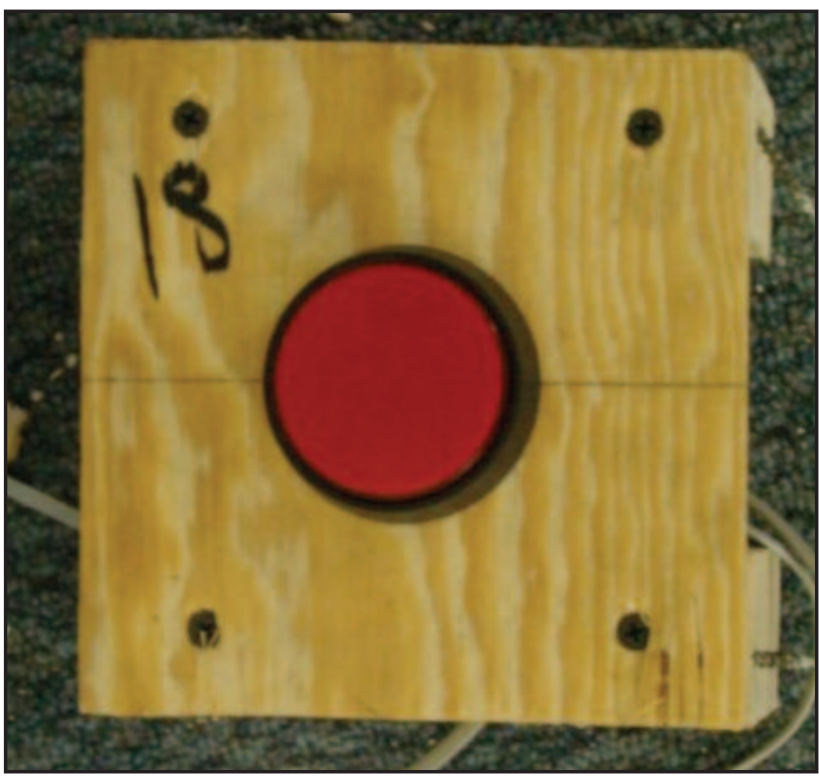

Figure 6: Electronic search marker 
Numbered wooden block markers were used on the floors without smoke present (the 9th floor directly below the fire and the upper floors above the 11th floor). Twenty blocks were distributed throughout each of these floors in pre-determined locations. Firefighters were instructed to collect the markers as they searched and then to deliver the twenty markers to people acting as timers on the floor to complete the search.

To ensure that there were sufficient SCBA cylinders for use in the experiments, Arlington County Fire and Rescue shared a cache of cylinders for the duration of the experiments. The cylinders were used by the stairwell/elevator support crew to supply the staging area and as extra cylinders that each firefighter took up with them in their initial ascent.

To make certain that crews had the capability to communicate without interruption, both Arlington County Fire and Rescue and Fairfax County Fire and Rescue provided a radio cache for use by the other fire departments throughout the study.

Finally, an experiment calendar was established and provided to participating departments. The calendar reflected 64 experiments to be conducted over 32 days ( 2 per day). All experiments were performed between the dates of May 15 and July 13, 2012. The 64 tests were divided into 4 sets of 16 experiments to match the 16 scenarios noted previously. The first set of 16 experiments was used as "shake-down" tests to refine the study protocols, timers, and building preparation. The remaining 48 experiments were carefully deployed for data collection, analysis, and reporting.

\subsection{Instrumentation}

Heavy smoke conditions present on the fire floor and the floor above the fire posed a significant problem for tracking firefighter crew tasks. In order to track the fire crews through these conditions, a recording system consisting of 46 momentary switches were installed throughout the two floors. Twenty-four were installed on the fire floor and 22 on the floor above. These buttons were positioned such that they could capture the time signal for critical events such as time of victim rescue and time when search and rescue operations were completed.

The momentary buttons were large red arcade-style momentary switches mounted onto wooden platforms (as shown in Figure 6). The button assemblies were securely fastened onto interior walls of cubicles and offices. As firefighters were searching, they were instructed to press and momentarily hold the buttons. When pressed the buttons sent a signal to the data acquisition system located on the floor above the fire.

All 46 buttons were hardwired via four conductor telephone wires to the data acquisition system. The data acquisition system (DAQ) used for the buttons consisted of:

\section{- 2 x Thermocouple-2095 32-Ch Terminal Block \\ - SCXI-1000 4 Slot Chassis \\ 2 x National Instruments SCXI-1102 32-Ch Thermocouple Input Amplifier \\ National Instruments SCXI-1600 USB Data Acquisition and Control Module}

When a button was pressed, a signal traveled through the DAQ system and created a timestamp. These timestamps were recorded onto a laptop and stored for later analysis. The information gathered from the buttons is presented later in the report.

\subsection{Fire and Smoke Simulation}

Due to the need for repeatability over the course of two months and the inability to ignite a fire within the high-rise building, fire and smoke simulators were used. On the fire floor, 5 BullEx Smart-Eye fire displays were coupled with 5 BullEx 3000 smoke generators. The smoke generators were tied to the fire displays such that the amount of smoke created and the size of the fire displayed followed the simulated fire model. The displays and smoke generators also reflected the impact of fire suppression by firefighters. When firefighters accomplished the corresponding task, the displays initialized a ramp-down feature and eventually turned off. As with a real fire, when the fire was completely extinguished, visibility conditions on the floor began to improve. More details about how the simulated fires were created can be found in Section 6.4 on Site Preparation. On the floor above the fire (Floor 11) one BullEx 3000 smoke generator was installed to simulate smoke movement through the high-rise.

\subsection{Safety Protocols}

Firefighter safety was a primary concern in conducting the high-rise field research. The fire departments participating in this study regularly conduct fire and rescue training for their staff and recruits compliant with NFPA and other industry standards and practices.

\section{Safety Personnel}

A Safety Officer was assigned to the experiments each day to assure compliance with NFPA standards. This position was filled primarily by a designated safety officer from the Howard County Fire and Rescue Department. The Safety Officer (Figure 7) participated in all crew orientation activities and daily briefings, and was actively involved in overseeing all experiments. The Safety Officer had full access to all floors of operation and had full authority to terminate any operation if any safety violation was observed.

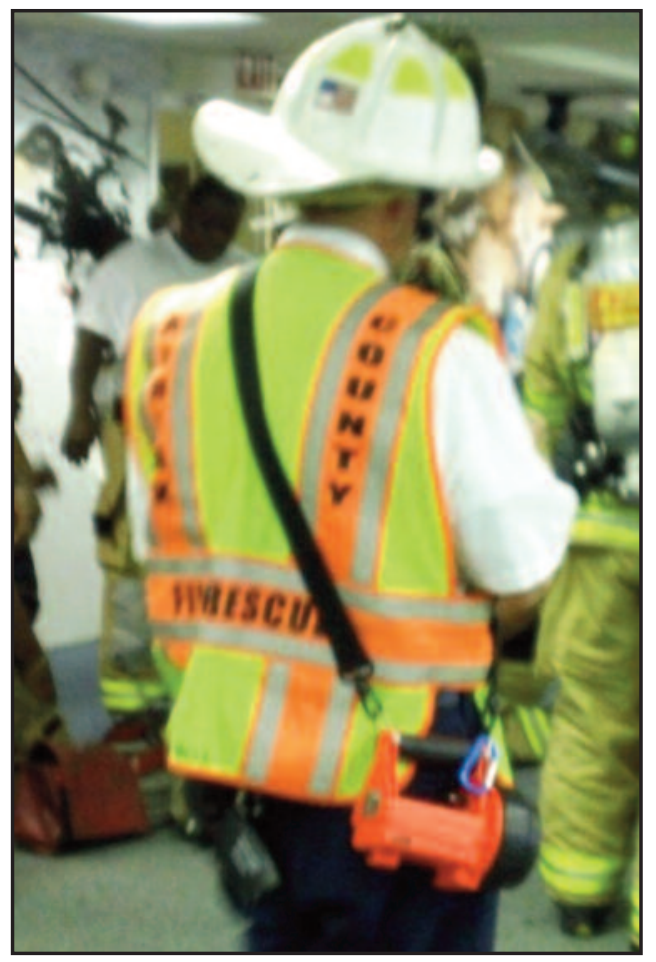

Figure 7: Safety officer 
In addition to the safety officer, the rapid intervention crew deployed within the experiments also functioned as a real RIC unit, available and expected to respond in the event of an actual firefighter health or safety emergency. Ambulance 1, operating the rehabilitation area in the experiments, was always staffed with a fully equipped 2-person Advanced Life Support (ALS) crew, which was also expected to respond in the event of an actual emergency. Finally, a dedicated ALS-staffed ambulance, not taking part in the actual experiment, was on standby at the site for all experiments.

During each morning orientation, crews were provided a radio code ("code blue") for signaling that a firefighter needed to drop out of the exercise for any reason. Interoperable radio communication was always available during the experiments for command and tactical operations and was also to be used should a code blue arise. Experiments were stopped for any action considered to be a protocol breach or safety concern.

In addition to the resources mentioned previously, researchers and timers were used for oversight and accountability for each experiment. Experimental protocols and methods were designed to assure the most controllable, safe, yet realistic high-rise fire environment possible.

\section{On air time}

Another safety protocol limited crews to $15 \mathrm{~min}$ 'on air' time for completing tasks. It was determined that $15 \mathrm{~min}$ was a reasonable lower bound for a firefighter using a 30-minute cylinder (despite performing similar tasks, some firefighters consume air faster than others depending upon several factors, such as physical fitness or stress level). Restaurant-style timers with vibration and flashing red lights were used to page crews when their 'on air' time reached $15 \mathrm{~min}$. Upon receiving the page or upon task completion, crew officers radioed division supervisors or Incident Command (IC) to request dispatch of a relief crew. Once relieved, the crews reported to the rehabilitation area located adjacent to the staging area on the 8th floor for a mandatory recovery period.

Air cylinders were refilled or replaced between experiments as needed and at the end of the test day to ensure crews were equipped to return to duty.

\section{Rehab}

A closely related priority was adequate rehabilitation. Adequate rehab was necessary to ensure firefighter safety and readiness to repeat experiments with equivalent performance. NFPA $1584^{\circledR}$ Standard on the Rehabilitation Process for Members During Emergency Operations and Training Exercises (NFPA 1584) ${ }^{21}$ expresses three stages of rehabilitation: preparedness, incident scene and training rehabilitation, and post-incident rehabilitation. The crew hold area (lobby level), the front of the building (shaded from sun), and the staging/rehab area on the 8th floor were cooled for crew rest and rehabilitation.

Throughout the experiments, as in a real fire event, food, water and electrolyte-rich drinks were available for crew intake. The importance of staying well-hydrated before, during and after the experiments was especially emphasized (Figure 8 to Figure 10).

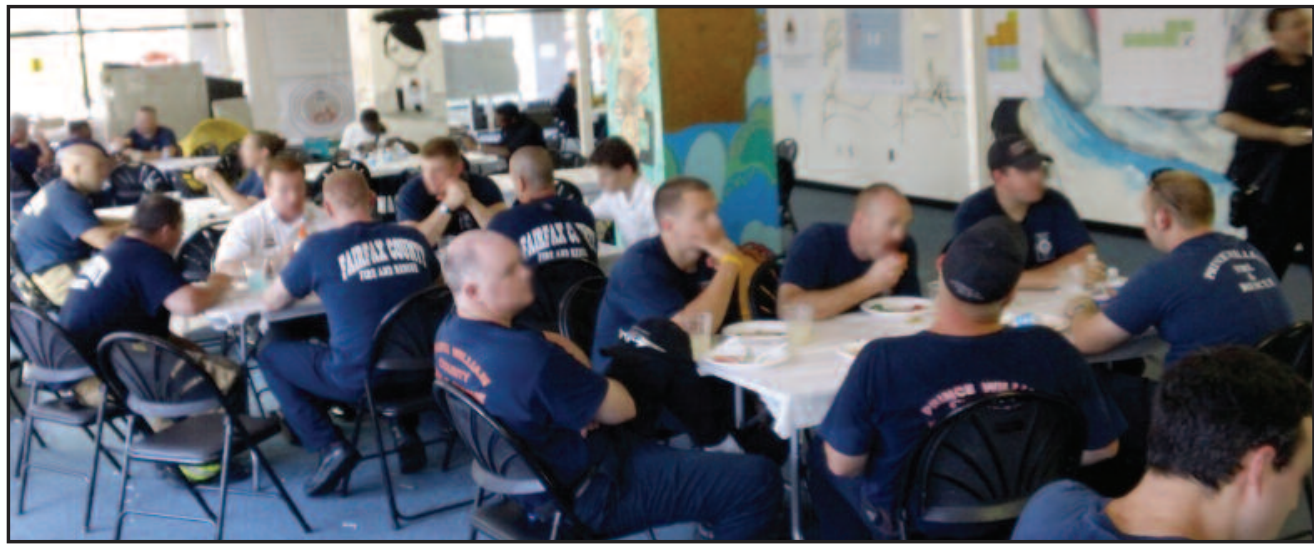

Figure 8: Crew rehab - Crew hold area

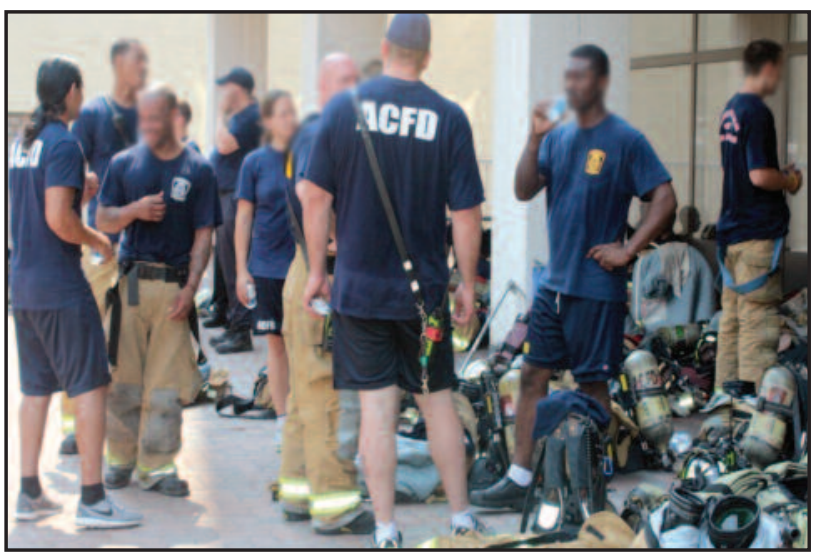

Figure 9: Crew rehab - Front of building

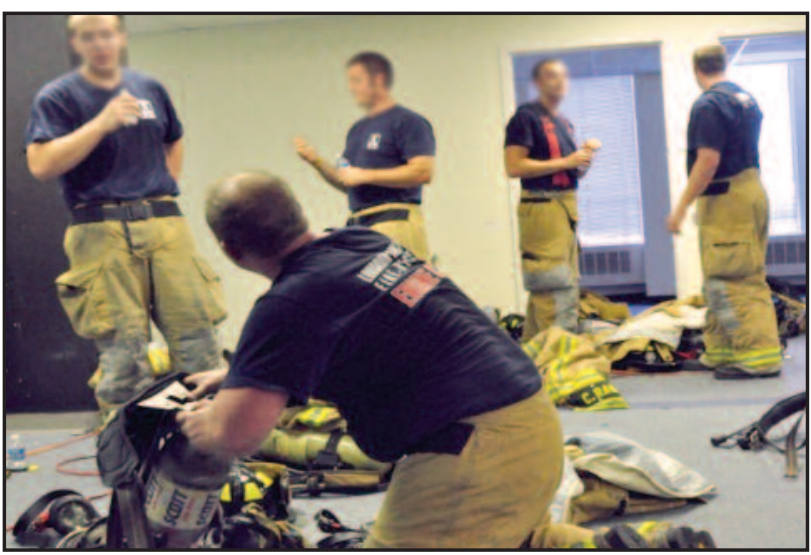

Figure 10: Crew rehab - 8th floor

21. NFPA Standard 1584: Recommended Practice on the Rehabilitation of Members Operating at Incident Scene Operations and Training Exercise provides comprehensive guidelines on developing rehab SOPs and performing the duties during emergency operations and training exercises. 


\section{Time-to-Task Experiments}

\subsection{On-Scene Fire Department Tasks}

The on-scene fire department time-to-task piece of the high-rise study focused on the tasks firefighters perform after they arrive on the scene of a high hazard commercial structure fire. A number of nationally recognized fire service subject matter experts were consulted during the development of the on-scene fire department tasks in order to ensure the broad applicability and appropriateness of the task distribution. Forty-eight total experiments were conducted to assess the time it took various crew sizes to complete the same tasks on technically similar fires in the same structure. The experiments compared the time to complete key tasks during a typical high-rise firefighting scenario using 3-, 4-, 5-, and 6-person crews. In addition to crew size, the experiments assessed the effects of alarm assignment (high vs. low) and vertical ascent mode (stairs vs. elevators). Therefore, 16 unique experiments $(4 \times 2 \times 2=16)$ were conducted in triplicate, totaling $48(16 \times 3)$ tests, as shown in the full replicate block in Table 1. A full replicate was completed in a randomized order (determined by randomization software) before a test configuration was repeated.

\subsection{Determination of Full-Alarm Assignment}

Fire departments deploy different numbers of apparatus to otherwise similar calls (often referred to as a full-alarm assignment). These differences reflect deployment decisions made at the department or community level. In order to best inform decision-making about response to high-rise building fires, full-alarm assignment configurations were gathered from metropolitan fire departments throughout the U.S. and Canada. As a pattern emerged, researchers were able to establish a low and high alarm sequence, included in the study as a primary variable named low/high alarm. Low alarm assignments consisted of 3 Engines, 3 Trucks, 2 Battalion Chiefs (with Aides), and 2 Ambulances. High alarm assignments consisted of 4 Engines, 4 Trucks, 2 Battalion Chiefs (with Aides), and 3 Ambulances.

\subsection{Crew Size}

For each experiment, the crew sizes studied included 3-, 4-, 5-, and 6-person crews assigned to each engine and truck dispatched.

Crew sizes reflect the members of the crew, both officers and firefighters, who actually engaged in completing tasks. In many departments, company officers are primarily responsible for crew command, crew safety, crew accountability, and communication with other operating units on scene, along with the IC. These officers do not directly engage in stretching hose lines, advancing and operating hose streams, normal truck operations including ventilation, or related tasks so that they can be available to focus on crew command, situational awareness and crew accountability. However, officers will assist in conducting searches and removing victims when necessary. These officer tasks are essential to firefighter safety, since studies show that situational awareness and human error are contributing factors in nearly $20 \%$ of the fireground line-of-duty deaths, and that $40 \%$ of firefighter injuries are attributed to situational awareness (Moore, 2006, 2008).

During the experiments, however, the researchers, timers, and safety officers fulfilled this officer role to assure safe tactical practices and protocol compliance. Further, during the planning stages of the experiments, it was determined that these officer tasks could not be effectively measured with the experimental methods used. Therefore, any department in which company officers operate in a safety/accountability role as described should consider the results and conclusions of this study as pertinent to firefighter task performance only.

\subsection{Number of Firefighters in the Experiments}

For each experiment, the number of firefighters was determined by the number of crew per apparatus, as the size of the full-alarm assignment was held constant for the experiments. ${ }^{22}$ Table 2 shows the total number of firefighters actually deployed for each of the 16 unique experimental configurations. The actual number of firefighters used in the experiments is fewer than the total calculated based on crew size and alarm size as described. This difference is due to the fact that later arriving crews on the high alarm deployment would have been assigned to base as tactical reserve for use in later tasks. Since individual experiments were stopped following the completion of a primary search and the rescue of victims on the fire floor and the floor above the fire, these resources weren't filled during the exercise. Unlike the field experiments, an actual high-rise fire would have engaged these additional resources to conduct secondary search, overhaul, and salvage.

22. Note that the on-scene staffing totals account for only the personnel assigned to "work" the fire. As previously explained, additional personnel were provided such as a staffed on-site medic and a safety officer specific to the experiments. They are not included in the staffing described here. 


\begin{tabular}{|c|c|c|c|c|c|}
\hline $\begin{array}{c}\text { Vertical Ascent } \\
\text { Mode }\end{array}$ & Crew Si e & $\begin{array}{c}\text { Si e of Full* } \\
\text { Alarm }\end{array}$ & $\begin{array}{c}\text { FFs } \\
\text { Engines/Trucks }\end{array}$ & $\begin{array}{c}\text { FFs } \\
\text { 3 BCs/Aides } \\
\text { and 3 Ambs }\end{array}$ & $\begin{array}{c}\text { Total Number } \\
\text { of FFs** }\end{array}$ \\
\hline Stairs & 3 & Low & 54 & 12 & 66 \\
\hline Stairs & 3 & High & 54 & 12 & 66 \\
\hline Stairs & 4 & Low & 72 & 12 & 84 \\
\hline Stairs & 4 & High & 72 & 12 & 84 \\
\hline Stairs & 5 & Low & 90 & 12 & 102 \\
\hline Stairs & 5 & High & 90 & 12 & 1202 \\
\hline Stairs & 6 & Low & 108 & 12 & 120 \\
\hline Stairs & 6 & High & 108 & 12 & 66 \\
\hline Elevators & 3 & Low & 54 & 12 & 66 \\
\hline Elevators & 3 & High & 54 & 12 & 84 \\
\hline Elevators & 4 & Low & 72 & 12 & 102 \\
\hline Elevators & 4 & High & 72 & 12 & 102 \\
\hline Elevators & 5 & Low & 90 & 12 & 120 \\
\hline Elevators & 5 & High & 90 & 12 & 120 \\
\hline Elevators & 6 & Low & 108 & 108 & \\
\hline Elevators & 6 & High & & & 84 \\
\hline
\end{tabular}

Table 2: Actual crew size for each experiment

* Low Alarm Threshold is 3 Engines, 3 Trucks, 2 Battalion Chiefs (with Aides), 2 Ambulances

High Alarm Threshold is 4 Engines, 4 Trucks, 2 Battalion Chiefs (with Aides), 3 Ambulances

${ }^{\star *}$ Firefighter count does not include experimental staff or support personnel, such as the safety officer and stand-by EMS crew.

\subsection{Department Participation}

The training exercises were conducted in Arlington County, VA at a 13 story commercial structure located at 223 23rd Street, Crystal City during the months of May through July 2012. All training took place in daylight between 8:00 am and 3:00 pm. Training was postponed for extreme heat and rescheduled for a later date following other scheduled experiments.

Participating departments considered the high-rise experiments a prime opportunity for incident command, company officer and firefighter training, as well as an opportunity for responding with neighboring jurisdictions to a multi-unit drill in a high hazard yet controlled environment. These departments committed a cache of engines, trucks, heavy rescues, ambulances, and battalion chiefs to the experiments. Some departments sent personnel separate from apparatus to ensure that sufficient numbers of firefighters and officers were available to conduct the training without depleting on-duty resources. Battalion chiefs alternated between the roles of Incident Commander, Division 10 (fire floor) and Division 11 (floor above the fire) supervisors. Figures 11 and 12 illustrate the scale of participation in the training.

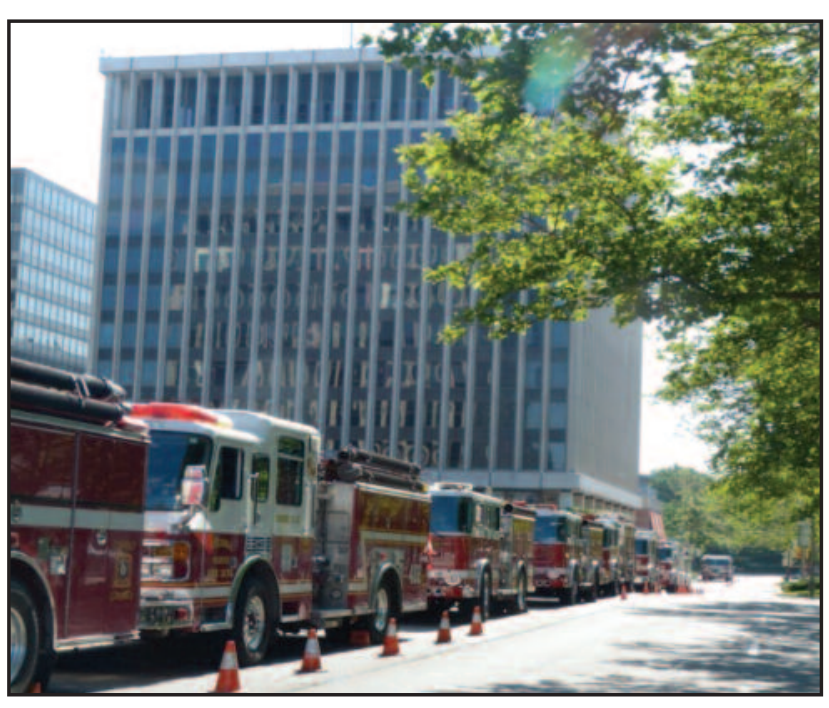

Figure 11: Apparatus parking on site

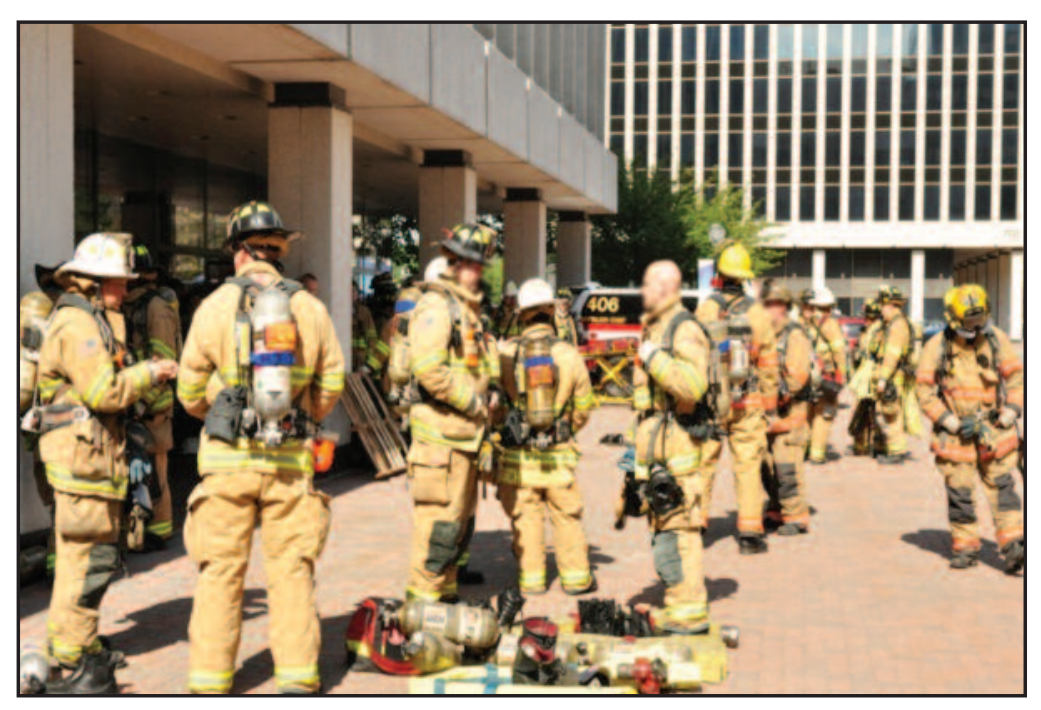

Figure 12: Crew resources 
All firefighters and officers were oriented to the experiments and the experimental response protocol each morning. Crews that normally operated together as a company were kept intact to assure typical operation for the crew during the scenarios.

Firefighters were added to or subtracted from crews based on the protocol performed. Crews were regularly combined on the spot and expected to perform as they would on any multi-unit response to a high hazard structure fire.

The allocation of resources by the participating departments made it possible to conduct back-to-back experiments each day by rotating firefighters between more labor intensive tasks, less intensive tasks and the rehabilitation areas.

\subsection{Officer and Crew Orientation}

All firefighters were required to attend an orientation each day prior to the beginning of the experiments (see Figure 13). The orientations were used to explain the purpose of the experiments, experimental procedures, task flow, division of labor between crews, radio communication, and milestone events in the scenario.

Daily orientations were conducted to assure that every study participant attended at least one session. Orientations included a description of the overall study objectives and the actual experiments in which they would be involved. Even though no live fire was used, the experiments were conducted consistent with the requirements of NFPA 1403®: Standard on Live Fire Training Evolutions (NFPA 1403) ${ }^{23}$. Full disclosure regarding the structure, the simulated fire and smoke, and the tasks to be completed was provided. Crews were also oriented to the fireground props, the instrumentation used for data collection, and the specific scenarios to be conducted. Every crew officer, Incident Commander, and Division Supervisor was provided a walkthrough of the structure during the daily orientation prior to the start of the experiments (Figure 14). Figures 15 through 27 show firefighters performing some of the tasks that were measured in the experiments.

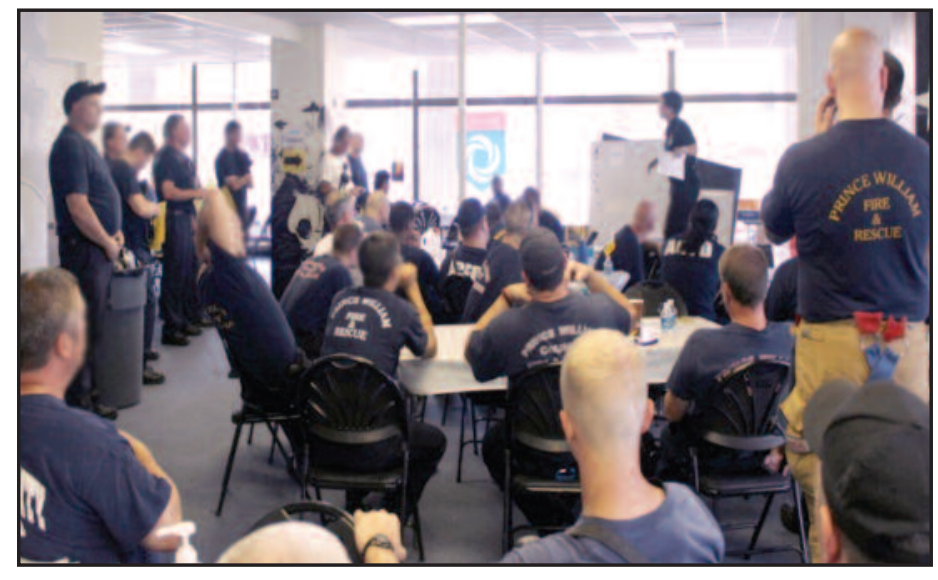

Figure 13: Daily crew orientation

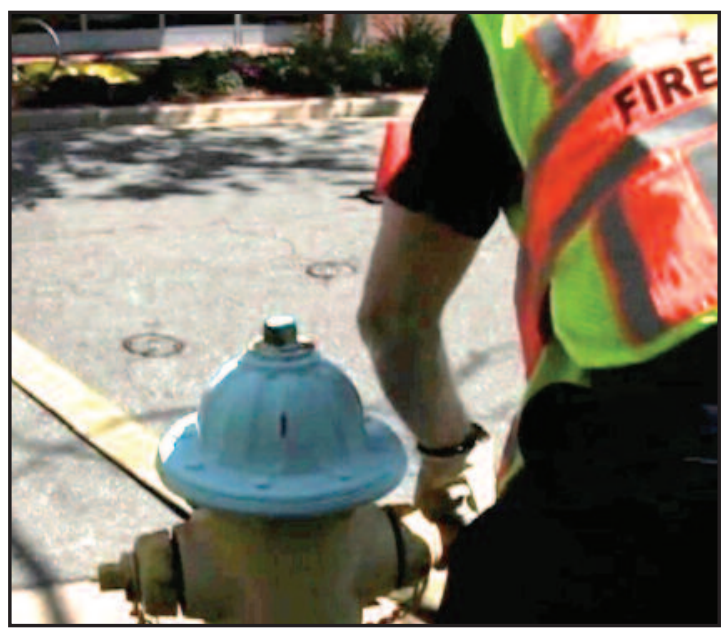

Figure 15: First Engine connects to hydrant

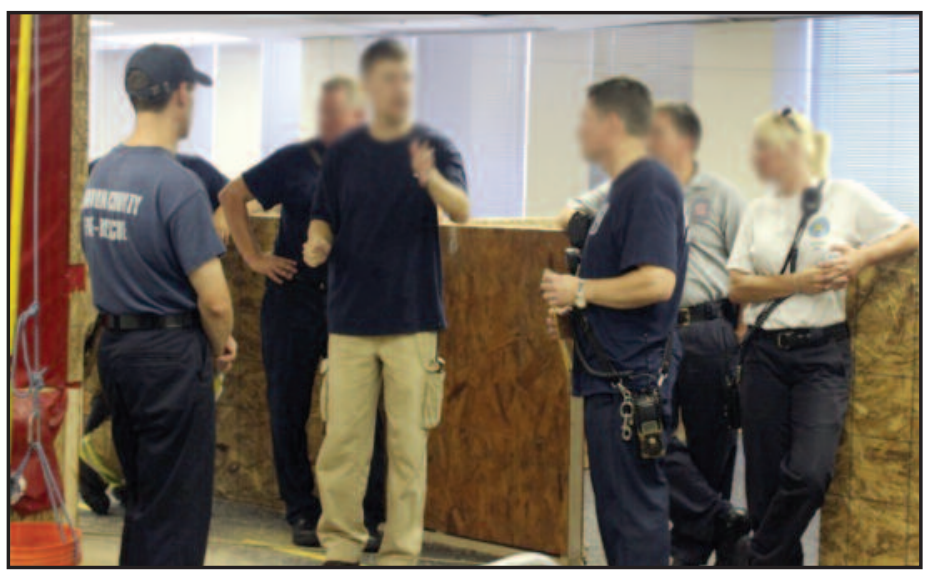

Figure 14: Officer walkthrough

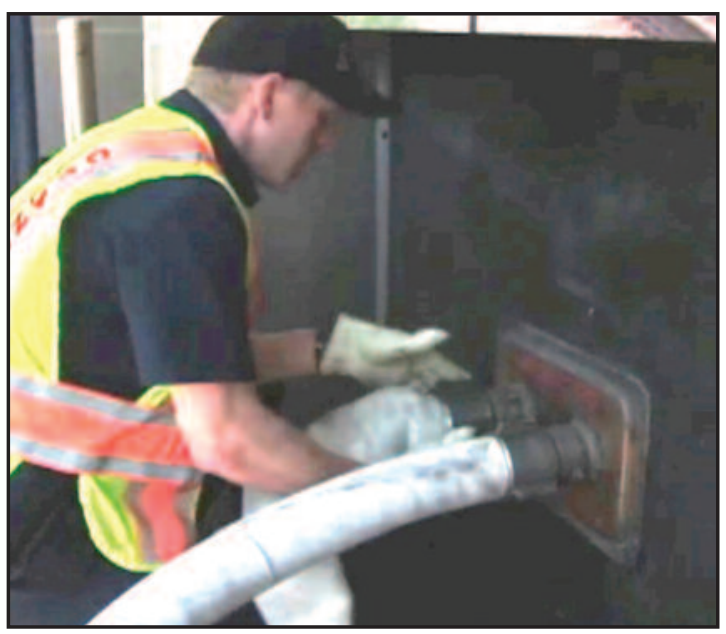

Figure 16: Driver connects to Siamese 

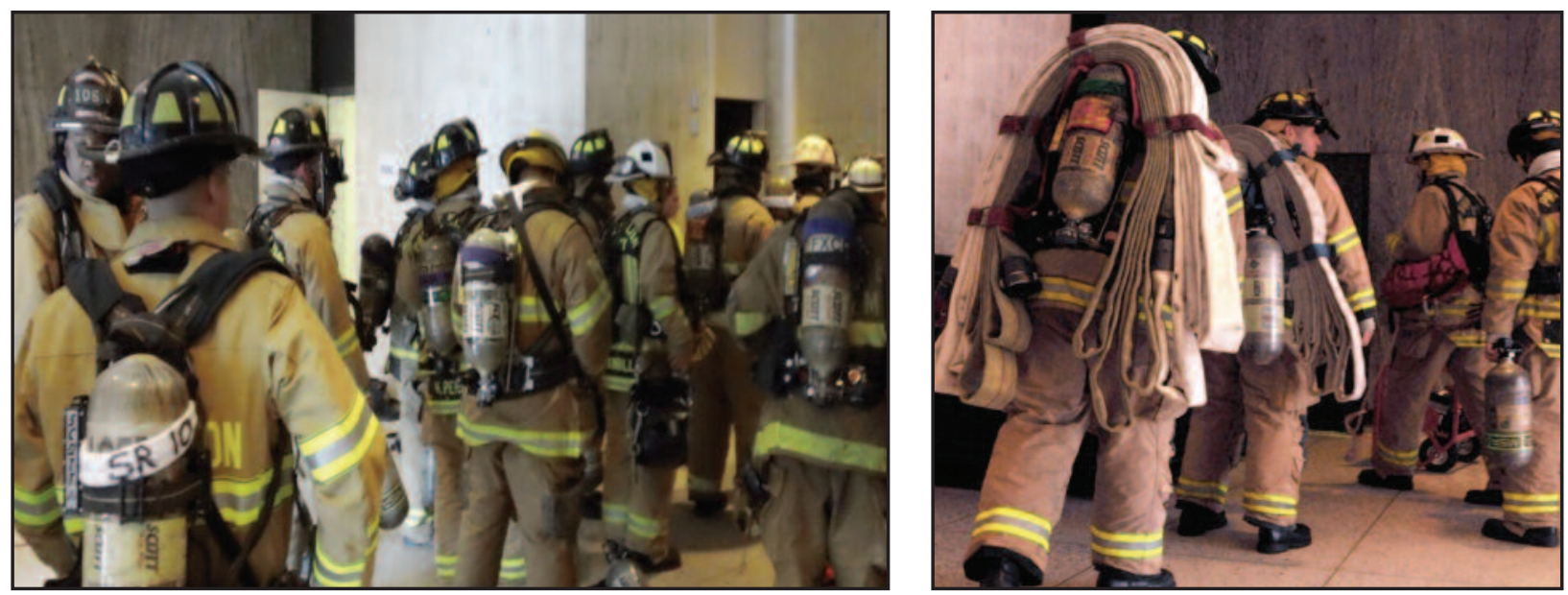

Figure 17: Crews arrive in lobby

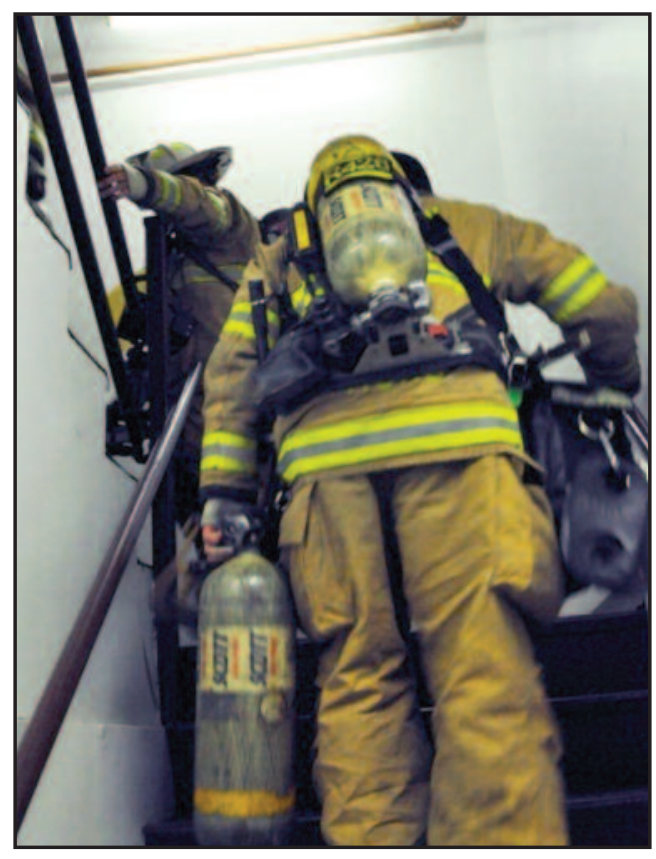

Figure 18: Crews ascend via stairs

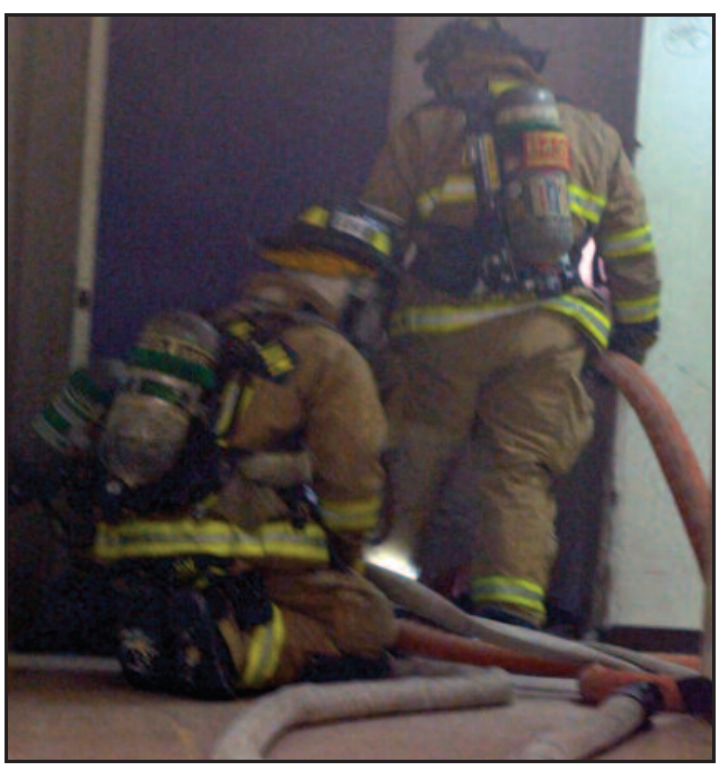

Figure 20: Firefighters assist in moving the attack line and second line on Fire Floor

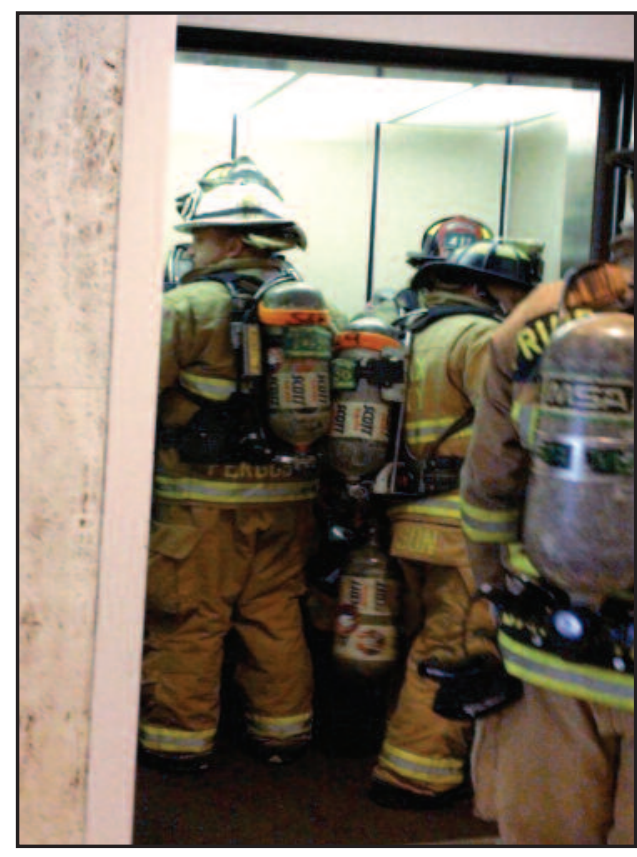

Figure 19: Crews ascend via elevator

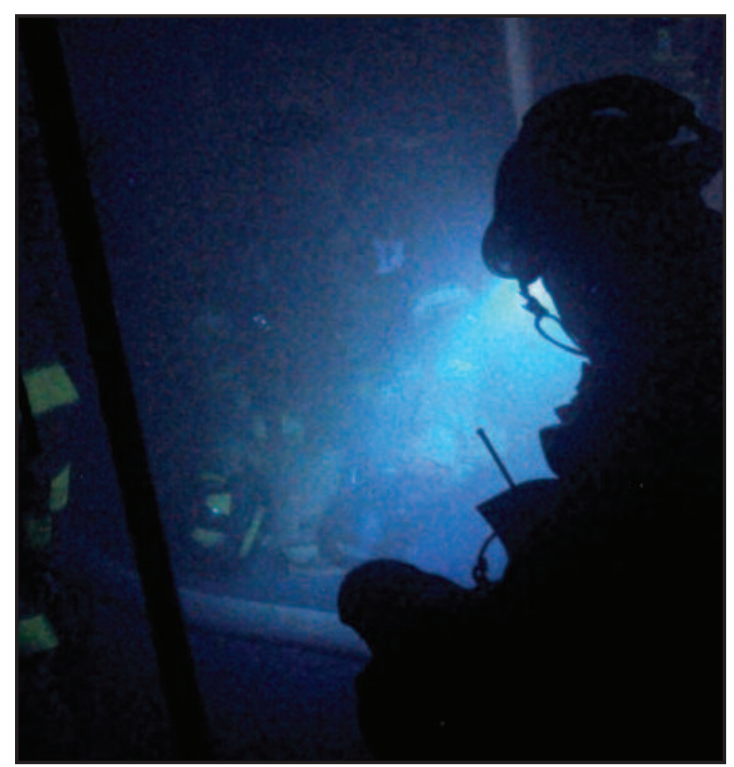

Figure 21: Establish line above the fire (Floor 11) 


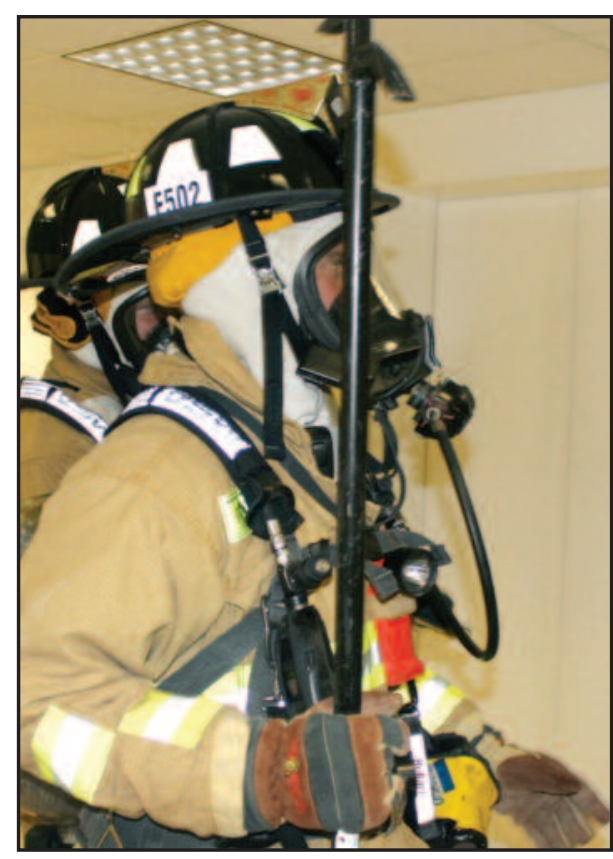

Figure 22: Search crew proceeds to fire floor

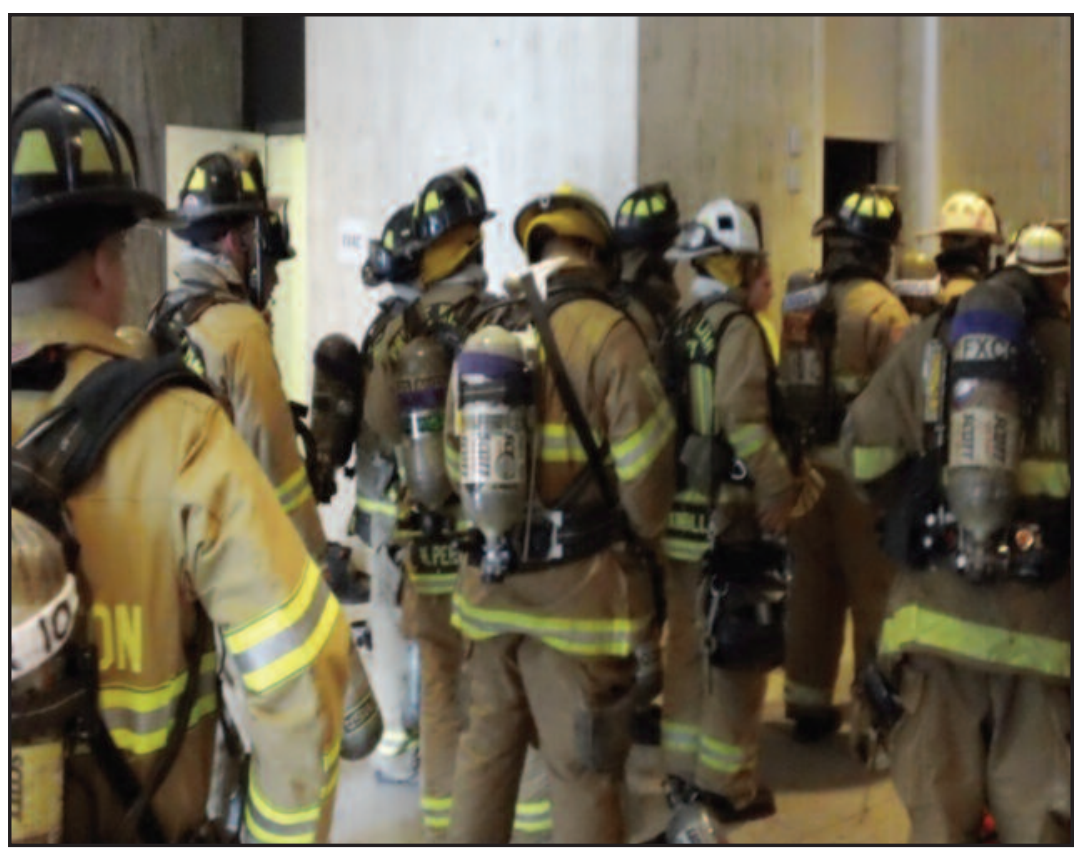

Figure 23: Search and rescue - Fire floor

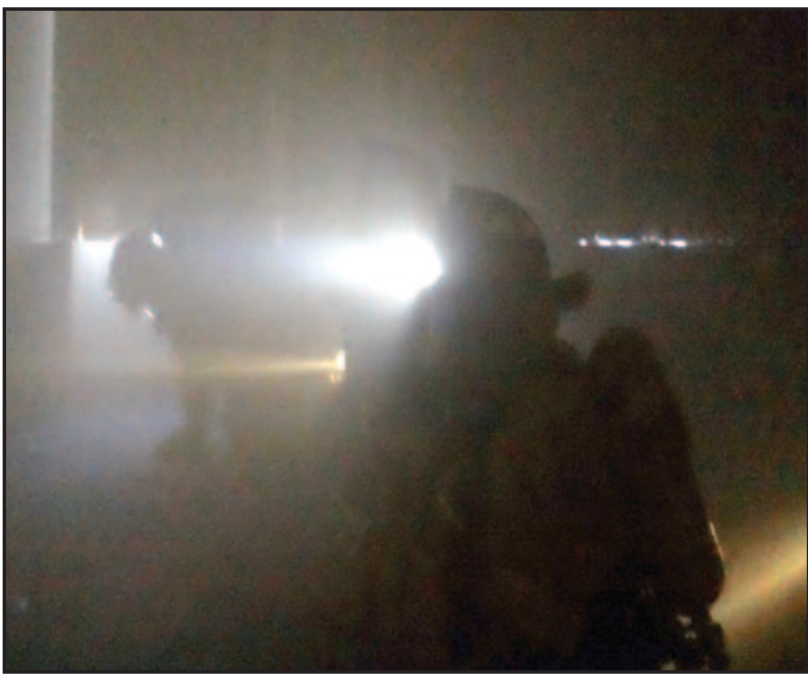

Figure 24: Search and rescue - Floor above fire

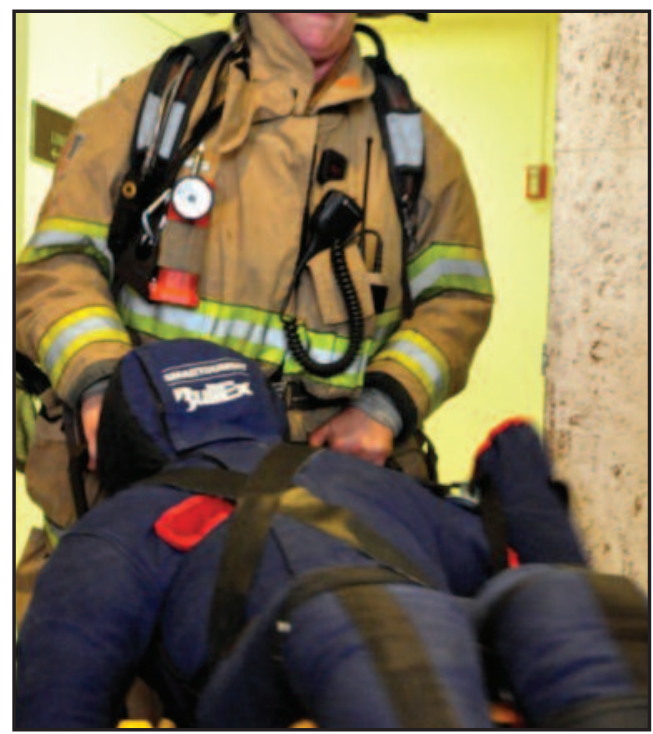

Figure 26: Victim descends via stairs

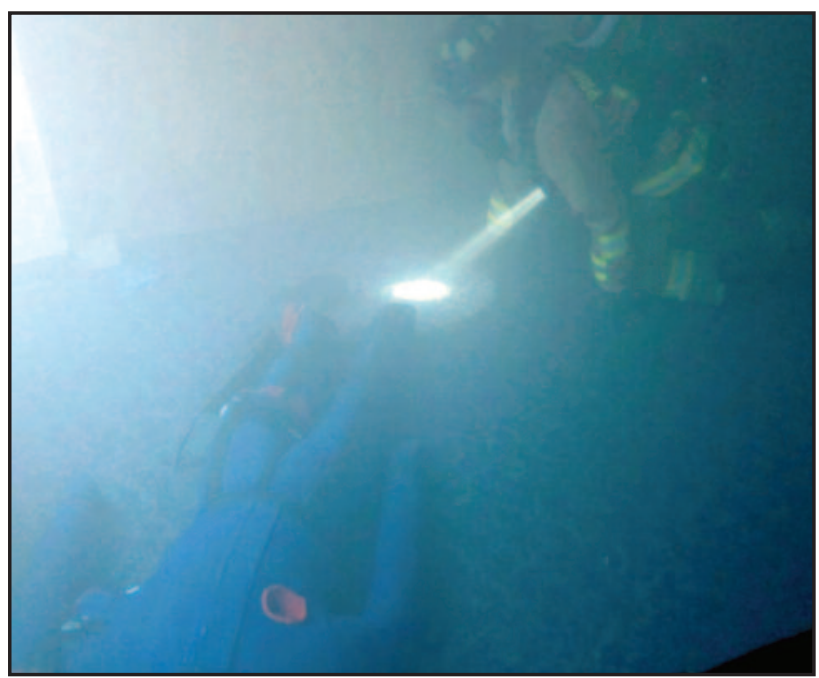

Figure 25: Victim located on floor above fire

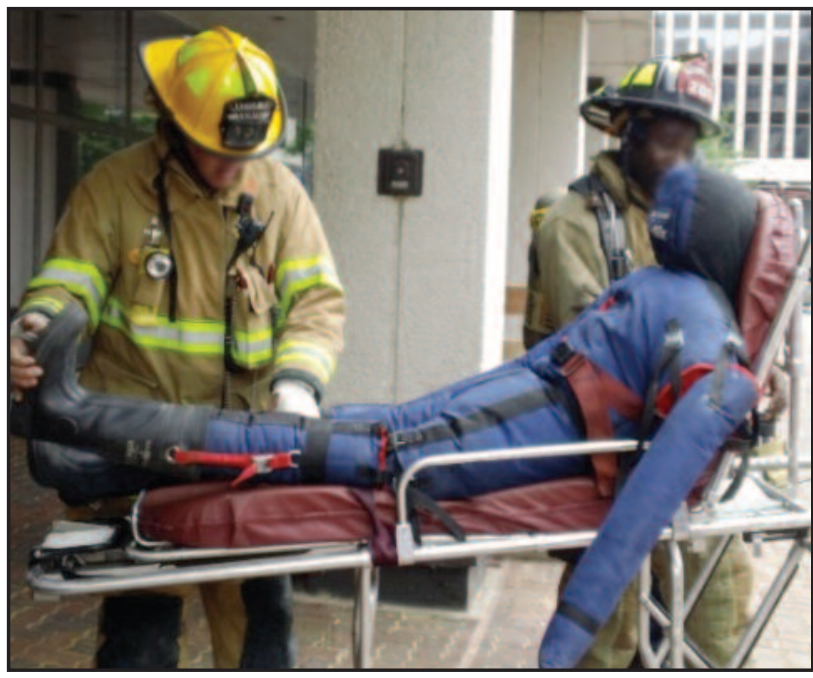

Figure 27: Victim removed from building 
Each task was assigned a standardized start and end marker for consistent data recording. For example, search and rescue start time was 'initial crew entry onto a floor to begin a search' and the end time for victim rescue was 'removal of the victim to the 8th floor stairwell landing'. The 38 tasks, including start and stop time markers, are shown in Table 3.

Table 3: Tasks and Measurement Parameters

\begin{tabular}{|c|c|c|c|}
\hline Tasks & Measurement Parameters & Tasks & Measurement Parameters \\
\hline 1. Initial Si e-up & $\begin{array}{l}\text { START > First Crew Goes to Work } \\
\text { STOP> Officer begins command } \\
\text { statement on scene }\end{array}$ & $\begin{array}{l}\text { 15. Second Line-Connect to } \\
\text { Standpipe }\end{array}$ & $\begin{array}{l}\text { START }>\text { Crew ascends from lobby } \\
\text { STOP }>\text { Second line connected to } \\
\text { wye valve }\end{array}$ \\
\hline 2. Establish Command & $\begin{array}{l}\text { START > First Crew Goes to Work } \\
\text { STOP> Command statement } \\
\text { completed }\end{array}$ & 16. Advance Second Line & $\begin{array}{l}\text { START> Second line no le } \\
\text { through 10th floor stairwell door } \\
\text { STOP }>\text { Second line-Water on Fire }\end{array}$ \\
\hline 3. Lobby Control & $\begin{array}{l}\text { START > First Crew Goes to Work } \\
\text { STOP }>\text { Attack/Evac } \\
\text { stairwells/elevators are located, } \\
\text { designated, and confirmed }\end{array}$ & 17. Fire Out & $\begin{array}{l}\text { START>Attack line no le } \\
\text { through 10th floor stairwell door } \\
\text { STOP }>\text { Attack and second lines } \\
\text { cross target threshold }\end{array}$ \\
\hline \multirow[t]{2}{*}{ 4. Establish IRIC } & \multirow{2}{*}{$\begin{array}{l}\text { TIME }>2 \text { crew members } \\
\text { assembled on 8th floor, not on } \\
\text { air, standing by and ready to assist } \\
\text { in the event of firefighter } \\
\text { emergency }\end{array}$} & $\begin{array}{l}\text { 18. Check for Fire Extension } \\
\text { on 10th floor }\end{array}$ & $\begin{array}{l}\text { START> Firefighter(s) touch pike pole } \\
\text { STOP> Thirty pulls on weighted } \\
\text { pike pole completed }\end{array}$ \\
\hline & & 19. Positive Pressure & TIME $>1 \mathrm{~min}$ after Fire Out \\
\hline \multirow[t]{3}{*}{ 5. Establish RIC (RIT) } & \multirow{3}{*}{$\begin{array}{l}\text { START > Crew enters } \\
\text { stairwell/elevator from lobby } \\
\text { STOP }>\text { Crew assembled on 8th } \\
\text { floor landing }\end{array}$} & & \\
\hline & & 20. Roof Ventilation & TIME $>1$ min after Fire Out \\
\hline & & 21. Search and Rescue Crew & START> Crew enters \\
\hline \multirow[t]{2}{*}{$\begin{array}{l}\text { 6. Establish Medical, } \\
\text { Rehab- Ambulance } 1\end{array}$} & \multirow{2}{*}{$\begin{array}{l}\text { START > Initial arrival, Ambulance } 1 \\
\text { START > Initial arrival, Ambulance } 1 \\
\text { STOP }>\text { Crew enters staging with } \\
\text { all equipment }\end{array}$} & & $\begin{array}{l}\text { STOP }>\text { Crew assembled on 8th } \\
\text { floor landing }\end{array}$ \\
\hline & & 22. Search and Rescue (Floor 10) & START> Crew through 10th floor \\
\hline \multirow[t]{2}{*}{ 7. Establish Medical, Ambulance-2 } & $\begin{array}{l}\text { START > Initial arrival, Ambulance } 2 \\
\text { STOP }>\text { Crew enters staging with } \\
\text { all equipment }\end{array}$ & & $\begin{array}{l}\text { STOP> Primary search on 10th } \\
\text { floor completed }\end{array}$ \\
\hline & & 23. Victim \#1 Found (Floor 10) & START> Crew through 10th floor \\
\hline \multirow[t]{2}{*}{ 8. Establish Medical, Ambulance-3 } & \multirow{2}{*}{$\begin{array}{l}\text { START> Initial arrival, Ambulance } 3 \\
\text { STOP }>\text { Crew enters staging with } \\
\text { all equipment }\end{array}$} & & $\begin{array}{l}\text { stairwell door } \\
\text { STOP }>\text { Victim found on 10th floor }\end{array}$ \\
\hline & & \multirow[t]{2}{*}{ 24. Victim \#1 Rescue (Floor 10) } & \multirow{2}{*}{$\begin{array}{l}\text { START > Victim found on 10th floor } \\
\text { STOP> Victim arrives on 8th floor } \\
\text { landing }\end{array}$} \\
\hline 9. Establish Staging & $\begin{array}{l}\text { START > Initial arrival, Truck } 3 \\
\text { STOP }>\text { Crew enters staging }\end{array}$ & & \\
\hline \multirow[t]{2}{*}{$\begin{array}{l}\text { 10. Establish Stairwell/Elevator } \\
\text { Support }\end{array}$} & \multirow{2}{*}{$\begin{array}{l}\text { START > Initial arrival, Truck } 5 \\
\text { STOP > } 35 \text { cylinders, extra search } \\
\text { rope and } 2 \text { coolers brought to } \\
\text { staging }\end{array}$} & 25. Victim \#1 Descent & $\begin{array}{l}\text { START }>\text { Victim exits 8th floor } \\
\text { STOP }>\text { Victim exits building }\end{array}$ \\
\hline & & \multirow[t]{2}{*}{ 26. Line Above Fire Crew Ascension } & \multirow{2}{*}{$\begin{array}{l}\text { START> Crew enters } \\
\text { stairwell/elevator from lobby } \\
\text { STOP }>\text { Crew assembled on 8th } \\
\text { floor landing }\end{array}$} \\
\hline \multirow[t]{2}{*}{ 11. Attack Crew Ascension } & \multirow{2}{*}{$\begin{array}{l}\text { START }>\text { Crew enters } \\
\text { stairwell/elevator from lobby } \\
\text { STOP }>\text { Crew assembled on 8th } \\
\text { floor landing }\end{array}$} & & \\
\hline & & 27. Line Above Fire-Connect & START> Crew ascends from lobby \\
\hline \multirow[t]{2}{*}{$\begin{array}{l}\text { 12. Attack Line-Connect to } \\
\text { Standpipe }\end{array}$} & \multirow{2}{*}{$\begin{array}{l}\text { START }>\text { Crew enters } \\
\text { stairwell/elevator from lobby } \\
\text { STOP }>\text { Attack line connected to } \\
\text { gated wye valve }\end{array}$} & & $\begin{array}{l}\text { STOP }>\text { Line above fire connected } \\
\text { to wye valve }\end{array}$ \\
\hline & & \multirow[t]{2}{*}{ 28. Advance Line Above Fire } & \multirow{2}{*}{$\begin{array}{l}\text { START > Line above fire no le } \\
\text { through 11th floor stairwell door } \\
\text { STOP }>\text { Line above fire at target } \\
\text { threshold }\end{array}$} \\
\hline \multirow[t]{2}{*}{ 13. Advance Attack Line } & \multirow{2}{*}{$\begin{array}{l}\text { START> Attack line no le } \\
\text { through } 10 \text { th floor stairwell door } \\
\text { STOP }>\text { Attack line-Water on Fire }\end{array}$} & & \\
\hline & & \multirow{2}{*}{$\begin{array}{l}\text { 29. Check for Fire Extension } \\
\text { on 11th floor }\end{array}$} & \multirow{2}{*}{$\begin{array}{l}\text { START > Firefighter(s) touch } \\
\text { weighted pike pole } \\
\text { STOP> Thirty pulls on pike pole } \\
\text { completed }\end{array}$} \\
\hline 14. Second Line Crew Ascension & $\begin{array}{l}\text { START }>\text { Crew enters } \\
\text { stairwell/elevator from lobby } \\
\text { STOP }>\text { Crew assembled on 8th } \\
\text { floor landing }\end{array}$ & & \\
\hline
\end{tabular}




\begin{tabular}{|l|l|}
\hline \multicolumn{1}{|l|}{ Tasks } & Measurement Parameters \\
\hline Ascension (Floor 11) & $\begin{array}{l}\text { START > Crew enters } \\
\text { stairwell/elevator from lobby } \\
\text { STOP> Crew assembled on 8th } \\
\text { floor landing }\end{array}$ \\
\hline 31. Search and Rescue (Floor 11) & $\begin{array}{l}\text { START> Crew through 11th floor } \\
\text { stairwell door } \\
\text { STOP> Primary search on 11th } \\
\text { floor completed }\end{array}$ \\
\hline 32. Victim \#2 Found (Floor 11) & $\begin{array}{l}\text { START> Crew through 11th floor } \\
\text { stairwell door } \\
\text { STOP> Victim found on 11th floor }\end{array}$ \\
\hline 33. Victim \#2 Rescue (Floor 11) & $\begin{array}{l}\text { START> Victim found on 11th } \\
\text { floor } \\
\text { STOP> Victim arrives on 8th floor } \\
\text { landing }\end{array}$ \\
\hline 34. Victim \#2 Descent & $\begin{array}{l}\text { START> Victim exits 8th floor } \\
\text { STOP> Victim exits building }\end{array}$ \\
\hline
\end{tabular}

\subsection{Other Tasks}

Similar to the residential fireground study (Averill et al., 2010), some fireground tasks were not included in the scope of this study. These include salvage and overhaul, mitigation of water, and removal of firefighting equipment from the building to the fire apparatus. From the time when the key tasks measured by this study have been completed to the point at which units dispatched to the incident can be returned to service may be a considerable period of time. This time period affects the delivery of fire department services since units are not available to respond to simultaneous incidents, and it should be accounted for in the design of community standards of cover.

\subsection{Data Collection: Standardized Control Measures}

Several measures were used to collect data, including radio communications, task timers, search buttons, and a number of video recordings. Performance was timed for each task in each scenario including selected critical tasks such as Water on Fire, Fire Out, Search Complete, Victim Found, and Victim Rescue. Data were collected for crew performance on each task. Individual firefighter time was not considered.

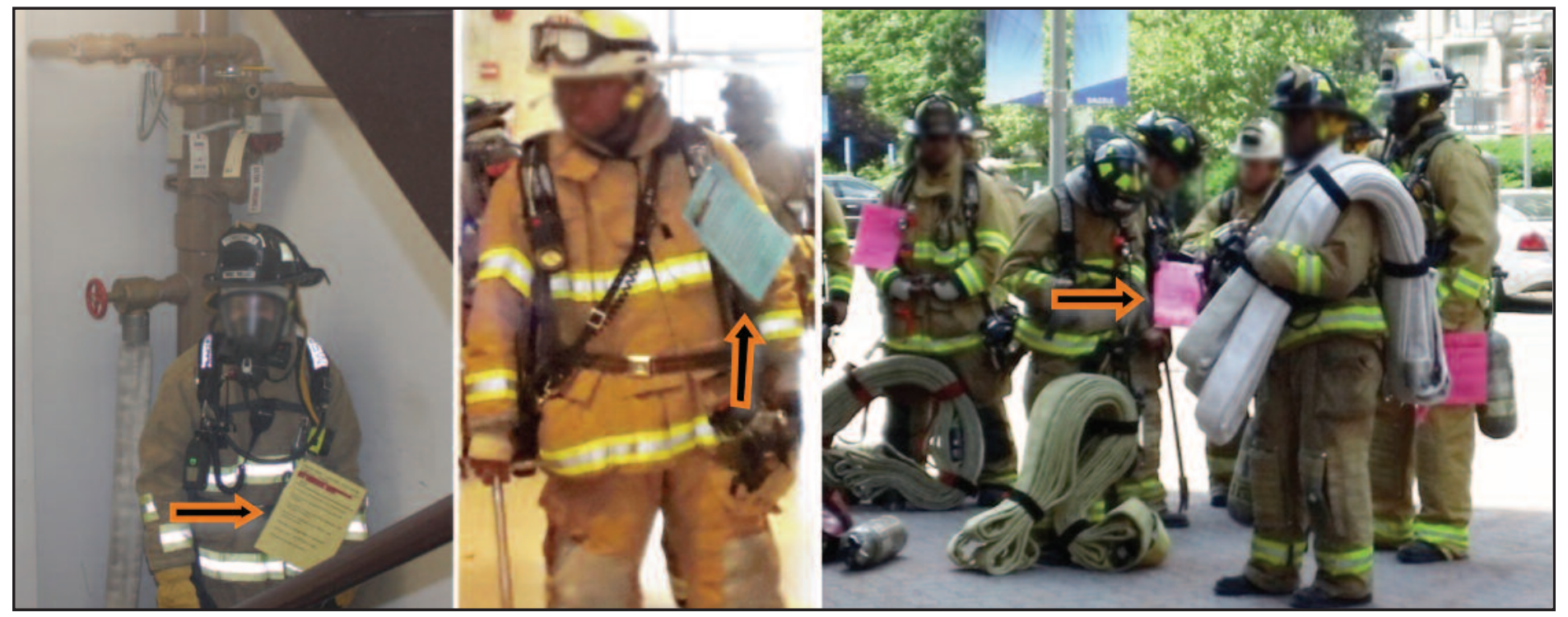

Figure 28: Crew cue cards 


\subsection{Radio Communications}

Interoperability and intraoperability of radio equipment used by all participating departments made it possible to use regular duty radios for communication during the experiments.

Company officers were instructed to use radios as they would in an actual incident while keeping unnecessary radio traffic to a minimum.

Arlington County Fire and Rescue Communications recorded all radio interaction as a means of data backup. Arlington County Communications assigned the study two exclusive channels. One channel was used by the researchers for starting, monitoring, and stopping each experiment while the other was designated the tactical command channel for use by study participants. Once all data quality control measures were complete, the communications records were overwritten as a routine procedure.

\subsection{Task Timers}

Eleven observers/timers, trained in the use of a standard stop watch with split-time feature, recorded time-to-task data for each field experiment, as shown in Figure 29. To ensure understanding of the observed tasks, firefighters were used as timers. Each timer was assigned specific tasks to observe and to record the start and end times. All timers wore high-visibility safety gear on the fireground. To enhance accuracy and consistency in recording times, the data recording sheets used color-coding for the tasks (see Appendix A). Each timer was assigned tasks coded in the same color. Due to the large number of firefighters participating in the experiments, crews wore color-coded "marker bands" around their SCBA bottles, as shown in Figure 30. These bands were coded to match specific categories of tasks corresponding with the timer data sheets and assisted the timers in identifying which crews performed tasks they were assigned to observe.

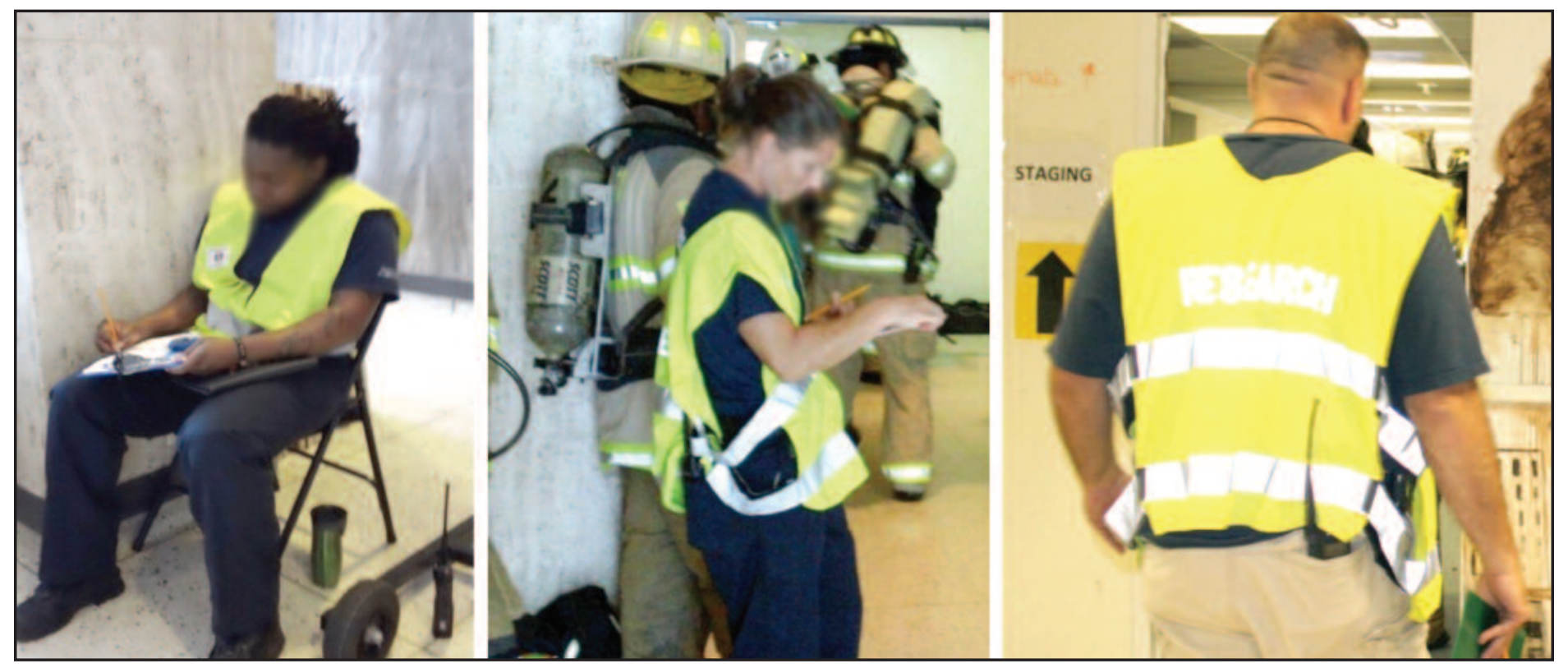

Figure 29: Task timers located throughout the high-rise structure

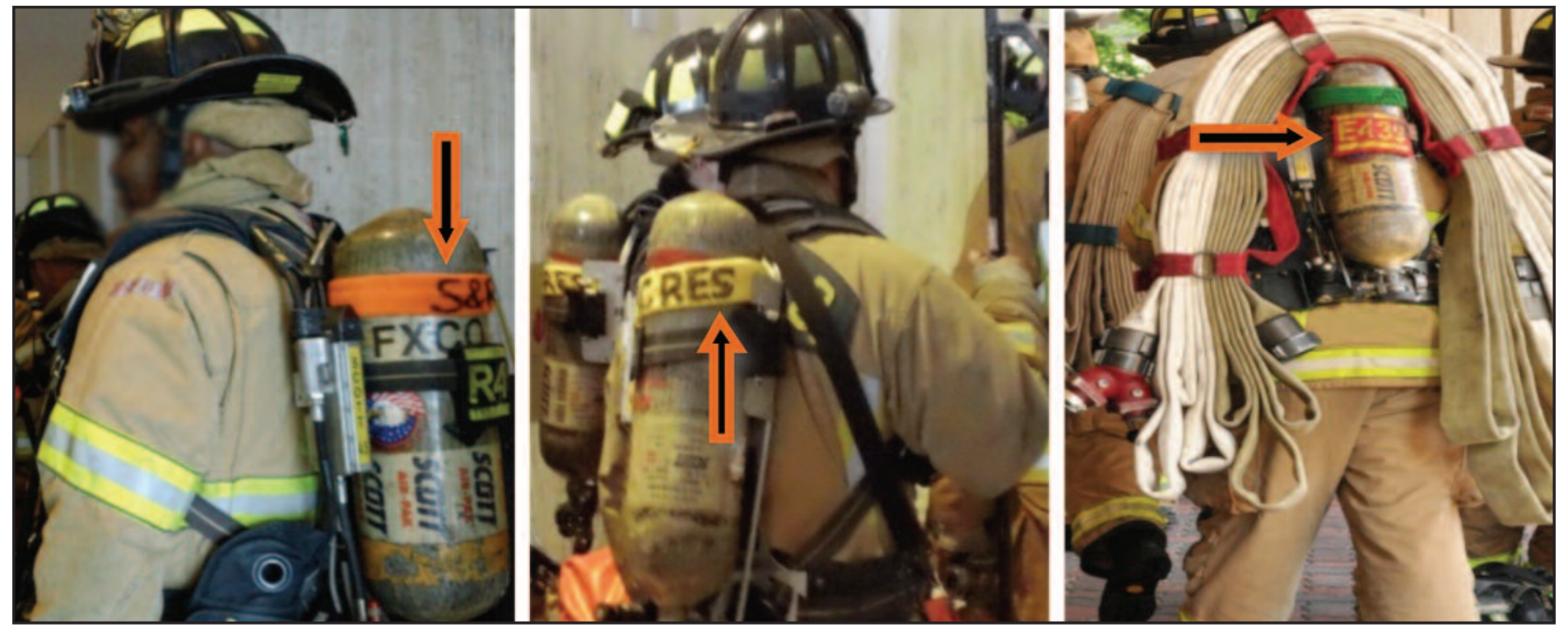

Figure 30: Task marker bands worn by firefighters during the experiments 
Timers/observers were also used for purposes of quality control and safety. They were allowed to direct crews and answer questions, as long as the experiment protocol was not affected. This local oversight was necessary since crews were working on several different floors simultaneously.

Timers were stationed on different floors in various parts of the building; therefore they were issued radios to allow them to hear the experiment "start time" and "stop time" commands and to maintain contact with the lead investigators in the event that there was a protocol breach or safety concern.

\subsection{Search Buttons}

Heavy smoke conditions on the fire floor and floor above prevented accurate tracking of search and rescue operations with timers. Instead, buttons were distributed at fixed locations on both floors. Firefighters performing searches were instructed to press the buttons as they encountered them along the prescribed search paths. When a button was pressed, the time of the press was recorded through a data acquisition system. From the locations of the buttons and the time each was pressed, a history of search and rescue progress on both floors was generated. This included Victim Rescue time (a button was located at the victim location) and Search Complete time (a button was located at the end of the search). Since the floor layouts of the fire floor and the floor above were different, the button placement on each floor was unique.

On the fire floor, 24 buttons were installed in the cubicles, nine buttons on the inside search loop and 15 buttons on the outside search loop. Figure 31 shows an isometric view of the fire floor with the location of each button marked. The image was created using Smokeview, a software tool developed by NIST to visualize FDS results. The victim was located at button 18 .

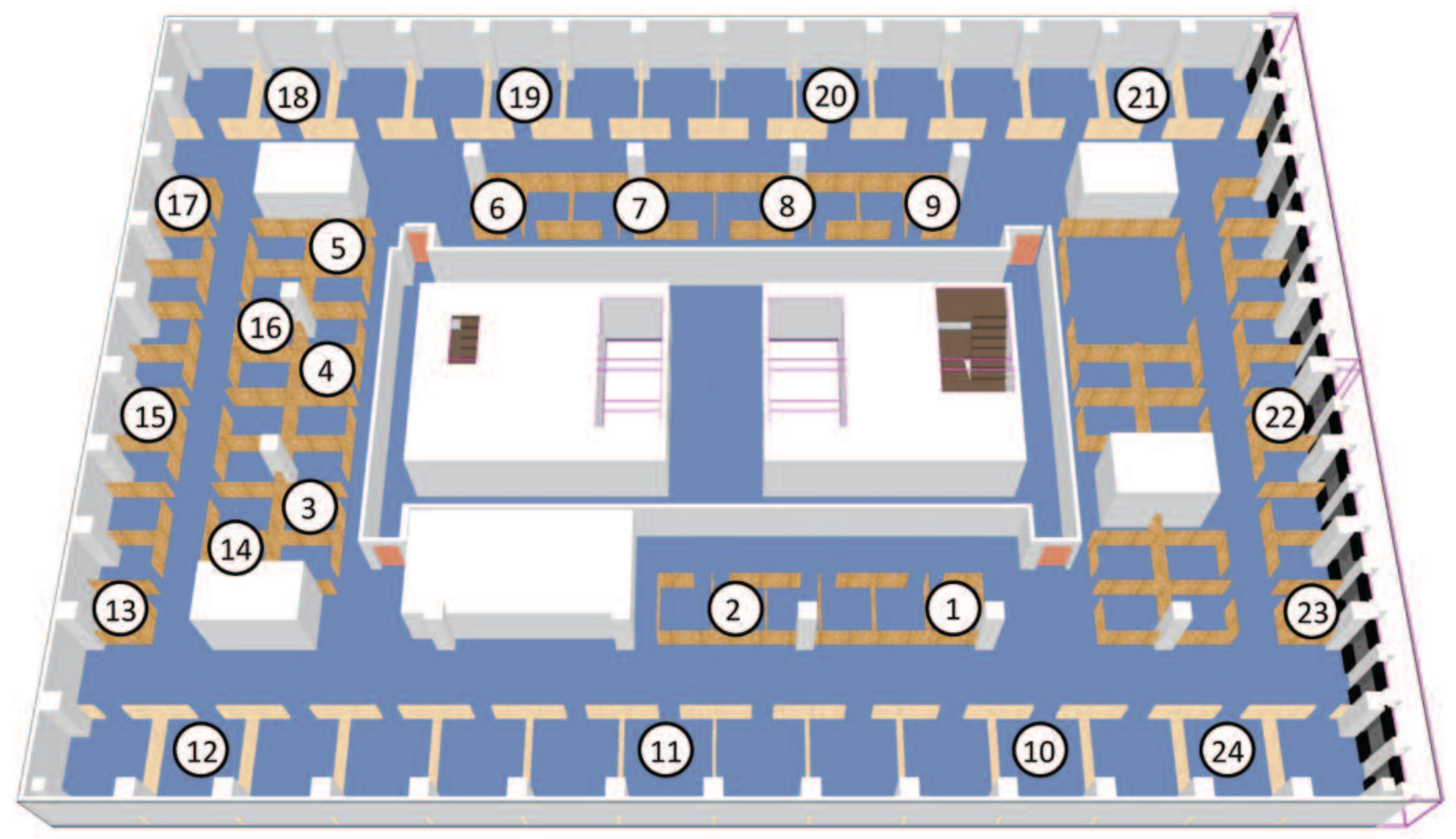

Figure 31: Search button locations on the fire floor 
On the floor above the fire (Floor 11), there were 22 buttons situated as shown in Figure 32. The conventional office configuration of Floor 11 (with floor to ceiling partitions) was quite different from the open-floor plan arrangement of Floor 10. As a result, buttons were installed along the walls of offices and connecting hallways. The victim on Floor 11 was located at button 15 .

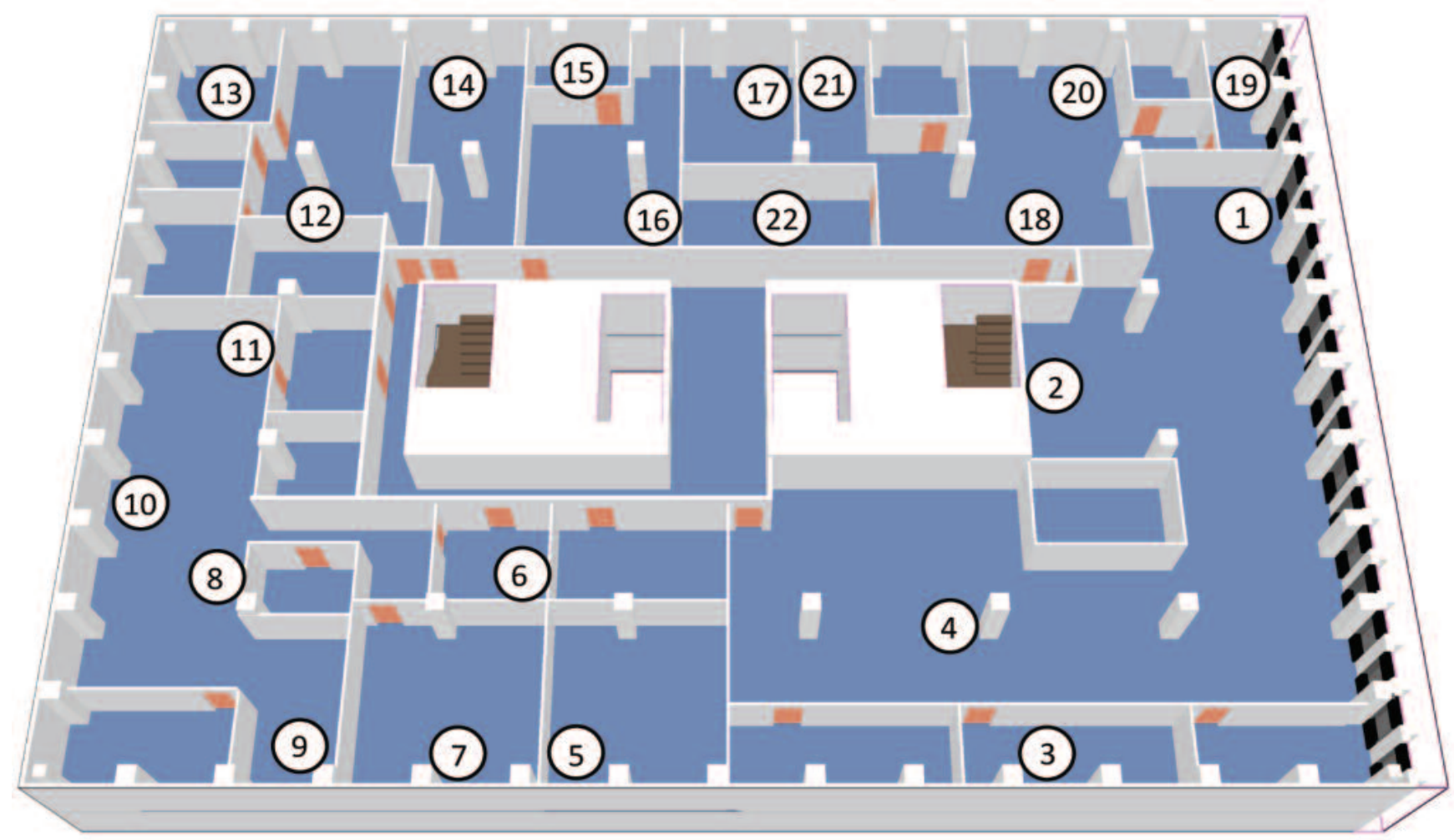

Figure 32: Search button locations on the floor above the fire (floor 11)

\subsection{Video Records}

In addition to the timers, video documentation was taken on one full set of high-rise scenarios. These video recordings provided a backup for timed tasks and for quality control. No fewer than six cameras were used to record fireground activity from varied vantage points. Video records were available for use in the data quality control process.

\subsection{Crew Assignments}

Crews from each participant department that regularly operated together were assigned to work as either engine or truck companies in each scenario. Depending on the scenario (crew size of $3,4,5$, or 6), firefighters were added or subtracted from regularly assigned crews to assemble the appropriate number for the scenario. This exercise, along with assigning different crews from different shifts and stations to the study on a daily basis, reduced learning from exact repetition of the same task and provided an opportunity for firefighters and officers from multiple departments to train for working together in a high hazard/ high risk environment.
Additionally, crews assigned to each responding company position in one scenario were assigned to another responding company position in subsequent scenarios, with the objective of minimizing learning from one experiment to another. For example, crews in the role of Engine 1 in a morning scenario might be assigned to the Engine 7 position in the afternoon.

\subsection{Response Time Assumptions}

Response time assumptions were made based on time objectives set forth in NFPA 1710, which is the nationally recognized consensus standard for career firefighter deployment and includes requirements for fire department arrival time, staffing levels, and fireground responsibilities.

In addition to the travel time and crew assembly time, the overall response time to a structure fire includes fire ignition, recognition, call to 9-1-1, call processing, dispatch, and turnout of responding crews. The various time segments assigned to the values in the overall response time were based on NFPA 1710 and NFPA $1221^{\circledR} \cdot{ }^{24}$

24. NFPA $1710^{\circledR}$ Organization and Deployment of Fire Suppression Operations, Emergency Medical Operations, and Special Operations to the Public by Career Fire Departments.

4.1.2.1 The fire department shall establish the following objectives: (1) Alarm handling time to be completed in accordance with 4.1.2.3. (2) $80 \mathrm{~s}$ for turnout time for fire and special operations response and 60 s turnout time for EMS response

4.1.2.3 Alarm Handling.

4.1.2.3.1 The fire department shall establish a performance objective of having an alarm answering time of not more than $15 \mathrm{~s}$ for at least $95 \%$ of the alarms received and not more than 40 s for at least $99 \%$ of the alarms received, as specified by NFPA 1221.

4.1.2.3.2 When the alarm is received at a public safety answering point (PSAP) and transferred to a secondary answering point or communication center, the agency responsible for the PSAP shall establish a performance objective of having an alarm transfer time of not more than $30 \mathrm{~s}$ for at least $95 \%$ of all alarms processed, as specified by NFPA 1221.

4.1.2.3.3 The fire department shall establish a performance objective of having an alarm processing time of not more than $60 \mathrm{~s}$ for at least $90 \%$ of the alarms and not more than 90 s for at least $99 \%$ of the alarms, as specified by NFPA 1221. 


\section{Additional time segments included in overall response time}

1) Fire ignition $=$ time ero

2) $30 \mathrm{~s}$ for recognition (detection of fire) and call to 9-1-1

3) $60 \mathrm{~s}$ for call processing/dispatch

4) $80 \mathrm{~s}$ for turnout

These time segments were added to the overall response time and considered in both time-to-task data analysis and fire modeling.

Responding company on-scene arrival times were calculated using ArcGIS (ArcInfo) geographical information system (GIS) software ${ }^{25}$ used for modeling emergency response. The address of the study location was entered into the GIS system along with all participating department station/company locations. The software was then used to map the response (travel time) of the appropriate companies to the scene.

After on-scene arrival, the 'time to engagement' or 'time to intervention' to stop the fire must be considered. This time period is referred to as 'assembly time', which is measured from the time apparatus wheels stop at the scene and includes the time for firefighters to gather equipment from the apparatus and the time for firefighters to walk to the building, get an assignment, and enter the building. To account for this additional time to intervention, $90 \mathrm{~s}$ was added to the travel time for each company. Therefore, the total time including detection, call processing, turnout, and travel time plus the on-scene assembly time is referred to here as the 'go-to-work' time. Figure 33 shows the measurement of time from fire ignition through the response and performance times to the completion of all firefighting tasks.

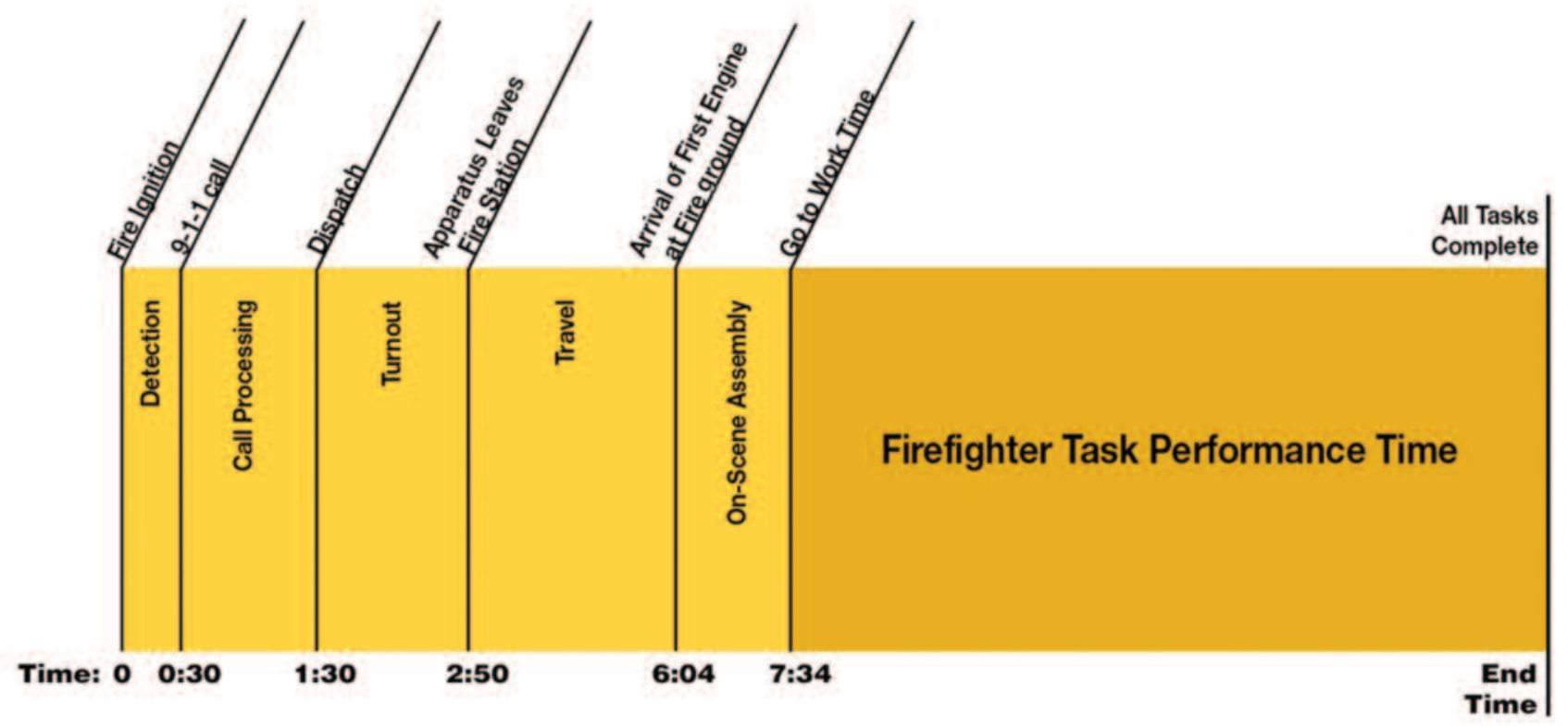

Figure 33: Timing for firefighter response and performance

4.1.2.4 The fire department shall establish a performance objective of not less than $90 \%$ for the achievement of each turnout time and travel time objective specified in 4.1.2.1. 25. Geographic information system (GIS) software lets users visualize, question, analyze, interpret, and understand geographical data to reveal relationships, patterns, and trends. GIS mapping software has a routing feature that projects travel times to a specific address from various multiple locations. GIS not only records travel time but also records the order of arrival of apparatus traveling to the address based on their origin. 
Table 4 shows the go-to-work time of responding units according to alarm size. The routes taken by the responding units are shown in the map in Figure 34. As noted previously in Table 2, the actual number of firefighters used in the experiments was fewer than the total calculated based on crew size and alarm size. This difference is due to the fact that later arriving crews on the high alarm deployment would have been assigned to base as tactical reserve for use in later tasks. Since individual experiments were ended following the completion of a primary search and the rescue of victims on the fire floor and the floor above the fire, these resources weren't used during the exercise. Unlike the field experiments, an actual high-rise fire would have engaged these additional resources to conduct search, overhaul, and salvage.

\begin{tabular}{|c|c|c|c|c|c|c|c|}
\hline \multicolumn{2}{|c|}{ HIGH } & \multicolumn{2}{|l|}{ LOW } & \multicolumn{2}{|c|}{ HIGH } & \multicolumn{2}{|c|}{ LOW } \\
\hline Engine 1 & $7: 34$ & Engine 1 & $7: 34$ & Ambulance $7^{*}$ & $16: 00$ & $\mathrm{BC} 3$ & $16: 00$ \\
\hline Truck 1 & $7: 34$ & Truck 1 & $7: 34$ & $\mathrm{BC} 3$ & $16: 00$ & Truck 5 & $16: 05$ \\
\hline Ambulance 1 & $7: 34$ & Ambulance 1 & $7: 34$ & Truck 5 & $16: 05$ & Engine 9 & $16: 17$ \\
\hline Engine 2 & $9: 03$ & Engine 2 & 9:03 & Ambulance $8^{*}$ & $16: 05$ & $\mathrm{BC} 4^{*}$ & $16: 17$ \\
\hline Ambulance 2 & $10: 20$ & Ambulance 2 & $10: 20$ & Engine 9 & $16: 17$ & Truck 6 & $16: 51$ \\
\hline Engine 3 & $11: 01$ & Engine 3 & $11: 01$ & Ambulance $9^{*}$ & $16: 17$ & $\mathrm{BC} 5^{\star}$ & $16: 51$ \\
\hline Truck 2 & $11: 01$ & Truck 2 & $11: 01$ & $\mathrm{BC} 4^{*}$ & $16: 17$ & Truck 7 & $17: 02$ \\
\hline Ambulance 3 & $11: 01$ & BC 1 & $11: 01$ & Engine $10^{\star}$ & $16: 29$ & Truck 8 & $17: 14$ \\
\hline $\mathrm{BC} 1$ & $11: 01$ & Truck 3 & $11: 58$ & Engine $11^{*}$ & $16: 41$ & Truck 9 & $17: 32$ \\
\hline Engine 4 & $11: 58$ & BC 2 & $12: 35$ & Engine $12^{*}$ & $16: 43$ & $\mathrm{BC} 6^{*}$ & $18: 26$ \\
\hline Truck 3 & $11: 58$ & Ambulance 3 & $14: 11$ & Truck 6 & $16: 51$ & & \\
\hline Truck 4 & $12: 17$ & Engine 4 & $15: 08$ & $\mathrm{BC} 5^{*}$ & $16: 51$ & & \\
\hline BC 2 & $12: 35$ & Engine 5 & $15: 27$ & Truck 7 & $17: 02$ & & \\
\hline Engine 5 & $15: 27$ & Truck 4 & $15: 27$ & Truck 8 & $17: 14$ & & \\
\hline Ambulance $4^{*}$ & $15: 27$ & Ambulance $4^{*}$ & $15: 27$ & Truck 9 & $17: 32$ & & \\
\hline Engine 6 & $15: 45$ & Engine 6 & $15: 45$ & Truck $10^{*}$ & $18: 21$ & & \\
\hline Ambulance $5^{\star}$ & $15: 45$ & Engine 7 & $15: 45$ & $\mathrm{BC} 6^{*}$ & $18: 26$ & & \\
\hline Engine 7 & $15: 45$ & Ambulance $5^{\star}$ & $15: 45$ & Truck $11^{*}$ & $18: 52$ & & \\
\hline Ambulance $6^{*}$ & $15: 45$ & Ambulance $6^{\star}$ & $15: 45$ & Truck $12^{*}$ & $19: 25$ & & \\
\hline Engine 8 & $16: 00$ & Engine 8 & $16: 00$ & ${ }^{*}$ not actually dis & tched $\mathrm{d}$ & periments & \\
\hline
\end{tabular}

Table 4: Go-to-work times calculated by GIS 


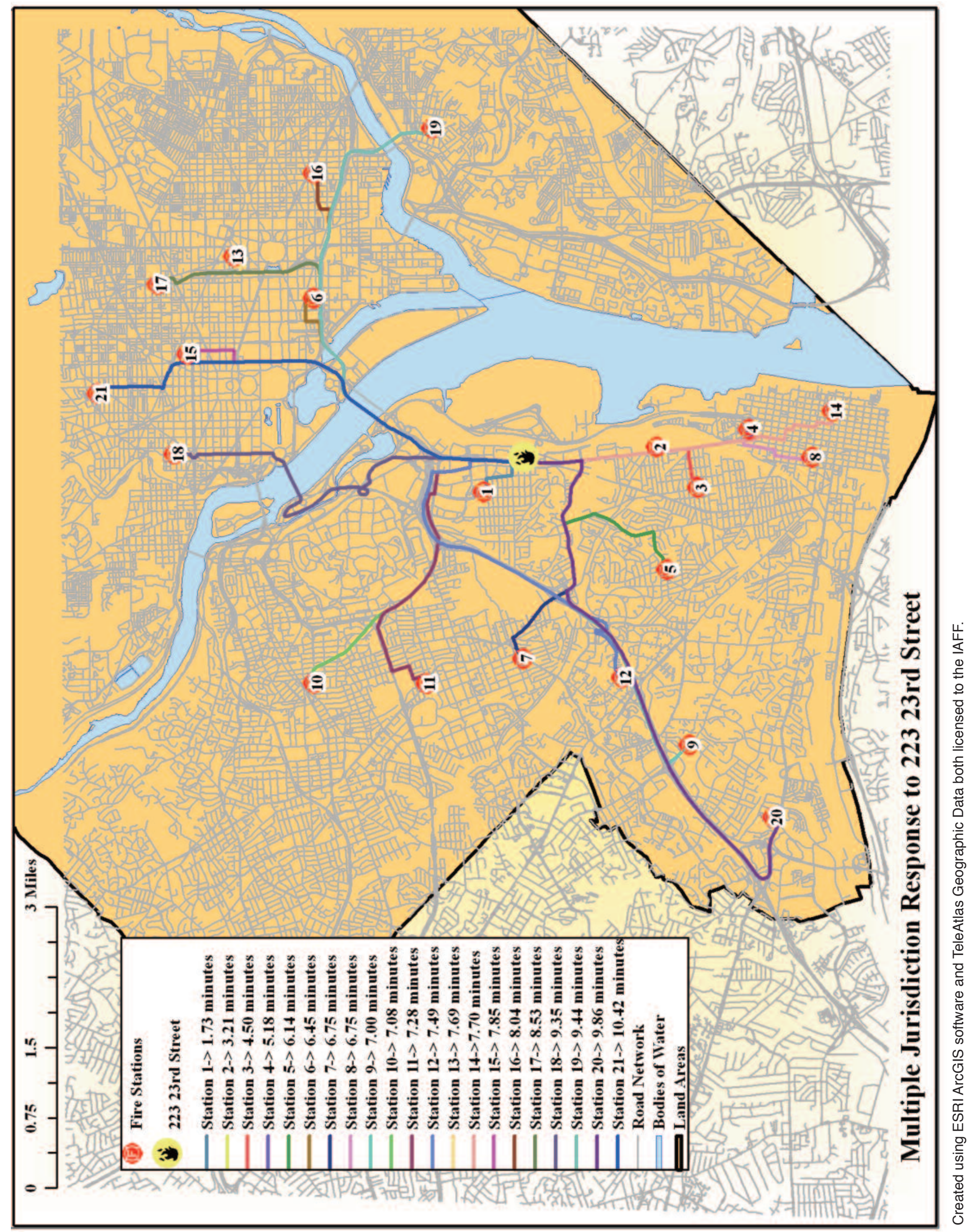




\section{Stages of High-Rise Fire Operations}

$\mathrm{T}$ he on-scene fire department task stage of the study focused on the tasks firefighters perform after they arrive on the scene of a high-hazard high-rise residential structure fire. A number of nationally recognized fire service experts were consulted during the development of the on-scene fire department tasks in order to ensure broad applicability and appropriateness of the task distribution. ${ }^{26}$

\subsection{Incident Command}

The high-rise fireground operations were managed and controlled using the Incident Command System (ICS). ${ }^{27}$ For each experiment, the officer on the first arriving company assumed Incident Command (IC). The officer maintained command until the arrival of the first Battalion Chief, at which time IC was transferred to the chief and the company officer assumed Lobby Control. Upon assuming command, the first Battalion Chief established the incident command post from his vehicle located in the front of the building, as shown in Figure 35. After assuming command, the IC confirmed the location and tasks of all companies that arrived prior to arrival. All subsequent crews entering the structure/fireground passed by the IC to check in and receive their assignments.

It was the responsibility of the IC to design and implement a basic fireground strategy, maintain overall situation awareness of progress toward critical objectives, and manage all equipment, personnel, procedures and communications operating within the high-rise structure. In order to manage the numerous assets, the IC and aide used a command board (shown in Figure 36). The chief's aide maintained the board while the IC maintained command via radio communication.

Upon arrival of Battalion Chief (BC) Two and Battalion Chief Three, the IC assigned them to supervise operations on the fire floor (10th floor) and the next most hazardous floor, the floor above the fire (11th floor). These two BC's became Division 10 and Division 11, respectively. All crews operating on these two floors then communicated directly with their respective division commander, who in turn communicated with the IC. Necessary communications included company task status, on-air supply status, and any necessary firefighter safety interaction. If crews operating in Division 10 or Division 11 required relief prior to task completion, relief was requested to the Division Supervisor. Request for relief crews were relayed from Division Supervisors to the IC then to the staging manager. The staging manager sent fresh crews to their assignments as instructed by the IC. All relieved crews were sent from their task area to Rehab (8th floor) for a minimum recuperation period of $15 \mathrm{~min}$.
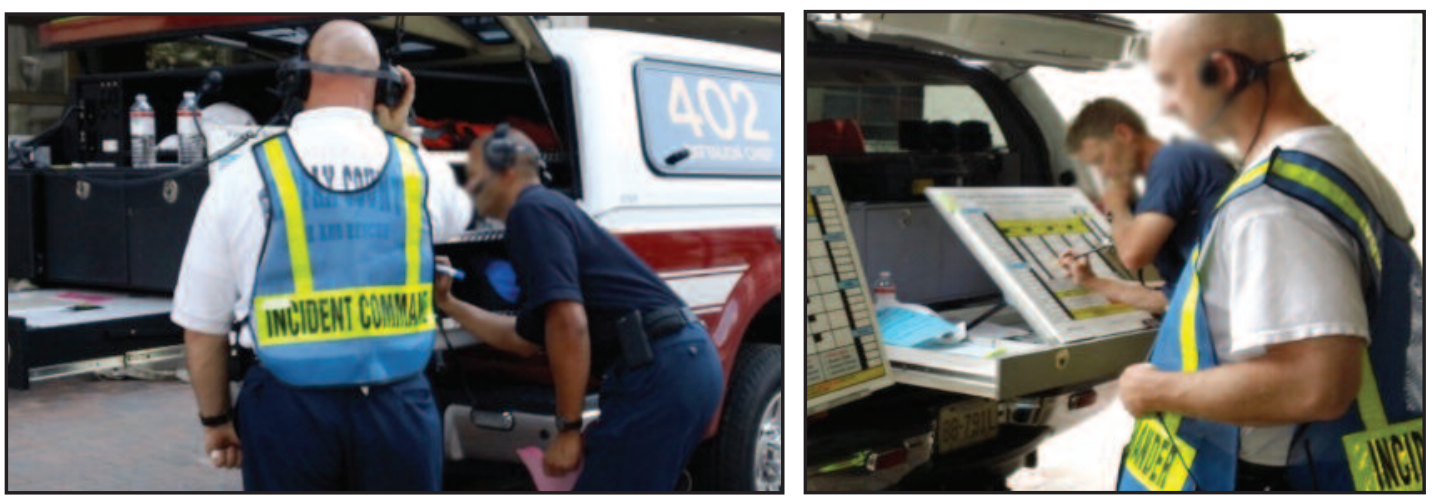

Figure 35: Incident Command post

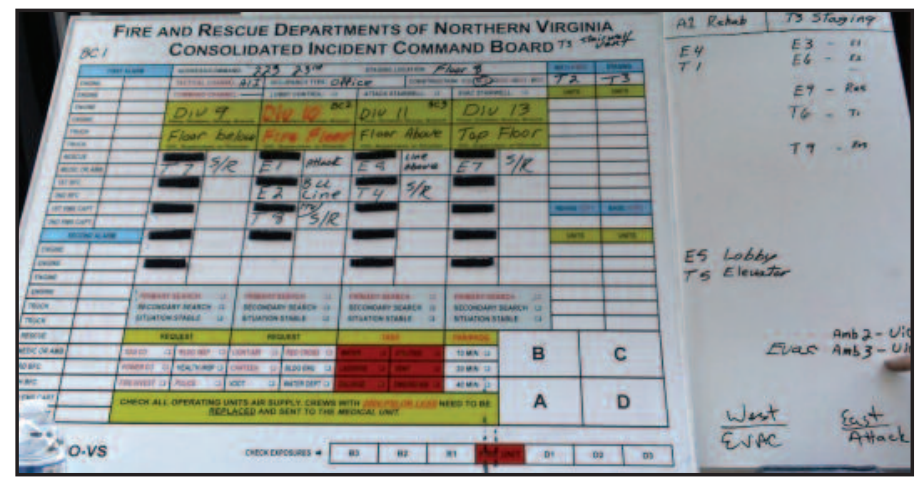

Figure 36: Incident Command board

26. Subject matter experts included Russ Sanders (NFPA, Louisville KY Chief, Retired), Dennis Compton (IFSTA, NFFF, Mesa, AZ Chief, Retired), Peter Van Dorpe (Training Chief Chicago, IL), David Rohr, (Chief Fairfax City, VA, Fairfax County, VA Operations Chief, Retired), Vincent Dunn (Deputy Chief FDNY, Retired), Ben Klaene (Cincinnati, OH Training Chief, Retired), James Walsh (Deputy Chief, Fairfax County, VA), Richard Bowers (Chief Montgomery County, MD) and Richard Travers (Deputy Chief FDNY, Retired).

27. The Incident Command System (ICS) is a standardized, on-scene, all-hazards incident management approach that allows for the integration of facilities, equipment, personnel, procedures, and communications operating within a common organizational structure. ICS also enables a coordinated response among various jurisdictions and functional agencies, both public and private, and establishes common processes for planning and managing resources. ICS is flexible and can be used for incidents of any type, scope, and complexity. ICS allows its users to adopt an integrated organizational structure to match the complexities and demands of single or multiple incidents. http://www.fema.gov/incident-command-system, January 2013. 
During the experiments, participating departments used the IC position as an intense training opportunity for their Battalion Chiefs, as well as those in line for promotion to that position. The high-rise structure fire experiments also provided the opportunity for multi-jurisdictional interaction for crews with command officers from nearby departments.

\subsection{Lobby Operations}

\section{Lobby Control}

The IC assigned an officer or firefighter to establish Lobby Control to provide critical intelligence, establish accountability, and control the building systems. Due to the dynamic nature of a high-rise event, the Lobby Control Officer must multi-task. Lobby Control was responsible for duties related to managing the stairwells, elevators, and the HVAC systems (see Figures 37 and 38). The duties of Lobby Control included the following.

Consulting with the building engineer to determine the presence and status of all key building systems, as well as any information about the status and nature of the fire

- Controlling or shutting down the HVAC system after consulting with the IC

- Assisting incident command post operations

- Locating all interior stairs.

- Preventing all building occupants and nonemergency personnel from entering the building

- Controlling, operating, and accounting for all elevators

- Directing incoming companies to the proper elevator or stairwell

Controlling the elevators and stairwells is the only way to effectively gain access to the upper floors of a high-rise building. Another important Lobby Control duty is to gain control of the HVAC system. If operated properly, the HVAC system can prevent heat, smoke, and toxic gases from spreading throughout the building and reaching more occupants than might otherwise be exposed. Conversely, if operated incorrectly, the HVAC system may spread the products of combustion well beyond the immediate fire area and into other occupied areas.

Throughout the experiments, Lobby Control was established by the officer or firefighter remaining in the lobby from the first arriving engine company. The position was then supplemented by the assigned crew upon arrival. As in an actual incident, all

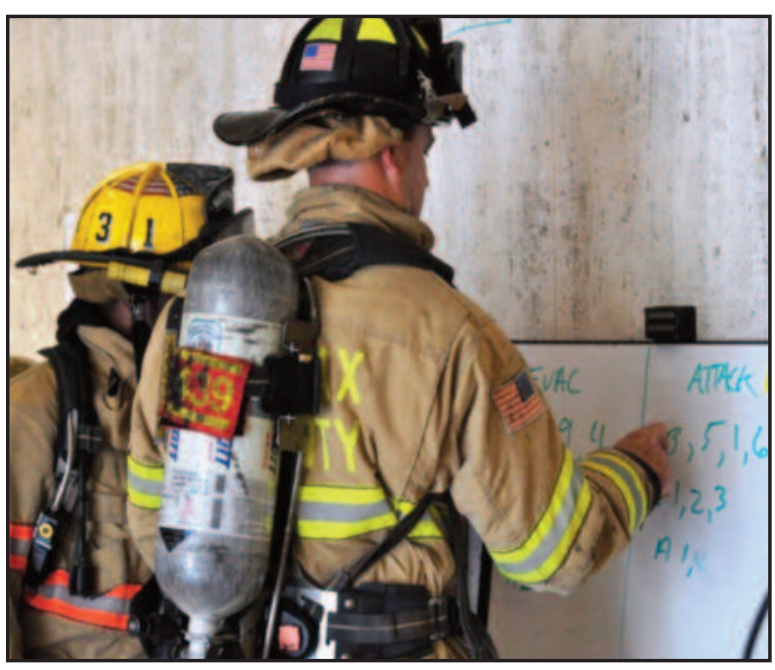

Figure 37: Lobby Control / Accountability

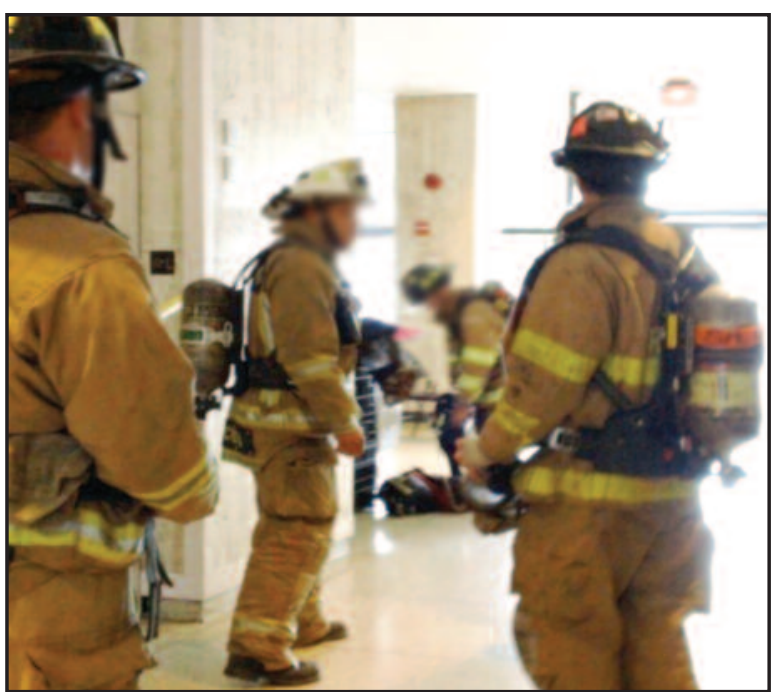

Figure 38: Lobby Control / Elevator access

incoming crews were required to stop at Lobby Control to confirm the location of the fire floor, staging floor, status of the elevators, designation of the attack stairwell, obtain building keys (if necessary), and then proceed to their assignment.

\section{Ventilation}

In a high-rise, more than any other type of building, quickly confining and extinguishing the fire are critical, as ventilation and evacuation options are limited. Once the fire is extinguished, the toxic products of combustion are no longer being produced, thereby reducing the hazard level to firefighters and occupants and making the operation more manageable overall. Another mode of ventilation in a high-rise fire is positive pressurization. Positive pressure ventilation (PPV) of a high-rise structure is achieved by placing fans at the base of a stairwell and blowing air into the structure, as illustrated in Figure 39. When appropriate openings or vents are used in the structure, the airflow produced by the fan exhausts contaminants to the outside. Fire departments use PPV as a means to ventilate contaminated atmospheres after initial knockdown and extinguishment of a fire. When configured properly, PPV fans can meet or exceed previously established performance criteria for fixed smoke control systems. Also, the stairs should be pressurized to reduce smoke infiltration. It should also be noted that the pressurized stairwell should be the stairwell of choice for occupant evacuation (Kerber \& Madrzykowski, 2007).

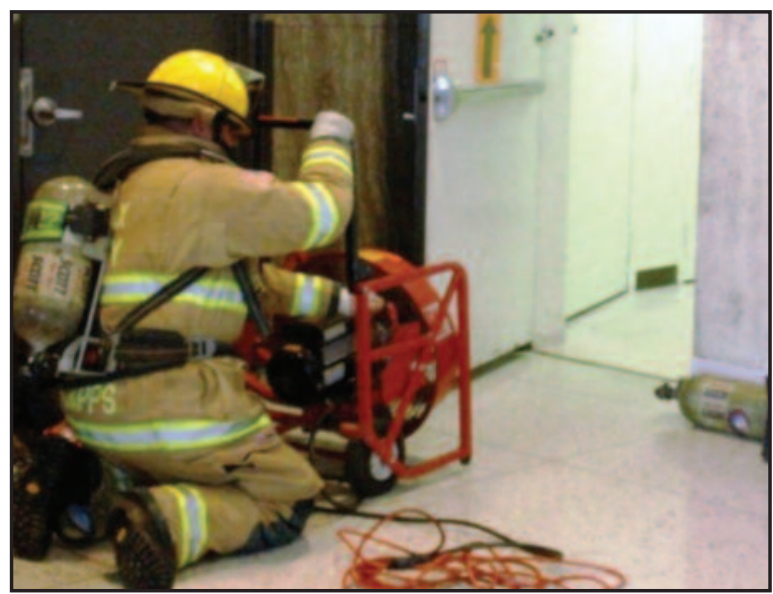

Figure 39: PPV fan ventilation 
During the experiments, the HVAC system was deemed nonoperational. Once the fire was extinguished, PPV was used to pressurize each stairwell. The roof hatch was opened to exhaust heat, smoke and other combustion products to the outside. Placement of the fans was handled by a firefighter from the first arriving truck.

\subsection{Staging and Rehabilitation on Floor 8}

\section{Staging}

In a high-rise structure fire, interior staging is set up in a safe area two or more floors below the fire floor. Arriving crews not directly assigned from the IC are typically dispatched to the staging area to await a specific assignment. The reason for the staging area is to provide a readily available reserve firefighting force, as well as reserve equipment and supplies. Having these resources available is a critical safety feature of high-rise response, since the event may worsen very quickly or require more resources than originally believed. Without resources near the fire floor, it may take a considerable period of time to move people and equipment from outside the building to the needed area.

The supervisor in charge of the staging area, or an aide, acts as the accountability officer for crews that are working outside of the staging area (see Figure 40). The Staging Officer maintains a continuous log of which companies are in Staging, which are in the rehabilitation area (Rehab), and which are working on the fire floor or in other areas of the structure. Tracking crew rotations can be a challenging, though absolutely vital, task.

During the experiments, depending on crew size, the third or fourth arriving truck company assumed the role of managing the staging area. The duties of the staging area crew in the experiments are listed below.

Report directly to the IC

- Assign crews as directed by the IC

- Maintain a record of the companies in Staging and in Rehab

- Maintain a record of the companies working in other areas of the building

Request additional resources to maintain a reserve force (as available)

Maintain an adequate supply of air cylinders, search rope, water, and other equipment, including EMS equipment as needed

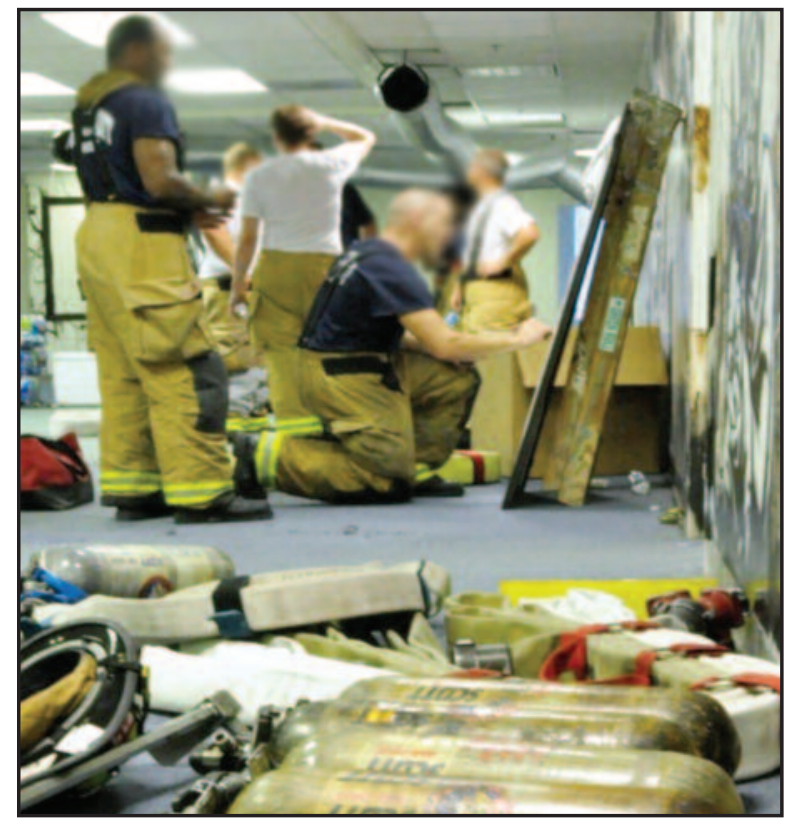

Figure 40: Staging management

\section{Rehabilitation}

A rehabilitation area may also be set up at the staging area. NFPA 1584 notes that a rehabilitation area should be established whenever emergency operations pose the risk of pushing personnel beyond a safe level of physical or mental endurance, particularly when crews may be reassigned multiple tasks during the same incident.

Specifically, NFPA 1584 provides a guideline that self-rehab with hydration occur for at least $10 \mathrm{~min}$ following the depletion of one 30-minute SCBA cylinder or after 20 min of intense work without wearing an SCBA. The standard further notes that firefighters must enter a formal rehab area, drink appropriate fluids, be medically evaluated, and rest for a minimum of $20 \mathrm{~min}$ following depletion of two 30-minute SCBA cylinders, depletion of one 45-minute or 60-minute SCBA cylinder, or following 40 mins of intense work without an SCBA.

The Rehab area in the high-rise field experiments was established adjacent to the staging area on the 8th floor. Firefighter/paramedics from the first arriving ambulance established and managed the Rehab area. The area was used to evaluate and assist personnel who had completed an assignment in an IDLH environment. Within the Rehab area, firefighters received a physical assessment, rest, hydration, evaluation, and treatment (if necessary), continual monitoring of their physical condition while in Rehab, transportation for additional evaluation (if necessary), and reassignment to Staging, when appropriate.

Time needed in Rehab is typically determined by the firefighter's level of physical conditioning, the atmospheric conditions, the nature of the activities the firefighter was performing before entering Rehab, and the time needed for adequate rehydration and/or eating. While NFPA 1584 requires $10 \mathrm{~min}$ of rehab for shorter work periods or 20 min for heavy work, as stated above, the training protocol observed was conservatively required to be 15 min. Firefighters operating in the experiments were also required to consume a minimum of one $12-\mathrm{oz}$ ( 0.36 liter) bottle of water before being sent to the staging area for possible reassignment.

\subsection{Logistics of Material Support (Stairs/Elevators)}

In high-rise operations, the logistical challenges of moving firefighters, air cylinders, hoses, nozzles, ropes, forcible entry tools, and EMS supplies increase with building height. If additional firefighters and equipment are not moved into staging and upper floors of the building early, early arriving crews will be unable to sustain initial operations. The easiest way to transport these resources is to use an elevator. If firefighters can safely use the elevators, fireground logistics are dramatically improved. However, if the elevator is not available or not safe to use, moving firefighters and supplies up 10,20, or more stories is an arduous task. Having equipment available in the staging area in a timely manner is a critical task, since later tasks may be delayed if the equipment is not available when needed.

The high-rise field experiment study protocol addressed the use of elevators, including circumstances in which it was unsafe for firefighters to use them. The protocol included alternative measures for getting needed equipment and firefighters to the fire floor when elevators could not be safely used.

Stairwell support was the procedure used to move supplies to the interior staging area when using elevators was not a safe option. Firefighters assigned to stairwell support ascended and distributed themselves evenly throughout the stairwell. Equipment and supplies were then transported up the stairway 
using a firefighter relay system. Equipment was taken to the interior staging area located two floors below the fire floor (8th floor). Stairwell support was one of the first assignments given when the building was without elevator service due to the location of the fire on an upper floor.

During the field experiments, both elevator and stairwell support tasks were included in the scenarios in an effort to measure the efficiency of each. A crew was assigned the task of elevator or stairwell support (Figure 41 and Figure 42) depending on the scenario being conducted. Elevator support was measured in the scenarios in which elevators were deemed safe for use, and stairwell support was measured in the scenarios in which the elevators were deemed unsafe for use.
Figure 41: Elevator support

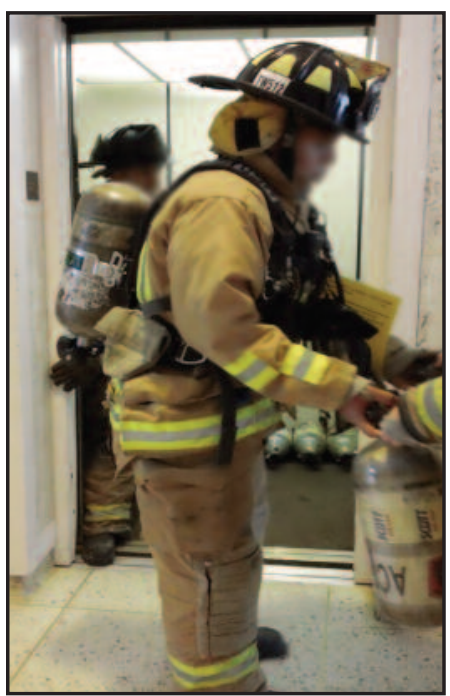

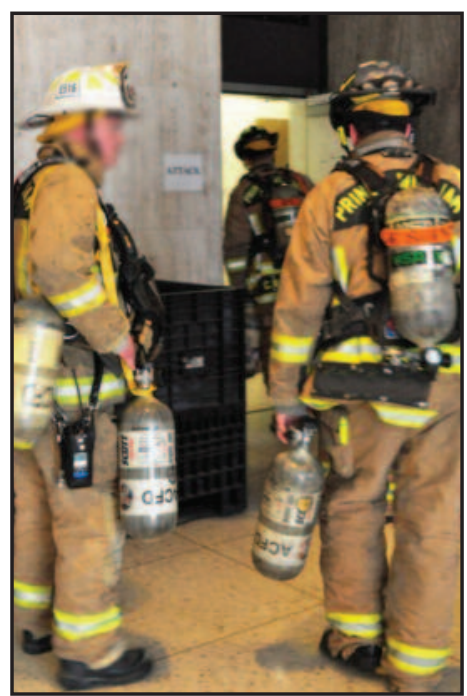

Figure 42: Stairwell support

\subsection{Ambulance Transport for Fire Victims}

The number of ambulances (Emergency Medical Services (EMS units)) needed to be available at a high-rise structure fire is dependent on the number of people assumed to occupy the structure and the number of firefighters operating at the incident. There should be at least one ambulance available at all times during a high-rise fire (IFSTA, 2011). Should one ambulance transport a victim or firefighter from the scene, another ambulance should be assigned by the IC to standby (Figure 43).

During the high-rise experiments, three ambulances/EMS Units were used in the experiment. Though most ambulances were staffed with two cross-trained firefighter/paramedics, all ambulances were ALS units staffed with a minimum of one firefighter/paramedic and one firefighter/EMT. The first arriving ALS crew was assigned to operate the Rehab area. The second and third arriving ambulance crews were sent to the staging area on the 8th floor. As the victims were located during the search and rescue operations, Ambulance 2 was assigned to transport the victim who was being moved to the 8 th floor from the fire floor (10th floor) by the search and rescue team and Ambulance 3 was assigned to transport the victim who was removed from the floor above the fire (11th floor) and transferred to the 8th floor by the search and rescue team.

Once assigned by the IC and staging manager, ambulance personnel responded to meet firefighters bringing the victims to the 8 th floor stairwell landing. When a victim was received by the firefighter/paramedics assigned to the ambulances, they proceeded to carry the victim down to the lobby of the high-rise structure via the elevator (Figure 44) or stairwell (Figure 45), according to the protocol governing the specific scenario. Once in the lobby, the EMS personnel exited the building with the victim. Time was recorded at the point that the victim was transferred to the ambulance crew on the 8th floor and when the crew had moved the victim to the exterior of the building.

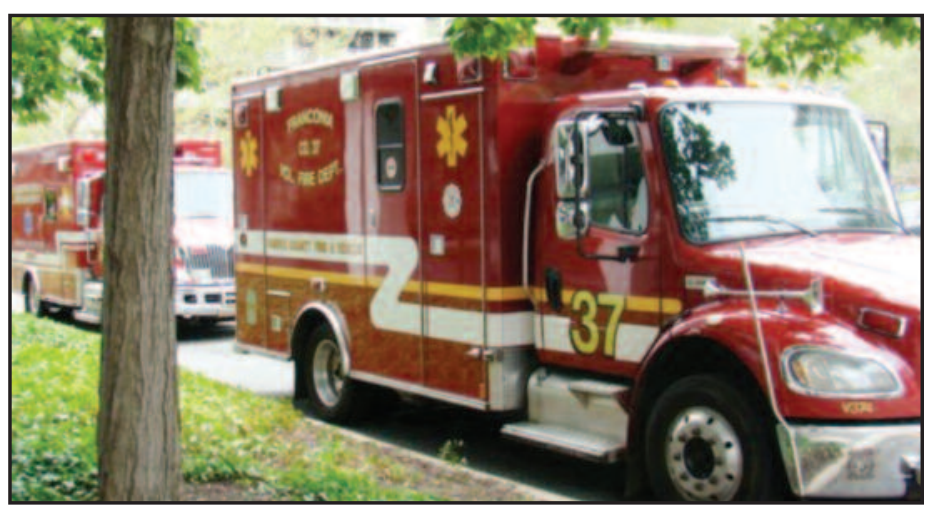

Figure 43: Ambulance standby at high-rise structure fire

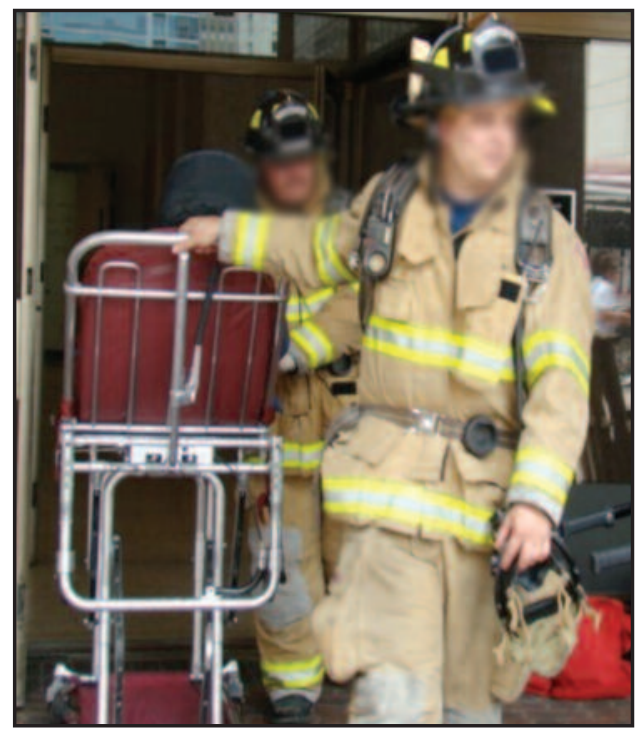

Figure 44: EMS personnel exiting with victim rescued using a wheeled stretcher by elevators

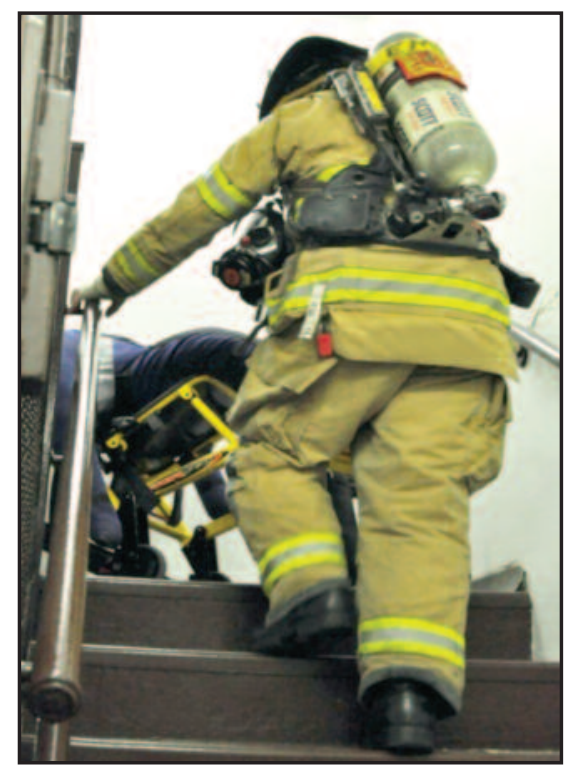

Figure 45: EMS personnel carry victim down stairs using a stair chair device 


\subsection{Floor 9, Floor 12, and Floor 13 Search and Rescue Operations}

Search and Rescue Operations on floor 9 (the floor below the fire) and upper floors (beyond Floor 11 - the floor above the fire) were assigned to later arriving crews after higher priority tasks in the more hazardous areas were underway. Along with search ropes, forcible entry tools were carried by search crews to force entry when necessary.

Search crews conducted a systematic search and collected blocks or markers in the areas as they were searched. Collection of the markers (blocks) was considered the equivalent of the typical simple marking system used by most fire departments of placing a chalk "X" on doors to rooms that had been searched and/or indicating that a whole floor had been searched by marking hallway doors or walls.

While the search crews were on air (any firefighter working above the 8th floor landing was on air), the searches on these floors revealed no smoke and no victims. Therefore, since there was no smoke, the searches were walking searches.

After completing a thorough search of their assigned floor, crews reported a search status to the IC and were then sent to the Rehab area.

\subsection{Floor 10 Suppression Operations}

Fire suppression is a top priority during structural firefighting operations. The ability to control the fire reduces the overall hazard level for the occupants and firefighters, as well as reducing property loss.

\section{Equipment}

Every suppression crew ascending to the fire floor carried equipment, including four $50 \mathrm{ft}(15 \mathrm{~m})$ sections of $2 \frac{1}{2}$ inch hose (a total of $200 \mathrm{ft}$ or $61 \mathrm{~m}$ ), a gated wye valve ${ }^{28}$ to connect to the building standpipe (riser) in the stairwell, and an extra air cylinder.

\section{Primary attack with 3-person crews}

With 3-person crews, the initial attack line on the fire floor was assigned to Engine 1 and Engine 2. Upon arrival on-scene, the Engine 1 crew left the apparatus driver outside to connect the hydrant to the engine pump (located on the street immediately outside the building) and to connect the engine pump to the building Siamese fitting in order to ensure adequate pressure for sprinklers (if applicable) and for fire suppression operations (standpipes). Throughout the incident the Engine 1 driver stayed with the engine in order to ensure that adequate pressure was continuously supplied to the standpipe and sprinkler systems. The remaining two firefighters entered the lobby of the building to begin an assessment of the situation. The Engine 1 officer assumed incident command in the lobby. The third Engine 1 firefighter awaited the arrival of Engine 2 to ascend the stairs as a composite, 4-person crew.

\section{Primary attack with 4-person crews}

With 4-person crews, the initial attack line on the fire floor was assigned to Engine 1 and Engine 2. As with all crew sizes, Engine 1 left the apparatus driver outside to connect the water supply. Throughout the incident, the Engine 1 driver stayed with the engine in order to ensure that adequate pressure was continuously supplied to the standpipe and sprinkler systems. The remaining three firefighters entered the lobby of the building to begin an assessment of the situation. As with 3-person crews, the Engine 1 officer assumed incident command in the lobby. The remaining two Engine 1 firefighters awaited the arrival of Engine 2 to ascend the stairs (thus ascending as a 6-person crew, consisting of two from $\mathrm{E} 1$ and four from $\mathrm{E} 2$ ).

\section{Primary attack with 5- and 6-person crews}

With 5-person and 6-person crews, the initial attack line on the fire floor was assigned entirely to Engine 1 . As with all crew sizes, the apparatus driver stayed outside to connect the water supply to the building. The remaining 4 (or 5) firefighters entered the lobby of the building to begin an assessment of the situation. Unlike 3-person or 4-person crews, Engine 1 left a firefighter to assume Lobby Control, while the officer stayed with the Engine $1 \mathrm{crew}$ to ascend to the fire floor. The remaining 3 (or 4) Engine 1 firefighters then immediately ascended the stairs (rather than waiting for Engine 2) to begin fire attack and suppression.

\section{Fire Floor Suppression Operations}

Upon arrival on the landing for Floor 9, the initial suppression company arrived at the standpipe, removed the pressure reducer and connected a pressure gauge. A firefighter then connected the gated wye valve to the building standpipe, connected a section of $2 \frac{1}{2}$ inch hose to the wye, and flaked out the line in the stairwell (following the stair from the 9th floor landing, past the 10th floor to the 11th floor landing, and back down to the 10th floor). Under real firefighting conditions, the hose line would be charged (filled with water) prior to making entry onto the 10th floor; since the experiments were not flowing water (in order to protect the building from the effects of water), the suppression team used a sand-filled $2 \frac{1}{2}$ inch hose line located immediately adjacent to the stairwell door in the core of the building (see Figure 46). The attack line was pulled from the stairwell through the elevator lobby and entered the fire compartment through a door in the opposite corner of the core. Entering the fire floor was always coordinated with Truck 1 (which did not commence search and rescue until the suppression team was ready to flow water). Upon entering the fire compartment, the initial attack line proceeded to the left and began suppression operations (as shown with the word "Start" in Figure 46). Suppression involved simply moving the nozzle of the hose line through the fire area to the seat of the fire (shown with the word "Stop" in Figure 46). Even after the fire was deemed "out", the hose lines were staffed for the duration of the experiment in order to protect the fire floor from a "rekindle" scenario, while search crews were operating in the environment. Each crew assigned to the attack or second line on the fire floor was allowed to remain on air for $15 \mathrm{~min}$, as described in section 6.7, and then called for relief.

\section{Suppression Technique}

Extending the hose lines on the fire floor (10th Floor) required crews to pull more than $200 \mathrm{ft}(51 \mathrm{~m})$ of $21 / 2$ inch hose, maneuvering through five right-angle corners. Each corner represented a significant friction point for hose line operations; thus, a crew member was typically left at each corner in order to pull the line through the corner and ease the effort of the crew members at the nozzle end of the line. This hose handling technique was discussed each day with engine crews in order to help ensure consistency during the tests. 


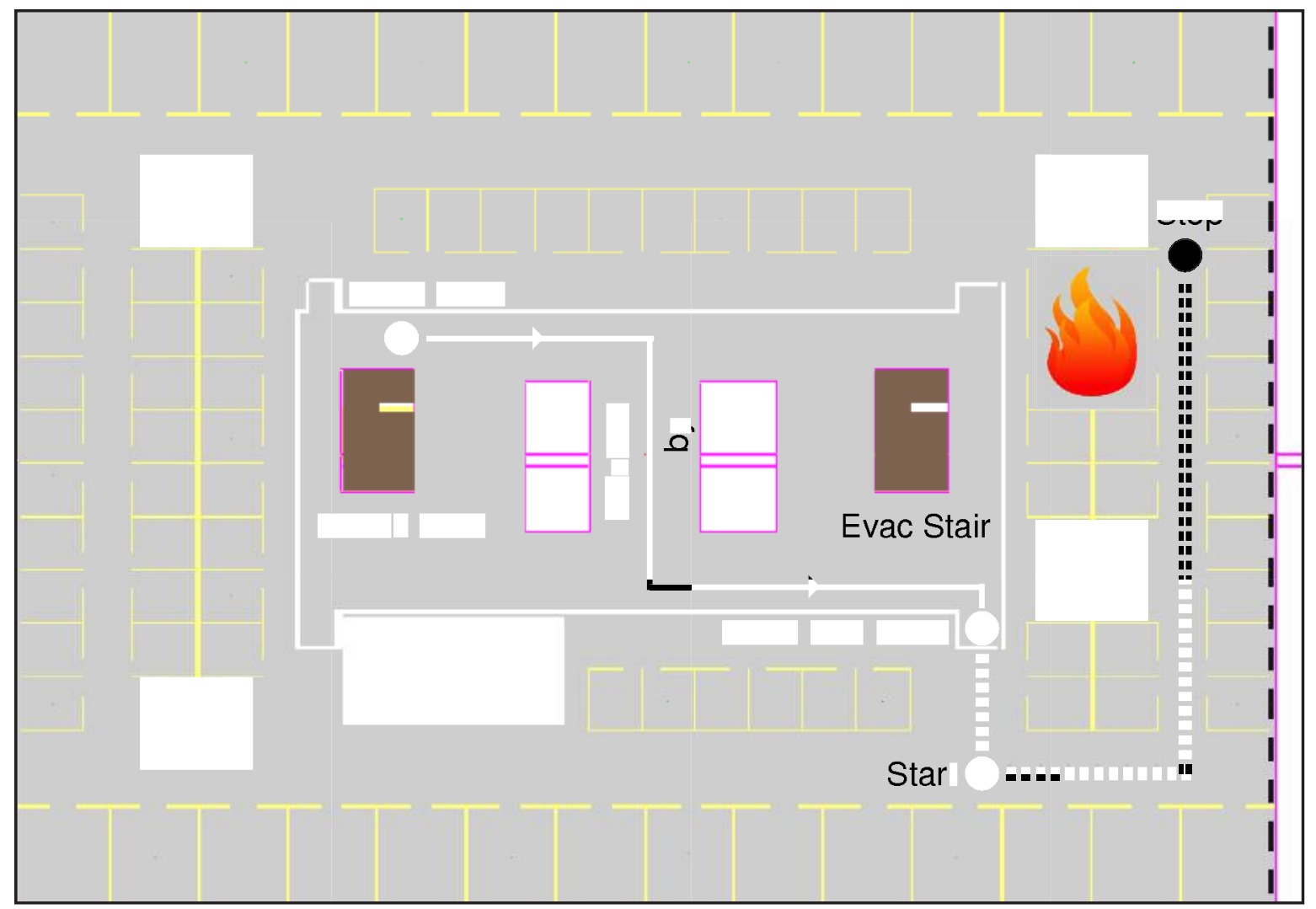

Figure 46: Pathway of hose lines from core through the fire area. The double dash lines represent the need for both hose lines to be present

\section{Second Attack Line}

For crew sizes of 3 and 4 persons, Engine 3 was assigned responsibility for deploying the second line on the fire floor. It is important to note that the second line was engaged in attack. The fire on Floor 10 was so large (more than $8 \mathrm{MW}$, involving several thousand square feet of fire involvement) that a single $2 \frac{1}{2} 2$ inch line would not have been sufficient to extinguish the fire. For 5 and 6-person crews, Engine 2 was assigned responsibility for the second line on Floor 10. Similar to the initial attack line, the second line crew connected a $21 / 2$ inch hose to the gated wye valve on the stairwell landing of Floor 9 and flaked the hose up and into Floor 10. The second line on Floor 10 was also a sand-filled 2 1/2 inch hose located immediately outside the stairwell door to the building core. The second line followed the same path to the seat of the fire as did the primary attack line. The fire was determined to be extinguished (Fire Out time) when the nozzle for the second line reached the seat of the fire as denoted by the word "Stop" in Figure 46. As with the primary attack line, the second line was subsequently staffed for the duration of the fire.

\subsection{Floor 10 Search and Rescue Operations}

Two significant efforts occurred in parallel on the fire floor: fire suppression and search and rescue. Fire suppression is typically the primary task of engine companies, while search and rescue operations are typically the responsibility of truck companies. The primary purpose of the search and rescue operation was to locate and extricate building occupants who may be trapped or otherwise require evacuation assistance. Consistent with prevailing common practices in the fire service, the suppression crews and rescue crews made entry to the fire compartment together. Having a hose line actively knocking down the fire is particularly important for the search and rescue companies because it reduces risks, including the likelihood of becoming trapped and/or overwhelmed by a growing fire. 

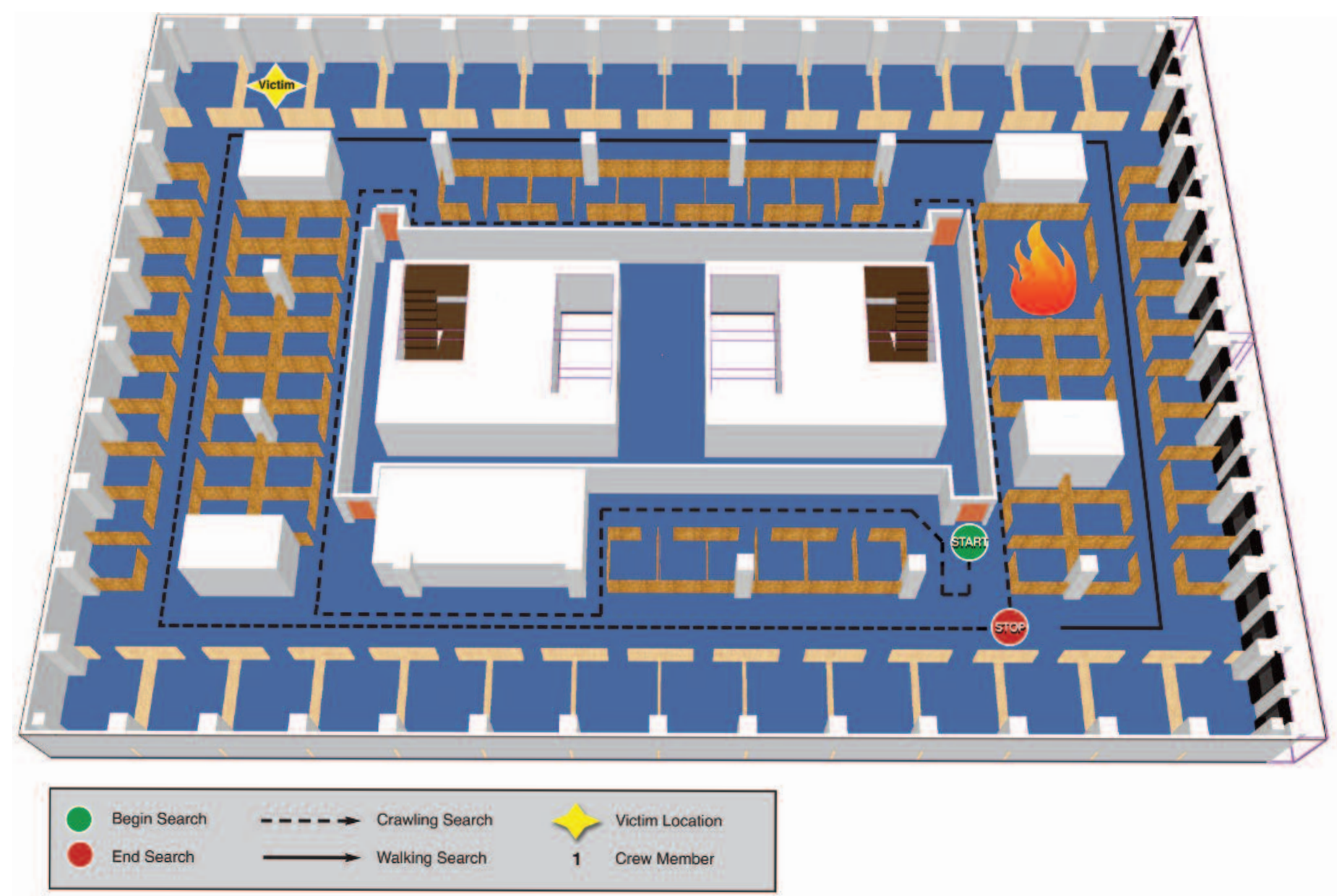

Figure 47: Schematic of the fire floor

\section{Overview of Fire Floor Search Patterns}

As shown in Figure 47, the fire floor (Floor 10) is an open-floor plan office layout. The core area contains two stairwells, four elevators, and a perimeter hallway with floor to ceiling walls separating the office space from the core area. This arrangement provides an area of protection from smoke and heat for evacuating occupants, as well as responding firefighters. Firefighter access to the fire floor was always through the attack stairwell. The crews then made entry to the floor from the doorway shown in Figure 47, roughly opposite the location of the attack stairwell. By maintaining a consistent entry point, Division 10 (Fire Floor Supervisor), could maintain crew accountability and know which resources were operating in the fire environment at any point in time. The arrangement of cubicles on the fire floor roughly formed two hallways that were continuous loops (referred to as the inner and outer loops for the purposes of these experiments).

\section{Search and Rescue Technique}

Search and rescue operations on the fire floor were always conducted under low visibility and simulated IDLH conditions. Thus, searches were conducted while crawling on hands and knees. The exception to crawling was when a crew used a search rope placed by a prior crew. Relief crews were allowed to hold a previously placed search line in their hand while they proceeded through a previously searched area until they found where the crew before them had stopped. Once the relief crew reached the end point of the prior crews' search area, as indicated by either a search bag or a search tool, they once again began to crawl.

As each crew depleted their air supply or search rope, they called for relief. Upon calling for relief, the search crew walked (again holding the search rope as a guide) back to the entry door and communicated with the Division 10 officer, and then reported to Rehab.

All searches used a right-hand search technique for consistency. ${ }^{29}$ The crew followed the inner (or outer) hallway, searching cubicles encountered along the way. As a crew encountered a cubicle, one firefighter stayed 'on the line' while another member of the crew searched the cubicle. Each firefighter was required to stay in visual, voice or rope contact with at least one other firefighter. Losing a search partner or the search rope is a dangerous situation for the search and rescue crew since it can lead to disorientation and difficulty in finding an exit from the floor. Once each cubicle was cleared, the crew proceeded to the next cubicle, clearing the hallway as they proceeded. Crews with three members could leapfrog cubicles, searching two simultaneously, while the officer or another firefighter continued to deploy the search line and maintain crew accountability.

While searching a cubicle, the firefighters used one of several common techniques. As each technique was roughly the same in 
terms of time to completion and energy expended, the study team did not prescribe a particular technique. One common technique was for the firefighter to crawl the perimeter of the cubicle, using the right-hand search pattern to search for a victim. A second technique was for the firefighter to assume a position in the center of the cubicle and then use a pike pole, axe, or Halligan tool to sweep the entire cubicle perimeter for a victim. A third technique was for the firefighter to assume a position in the center of the cubicle and then use their leg to sweep the perimeter of the cubicle in order to find a victim. These three techniques all implemented a tactile strategy for locating victims, which is critical under low visibility conditions. The primary tactile strategy was supplemented by a secondary strategy of flashlights/headlights and visual searches in the limited circumstances when the smoke conditions allowed for local visibility.

\section{Search Lines (Tag lines)}

Search and rescue companies utilize search lines (rope) for three reasons. First, the search line provides an assured path back to the point-of-entry on the fire floor. Second, the search line provides relief crews with a physical indicator of which portions of the floor have been previously searched. Third, the length of the search line (typically $200 \mathrm{ft}(61 \mathrm{~m})$ ), when combined with effective air management, helps search crews consider when the crew has performed their task and should call for a relief crew. Typically, search and rescue crews deploy search lines as they proceed through the structure. Initial shake-down tests revealed a fair degree of variation in search line or rope deployment from one crew to another. Therefore, in order to improve experimental reproducibility, search lines were pre-positioned on the fire floor (see discussion of deployment patterns below).

\section{Search and Rescue Relief}

In the high-rise experiments, search and rescue companies completed their assigned task and called for a relief crew under one of three conditions:

A member of the company had a low-air indicator on their bottle.

- The handheld timer ${ }^{30}$ activated, indicating that the company was nearly out of air.

- The company deployed and searched $200 \mathrm{ft}$ (61 m) of search line.

The company officer radioed the Battalion Chief in charge of fire floor operations (Division 10) to request relief. Division 10 acknowledged the request and then communicated with the Incident Commander in order to have a relief crew sent to the fire floor from Staging. Upon calling for relief, the company would either leave the rope bag or leave a search tool (such as an axe, Halligan tool, or pike pole) in order to mark the end of their search, so that the relief company could locate the transition point between searched and unsearched floor space. The company then followed the search line back to the point of entry for the floor. Upon reaching the entry door to the fire compartment, the company officer conducted a face-to-face discussion with either Division 10 or with the officer from the relief crew (or both). The purpose of the discussion was to summarize what activities were accomplished, provide a sense of direction and/or distance to the point where activities terminated, and any other task-relevant information, such as the location of any special hazards. Once the face-to-face discussion was completed, the company exiting the fire floor reported to the designated rehabilitation floor in order to obtain fluids, rest, and be physically assessed for fitness to return to operations.

\section{Search and Rescue Search Strategy}

Crew size has a dramatic impact on the manner of crew deployment for search and rescue operations. This is due to the ability of larger search and rescue companies to split into multiple crews and search the floor area in parallel, rather than in series. Since all fireground operations in IDLH environments must be conducted with at least two persons for safety and accountability, a search and rescue crew can form two teams only when there are at least four members. Five-person companies can form a team of two firefighters and a team of three firefighters. While 6-person crews can typically form three 2-person crews, during the experiments, the nature of the search on the fire floor dictated the formation of two teams of 3 firefighters. One crew retained the base company moniker (e.g., Truck 6), while the second crew adopted an X-ray suffix (e.g., Truck 6 X-Ray) in order to distinguish the crews, particularly for radio traffic purposes.

A complicating reality for the first arriving truck crew was the assignment of one member to establish Lobby Control and establish positive pressure ventilation in the two stairwells serving the building. As a consequence, the first arriving crew had one less member than subsequent arriving search and rescue teams. This assignment had a significant impact on the operational effectiveness of some crew configurations, as described below. 


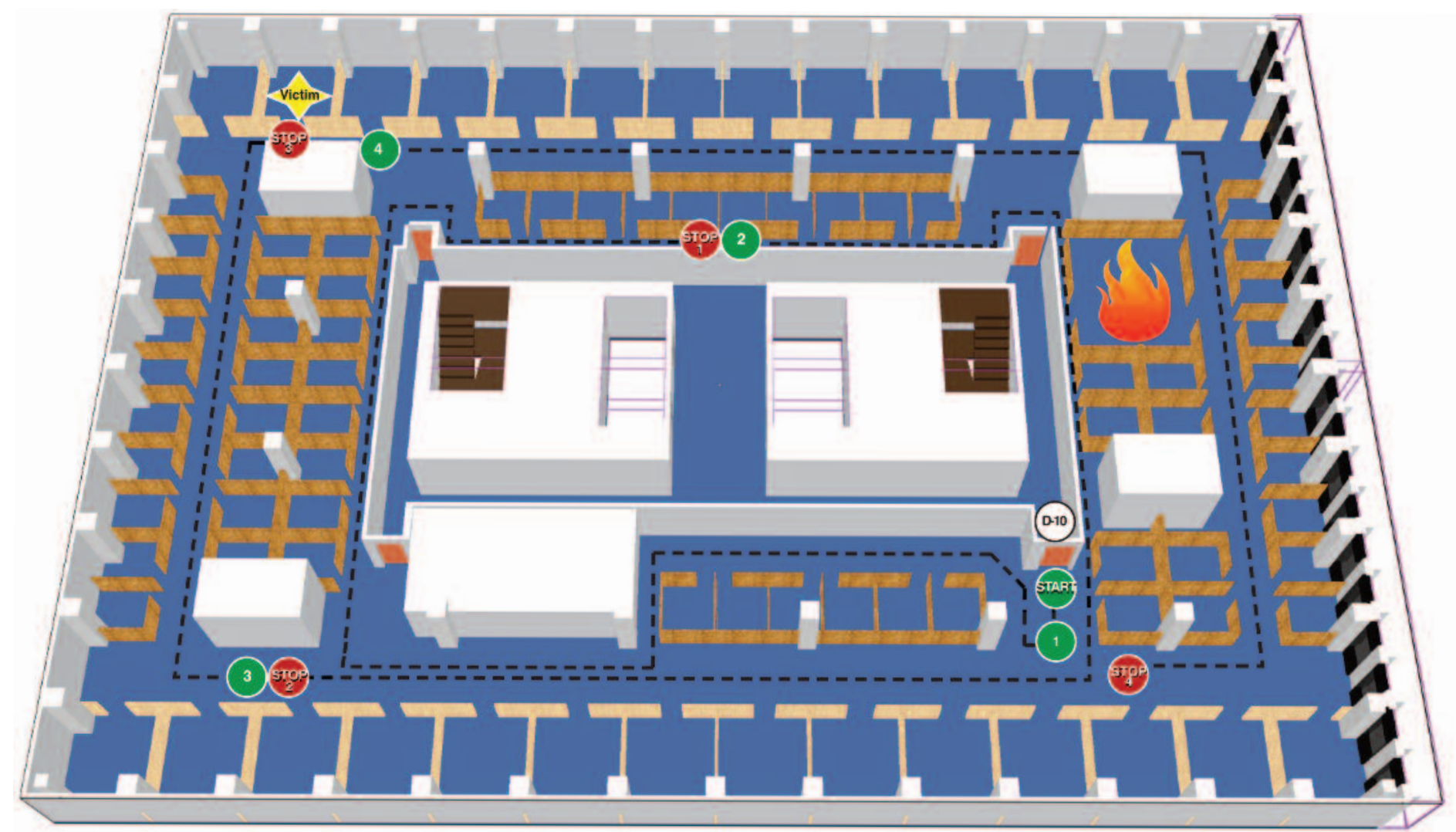

Figure 48: Fire floor search by 3-person crew

\section{Three-Person Search Crews}

The first-arriving 3-person crew (Truck 1) left one member in the lobby for PPV in the stairwells (as described above). Thus, Truck 1 became a two-person crew for the remainder of the experiment. After completing the Check for Fire Extension task, Truck 1 commenced a right-hand search on the inner loop of the fire floor, at the location in Figure 48 marked by Symbol 1 (the number 1 in a green circle). In most circumstances, Truck 1 searched for $200 \mathrm{ft}(61 \mathrm{~m})$ and called for relief upon reaching the end of the search rope (marked by Symbol 2). No crew was allowed by convention to search more than $200 \mathrm{ft}(61 \mathrm{~m})$. The relief crew operating with 3 firefighters followed the inside loop search rope, starting the search once the search bag (or search tool) left by Truck 1 at Symbol 2 was located. As the second search crew completed the inside loop search, the search was continued by crossing to the outside loop and commencing to the right. The second search crew typically completed their search when they ran out of rope at Symbol 3 in Figure 48. After calling for relief and leaving an indicator for the next search crew, the second crew followed the search line back to the entry door, roughly $60 \mathrm{ft}$ (20 $\mathrm{m}$ ) from their stop point, and communicated with Division 10 and/or the relief crew. Division 10 was located at the symbol with "D10" within a white circle. The relief company proceeded to the end of the search rope located along the outer loop at Symbol 3 and resumed the search. The third crew typically encountered the fire floor victim, represented in Figure 48 and subsequent figures by the word "Victim" in a yellow star. Procedures for victim rescue have been described previously in this report. As the victim rescue required the effort of all 3 crew members, a fourth search company was called to the fire floor when the victim was located. With only three full alarms available for this incident response, the only search crew available was one that had completed a prior task, had been processed through Rehab, and had been declared fit for return to operations by the ALS medic crew. The final search crew on the fire floor followed the outer loop search line from the entry door to the location where the victim was found (Symbol 4) and completed the fire floor search. The location of the search completion is shown by the Stop symbol in Figure 48. 


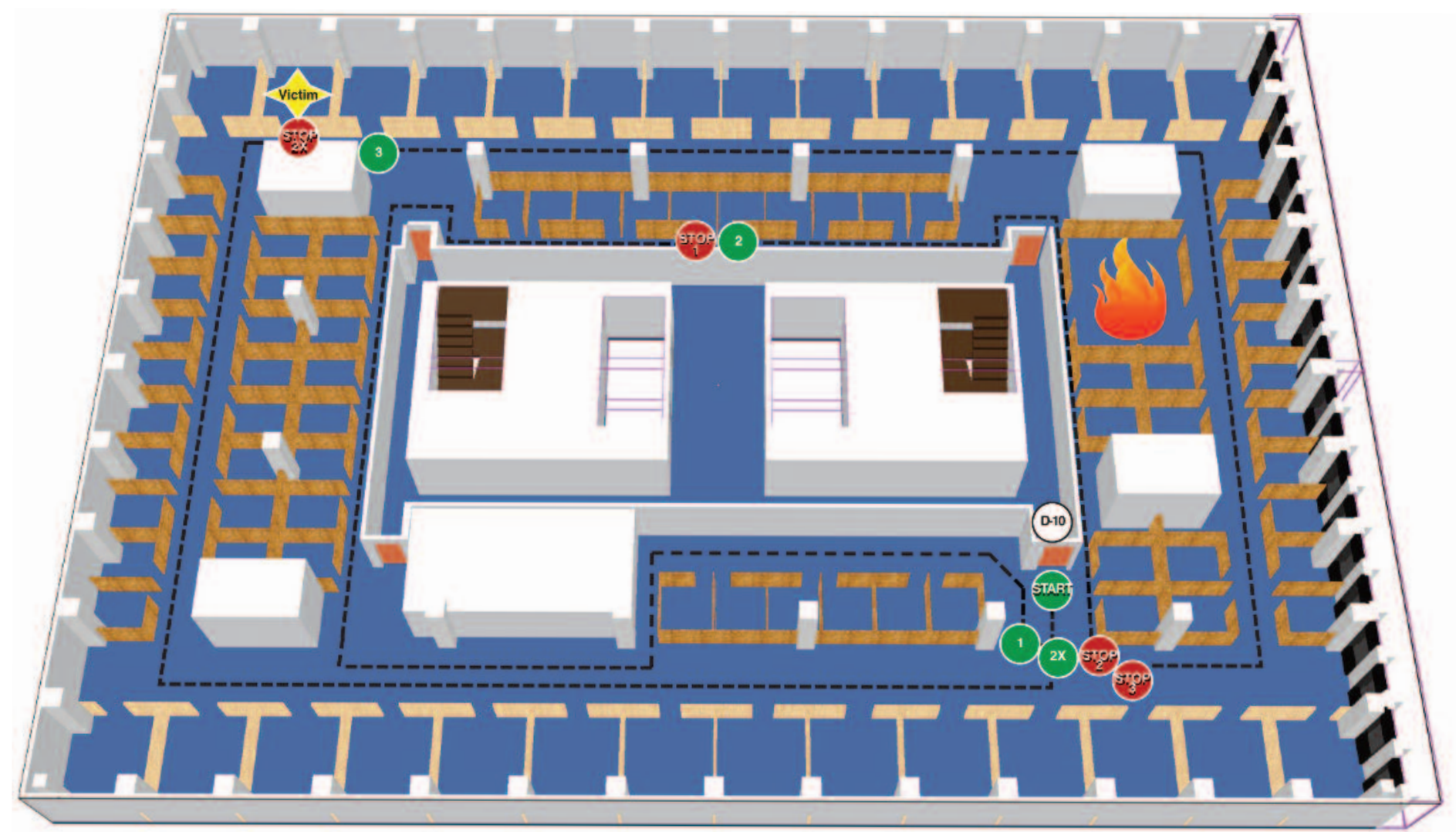

Figure 49: Fire floor search by 4-person crew

\section{Four-person Search Crews}

The first-arriving 4-person crew (Truck 1) left one member in the lobby to handle PPV for the stairwells (as described previously). Thus, Truck 1 operated as a 3-person crew for the remainder of the experiment. After completing the Check for Fire Extension task, Truck 1 commenced a right-hand search on the inner loop at Symbol 1 in Figure 49. In most circumstances, Truck 1 searched for $200 \mathrm{ft}(61 \mathrm{~m})$ and then called for relief upon reaching the end of the search rope at Symbol 2. The relief crew, operating with four personnel, split into two search teams (one team on the inside loop and one team on the outside loop). The relief crew on the inside loop followed the search rope previously searched by Truck 1 until they located the search bag (or search tool) left by Truck 1 at Symbol 2. The relief crew then continued searching until the inner loop search was complete. Once complete, the crew on the inner loop waited briefly to reassemble with the X-Ray team. The relief X-Ray crew commenced the outer loop search at Symbol 2X, typically finding the incapacitated victim at the star. Upon locating the victim, the X-Ray crew informed Division 10 that a victim had been located and that an ambulance team was needed to meet them in the stairwell. In addition, as an ambulance was requested, relief was also requested so that another crew could be sent to complete the search on the fire floor. The second crew members all met in the stairwell to maintain full crew accountability. The crew carried the victim to meet the EMS crew on the 8th floor and then proceeded to Rehab. A third crew then relieved the second and completed the search of the outer loop on the fire floor from Symbol 3 as a 4-person crew. The location of the search completion is shown by a stop sign in Figure 49. 


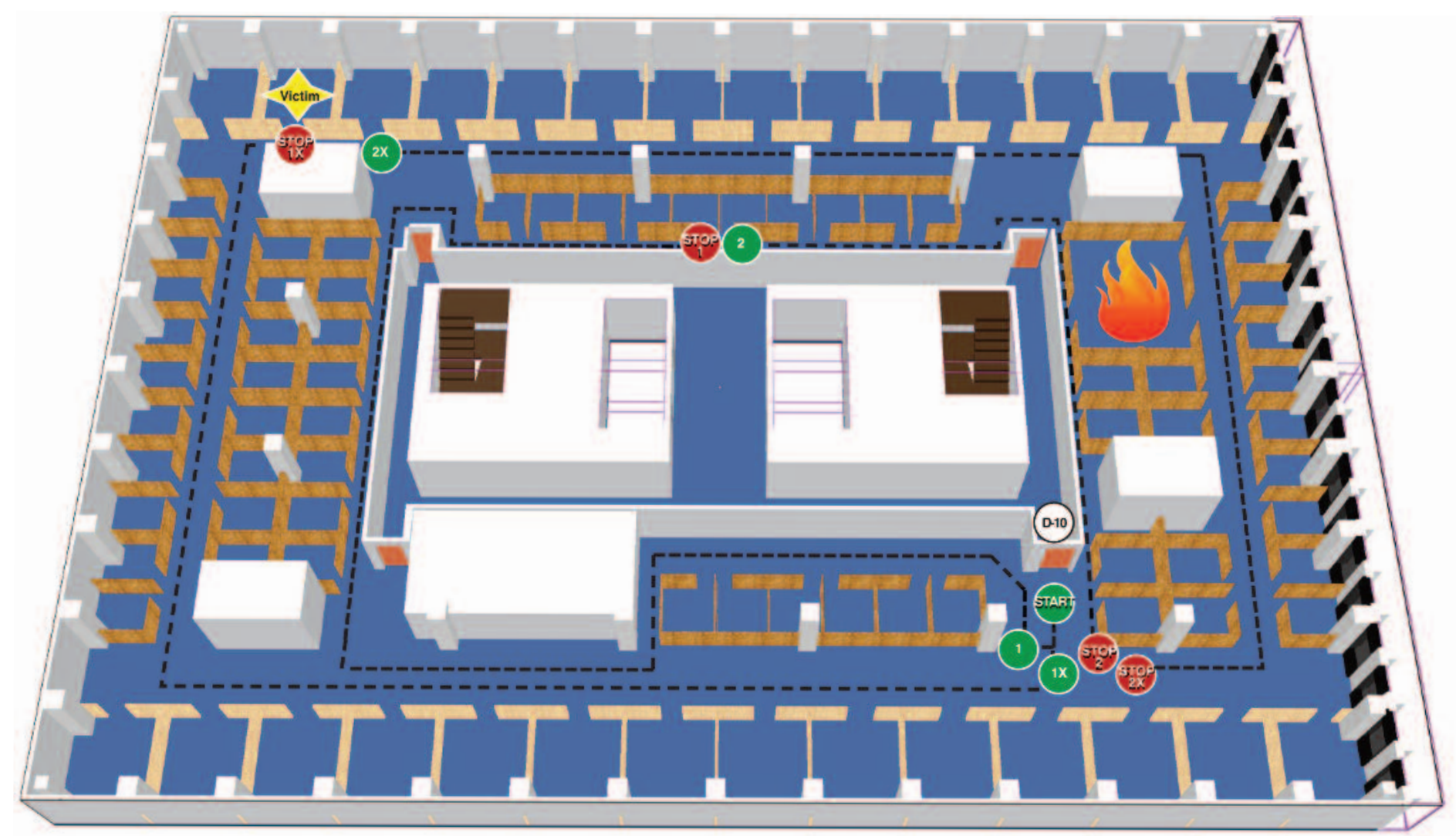

Figure 50: Fire floor search by 5- or 6-person crew

\section{Five-person Search Crews}

The first-arriving 5-person crew (Truck 1) left one member in the lobby to handle PPV in the stairwells (as described above). Thus, Truck 1 operated as a 4-person crew for the remainder of the experiment. Upon arrival on the fire floor, Truck 1

immediately split into Truck 1 and Truck 1 X-Ray crews. Truck 1 completed the Check for Fire Extension task, and then started a right-hand search on the inner loop, beginning at Symbol 1 in Figure 50. Truck 1 typically searched for $200 \mathrm{ft}(61 \mathrm{~m})$ and called for relief upon reaching the end of the search rope at Symbol 2. Meanwhile, Truck $1 \mathrm{X}$-Ray commenced the outer loop search at Symbol 1X, typically finding the incapacitated victim at the star. Upon locating the victim, Truck $1 \mathrm{X}$-Ray informed Division 10 that a victim had been located and that an ambulance crew was needed to meet them in the stairwell. In addition, relief was requested in order to complete the search on the fire floor. Truck 1 met the X-Ray team in the stairwell to maintain full crew accountability. The crew delivered the victim to the EMS crew on the 8th floor landing and then proceeded to Rehab. The relief crew, operating with all 5 personnel, split into two search teams (one team with 2 persons on the inside loop and the other team (X-Ray) with 3 persons on the outside loop). The crew on the inside loop followed the search rope until they located the search bag (or search tool) left by the previous crew then continued searching until the inner loop search was complete. Upon completion, the crew waited briefly to reassemble with the X-Ray team. The X-Ray crew typically completed the search of the outer loop on the fire floor.

\section{Six-person Search Crews}

The first-arriving 6-person crew (Truck 1) left one member in the lobby to handle PPV in the stairwells (as described previously). Thus, Truck 1 operated as a 5-person crew for the remainder of the experiment. Upon arrival on the fire floor, Truck 1 immediately split into Truck 1 and Truck 1 X-Ray crews. Truck 1 completed the Check for Fire Extension task, and then started a right-hand search on the inner loop, beginning at Symbol 1 in Figure 50. Truck 1 typically searched for $200 \mathrm{ft}(61 \mathrm{~m})$ and called for relief upon reaching the end of the search rope at Symbol 2. Meanwhile, Truck $1 \mathrm{X}$-Ray commenced the outer loop search at Symbol 1X, typically finding the incapacitated victim at the star. Upon locating the victim, Truck $1 \mathrm{X}$-Ray informed Division 10 that a victim had been located and that an ambulance crew was needed to meet them in the stairwell. In addition, relief was requested in order to complete the search on the fire floor. Truck 1 met the X-Ray team in the stairwell to maintain full crew accountability. The crew delivered the victim to the EMS crew on the 8th floor landing and then proceeded to Rehab. The relief crew, operating with all 6 personnel, split into two search teams with 3 persons each. The crew on the inside loop followed the search rope until they located the search bag (or search tool) left by the previous crew at Symbol 2, then continued searching until the inner loop search was complete. Upon completion, the crew waited briefly to reassemble with the X-Ray team. The X-Ray crew typically completed the search of the outer loop on the fire floor from Symbol 2X. 


\section{Victim Rescue}

The victim on the fire floor was rescued using a proper rescue technique requiring two firefighters, one of whom used webbing to lift the victim under the shoulders, and the other who lifted the victim's legs. Once properly packaged, the firefighters proceeded to the evacuation stairwell and moved down to Floor 8 where they were met by an EMS crew. At that time, emergency care was transferred to the EMS crew and the two firefighters proceeded to Rehab.

\section{Management of Fire Floor Operations}

The second arriving Battalion Chief (BC2) was assigned the responsibility for managing the fire floor operations. Upon arriving on the fire floor, BC2 became Division 10. Division 10 also had an aide to assist in managing and recording the flow of personnel and information. Due to travel times, BC2 and the aide arrived on the fire floor and established Division 10 shortly after the fire floor operations were initiated by Engine 1, Truck 1, and Engine 2. Upon establishing Division 10, all fire floor communications were directed through Division 10 in order to limit the radio traffic to the Incident Commander. The location of Division 10 is shown by the symbol "D10" in a white circle in Figure 48 through Figure 50.

A key task for Division 10 was crew accountability. With the exception of the early arriving crews, all companies performed a face-to-face check-in with Division 10 before entering the fire compartment and all units performed a face-to-face check-out with Division 10 as they left the fire compartment to ensure accountability for all firefighters.

\subsection{Floor 11 Suppression Operations}

In addition to extinguishing the fire, two of the primary objectives of high-rise fireground operations are to conduct a primary search on the fire floor and the floors above and to check for fire extension on the floor above the fire.

The floor above the fire is considered a high hazard environment due to the threat of rapid fire and smoke spread vertically.

Occupants on the fire floor and above are normally in the greatest danger due to the probability of fire extension. In many buildings fire-resistive construction should contain the body of a fire to the fire floor; however, there is a need to deploy at least one precautionary hose line on the floor above the fire.

During the high-rise experiments, a $2 \frac{1}{2}$ inch hose line was deployed to the 11th floor (immediately above the fire) as a precaution. Additionally, the suppression crew also pulled ceiling tiles to check for fire extension in the ceiling. Although crews responding to the 11th floor encountered heavy smoke, there was no fire extension. To assure the safety of search crews, fire suppression crews stood by in the area above the location of the fire for the duration of the search on the 11th floor.

\subsection{Floor 11 Search and Rescue Operations}

\section{Overview of Floor 11 Search Patterns}

The 11th floor was a completely different environment from the fire floor. Floor 11 was highly compartmentalized with four distinct, fully segregated areas. Figure 51 shows a bird's eye view of floor 11. As shown, there are a few areas of open floor space with many offices dispersed throughout.

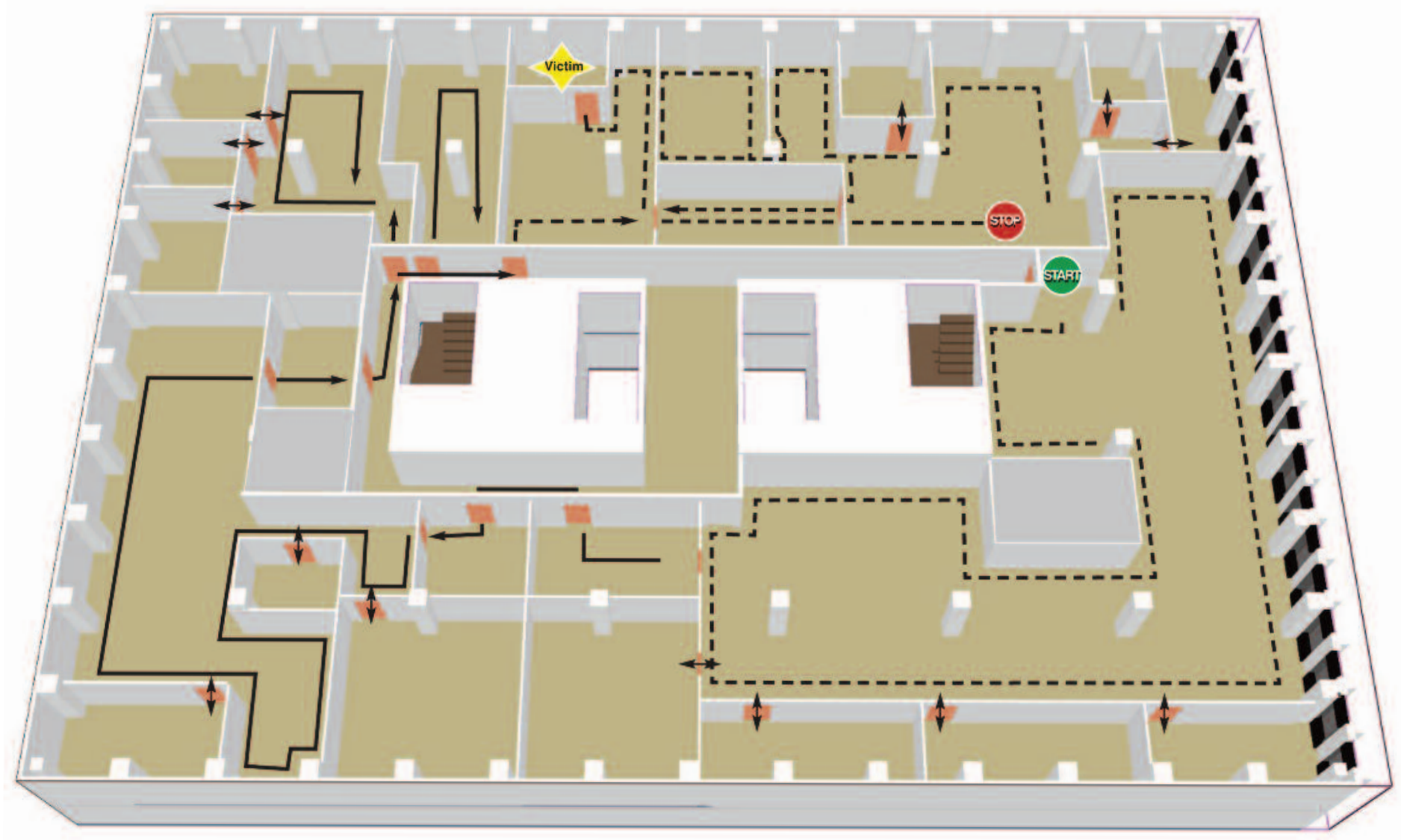

Begin Search End Search

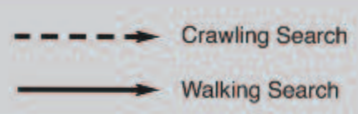

Walking Search
Victim Location

Crew Member 


\section{Search and Rescue Technique}

The search and rescue operations on the floor above the fire were different from those on the fire floor. All crews entered the floor though the door directly east of the evacuation stairwell and depending on crew size, began either a right hand search or both a left and right hand search. As with floor 10, crews could only split to search if they maintained two persons in each team. While the first and last sections searched were near zero visibility, the remainder of the floor had good visibility. Fire crews used search lines (ropes) and crawled in areas of low visibility (first and last sections) and walked in areas of good visibility (middle section).

\section{Search and Rescue Relief}

As on floor 10, when crews needed relief they radioed the Division Commander. On Floor 11, Division 11 was established by BC3.

The need for crew relief was dictated by three conditions:

A member of the company had a low-air indicator on their bottle.

- The handheld timer activated, indicating that the company was out of air.

The company deployed $200 \mathrm{ft}(61 \mathrm{~m})$ of search line.

The company officer radioed the Battalion Chief in charge of fire floor operations (Division 11) to request relief. Division 11 acknowledged the request and then communicated with the Incident Commander in order to have a relief crew sent to the floor above the fire from staging. Upon calling for relief, the company would either leave the rope bag or leave a search tool (such as an axe, Halligan tool, or pike pole) in order to mark the end of their search so that the relief company could locate the transition point between searched and unsearched floor space. The company then followed the search line back to the point of entry for the floor. Upon reaching the entry door to the interior compartment, the company officer conducted a face-to-face discussion with either Division 11 or with the officer from the relief crew (or both). The purpose of the discussion was to summarize what activities were accomplished, provide a sense of direction and/or distance to the point where activities terminated, and any other task-relevant information, such as the location of any special hazards. Once the face-to-face discussion was completed, the company exiting the floor reported to Rehab in order to obtain fluids, rest, and be physically assessed for fitness to return to operations.

\section{Search and Rescue Crew Deployment}

In all scenarios, an engine and a truck were assigned to the floor above the fire upon their arrival. All relief crews to Floor 11 were sent from Staging.

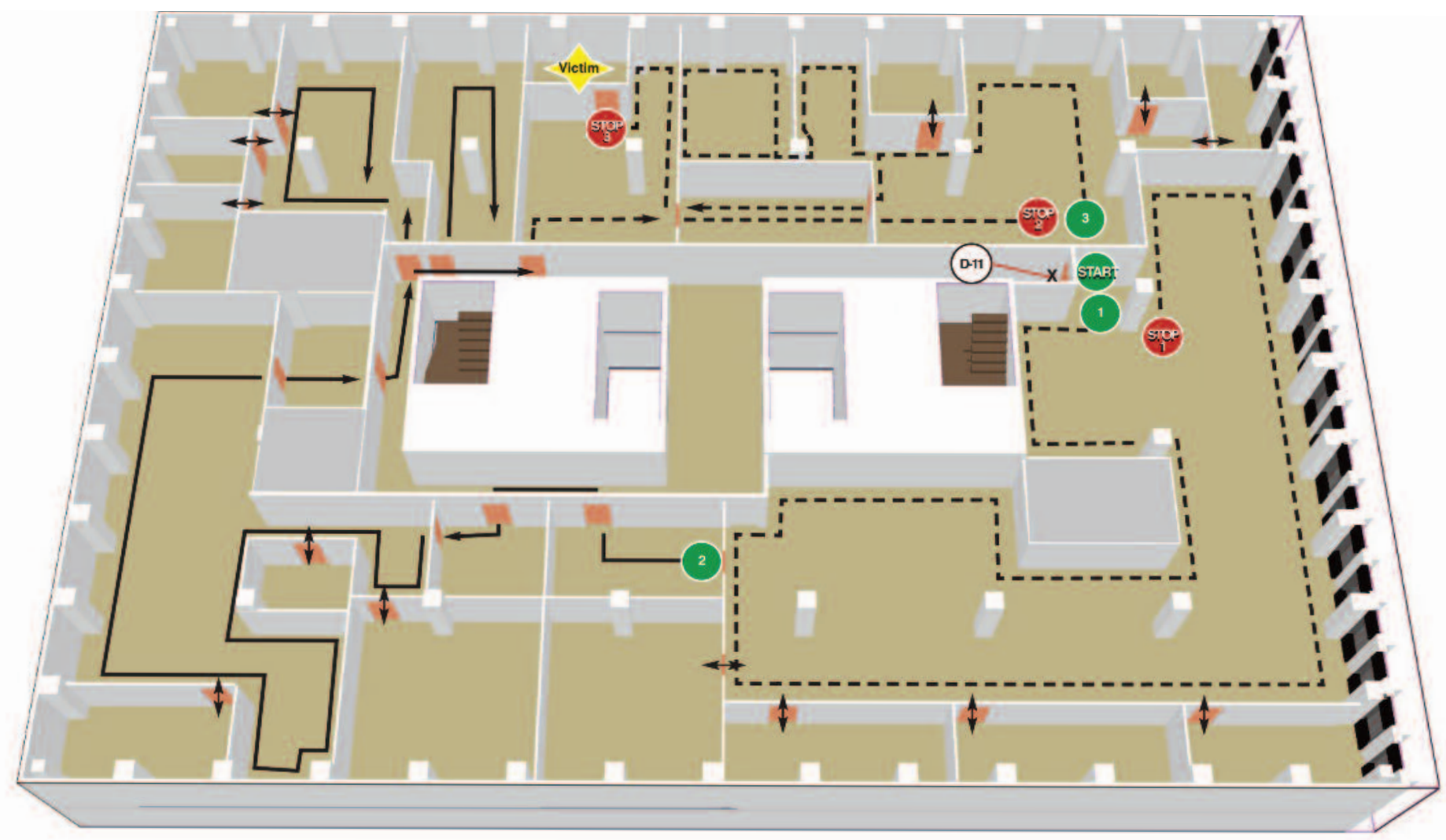

Figure 52: Floor 11 search by 3-person crew

\section{Three-person crews}

Since 3-person crews must maintain crew integrity at all times (cannot split into two teams), the first crew started with a right-hand search of the first compartment. The start of the first truck crew's search is shown in Figure 52 as the number 1 in a green circle, or Symbol 1. After searching this first compartment, (ending at the number 1 in a red Stop sign) the initial truck crew was out of rope, and often out of 'on air' time. At this point, the first crew would communicate the extent of their search to Division 11 and request relief. The next crew on the floor began their search at the location shown by Symbol 2. As indicated by the line style, this second crew began their search by crawling, but as they entered the inner core of the building beyond the first compartment the visibility conditions improved, allowing them to walk the next portion of their search. After covering the west end of the building, the second crew encountered another compartment of heavy smoke, causing them to revert back to crawling. Very shortly after entering this area, at Symbol Stop 2, the second crew ran out of search rope, requested relief and proceeded to Rehab. The final crew continued where the second left off at Symbol 3 and finished their search by finding the incapacitated victim (represented in Figure 52 as the word "Victim" in a yellow star). The search crew then carried the victim to the 8th floor, where they were met by the EMS crew. Meanwhile, Division 11 requested another search crew from staging to complete the search on Floor 11. 


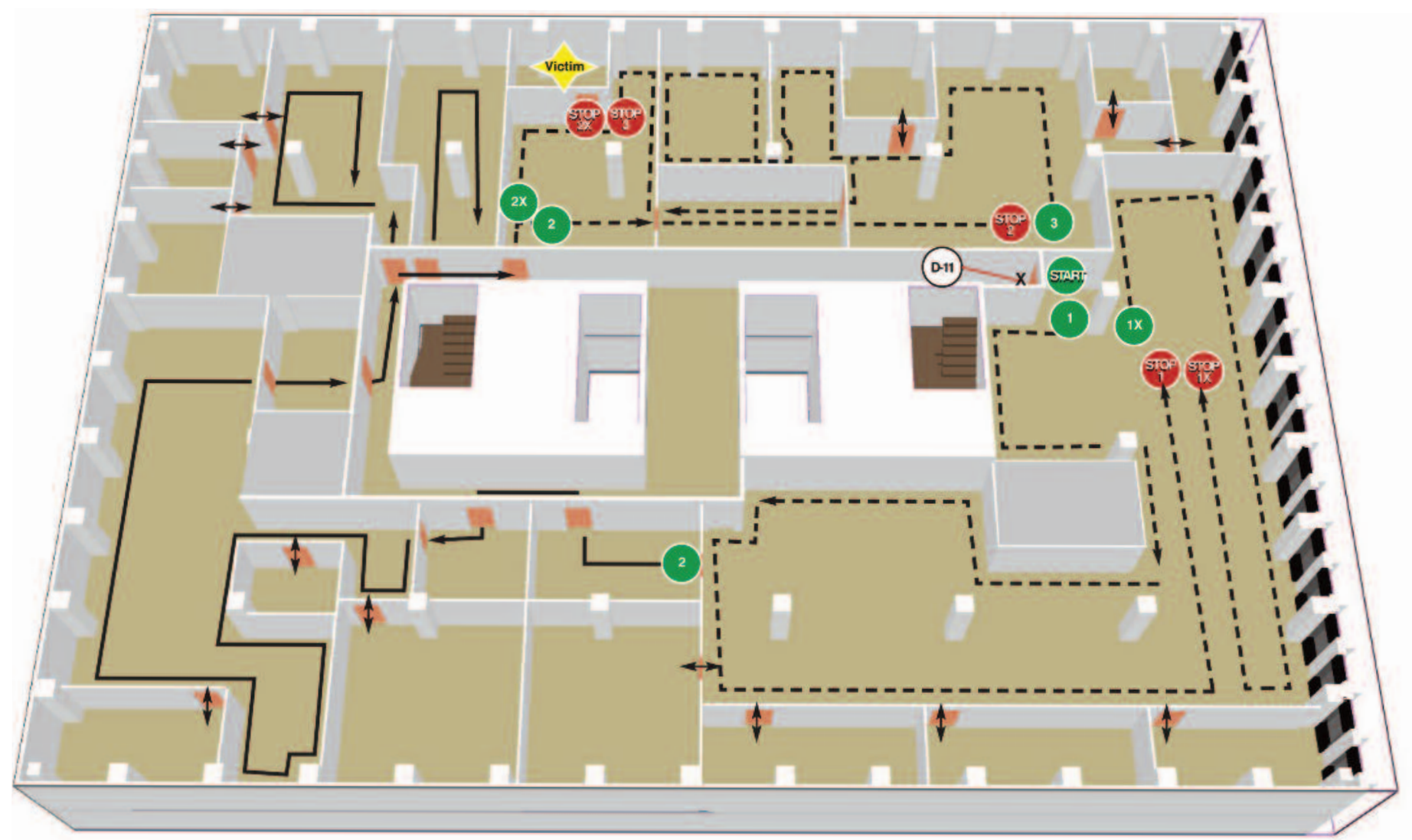

Figure 53: Floor 11 search by 4-person crew

\section{Four-person crews}

Since 4-person crews are able to split into two crews and still maintain a minimum crew size of two, the initial truck crew entering Floor 11 could begin both right-hand and left-hand searches, denoted by Symbols 1 and $1 \mathrm{X}$ in Figure 53. Due to the width of the first compartment, the initial crew was unable to span the entire compartment and was required to trace back along their search rope and cover the center of the floor. After completing the search of the first compartment, the first truck company ran out of rope and depleted their 'on air' time at Symbols Stop 1/1X and needed to be replaced. Working through Division 11, located at the symbol with "D11" within a white circle, the crew called for relief and then proceeded to Rehab. The next crew on the floor picked up at the start point shown as the isolated Symbol 2 in Figure 53. As with the second crew for the 3-person crews, the initial section of the search had limited visibility, but after the first compartment visibility improved. When the second crew reached the last compartment, the visibility again decreased, forcing a crawling search. At this point, the crew split into two crews of two. One crew began a right hand search at Symbol 2 while the second crew (X-Ray) began a left hand search at the nearby Symbol 2X. During this portion of the search, the X-Ray crew encountered the incapacitated victim at the star, forcing both firefighters to leave the floor with the victim. The remaining two members of the full crew continued to search until they ran out of rope or 'on air' time at Symbol Stop 2. When relief was called, a final crew was sent in to finish searching the remaining area from Symbol 3 to Symbol Stop 3. 


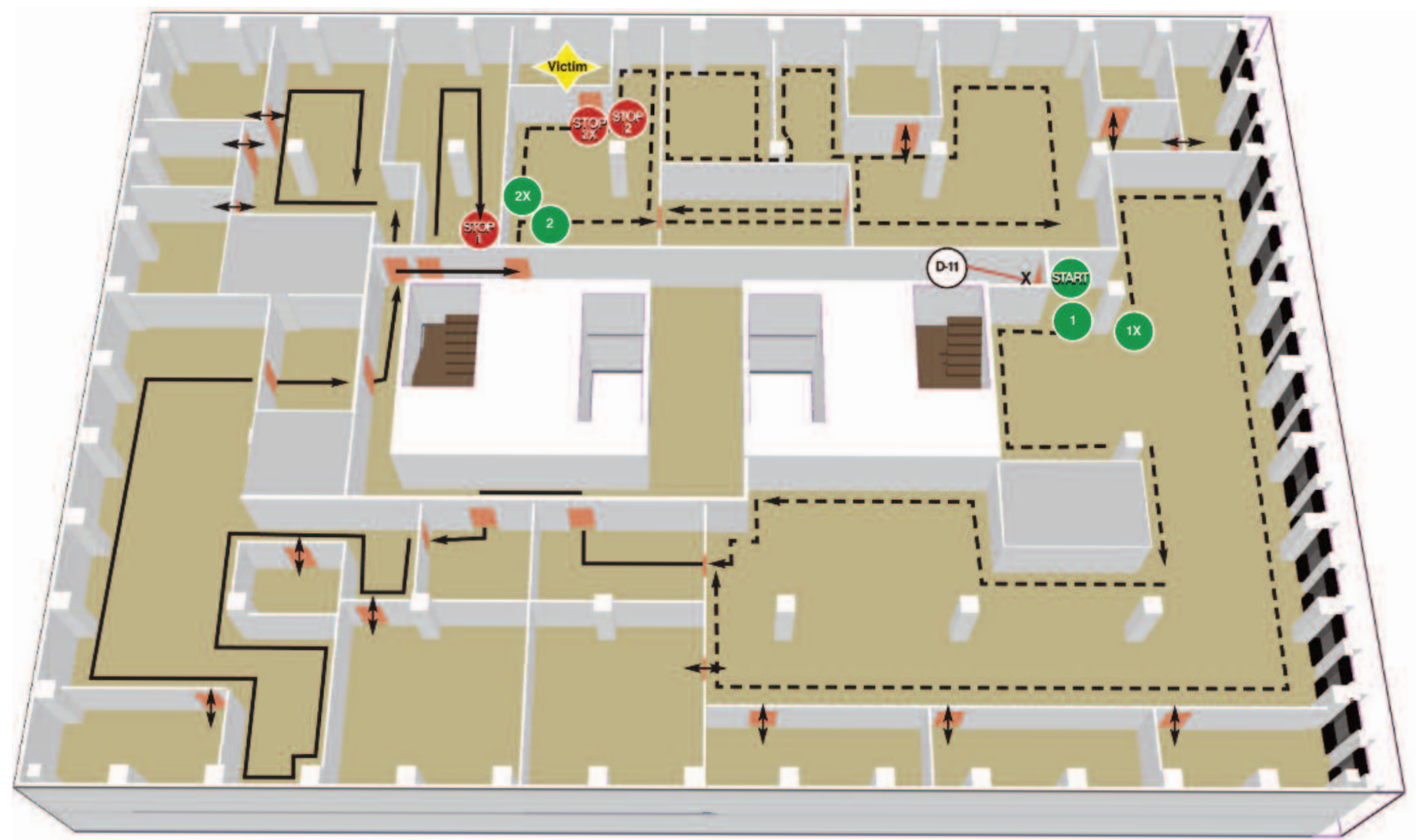

Figure 54: Floor 11 search by 5-person crew

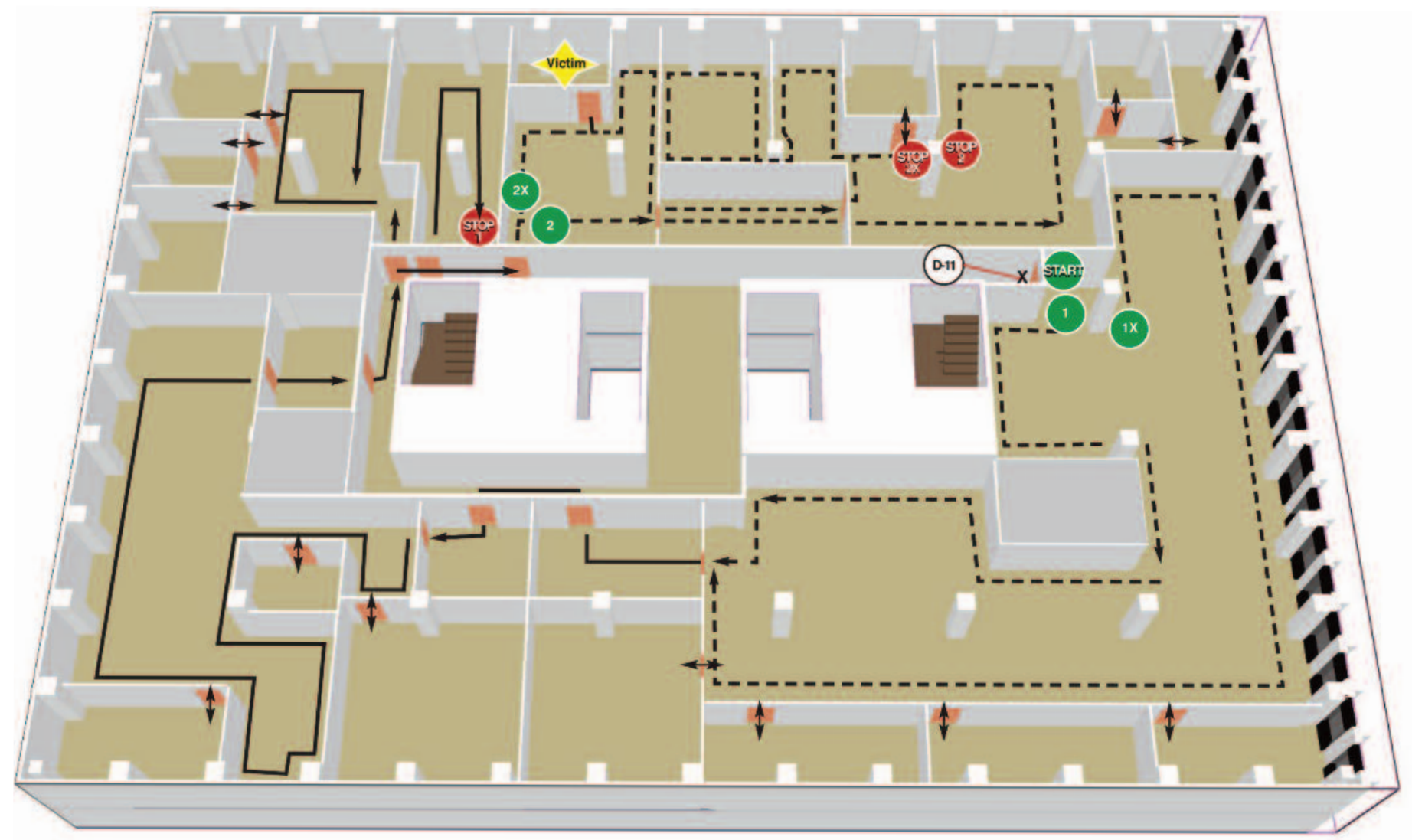

Figure 55: Floor 11 search by 6-person crew 
Five- and six-person crews

As shown in Figure 54 and Figure 55, the initial crew for 5-person and 6-person crews behaved the same. The initial crew made entry onto the floor and began a right- and left-hand search of the first compartment, starting at Symbol 1 and Symbol 1X respectively. Unlike the 4-person crews, the 5-person and 6-person crews were able to span the entire width of the compartment, which allowed them to progress past the first compartment without the need for relief. This first crew of 5 or 6 firefighters was able to search beyond the point where the first crews of 3 and 4 persons had to stop. The larger crews continued forward to search the floor until they ran out of search rope or 'on air' time at Symbol Stop 1. The first truck crew made it as far as the door into the last compartment. From this point the 5-person and 6-person crews differ, so each will be individually discussed.

The final 5-person crew

Upon entry to the last compartment at Symbols 2/2X in Figure 54 , the five-person crew split into two crews. The X-Ray crew had 2 firefighters, leaving 3 for the other crew. The X-Ray crew found the victim shortly after entry. The X-Ray crew then packaged the victim, transported the victim down to meet the EMS crew on the 8 th floor and then went to Rehab. The remaining members of the second crew finished the search of the floor.

\section{The final 6-person crew}

As with the 5-person crew, the second truck crew made entry into the last compartment at the location marked by Symbols $2 / 2 \mathrm{X}$ in Figure 55. The major difference between the 5-person and 6-person crews at this point is that with 6 members, the X-Ray crew could transport the victim down to the 8th floor to meet EMS while leaving 4 firefighters to complete the search. From here the remaining crew split again into two crews of two and finished the remaining search.

\section{Victim Rescue}

The victim on the floor above the fire was rescued using the same method as for the victim on the fire floor. Proper rescue technique required two firefighters, one using webbing to lift the victim under the shoulders, and the second firefighter to lift the victim's legs. Once properly packaged, the firefighters proceeded to the evacuation stairwell and moved down to Floor 8 where they were met by an EMS crew. At this time, emergency care was transferred to the EMS crew and the two firefighters proceeded to Rehab.

\section{Management of Floor 11 Operations}

As noted previously, BC3, upon arrival to the scene, was assigned to Division 11. Within this role, Division 11 was responsible for all operations and communications on Floor 11. When Division 11 made entry to the 11th floor, crews were already manning a hose line above the fire, and a crew was already searching the floor. Division 11 established the area just outside of the evacuation stairwell as his command area, marked by the symbol "D11" in a white circle in Figure 52 through Figure 55, since all crews must move through this stairwell to access the floor. This location allowed face-to-face exchange with crews entering and exiting the area. The face-to-face communications with arriving and departing crews also allowed Division 11 to know the progress being made on the floor. While there was heavy smoke, there was no fire extension onto the floor. However, the hose line was always staffed while a search crew was operating on the floor. 


\section{Analysis of Experimental Results}

T his section describes the analytic approaches used to address the research objectives of the study. First the statistical methods used to analyze the high-rise fireground time-to-task observations are presented. Then the time-to-task data and the fire modeling data are combined to assess crew performance in relation to tenability within the structure.

\subsection{Time-to-Task Analysis}

Time-to-task data were compiled into an analytic database. The data were reviewed for logical consistency, outliers, and missing entries. A small amount of editing was performed to clean up the data. Because all experiments were conducted in triplicate, missing data were readily apparent and were reviewed against the recorded radio tape information. Missing data were replaced by times calculated from the radio recordings. Where radio documentation proved inadequate, missing data were entered using the mean task time from the corresponding two observed field replicates. The amount of data requiring such imputation was minimal (less than $0.05 \%$ ).

\subsection{Data Queries}

The methods used to analyze the time-to-task data were driven by the principal goal of assessing the effects of crew size on task timing measures (i.e., start, duration, end) for critical steps in the high-rise response, while controlling for ascent mode and alarm size. This research goal motivated the development of five specific research questions (see Figure 56) that in turn motivated specific statistical models and analyses for generating inference. The critical tasks are presented in Table 5.

\section{Time-to-Task Research Questions}

1) How do crew si e, ascent mode (stairs vs. elevator) and si e of full alarm assignment (i.e., alarm si e low versus high) affect overall (i.e., start to completion) response timing?
a. How do variations in crew si e affect overall response timing?
b. How much does ascent mode affect overall timing?
c. How much does si e of full alarm assignment affect overall response timing?
d. How do overall response times vary by combinations of crew si e, ascent, and alarm si e?

Figure 56: Time-to-Task Research Questions

\begin{tabular}{|l|l|}
\hline$\#$ & Critical Task: \\
\hline 1 & Advance Attack Line \\
\hline 2 & Advance Second Line \\
\hline 3 & Fire Out \\
\hline 4 & Establish Stairwell/Elevator Support \\
\hline 5 & Search and Rescue 10th Floor \\
\hline 6 & Victim \#1 Found \\
\hline 7 & Victim \#1 Rescue \\
\hline 8 & Victim \#1 Descent \\
\hline 9 & Search and Rescue 11th Floor \\
\hline 10 & Victim \#2 Found \\
\hline 11 & Victim \#2 Rescue \\
\hline 12 & Victim \#2 Descent \\
\hline 13 & Advance Line Above Fire \\
\hline 14 & All Tasks Complete (timer sheet) \\
\hline
\end{tabular}

Table 5: Critical tasks considered in analysis

\subsection{Statistical Methods - Time to Task}

The analysis of the time-to-task data involved a sequence of multiple linear regressions using Ordinary Least Squares to generate and test the effects of crew size, alarm size and ascent mode on timings. The regressions were of the form:

$$
y_{i}=\beta_{0}+\beta_{1} x_{i 1}+\beta_{2} x_{i 2}+\ldots+\beta_{k} x_{i k}+\varepsilon_{i}
$$

Where $y_{i}$ represents the ith dependent/outcome variable, $x_{i k}$ denotes the factors such as vertical ascent mode and crew size whose effects on $y_{i}$ are being tested, and $\beta_{k}$ is the set of regression coefficients that minimizes the set of errors $\varepsilon_{i}$. The three potential dependent (outcome) variables for each critical task listed in Table 5 were:

Begin time (i.e., the time at which a task commenced), Duration time (i.e., the time it took to complete the task), and End time (i.e., the time of task completion).

Three sets of independent variables were included: crew size (3, $4,5,6)$; ascent mode (elevator, stairs) and alarm size (low, high). Dummy variables were used for these controlling factors in the regressions. Regressions were performed for each task-outcome combination, so that the impacts of crew, alarm and ascent could be examined by task individually and in combination (according to the research question being addressed). These regressions permitted the development of specific contrasts (e.g., to look at 
the effects of incremental changes in crew size) that were used to address the research questions posed in Figure 56.

\subsection{Regression Analyses}

\section{Regression results}

Appendices B1 to B3 present the regression results used to generate the findings in this chapter. Regression models were developed for each relevant critical task and outcome combination. Additional regressions were performed to explore the effects of specific combinations of crew and alarm size as well as combinations of ascent mode and alarm size. The regression results include the coefficient values, their standard errors and their corresponding levels of significance.

Rather than detailing each of the lengthy lists of coefficients found to be significant, specific summaries of these results were compiled in order to address the primary research questions presented in this chapter. Appendices $\mathrm{C} 1$ to $\mathrm{C} 4$ provide a detailed summary of regression findings, and Appendix C5 presents a tabular summary of all statistically significant findings.

\section{Overview of Time-to-Task}

As a segue to the statistical regression analyses, an overview of the time-to-task results for the different crew sizes used in these experiments is presented, along with an examination of how each result varied when introducing the two other factors that were variables in the study — vertical ascent method and alarm size.

Figure 57 presents the overall average times to completion for each crew size tested in the field experiments. As one would expect, the time to completion decreases as crew size increases. Three-person crews took an average of about an hour to complete their fire response, while crews of 6 firefighters required a mean time of just under $40 \mathrm{~min}$ for completion. The performance of crews with 4 and 5 firefighters were between these two values, with crew size 5 taking about 2 min longer than crew size 6 , and crew size 4 taking about 9 min longer than crew size 5 but 12 min less time than crew size 3 . The findings from the crew size analysis suggest that size does matter when it comes to number of firefighters assigned to crews. Even the increment of a single firefighter can have a positive impact on the start, duration and completion of varied critical tasks. Incrementing crew size by two is also beneficial. The most sizeable gains were seen

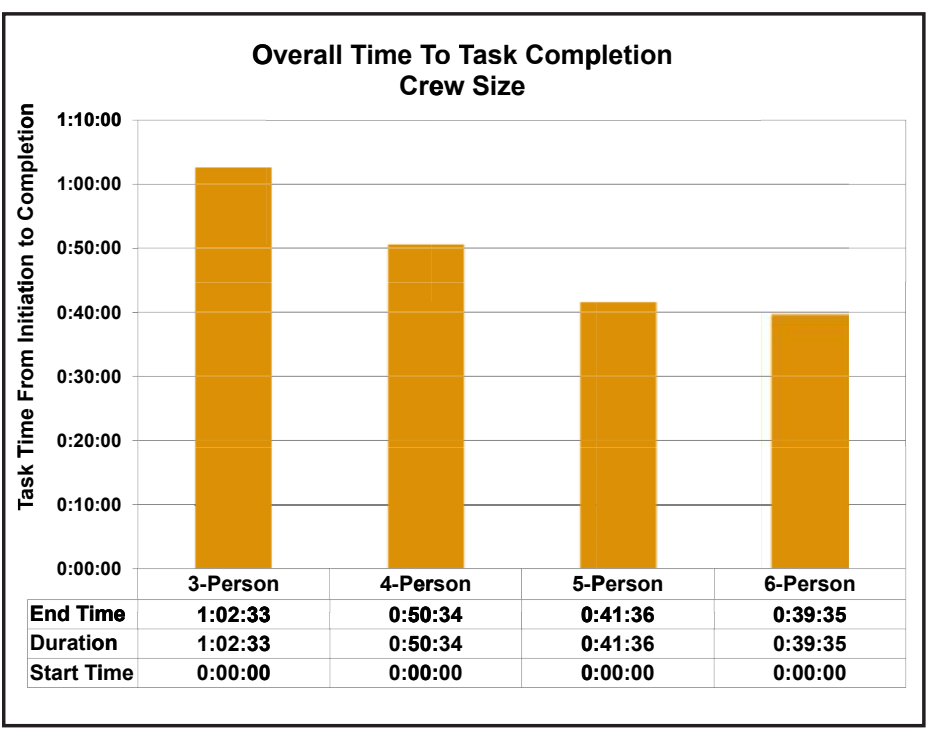

Figure 57: Time to complete all tasks by crew size when incrementing from a smaller crew size to a larger crew size, e.g., 3 to 4,4 to 5 , or 3 to 5 .

Figure 58 presents the average completion times by ascent mode for each crew size. The patterns across crew size are similar by mode. The use of elevators reduced overall completion time relative to ascent by stairs. For any given crew size, the reduction in the time to complete all tasks attributed to ascent mode was roughly in the $3 \mathrm{~min}$ to $5 \mathrm{~min}$ range in favor of elevators.

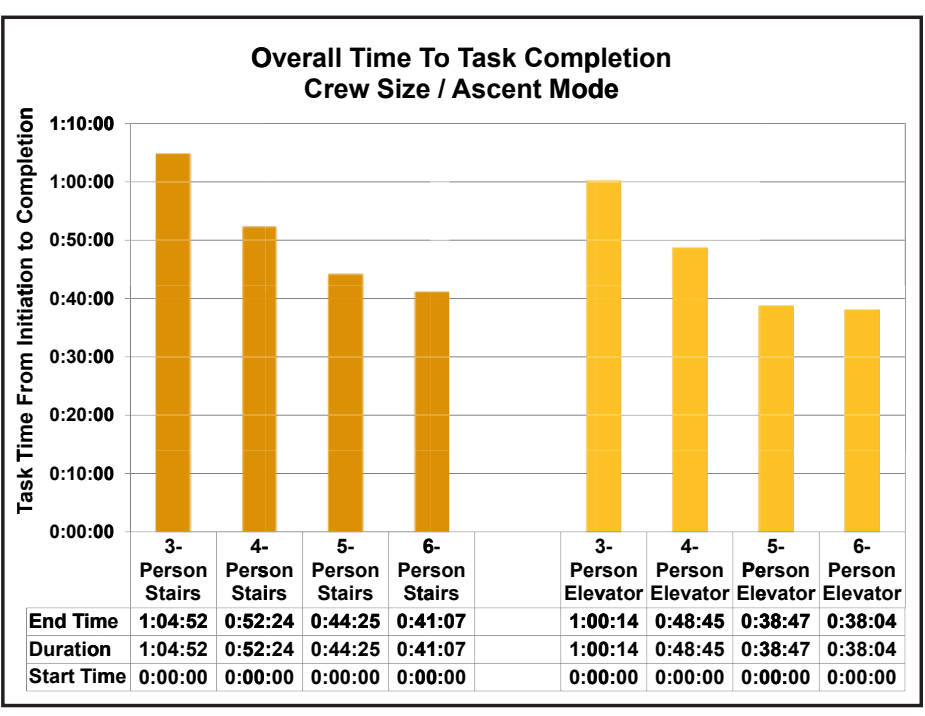

Figure 58: Time to complete by crew size and ascent mode

Figure 59 presents the average completion times by crew size and alarm size (low vs. high). Again, the patterns by alarm size are similar across crew sizes. For three of the four crew sizes $(4,5$, and 6 ) average completion times are slightly shorter for high response compared to low response, with differences ranging from just over a half minute to about $3 \mathrm{~min}$. While these results suggest little impact of alarm size on overall time to completion for a given crew size, it does leave open the possibility that specific combinations of crew size and alarm size may outperform others - e.g., 4/high versus 5/low. This possibility will be explored later in the regression analyses.

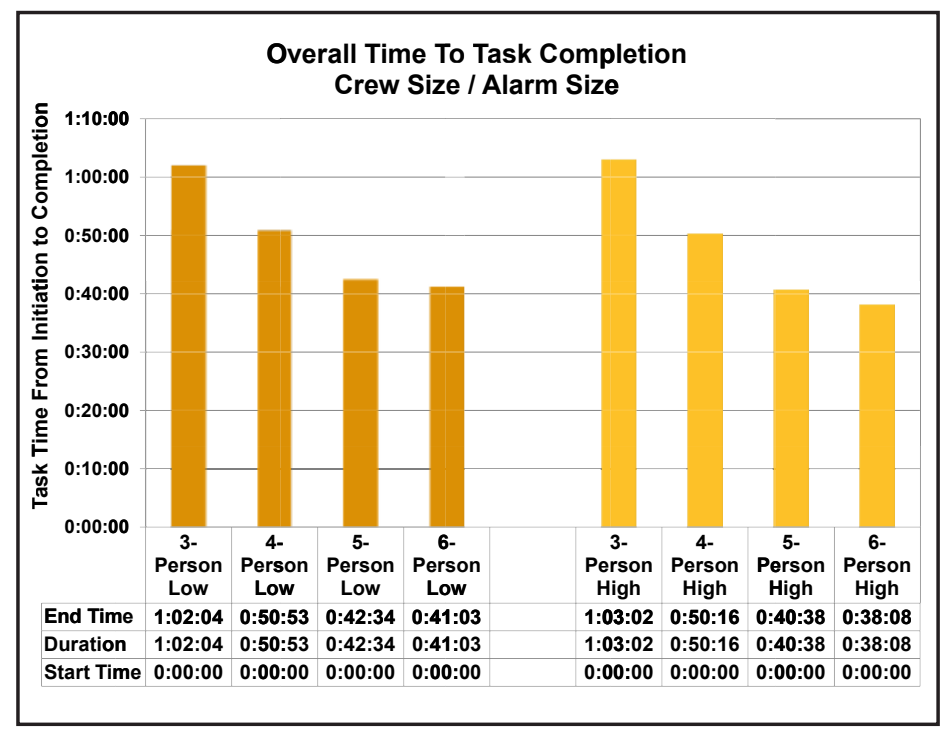

Figure 59: Time to complete all tasks by crew size and alarm size 


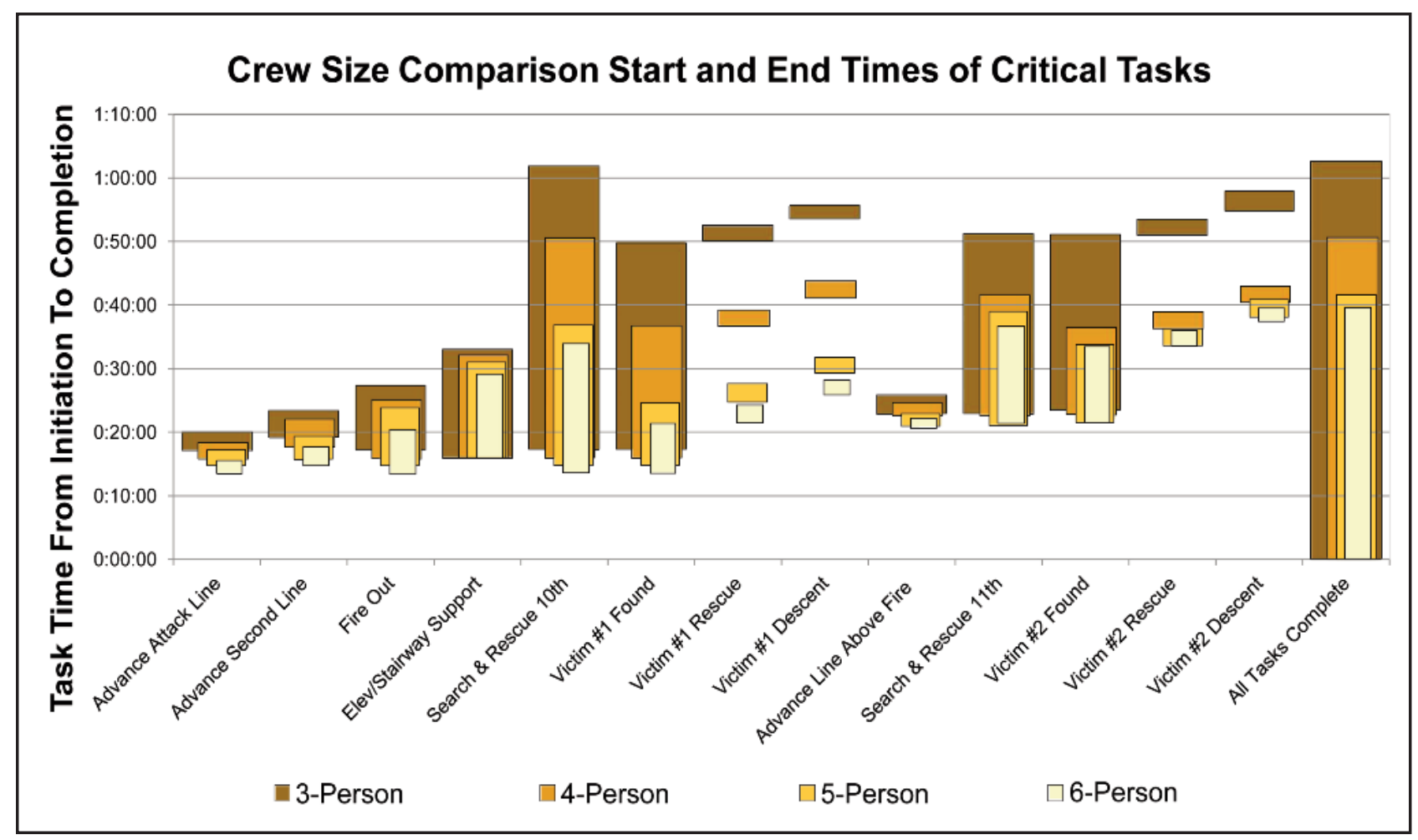

Figure 60: Start and end timing of critical tasks by crew size

\section{Critical Tasks}

In this section the subset of tasks deemed 'critical' in the firefighter high-rise response is examined. Different timing outcomes (begin, duration, end) are important to consider for the subset of tasks, listed in Table 5. An overview of critical task timings is presented by crew size in Figure 60, which shows the overall average start and completion times for each crew size and critical task, including overall completion time. The overall patterns for a given critical task show fairly consistently that the average time to perform a task diminishes as crew size increases. The obvious exceptions are for Victim (\#1 and \#2) Rescue and Descent, since the same number of firefighters were carrying the victim during these tasks regardless of overall crew size. Note the cascading start times by crew size for the early critical tasks -Advance Attack Line, Advance Second Line, Fire Out, Search and Rescue, and Victim Found. Note also that the time differentials by crew size are very pronounced for both victim rescues. With shorter durations of these critical tasks for larger crew sizes, the net result is seen in the right hand side of the graph — All Task Complete times are substantially reduced for crew size of 6 compared to 5, 5 compared to 4, etc. The greatest improvements in All Task Complete time occurred for time differences between 3-person and 4-person crews and between 4-person and 5-person crews.

\subsection{Search Buttons}

The data generated from the search button presses provided time-based firefighter location information. This information gave insight into the effects of crew size changes and, when applicable, crew-splitting. Figure 61 shows the search time-history of a 3-person crew that used the stairs. The horizontal axis represents time, where the initial time is the start of the experiment. The vertical axis represents the button numbers. In Figure 61, the circles represent buttons that existed on the interior search loop (buttons \#1-9) and the diamonds represent buttons

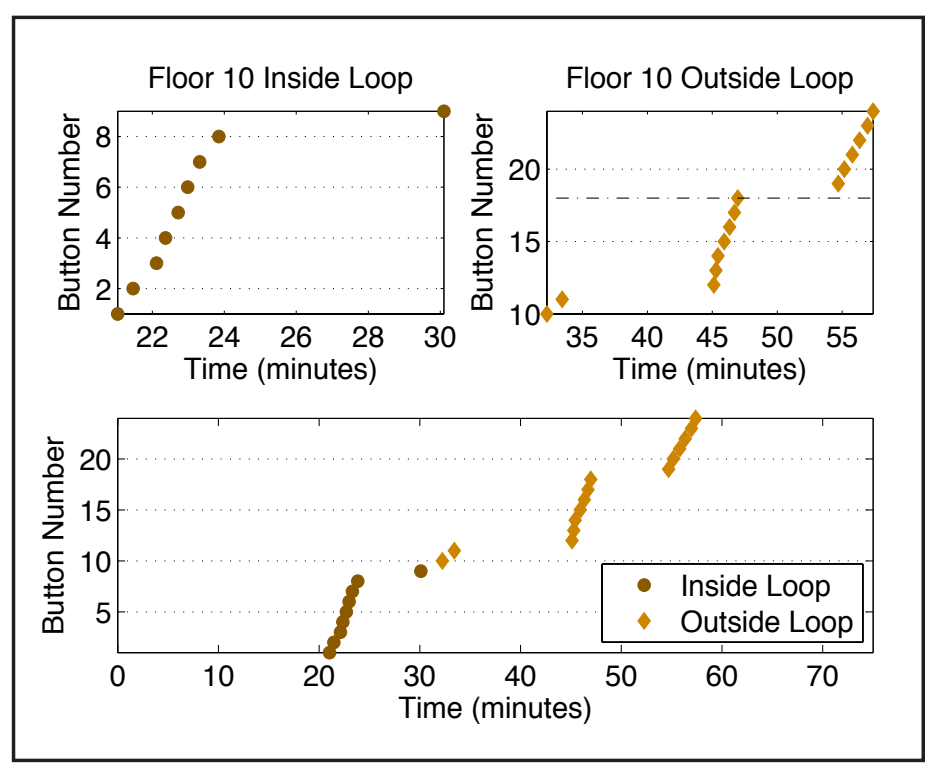

Figure 61: Button presses as a function of time on the fire floor for a 3-person crew using the stairs.

on the outer search loop (buttons \#10-24). The top left plot shows only the inside search loop buttons pressed whereas the top right plot shows only the outside search loop buttons pressed. The bottom plot is the combined inside and outside search data.

Examination of the button data as a function of time shows that there were distinct periods of time when no buttons were pressed. These extended time gaps represent an exchange of crews (one crew reported to Rehab, while another crew was dispatched from Staging and began searching where the other left off). As mentioned previously, a crew change occurred if the searching crew ran out of air, reached the end of a tag line, or found a victim. Due to the distinct inside and outside search loops, a 
detailed analysis of the button data in this report will be focused on the fire floor.

For a 3-person crew, the first button press occurred approximately 21 min into the experiment, as indicated in Figure 61 (refer to button locations in Figure 31). The first-in truck company (Truck 1) searched the fire floor until button 8 was pressed at approximately $24 \mathrm{~min}$. Between presses of button 8 and button 9, there was an extended gap in time of approximately 6 min. This gap represents a crew change as Truck 1 either reached the end of the search rope or exceeded their time on supply air. The second truck assigned to the search picked up where the first truck company left off; their first button press was button 9 . Because button 9 represents completion of the interior search, the second-in truck started the search of the outer loop, pressing buttons 10 and 11 . This is shown in both the top right and bottom figures. The time gaps between the button presses of 11 and 12 as well as 18 and 19 indicate that two more crews were required to complete the search of the fire floor. For this particular experiment, four 3-person crews were required to complete the search.

Similar examination of the fire floor button data for all of the crew size configurations can be used to determine how many crews were needed to complete search and rescue operations. Table 6 shows the average number of crews required to complete the search operations for each crew size examined in the experiments. This average number of required crews was calculated by including high and low deployments as well as stair and elevator ascents.

\begin{tabular}{|l|c|c|}
\hline Crew Si e & $\begin{array}{c}\text { Average Number } \\
\text { of Crews to Complete } \\
\text { Search on Fire Floor* }\end{array}$ & $\begin{array}{c}\text { Total Number of } \\
\text { Firefighters Actively } \\
\text { Searching the Floor }\end{array}$ \\
\hline 3-person & 4 & 11 \\
\hline 4-person & 3 & 11 \\
\hline 5-person & 2 & 9 \\
\hline 6-person & 2 & 11 \\
\hline
\end{tabular}

* Rounded to the nearest whole number of crews

Table 6: Average number of crews required to complete search and rescue on the fire floor for each crew size

Table 6 shows that as the crew sizes increased from 3-person to 4-person, the average number of crews required to complete the search decreased from 4 to 3 . The drop in number of crews between 3-person and 4-person crews was a result of crew-splitting for the second-in truck crew. The 4-person crew on the second-in truck could split into two groups of two firefighters to concurrently complete the search on the inside search loop and begin searching the outside loop. This result is shown in Figure 62.

Figure 62 shows that the first-in truck company was able to hit the first 5 buttons before leaving the fire floor for relief. Though technically a 4-person crew, one firefighter remained in the lobby to pressurize the stairwells with PPV, leaving 3 to ascend for search activity. Therefore, the first-in truck company was really a 3 -person crew and could not split into two teams. The second-in truck company, however, ascended to the fire floor as a full
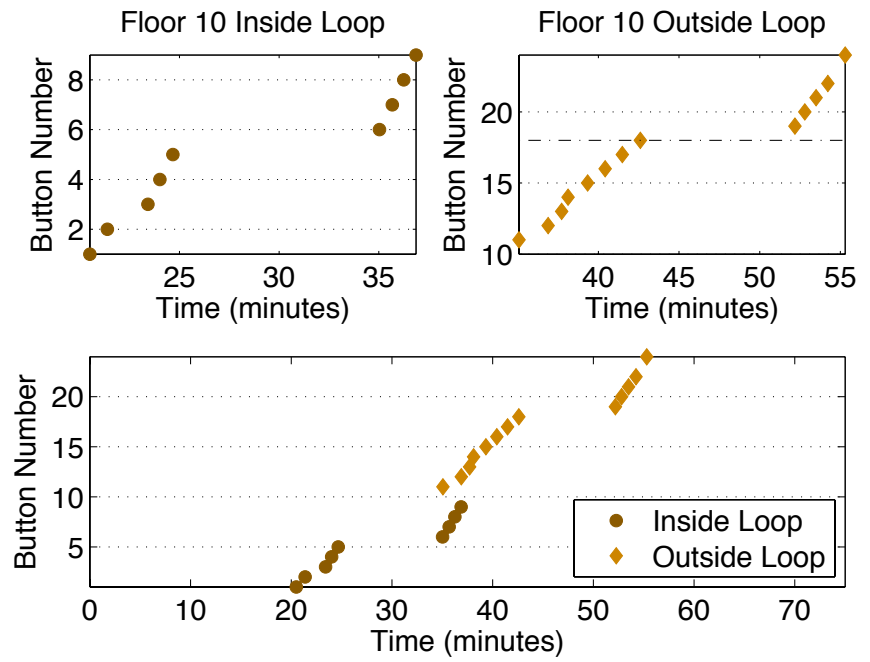

Figure 62: Button presses as a function of time on the fire floor for a 4-person crew using the stairs

4-person crew. They were able to split and search the inside loop and the outside loop, as shown by the bottom plot. Two firefighters resumed searching the inside loop (button 6) and were able to complete the loop (button 9). The remaining two firefighters started searching the outside loop and reached the victim (button 18). A third crew completed the remaining length of the outside loop. A full complement of 4-person crews was able to complete the search using fewer crews than a complement of 3-person crews, because of the ability to split crews into two teams during the search. The advantages of splitting crews were even more evident in the 5-person and 6-person crews because the first-in trucks arrived on the fire floor with a working 4-person and 5 -person crew, respectively. This allowed both initial crews to split and begin searching the inside and outside loops simultaneously upon arrival. Figure 63 and Figure 64 show the button history of 5-person and 6-person crews that used the stairs.

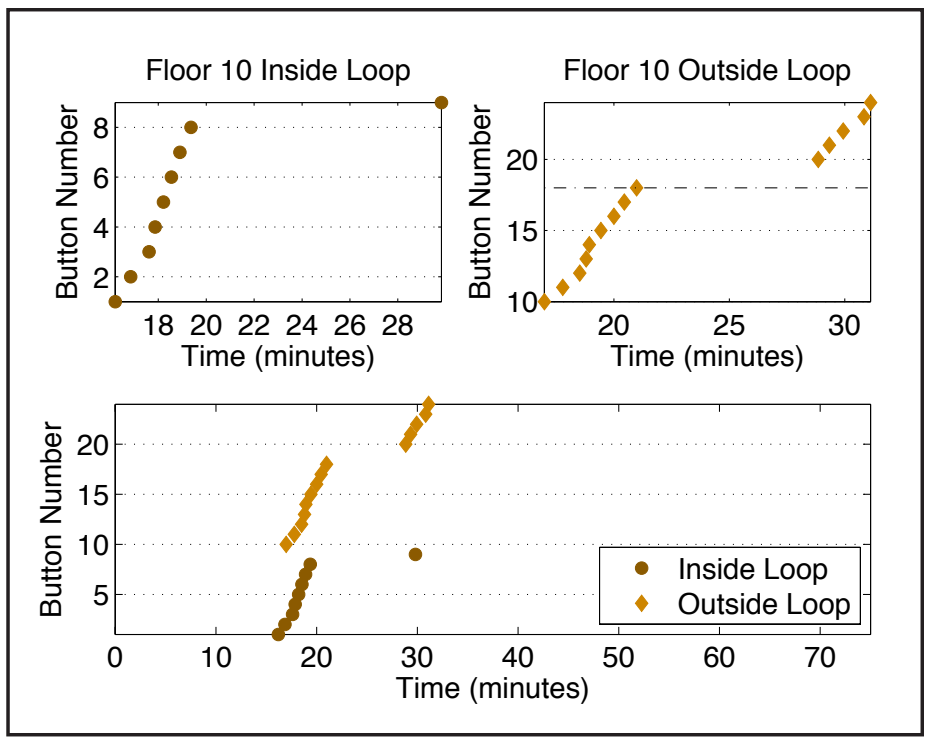

Figure 63: Button presses as a function of time on the fire floor for a 5-person crew using the stairs 


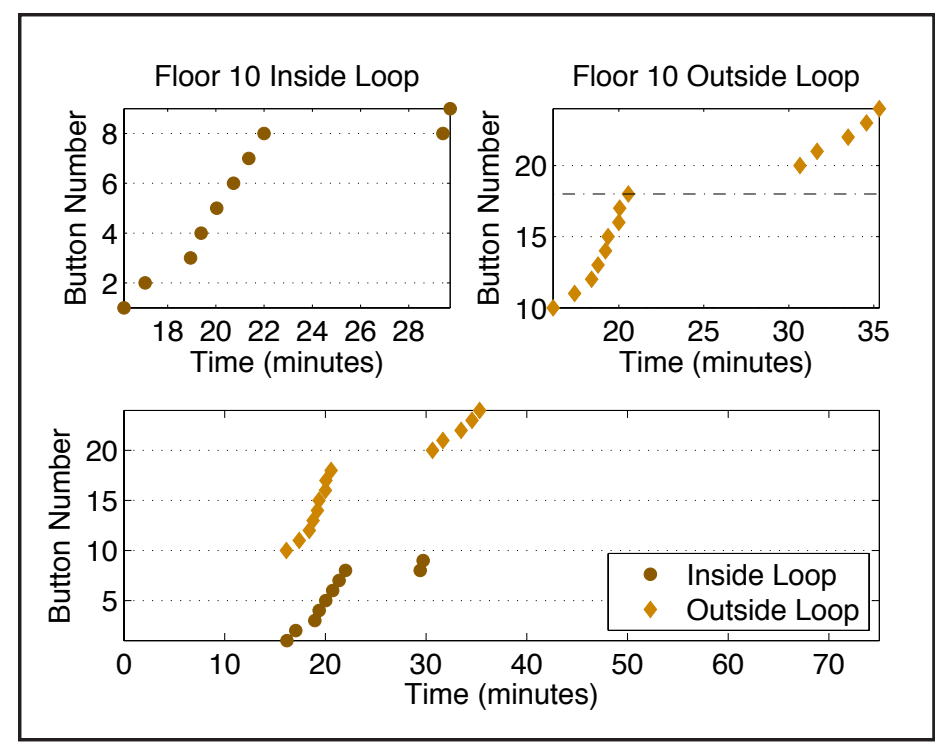

Figure 64: Button presses as a function of time on the fire floor for a 6 -person crew using the stairs

Table 6 shows that both the average 5-person and 6-person crews are more time-efficient, requiring the same number of crews (one less than 4-person crews) to complete the fire floor search. Figure 63 and Figure 64 show that both crew sizes could initially split, so the differences between the 5-person and 6-person crews were due to the additional firefighter on the search crew searching the outer loop.

The button data provided insight into the total time actively spent searching, which the timer data did not. Timer data provided information on the duration of the search and rescue actions; however, that time also included all of the gaps associated with crew changes. Using the button data, these time gaps could be subtracted from the total search duration time to determine an active search time. The active search time was defined to include the entire time a particular crew spent searching the floor. For example, consider Figure 63 for the 5-person crew. The bottom

\begin{tabular}{|l|c|c|}
\hline Crew Si e & $\begin{array}{c}\text { Average Time of } \\
\text { Active Searching on } \\
\text { Fire Floor (MM:SS) }\end{array}$ & $\begin{array}{c}\text { Standard Deviation } \\
\text { of Active Searching } \\
\text { on Fire Floor (MM:SS) }\end{array}$ \\
\hline 3-person & $15: 28$ & $3: 19$ \\
\hline 4-person & $14: 26$ & $2: 51$ \\
\hline 5-person & $9: 59$ & $1: 55$ \\
\hline 6-person & $9: 57$ & $2: 08$ \\
\hline
\end{tabular}

Table 7: Average and standard deviation of time spent actively searching the fire floor as a function of crew size plot shows that the first crew was split into two subgroups to search the inner and outer loop at the same time. The upper two plots show that the first button pressed was button 1 on the inside loop. The last button pressed was button 18 on the outside loop. Therefore, the time spent actively searching was the length of time between those button presses. This analysis was independent of when the first crews arrived on the floor or the length of time it took for a relief crew to arrive. Therefore, it allowed for the effects of both deployment size and ascent method to be examined together. Table 7 shows the average time spent actively searching the fire floor by each crew size and the standard deviation of the time measurements.

Similar to Table 6, this table shows that there was a gain in performance when crews can split. Most notably, the largest gain occurred between 4-person and 5-person crews. As stated earlier, a 5-person crew was large enough to split the first-in crew. Time spent actively searching by the 5-person and 6-person were nominally the same. Differences here are again attributed to the extra firefighter on the outer loop. The fire floor button data showed that because of crew splitting, 5-person and 6-person crews required the lowest number of crews to complete the search and spent the least amount of time actively searching the floor.

\subsection{Measurement Uncertainty}

The measurements of length and time taken in these experiments have unique components of uncertainty that must be evaluated in order to determine the significance of the results. Appendix F summarizes the uncertainty of key measurements taken during the experiments. Importantly, the magnitudes of uncertainties associated with these measurements have no impact on the statistical inferences presented in this report. 


\section{Time-to-Task Results}

\subsection{How to Interpret \\ Time-to-Task Graphs}

igure 65 presents a sample

time-to-task analysis. Each crew size has a column graphic showing the start time and completion time for the task. Visually, columns starting lower on the graph depict deployment

configurations that resulted in earlier start times. The height of the column graphic is a visualization of the duration of the task, with taller columns indicating longer times-to-task completion. Times are also shown in a table below the graph. If subtracting the start time from the end time yields a result that differs from the printed duration by one second, it is the result of rounding fractional seconds to the nearest whole second. Where vertical response mode or alarm size were statistically significant, the effects are graphed separately. All differential outcomes described in this chapter are statistically significant at the $95 \%$ confidence level or better. Where time differences due to vertical response mode or alarm size were not statistically significant, as in this sample, the data were combined by crew size.

Time is represented on the $y$-axis with 0:00:00 denoting fire ignition time. (See Table 4 and Figure 33.)

Unless otherwise stated, differences are for end times of the relevant task.

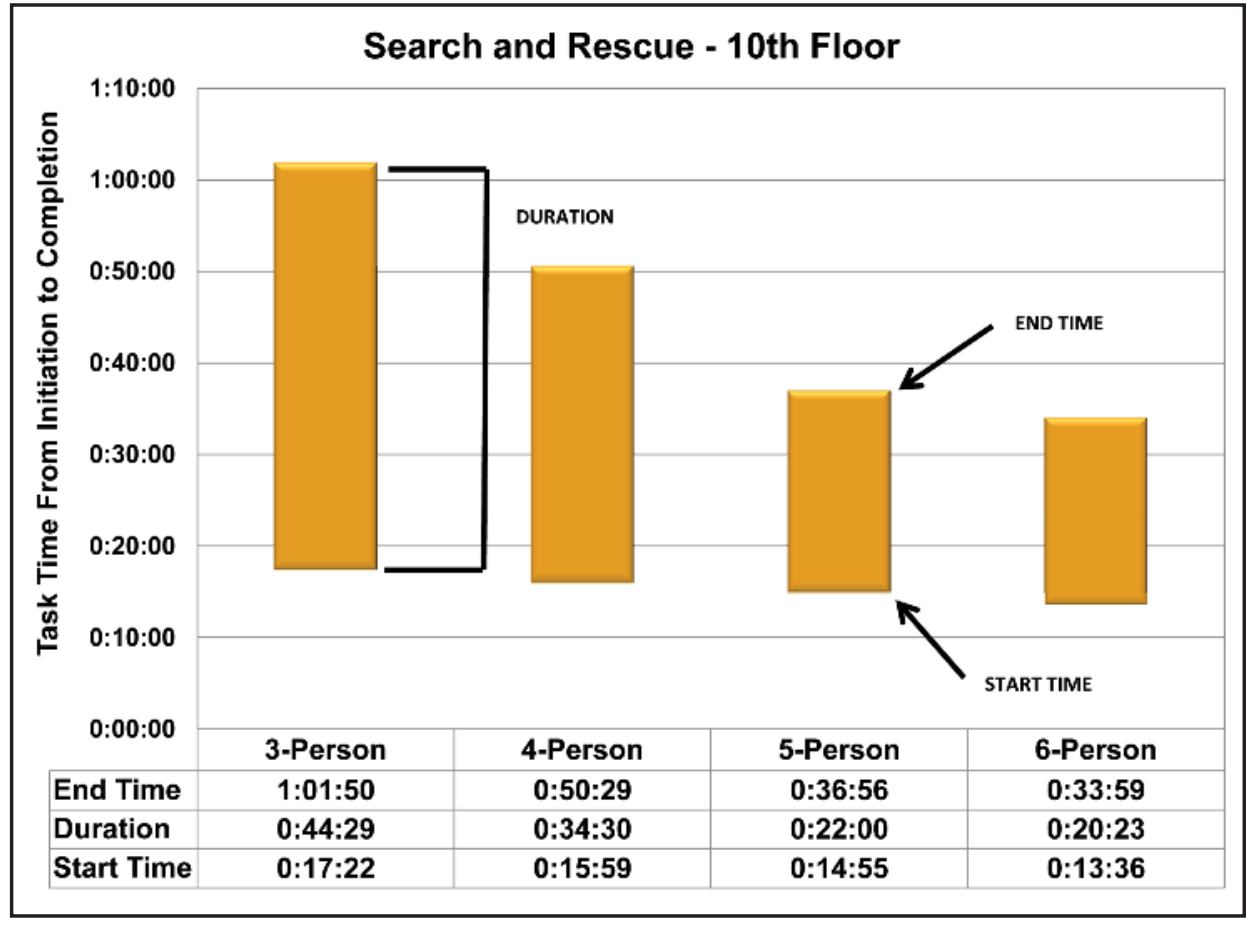

Figure 65: Sample time-to-task graph 


\subsection{Overall Time to Task Completion and Crew Size}

Overall scene time is the time that firefighters are actually engaged in tasks on the scene of a structure fire and are unavailable for dispatch to other incidents. The times noted do not include some tasks such as salvage, overhaul and secondary search of the structure as these were not included in the field experiments.

The graphs in Figure 66 through Figure 69 show average times for each critical task by crew size. Percentage calculations for all the charts were based on the overall time from detection of the incident to the completion of all on-scene tasks. Time to detection, call processing time, and travel time may vary across jurisdictions. The times used in this report are considered conservative.

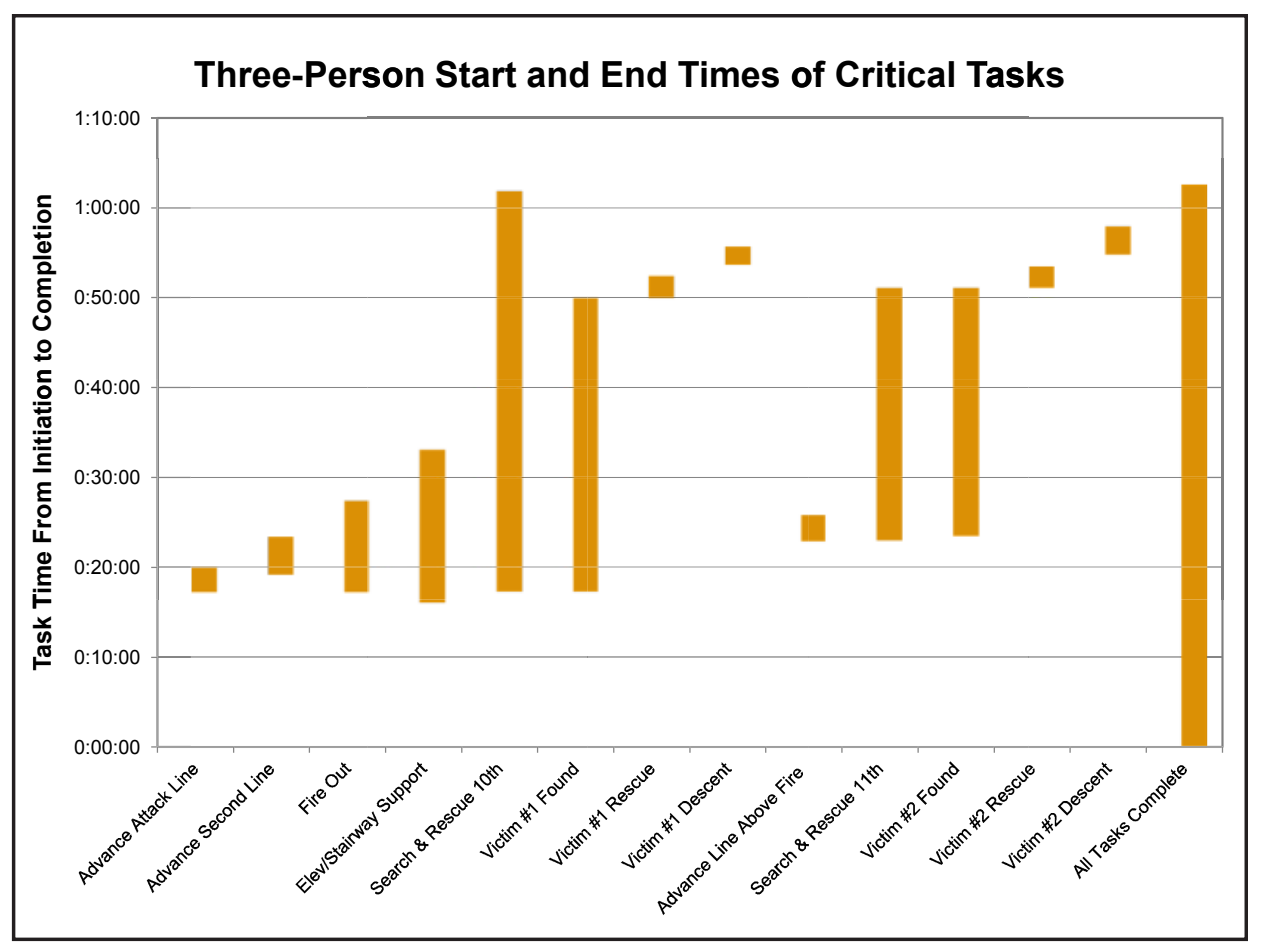

Figure 66: Average start and end times of critical tasks for a 3-person crew

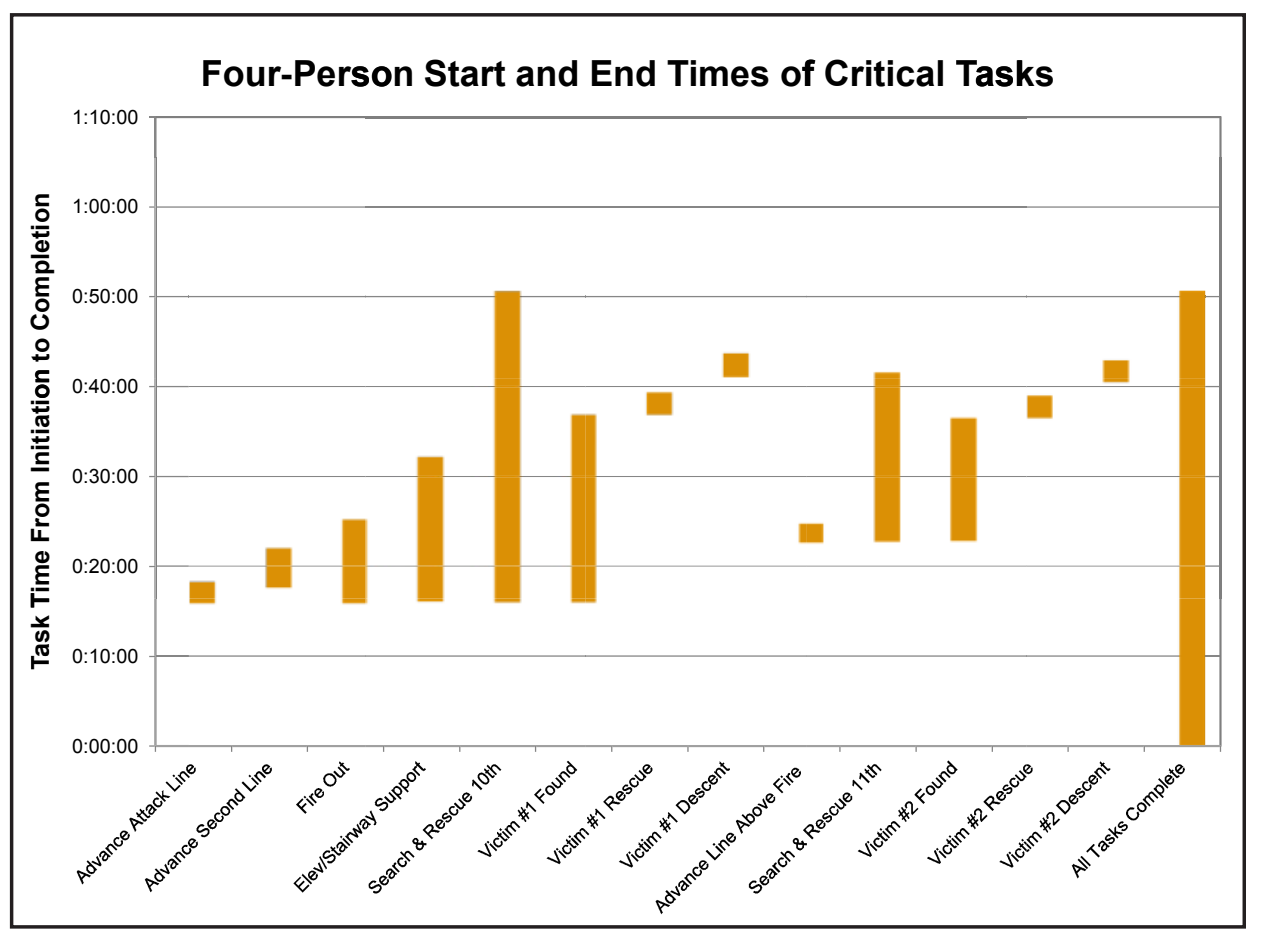

Figure 67: Average start and end times of critical tasks for a 4-person crew 


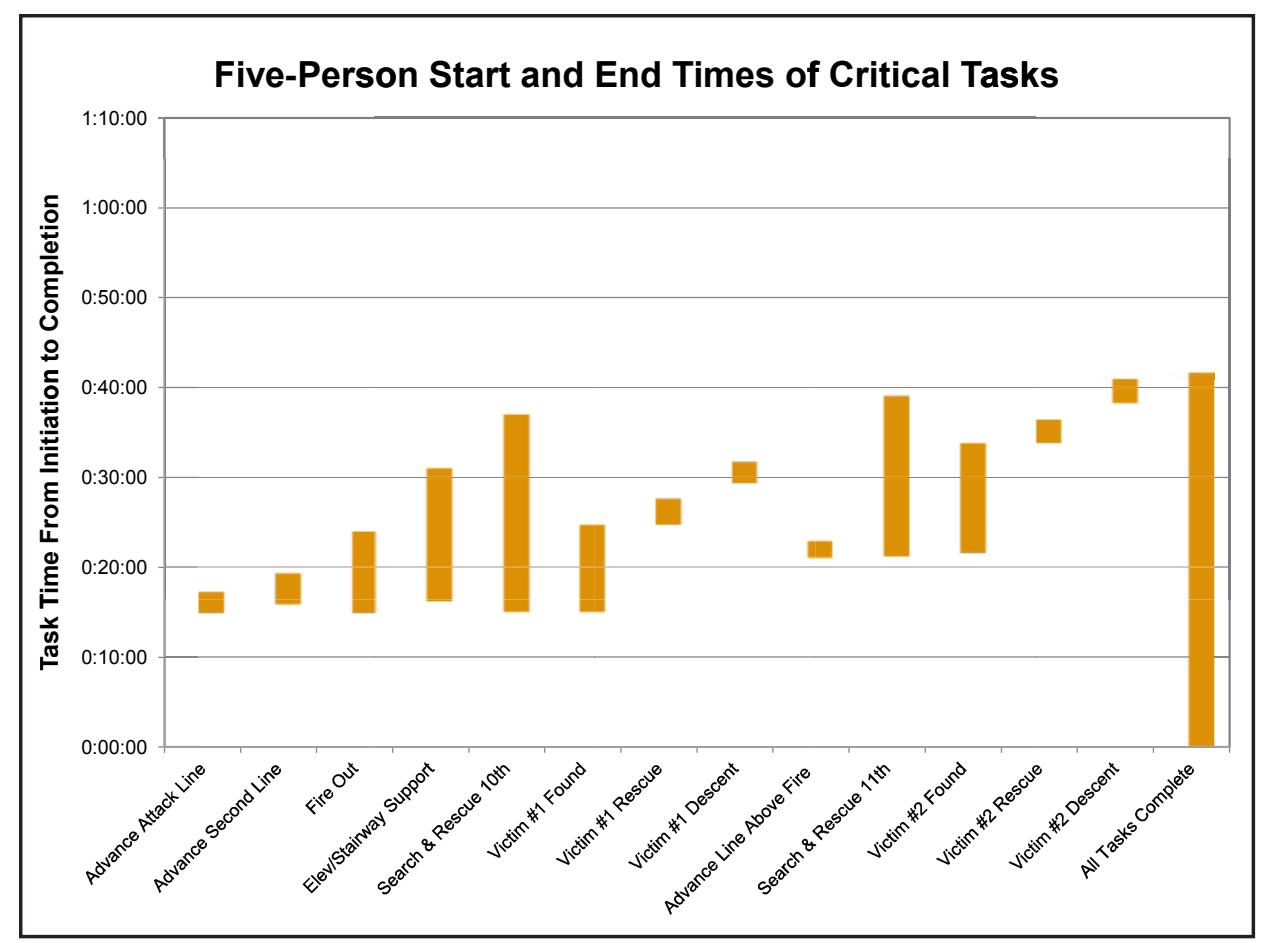

Figure 68: Average start and end times of critical tasks for a 5-person crew

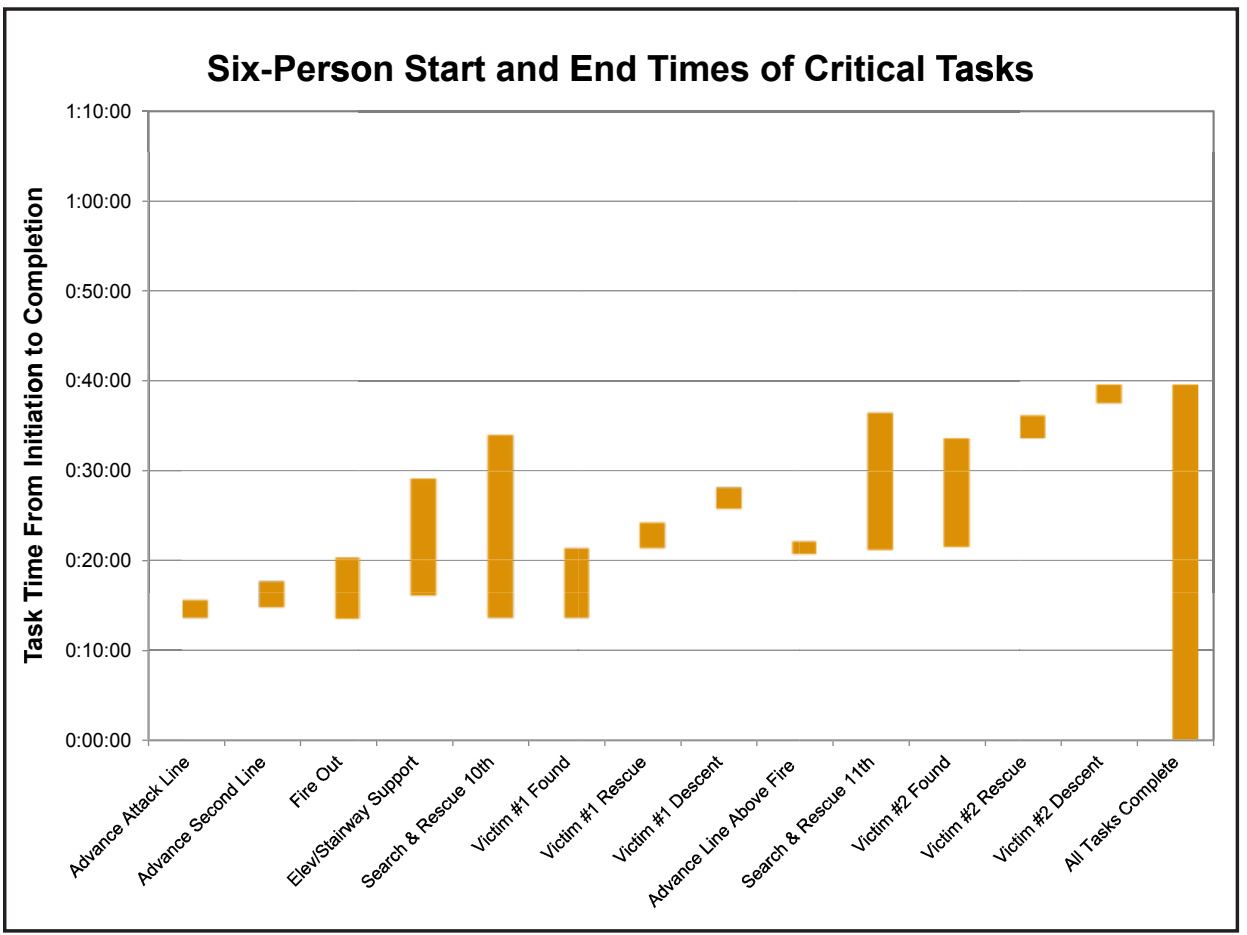

Figure 69: Average start and end times of critical tasks for a 6-person crew 


\subsection{Advance Attack Line}

Putting water on the fire is one of the most important tasks on the fireground. Before water can be put on a fire however, a hose line must be stretched from the standpipe in the stairwell to the compartment where the fire is burning. Figure 70 measures the interval from the start to the end of the task Advance Attack Line.

The time differences increased with increasing crew size. From the initiation of on-scene firefighting activities, 4-person crews were 1 min $42 \mathrm{~s} \mathrm{(8.5 \% )} \mathrm{faster} \mathrm{than}$ 3 -person crews to stretch the hose line. Five-person crews were 2 min 47 s (13.9\%) faster than 3 -person crews. The most notable comparison is between 6-person crews and 3-person crews. The 6-person crews were 4 min 28 s (22.3 \%) faster in task completion time.

\subsection{Advance Second Line}

The size of the fire required two $2 \frac{1}{2}$ inch lines to fully suppress, therefore a second hose line had to be advanced from the standpipe in the stairwell to the fire. Figure 71 measures the interval from the start of the task Advance Second Line to the end of this task.

From the initiation of on-scene firefighting activities, 5-person crews were 4 min 4 s (17.4\%) faster than 3-person crews and 2 min $43 \mathrm{~s}$ (12.3\%) faster than 4-person crews to stretch the second line. Finally, the most notable comparison was between 6-person crews and 3-person crews. The 6-person crews were faster by 5 min $38 \mathrm{~s}(24.1 \%)$ in task completion time.

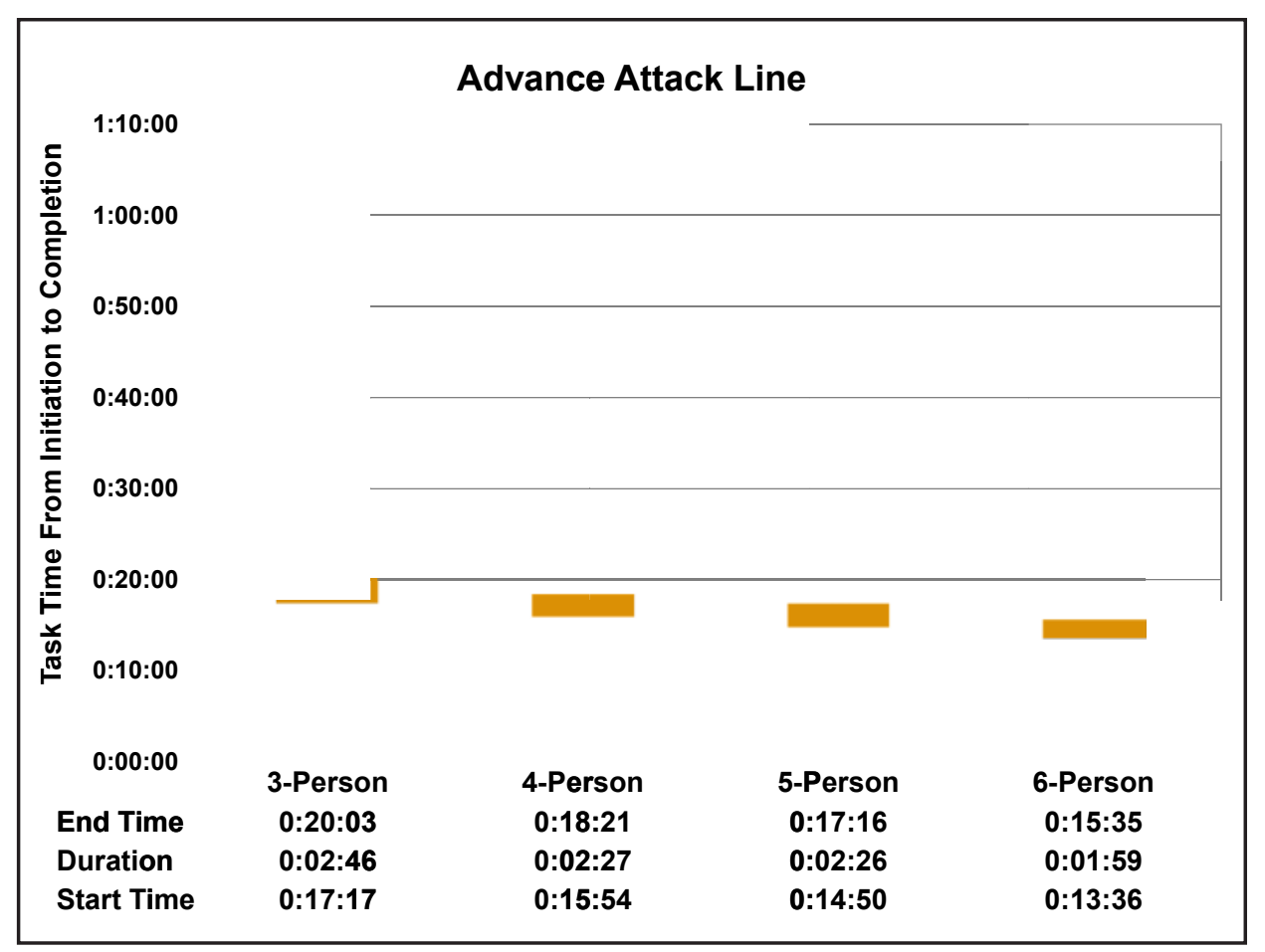

Figure 70: Advance Attack Line on Fire Floor

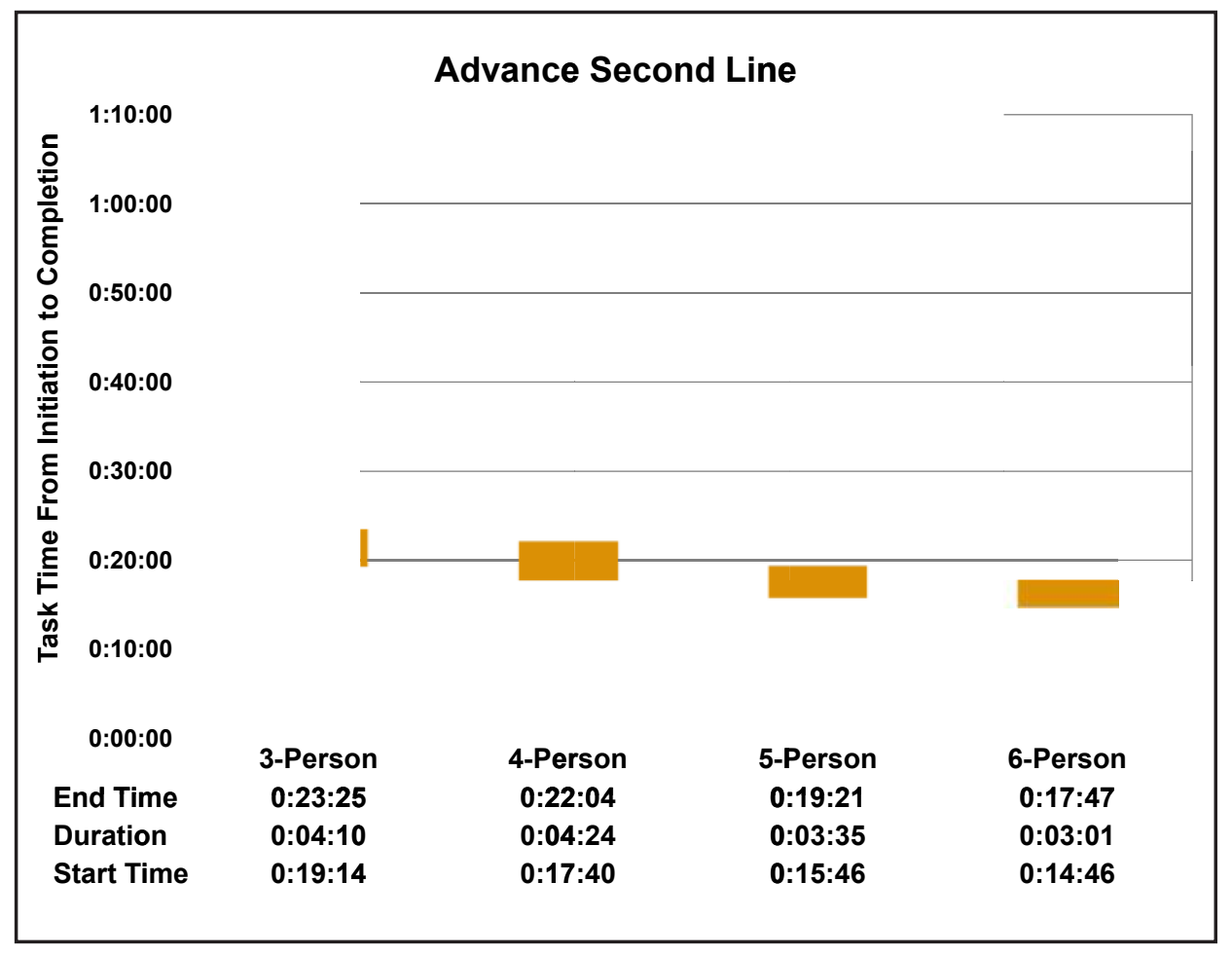

Figure 71: Advance Second Line on Fire Floor 


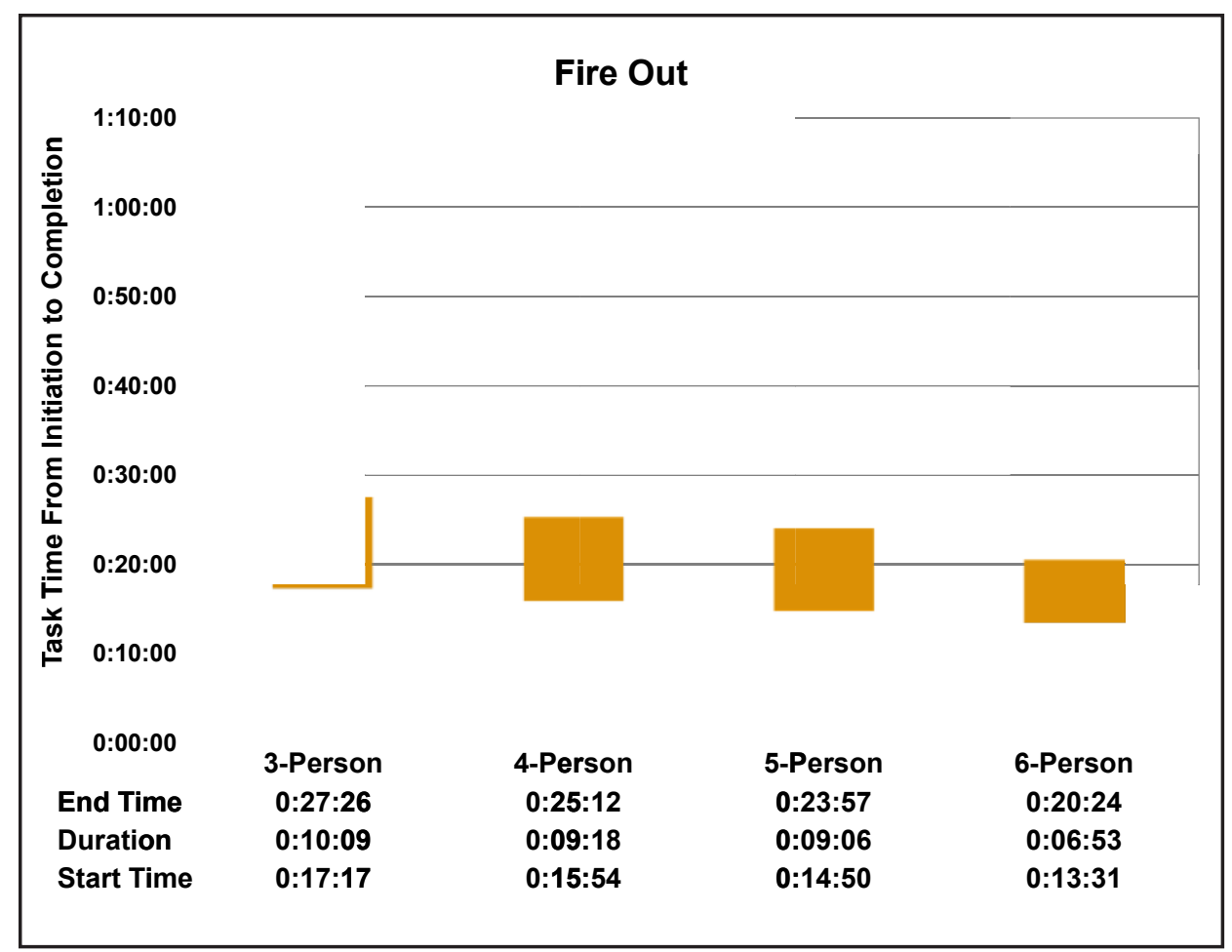

Figure 72: Fire Out

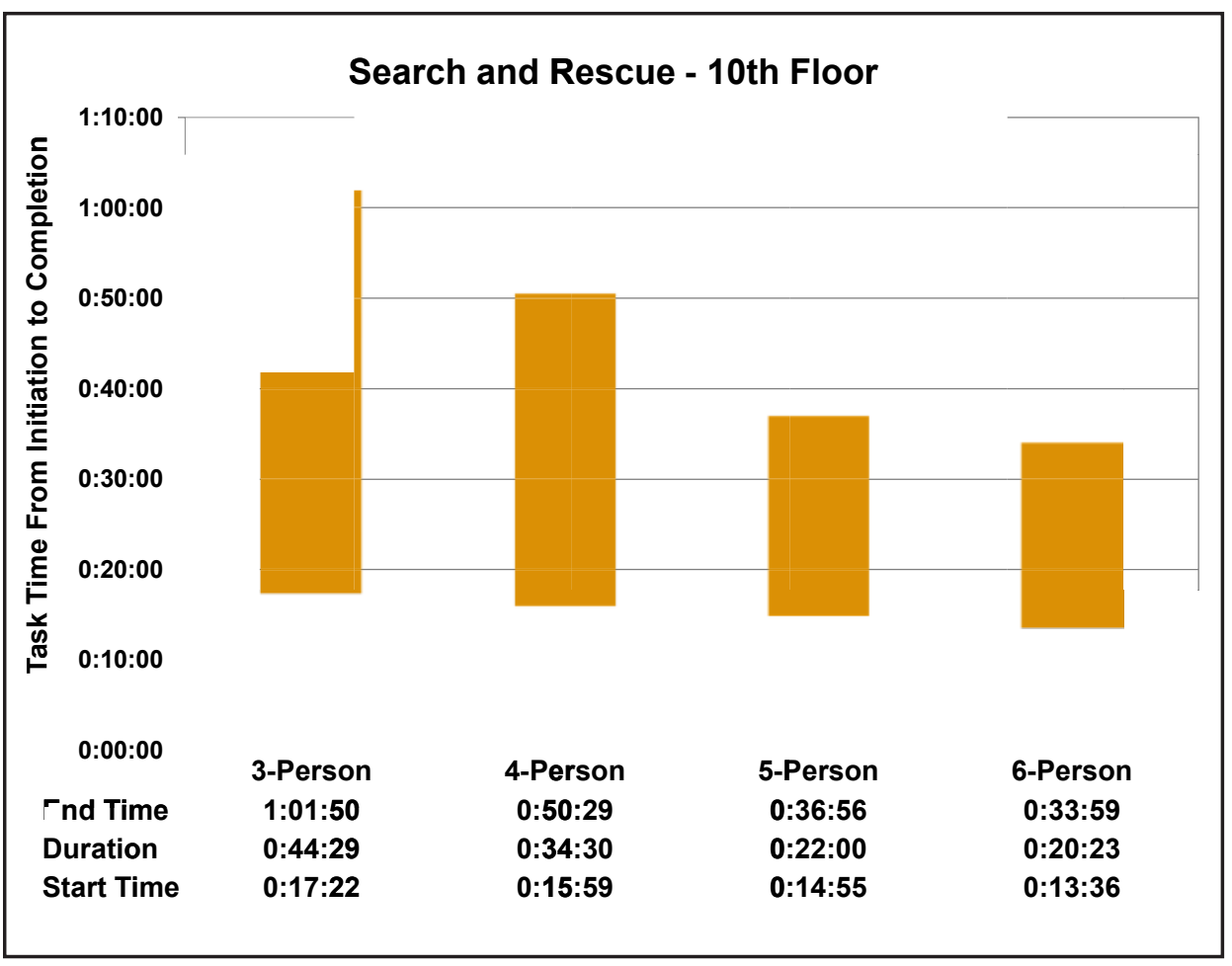

Figure 73: Search and Rescue on Fire Floor (10th)

\subsection{Fire Out}

Extinguishing the fire out is critical to reducing risk to both firefighters entering the structure and to occupants. Fire Out, in the study, was defined as having both the attack line and the second hose line in place. As shown in Figure 72, the 4-person crews were 2 min $14 \mathrm{~s}(8.1 \%)$ faster in the Fire Out time compared to 3 -person crews. The 5-person crews were $1 \mathrm{~min} 15 \mathrm{~s}$ (5.0 $\%$ ) faster than 4-person crews and $3 \mathrm{~min}$ $29 \mathrm{~s}(12.7 \%)$ faster than 3 -person crews. The 6-person crews finished 7 min $2 \mathrm{~s}$ (25.6\%) faster than the 3 -person crews.

10.6 Search and Rescue 10th Floor The fire floor in the experiments measured $30,000 \mathrm{sq} \mathrm{ft}\left(2800 \mathrm{~m}^{2}\right)$ and contained 96 cubicles. Figure 73 summarizes the amount of time that crews took to start and complete the search on the fire floor. The 4-person crew started the search 1 min $23 \mathrm{~s}(7.8 \%)$ faster than the 3-person crew and completed the search and rescue 11 min $21 \mathrm{~s}(18.4 \%)$ faster than the 3-person crews. The 5 -person crews started the search 1 min $4 \mathrm{~s}$ $(6.7 \%)$ faster than the 4-person crews and 2 min $27 \mathrm{~s}(14.1 \%)$ faster than the 3-person crew. Additionally, 5- person crews completed the search faster than the 4- and 3-person crews by 13 min $34 \mathrm{~s}$ (26.8 $\%)$ and 24 min $55 \mathrm{~s} \mathrm{(40.3 \% )} \mathrm{respectively.}$ Six-person crews had the best times, starting the search 1 min $19 \mathrm{~s}(8.8 \%)$ faster and completing the search $2 \mathrm{~min} 57$ $s$ faster than 5 -person crews $(8.0 \%)$. The greatest difference in search times was between 6- and 3-person crews. Six-person crews started the search on the fire floor 3 $\min 46 \mathrm{~s}(21.7 \%)$ faster and completed the search 27 min $51 \mathrm{~s} \mathrm{(45.0 \% )} \mathrm{faster} \mathrm{than} \mathrm{the}$ 3 -person crews. 


\subsection{Victim \#1 Found}

There was a single victim located on the fire floor that was found and rescued by all crews. Figure 74 shows the time taken by crews to locate the victim on the fire floor. A 5-person crew operating in the high-rise structure located the victim $25 \mathrm{~min} 18 \mathrm{~s}$ (50.6\%) faster than a 3-person crew and 12 min $7 \mathrm{~s}(32.9 \%)$ faster than a 4-person crew. A 6-person crew located the victim on the fire floor $28 \mathrm{~min} 33 \mathrm{~s}(57.1 \%)$ faster than the 3-person crew, 15 min $21 \mathrm{~s}$ $(41.7 \%)$ faster than the 4-person crew, and $3 \mathrm{~min} 14 \mathrm{~s}(13.2 \%)$ faster than a 5-person crew.

\subsection{Victim \#1 Rescue}

Since the experiment protocol dictated that each victim be rescued by two firefighters, one at the shoulders and one at the legs, the duration of the victim rescue varies only slightly as seen in Figure 75. However, the start and end time of victim rescue is significantly different between crews. Four-person crews removed the victim from the IDLH environment 13 min $11 \mathrm{~s} \mathrm{(25.1 \% )} \mathrm{faster}$ than a 3-person crew. Likewise, 5-person crews were able to remove the victim from the fire environment 11 min 39 s $(29.7 \%)$ faster than the 4- person crews while 6-person crews removed the victim from the environment $14 \mathrm{~min} 58 \mathrm{~s}(38.1 \%)$ faster than the 4-person crews and $3 \mathrm{~min}$ 19 s $(12.0 \%)$ faster than the 5 -person crews.

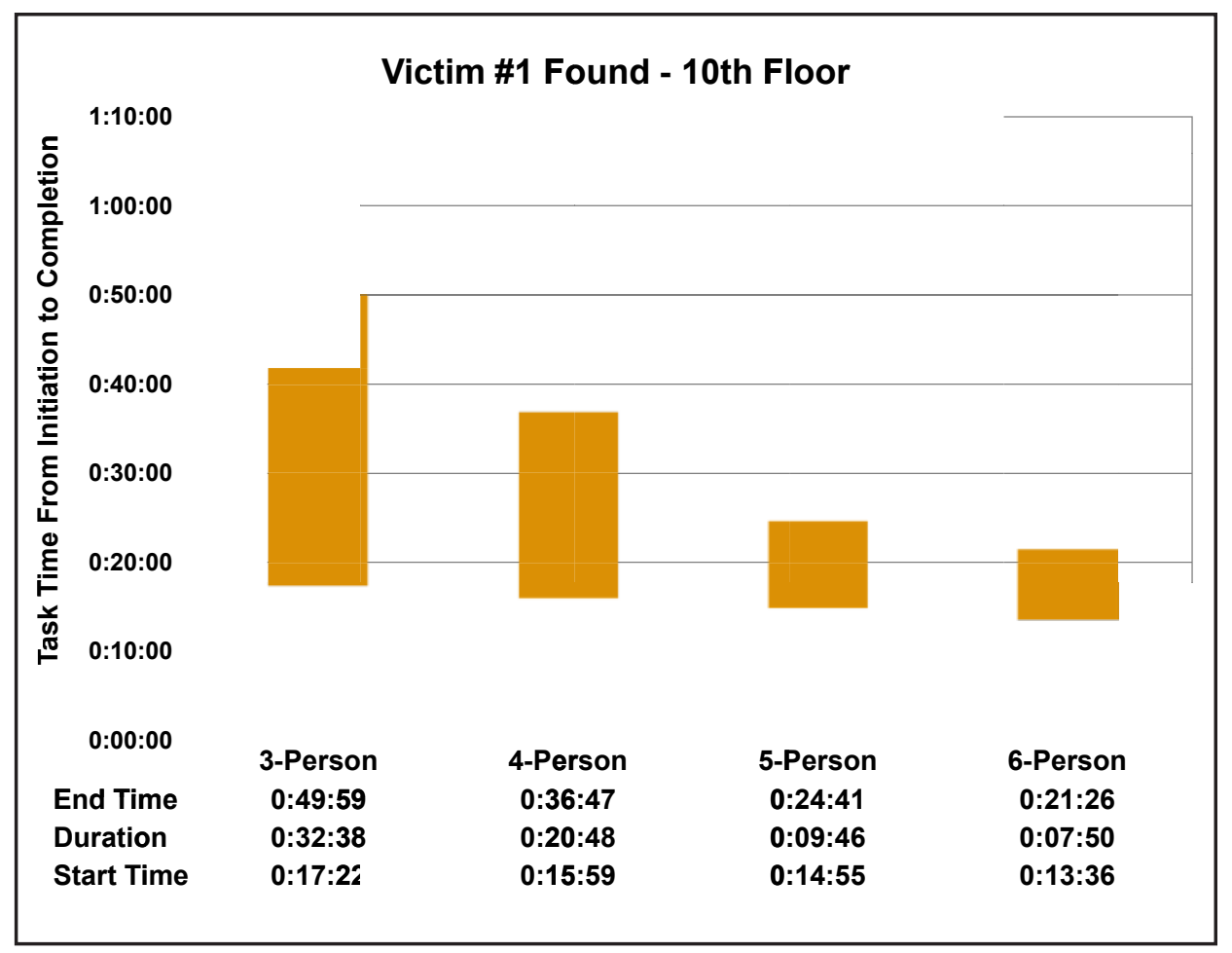

Figure 74: Victim \#1 Found on Fire Floor

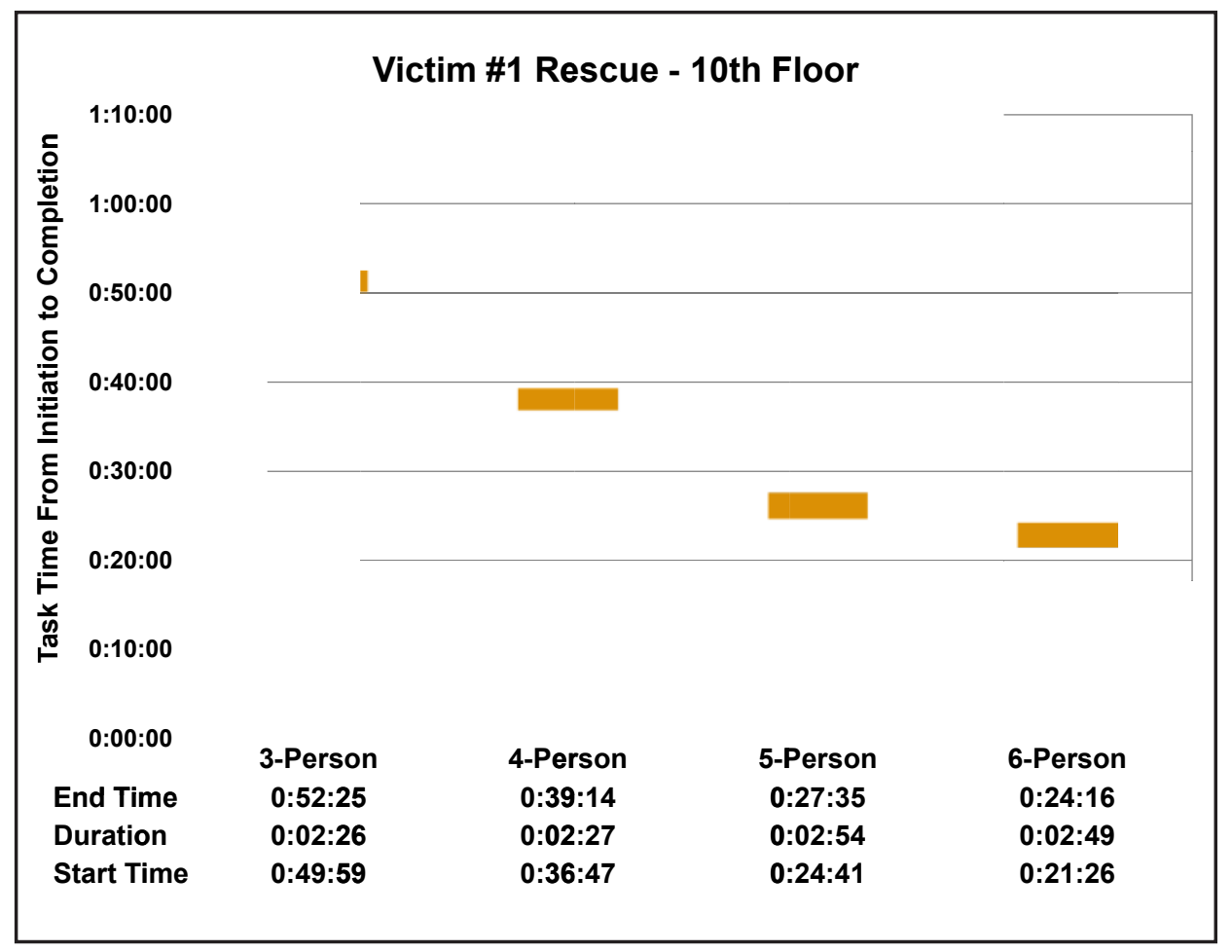

Figure 75: Victim \#1 Rescue - Removed from IDLH atmosphere 


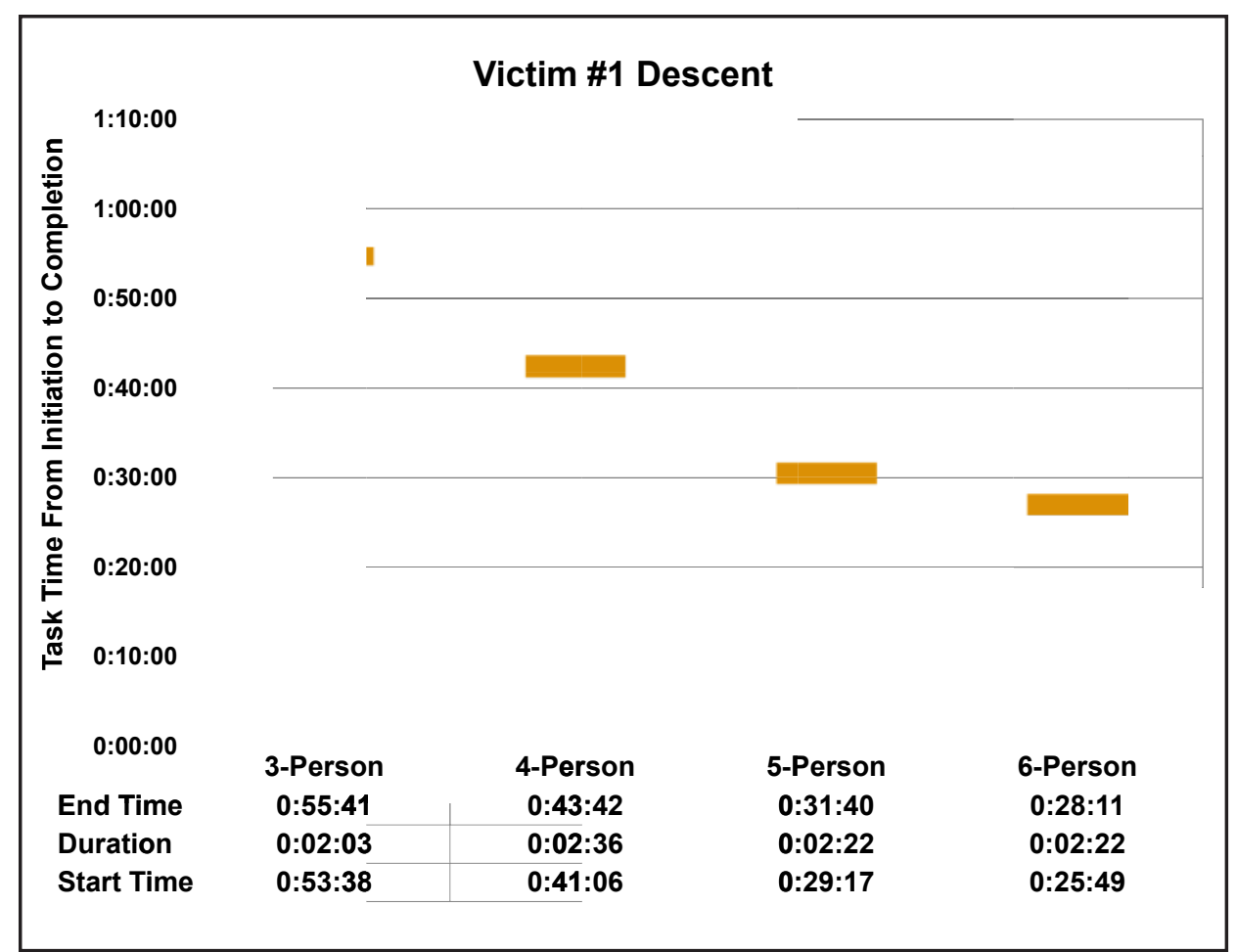

Figure 76: Victim \#1 Descent

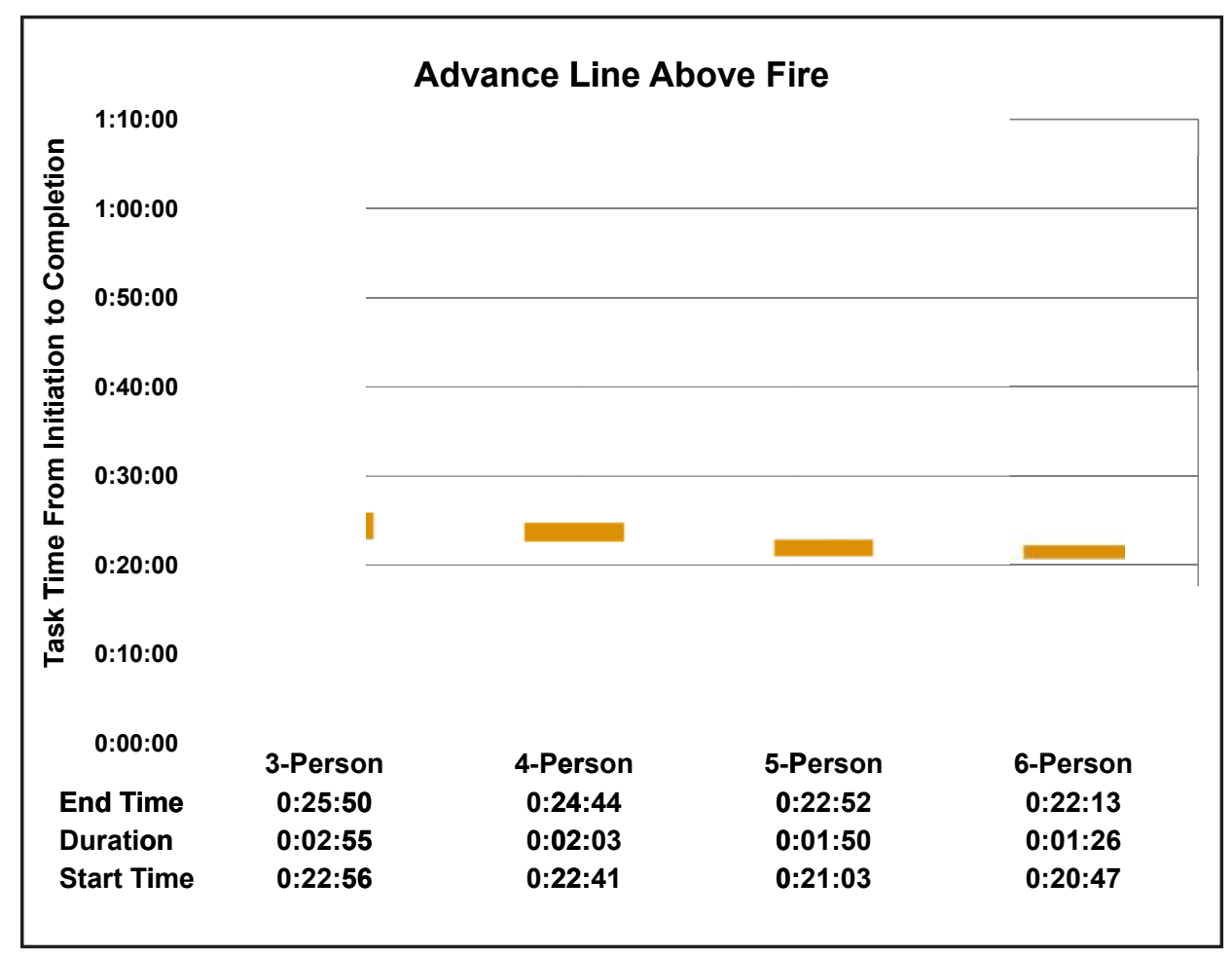

Figure 77: Advance Line on Floor Above the Fire (11th)

\subsection{Victim \#1 Descent}

Experiment protocols required that an EMS crew of two carried the victim out of the building. Each crew was to descend via stairs or elevator depending on the scenario being tested, so duration times did not vary greatly between overall crew sizes studied as shown in Figure 76. The start and end times of this task did vary. Four-person crews were able to facilitate the victim descent and exit from the building 11 min $59 \mathrm{~s}(21.5 \%)$ faster than 3 -person crews. Five-person crews were able to facilitate victim descent $11 \mathrm{~min} 39 \mathrm{~s}$ ( $27.5 \%$ ) faster than 4-person crews and 24 min $1 \mathrm{~s}(43.1 \%)$ faster than 3-person crews while 6-person crews were $3 \mathrm{~min}$ and $19 \mathrm{~s}$ faster than 5-person crews (11.0 $\%)$. Additionally, victim descent occurred $4 \mathrm{~min} 42 \mathrm{~s}$ more quickly for crews using the elevator rather than the stairs.

\subsection{Advance Line Above the Fire (11th Floor)}

In a high-rise structure, it is essential to place a hose line on the floor above the fire floor to fight potential vertical fire spread. Figure 77 measures the interval from the start of the task Advance Line Above the Fire to the end of this task. Comparing crew sizes, 5-person crews were 2 min $58 \mathrm{~s}$ $(11.5 \%)$ faster than a 3 -person crew to complete the same task. The most notable comparison was between 6-person crews and 3-person crews. The 6-persons crews were $3 \mathrm{~min} 37 \mathrm{~s}(14.0 \%)$ faster in task completion time. 
10.11 Search and Rescue 11th Floor

The floor above the fire was separated into a number of conference rooms and offices that had to be searched by each crew. The area of the floor measured $30,000 \mathrm{sq} \mathrm{ft}\left(2800 \mathrm{~m}^{2}\right)$. Figure 78 summarizes the times that crews took to start and complete the search on the floor above the fire. During the experiments, the 4-person crews completed the search 9 min $31 \mathrm{~s}(18.6 \%)$ faster than the 3-person crews. Meanwhile, the 5-person crews started a primary search/rescue $1 \mathrm{~min} 34 \mathrm{~s}$ $(6.8 \%)$ faster than the 4-person crews and completed the search 2 min $37 \mathrm{~s}(6.3 \%)$ faster than the 4-person crews. In the same structure, the 6-person crews also started the search 1 min $30 \mathrm{~s}(6.6 \%)$ faster than the 4-person crews but completed the search $5 \min 8 \mathrm{~s}(12.3 \%)$ faster than the 4-person crews.

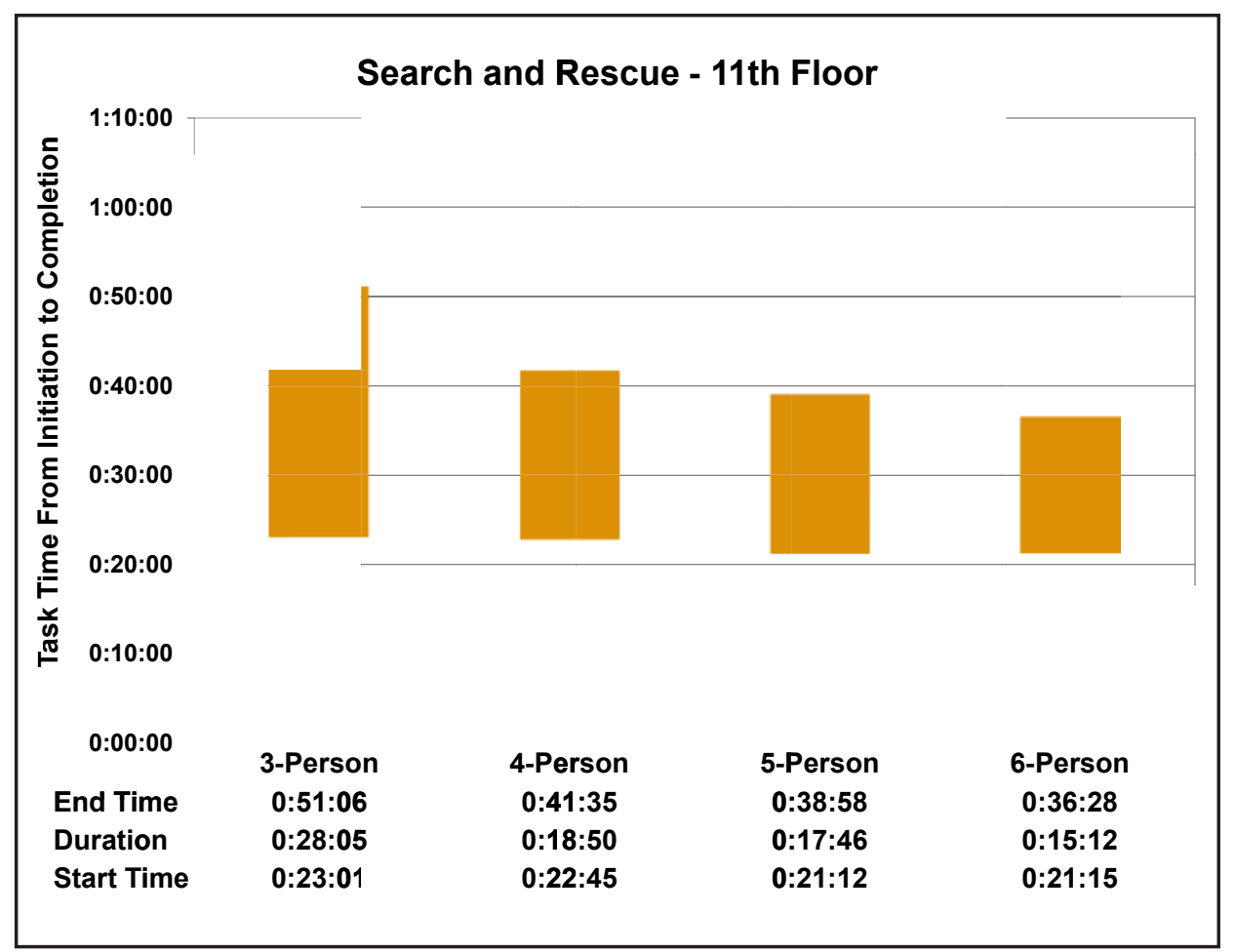

Figure 78: Search and Rescue on Floor Above the Fire (11th)

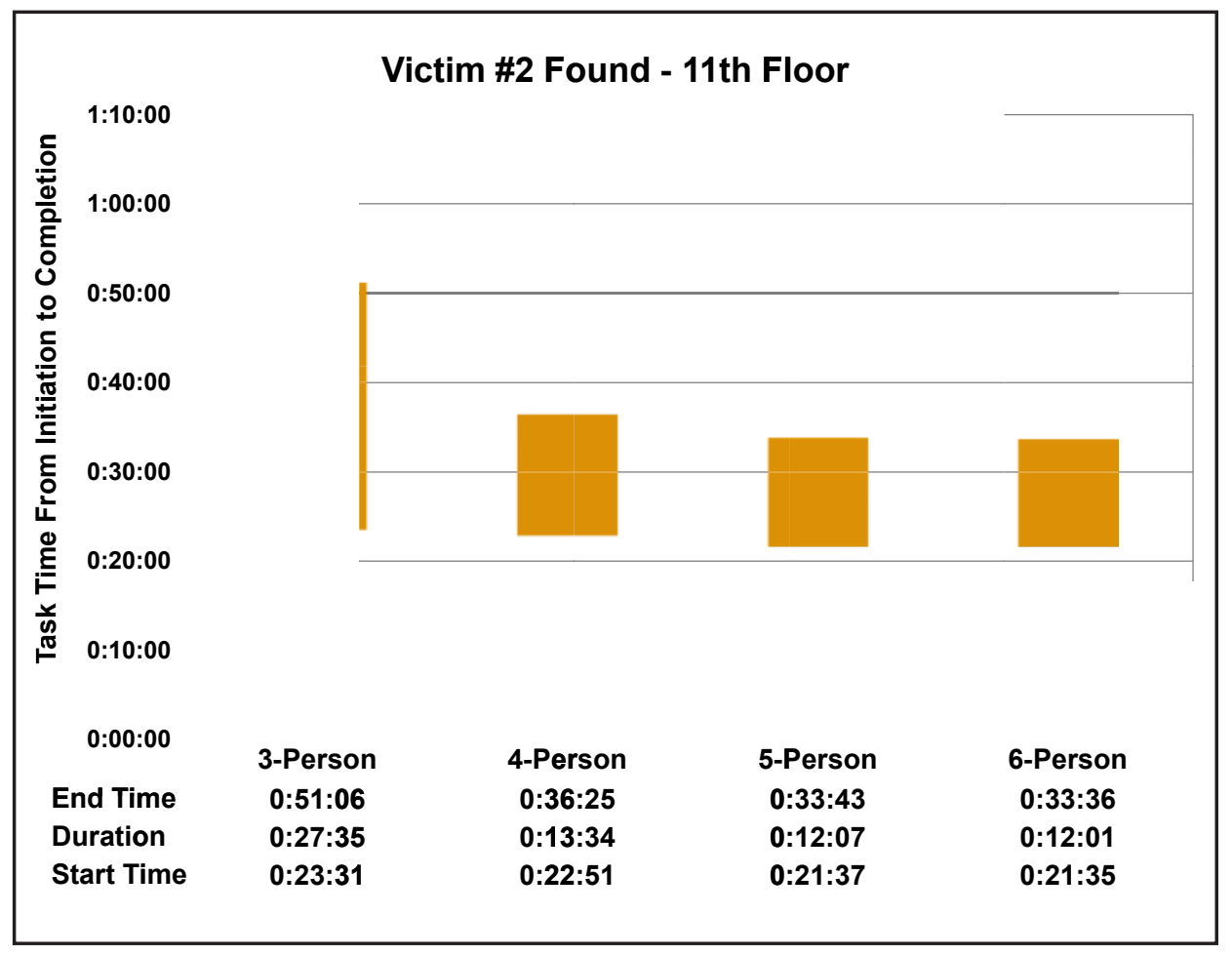

Figure 79: Victim \#2 Found on Floor Above the Fire 


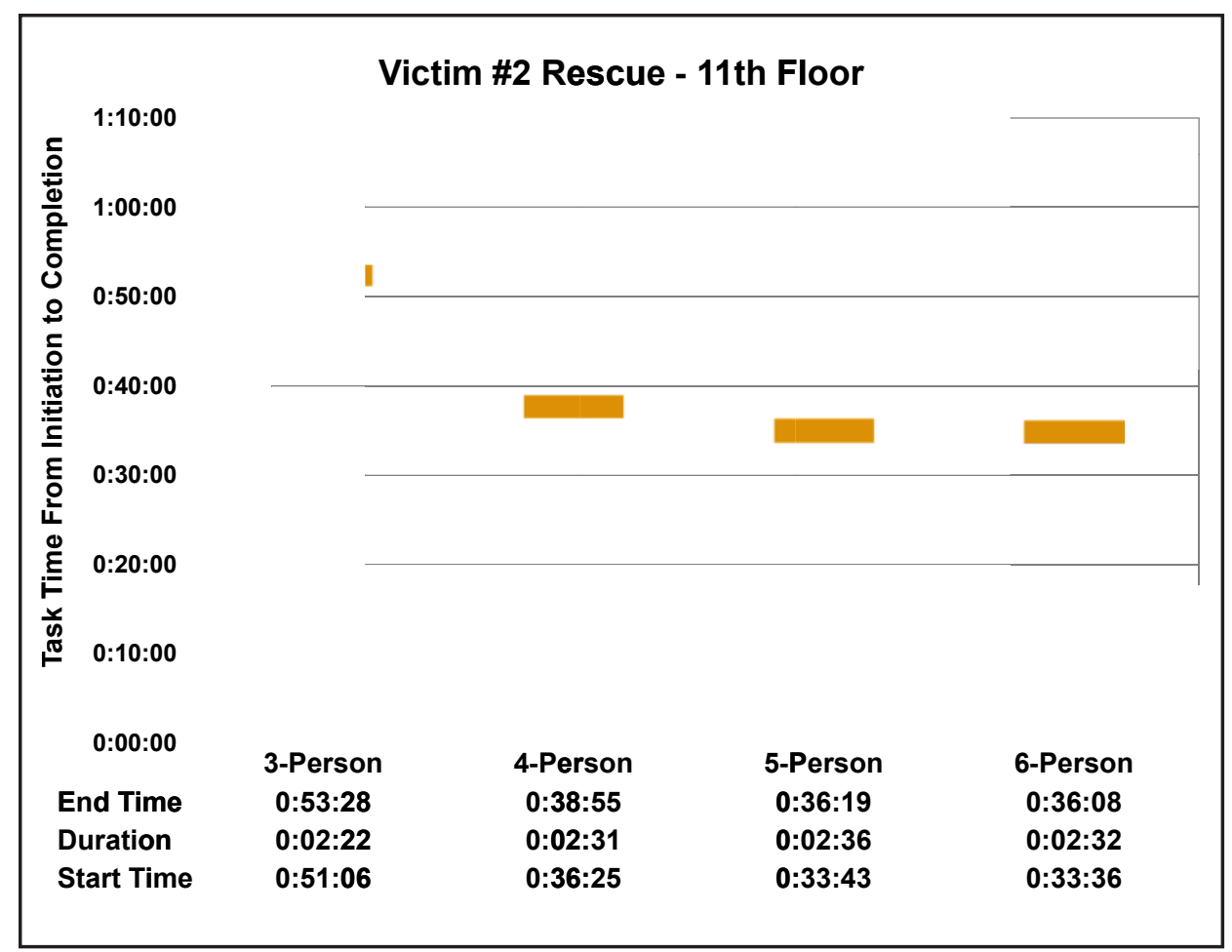

Figure 80: Victim \#2 Rescued from Floor Above the Fire

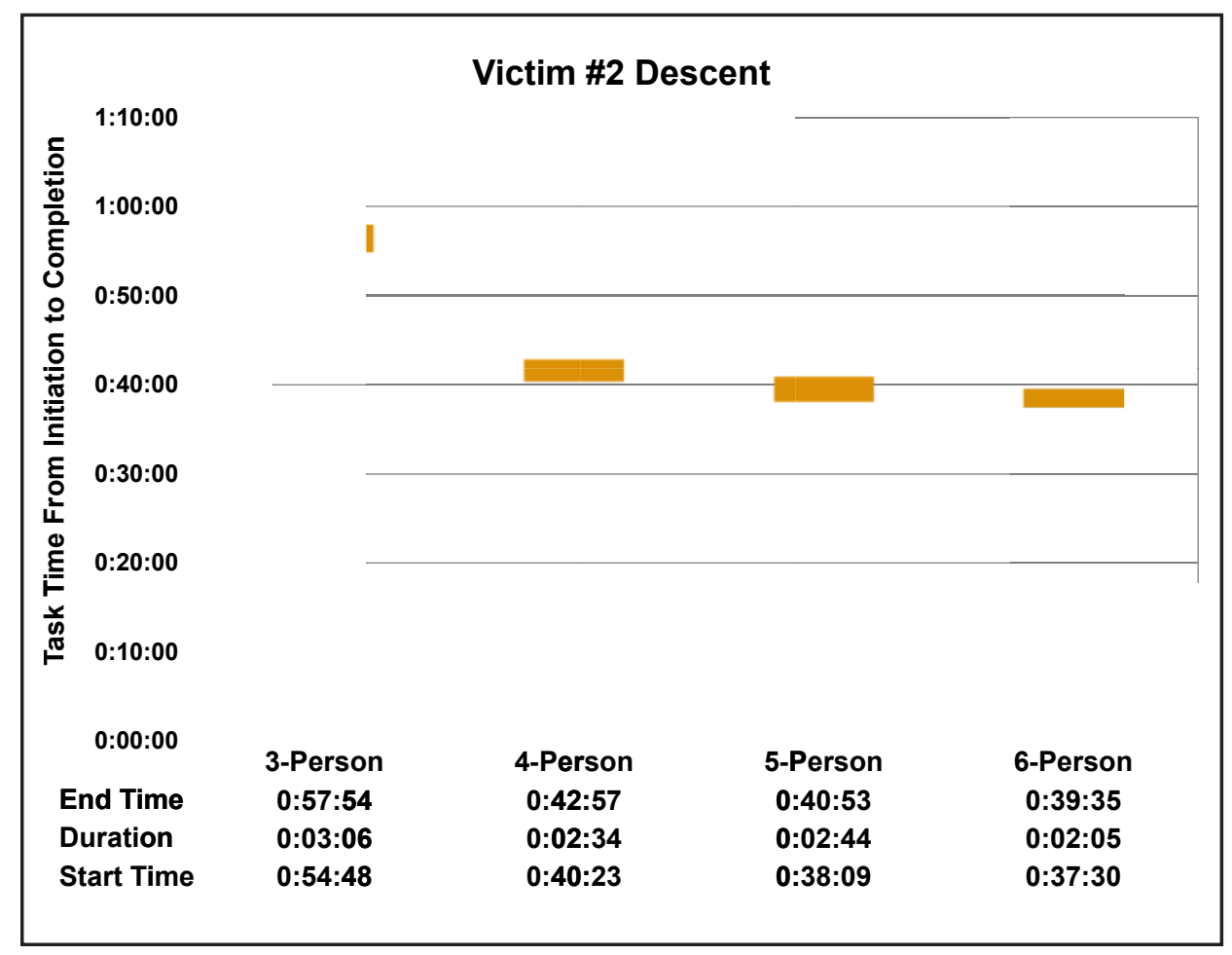

Figure 81: Victim \#2 Descent

\subsection{Victim \#2 Rescued}

Since the experiment protocol dictated that each victim be rescued by two firefighters, one at the shoulders and one at the legs, the duration of the rescue of Victim \#2 varies only slightly by crew size as seen in Figure 80. However, the start and end time of victim rescue is significantly different between crews due to performance on previous tasks. Four-person crews removed Victim \#2 from the IDLH environment 14 min $33 \mathrm{~s}$ $(27.2 \%)$ faster than a 3 -person crew. Five-person crews were able to remove Victim \#2 from the fire environment 17 min $9 \mathrm{~s}$ faster $(32.1 \%)$ than 3-person crews and 2 min $36 \mathrm{~s}$ faster than the 4-person crews $(6.7 \%)$. Similarly, the 6-person crews rescued the victim 2 min $48 \mathrm{~s}(7.1 \%)$ faster than 4-person crews.

\subsection{Victim \#2 Descent}

As with Victim \#1, experiment protocols required that an EMS crew of two carried the victim out of the building. Each crew was to descend via stairs or elevator depending on the scenario being tested, so duration times did not vary greatly between overall crew sizes studied as seen in Figure 81. The start and end times of this task did vary. Five-person crews were able to facilitate the victim descent $2 \min 4$ $\mathrm{s}(4.8 \%)$ faster than 4-person crews and 17 min $1 \mathrm{~s}(29.4 \%)$ faster than 3-person crews. Six-person crews were able to facilitate victim descent 3 min 21 s $(7.8 \%)$ faster than 4-person crews. Additionally, victim descent occurred nearly 6 min more quickly for crews using the elevator rather than stairs. 


\subsection{Simultaneous Operations by Crew Size}

The 38 fireground tasks included in the experiments were completed by all crews regardless of crew size. However, as on an actual fireground, assignments of crews and the tactics used on each task varied based on the availability of firefighters to complete the task. For example, search patterns used on the fire floor and floor above the fire were dependent on the number of firefighters available on the crew assigned to complete the task. Figure 82 through Figure 85 show how tasks were simultaneously or sequentially performed based on the number of firefighters available on scene. ${ }^{31}$

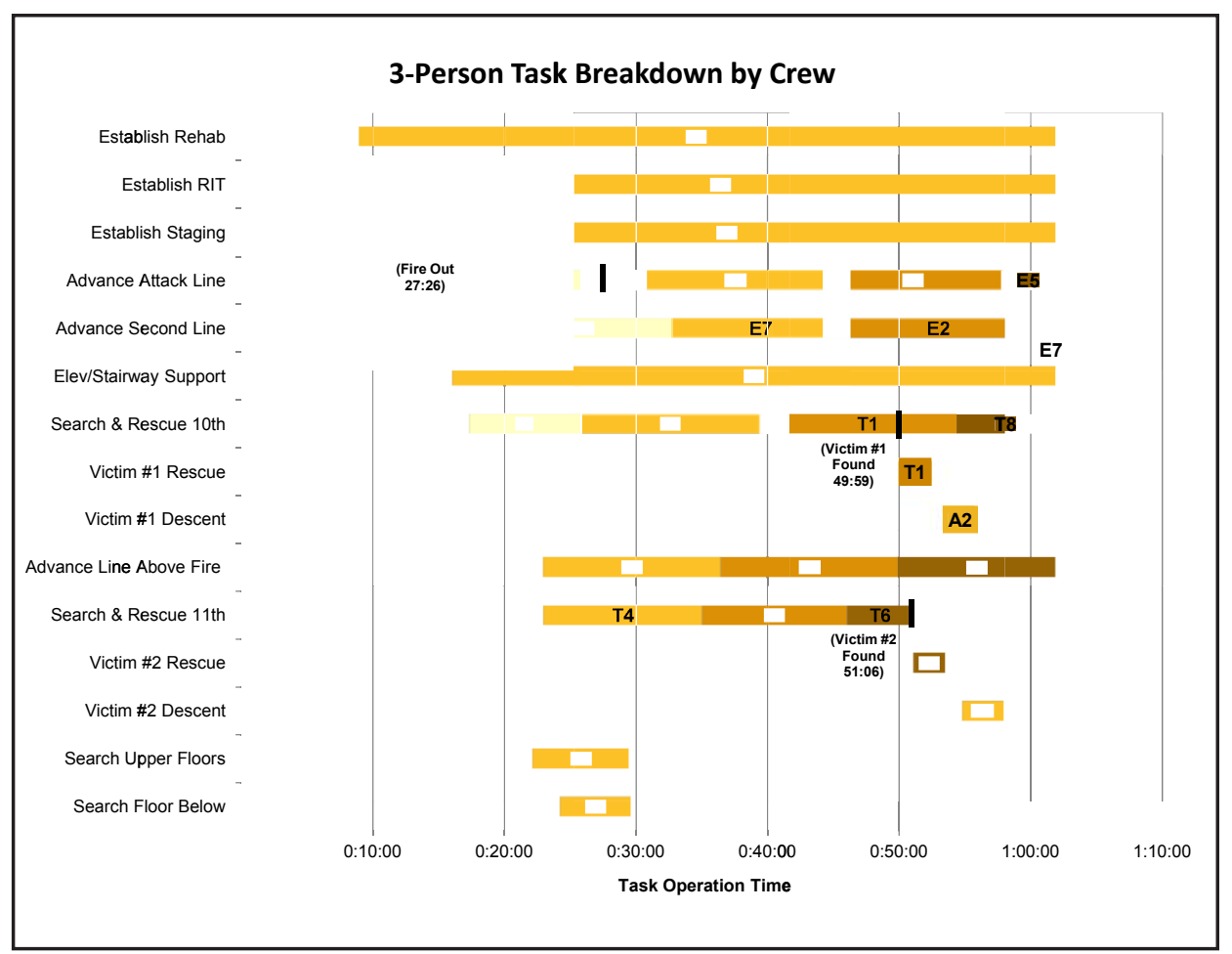

Figure 82: 3-person crew task operation

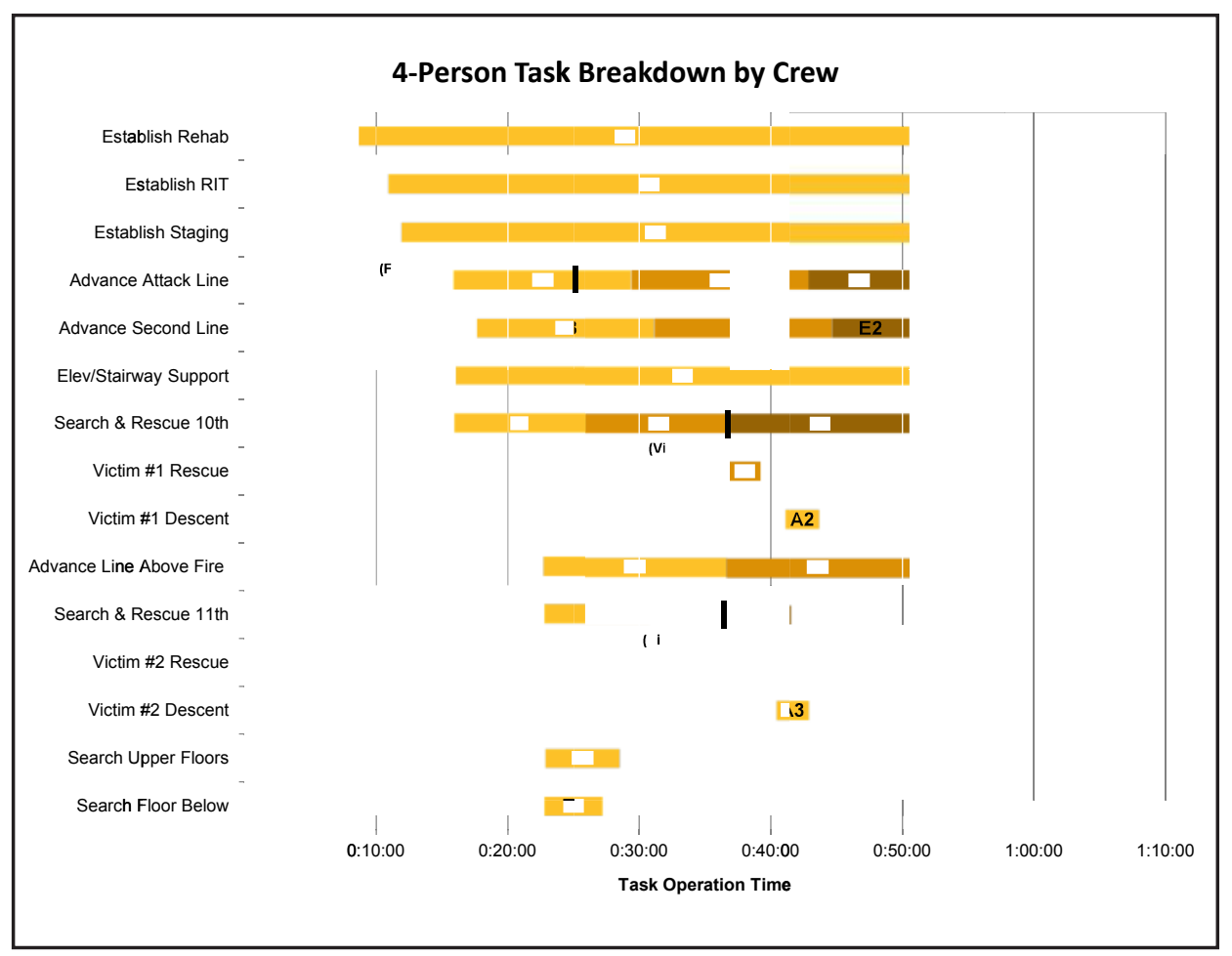

Figure 83: 4-person crew task operation 


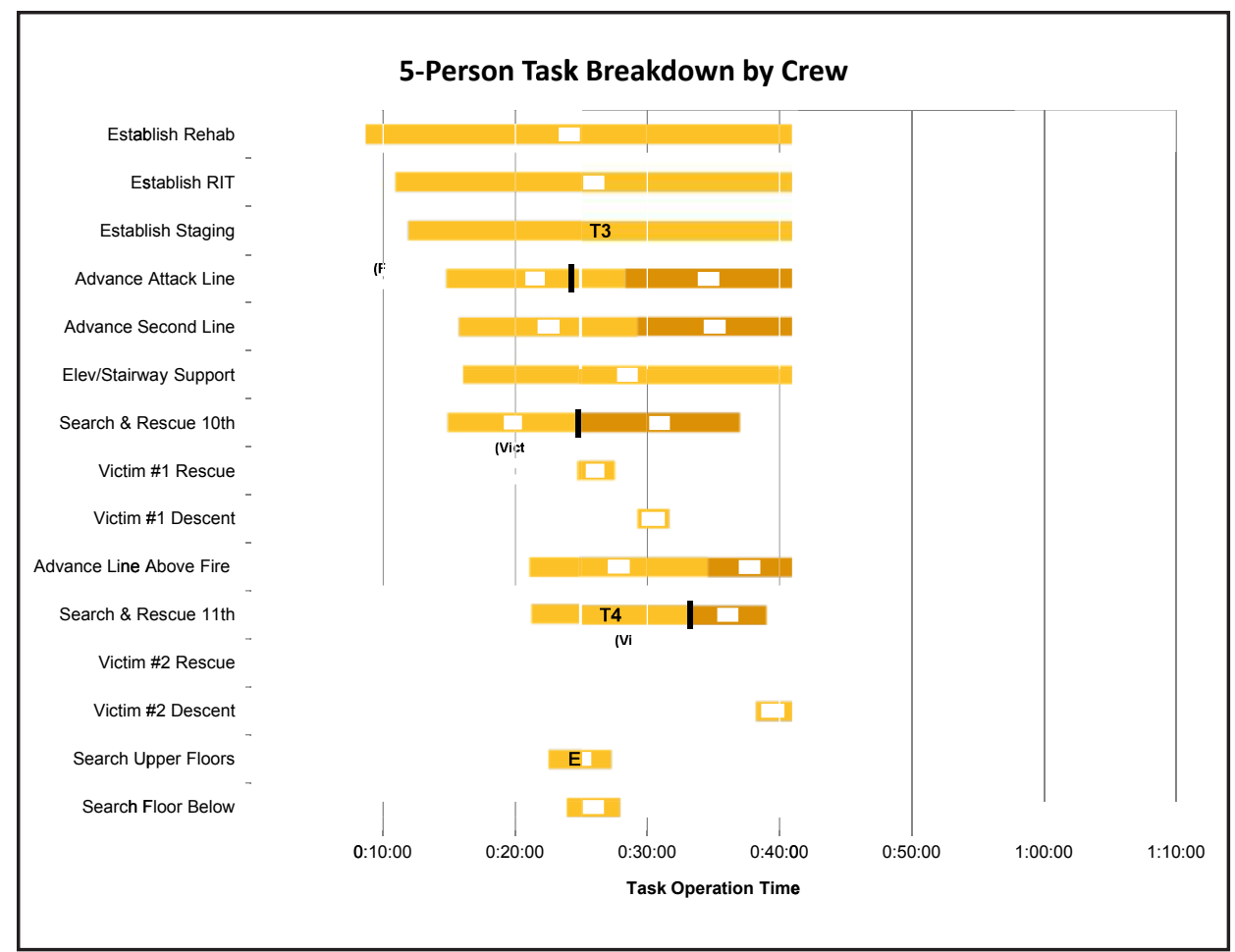

Figure 84: 5-person crew task operation

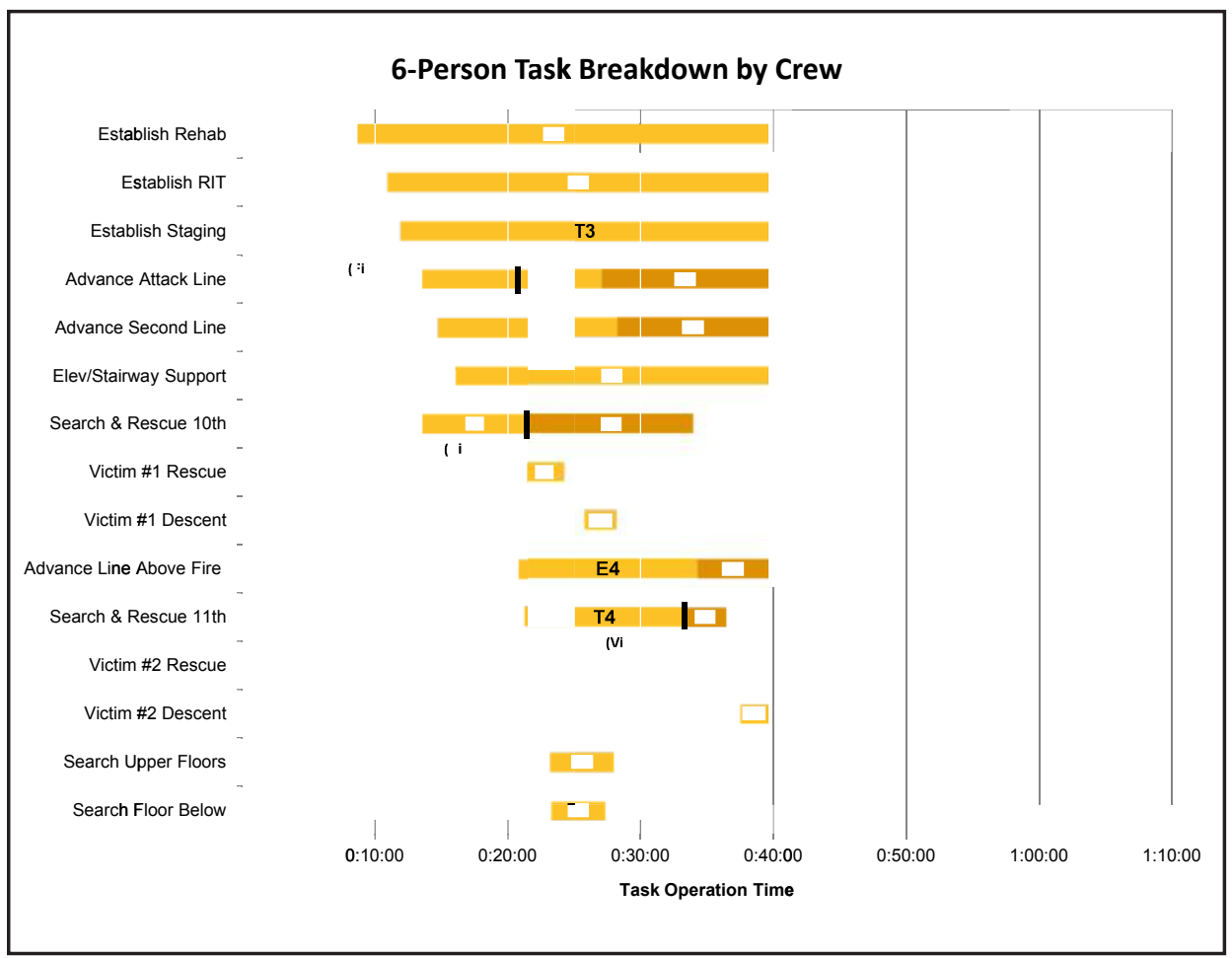

Figure 85: 6-person crew task operation 


\subsection{Summary of Regression Results}

\section{Crew Size}

Appendix $\mathrm{C} 1$ uses the results of regression analysis to show the incremental effects of crew size on critical tasks. Time increments analyzed include the begin time, duration and end time of each critical task. The results control for alarm size and ascent mode, so the results indicate the net impact of crew size change alone.

Generally, going from 3-person to 4-person crews had a large impact on begin time to advancing the attack line, advancing the second line, and starting search and rescue. Time reductions were in the range of $1 \mathrm{~min}$ to $2 \mathrm{~min}$. Going from 4-person to 5-person crews showed significant time reductions in begin times all critical tasks in the range of $1 \mathrm{~min}$ to $2 \mathrm{~min}$. Increasing crew size from 5-person to 6-person crews showed a significant reduction, just over $1 \mathrm{~min}$, in the begin time to advance the attack and second lines and for search and rescue on the fire floor (10th floor).

When assessing task end times and incrementing crew size by a single firefighter (i.e., 3 to 4,4 to 5 , and 5 to 6 ), the largest time improvements are seen when going from crew size 3 to 4 . As firefighter crews navigate the later tasks, the improvements reach the $10 \mathrm{~min}$ to $15 \mathrm{~min}$ range. Very large improvements are seen for the 10th Floor Search and Victim \#1 Rescue tasks (over $11 \mathrm{~min}$ ) when incrementing crew size from 4 to 5 . The improvements in All Tasks Complete end times are substantial ( 9 min to $12 \mathrm{~min}$ ) when incrementing crew size from 3 to 4 or from 4 to 5 .

Increasing crews by 2 firefighters resulted in an even larger reduction in task times. Increasing from 3 to 5 firefighters or from 4 to 6 firefighters per crew showed the largest improvements in begin times for critical tasks, ranging from $1 \mathrm{~min}$ to $25 \mathrm{~min}$.

\section{Fire Service Access Elevators}

All Tasks Complete occurred over 4 min more quickly when the elevators were utilized compared to stairs. Begin times for nearly every task above ground level and nearly all end times were reduced compared to stair ascent. Most of the reductions due to elevator usage were in the 2 min to 4 min range, with a few obvious exceptions in that using fire service access elevators more dramatically reduced times associated with upward and downward transport of people or equipment. Using elevators to transport air bottles and other equipment from the lobby to Staging allowed completion of Establishment of Stairwell Support over 10 min more quickly than moving the equipment manually up the stairs. Additionally, the transport of both Victim \#1 and Victim \#2 from Staging to the outside of the building was faster when using the elevators (compared to the stairs), by 2 min $41 \mathrm{~s}$ and $3 \mathrm{~min} 19 \mathrm{~s}$, respectively. As a result, except for Establishment of Stairwell Support and Victim Descent, there were no ascent mode differences for task duration.

\section{Combining Alarm Size and Ascent Mode}

The results of regression analysis to compare high and low alarm size and to compare elevator to stairs ascent for all critical tasks and associated outcomes are presented in Appendix C2. Because alarm size and ascent mode are design factors built into the field experiment in order to examine crew size effects, a summary of the findings in this section is provided rather than a detailed assessment. With regard to alarm size, five of the eight significant differences between high and low alarm sizes involved reductions of task end times ranging between $1 \mathrm{~min}$ and $3 \mathrm{~min}$ in favor of the high alarm size response. These tasks were Primary Search of

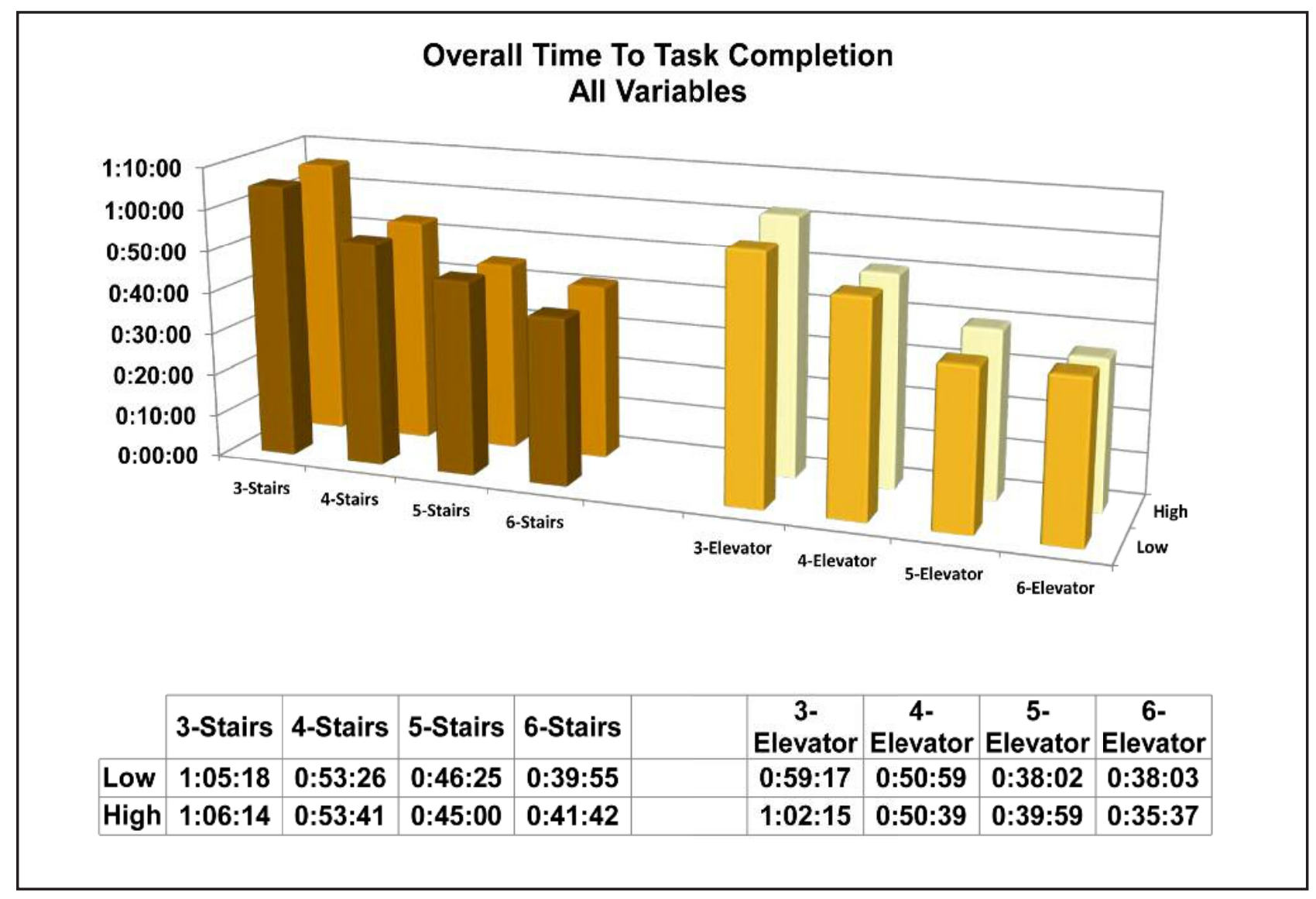

Figure 86: All Tasks Complete comparing all three study variables 
Floor 11, the three rescue tasks related to Victim \#2 (Find, Rescue, Descent), and Advancing Line Above Fire.

Comparing elevator to stair ascent, statistically significant reductions in time were seen in favor of elevator use for over two thirds of the critical task outcomes. The most sizeable time reduction for the elevator was for Establishing Stairwell Support, with reduction of nearly $10 \mathrm{~min}$ in duration and end time. Other notable reductions in favor of elevator use were for Victim Descent at $4 \mathrm{~min} 42 \mathrm{~s}$ for Victim \#1 and $5 \mathrm{~min} 30 \mathrm{~s}$ for Victim \#2. When other significant reductions occurred they were smaller, in the range of about $1 \mathrm{~min}$ to $4 \mathrm{~min}$ (see Appendix C2 for details). The assessment of alarm response and ascent mode confirmed the logical direction of differences (i.e., high alarm size faster than low, elevator faster than stairs) when they were found to exist.

A visual comparison of All Tasks Complete for all three study variables: crew size, ascent mode (stairs vs. elevators), and alarm size is presented in Figure 86.

\section{Combining Alarm Size and Crew Size}

Given the findings from the crew size analysis that adding one or two firefighters to a crew could on average achieve substantial task time decreases, a logical question is whether the meaningful benefits of a higher crew size could be realized by implementing a higher alarm response at a smaller crew size (e.g., high/4 compared to low/5). The hope might be that a high response with lower crew size would yield similar results in task timing to that of a low response with higher crew size.

Appendix C3 presents regression results that compare the effect of high response with lower crew size compared to a low response with higher crew size. These results were also compiled in separate summary tables for begin, duration, and end times. Appendix D Table 20 provides a summary of findings for begin times of critical tasks. The analysis shows that statistically significant differences in begin times occurred for a low alarm response with 4 or 5 crew size compared to high response with crew sizes of 3 and 4, respectively. As seen in Figures 87 through 89 , there are significant reductions in begin times for all critical tasks from Advance Attack Line through Victim \#1 Descent. Sizeable reductions of about $12 \mathrm{~min}$ are also seen for Victim \#2 Rescue and Descent. This suggests that a low alarm response with crews of size 4 or 5 outperforms a high response with crew sizes smaller by one firefighter. When significant decreases occur, they are in the range of $1 \mathrm{~min}$ to just under $2 \mathrm{~min}$.

It is noteworthy that this pattern does not hold for low response with a crew size of 6 compared to high response with a crew size of 5. The observed differences in begin times were significantly higher for Primary Search of Floor 11 and Advance Line Above Fire.

Figure 87 through 89 also provide a graphical summary of findings for tasks deemed critical for duration times. Although less than a third of the comparisons were statistically significant, when they occurred, they tended to be prominent. For Search of the 10th Floor and Victim \#1 Rescue, a low response with crew size 4 showed about $11 \mathrm{~min}$ to $13 \mathrm{~min}$ reductions compared to that of a high response with crew size 3 . And for low response with crew size 5 , the duration time reductions were $10 \mathrm{~min}$ to 13 min compared to a high response with crew size 4 . The low/4 combination revealed an $8 \mathrm{~min}$ reduction in duration time for Primary Search of Floor 11 and about a 12 min reduction for Victim \#2 Rescue compared to a high response with crew size 3. Similarly large reductions in duration time appear for All Tasks Complete under these two response crew size scenarios. The low/ 6 combination shows three significant duration reductions compared to high/5: a 2 min 12 s reduction for Fire Out and a 2 min 30 s reduction for Primary Search of Floor 11, and 1 min reduction for Victim \#2 Descent. (See Table 21 in Appendix D for a summary of findings.) 
Finally, Figures 87 through 89 present critical task contrasts of low alarm size to that of a high alarm size with one less crew on staff. Over half of the task end time comparisons were statistically different when comparing the low/4 and low/5 combinations against their counterparts (i.e., high/4 and high/5, respectively). When significant, low alarm size with crew sizes 4 and 5 displayed end time reductions of $2 \mathrm{~min}$ to over $14 \mathrm{~min}$. The low/4 combination showed significant end time reductions compared to high/3 for 11 of the 14 critical tasks in this analysis. For the low/5 combination, 6 of 14 tasks showed significant reductions in end time compared to high/4. Only 2 of 14 tasks exhibited significantly lower end times for the low/6 combination relative to that of high/5. Generally, for critical task end times, reductions were most pronounced when comparing low/4 to high/3, followed by low/5 compared to high/4. The low/ 6 combination featured the smallest reductions compared to the high/5. (See Appendix D Table 22 for a summary of end time analysis.)

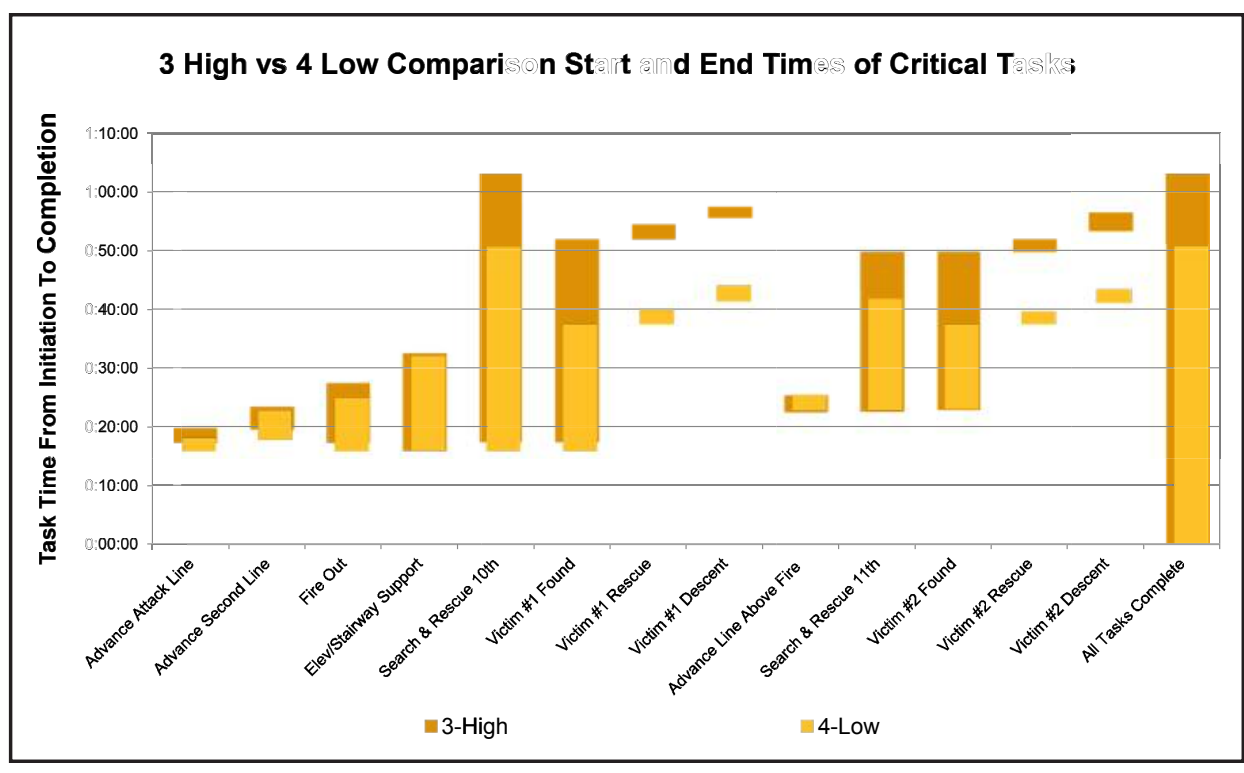

Figure 87: Comparison of 3 high/4 low start, end, and duration times

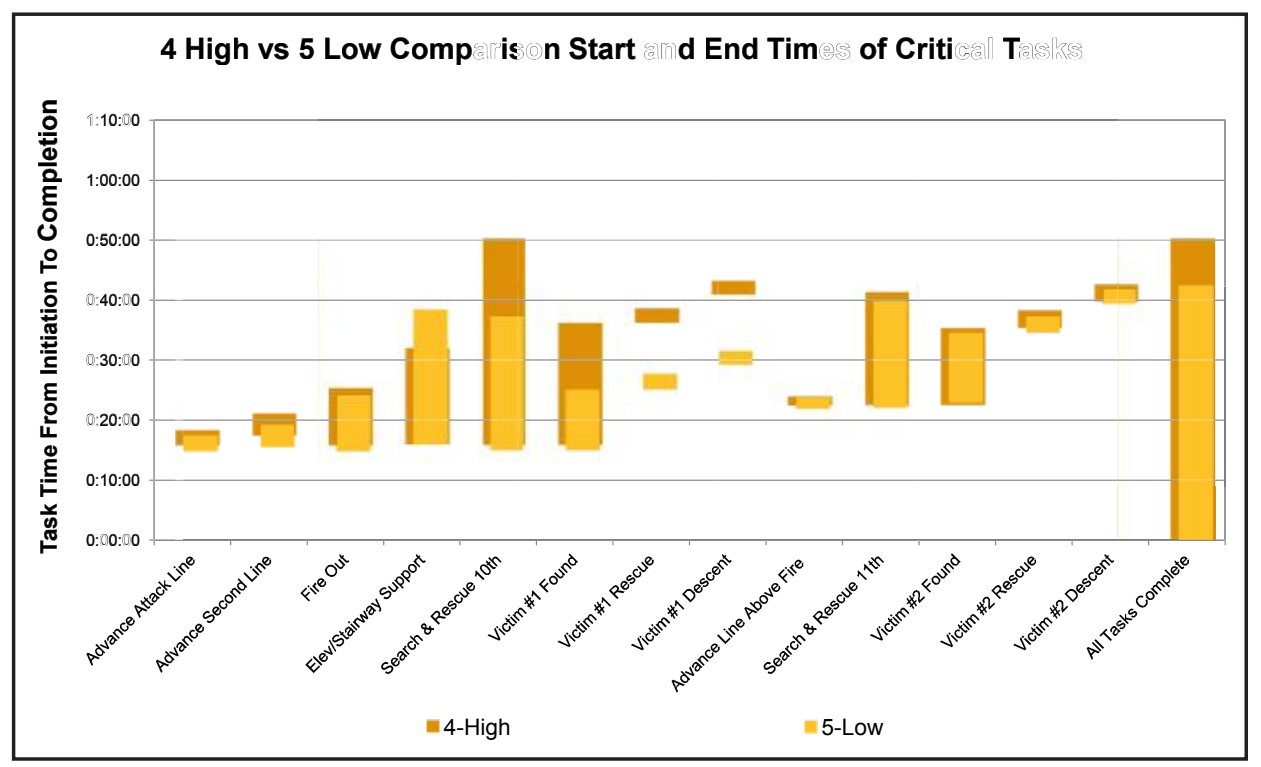

Figure 88: Comparison of 4 high/5 low start, end, and duration times

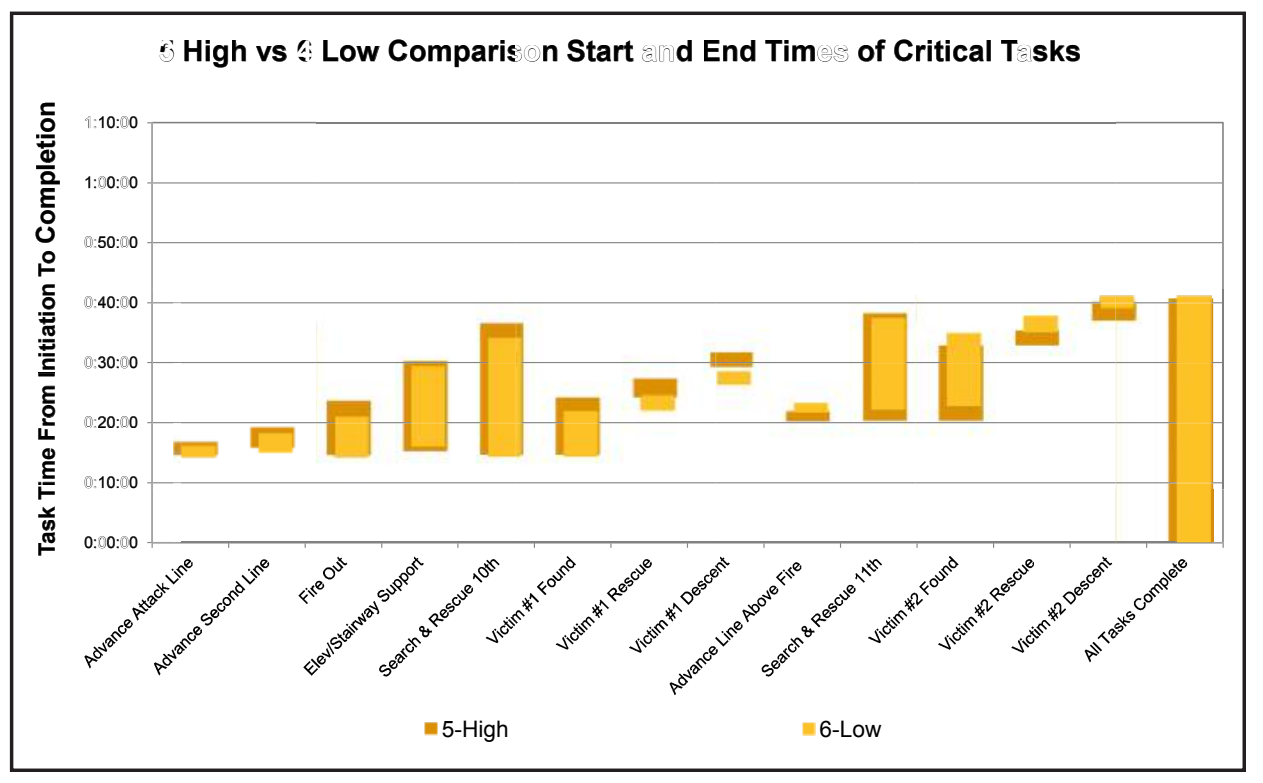

Figure 89: Comparison of 5 high/6 low start, end, and duration times 
In summary, the analysis of the alarm response and crew size combinations suggests that the benefits of crew size increases (in terms of reduced begin, duration and end times for critical tasks) are fairly robust. Low alarm response with a higher crew size tends to be more favorable in critical task timings than the corresponding timings for a high alarm response with a crew size of one less firefighter.

\section{Combining Alarm Response and Ascent Mode}

Appendix C4 presents regression results that compare several combinations of alarm size, (high or low) and ascent mode (stairs or elevator).

Stairs/High vs. Stairs/Low; this scenario focuses on stair ascent and examines the impact of high versus low alarm size;

Elevator/High vs. Elevator/Low; this scenario focuses on elevator ascent and explores the effect of high versus low alarm size.
Stairs/High vs. Stairs/Low - The alarm size had virtually no effect for critical task timings, with the exception of Primary Search of the Floor Above the Fire (Floor 11) and Victim \#2 Rescue. High alarm size realized a mean reduction in the range of $1 \mathrm{~min}$ to $4 \mathrm{~min}$ for these tasks. All Tasks Complete was also significantly shorter for high alarm size by 3 min. No other task timing comparisons were statistically different.

Elevator/High vs. Elevator/Low - In the elevator scenarios, high alarm size led to eight significantly lower timings than did a low alarm response. Results showed $45 \mathrm{~s}$ reductions in begin time for Fire Out, Primary Search of Fire Floor 10, and Victim \#1 Found. Small reductions of just over 1 min were noted in begin times for Search and Rescue 11th floor and Victim \#2 Found. Small reductions of $30 \mathrm{~s}$ to $2 \mathrm{~min}$ were also noted for times related to Advance Line Above Fire. No other task timing comparisons were statistically different. 


\subsection{Purpose of Fire Modeling}

The time to task data from the firefighter exercises provided critical data regarding the timing of key fireground tasks.

However, as the high-rise building could not accommodate live fire tests, there was no experimental data on the tenability of the fire floor for each of the different response configurations. To couple thermal and tenability issues associated with real fire behavior to the experimental time to task data, a computer fire model was used. An advantage of using a computer model is that the hazardous conditions can be simulated, thereby minimizing the risk to the safety of the firefighters, timers, and other personnel present during exercises.

\subsection{Research Question: Time to Untenable Conditions}

More specifically, computer fire modeling was conducted to assess the following research questions:

\section{Fire Modeling Research Questions}

1) How do performance times resulting from different combinations of crew si e, alarm si e vertical ascent, and fixed fire sprinkler systems affect the development of standard fire growth scenarios?

2) How do crew si e, alarm si e, vertical ascent, and fixed fire sprinklers affect the resulting interior tenability on the fire floor?

The quantitative methods used to generate the analytic data for this portion of the high-rise research study are discussed below.

\subsection{The NIST Fire Dynamics Simulator}

The computational model selected for the analysis in this project is the NIST Fire Dynamics Simulator (FDS). FDS is a

physics-based computational fluid dynamics program with an emphasis on smoke and heat transport from fire (McGrattan et al. 2012). The version of FDS used for the simulations in this report is FDS 6 RC1 (sub-version \#13844). The visualization component to FDS is Smokeview. Smokeview allows users to visualize the three-dimensional environment created by FDS as well as the data generated by the model in two and three dimensions when applicable. Smokeview Version 6.0.11 (sub-version \#14315) is used for this report.

\section{Tenability Due to Fire Gases}

FDS is used to calculate the change in interior conditions (spatially and temporally) of the high-rise due to the presence of fire. This analysis focuses on the tenability (the likelihood that persons exposed to a specific dose of toxic products will be capable of escaping) of the fire floor. To characterize the accumulated hazard associated with inhalation of gases typical of combustion products, a time-integrated value known as the fractional effective dose (FED) is used. FED is an international standard, maintained by the International Standards Organization (ISO) and documented in ISO document 13571. FED is a probabilistic quantity used to estimate the impact of toxic gases on humans (ISO 2007). For this study, FED includes the impact of excess carbon monoxide and carbon dioxide inhalation and oxygen depletion. Additional gases such as cyanide, nitric oxide and irritants were not included in the calculation of the FED value as they tend to be of secondary importance compared to carbon monoxide, carbon dioxide, and oxygen..$^{32}$ Depending on the fuels present, neglecting these species may affect the FED for occupants, potentially raising the FED value. Additionally, smoke density is a commonly used tenability criterion, since it may limit the ability of an occupant to find their way to an exit. However, it is assumed for this analysis that occupants are in a single fixed location for the duration of the fire event; therefore, smoke density is neglected.

FED values are generally divided by three thresholds as they relate to the potential for certain portions of the population to become incapacitated. There exists uncertainty in the correlation of FED values and the percent of the population affected. See Appendix F for uncertainty information. Incapacitation is defined to be the point at which a person can no longer escape the hazardous area on his/her own. The lowest FED threshold is 0.3 , which typically relates to the most sensitive populations: elderly, young, or those with compromised immune systems. The lowest threshold group encompasses approximately $11 \%$ of the population. The second threshold occurs at an FED value of 1.0, which represents the level at which the median or $50 \%$ of the population is likely to become incapacitated. An FED value of 3.0 represents the upper threshold for tolerance to combustion gas inhalation. This formulation of FED assumes that the potential victim remains stationary over the course of the simulation. Adding movement capabilities to FED calculations is still under development. Table 8 shows the four bins created by the three threshold limits and the percentage of the population likely to become incapacitated.

\begin{tabular}{|c|c|c|}
\hline $\begin{array}{c}\text { FED Value } \\
\text { Range }\end{array}$ & $\begin{array}{c}\text { Estimated } \\
\text { Population Range } \\
\text { of Incapacitation }\end{array}$ & $\begin{array}{c}\text { FDS-Smokeview } \\
\text { Coloring }\end{array}$ \\
\hline $0.0<$ FED $\leq 0.3$ & $0.0<\% \leq 11$ & \\
\hline $0.3<$ FED $\leq 1.0$ & $11<\% \leq 50$ & $\square$ \\
\hline $1.0<$ FED $\leq 3.0$ & $50<\% \leq 89$ & \\
\hline FED $>3.0$ & $\%>89$ & \\
\hline
\end{tabular}

Table 8: Relating FED values to percentage of population likely to be incapacitated and indicating coloring scheme for visualization.

32. From the ISO 13571 document: "All available evidence supports the working hypothesis that, in typical fire atmospheres, CO and HCN are the only asphyxiant combustion products that exert a significant effect on the time available for escape." However, oxygen is important if the levels fall below $13 \%$, while carbon dioxide levels above $2 \%$ have a hyperventilating effect that exacerbates the impact of $\mathrm{CO}$ uptake. $\mathrm{HCN}$ was neglected in this analysis due to the lack of reliable input data for the model. These assumptions all lead to an underestimate of the effects of the fire on occupants in this analysis. 
FDS and Smokeview can be used to calculate FED values as a singular point in space, a two-dimensional plane, or three-dimensional surfaces. In this report, FED values are calculated for two-dimensional planes covering the entire 10th floor at elevations of $3 \mathrm{ft}(0.91 \mathrm{~m})$ and $5 \mathrm{ft}(1.5 \mathrm{~m})$. To distinguish between threshold levels within a plane, each range of FED values has a unique color identifier. The third column in Table 8 shows the colors used to identify each FED range. Note that FED is a cumulative quantity. The FED value will continue to increase as long as a potential victim remains exposed to hazardous conditions. Even if the conditions return to ambient, the FED value will remain constant at its peak for a significant period of time or until the potential victim receives medical attention.

\subsection{Development of the Design Fires for FDS Simulations}

A crucial element of computer fire modeling is the selection of a design fire. A design fire curve quantifies how a fire grows and decays over some pre-defined period of time (Hadjisophocleous 2008). For these experiments, the fire floor was configured to be an open floor plan comprised of typical workspace cubicles; therefore, a cubicle fire was determined to be the most likely fire scenario.

Since fires were not an explicit part of the experiments, determining the appropriate characteristics for the design fires was paramount. The necessary design fire parameters are the fuel, peak fire size, growth rate and duration. These parameters allow for the construction of a design fire curve. One way to construct the design fire curve is to use experimental data to determine values for the critical parameters. In this report, two NIST reports that documented experiments involving cubicle fires were used (Ohlemiller et al. 2005, Madrzykowski et al. 2004).

While data from fire experiments provides validity to the modeling calculations, not all fires grow at the exact rate observed in the experiments. The growth rate of the fire is critical as it significantly affects the hazard level faced by the occupants and firefighters upon arrival to the fire floor; slower growing fires are less hazardous upon arrival than faster growing fires. Therefore, the design curve used in this high-rise study was idealized in two ways: assuming three simple phases for the fire development, and varying the growth rate to bound the problem. First, the Society of Fire Protection Engineers (SFPE) Handbook describes three basic phases to fire development: growth, steady burning, and decay (Hadjisophocleous 2008). These phases can be clearly seen in the experimental data shown in Figure 90 as the heat release rate (HRR) increases to a peak, burns steadily for a period of time, and then decays as the available fuel is consumed. Second, in order to bound the range of hazards associated with potential cubicle fires, three different $t$-squared fire growth rates were chosen. For a t-squared growth rate fire, the change in HRR is given by the square of time multiplied by a prefactor. By changing the value of the prefactor from a small number (slow growth) to a larger number (fast growth), the growth of the HRR can be bounded. Slow, medium, and fast t-squared growth rate fires were used as defined by the SFPE Handbook (Alpert 2008). The SFPE Handbook defines the rates by the time it takes for the HRR to reach $1 \mathrm{MW}$ as shown in Table 9.

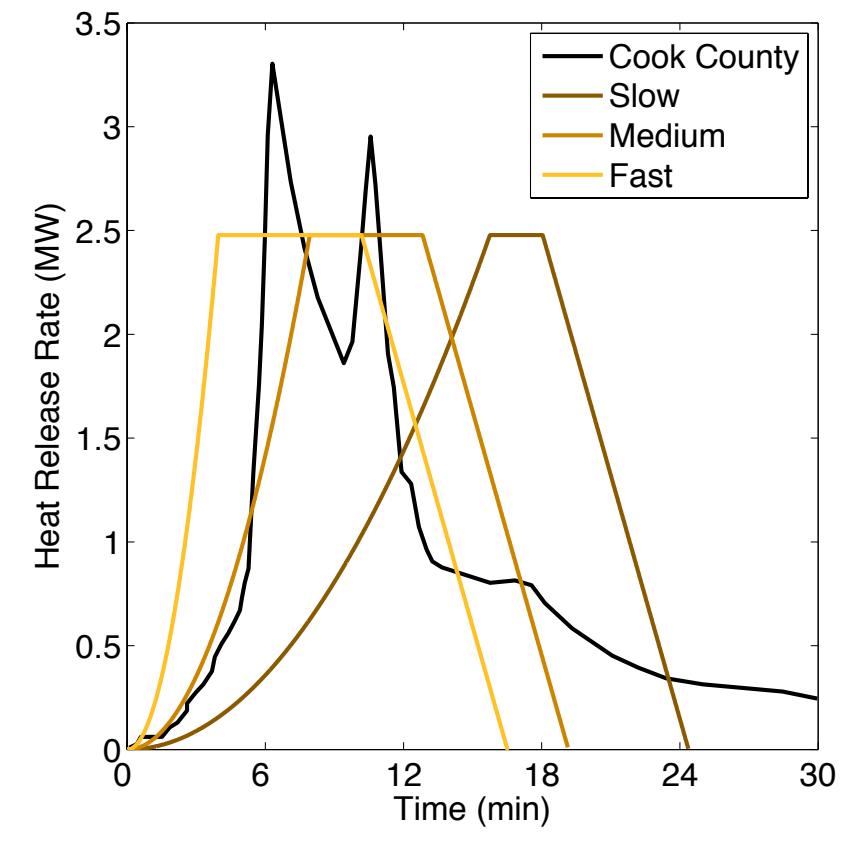

Figure 90: Heat release rate versus time curve of a typical single cubicle fire from the Cook County Administration building compared to design curves of slow, medium, and fast growth (Madrzykowski et al. 2004).

\begin{tabular}{|c|c|c|}
\hline $\begin{array}{c}\mathrm{t}^{2} \text { Fire Growth } \\
\text { Rate }\end{array}$ & $\begin{array}{c}\text { Sprinkler Activation } \\
\text { Time (MM:SS) }\end{array}$ & $\begin{array}{c}\text { Peak HRR Value } \\
\text { (MW) }\end{array}$ \\
\hline Slow & $4: 54$ & 0.41 \\
\hline Medium & $2: 45$ & 0.64 \\
\hline Fast & $2: 15$ & 1.69 \\
\hline
\end{tabular}

Table 9: Time in minutes and seconds (MM:SS) for different growth rates to reach specified HRR values.

The three growth rates were used to simulate different degrees of hazard, with HRR increased until it reached a maximum value. This value was set at $75 \%$ of the peak HRR value from the experimental data, which was approximately $2.5 \mathrm{MW}$ (Madrzykowski et al. 2004). The HRR remained fixed at this peak value until $70 \%$ of the total energy in the fuel had been released. At this point, the HRR began to decay linearly to $0 \mathrm{~kW}$. These thresholds were selected to best approximate the rate of energy release by the experimental data, while still using simplified design fire curves. Figure 90 shows the design curves for the slow, medium, and fast growth rates compared to the experimentally measured HRR data.

Note that according to Figure 90 the medium rate fire growth curve is a high quality fit to the experimental HRR curve from Madrzykowski (2004).

The design fire curve described above addressed only a single cubicle burning. Prior to fire department intervention, the fire spread to more than one cubicle in all scenarios. The experimental data were used to determine a critical ignition criterion for nearby cubicles. Once the fire spreads to nearby cubicles, the HRR is simply additive. For additional information on the design fire development, see Appendix G. 
Presently, fire suppression is not directly calculated within FDS. In order to simulate a fire in which suppression is applied by firefighters, the design fire curves were modified to represent the timing and outcome of suppression. An exponential decay was used to ramp down the design fire HRR from its value at Water on Fire time to $50 \mathrm{~kW}$ at Fire Out time and then to $0 \mathrm{~kW}$ two seconds later.

One limitation of this approach is that the suppression time is assumed to be independent of the HRR value at the time of water on fire. Whether the HRR is $1 \mathrm{MW}$ or $10 \mathrm{MW}$ at the time of water on fire, the magnitude of HRR will decay to $50 \mathrm{~kW}$ at the Fire Out time derived from the time-to-task exercises. A larger fire may take longer to extinguish; however, an experimental basis for the relationship between fire size and time to suppression does not exist. Here, engineering judgment is used.

A second assumption is that two $21 / 2$ inch hose lines will provide sufficient water flow to extinguish the fire. In practice, some of the design fires may be too large for the water supply, preventing firefighters from reducing the HRR.

The source of data for the suppression algorithm is the time-to-task data for the engine crews advancing the primary and second hose lines on the fire floor. These times can be influenced by the crew size and the method for ascending from the lobby to Staging (stairs/elevators). Table 10 shows the average times for Water on Fire and Fire Out for the replicates of each crew size and ascent method, holding deployment (high/low) constant. The rows in the table are sorted by time to water on fire, from longest time to shortest.

The first takeaway from Table 10 is that, for each crew size, firefighters would be able to get water on the fire and put the fire out faster when taking the elevator to the staging area versus taking the stairs. The second key point comes from examining the impact of stairs versus elevators across crew sizes. Table 10 shows that the average water on fire time for a 4-person crew using the stairs is longer than the average water on fire time for a 3-person crew using the elevator. The average 4-person crew taking the elevator is also faster in getting water on the fire than the average 5-person crew and 6-person crew taking the stairs. The third takeaway from the table is that the larger crews can make up the

\begin{tabular}{|c|c|c|c|}
\hline Crew Size & Ascent Method & $\begin{array}{c}\text { Average Water on } \\
\text { Fire Time (MM:SS) }\end{array}$ & $\begin{array}{c}\text { Average Fire Out } \\
\text { Time (MM:SS) }\end{array}$ \\
\hline 3 & Stairs & $18: 48$ & $28: 04$ \\
\hline 4 & Stairs & $17: 01$ & $26: 22$ \\
\hline 3 & Elevator & $15: 45$ & $26: 48$ \\
\hline 5 & Stairs & $15: 19$ & $24: 33$ \\
\hline 6 & Stairs & $14: 52$ & $21: 17$ \\
\hline 4 & Elevator & $14: 47$ & $24: 02$ \\
\hline 5 & Elevator & $14: 21$ & $23: 20$ \\
\hline 6 & Elevator & $12: 10$ & $19: 32$ \\
\hline
\end{tabular}

Table 10: Comparison of the impact of crew size and ascent method on average firefighter suppression time gains associated with using the elevators instead of the stairs. The 6-person crew using the stairs has a faster average Fire Out time compared to the 4-person and 5-person crews that had faster water on fire times.

\subsection{Ventilation}

Ventilating through exterior windows is not commonly a tactic in high-rise firefighting operations. This is due to the fact that broken glass on an upper floor can injure people and damage equipment (such as hose lines) on the ground below the windows, as well as creating the potential for a wind-driven fire scenario. Therefore, the only window breakage captured in the simulations was due to thermal effects from the fire. The thermal breakage model uses a critical temperature difference across the windowpane as the basis for failure (Pagni and Joshi 1991). Exact thermal properties of the glass installed in the high-rise were not known, so assumptions were made based on typical pane glass. Based on the thermal breakage model from Pagni using assumed properties of the glass, the breakage criteria was set at a temperature gradient of $100{ }^{\circ} \mathrm{C}$. The temperature gradient was measured by taking the difference between two point sources located on the exposed and shielded areas of the windows, respectively. If the breakage criterion was met, the entire window was removed.

In an effort to keep the variations between simulations limited to the three study variables, ventilation was held constant. The order and timing of windows breaking was based on the simulation of a 6-person crew that used the elevator to reach a fast growth rate fire. This scenario represents both the largest fire size and the crew size that gets water on the fire fastest. In total, ten windows on the East side of the structure broke.

\subsection{Fixed Fire Sprinkler Systems}

A properly engineered and maintained fire sprinkler system can be highly effective in limiting the size of an unwanted building fire. Sprinkler systems are effective at protecting building occupants, firefighters, and property. According to the NFPA, a working sprinkler system is $96 \%$ effective at controlling the growth and spread of fires in structures (NFPA 2006). Due to a number of high-profile fires in high-rise buildings and considering their demonstrated effectiveness, sprinkler systems are often required in new high-rise buildings and many jurisdictions have required existing high-rise buildings to be retrofit with sprinkler systems.

However, sprinkler systems are not found in all high-rise buildings. According to the NFPA (NFPA 2011), $41 \%$ of high-rise office buildings are not protected by sprinkler systems (compared to $25 \%$ of high-rise "care of sick" facilities, $45 \%$ of high-rise hotels and $54 \%$ of high-rise apartment buildings). Therefore, much of this report is focused on analysis of fire department deployment configurations responding to fires in an unsprinklered high-rise building. 
Note, however, that sprinkler systems are designed to control fires rather than to suppress them. Fire department response is still required even in fully-sprinklered high-rises in order to extinguish the fire, to search for and rescue occupants requiring assistance, and to control the sprinklers (limiting water damage). NFPA estimates that sprinkler systems fail to operate in $7 \%$ of structure fires (one of every fourteen fires) primarily due to human error. A full two-thirds ( $65 \%$ ) of the sprinkler failures were because the system had been shut off before the fire. Another one-sixth (16\%) occurred because manual intervention defeated the system, for example, by shutting off the sprinklers prematurely. Lack of maintenance accounted for $11 \%$ of the sprinkler failures and $5 \%$ occurred because the wrong type of system was present. Nearly all failures were therefore entirely or primarily problems of human action. Only $3 \%$ involved damage to system components." (NFPA 2006) Therefore, even when a large proportion of high-rise buildings within a jurisdiction are protected by sprinkler systems, the fire department should be prepared to deploy resources to hazards consistent with unsprinklered fires.

The computer fire modeling simulations assuming a sprinkler system were conducted with a specified typical sprinkler array located above the cubicle in which the fire starts. The sprinkler locations and activation properties used in the simulations were based on conventional response sprinklers and typical sprinkler locations. The sprinklers were set to activate when the surrounding gas temperature reached $74^{\circ} \mathrm{C}$.

For these simulations, sprinklers were assumed to be able to contain but not extinguish the fire. Containment was achieved in the model by fixing the HRR value from the time of sprinkler activation until the time when firefighter suppressive actions caused the HRR to decay. The slow, medium, and fast fires have unique sprinkler activation times and therefore have unique plateau/peak values of HRR.

The impact of the sprinklers is seen in Table 11, where the peak HRR value for the fast fire of approximately $1.7 \mathrm{MW}$ is less than the peak of a single cubicle fire of approximately 2.5 MW (as shown in Figure 90).

Note that since the HRR in simulations with sprinklers is less than in the non-sprinklered cases, the ventilation must be

\begin{tabular}{|c|c|c|}
\hline $\begin{array}{c}\mathrm{t}^{2} \text { Fire Growth } \\
\text { Rate }\end{array}$ & $\begin{array}{c}\text { Sprinkler Activation } \\
\text { Time (MM:SS) }\end{array}$ & $\begin{array}{c}\text { Peak HRR } \\
\text { Value (MW) }\end{array}$ \\
\hline Slow & $4: 54$ & 0.41 \\
\hline Medium & $2: 45$ & 0.64 \\
\hline Fast & $2: 15$ & 1.69 \\
\hline
\end{tabular}

Table 11: Sprinkler activation time and peak HRR value for slow, medium, and fast growth rate fires

recalculated. Ventilation in the sprinkler simulations followed the same procedure and criteria as in the non-sprinklered cases. By the time sprinklers activate, a total of two windows have broken.

Given a properly engineered and functioning sprinkler system, computer fire modeling results in this report reveal significantly lower temperatures, toxic species concentrations, and smoke obscuration when the sprinkler system is able to control fire growth and spread. As a result, when the sprinkler system operates, these results confirm that the total risk to occupants and firefighters is greatly lessened across all deployment configurations.

\subsection{Fire Modeling Results}

\section{Effects on Fire Development}

The time advantages gained by larger engine crew sizes and/or by using elevators impacted the interior conditions on the fire floor (i.e., temperature, visibility, toxicity, etc.). For medium growth rate fires, entering firefighters encountered fires between 5 MW to $11 \mathrm{MW}$ in size, depending on crew configuration and ascent method. This range in fire size can be visualized as the equivalent of two cubicles on fire for a 6-person crew versus five cubicles on fire for a 3-person crew, as demonstrated in Figure 91. For a fast fire, the fire size in terms of cubicles on fire increased from two for 6-person crews to eight for 3-person crews (13 MW to $20 \mathrm{MW}$ ). For a slow growth fire, all crew sizes got water on the fire before the fire grew past two cubicles (2.5 MW to $5 \mathrm{MW}$ ).

Due to the length of time for which these fires burn (between 12 min and $28 \mathrm{~min}$ on average), the number of cubicles burning at

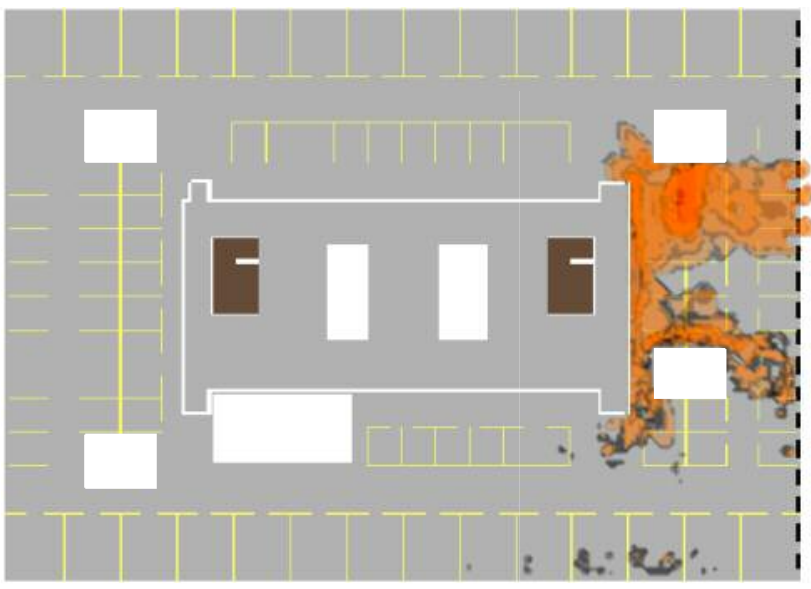

3-Person Stairs

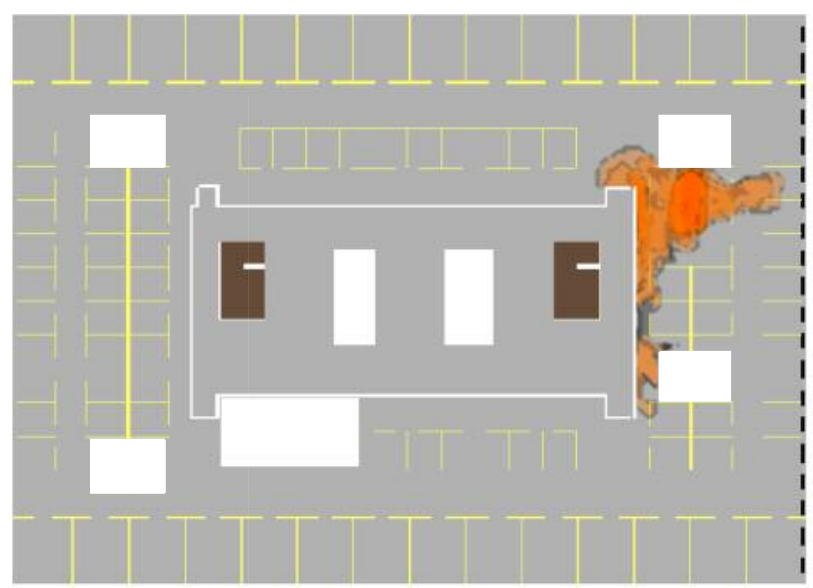

6-Person Elevator

Figure 91: Visualization of HRR for a medium growth fire for a 3-person crew using the stairs (left) and a 6-person crew using the elevators (right) at the time firefighters make entry to the floor 
any given time can vary. Depending on the growth rate, some cubicles may be in the growth phase, some in the steady burning phase, and some in the decay phase. As a result, the instantaneous HRR value may be higher at an earlier time, even though the conditions on the fire floor may be worse as more total heat has been released. Consider the HRR curves for a slow, medium, and fast growth fire for a 6-person crew using the elevators in Figure 92.

Focusing on the fast growth rate fire (top curve), the HRR reaches a steady rate of approximately $5 \mathrm{MW}$ (two cubicles) at

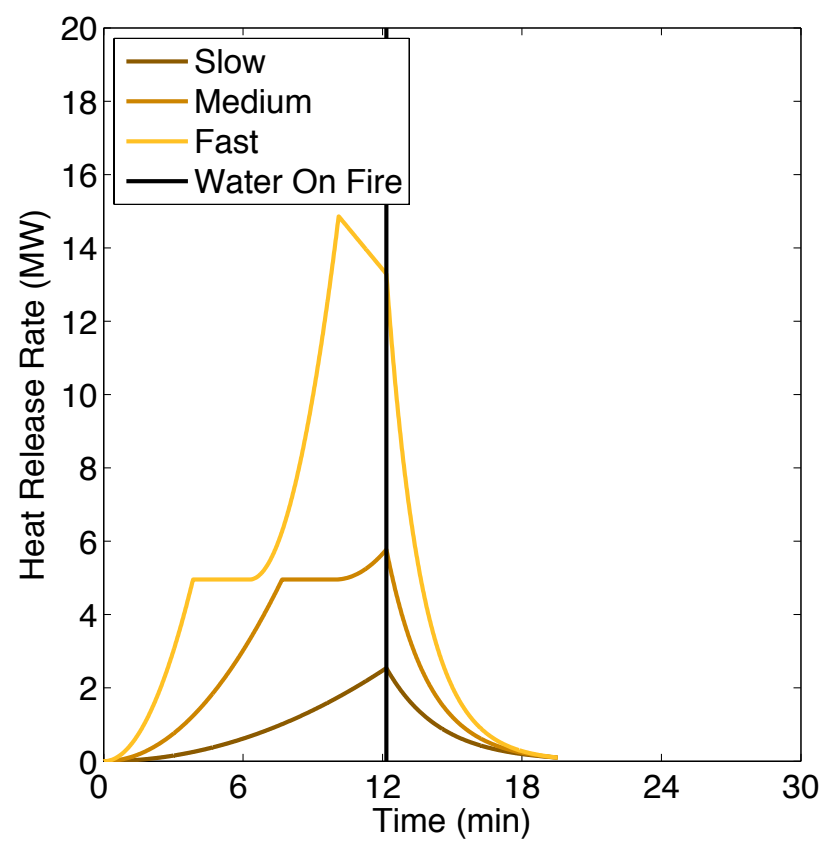

Figure 92: HRR curves for the three growth fires for a 6-person crew taking the elevator. The vertical line represents the Water on Fire time and the start of firefighter suppression

\begin{tabular}{|c|c|c|c|c|}
\hline \multirow{2}{*}{ Crew Size } & \multirow{2}{*}{ Ascent Method } & \multicolumn{3}{|c|}{ Total Heat Release by Fire Growth Rate (GJ) } \\
\cline { 3 - 5 } & & Slow & Medium & Fast \\
\hline 3 & Stairs & 3.0 & 7.1 & 13.7 \\
\hline 4 & Stairs & 2.4 & 5.9 & 11.7 \\
\hline 5 & Stairs & 1.9 & 4.7 & 9.9 \\
\hline 6 & Stairs & 1.8 & 4.4 & 9.4 \\
\hline \hline 3 & Elevator & 2.1 & 5.2 & 10.8 \\
\hline 4 & Elevator & 1.7 & 4.2 & 9.0 \\
\hline 5 & Elevator & 1.6 & 4.0 & 8.8 \\
\hline 6 & Elevator & 1.0 & 3.0 & 6.6 \\
\hline
\end{tabular}

Table 12: Comparison of the impact of crew size and ascent method on total heat release prior to suppression actions about $3 \mathrm{~min}$. There is steady burning at $5 \mathrm{MW}$ until the fire ramps up to $15 \mathrm{MW}$ shortly after $6 \mathrm{~min}$. The steep ramp indicates that the fire has spread from the two original cubicles to four additional cubicles. From the peak value of $15 \mathrm{MW}$, the HRR decays to approximately $13 \mathrm{MW}$ at $12 \mathrm{~min}$. This decay occurs because the initial two cubicles are in the decay portion of the HRR curve, as shown by the experimental cubicle fire plotted in Figure 90.

The area under the curve in Figure 92 represents the total amount of heat released. Moving left to right on the plot represents an increase in total heat release even if the instantaneous value of HRR might be decreasing. Additionally, the fuel load in the high-rise is sufficient such that burning will continue without intervention. Therefore, prior to suppression, it is more informative to consider the total heat release at the time of water on fire than the instantaneous values.

Table 12 shows the total heat released by the fire from the time of ignition until suppression actions begin, as a function of crew size, ascent method, and fire growth rate. The units of the values are GJ, where a joule is a standard measure of energy.

The values shown in Table 12 are sorted by the amount of heat released at the time of entry to the fire floor, which illustrates the impact of getting to the fire faster. Moving up and down a column of this table shows the impact of crew configuration and ascent method on total heat release. Moving left to right shows the impact of varying the fire growth rate holding configuration and ascent method constant. Referring back to Table 10, a 6-person crew taking the elevator gets water on the fire $6 \mathrm{~min} 38 \mathrm{~s}$ faster than a 3-person crew taking the stairs. This time difference results in the 3-person crew facing interior conditions with total heat release from 2 to 3 times greater than that faced by the 6-person crew, depending on the fire growth rate. 
Tenability Results for $\mathrm{CO}, \mathrm{O} 2, \mathrm{CO} 2$

On the fire floor, the victim was located in the cubicle containing button 18. The local fractional effective dose (FED) of toxic gases experienced by the victim during these hypothetical fires can be calculated based on the average time at which button 18 was pressed during experiments. In order to study the effects of crew size and ascent method, data from high and low

deployments were combined. FED values at the time and location of victim rescue for the three fire growth rates are shown in Table 13 as a function of crew size and ascent method.

In a slow growth rate fire, all crew configurations and ascents could get to the victim before the lowest tenability threshold was reached. As the fire growth rate increased to medium or fast, the tenability was worse. In a medium growth rate fire, FED values for the victim were above 1.0 (50\% threshold for incapacitation) in the case of 3-person crews using the stairs only. In a fast-growing fire, however, FED was above 1.0 for all firefighter configurations except 6-person crews using the elevator. In this case, 3-person crews using either ascent method failed to reach the victim until the FED was greater than 3.0, for which the likelihood for incapacitation is greater than $89 \%$.

The time-to-task experiments used a pre-determined, fixed victim location in order to ensure repeatability and to evaluate the impact of the three main study variables. In reality, a victim can be located in any of the cubicles. It is therefore important to know how FED evolves throughout the entire fire floor as a function of time.

\begin{tabular}{|c|c|c|c|c|}
\hline \multirow{2}{*}{$\begin{array}{c}\text { Ascent and } \\
\text { Crew Size }\end{array}$} & \multirow{2}{*}{$\begin{array}{c}\text { Victim Rescue } \\
\text { Time (MM:SS) }\end{array}$} & Slow & Medium & Fast \\
\cline { 3 - 5 } & $51: 34$ & 0.26 & 1.22 & 4.29 \\
\hline $3 S$ & $37: 44$ & 0.15 & 0.68 & 2.92 \\
\hline $4 S$ & $25: 09$ & 0.06 & 0.29 & 1.62 \\
\hline $5 S$ & $22: 23$ & 0.05 & 0.21 & 1.25 \\
\hline $6 S$ & $48: 55$ & 0.20 & 0.78 & 3.36 \\
\hline $3 E$ & $35: 50$ & 0.15 & 0.48 & 2.36 \\
\hline $4 \mathrm{E}$ & $24: 12$ & 0.06 & 0.27 & 1.54 \\
\hline $5 \mathrm{E}$ & $20: 30$ & 0.03 & 0.14 & 0.68 \\
\hline $6 \mathrm{E}$ & & & & \\
\hline
\end{tabular}

Table 13: FED at the time of victim rescue on the fire floor as a function of crew size, ascent method, and fire growth rate. See Table 8 for discussion of colors. 


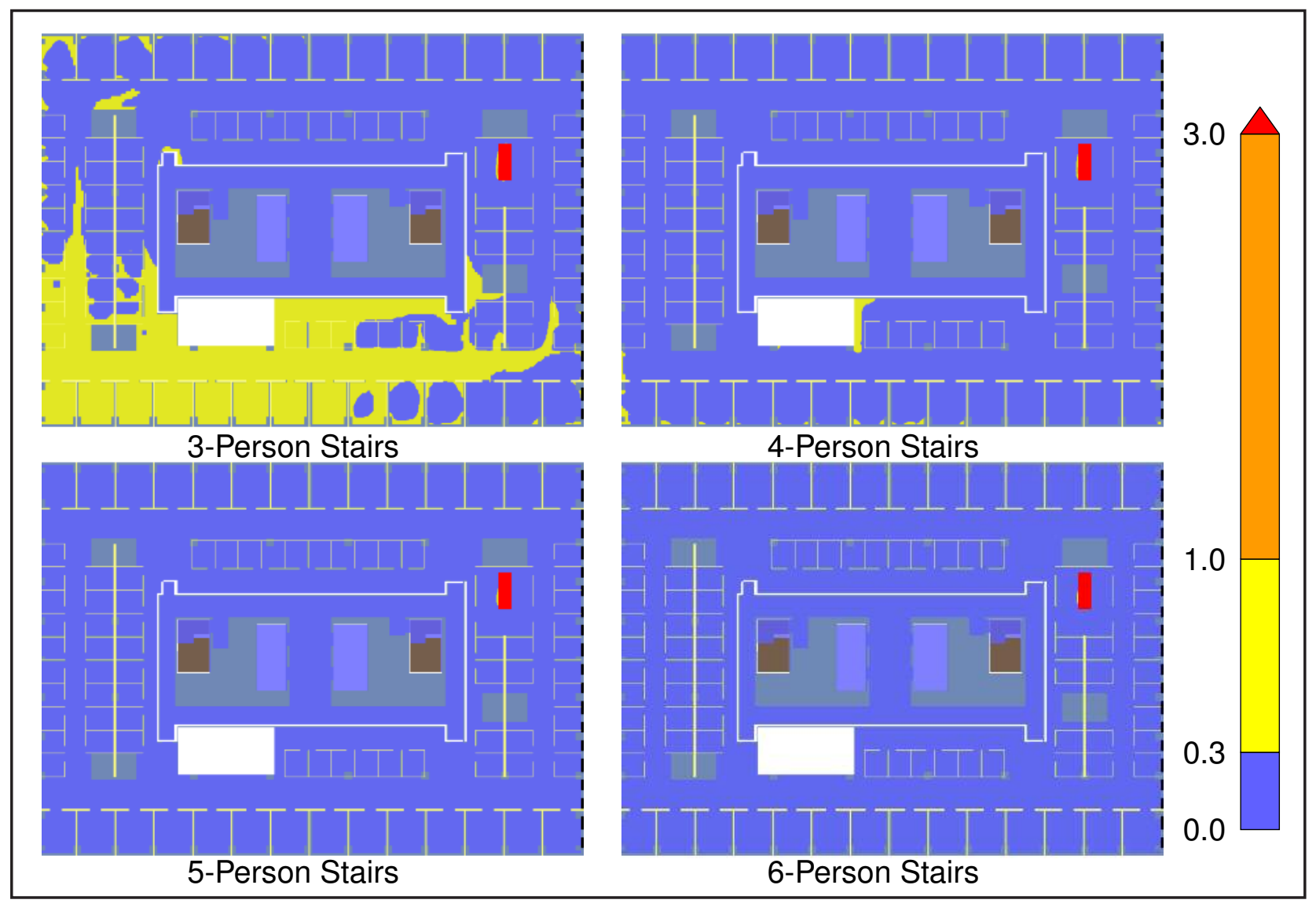

Figure 93: FED contours at an elevation of $3 \mathrm{ft}(0.9 \mathrm{~m})$ on the fire floor for a medium growth non-sprinklered fire at the time of firefighter entry

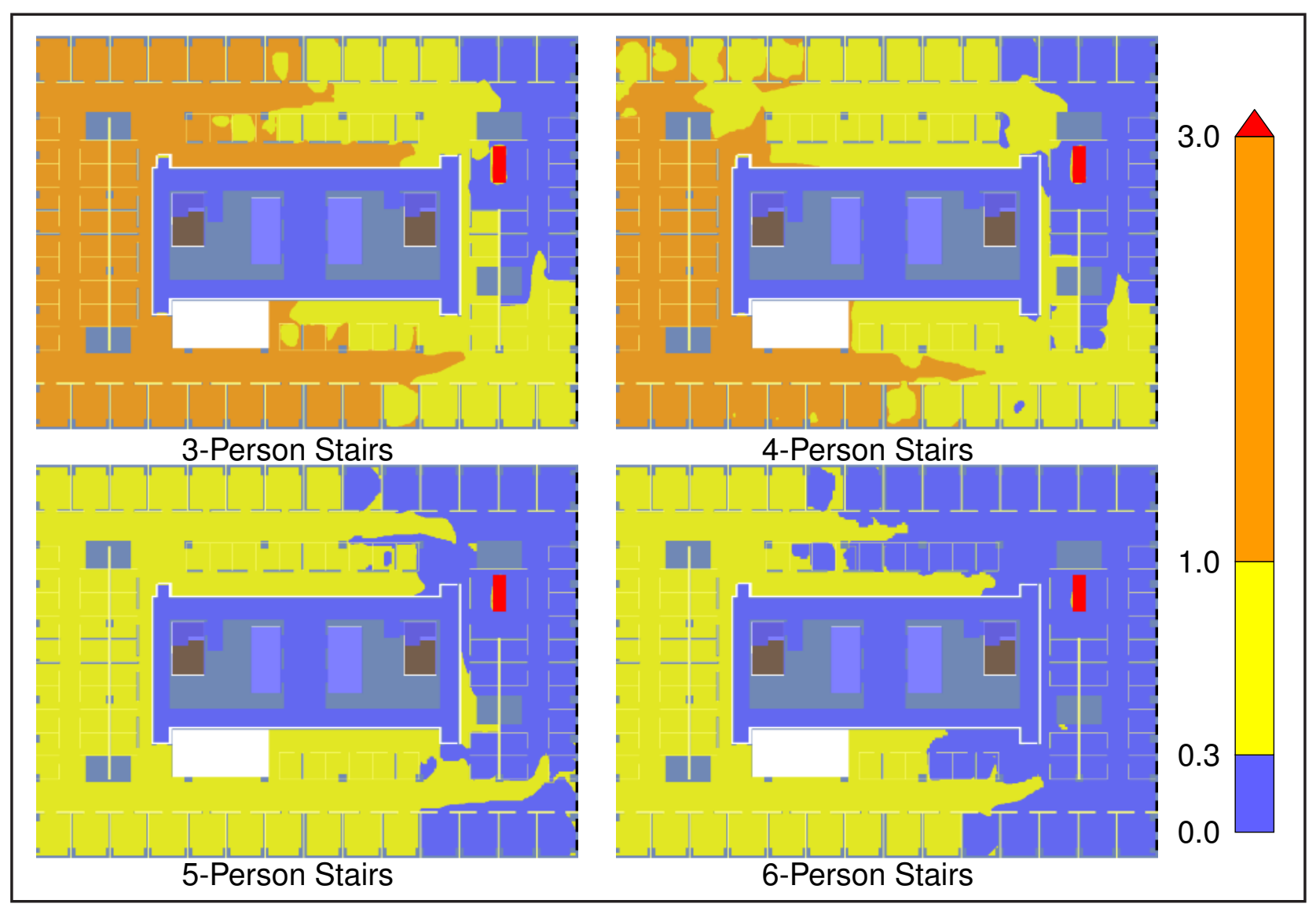

Figure 94: FED contours at an elevation of $3 \mathrm{ft}(0.9 \mathrm{~m})$ on the fire floor for a medium growth non-sprinklered fire at the time the search is complete 
Figure 93 shows FED contours at an elevation of $3 \mathrm{ft}(0.9 \mathrm{~m})$ on the fire floor at the time of firefighter entry on the floor for each crew size taking the stairs, assuming a medium growth rate fire. (Recall from Figure 90 that the experimental cubicle fire data best follows a medium growth rate.) When firefighters in the 3-person crew entered the fire floor, FED values in the cubicles along the south wall were between 0.3 and 1.0. Values in this range impact between $11 \%$ and $50 \%$ of the population. For the larger crew sizes, the majority of the floor area remained below FED values of 0.3 .

Figure 94 compares the FED values on the fire floor under the same experimental conditions at the time the search is completed. As expected, the hazard at this time decreases as crew size increases. For both 3-person and 4-person crews, a significant area on the fire floor is above an FED of 1.0. The 5-person and 6-person crews encounter FED levels above 0.3, but no regions rise above an FED of 1.0.
To better quantify the FED on the fire floor, the percentage of floor area in each FED band is examined. The stacked bar charts in Figure 95 show area percentages from the highest FED range to the lowest for crews using the stairs, summing to $100 \%$. The charts allow comparison of different crew sizes on tenability of the entire floor at three critical times unique to each test configuration. By the time a 3-person crew has completed the search for victims, approximately $45 \%$ of the floor area has an FED greater than 1.0 and less than 3.0. This can be compared to a search completed by a 6-person crew, at which time the FED for $45 \%$ of the floor area is greater than 0.3 but less than 1.0.
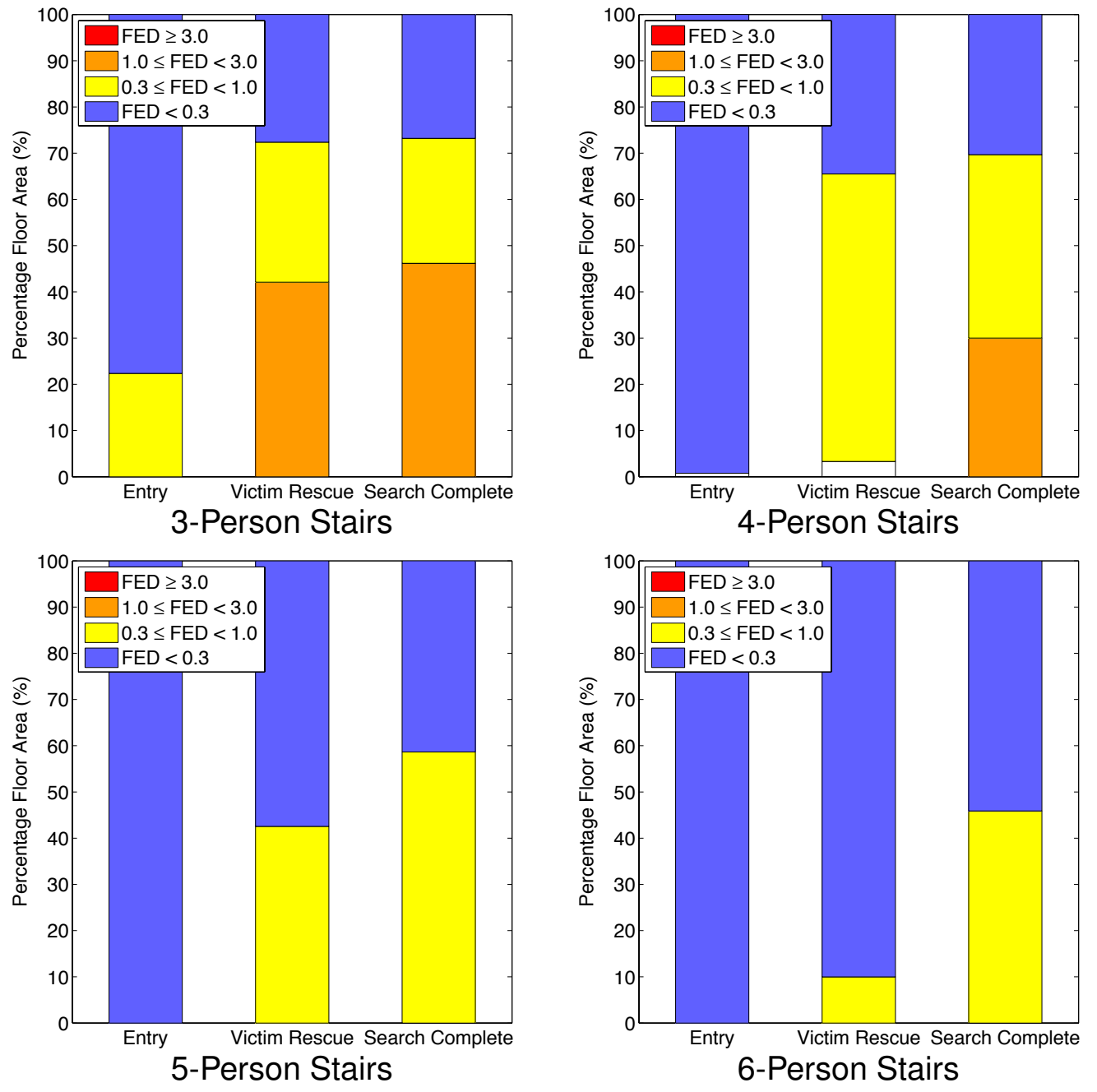

Figure 95: FED area percentages of the fire floor at an altitude of $3 \mathrm{ft}(0.9 \mathrm{~m})$ at times of entry, victim rescue, and search complete for a non-sprinklered medium growth fire. See Table 8 for discussion of colors. 
Victims may be situated anywhere on the fire floor. Therefore, in order to generalize the determination of FED at the time of rescue to victims in places other than the cubicle marked by button 18 , the non-uniform contours of FED on the fire floor can be combined with the experimental time-to-task data by calculating FED values at the time and location of button press for each

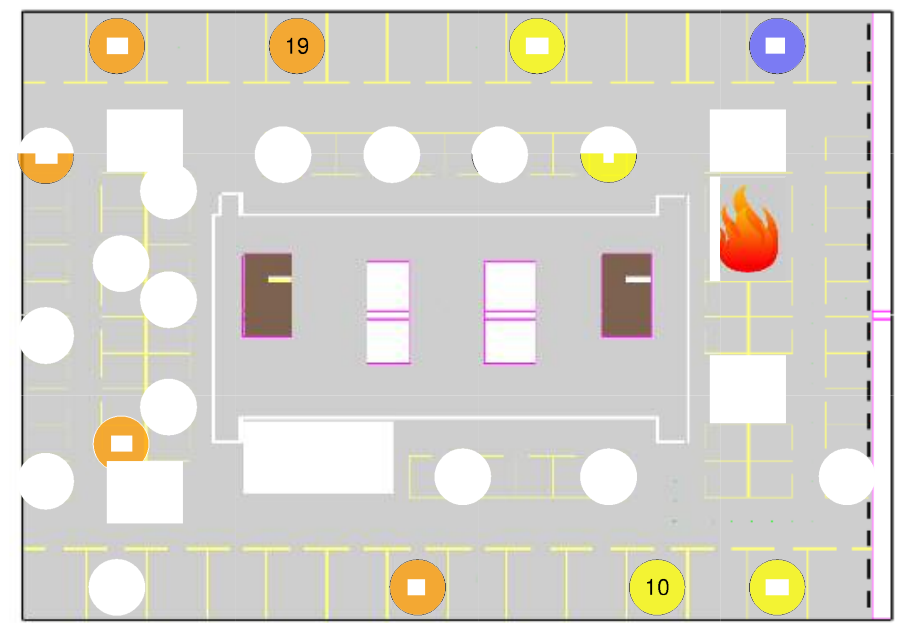

Figure 96: FED at button press - Crew size of 3 using stairs

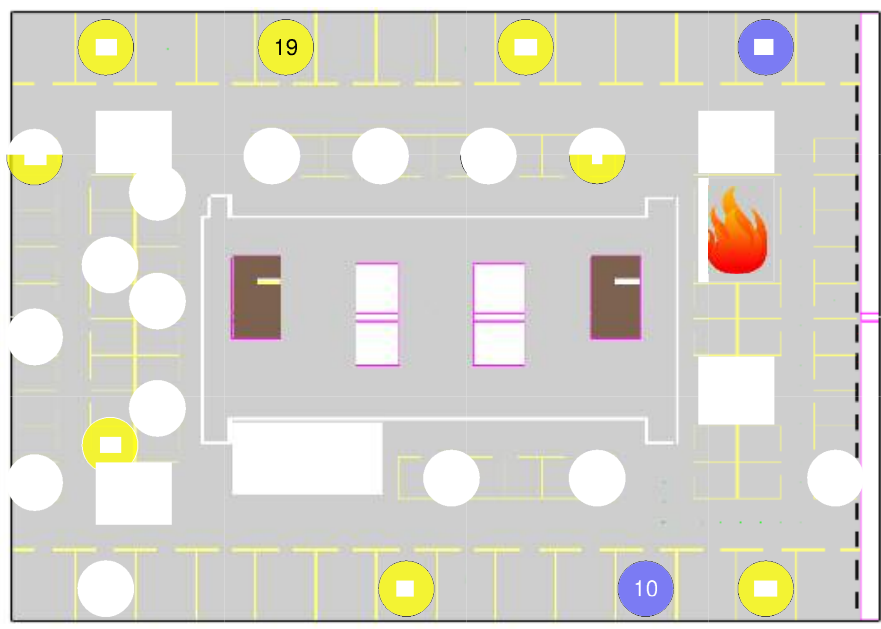

Figure 98: FED at button press - Crew size of 4 using stairs

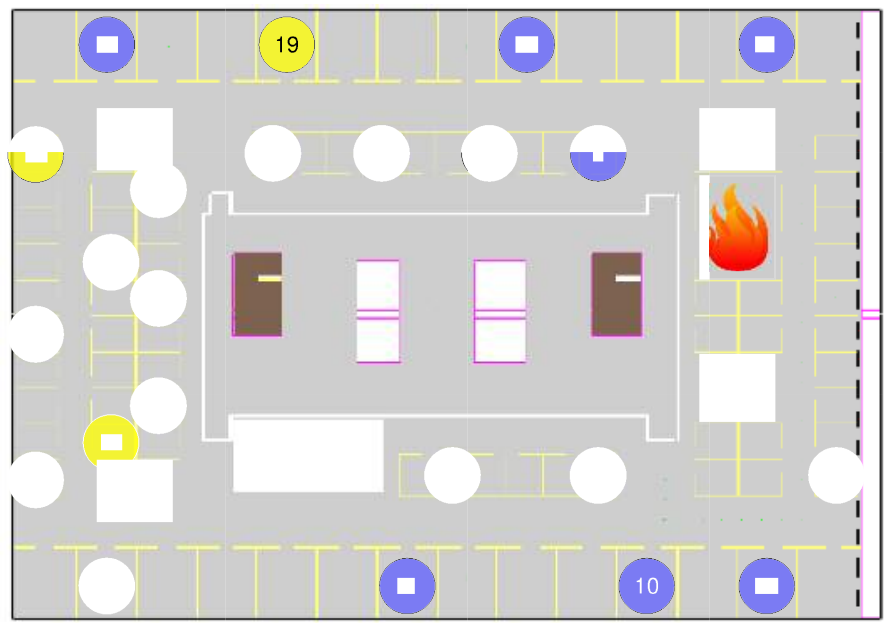

Figure 100: FED at button press - Crew size of 5 using stairs experimental configuration. Figure 96 through Figure 103 show the FED values on the fire floor at the time of the average button press as a function of crew size and means of ascent for a medium growth rate fire. These figures show the ascent by stairs and elevator in each of the crew sizes studied.

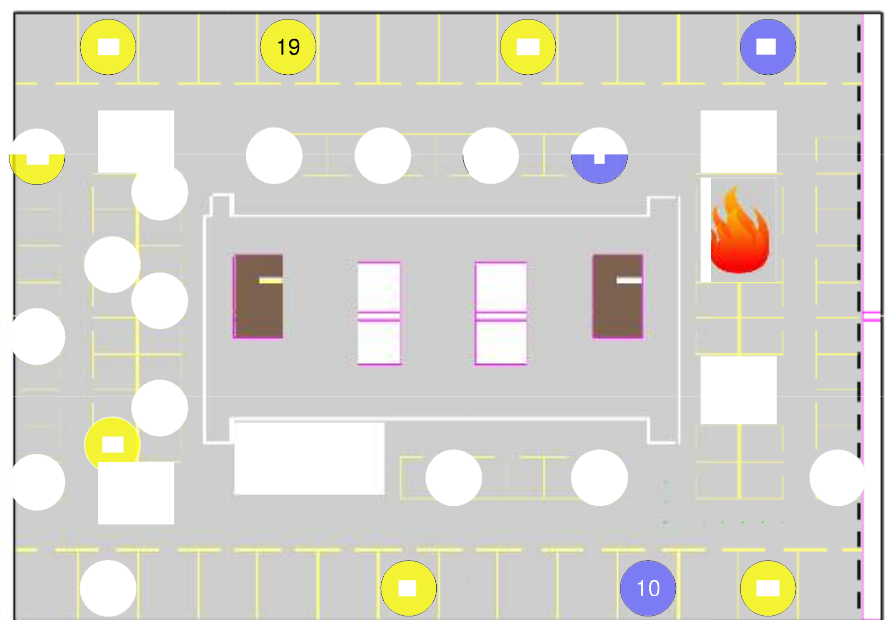

Figure 97: FED at button press - Crew size of 3 using elevator

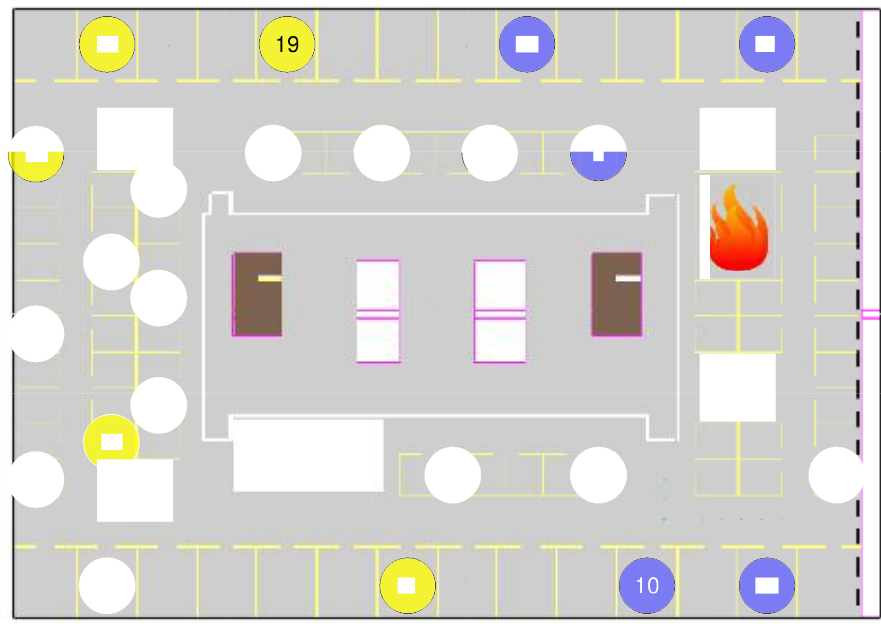

Figure 99: FED at button press - Crew size of 4 using elevator

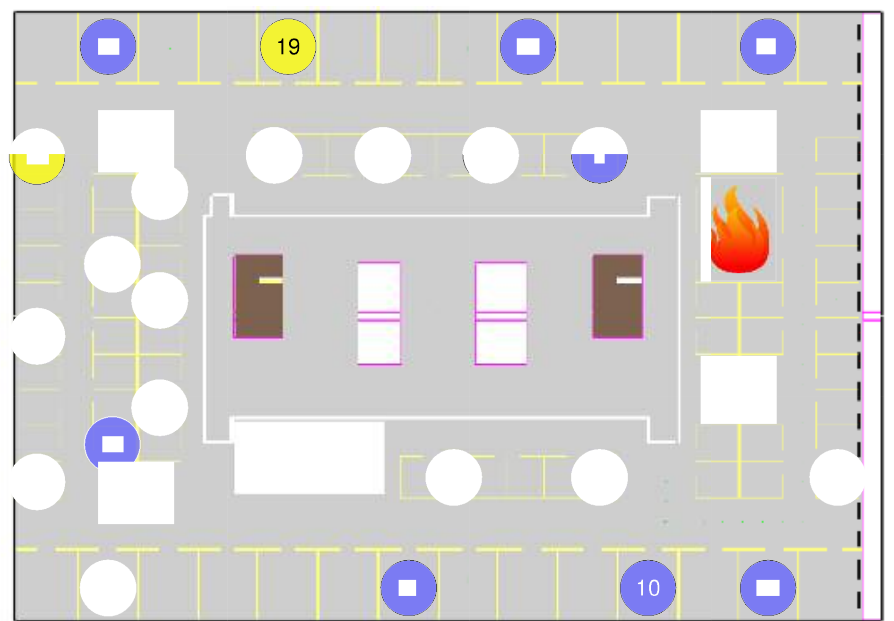

Figure 101: FED at button press - Crew size of 5 using elevator 


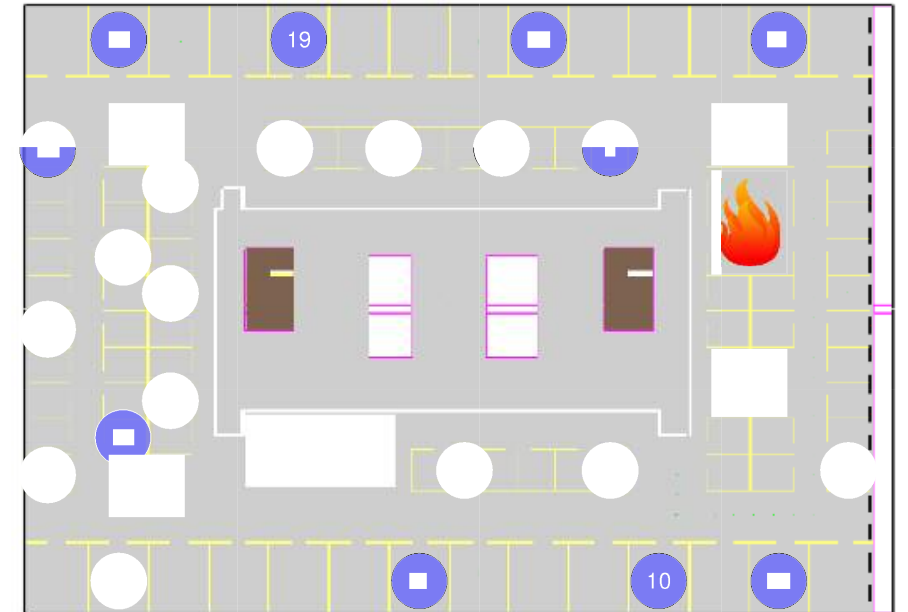

Figure 102: FED at button press - Crew size of 6 using stairs

The treatment of buttons located near the fire in Figure 96 through Figure 103 should be interpreted with caution. Note that button 22 is not included in these figures. Due to the location of button 22 relative to the fire location and ventilation through the windows, the FED value remains near ambient while the thermal conditions are not survivable. As a result, presenting FED information on this button location would be misleading with respect to the rest of the fire floor and the variables of study in this experiment. A similar argument could be made for buttons 21 and 23 due to their proximity to the windows and fire. However, the large interior columns between these buttons and the fire

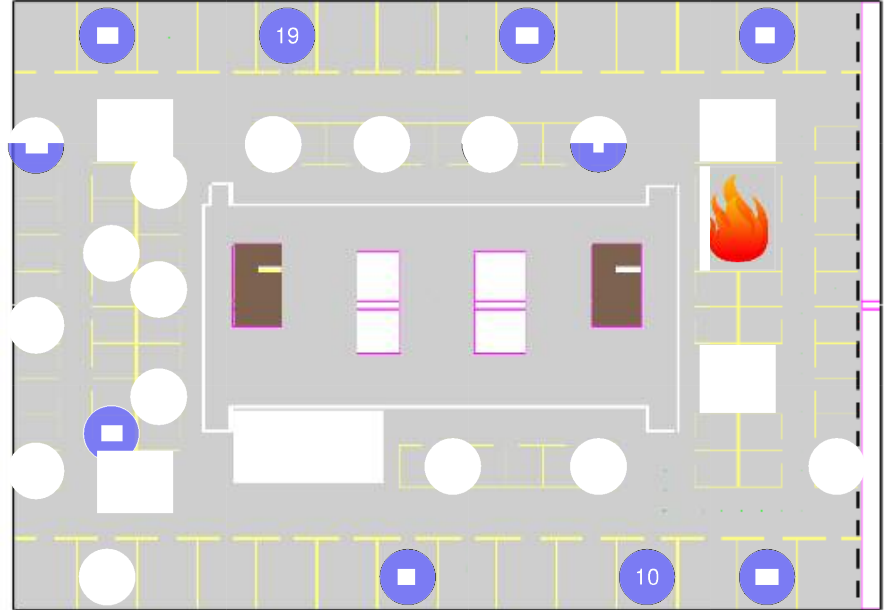

Figure 103: FED at button press - Crew size of 6 using elevator

minimize thermal effects. For completeness these buttons are included in the tenability analysis, although it must be noted that the thermal component should be considered.

The survivability advantage provided by firefighter ascent using elevators rather than stairs is demonstrated by comparing each figure in the right column above to the figure for the same crew size in the left column. Scanning down each column of figures shows that for a given ascent method, a larger crew reaches potential victims when the FED is lower. To better quantify the impact, Table 14 shows the FED values that correspond to each of the data points in Figure 96 through Figure 103.

\begin{tabular}{|c|c|c|c|c|c|c|c|c|}
\hline \multirow{2}{*}{$\begin{array}{l}\text { Button } \\
\text { Number }\end{array}$} & \multicolumn{8}{|c|}{ FED by Crew Ascent and Size } \\
\hline & $3 S$ & $4 S$ & $5 S$ & $6 S$ & $3 \mathrm{E}$ & $4 E$ & $5 E$ & $6 \mathrm{E}$ \\
\hline 1 & 0.46 & 0.28 & 0.19 & 0.15 & 0.21 & 0.16 & 0.14 & 0.10 \\
\hline 2 & 0.43 & 0.26 & 0.18 & 0.14 & 0.20 & 0.15 & 0.13 & 0.09 \\
\hline 3 & 0.55 & 0.43 & 0.26 & 0.19 & 0.29 & 0.20 & 0.19 & 0.14 \\
\hline 4 & 0.64 & 0.42 & 0.26 & 0.19 & 0.28 & 0.20 & 0.19 & 0.15 \\
\hline 5 & 0.65 & 0.43 & 0.27 & 0.19 & 0.37 & 0.21 & 0.20 & 0.15 \\
\hline 6 & 0.66 & 0.51 & 0.32 & 0.20 & 0.39 & 0.21 & 0.22 & 0.17 \\
\hline 7 & 0.56 & 0.40 & 0.27 & 0.14 & 0.31 & 0.19 & 0.16 & 0.12 \\
\hline 8 & 0.50 & 0.28 & 0.21 & 0.11 & 0.27 & 0.15 & 0.12 & 0.11 \\
\hline 9 & 0.47 & 0.31 & 0.22 & 0.16 & 0.27 & 0.16 & 0.14 & 0.12 \\
\hline 10 & 0.39 & 0.23 & 0.08 & 0.07 & 0.23 & 0.14 & 0.09 & 0.05 \\
\hline 11 & 1.14 & 0.86 & 0.27 & 0.16 & 0.82 & 0.49 & 0.19 & 0.11 \\
\hline 12 & 1.59 & 0.93 & 0.36 & 0.23 & 0.96 & 0.55 & 0.29 & 0.15 \\
\hline 13 & 1.59 & 0.87 & 0.33 & 0.22 & 0.93 & 0.52 & 0.28 & 0.15 \\
\hline 14 & 1.48 & 0.79 & 0.28 & 0.18 & 0.88 & 0.48 & 0.22 & 0.13 \\
\hline 15 & 1.32 & 0.71 & 0.28 & 0.19 & 0.84 & 0.45 & 0.25 & 0.14 \\
\hline 16 & 1.52 & 0.84 & 0.30 & 0.20 & 0.93 & 0.52 & 0.27 & 0.14 \\
\hline 17 & 1.29 & 0.67 & 0.30 & 0.18 & 0.83 & 0.45 & 0.24 & 0.13 \\
\hline 18 & 1.22 & 0.68 & 0.28 & 0.21 & 0.78 & 0.48 & 0.27 & 0.14 \\
\hline 19 & 1.29 & 0.88 & 0.43 & 0.28 & 0.85 & 0.53 & 0.37 & 0.19 \\
\hline 20 & 0.71 & 0.44 & 0.20 & 0.15 & 0.47 & 0.29 & 0.17 & 0.11 \\
\hline 21 & 0.18 & 0.14 & 0.09 & 0.06 & 0.14 & 0.11 & 0.08 & 0.05 \\
\hline 23 & 0.71 & 0.51 & 0.31 & 0.19 & 0.49 & 0.30 & 0.23 & 0.12 \\
\hline 24 & 0.63 & 0.42 & 0.26 & 0.18 & 0.39 & 0.28 & 0.21 & 0.12 \\
\hline
\end{tabular}

Table 14: FED at the time of button press on the fire floor, as a function of crew size and ascent method for a medium growth rate fire without sprinklers 
Table 14 shows the impact of crew size and ascent method on FED at each of the point locations (buttons) where there was a time history of firefighter position. The highest FED values, indicating greater tenability hazards, are typically related to the smaller crew sizes. There are some outliers, specifically when examining 3-person elevators versus 4-person stairs. The 3-person crews show lower FED values than the 4-person crews on the inner loop buttons (1-9) because the search advantage of a 4-person crew does not show up until the second crew arrives and can split. These differences are also seen on the inner loop buttons for a 5-person crew using the stairs versus a 4-person crew using the elevators. However, as the search proceeds in time, the 5 -person crew can reach the outer loop buttons at lower FED values than the 4-person crew. Both 6-person crews can search the entire floor without having any of the potential victim locations exceed an FED value of 0.3 , which is the lowest FED threshold. Also note that FED values at button 21 remain below the lowest threshold despite being among the last buttons to be pressed. As discussed previously, this is because the button is located near the windows that break due to thermal failure. Ventilation allows for toxic gases to exit the structure; however, the low FED values do not mean that this area is tenable.

\begin{tabular}{|c|c|c|c|c|c|c|c|c|}
\hline \multirow{2}{*}{$\begin{array}{l}\text { Button } \\
\text { Number }\end{array}$} & \multicolumn{8}{|c|}{ FED by Crew Ascent and Size } \\
\hline & $3 S$ & $4 S$ & $5 S$ & $6 S$ & $3 E$ & $4 \mathrm{E}$ & $5 E$ & $6 \mathrm{E}$ \\
\hline 1 & 0.04 & 0.03 & 0.03 & 0.03 & 0.03 & 0.03 & 0.01 & 0.02 \\
\hline 2 & 0.04 & 0.03 & 0.03 & 0.02 & 0.03 & 0.02 & 0.01 & 0.02 \\
\hline 3 & 0.05 & 0.04 & 0.03 & 0.03 & 0.03 & 0.03 & 0.01 & 0.03 \\
\hline 4 & 0.05 & 0.04 & 0.03 & 0.03 & 0.03 & 0.03 & 0.01 & 0.03 \\
\hline 5 & 0.06 & 0.05 & 0.04 & 0.03 & 0.04 & 0.03 & 0.01 & 0.03 \\
\hline 6 & 0.06 & 0.06 & 0.04 & 0.03 & 0.05 & 0.03 & 0.01 & 0.04 \\
\hline 7 & 0.06 & 0.05 & 0.04 & 0.03 & 0.04 & 0.04 & 0.01 & 0.03 \\
\hline 8 & 0.08 & 0.05 & 0.05 & 0.03 & 0.05 & 0.04 & 0.01 & 0.04 \\
\hline 9 & 0.07 & 0.06 & 0.05 & 0.04 & 0.05 & 0.04 & 0.01 & 0.03 \\
\hline 10 & 0.06 & 0.04 & 0.02 & 0.01 & 0.05 & 0.03 & 0.01 & 0.01 \\
\hline 11 & 0.09 & 0.08 & 0.03 & 0.02 & 0.10 & 0.06 & 0.01 & 0.02 \\
\hline 12 & 0.14 & 0.09 & 0.04 & 0.03 & 0.13 & 0.07 & 0.02 & 0.03 \\
\hline 13 & 0.15 & 0.09 & 0.04 & 0.03 & 0.13 & 0.07 & 0.02 & 0.03 \\
\hline 14 & 0.15 & 0.09 & 0.03 & 0.03 & 0.13 & 0.07 & 0.02 & 0.02 \\
\hline 15 & 0.16 & 0.09 & 0.04 & 0.03 & 0.14 & 0.08 & 0.02 & 0.03 \\
\hline 16 & 0.16 & 0.09 & 0.04 & 0.03 & 0.13 & 0.07 & 0.02 & 0.03 \\
\hline 17 & 0.16 & 0.09 & 0.04 & 0.03 & 0.13 & 0.08 & 0.02 & 0.03 \\
\hline 18 & 0.17 & 0.10 & 0.04 & 0.04 & 0.14 & 0.09 & 0.02 & 0.03 \\
\hline 19 & 0.20 & 0.15 & 0.07 & 0.06 & 0.16 & 0.11 & 0.02 & 0.06 \\
\hline 20 & 0.16 & 0.12 & 0.06 & 0.05 & 0.14 & 0.10 & 0.02 & 0.05 \\
\hline 21 & 0.13 & 0.10 & 0.05 & 0.04 & 0.12 & 0.08 & 0.01 & 0.04 \\
\hline 23 & 0.12 & 0.09 & 0.06 & 0.04 & 0.10 & 0.08 & 0.00 & 0.04 \\
\hline 24 & 0.11 & 0.09 & 0.07 & 0.05 & 0.10 & 0.07 & 0.02 & 0.05 \\
\hline
\end{tabular}

Table 15: FED at $3 \mathrm{ft}$ elevation at the time of each fire floor button press, as a function of crew size and ascent method for a medium growth rate fire with sprinklers 


\section{Sprinklers}

The FED analysis above was conducted for a non-sprinklered structure. To examine the impact of sprinklers on the tenability of the fire floor, Table 15 lists the FED values at each button for each crew configuration for a sprinklered medium growth fire. In this case, the FED values remain well below the first threshold value of 0.3 for all crew sizes and ascent methods. Tenability is greatly improved compared to the non-sprinklered fires, as is expected for cases in which sprinklers limit the fire growth. Holding everything else constant, smaller fires produce a lower amount of toxic gases and thus decrease FED values.

\subsection{Building Evacuation}

The high-rise time-to-task experiments were conducted in a building without occupants present. Depending on the time of day, a typical commercial high-rise may have hundreds or thousands of occupants. A complete evacuation of occupants could require significant time. This is important for the time-to-task experiments because during this evacuation time period the occupants may interact in an unknown way with the arriving firefighters, particularly in the stairs. If evacuation is not complete prior to firefighter arrival, the presence of evacuees in stairwells will impede the progress of ascending firefighters. This would result in longer ascent times than those measured in the experiments. Therefore, the times determined in the experiments are considered a conservative (best-case) scenario.

The length of overlap time when occupants are exiting and firefighters are entering is important for understanding the degree to which the experimental times may be delayed (i.e., how many of the arriving crews are impacted). To determine a reference time for expected complete evacuation, the NIST Egress Estimator model was used (Reneke et al., 2013). The Egress Estimator uses separate calculations to estimate the egress times for stairwell evacuation and elevator evacuation; both have been validated against data from experiments similar to the high-rise scenario evaluated in this report. The stairwell model is based on algorithms from the SFPE Handbook (Nelson and Mowrer, 2002) and the elevator model is based on the ELVAC model (Klote, 1993). The maximum time between the model results is taken to determine a conservative overall building evacuation time.

For egress analysis of the high-rise building used for these experiments, two occupant loads were used: 100 occupants per floor and 200 occupants per floor. The 100 occupants-per-floor load was determined based on the open-floor plan cubicle construction that was completed for the fire floor (counting the number of desks). The 200 occupants-per-floor load was based on a recommendation from Muha that a general office space should have a load of 1 person per $150 \mathrm{sq} \mathrm{ft}\left(19.9 \mathrm{~m}^{2}\right)$ (Muha, 2012). For the 30,000 square foot $\left(2800 \mathrm{~m}^{2}\right)$ floor plan, this loading results in 200 occupants per floor. Using the Egress Estimator model, the total evacuation time was calculated for the two occupant loads and two egress criteria. The first criterion was that all of the occupants would exit the high-rise via one of the two available stairwells. The second criterion was that $25 \%$ of the occupants on each floor would use two of the four elevators while the remaining $75 \%$ of the occupants would use the stairs. Two of the elevators were reserved for firefighter use. Table 16 shows the time for total evacuation for the two occupant loads and two evacuation configurations.

In these experiments, firefighters enter the lobby 7 min $4 \mathrm{~s}$ after first detection and the start of evacuation. This time includes $60 \mathrm{~s}$ for alarm processing (NFPA 2010), $80 \mathrm{~s}$ for turnout time (NFPA 2010), and $4 \mathrm{~min} 44 \mathrm{~s}$ for travel time, unloading gear, and walking to the lobby. For full occupancy at the 100 occupant per floor loading, there can be approximately $5 \mathrm{~min}$ to $8 \mathrm{~min}$ where ascending firefighters may face counter-flow conditions from evacuating occupants. For the higher loading density the amount of counter-flow can increase to between $15 \mathrm{~min}$ and $22 \mathrm{~min}$. Depending on the duration of counter-flow, ascending firefighters using the stairs may be slowed. Therefore, the experiments that compare the impact of stairs versus elevators are a best-case comparison, as actual time differences may be more significant.

\begin{tabular}{|c|c|c|}
\hline $\begin{array}{c}\text { Average Occupant } \\
\text { Load per Floor }\end{array}$ & $\begin{array}{c}\text { Evacuation } \\
\text { Configuration }\end{array}$ & $\begin{array}{c}\text { Evacuation Time } \\
\text { (MM:SS) }\end{array}$ \\
\hline 100 & Stairs Only & $15: 10$ \\
\hline 100 & Stairs $+25 \%$ Elevators & $12: 28$ \\
\hline 200 & Stairs Only & $29: 28$ \\
\hline 200 & Stairs $+25 \%$ Elevators & $22: 19$ \\
\hline
\end{tabular}

Table 16: Total evacuation time as a function of occupant load and evacuation configuration 


\section{Physiological Effects on Firefighters}

eports on firefighter fatalities consistently document overexertion/overstrain as the leading cause of line-of-duty

fatalities (NIOSH, 2013). There is strong epidemiological evidence that heavy physical exertion can trigger sudden cardiac events (Mittleman et al, 1993; Albert et al, 2000). Therefore, information about the effect of crew size and vertical response mode on physiological strain is very valuable.

During the planning of the high-rise fireground experiments, as with the Residential Fireground Field Experiments, investigators at Skidmore College recognized an opportunity to conduct an independent study on the relationship between firefighter deployment configurations, vertical response mode and firefighter heart rates.

Investigators were able to leverage the resources of the high-rise field experiments to conduct a separate analysis of the cardiac strain on firefighters in the high hazard environment.

The results of this study are compiled in a separate report. 


\section{Study Limitations}

$\mathrm{T}$ he scope of this study is limited to understanding the relative influence of deployment variables to a working high-rise structure fire. The applicability of the conclusions from this report to low hazard residential, outside fires, natural disaster response, HAZMAT or other technical responses has not been assessed and should not be extrapolated from this report.

Fire Out times were determined by the time at which engine crews moved the nozzle of the second line on the fire floor to the origin of the fire. As the experiments could not utilize live fires, the effect of fire size on suppression time was not captured. The fire modeling results indicated that smaller crew sizes would face a larger fire; for example a 3-person crew would face a fire of 11 MW, while the 6-person crew would face a fire of $5 \mathrm{MW}$. Therefore, the time to fully suppress the fire (and the total toxic dose received by trapped occupants) is likely underestimated by an unknown amount in these experiments.

The total number of apparatus responding for each high-rise experiment was held constant at 9 engines, 9 trucks, 3 ambulances, and 3 battalion chiefs for all crew size configurations. The apparatus were dispatched in the high/low alarm groupings as described in the report in Table 5. The effect of deploying more or fewer than this total number of apparatus was not evaluated.

Fireground operations in these experiments were conducted under best-case scenario conditions without many of the complications that can occur in real fireground environments. These complications can include, but are not limited to inclement weather, nighttime operation, building security infrastructure that may impede building access, elevator operation failure, communications failures (both technological and interpersonal), or power / fire technology failures.

Since the field experiments were conducted in a vacant building, the counter flow effects of evacuating occupants on firefighter ascent were not measured. Therefore, the recorded stairwell ascent times are likely underestimated.

Fire crews participating in the experiments were provided by 13 different fire and rescue departments, each with their own protocols, fireground tactics, training regimes, and equipment. Standardized protocols were developed and implemented for the high-rise experiments. Though similar, the protocols differed from those used by the participating departments. Daily orientation and crew cue cards were used to minimize the effects of protocol differences. Since there is more than one effective way to perform many of the required tasks on the high-rise fireground, attempts to generalize the results from these experiments to individual departments must take into account tactics and equipment that vary from those used in the experiments.

The fire crews that participated in the experiments typically operate using 3-person and 4-person staffing. Therefore, the effectiveness of the 5-person and 6-person operations may have been influenced by a lack of experience in operating at those staffing levels. Standardizing and assigning tasks likely minimized the impact of this factor, although the actual influence on the results is unknown.

Although efforts were made to minimize the effect of learning across experiments, some participants took part in more than one experiment, while others did not.
Though actual fire could not be used during the experiments, all operations were handled according to NFPA 1403 and every attempt was made to ensure the highest possible degree of realism. Realistic digital fire displays and simulated smoke were used on the fire floor and the floor above the fire. The quality of simulated smoke was exceptional in replicating visual obscuration for both fire attack and search and rescue operations. Digital fire displays were also exceptional in visual simulation of live fire, although thermal effects were lacking. Firefighters were informed of all simulations and were asked to work and move as if they were in a real fire environment. Though the instruction likely minimized the effect, there was no ability to measure the actual impact the use of simulation had on the results.

Though there was smoke breech to the floor above the fire, fire spread beyond the floor of origin was not considered in the tests or the fire models. Therefore, the size of the fire and the risk to the firefighter may be somewhat underestimated for fast growing fires or slower response configurations.

The scale and magnitude of the high-rise building was fixed. Fire department response to ultra-high-rise buildings (e.g., buildings greater than $420 \mathrm{ft}(128 \mathrm{~m})$ in height) may require customized planning and resource allocation.

Radio communications throughout the experiments were facilitated through two dedicated frequencies and interoperable portable radios assigned to all crews. Typically, a high-rise response, as for other structural fires, uses at least two radio frequencies on the fireground-one for command and one for tactical communication. Since one of the channels provided for the experiments was used by researchers to communicate during the experiments, only one channel remained for use in both command and tactical communication. Even though both fire officers and incident commanders were asked to minimize radio communications to include protocol and critical needs only, the simultaneous operations on multiple floors often caused delay in officers' communication with division supervisors and command. The influence of limited radio communication was not evaluated.

The National Incident Management System (NIMS) was used throughout the experiments. It was assumed that chief officers assigned to the experiments know and frequently use the system in actual operations. Therefore, there was no training or orientation to the NIMS during the experiments. In an effort to control variance in technique from chief to chief, each IC was given a specific protocol to follow for crew assignments and was advised to hand off the operations sections in the two most hazardous areas (on the fire floor and floor above the fire) early in the incident, so that other priorities could be addressed.

Due to the magnitude of the experiments, fireground operations record keeping was essential. To limit variance in record keeping, each IC had an assigned aide who recorded situation reports, resource status and company assignments. Units operating on the fire floor and the floor above the fire were managed by operations section chiefs, who documented which companies were active on their assigned floor. Effective operation of the Chiefs assigned to these positions was dependent on their understanding and experience with the NIMS and was not measured. 


\section{Conclusions}

O verall, the results of this study show that the number of fire service crew members in each company responding to a fire in a 30,000 square foot, thirteen-story structure had a dramatic effect on the crew's ability to protect lives and property. Three significant conclusions are discussed below.

First, when responding to a medium growth rate fire on the 10 th floor, 3-person crews ascending to the fire floor confronted an environment where the fire had released $60 \%$ more heat energy than the fire encountered by the 6-person crews. Unfortunately, larger fires expose firefighters to greater risks and are more challenging to suppress.

Second, larger fires produce more risk exposure for building occupants. In general, occupants being rescued by smaller crew sizes and by crews that used the stairs rather than the elevators were exposed to significantly greater dose of toxins from the fire. While the exact risk exposure for an occupant will depend on the fire growth rate, their proximity to the fire, and the floor on which the fire is located, it is clear that on-scene deployment decisions can have a dramatic impact in determining the fate of building occupants.

Third, the study confirmed that a properly engineered and operational fire sprinkler system drastically reduces the risk exposure for both the building occupants and the firefighters. While this has been well understood for many years and most new high-rise buildings are constructed with fire sprinkler protection, NFPA estimates that 41 percent of U.S. high-rise office buildings, 45 percent of high-rise hotels, and 54 percent of high-rise apartment buildings are not equipped with sprinklers. Moreover, sprinkler systems fail in about one in 14 fires. Thus, fire departments should be prepared to manage the risks associated with unsprinklered high-rise building fires.

Forty-eight laboratory and full-scale simulated fire experiments were conducted to determine the impact of crew size, alarm size and vertical response mode times on firefighter task completion times responding to a high hazard high-rise commercial structure fire. This report quantifies the effects of changes to staffing, alarm size and/or vertical response mode for high hazard high-rise commercial firefighting operations. While resource deployment is addressed in the context of a high-rise structure type and high-risk level, it is recognized that public policy decisions regarding the cost-benefit of specific deployment decisions are a function of many factors including geography, available resources, community expectations, and local hazards and risks. Though this report contributes significant knowledge to community and fire service leaders in regard to effective resource deployment for fire suppression, other factors contributing to policy decisions are not addressed.

The objective of the experiments was to determine the relative effects of crew size, alarm size, and vertical response mode on the task completion times of the firefighting crews relative to intervention times and the likelihood of occupant rescue. The experimental results for each of these factors are discussed below.

Of the 38 high-rise fireground tasks measured during the experiments, the following were determined to have especially significant impact on the firefighting operations. Differential outcomes based on variation of crew size, alarm size, and/or vertical response mode times are discussed below.

\section{Overall Scene Time}

Overall scene time is the time that firefighters are actually engaged in tasks on the scene of a structure fire and are unavailable for dispatch to other incidents. The times noted do not include some tasks such as salvage, overhaul and secondary search of the structure. During the experiments, this time included typical operational tasks with the exception of overhaul and salvage. The time to completion of all tasks decreased as crew size increased. Three-person crews took an average of about an hour to complete the fire response, whereas crews of 6 firefighters required a mean time of just under $37 \mathrm{~min}$ for completion. The performance of crews sized 4 and 5 were in-between, with a crew size of 5 taking about 2 min longer than a crew size of 6 , and a crew size of 4 taking about 9 min longer than a crew size of 5 but $12 \mathrm{~min}$ shorter than a crew size of 3 . Therefore, All Tasks Complete times were substantially reduced for crew sizes of 5 compared to 4 , and 4 compared to 3 .

\section{Advance Attack Line}

As firefighters engage on a fireground, putting water on the fire is one of the most important tasks. Extinguishing the fire is necessary to reduce the continuously escalating risks from fire heat release and the toxic products of combustion. Before water can be put on a fire however, a hose line must be stretched from the standpipe in the stairwell to the compartment where the fire is burning. Comparing each crew size to a 3-person crew, the time differences increased with increasing crew size. From the initiation of on-scene firefighting activities, a 3-person crew took 1 min $43 \mathrm{~s}(8.5 \%)$ longer than a 4-person crew to stretch the hose line. A 3-person crew took 2 min 47 s (13.9\%) longer than a 5 -person crew to complete the same task. Finally, the most notable difference was between a 3-person crew and a 6-person crew, with a 4 min $28 \mathrm{~s}(22.3 \%)$ task completion time difference.

\section{Advance Second Line}

The size of the fire in the experiments required two $2 \frac{1}{2}$ inch hose lines to fully suppress, therefore a second hose line had to be advanced from the standpipe in the stairwell to the fire to assure adequate water flow to extinguish. A 4-person crew took 2 min $43 \mathrm{~s}$ $(12.3 \%)$ longer than a 5-person crew to complete the same task. Finally, the most notable comparison was between a 4-person crew and a 6-person crew, with a $5 \mathrm{~min} 38 \mathrm{~s}$ (24.1) difference in task completion time.

\section{Fire Out}

Getting the fire out is critical to reducing risk to both firefighters entering the structure and to trapped occupants. Based on fuel available to burn, heat release accelerates and products of combustion continue to poison the environment until the fire is out. Fire Out, in the study, was defined as having both the attack line and the second hose line in place. There was a $1 \mathrm{~min} 15 \mathrm{~s}(5.0$ $\%$ ) difference in the Fire Out time between the 4- and 5-person crews and a 3 min $29 \mathrm{~s}(12.7 \%$ ) difference between the 5-person and 3 -person crews). There was an additional 3 min 33 s (17\%) difference in the Fire Out time between the 5- and 6-person crews. The 6-person crews extinguished the fire $7 \min 2 \mathrm{~s}(25.6 \%)$ faster than 3-person crews. 


\section{Search and Rescue 10th Floor}

The fire floor in the experiments measured 30,000 sq ft (2800 $\mathrm{m}^{2}$ ) and contained 96 cubicles. Responding firefighters must search every inch of the fire floor and adjacent stairwells to assure that occupants have not been trapped, disabled or killed due to the toxic environment or direct fire. In the high hazard high-rise commercial building, the 4-person crews started the search and rescue 1 min $23 \mathrm{~s}(7.8 \%)$ earlier than the 3-person crews and completed the search $11 \mathrm{~min}$ and $21 \mathrm{~s}(17.4 \%)$ faster than the 3 -person crews. In the same structure, the 5 -person crews started the search $1 \mathrm{~min} 4 \mathrm{~s}$ (6.7\%) earlier than the 4-person crews and 2 $\min 27 \mathrm{~s}(14.1 \%)$ faster than the 3-person crews. Additionally, 5 -person crews completed the search faster than the 4 - and 3 -person crews by $13 \mathrm{~min}$ and $34 \mathrm{~s} \mathrm{(26.8 \% )} \mathrm{and} 24 \mathrm{~min} 55 \mathrm{~s}$ (40.3 $\%)$ respectively. Six-person crews had the fastest performance, starting the search $1 \mathrm{~min} 19 \mathrm{~s}(8.8 \%)$ faster and completing the search 2 min $57 \mathrm{~s}(8.0 \%)$ faster than 5 -person crews. The greatest difference in search times was between 6- and 3-person crews. Six-person crews started the search on the fire floor $3 \mathrm{~min} 46 \mathrm{~s}$ (21.7 \%) faster and completed the search 27 min 51 s (45\%) faster than the 3 -person crews.

\section{Victim \#1 Found}

There was a single victim located on the fire floor. The victim was found and rescued by all crews. A 5-person crew operating in the high-rise structure located the victim on the fire floor $25 \mathrm{~min}$ $18 \mathrm{~s}(50.6 \%)$ faster than 3-person crews and $12 \min 7 \mathrm{~s}(32.9 \%)$ faster than a 4-person crew. Likewise, 6-person crews located the victim on the fire floor $28 \mathrm{~min} 33 \mathrm{~s}(57.1 \%)$ faster than the 3 -person crews, 15 min $21 \mathrm{~s}$ (41.7 \%) faster than the 4-person crew, and 3 min $14 \mathrm{~s} \mathrm{(13.2 \% )} \mathrm{faster} \mathrm{than} \mathrm{5-person} \mathrm{crews.}$

\section{Victim \#1 Rescue}

Victim rescue requires at least two firefighters to carry a victim away from the hazardous environment to safety and emergency medical care. Removing a viable victim from the environment increases the chance of survival. Five-person crews were able to remove the victim from the fire environment $11 \mathrm{~min} 39 \mathrm{~s}(29.7 \%)$ faster than the 4-person crews, while 6-person crews removed the victim from the environment $14 \mathrm{~min} 58 \mathrm{~s}(38.1 \%)$ faster than the 4-person crews and 3 min $19 \mathrm{~s}(12.0 \%)$ faster than the 5-person crews.

\section{Victim \#1 Descent}

Getting the victim out of the IDLH environment is not enough. The victim must be removed from the building and when necessary, transported to a hospital for examination and definitive care.Five-person crews were able to facilitate victim descent 11 $\min 39 \mathrm{~s}(27.5 \%)$ faster than 4-person crews and $24 \min (43.1 \%)$ faster than 3-person crews, while 6-person crews were 3 min $19 \mathrm{~s}$ (11.0\%) faster than 5-person crews.

\section{Advance Line Above the Fire (11th Floor)}

In a high-rise structure, it is essential to stretch a charged hose line from the standpipe in the stairwell to the area above the fire on the floor above the fire to extinguish fire that has spread vertically from the floor of origin. A 3-person crew took 2 min $58 \mathrm{~s}$ $(11.5 \%)$ longer than 5-person crews to complete the same task. While the most notable comparison was between 3-person crews and a 6-person crew, with a 3 min $37 \mathrm{~s}(14.0 \%)$ difference in task completion time.

\section{Search and Rescue 11th Floor}

The floor above the fire was separated into a number of conference rooms and offices that had to be searched by each crew. The area of the floor measured 30,000 sq ft $\left(2800 \mathrm{~m}^{2}\right)$. Responding firefighters must search every inch of the floor above the fire and adjacent stairwells to determine whether occupants have been trapped, disabled or killed due to the toxic environment. The area of the floor measured $30,000 \mathrm{sq} \mathrm{ft}\left(2800 \mathrm{~m}^{2}\right)$. During the experiments, the 4-person crews completed the search $9 \min 31 \mathrm{~s}$ (18.6\%) faster than the 3-person crews. Meanwhile, the 5-person crews started a primary search/rescue $1 \mathrm{~min} 34 \mathrm{~s}$ (6.8\%) faster than the 4-person crews and completed the search 2 min $37 \mathrm{~s}(6.3$ $\%)$ faster than the 4-person crews. In the same structure, the 6-person crews also started the search $1 \mathrm{~min} 30 \mathrm{~s}(6.6 \%)$ faster than the 4-person crews but completed the search $5 \mathrm{~min} 8 \mathrm{~s}(12.3$ $\%$ ) faster than the 4-person crews.

\section{Victim \#2 Found}

There was a single disabled victim located on the floor above the fire. The victim was found and rescued by all crews. A 5-person crew operating in the high-rise structure located the second victim $17 \min 23 \mathrm{~s}(34 \%)$ faster than a 3-person crew and $2 \min 41 \mathrm{~s} \mathrm{(7.4}$ $\%)$ faster than a 4-person crew. Likewise, a 6-person crew located the second victim on the floor above the fire $2 \mathrm{~min} 48 \mathrm{~s}(7.7 \%)$ faster than the 4-person crew.

\section{Victim \#2 Rescued}

In addition to the victim on the fire floor, a second victim was located on the floor above the fire. Each crew operating on this floor was tasked with locating and rescuing the victim. Victim rescue requires at least two firefighters to carry a victim away from the hazardous environment to safety and emergency medical care. Removing a viable victim from the environment increases the chance of survival. Five-person crews were able to remove the second victim from the fire environment $17 \min 9 \mathrm{~s}(32.1 \%)$ faster than 3-person crews and 2 min $36 \mathrm{~s}(6.7 \%)$ faster than the 4-person crews. Similarly, the 6-person crews rescued the victim 2 $\min 48 \mathrm{~s}(7.1 \%)$ faster than 4 -person crews.

\section{Victim \#2 Descent}

Getting a victim out of the IDLH environment is not enough. All victims must be removed from the building and when necessary, transported to a hospital for examination and definitive care. Six-person crews were able to facilitate victim descent $3 \mathrm{~min}$ $21 \mathrm{~s}(7.8 \%)$ faster than 4-person crews. Additionally, victim descent occurred nearly 6 min more quickly for crews using the elevator rather than the stairs.

\section{Summary of Regression Analysis}

\section{Crew Size}

Generally, going from 3-person to 4-person crews significantly reduced the time to begin advancing the attack line, to begin advancing the second line, and to begin search and rescue. Task begin-time reductions were in the range of $1 \mathrm{~min} 30 \mathrm{~s}$. Going from 4-person to 5-person crews resulted in significant time reductions to begin each critical task in the range of 1 min to 2 min. Increasing crew size from 5-person to 6-person crews showed significant reductions in begin time, just over $1 \mathrm{~min}$, to advance the attack and second lines and for search and rescue on the fire floor (10th floor).

When assessing task end times and incrementing crew size by a single firefighter (i.e., 3 to 4,4 to 5 , and 5 to 6 ), the largest time 
improvements in time are seen when going from crew size 3 to 4 . As firefighter crews navigate the later tasks, the time improvements reach the $10 \mathrm{~min}$ to $15 \mathrm{~min}$ range. Very large time improvements are seen for the 10th Floor Search and Victim \#1 Rescue tasks (over $11 \mathrm{~min}$ ) when incrementing crew size from 4 to 5 . The improvements in All Tasks Complete end times are substantial (9.5 min to $12 \mathrm{~min}$ ) when incrementing crew size from 3 to 4 or from 4 to 5 .

\section{Fire Service Access Elevators}

All Tasks Complete occurred over 4 min more quickly when the elevators were utilized compared to stairs. Begin times for nearly every task above ground level and nearly all end times were reduced compared to stair ascent. Most of the reductions due to elevator usage were in the $2 \mathrm{~min}$ to $4 \mathrm{~min}$ range, with a few obvious exceptions in that using fire service access elevators more dramatically reduced times associated with upward and downward transport of people or equipment. Using elevators to transport air bottles and other equipment from the lobby to Staging allowed completion of Establishment of Stairwell Support over 10 min more quickly than moving the equipment manually up the stairs. Additionally, the transport of both Victim \#1 and Victim \#2 from Staging to the outside of the building was faster when using the elevators (compared to the stairs), by 2 min $41 \mathrm{~s}$ and 3 min 19 s, respectively. As a result, except for Establishment of Stairwell Support and Victim Descent, there were no ascent mode differences for task duration.

\section{Alarm Size and Ascent Mode}

About one third of the 27 distinct outcome-task comparisons between high and low alarm size were statistically significant. Six of the eight significant differences between high and low alarm sizes involved reductions of task begin times ranging from $1 \frac{1}{2}$ $\min$ to $2 \frac{1}{2} \mathrm{~min}$ in favor of the high alarm size response. These task begin times involved Primary Search of Floor 11, Victim \#2 Rescue, and Advance Line Above Fire.

\section{Combining Alarm Size and Crew Size}

Given the findings from the crew size analysis that adding one or two firefighters to a crew could generally achieve substantial task time decreases, a logical question is whether the meaningful benefits of a higher crew size could be realized by implementing a higher alarm response at a smaller crew size (e.g., 4/high compared to 5/low). The hypothesis might be that a high response with lower crew size might yield similar results in task timing to that of a low response with higher crew size.

Regression results shown in Appendix C3, comparing the effect of a high response with lower crew size to a low response with higher crew size showed that statistically significant differences occurred for low alarm response with 4 or 5 crew size compared to high response with crew sizes of 3 and 4 , respectively. There were significant reductions in begin times for all critical tasks from Advance Attack Line through Victim \#1 Rescue. Sizeable reductions of about 12 min were also seen for Victim \#2 Rescue. These results suggest that a low alarm response with crews of size 4 or 5 outperforms a high alarm response with crew sizes smaller by 1 firefighter. When decreases occurred, they were in the range of 1 to just under $2 \mathrm{~min}$. This pattern did not hold for low alarm response with crew size 6 compared to high response with crew size 5 . The observed differences in times were significantly higher for Search of Floor 11 and Advance Line Above Fire.

\section{Combining Alarm Response and Ascent}

In comparing different combinations of alarm response (high, low) and ascent mode (stairs, elevator), results contrast several combinations of alarm size and ascent mode.

Stairs/High vs. Stairs/Low - The alarm size had virtually no effect for critical task timings, with the exception of Search of the Floor Above the Fire (Floor 11) and Victim \#2 Rescue. High alarm size realized a mean reduction in the range of $1.5 \mathrm{~min}$ to 3.4 min for these tasks. The All Tasks Complete was also significantly smaller for high alarm size by $3 \mathrm{~min}$. No other task timing comparisons were statistically different.

Elevator/High vs. Elevator/Low - In the elevator scenarios, high alarm size led to eight significantly lower timings than did a low alarm response. Results show a 45 s reduction in begin time for Fire Out, Search of Fire Floor 10, and Victim \#1 Found. Small reductions of just over 1 min were noted in begin times for Search Floor 11 and Victim \#2 Found. Small reductions of $30 \mathrm{~s}$ to $2 \mathrm{~min}$ were also noted for times related to Advance Line Above Fire. No other task timing comparisons were statistically different.

\section{Fire Modeling Results}

In order to assess the hazard to occupants and firefighters as a consequence of different deployment configurations, computer fire modeling was performed. Three different 'standard' fires were simulated using the NIST Fire Dynamics Simulator (FDS) model. The three fires, characterized in the Handbook of the Society of Fire Protection Engineers (Hadjisophocleous and Mehaffey 2008) as slow, medium, and fast, ${ }^{33}$ grew exponentially with time and had burning characteristics similar to the experimental results of typical office cubicle fires (Madrzykowski et al. 2004)

Computer fire modeling demonstrated the effectiveness of a working fire sprinkler system: the FED values remained well below the threshold value of 0.3 regardless of crew size and ascent method. Thus, the overall hazard is greatly improved compared to the non-sprinklered fires for both firefighters and occupants. According to the National Fire Protection Association, a working sprinkler system is $96 \%$ effective at controlling the growth and spread of fires in structures (NFPA 2006). Due to a number of high-profile fires in high-rise buildings and considering their demonstrated effectiveness, sprinkler systems are often required in new high-rise buildings and many jurisdictions have required existing high-rise buildings to be retrofit with sprinkler systems.

However, sprinkler systems are not in all high-rise buildings. According to the National Fire Protection Association (NFPA 2011), $41 \%$ of high-rise office buildings are not protected by sprinkler systems (compared to $25 \%$ of high-rise "care of sick" facilities, $45 \%$ of high-rise hotels and $54 \%$ of high-rise apartment buildings). Therefore, much of this report is focused on analysis of fire department deployment configurations responding to fires in an unsprinklered high-rise building. 
Sprinkler systems are designed to control, rather than suppress fires. Fire department response is still required even in fully-sprinklered high-rises in order to extinguish the fire, search for and rescue occupants requiring assistance, and to control the sprinklers (limiting water damage). NFPA estimates that sprinkler systems fail to operate in $7 \%$ of structure fires (one of every fourteen fires) primarily due to human error. "Two-thirds $(65 \%)$ of the sprinklers failed to operate because the system had been shut off before the fire. Another one-sixth (16\%) failed because manual intervention defeated the system, for example, by shutting off the sprinklers prematurely. Lack of maintenance accounted for $11 \%$ of the sprinkler failures to operate and $5 \%$ occurred because the wrong type of system was present. Only 3 $\%$ of sprinkler failures involved damage to system components." (NFPA 2006)

Therefore, even if a large proportion of high-rise buildings in a local jurisdiction are protected by sprinkler systems, a fire department should be prepared to deploy resources to hazards consistent with unsprinklered structure fires.

For unsprinklered scenarios, the time advantages gained by larger engine crew sizes and by using elevators versus stairs impacted the calculated interior conditions, including temperature, visibility, and toxicity on the fire floor. For medium growth rate fires, firefighters entering the environment would encounter fires between $5 \mathrm{MW}$ to $11 \mathrm{MW}$ in size, depending on crew configuration and ascent method. This range in fire size can be approximately visualized as the equivalent of two cubicles on fire for a 6-person crew versus five cubicles on fire for a 3-person crew.

The calculations suggest that crew size and vertical ascent mode can significantly affect the likelihood of a successful rescue of victims on the fire floor. For victim rescue times discussed above, $\mathrm{FED}^{34}$ values in the cubicle where the victim was located ranged from 0.14 (6-person crew using the elevator) to 1.22 (3-person crew using the stairs). An FED value of less than 0.3 indicates that less than $11 \%$ of the population would be incapacitated by the toxic exposure, while an FED value of greater than 1.0 indicates that greater than $50 \%$ of the population would be incapacitated by the specific toxic exposure. Consistently, smaller crew sizes resulted in greater exposure to combustion products compared to larger crew sizes. Additionally, using the stairs delayed rescue and resulted in higher toxic exposure when compared to using the elevators.

\section{Evacuation Effects}

The high-rise time-to-task experiments were conducted in a vacant building. The only people present during the experiments were active participants or observers. Depending on the time of day, a typical commercial high-rise is not likely to be empty. A complete evacuation of occupants could require significant time. This is important for the time-to-task experiments, because depending on the local fire code, evacuating occupants would use the stairs and/or elevators. If evacuation is not complete prior to firefighter arrival, the presence of evacuees in stairwells would impede the progress of ascending firefighters. This would result in longer ascent times than those measured in the experiments. Therefore, these experiments are considered a best-case scenario.

\section{Summary}

The purpose of this report, in combination with the Residential Fireground Report, is to provide fire chiefs and local decision-makers with science-based relationships between deployment variables (crew size, alarm size, and ascent mode) and the resulting service outcomes, including risk to building occupants and firefighters, as well as the potential for property loss.

The results of these field experiments contribute to the body of knowledge about the fire service. First, the results establish a technical basis for the consideration of company crew size, alarm size and vertical response mode to NFPA 1710. The results also provide valid measures of total effective response force assembly on scene for high-rise fireground operations, as well as the expected performance of time-to-critical-task measures for high hazard high-rise commercial structure fires. Additionally, the results provide tenability measures associated with occupant exposure rates to the range of fires considered in this study involving a large (20 MW) fire on the 10th floor of a high-rise. The results of the project will also inform code provisions in Section 403 of the 2009 International Building Code which require fire service access elevators in new construction over $120 \mathrm{ft}(36.6 \mathrm{~m})$. 


\section{Future Research}

n order to realize improved firefighter safety and effectiveness, resource allocation and the deployment of resources on fire incidents of all hazard levels need consideration.

Future research should use similar methods for evaluating firefighter resource deployment to extend the findings of this report to quantify the effects of crew size and firefighter assembly times on fires in medium hazard and other high hazard structures, including multiple-family residences, schools, hospitals, places of assembly, warehouses, mercantile establishments consisting of a row of stores and restaurants, night clubs, warehouse facilities, responses to large scale non-fire incidents, or technical rescue operations.

Additionally, resource deployment to multiple-casualty disasters should be studied to provide insight into levels of risks specific to individual communities and to recommend resource deployment proportionate to such risk. Future studies should continue to investigate the effects of resource deployment on the safety of both firefighters and the civilian population to better inform public policy.

Investigating firefighter and civilian safety should also include future research to assess counter-flow relationships between firefighters entering and occupants exiting stairwells during evacuation in a variety of structures.
Additional research is necessary to understand the economics of improving fire resilience of structures within communities including costs vs. benefit, total economic burden, role of fire protection via fire service response, fire-resistant design, and/or fire-resistant materials.

Future research is needed in the area of suppression effectiveness based on the relationship between crew size and fire suppression time for a range of different fires.

Future research opportunities should also consider EMS response to multiple casualties in high-rise fires or other high hazard occupancies.

Finally, research is needed in the area of radio communication during a high hazard incident, when hundreds of firefighters are at risk and communication is often profuse, interrupted, misunderstood, garbled and/or chaotic. These issues extend beyond the research currently underway in regard to radio equipment and frequencies, which play a vital role in firefighter safety on the fire ground. 


\section{Acknowledgments}

A project of this magnitude extends significantly beyond the capabilities and expertise of the report authors. The following groups and individuals were instrumental in the success of the high-rise fireground field experiments.

Subject Matter Experts — Russ Sanders, NFPA, Louisville KY Chief Retired; Dennis Compton, IFSTA, NFFF, Mesa, AZ Chief Retired; Peter Van Dorpe, Training Chief Chicago, IL; David Rohr, Chief Fairfax City, VA/ Fairfax County, VA Operations Chief, Retired; Vincent Dunn, Deputy Chief FDNY, Retired; Ben Klaene, Cincinnati, OH Training Chief, Retired; James Walsh, Deputy Chief, Fairfax County, VA; Richard Bowers, Chief Montgomery County, MD; and Richard Travers, Deputy Chief, FDNY, Retired.

NIST Instrumentation Personnel — Michael Selepak, Artur Chernovsky, and Scott Bareham.

IAFF Staff and Interns - Data Entry/ Timers/Physiological Monitors - Ron Benedict, Jamie Grimes, Mike Haertel, Joe Bertoni, Alexa Sherman, and Devon Wolcott.

IAFF Graphic Design — Kristin Hazlett

Chief James Schwartz, Arlington County Fire and Rescue Department for supporting this study to an unprecedented degree.

- Vornado/Charles E. Smith, Washington Division Vornado Realty Trust — Building Contact, Tanya Seyfert.

- Vornado/ Charles E. Smith, Washington Division Vornado Realty Trust Building Engineers - Dave McCann, Mike Fitzgerald, Nate Ford, Keith White, Carlton Carter ('CC').

BullEx, Inc., digital fire and simulated smoke display equipment — Ryan O’Donnell, President, John Blackburn, Inventor, Simon Balint, VP of Sales.

http://www.bullexsafety.com/about.aspx

Firefighting Crew Resources - David Rohr, Chief Fairfax City, VA/Fairfax County, VA Operations Chief, Retired.

Experiment Logistics and Monitoring - Richard Merrell, Fairfax County Fire and Rescue Department.

Building Construction and Props - Randy Carter, Chris Hinkle, Chad McDonald, Joey Fuller III, Brady Miller, and James Falise from Montgomery County Fire and Rescue Department and Richard Merrell from Fairfax County Fire and Rescue Department.
- Experiment Props, Radios and Apparatus Parking — Charles Kramaric, Arlington County Fire and Rescue Department and J.J. Walsh, Fairfax County Fire and Rescue Department.

The Fire Chiefs and Operations Chiefs of the participating departments along with the dedicated Fire Officers and Firefighters who performed the difficult work of high-rise structural firefighting safely and courageously.

- Arlington County Fire Department

- Alexandria Fire Department

- Fairfax County Fire and Rescue Department

- Prince William County Fire and Rescue Department

- District of Columbia Fire and EMS Department

- Montgomery County Fire and Rescue Services

- Howard County Department of Fire and Rescue Services

- Fairfax City Fire and Rescue Department

- Prince George's County Fire/EMS Department

- Loudoun County Fire and Rescue Department

- Manassas City Fire and Rescue Department

- Stafford County Fire and Rescue Department

Timers - dedicated fire officers and firefighters from Arlington County Fire and Rescue Department, Fairfax County Fire and Rescue Department, Fairfax City Fire and Rescue, Prince George's County Fire/EMS Department, and Alexandria Fire Department assigned to the project for data collection.

Metropolitan Washington Airports Authority — for the parking area to stage several pieces of fire apparatus during the experiments.

- Skidmore College — Denise Smith, Skidmore College and Jeannie Haller, Skidmore College for supplemental physiological study to bolster the significance of the main study results.

- Zephyr - Ben Morris for physiological status monitoring (PSM) high-rise operational environment.

Finally, the study investigators wish to express our gratitude to the public safety officials and the citizens of Arlington County, Virginia for their support of the project. 
Albert, C.M., Mittleman, M.A., Chae, C.U., Lee, I.M., Hennekens, C.H.and Manson, J.E. (2000). Triggering of sudden death from cardiac causes by vigorous exertion. New England Journal of Medicine, Vol. 343, No. 19, 1355-1361.

Alpert, R. (2008). Chapter 2-2: Ceiling Jet Fires. SFPE Handbook of Fire Protection Engineering, 4th ed. National Fire Protection Association, Quincy, MA.

Allen, K., Rushowy, K., and Poisson, J., High-rise blaze strands 1,200 people. The Star.com, Published on Sat Sep 252010. http://www.thestar.com/news/gta/2010/09/25/highrise_blaze_st rands_1200_people.html.April 4, 2013.

Averill, J.D., Moore-Merrell, L., Baroway, A., Santos, R., Peacock, R., Notarianni, K. and Wissoker, D. (2010). Report on Residential Fireground Field Experiments. NIST Technical Note 1661. National Institute of Standards and Technology, Gaithersburg, MD, April 2010.

- Babrauskas,V. and Krasny, J. (1985). Fire Behavior of Upholstered Furniture. NBS Monograph 173. National Bureau of Standards (currently National Institute of Standards and Technology), Gaithersburg, MD, November 1985.

- Babrauskas, V. (2008). Chapter 3-1: Heat Release Rates. SFPE Handbook of Fire Protection Engineering, 4th ed. National Fire Protection Association, Quincy, MA.

BBC News - Asia Pacific. (2010). Shanghai high-rise flats fire leaves dozens dead. November 15, 2010.

http://www.bbc.co.uk/news/world-asia-pacific-11759276.

Best, R. (2013). Investigation report on the MGM hotel fire, National Fire Investigation Association, February 2013. http://www.nfpa.org/assets/files/Press\%20Room/LasVegasMG MGrand.pdf.

Calfee, M. (2013). Looking Back: The Winecoff Hotel Fire Tragedy. February 2013.

http://my.firefighternation.com/profiles/blogs/historic-loss-of-li fe-the? $q=$ profiles/blogs/historic-loss-of-life-the.

- Chicago Tribune News. (2012). High-rise fire victim: 'When elevator door opened ... she just got blasted." Chicago Tribune News, January 8, 2012.

http://articles.chicagotribune.com/2012-01-08/news/chi-fire-re ported-at-lake-shore-drive-high-rise-20120108_1_smoke-inhal ation-elevator-high-rise-apartment, February 2013.

Chubb, M. and Caldwell, J. (1995). Tragedy in a Residential High-Rise. Memphis, Tennessee,March 1995.

http://www.fireengineering.com/articles/print/volume-148/issu e-3/features/tragedy-in-a-residential-high-rise-memphis-tennes see.html, February 2013.

Chubb, M.; Jennings, C., Routley, J.G. (1991). High-rise Office Building Fire One Meridian Plaza Philadelphia, Pennsylvania.
United States Fire Administration Fire Investigations Program. FEMA and USFA, December 18, 1991.

http://www.interfire.org/res_file/pdf/Tr-049.pdf, February 2013.

- Clark County Fire Department

http://fire.co.clark.nv.us/(S(bnshla55amedp345ps5ylj55))/MG

M.aspx. February, 2013.

Cornell University, ILR School, Kheel Center, Remembering the 1911 Triangle Factory Fire;

http://www.ilr.cornell.edu/trianglefire/supplemental/timeline.ht $\mathrm{ml}$, February 2011.

- ctvtoronto.ca. High-rise evacuated after major fire injures 14. Published Friday, September 24, 2010 11:46PM EDT. http://toronto.ctvnews.ca/highrise-evacuated-after-major-fire-i njures-14-1.556369\#ixzz2PYIbhj98 . April 4, 2013

DCA (1991). Technical Bulletin 133, Flammability Test Procedure for Seating Furniture for Use in Public Occupancies, State of California, Department of Consumer Affairs, Bureau of Home Furnishings and Thermal Insulation, North Highlands, CA, January 1991.

Gust, J.C., Graham, R.M., Lombardi, M.A. (2009).NIST Recommended Practice Guide for Stopwatch and Timer Calibrations (2009 edition). NIST Special Publication 960-12. National Institute of Standards and Technology, Gaithersburg, MD, January 2009.

Hadjisophocleous, G.V. and Mehaffey, J.R. (2008). Chapter 5-11: Fire Scenarios. SFPE Handbook of Fire Protection Engineering, 4th ed. National Fire Protection Association, Quincy, MA.

- Hall, J.R. (2011). High-Rise Building Fires. NFPA.

Hamins, A., Bundy, M. and Dillon, S. (2005). Characterization of Candle Flames. Journal of Fire Protection Engineering, Vol. 15, November 2005.

- International Association of Fire Training Association. (2010). Structural Fire Fighting: Initial Response, Strategy and Tactics. Fire Protection Publications, 2nd Edition, p 162. October 2010.

International Association of Fire Training Association. (2011). Structural Fire Fighting: High-Rise Fire Fighting. Fire Protection Publications, 2nd Edition, p 14. July 2011.

ISO 13571: Life-threatening Components of Fire — Guidelines for the Estimation of Time Available for Escape Using Fire Data. (2007). International Standards Organization, Geneva.

Karter, M.J. Jr. (2008). U.S. Fire Loss for 2007. NFPA Journal, September/October 2008.

Karter, M.J. Jr. (2012) Firefighter Injuries in the United States. NFPA, October 2012 
Kerber, S. and Madrzykowski, D. (2007). Evaluating Positive Pressure Ventilation In Large Structures: High-Rise Fire Experiments. NISTIR 7468. National Institute of Standards and Technology, Gaithersburg, MD, November 2007. http://fire.nist.gov/bfrlpubs/fire07/PDF/f07055.pdf

Klaene, B. and Sanders, R. (2007). Structural Firefighting: Strategies and Tactics- High-Rise. Jones and Bartlett, November 2007.

Klote, J.H. (1993). Method for Calculation of Elevator Evacuation Time. Journal of Fire Protection Engineering. Vol. 5, No. 3, 86-89.

- Los Angeles Fire Department, Historical Archive. (1988). First Interstate Bank Fire. May 4, 1988.

http://www.lafire.com/famous_fires/1988-0504_1stInterstateFir e/050488_InterstateFire.htm, February 2013.

- Madrzykowski D. and Walton W.D. (2004). Cook County Administration Building Fire, 69 West Washington, Chicago, Illinois, October 17, 2003: Heat Release Rate Experiments and FDS Simulations. NIST Special Publication SP-1021. National Institute of Standards and Technology, Gaithersburg, MD, July 2004.

Madrzykowski, D. (2008). Impact of a Residential Sprinkler on the Heat Release Rate of a Christmas Tree Fire. NISTIR 7506. National Institute of Standards and Technology, Gaithersburg, MD, May 2008.

Madrzykowski, D. and Kerber, S. (2008). Sofa Heat Release Rate Experiments, National Institute of Standards and Technology, Gaithersburg, MD, 2008.

Madrzykowski, D. and Kerber, S. (2009). Fire Fighting Tactics Under Wind Driven Conditions: Laboratory Experiments. NIST TN 1618. National Institute of Standards and Technology, Gaithersburg, MD, January 2009.

McGrattan, K.M., McDermott, R., Hostikka, S. and Floyd, J.E. (2012). Fire Dynamics Simulator, User's Guide Version 6 (SVN 13844). NIST Special Publication 1019. National Institute of Standards and Technology, Gaithersburg, MD, November 2012.

- McGrattan, K.M., McDermott, R., Hostikka, S. and Floyd, J.E. (2012b). Fire Dynamics Simulator, Technical Reference Guide, Volume 2: Verification. Version 6 (SVN 13844). NIST Special Publication 1019. National Institute of Standards and Technology, Gaithersburg, MD, November 2012.

- McGrattan, K.M., McDermott, R., Hostikka, S. and Floyd, J.E. (2012c). Fire Dynamics Simulator, Technical Reference Guide, Volume 2: Experimental Validation. Version 6 (SVN 13844). NIST Special Publication 1019. National Institute of Standards and Technology, Gaithersburg, MD, November 2012.

Mittleman, M.A, Maclure, M., Tofler, G.H., Sherwood, J.B., Goldberg, R.J. and Muller, J.E. (1993). Triggering of acute myocardial infarction by heavy physical exertion. New England Journal of Medicine, Vol. 329, No. 23, 1677-1683.
- Moore-Merrell, L., Zhou, D., McDonald, S., Fisher, E. and Moore, J. (2008). Contributing Factors to Firefighter Line-of-Duty Deaths in the United States. Journal of Fire Service Leadership and Management, Vol. 2, No. 2, 3-23.

Moore-Merrell, L., Zhou, D., McDonald-Valentine, S., Goldstein, R. and Slocum, C. (2009). Contributing Factors to Firefighter Line-of-Duty Injury in Metropolitan Fire Departments in the United States. Journal of Fire Service Leadership and Management, Vol. 3, No. 1, 25-36.

Muha, T. (2012). Evaluating Occupant Load Factors for Business Operations. Report. Worcester Polytechnic University, Worcester, MA, April 2012.

National Incident Management System (NIMS) Fact Sheet. Department of Homeland Security (DHS)/FEMA. http://www.fema.gov/pdf/emergency/nims/NIMSFactSheet.pdf , February 2013.

Nelson, H.E. and Mowrer, F.W. (2002). Chapter 3-14: Emergency Movement. SFPE Handbook of Fire Protection Engineering, 3rd ed. National Fire Protection Association, Quincy, MA.

NFPA 1221: Standard for the Installation, Maintenance, and Use of Emergency Services Communications Systems. (2010). National Fire Protection Association, Inc., One Batterymarch Park, Quincy, Massachusetts.

NFPA 1403: Standard on Live Fire Training Evolutions. (2012). National Fire Protection Association, Inc., One Batterymarch Park, Quincy, Massachusetts.

NFPA 1584: Standard on the Rehabilitation Process for Members During Emergency Operations and Training Exercises. (2008). National Fire Protection Association, Inc., One Batterymarch Park, Quincy, Massachusetts.

NFPA 1710: Standard for the Organization and Deployment of Fire Suppression Operations, Emergency Medical Operations, and Special Operations to the Public by Career Fire Departments. (2010). National Fire Protection Association, Inc., One Batterymarch Park, Quincy, Massachusetts.

NFPA Fire Protection Handbook, 20th Edition, Copyright (C) 2008 NFPA

NIOSH: Fire Fighter Fatality Investigation and Prevention Program. http://www.cdc.gov/niosh/fire/ March, 2013.

- Ohlemiller,T.J., Mulholland, G.W., Maranghides, A., Filliben, J.J. and Gann, R.G. (2005). Fire Tests of Single Office Workstation. NIST NCSTAR 1-5C. National Institute of Standards and Technology, Gaithersburg, MD, September 2005.

OSHA. (1995). Letter to Thomas N. Cooper, Purdue University, from Paula O. White, Director of Federal-State Operations, U.S. Department of Labor, Occupational Safety \& Health Administration, November 1, 1995. 
Pagni, P.J. and Joshi, A.A. (1991). Glass Breaking In Fires. Fire Safety Science 3: 791-802.

- The New York Times. (1991). Philadelphia Tower Set Ablaze by Rags, Commissioner Says. The New York Times, April 11, 1991. http://query.nytimes.com/gst/fullpage.html?res=9D0CE1D6113 CF932A25757C0A967958260, February 2013.

Reneke, P.A., Tofilo, P., Peacock, R.D. and Hoskins, B.L. (2012). Simple Estimates of Combined Stairwell / Elevator Egress in Buildings. NIST Technical Note 1722. National Institute of Standards and Technology, Gaithersburg, MD.

Routley, J.G. (1988). Technical Report Series: Interstate Bank Building Fire. U.S. Fire Administration/FEMA. Contract EMW-8-4321.

http://www.webcitation.org/query?url=http $\% 3 \mathrm{~A} \% 2 \mathrm{~F} \% 2 \mathrm{Fwww}$. lafire.com $\% 2$ Ffamous_fires $\% 2 \mathrm{~F} 880504$ 1stInterstateFire $\% 2 \mathrm{FF}$ EMA-TecReport\%2FFEMA-report.htm\&date=2010-07-13, February 2013

Stroup, D.W., DeLauter, L., Lee, J. and Roadarmel, G. (1999). Scotch Pine Christmas Tree Fire Tests, Report of Test FR 4010. National Institute of Standards and Technology, Gaithersburg, MD, December 1999.

Stroup, D.W., DeLauter, L., Lee, J. and Roadarmel, G. (2001). Upholstered Chair Fire Test Using a California TB 133 Burner Ignition Source, Report of Test FR 4012. National Institute of Standards and Technology, Gaithersburg, MD, December 2001.

Stroup, D.W. and Madrzykowski, D. (2003). Heat Release Rate Tests of Plastic Trash Can Containers, Report of Test FR 4018. National Institute of Standards and Technology, Gaithersburg, MD, April 2003.
Tewarson, A. (2008). Chapter 3-4: Generation of Heat and Gaseous, Liquid, and Solid Products in Fires. SFPE Handbook of Fire Protection Engineering, 4th ed. National Fire Protection Association, Quincy, MA.

TriData Corporation. (2005). The Economic Consequences of Firefighter Injuries and Their Prevention, Final Report. National Institute of Standards and Technology, U.S. Department of Commerce, Gaithersburg, MD. 2005.

- U.S. Fire Administration. (1989). Technical Report Series: Five-Fatality High-Rise Office Building Fire Atlanta, Georgia, USFA-TR-033, June 1989.

http://www.usfa.fema.gov/downloads/pdf/publications/tr-033.p df, February 2013.

U.S. Fire Administration. (1988). Technical Report Series: Apartment Building Fire East 50th Street, New York City. USFA-TR-019, January 1988.

U.S. Fire Administration. (1993). Technical Report Series: The World Trade Center Bombing: Report and Analysis, New York City, New York. USFA-TR-076, February 1993.

USNRC (2007). Verification and Validation of Selected Fire Models for Nuclear Power Plant Applications. Volume 2: Experimental Uncertainty. Washington, DC: United States Nuclear Regulatory Commission. p. 1824.

Winecoff Hotel. (2013). Tragedy In The South: The Winecoff Hotel Fire of 1946. A documentary depicting the horror of our country's deadliest hotel fire.

http://www.winecoffhotelfire.com/index.html, February 2013. 


\section{Time-to-Task Data Collection Chart - High-Rise Operations}

(Chart colors denote tasks assigned to individual timers)

\begin{tabular}{|c|c|c|}
\hline Task & Start & Stop \\
\hline Position Engine & Wheels stopped, brake engaged (4:44) & Chocks in place \\
\hline Connect to Standpipe & $\begin{array}{l}\text { Touch hose at engine to hook into } \\
\text { Siamese }\end{array}$ & $\begin{array}{l}\text { Both hose lines connected to standpipe } \\
\text { Siamese connection }\end{array}$ \\
\hline Test Hydrant & & Test hydrant - Water flowing from hydrant \\
\hline Connect to Hydrant & & $\begin{array}{l}\text { Connection from pumper to hydrant } \\
\text { complete }\end{array}$ \\
\hline $\begin{array}{l}\text { Lines Charged (hydrant to pumper and } \\
\text { pumper to Siamese) }\end{array}$ & & Both lines charged \\
\hline $\begin{array}{l}\text { Initial Size-up } \\
\text { (Check off each Task } \\
\text { as completed }\end{array}$ & $\begin{array}{l}\text { First Dispatch (0:00) } \\
\square T a l k \text { with building manager } \\
\square C h e c k \text { alarm panel - locate fire and } \\
\text { check status of building systems and } \\
\text { HVAC }\end{array}$ & $\begin{array}{l}\text { 口Access building keys } \\
\text { पLocate fire control room } \\
\text { Give command statement (time stamp) }\end{array}$ \\
\hline Establish Command & First Dispatch (0:00) & $\begin{array}{l}\text { Officer crosses threshold, makes } \\
\text { command statement (time stamp) }\end{array}$ \\
\hline Lobby Control & First Dispatch (0:00) & $\begin{array}{l}\text { Key elevator for recall (check to assure } \\
\text { they are all down) (time stamp) } \\
\text { Verify status of elevators, pop panel from } \\
\text { each and check with flashlight, and report } \\
\text { whether or not elevators are functioning } \\
\text { (time stamp) } \\
\text { Attack and evacuation stairwells/elevators } \\
\text { are located, designated, and confirmed } \\
\text { (time stamp) }\end{array}$ \\
\hline Establish IRIC & & $\begin{array}{l}2 \text { crew members assembled on } 8^{\text {th }} \text { floor, } \\
\text { not on air, standing by and ready to assist } \\
\text { in the event of firefighter emergency }\end{array}$ \\
\hline
\end{tabular}




\begin{tabular}{|c|c|c|}
\hline Establish RIT & $\begin{array}{l}\text { Crew enters stairwell/elevator from } \\
\text { lobby }\end{array}$ & Crew assembled on $8^{\text {th }}$ floor landing \\
\hline Establish Medical, Rehab- Ambulance 1 & Initial arrival, Ambulance 1 & Crew enters staging with all equipment \\
\hline Establish Medical, Ambulance 2 & Initial arrival, Ambulance 2 & Crew enters staging with all equipment \\
\hline Establish Medical, Ambulance 3 & Initial arrival, Ambulance 3 & Crew enters staging with all equipment \\
\hline Establish Staging & Initial arrival, Truck 3 & Crew enters staging \\
\hline Establish Stairwell/Elevator Support & Initial arrival, Truck 5 & $\begin{array}{l}35 \text { air cylinders, extra search rope, and } 2 \\
\text { coolers brought to staging }\end{array}$ \\
\hline Attack Crew Ascension & $\begin{array}{l}\text { Crew enters stairwell/elevator from } \\
\text { lobby }\end{array}$ & Crew assembled on $8^{\text {th }}$ floor landing \\
\hline Attack Line-Connect To Standpipe & $\begin{array}{l}\text { Crew enters stairwell/elevator from } \\
\text { lobby }\end{array}$ & Attack line connected to gated wye valve \\
\hline Advance Attack Line & $\begin{array}{l}\text { Attack line nozzle through } 10^{\text {th }} \text { floor } \\
\text { stairwell door }\end{array}$ & Attack line- Water on Fire \\
\hline Second Line Crew Ascension & Crew enter stairwell/elevator from lobby & Crew assembled on $8^{\text {th }}$ floor landing \\
\hline Second Line-Connect to Standpipe & $\begin{array}{l}\text { Crew enters stairwell/elevator from } \\
\text { lobby }\end{array}$ & Second line connected to wye valve \\
\hline Advance Second Line & $\begin{array}{l}\text { Second line nozzle through } 10^{\text {th }} \text { floor } \\
\text { stairwell door }\end{array}$ & Second line-Water on Fire \\
\hline Fire Out & $\begin{array}{l}\text { Attack line nozzle through } 10^{\text {th }} \text { floor } \\
\text { stairwell door }\end{array}$ & $\begin{array}{l}\text { Attack and second lines cross target } \\
\text { threshold }\end{array}$ \\
\hline Check for Fire Extension on $10^{\text {th }}$ Floor & Firefighters touch pike pole & $\begin{array}{l}\text { Thirty pulls on weighted pike pole } \\
\text { completed }\end{array}$ \\
\hline Positive Pressure Ventilation-Fans & & $1 \mathrm{~min}$ after Fire Out (time stamp) \\
\hline Roof Ventilation & & 1 min after Fire Out (time stamp) \\
\hline
\end{tabular}




\begin{tabular}{|c|c|c|}
\hline $\begin{array}{l}\text { Search and Rescue Crew Ascension (Floor } \\
\text { 10) }\end{array}$ & $\begin{array}{l}\text { Crew enters stairwell/elevator from } \\
\text { lobby }\end{array}$ & Crew assembled on $8^{\text {th }}$ floor landing \\
\hline Search and Rescue (Floor 10) & Crew through $10^{\text {th }}$ floor stairwell door & Primary search on $10^{\text {th }}$ floor completed \\
\hline Victim \#1 Found (Floor 10) & Crew through $10^{\text {th }}$ floor stairwell door & Victim found on $10^{\text {th }}$ floor \\
\hline Victim \#1 Rescue (Floor 10) & Victim found on $10^{\text {th }}$ floor & Victim arrives on $8^{\text {th }}$ floor landing \\
\hline Victim \#1 Descent & Victim exits $8^{\text {th }}$ floor & Victim exits building \\
\hline Line Above Fire Crew Ascension & $\begin{array}{l}\text { Crew enters stairwell/elevator from } \\
\text { lobby }\end{array}$ & $\begin{array}{l}\text { All personnel assembled on } 8^{\text {th }} \text { floor } \\
\text { landing (stairwell) }\end{array}$ \\
\hline Line Above Fire-Connect to Standpipe & Crew ascends from lobby & Line above fire connected to wye valve \\
\hline Advance Line Above Fire & $\begin{array}{l}\text { Line above fire nozzle through } 11^{\text {th }} \text { floor } \\
\text { stairwell door }\end{array}$ & Line above fire at target threshold \\
\hline Check for Fire Extension on $11^{\text {th }}$ floor & Firefighter(s) touch weighted pike pole & Thirty pulls on pike pole completed \\
\hline $\begin{array}{l}\text { Search and Rescue Crew Ascension (Floor } \\
\text { 11) }\end{array}$ & Crew enter stairwell/elevator from lobby & $\begin{array}{l}\text { All personnel assembled on } 8^{\text {th }} \text { floor } \\
\text { landing (stairwell) }\end{array}$ \\
\hline Search and Rescue (Floor 11) & Crew through $11^{\text {th }}$ floor stairwell door & Primary search on $11^{\text {th }}$ floor completed \\
\hline Victim \#2 Found (Floor 11) & Crew through $11^{\text {th }}$ floor stairwell door & Victim found on $11^{\text {th }}$ floor \\
\hline Victim \#2 Rescue (Floor 11) & Victim found on $11^{\text {th }}$ floor & Victim arrives on $8^{\text {th }}$ floor landing \\
\hline Victim \#2 Descent (Staging to Lobby) & Victim exits $8^{\text {th }}$ floor & Victim exits building \\
\hline $\begin{array}{l}\text { Search and Rescue (Upper Floor) Crew } \\
\text { Ascension }\end{array}$ & $\begin{array}{l}\text { Crew enters stairwell/elevator from } \\
\text { lobby }\end{array}$ & Crew assembled on $8^{\text {th }}$ floor landing \\
\hline Search and Rescue (Upper Floor) & Crew through upper floor stairwell door & $\begin{array}{l}\text { Search completed (must have all } 20 \text { prop } \\
\text { markers) }\end{array}$ \\
\hline $\begin{array}{l}\text { Search and Rescue Crew Ascension (Floor } \\
\text { 9) }\end{array}$ & $\begin{array}{l}\text { Crew enters stairwell/elevator from } \\
\text { lobby }\end{array}$ & Crew assembled on $8^{\text {th }}$ floor landing \\
\hline Search and Rescue (Floor 9) & Crew through $9^{\text {th }}$ floor stairwell door & $\begin{array}{l}\text { Search completed (must have all } 20 \text { prop } \\
\text { markers) }\end{array}$ \\
\hline
\end{tabular}




\section{Company Protocols: Crew Size of 3}

COMPANY

Engine 1

\section{TASK ASSIGNMENTS}

Assume command

-Access building keys

-Locate fire control room

-Check alarm panel

-Give command statement

-Check status of elevators

-Designate attack and evacuation stairwells

Join Engine 2 to form attack crew

-Connect to gated wye valve on 9th floor

-Advance attack line to 10th floor

-Simulate water on fire

-Maintain sustained attack

Truck 1

Establish Initial Rapid Intervention Crew

Once Rapid Intervention Team is established, begin search and rescue on fire floor

-Check for extension, complete 30 reps on weighted pike pole

-Follow search pattern on floor, hit bu ers

-If victim is found, evacuate victim to 8th floor

\begin{tabular}{l|l}
\hline Ambulance 1 & Establish firefighter rehab on 8th floor \\
\hline Engine 2 & Join Engine 1 to join attack crew \\
& -Connect to gated wye valve on 9th floor \\
& -Advance attack line to 10th floor \\
& -Simulate water on fire \\
& -Maintain sustained attack \\
\hline Ambulance 2 & Report to 8th floor staging area \\
& -Package and transport Victim \#1 \\
\hline Engine 3 & Establish back up line \\
& -Connect to gated wye valve on 9th floor \\
& -Advance back up line to 10th floor \\
& -Simulate water on fire \\
& -Maintain sustained attack \\
\hline Truck 2 & Establish Rapid Intervention Team on 8th floor \\
\hline Ambulance 3 & Report to 8th floor staging area \\
\hline BC 1 & -Package and transport Victim \#2 \\
\hline Engine 4 & Incident Command \\
\hline Truck 3 & Relieve attack line crew \\
\hline Truck 4 & Establish staging on 8th floor \\
\hline & Search and rescue, floor above fire \\
\hline & -Follow search pattern on floor, hit bu ers \\
& -If victim is found, evacuate victim to 8th floor \\
\hline
\end{tabular}


\begin{tabular}{l|l} 
Engine 5 & Establish 3rd line to floor above the fire
\end{tabular}

-Connect to standpipe on 11th floor

-Advance 3rd line to compartment above the fire

-Check for extension, complete 30 reps on weighted pike pole

\begin{tabular}{|c|c|}
\hline Ambulance 4 & Report to staging \\
\hline Engine 6 & $\begin{array}{l}\text { Lobby control } \\
\text {-Establish base outside of building } \\
\text {-Logistical support } \\
\text {-Check fire control room }\end{array}$ \\
\hline Ambulance 5 & Report to base \\
\hline Engine 7 & Relieve back up line crew \\
\hline Ambulance 6 & Report to base \\
\hline Engine 8 & $\begin{array}{l}\text { Search and rescue upper floor (13th) } \\
\text {-Follow search pattern on floor } \\
\text {-Gather all } 20 \text { markers }\end{array}$ \\
\hline Ambulance 7 & Report to base \\
\hline BC 3 & Division 11 Command \\
\hline Truck 5 & $\begin{array}{l}\text { Elevator/stairwell support } \\
\text {-Transport } 35 \text { air cylinders and } 2 \text { water coolers to staging }\end{array}$ \\
\hline Ambulance 8 & Report to base \\
\hline Engine 9 & Relieve 3rd line crew \\
\hline Ambulance 9 & Report to base \\
\hline $\mathrm{BC} 4$ & Ventilation group supervisor \\
\hline Engine 10 & Report to staging, tactical reserve \\
\hline Engine 11 & Report to staging, tactical reserve \\
\hline Engine 12 & Report to base \\
\hline Truck 6 & $\begin{array}{l}\text { Ventilation of attack stairwell } \\
\text {-Proceed to roof and burp hatch when "Fire Out" is heard }\end{array}$ \\
\hline BC 5 & Report to staging \\
\hline Truck 7 & $\begin{array}{l}\text { Relieve search and rescue crew on fire floor } \\
\text {-If victim is found, evacuate victim to 8th floor }\end{array}$ \\
\hline Truck 8 & $\begin{array}{l}\text { Search and rescue lower floor (9th) } \\
\text {-Follow search pattern on floor } \\
\text {-Gather all } 20 \text { markers }\end{array}$ \\
\hline Truck 9 & $\begin{array}{l}\text { Relieve search and rescue crew on floor above fire } \\
\text {-If victim is found, evacuate victim to 8th floor }\end{array}$ \\
\hline Truck 10 & Report to staging, tactical reserve \\
\hline $\mathrm{BC} 6$ & Report to staging, tactical reserve \\
\hline Truck 11 & Report to staging, tactical reserve \\
\hline Truck 12 & Report to base \\
\hline
\end{tabular}




\section{Company Protocols: Crew Size of 4}

\begin{tabular}{|c|c|}
\hline COMPANY & TASK ASSIGNMENTS \\
\hline Engine 1 & $\begin{array}{l}\text { Assume command } \\
\text {-Access building keys } \\
\text {-Locate fire control room } \\
\text {-Check alarm panel } \\
\text {-Give command statement } \\
\text {-Check status of elevators } \\
\text {-Designate attack and evacuation stairwells } \\
\text { Join Engine } 2 \text { to form attack crew } \\
\text {-Connect to gated wye valve on 9th floor } \\
\text {-Advance attack line to 10th floor } \\
\text {-Simulate water on fire } \\
\text {-Maintain sustained attack }\end{array}$ \\
\hline Truck 1 & $\begin{array}{l}\text { Search and rescue. 10th floor } \\
\text {-Check for extension, complete } 30 \text { reps on weighted pike pole } \\
\text {-Follow search pattern on floor, hit bu ers } \\
\text {-If victim is found, evacuate victim to 8th floor }\end{array}$ \\
\hline Ambulance 1 & Establish firefighter rehab on 8th floor \\
\hline Engine 2 & Establish Initial Rapid Intervention Crew \\
\hline & $\begin{array}{l}\text { Once Rapid Intervention Team is established, join Engine } 1 \text { to form attack crew } \\
\text {-Connect to gated wye valve on 9th floor } \\
\text {-Advance attack line to 10th floor } \\
\text {-Simulate water on fire } \\
\text {-Maintain sustained attack }\end{array}$ \\
\hline Ambulance 2 & $\begin{array}{l}\text { Report to 8th floor staging area } \\
\text {-Package and transport Victim \#1 }\end{array}$ \\
\hline Engine 3 & $\begin{array}{l}\text { Establish back up line } \\
\text {-Connect to gated wye valve on } 9 \text { th floor } \\
\text {-Advance back up line to } 10 \text { th floor } \\
\text {-Simulate water on fire } \\
\text {-Maintain sustained attack }\end{array}$ \\
\hline Truck 2 & Establish Rapid Intervention Team on 8th floor \\
\hline Ambulance 3 & $\begin{array}{l}\text { Report to 8th floor staging area } \\
\text {-Package and transport Victim \#2 }\end{array}$ \\
\hline $\mathrm{BC} 1$ & Incident Command \\
\hline Engine 4 & Relieve attack line crew \\
\hline Truck 3 & $\begin{array}{l}\text { Establish staging on 8th floor } \\
\text { Ventilation of attack stairwell }\end{array}$ \\
\hline Truck 4 & $\begin{array}{l}\text {-Proceed to roof and burp hatch when "Fire Out" is heard } \\
\text { Search and rescue, floor above fire } \\
\text {-Follow search pattern on floor, hit bu ers } \\
\text {-If victim is found, evacuate victim to 8th floor }\end{array}$ \\
\hline
\end{tabular}




\begin{tabular}{|c|c|}
\hline BC 2 & Division 10 Command \\
\hline Engine 5 & $\begin{array}{l}\text { Establish 3rd line to floor above the fire } \\
\text {-Connect to standpipe on } 11 \text { th floor } \\
\text {-Advance 3rd line to compartment above the fire } \\
\text {-Check for extension, complete } 30 \text { reps on weighted pike pole }\end{array}$ \\
\hline Ambulance 4 & Report to staging \\
\hline Engine 6 & $\begin{array}{l}\text { Lobby control } \\
\text {-Establish base outside of building } \\
\text {-Logistical support } \\
\text {-Check fire control room }\end{array}$ \\
\hline Ambulance 5 & Report to base \\
\hline Engine 7 & Relieve back up line crew \\
\hline Ambulance 6 & Report to base \\
\hline Engine 8 & $\begin{array}{l}\text { Search and rescue upper floor (13th) } \\
\text {-Follow search pattern on floor } \\
\text {-Gather all } 20 \text { markers }\end{array}$ \\
\hline Ambulance 7 & Report to base \\
\hline $\mathrm{BC} 3$ & Division 11 Command \\
\hline Truck 5 & $\begin{array}{l}\text { Elevator/stairwell support } \\
\text {-Transport } 35 \text { air cylinders and } 2 \text { water coolers to staging }\end{array}$ \\
\hline Ambulance 8 & Report to base \\
\hline Engine 9 & Relieve 3rd line crew \\
\hline Ambulance 9 & Report to base \\
\hline $\mathrm{BC} 4$ & Ventilation group supervisor \\
\hline Engine 10 & Report to staging, tactical reserve \\
\hline Engine 11 & Report to staging, tactical reserve \\
\hline Engine 12 & Report to base \\
\hline Truck 6 & $\begin{array}{l}\text { Relieve search and rescue crew on fire floor } \\
\text {-If victim is found, evacuate victim to 8th floor }\end{array}$ \\
\hline $\mathrm{BC} 5$ & Report to staging \\
\hline Truck 7 & Search and rescue lower floor (9th) \\
\hline & $\begin{array}{l}\text {-Follow search pattern on floor } \\
\text {-Gather all } 20 \text { markers }\end{array}$ \\
\hline Truck 8 & $\begin{array}{l}\text { Relieve search and rescue crew on floor above fire } \\
\text {-If victim is found, evacuate victim to 8th floor }\end{array}$ \\
\hline Truck 9 & Report to staging, tactical reserve \\
\hline Truck 10 & Report to staging, tactical reserve \\
\hline $\mathrm{BC} 6$ & Report to staging, tactical reserve \\
\hline Truck 11 & Report to base \\
\hline Truck 12 & Report to base \\
\hline
\end{tabular}


Company Protocols: Crew Size of 5

\begin{tabular}{|c|c|}
\hline COMPANY & TASK ASSIGNMENTS \\
\hline Engine 1 & $\begin{array}{l}\text { Assume command } \\
\text {-Access building keys } \\
\text {-Locate fire control room } \\
\text {-Check alarm panel } \\
\text {-Give command statement } \\
\text {-Check status of elevators } \\
\text {-Designate attack and evacuation stairwells } \\
\text { Join Engine } 2 \text { to form attack crew } \\
\text {-Connect to gated wye valve on 9th floor } \\
\text {-Advance attack line to 10th floor } \\
\text {-Simulate water on fire } \\
\text {-Maintain sustained attack }\end{array}$ \\
\hline Truck 1 & $\begin{array}{l}\text { Establish Initial Rapid Intervention Crew } \\
\text { Once Rapid Intervention Team is established, begin search and rescue on fire floor } \\
\text {-Check for extension, complete } 30 \text { reps on weighted pike pole } \\
\text {-Follow search pattern on floor, hit bu ers } \\
\text {-If victim is found, evacuate victim to 8th floor }\end{array}$ \\
\hline Ambulance 1 & Establish firefighter rehab on 8th floor \\
\hline Engine 2 & $\begin{array}{l}\text { Establish back up line } \\
\text {-Connect to gated wye valve on } 9 \text { th floor } \\
\text {-Advance back up line to } 10 \text { th floor } \\
\text {-Simulate water on fire } \\
\text {-Maintain sustained attack }\end{array}$ \\
\hline Ambulance 2 & $\begin{array}{l}\text { Report to 8th floor staging area } \\
\text {-Package and transport Victim \#1 }\end{array}$ \\
\hline Engine 3 & Relieve attack line crew \\
\hline Truck 2 & Establish Rapid Intervention Team on 8th floor \\
\hline Ambulance 3 & $\begin{array}{l}\text { Report to 8th floor staging area } \\
\text {-Package and transport Victim \#2 }\end{array}$ \\
\hline $\mathrm{BC} 1$ & Incident Command \\
\hline Engine 4 & $\begin{array}{l}\text { Establish 3rd line to floor above the fire } \\
\text {-Connect to standpipe on 11th floor } \\
\text {-Advance 3rd line to compartment above the fire } \\
\text {-Check for extension, complete } 30 \text { reps on weighted pike pole }\end{array}$ \\
\hline Truck 3 & $\begin{array}{l}\text { Establish staging on 8th floor } \\
\text { Ventilation of attack stairwell } \\
\text {-Proceed to roof and burp hatch when "Fire Out" is heard }\end{array}$ \\
\hline
\end{tabular}




\begin{tabular}{|c|c|}
\hline Truck 4 & $\begin{array}{l}\text { Search and rescue, floor above fire } \\
\text {-Follow search pattern on floor, hit bu ers } \\
\text {-If victim is found, evacuate victim to 8th floor }\end{array}$ \\
\hline $\mathrm{BC} 2$ & Division 10 Command \\
\hline Engine 5 & $\begin{array}{l}\text { Lobby control } \\
\text {-Establish base outside of building } \\
\text {-Logistical support } \\
\text {-Check fire control room }\end{array}$ \\
\hline Ambulance 4 & Report to staging \\
\hline Engine 6 & Relieve back up line crew \\
\hline Ambulance 5 & Report to base \\
\hline Engine 7 & $\begin{array}{l}\text { Search and rescue upper floor (13th) } \\
\text {-Follow search pattern on floor } \\
\text {-Gather all } 20 \text { markers }\end{array}$ \\
\hline Ambulance 6 & Report to base \\
\hline Engine 8 & Relieve 3rd line crew \\
\hline Ambulance 7 & Report to base \\
\hline $\mathrm{BC} 3$ & Division 11 Command \\
\hline Truck 5 & $\begin{array}{l}\text { Elevator/stairwell support } \\
\text {-Transport } 35 \text { air cylinders and } 2 \text { water coolers to staging }\end{array}$ \\
\hline Ambulance 8 & Report to base \\
\hline Engine 9 & Report to staging, tactical reserve \\
\hline Ambulance 9 & Report to base \\
\hline $\mathrm{BC} 4$ & Ventilation group supervisor \\
\hline Engine 10 & Report to staging, tactical reserve \\
\hline Engine 11 & Report to base \\
\hline Engine 12 & Report to base \\
\hline Truck 6 & $\begin{array}{l}\text { Relieve search and rescue crew on fire floor } \\
\text {-If victim is found, evacuate victim to 8th floor }\end{array}$ \\
\hline BC 5 & Report to staging \\
\hline Truck 7 & $\begin{array}{l}\text { Search and rescue lower floor (9th) } \\
\text {-Follow search pattern on floor } \\
\text {-Gather all } 20 \text { markers }\end{array}$ \\
\hline Truck 8 & $\begin{array}{l}\text { Relieve search and rescue crew on floor above fire } \\
\text {-If victim is found, evacuate victim to 8th floor }\end{array}$ \\
\hline Truck 9 & Report to staging, tactical reserve \\
\hline Truck 10 & Report to staging, tactical reserve \\
\hline BC 6 & Report to staging, tactical reserve \\
\hline Truck 11 & Report to base \\
\hline Truck 12 & Report to base \\
\hline
\end{tabular}




\section{Company Protocols: Crew Size of 6}

\begin{tabular}{|c|c|}
\hline COMPANY & TASK ASSIGNMENTS \\
\hline Engine 1 & $\begin{array}{l}\text { Assume command } \\
\text {-Access building keys } \\
\text {-Locate fire control room } \\
\text {-Check alarm panel } \\
\text {-Give command statement } \\
\text {-Check status of elevators } \\
\text {-Designate attack and evacuation stairwells } \\
\text { Join Engine } 2 \text { to form attack crew } \\
\text {-Connect to gated wye valve on 9th floor } \\
\text {-Advance attack line to 10th floor } \\
\text {-Simulate water on fire } \\
\text {-Maintain sustained attack }\end{array}$ \\
\hline Truck 1 & $\begin{array}{l}\text { Establish Initial Rapid Intervention Crew } \\
\text { Once Rapid Intervention Team is established, begin search and rescue on fire floor } \\
\text {-Check for extension, complete } 30 \text { reps on weighted pike pole } \\
\text {-Follow search pattern on floor, hit bu ers } \\
\text {-If victim is found, evacuate victim to 8th floor }\end{array}$ \\
\hline Ambulance 1 & Establish firefighter rehab on 8th floor \\
\hline Engine 2 & $\begin{array}{l}\text { Establish back up line } \\
\text {-Connect to gated wye valve on 9th floor } \\
\text {-Advance back up line to } 10 \text { th floor } \\
\text {-Simulate water on fire } \\
\text {-Maintain sustained attack }\end{array}$ \\
\hline Ambulance 2 & $\begin{array}{l}\text { Report to 8th floor staging area } \\
\text {-Package and transport Victim \#1 }\end{array}$ \\
\hline Engine 3 & Relieve attack line crew \\
\hline Truck 2 & Establish Rapid Intervention Team on 8th floor \\
\hline Ambulance 3 & $\begin{array}{l}\text { Report to 8th floor staging area } \\
\text {-Package and transport Victim \#2 }\end{array}$ \\
\hline $\mathrm{BC} 1$ & Incident Command \\
\hline Engine 4 & $\begin{array}{l}\text { Establish 3rd line to floor above the fire } \\
\text {-Connect to standpipe on 11th floor } \\
\text {-Advance 3rd line to compartment above the fire } \\
\text {-Check for extension, complete } 30 \text { reps on weighted pike pole }\end{array}$ \\
\hline Truck 3 & $\begin{array}{l}\text { Establish staging on 8th floor } \\
\text { Ventilation of attack stairwell } \\
\text {-Proceed to roof and burp hatch when "Fire Out" is heard }\end{array}$ \\
\hline
\end{tabular}




\begin{tabular}{|c|c|}
\hline Truck 4 & $\begin{array}{l}\text { Search and rescue, floor above fire } \\
\text {-Follow search pattern on floor, hit bu ers } \\
\text {-If victim is found, evacuate victim to 8th floor }\end{array}$ \\
\hline $\mathrm{BC} 2$ & Division 10 Command \\
\hline Engine 5 & $\begin{array}{l}\text { Lobby control } \\
\text {-Establish base outside of building } \\
\text {-Logistical support } \\
\text {-Check fire control room }\end{array}$ \\
\hline Ambulance 4 & Report to staging \\
\hline Engine 6 & Relieve back up line crew \\
\hline Ambulance 5 & Report to base \\
\hline Engine 7 & $\begin{array}{l}\text { Search and rescue upper floor (13th) } \\
\text {-Follow search pattern on floor } \\
\text {-Gather all } 20 \text { markers }\end{array}$ \\
\hline Ambulance 6 & Report to base \\
\hline Engine 8 & Relieve 3rd line crew \\
\hline Ambulance 7 & Report to base \\
\hline $\mathrm{BC} 3$ & Division 11 Command \\
\hline Truck 5 & $\begin{array}{l}\text { Elevator/stairwell support } \\
\text {-Transport } 35 \text { air cylinders and } 2 \text { water coolers to staging }\end{array}$ \\
\hline Ambulance 8 & Report to base \\
\hline Engine 9 & Report to staging, tactical reserve \\
\hline Ambulance 9 & Report to base \\
\hline $\mathrm{BC} 4$ & Ventilation group supervisor \\
\hline Engine 10 & Report to staging, tactical reserve \\
\hline Engine 11 & Report to base \\
\hline Engine 12 & Report to base \\
\hline Truck 6 & $\begin{array}{l}\text { Relieve search and rescue crew on fire floor } \\
\text {-If victim is found, evacuate victim to 8th floor }\end{array}$ \\
\hline BC 5 & Report to staging \\
\hline Truck 7 & $\begin{array}{l}\text { Search and rescue lower floor (9th) } \\
\text {-Follow search pattern on floor } \\
\text {-Gather all } 20 \text { markers }\end{array}$ \\
\hline Truck 8 & $\begin{array}{l}\text { Relieve search and rescue crew on floor above fire } \\
\text {-If victim is found, evacuate victim to 8th floor }\end{array}$ \\
\hline Truck 9 & Report to staging, tactical reserve \\
\hline Truck 10 & Report to staging, tactical reserve \\
\hline BC 6 & Report to staging, tactical reserve \\
\hline Truck 11 & Report to base \\
\hline Truck 12 & Report to base \\
\hline
\end{tabular}




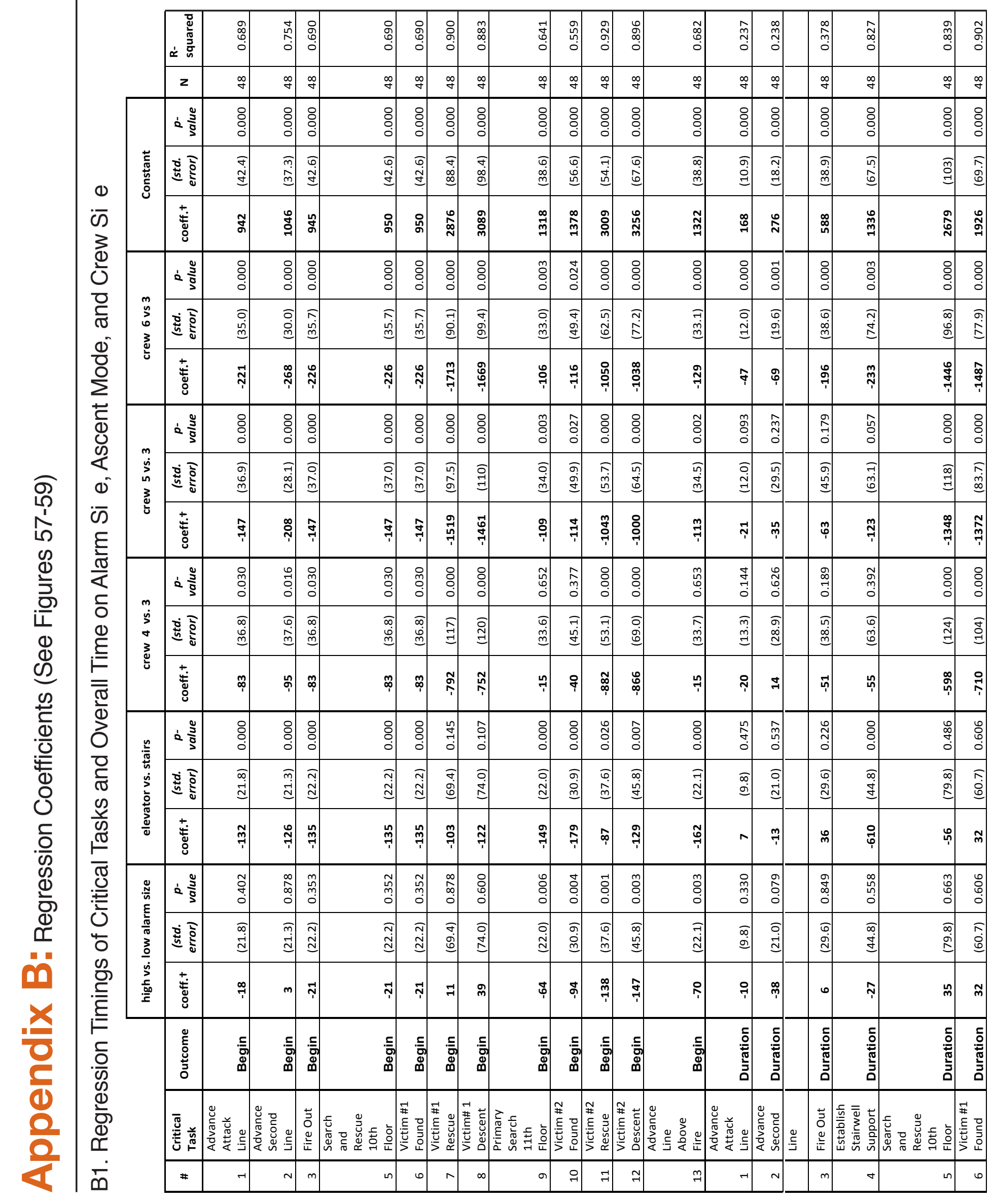




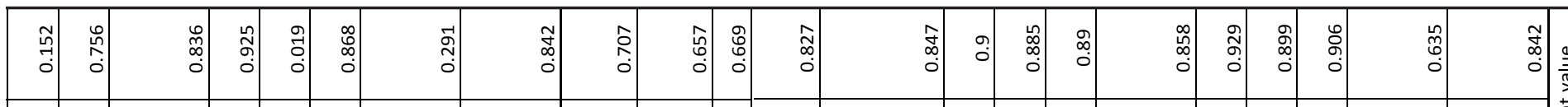

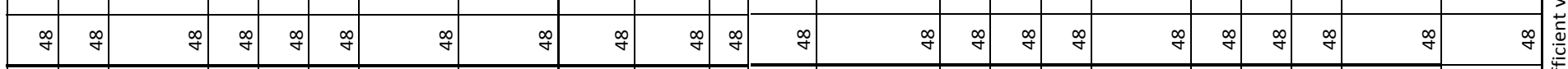

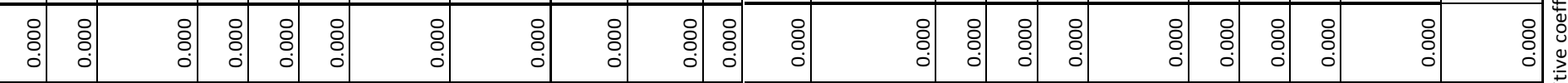

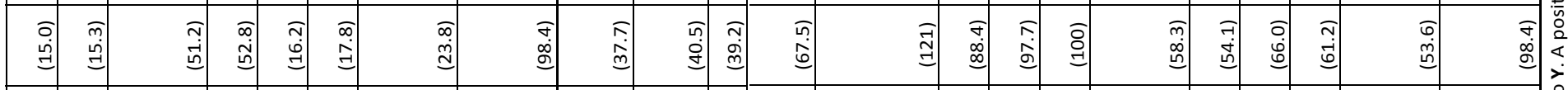

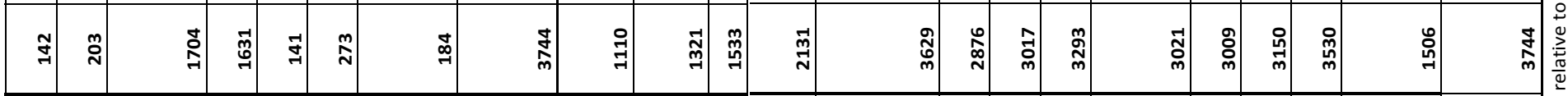

\begin{tabular}{|c|c|c|c|c|c|c|c|c|c|c|c|c|c|c|c|c|c|c|c|}
\hline 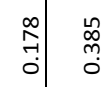 & $\begin{array}{l}0 \\
\\
\end{array}$ & \begin{tabular}{l}
0 \\
$\stackrel{0}{0}$ \\
\hdashline
\end{tabular} & 竞 & 苂 & $\vec{\circ}$ & : & : & $\begin{array}{l}0 \\
: \\
: \\
:\end{array}$ & on & $\begin{array}{l}0 \\
: \\
0 \\
0\end{array}$ & $\begin{array}{l}0 \\
: \\
:\end{array}$ & $\begin{array}{l}: \\
: \\
:\end{array}$ & $\begin{array}{l}0 \\
: \\
:\end{array}$ & $\stackrel{\circ}{\circ}$ & a & 웅 & ঃ & \begin{tabular}{l}
0 \\
$:$ \\
$:$ \\
\hdashline
\end{tabular} & $\begin{array}{l}: \\
: \\
:\end{array}$ \\
\hline 氞 & 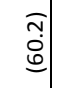 & 奋 & $\begin{array}{l}\sqrt[n]{0} \\
\stackrel{\Xi}{=}\end{array}$ & $\begin{array}{l}\bar{n} \\
\stackrel{0}{6}\end{array}$ & $\begin{array}{l}\widehat{m} \\
\stackrel{d}{d}\end{array}$ & $\begin{array}{c}\mathbb{R} \\
\text { 岁 }\end{array}$ & $\begin{array}{l}\bar{o} \\
\text { d } \\
\end{array}$ & 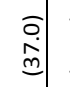 & $\begin{array}{l}\widehat{T} \\
\mathfrak{d} \\
\end{array}$ & : & 势 & & a & $\begin{array}{l}\bar{n} \\
\underline{j}\end{array}$ & $\stackrel{\widehat{n}}{\underline{Q}}$ & (2) & ed & $\begin{array}{l}0 \\
0 \\
\dot{y}\end{array}$ & : \\
\hline
\end{tabular}

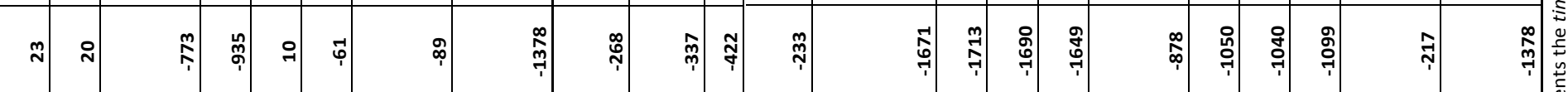

\begin{tabular}{|c|c|c|c|c|c|c|c|c|c|c|c|c|c|c|c|c|c|c|c|}
\hline 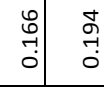 & $\begin{array}{l}: \\
\vdots \\
\end{array}$ & & 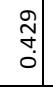 & 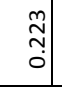 & $\begin{array}{l}\text { o } \\
0 \\
0\end{array}$ & $\begin{array}{l}0 \\
: \\
0\end{array}$ & $\begin{array}{l}\circ \\
\vdots \\
\end{array}$ & & $\begin{array}{l}\hat{H} \\
0 \\
0\end{array}$ & & $\begin{array}{l}0 \\
\vdots \\
0\end{array}$ & & $\begin{array}{l}: \\
: \\
\end{array}$ & $\begin{array}{r}\circ \\
\vdots \\
\end{array}$ & $\begin{array}{l}0 \\
\vdots \\
\end{array}$ & $\begin{array}{l}: \\
\vdots \\
\end{array}$ & $\stackrel{\circ}{\circ}$ & $\begin{array}{l}: \\
0 \\
0\end{array}$ & ঃ \\
\hline 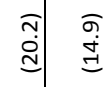 & $\begin{array}{l}\widehat{\infty} \\
\dot{0} \\
\dot{0}\end{array}$ & $\begin{array}{l}\bar{n} \\
\mathfrak{d} \\
\underline{w}\end{array}$ & 弪 & 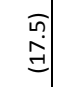 & $\begin{array}{l}\vec{I} \\
\vec{d}\end{array}$ & $\begin{array}{l}\overrightarrow{0} \\
\dot{f} \\
\dot{d}\end{array}$ & $\begin{array}{l}\vec{ज} \\
\stackrel{\rho}{m}\end{array}$ & 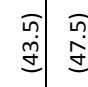 & $\begin{array}{l}\overrightarrow{\hat{\oplus}} \\
\stackrel{\dot{\theta}}{9}\end{array}$ & 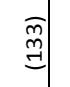 & $\begin{array}{l}\bar{n} \\
\vdots \\
a\end{array}$ & & 㞵 & 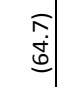 & 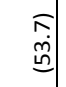 & 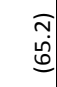 & $\begin{array}{l}\bar{\partial} \\
\dot{0} \\
\underline{0}\end{array}$ & $\begin{array}{l}\widehat{m} \\
\dot{y} \\
\dot{f}\end{array}$ & $\begin{array}{l}\widehat{\Phi} \\
\stackrel{0}{0}\end{array}$ \\
\hline
\end{tabular}

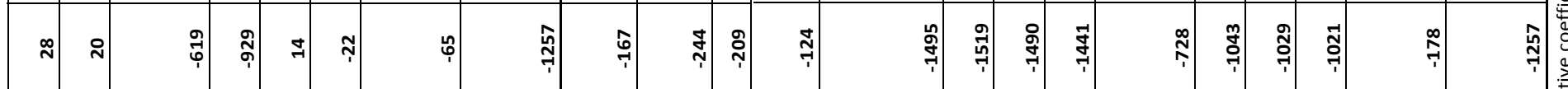

\begin{tabular}{|c|c|c|c|c|c|c|c|c|c|c|c|c|c|c|c|c|c|c|c|}
\hline \begin{tabular}{l|l}
$\substack{f \\
0 \\
0 \\
0}$ & 0 \\
0
\end{tabular} & $\begin{array}{l}0 \\
\\
o \\
\end{array}$ & $\begin{array}{l}0 \\
0 \\
0 \\
0\end{array}$ & $\begin{array}{l}\text { t. } \\
\stackrel{0}{0} \\
0\end{array}$ & $\begin{array}{l}0 \\
\text { o. } \\
\text { ô. } \\
0\end{array}$ & $\begin{array}{l}0 \\
0 \\
0 \\
0 \\
0\end{array}$ & $\begin{array}{l}0 \\
: \\
0 \\
0\end{array}$ & $\vec{\vdots}$ & 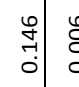 & ָ̃ & $\begin{array}{l}\circ \\
: \\
:\end{array}$ & $\begin{array}{l}\circ \\
\vdots \\
0\end{array}$ & $\begin{array}{ll}0 & 0 \\
0 & 0\end{array}$ & $\begin{array}{l}0 \\
: \\
: \\
:\end{array}$ & \begin{tabular}{l|l} 
& $\circ$ \\
$\vdots$ \\
\hdashline
\end{tabular} & $\begin{array}{l}0 \\
: \\
\vdots \\
\end{array}$ & $\stackrel{\circ}{\circ}$ & $\stackrel{\circ}{\circ}$ & 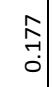 & $\begin{array}{l}0 \\
0 \\
0\end{array}$ \\
\hline 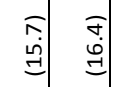 & 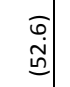 & 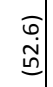 & ज्ञ & : & $\begin{array}{l}\widehat{m} \\
\stackrel{d}{d}\end{array}$ & : & $\underset{\substack{\infty \\
\tilde{n}}}{\mathbb{p}}$ & 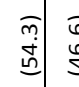 & $\begin{array}{l}\widehat{\sigma} \\
\stackrel{\sigma}{\dot{\rho}}\end{array}$ & 离 & $\widehat{\Xi}$ & & 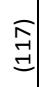 & $\begin{array}{l}\mid \overrightarrow{0} \\
\overrightarrow{0} \\
\end{array}$ & नि: & 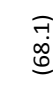 & 焉 & 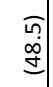 & \\
\hline
\end{tabular}

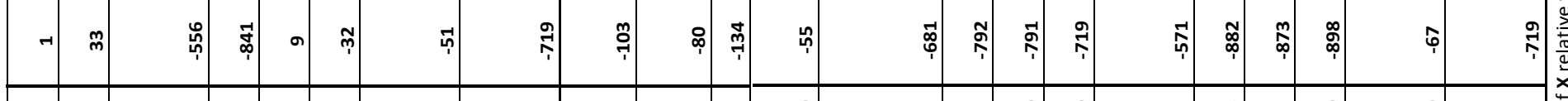

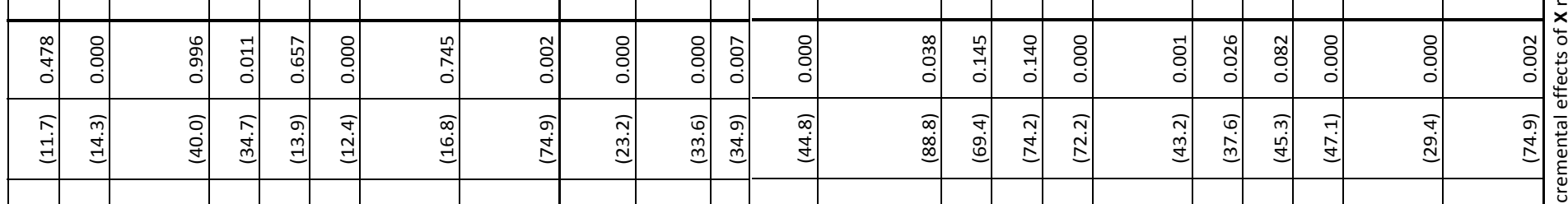

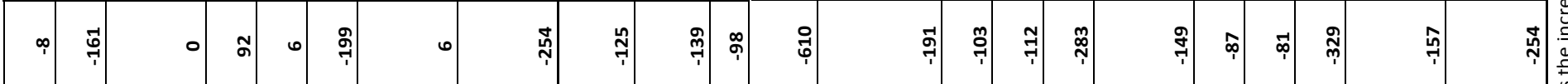

\begin{tabular}{|c|c|c|c|c|c|c|c|c|c|c|c|c|c|c|c|c|c|c|c|c|}
\hline $\begin{array}{l}m \\
y \\
0 \\
0\end{array}$ & : & $\begin{array}{c}0 \\
0 \\
0 \\
0\end{array}$ & 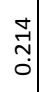 & 望| & 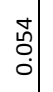 & 竞 & 络 & $\begin{array}{l}\tilde{N} \\
\stackrel{\tilde{N}}{0}\end{array}$ & \begin{tabular}{c|c}
$\stackrel{0}{0}$ & $\stackrel{0}{0}$ \\
0 & 0
\end{tabular} & 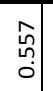 & 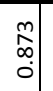 & . & : & 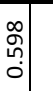 & 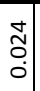 & : & : & 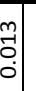 & | & |ct \\
\hline 胥 & 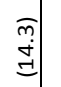 & $\begin{array}{l}\widehat{o} \\
\dot{d} \\
\dot{d}\end{array}$ & 胥 & ঐ̊. & 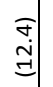 & $\begin{array}{l}\widehat{a} \\
\stackrel{0}{0}\end{array}$ & 可 & $\begin{array}{l}\widehat{\widetilde{d}} \\
\tilde{d}\end{array}$ & 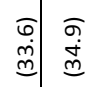 & $\begin{array}{l}\widehat{\infty} \\
\dot{q} \\
\dot{q}\end{array}$ & $\begin{array}{l}\widehat{\infty} \\
\infty \\
\infty \\
\infty\end{array}$ & 宛 & & ה్తి & \pm & : & 商 & 高 & $\simeq$ & 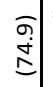 \\
\hline$f$ & 7 & $\hat{m}$ & $f$ & 4 & $\stackrel{n}{\sim}$ & $\stackrel{\mathscr{T}}{\sim}$ & $\stackrel{\infty}{\varphi}$ & $\stackrel{\infty}{\sim}$ & $\stackrel{m}{p} \stackrel{?}{?}$ & $\hat{\imath}$ & \pm & 7 & $\stackrel{\infty}{\sim}$ & $\stackrel{\infty}{m}$ & : & 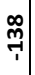 & | & | & के & "ا \\
\hline 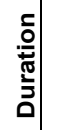 & 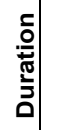 & 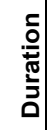 & 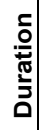 & 产 & 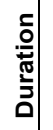 & 䐻 & 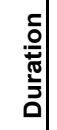 & 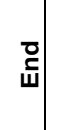 & 离 & 总 & 离 & 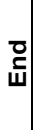 & 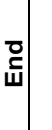 & 离| & $\overline{\mathrm{w}}$ & 总 & & 密 & 离 & 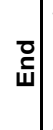 \\
\hline
\end{tabular}

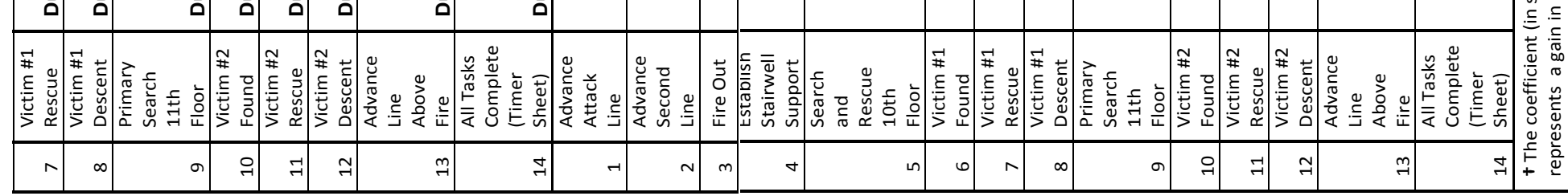




\begin{tabular}{|c|c|c|c|c|c|c|c|c|c|c|c|c|c|c|c|c|c|c|c|c|}
\hline & 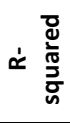 & $\begin{array}{l}\stackrel{2}{2} \\
0\end{array}$ & $\begin{array}{l}\stackrel{0}{0} \\
\stackrel{0}{0}\end{array}$ & 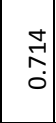 & 离 & 离 & $\begin{array}{l}\text { वे } \\
\text { के }\end{array}$ & $\begin{array}{l}\stackrel{0}{\infty} \\
\stackrel{0}{0}\end{array}$ & 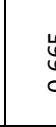 & | & 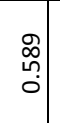 & $\begin{array}{l}\text { ô. } \\
\text { o. }\end{array}$ & $\begin{array}{c}\text { â } \\
\text { o. } \\
0\end{array}$ & $\begin{array}{l}0 \\
\stackrel{0}{0} \\
0\end{array}$ & $\begin{array}{c}\tilde{N} \\
\tilde{m} \\
0\end{array}$ & 㞻| & $\mid \begin{array}{c}\tilde{D} \\
\tilde{o} \\
0\end{array}$ & $\begin{array}{l}\infty \\
\infty \\
\infty \\
0\end{array}$ & $\begin{array}{c}\mathcal{N} \\
0 \\
0 \\
0\end{array}$ & $\begin{array}{l}\text { ने } \\
\text { o. }\end{array}$ \\
\hline & z & $\stackrel{\infty}{+}$ & $\stackrel{\infty}{\rightarrow}$ & $\stackrel{\infty}{+}$ & $\stackrel{\infty}{+}$ & $\stackrel{\infty}{+}$ & $\stackrel{\infty}{+}$ & $\stackrel{\infty}{+}$ & 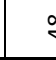 & $\infty$ & $\stackrel{\infty}{\sim}$ & $\stackrel{\infty}{\rightarrow}$ & $\stackrel{\infty}{\sigma}$ & $\stackrel{\infty}{\sigma}$ & $\stackrel{\infty}{\sigma}$ & $\stackrel{\infty}{\sigma}$ & $\stackrel{\infty}{\sigma}$ & $\stackrel{\infty}{\stackrel{\circ}{f}}$ & $\stackrel{\infty}{\sigma}$ & $\stackrel{\infty}{\sigma}$ \\
\hline \multirow{3}{*}{ 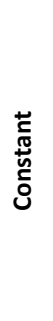 } & $2 \frac{\grave{z}}{3}$ & $\begin{array}{l}8 \\
\circ \\
\circ \\
\end{array}$ & $\begin{array}{l}8 \\
0 \\
0 \\
\end{array}$ & $\begin{array}{l}0 \\
0 \\
0 \\
\end{array}$ & $\begin{array}{l}\text { : } \\
\circ \\
\end{array}$ & $\begin{array}{l}8 \\
0 \\
0\end{array}$ & $\begin{array}{l}\text { : } \\
\circ \\
\end{array}$ & $\begin{array}{l}\text { : } \\
\circ \\
\end{array}$ & 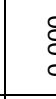 & 8 & $\begin{array}{l}\text { ò } \\
\text { : } \\
\end{array}$ & $\begin{array}{l}8 \\
0 \\
0 \\
\end{array}$ & $\begin{array}{l}0 \\
0 \\
0 \\
\end{array}$ & $\begin{array}{l}\circ \\
\text { o. } \\
0\end{array}$ & $\begin{array}{l}\text { o } \\
0 \\
0 \\
\end{array}$ & ০০ & \begin{tabular}{|l|}
0 \\
0 \\
0 \\
\end{tabular} & $\begin{array}{l}8 \\
0 \\
0 \\
\end{array}$ & $\begin{array}{l}0 \\
0 \\
0 \\
\end{array}$ & $\begin{array}{l}8 \\
0 \\
\end{array}$ \\
\hline & 它 & $\begin{array}{l}\sigma \\
\infty \\
0 \\
0\end{array}$ & $\begin{array}{c}\mathbb{E} \\
\dot{ \pm}\end{array}$ & $\begin{array}{l}\text { ô } \\
\dot{\sigma} \\
\text { ம. }\end{array}$ & $\begin{array}{l}\text { oे } \\
\text { in }\end{array}$ & $\begin{array}{l}\text { oे } \\
\text { in }\end{array}$ & $\begin{array}{l}0 \\
\stackrel{0}{0} \\
\stackrel{्}{-}\end{array}$ & $\begin{array}{l}\infty \\
\text { ம் } \\
\stackrel{्}{=}\end{array}$ & 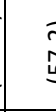 & ר్ & 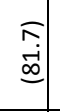 & $\begin{array}{l}\hat{R} \\
\dot{5} \\
\vdots\end{array}$ & $\begin{array}{l}\sigma \\
\tilde{n} \\
\Omega \\
\Omega\end{array}$ & 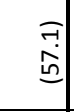 & $\begin{array}{l}\widehat{n} \\
\stackrel{\Xi}{\Xi} \\
\end{array}$ & $\begin{array}{l}\widehat{n} \\
\stackrel{\Xi}{\Xi}\end{array}$ & नि: & $\begin{array}{l}\widehat{a} \\
\hat{a}\end{array}$ & $\begin{array}{l}\widehat{ָ} \\
\stackrel{\leftrightarrow}{\Xi} \\
\Xi\end{array}$ & \\
\hline & 蓠 & స్ & స్త్ర & $\stackrel{\llcorner}{\Omega}$ & ळ్ & ஜू & $\stackrel{\vec{d}}{\stackrel{N}{*}}$ & ঃి & 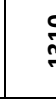 & t. & 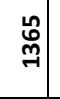 & 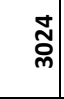 & 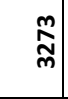 & $\underset{m}{\tilde{m}}$ & $\stackrel{\infty}{\rightarrow}$ & $\stackrel{0}{\sim}$ & 员 & 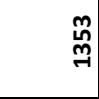 & : & 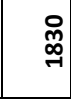 \\
\hline \multirow{3}{*}{ 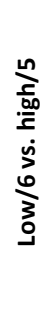 } & $2 \frac{\beth}{3}$ & $\stackrel{\substack{0 \\
0 \\
0}}{0}$ & $\begin{array}{l}0 \\
\stackrel{n}{0} \\
0 \\
\end{array}$ & $\begin{array}{l}\infty \\
0 \\
0 \\
0 \\
0\end{array}$ & 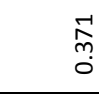 & 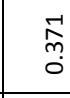 & $\begin{array}{l}\infty \\
\stackrel{0}{0} \\
\stackrel{0}{0}\end{array}$ & $\stackrel{m}{\stackrel{m}{0}}$ & $\dot{\delta}$ & : & $\begin{array}{l}\hat{n} \\
0 \\
0\end{array}$ & $\begin{array}{l}\text { స్ } \\
\text { : }\end{array}$ & $\begin{array}{l}\stackrel{\infty}{0} \\
\stackrel{0}{0} \\
0\end{array}$ & $\begin{array}{l}\hat{f} \\
0 \\
0\end{array}$ & $\begin{array}{l}0 \\
0 \\
0 \\
0\end{array}$ & 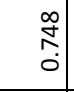 & $\begin{array}{l}0 \\
0 \\
0 \\
0\end{array}$ & $\begin{array}{c}0 \\
\stackrel{\infty}{0} \\
0 \\
0\end{array}$ & 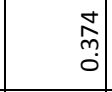 & \begin{tabular}{c}
$\infty$ \\
$\stackrel{\infty}{0}$ \\
\hdashline \\
0
\end{tabular} \\
\hline & 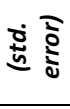 & $\begin{array}{l}\widehat{\infty} \\
\stackrel{\Xi}{\Xi}\end{array}$ & 㸃 & $\begin{array}{l}\tilde{f} \\
\dot{0} \\
\end{array}$ & $\begin{array}{l}\bar{f} \\
\dot{0} \\
\dot{m}\end{array}$ & $\begin{array}{l}\bar{f} \\
\dot{m} \\
\end{array}$ & $\begin{array}{l}\text { In } \\
\text { जं }\end{array}$ & $\begin{array}{l}0 \\
\infty \\
\infty \\
\Xi \\
=\end{array}$ & 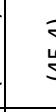 & f. & $\begin{array}{l}\bar{\sigma} \\
\dot{\rho} \\
.\end{array}$ & 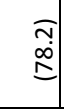 & $\begin{array}{c}\widehat{N} \\
\stackrel{\infty}{\infty} \\
\underline{\infty}\end{array}$ & $\begin{array}{c}\widehat{\widehat{y}} \\
\dot{g} \\
\dot{y}\end{array}$ & 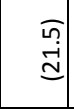 & $\begin{array}{l}7 \\
\stackrel{-}{0} \\
\stackrel{0}{0}\end{array}$ & $\begin{array}{l}\widehat{\sigma} \\
\dot{\omega} \\
\underline{\omega}\end{array}$ & $\begin{array}{l}\widehat{\infty} \\
\dot{\infty} \\
\infty\end{array}$ & $\begin{array}{c}\bar{m} \\
\stackrel{n}{=} \\
\end{array}$ & 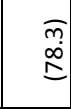 \\
\hline & 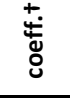 & $\stackrel{\infty}{\sim}$ & కุ? & $\stackrel{\infty}{\sim}$ & $\stackrel{\infty}{\uparrow}$ & $\stackrel{\infty}{\sim}$ & $\stackrel{m}{\rightarrow}$ & $\stackrel{-0}{\rightarrow}$ & $£$ & ב. & $\mathbf{F}$ & $\stackrel{\sim}{\underset{\sim}{*}}$ & $\stackrel{\widetilde{A}}{\mathbb{*}}$ & ๓ & בְ & $\dddot{7}$ & $\stackrel{\substack{m \\
\rightarrow}}{\rightarrow}$ & क़े & $\stackrel{9}{7}$ & ํำ \\
\hline \multirow{3}{*}{ 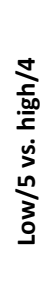 } & $\sum \frac{\stackrel{2}{g}}{g}$ & $\begin{array}{l}\hat{0} \\
0 \\
0\end{array}$ & $\begin{array}{l}0 \\
\vdots \\
0 \\
\end{array}$ & $\begin{array}{l}0 \\
0 \\
0 \\
0\end{array}$ & $\begin{array}{l}\text { : } \\
\text { : } \\
\end{array}$ & $\begin{array}{l}\text { : } \\
\text { : }\end{array}$ & ঃ. & ঃ & 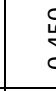 & : & 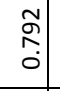 & $\begin{array}{l}0 \\
f \\
0 \\
0\end{array}$ & $\begin{array}{l}\mathscr{0} \\
\infty \\
0 \\
0 \\
0\end{array}$ & $\begin{array}{c}0 \\
\infty \\
m \\
0 \\
0\end{array}$ & $\begin{array}{l}\mathbf{d} \\
\stackrel{\Delta}{0} \\
0 \\
\end{array}$ & $\begin{array}{l}\stackrel{\circ}{\circ} \\
\stackrel{2}{0} \\
\end{array}$ & $\mid \begin{array}{l}0 \\
\vdots \\
0 \\
0\end{array}$ & $\begin{array}{l}0 \\
\stackrel{7}{7} \\
0\end{array}$ & $\begin{array}{l}-1 \\
0 \\
0\end{array}$ & $\begin{array}{l}8 \\
0 \\
0\end{array}$ \\
\hline & 这 & $\begin{array}{l}\widehat{m} \\
\stackrel{m}{\sim}\end{array}$ & $\begin{array}{l}\stackrel{o}{d} \\
\stackrel{d}{d}\end{array}$ & $\begin{array}{l}\widehat{\omega} \\
\dot{m} \\
\tilde{m}\end{array}$ & 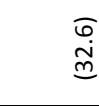 & 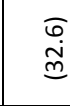 & $\begin{array}{l}\bar{m} \\
\vec{m} \\
\stackrel{\Xi}{=}\end{array}$ & $\begin{array}{l}\text { ō } \\
\text { m } \\
\stackrel{\Xi}{=}\end{array}$ & 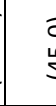 & b. & $\begin{array}{l}\text { aे. } \\
\text { o. }\end{array}$ & $\begin{array}{l}\widehat{m} \\
\dot{f} \\
\dot{0}\end{array}$ & $\begin{array}{c}\widehat{m} \\
\dot{\infty} \\
\infty\end{array}$ & $\begin{array}{l}\sigma \\
\dot{y} \\
\end{array}$ & $\begin{array}{l}\widehat{\sigma} \\
\stackrel{\Xi}{\Xi}\end{array}$ & $\begin{array}{l}0 \\
\text { ọ. } \\
\text { ஸn }\end{array}$ & $\begin{array}{c}\vec{a} \\
0 \\
0 \\
0\end{array}$ & $\stackrel{m}{\vec{s}}$ & $\begin{array}{l}\text { a } \\
\dot{0} \\
\stackrel{d}{d}\end{array}$ & $\begin{array}{l}\bar{f} \\
\stackrel{g}{\Xi}\end{array}$ \\
\hline & 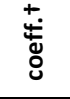 & ตุ & $\underset{7}{7}$ & ?ุ & ?ุ & ใุ & ధ్ & ఫ్రి & i & $\vec{m}$ & $\underset{-1}{0}$ & ధำ & 7 & शి & $\sigma$ & 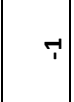 & $\bar{\top}$ & :ึ & $\stackrel{\infty}{\rightarrow}$ & ర్రి \\
\hline \multirow{3}{*}{ 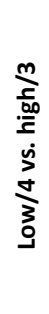 } & 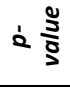 & $\begin{array}{l}\text { N} \\
0 \\
0 \\
0\end{array}$ & $\begin{array}{l}\hat{1} \\
0 \\
0 \\
\end{array}$ & $\begin{array}{l}5 \\
0 \\
0 \\
0\end{array}$ & $\begin{array}{l}5 \\
0 \\
0 \\
0\end{array}$ & $\begin{array}{l}5 \\
0 \\
0 \\
0\end{array}$ & $\begin{array}{l}\text { ¿ } \\
\text { ¿ }\end{array}$ & ঃ & $\vdots$ & : & $\begin{array}{l}\Omega \\
0 \\
0 \\
0 \\
\end{array}$ & $\begin{array}{l}8 \\
\vdots \\
0 \\
\end{array}$ & $\begin{array}{l}8 \\
\vdots \\
0 \\
\end{array}$ & $\begin{array}{l}\hat{n} \\
\hat{0} \\
0\end{array}$ & $\begin{array}{l}0 \\
\text { h. } \\
0 \\
0\end{array}$ & $\begin{array}{l}\overrightarrow{7} \\
0\end{array}$ & $\mid \begin{array}{l}\text { t } \\
\stackrel{0}{0} \\
0\end{array}$ & $\begin{array}{c}\text { I } \\
0 \\
0\end{array}$ & $\begin{array}{l}\overrightarrow{0} \\
0 \\
0\end{array}$ & $\begin{array}{l}8 \\
: \\
0\end{array}$ \\
\hline & 透 & 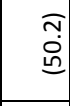 & $\begin{array}{l}0 \\
\dot{0} \\
\dot{0} \\
0\end{array}$ & $\begin{array}{l}\sigma \\
g \\
g\end{array}$ & $\begin{array}{l}\sigma \\
\text { gे }\end{array}$ & बू & $\begin{array}{l}\bar{\infty} \\
\infty \\
\infty \\
\Xi\end{array}$ & 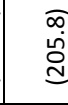 & 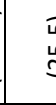 & ڤُ. & $\begin{array}{l}0 \\
\infty \\
0 \\
\stackrel{\infty}{0}\end{array}$ & $\begin{array}{l}\widehat{N} \\
\mathbb{S} \\
\end{array}$ & 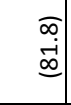 & $\begin{array}{l}\widehat{\sim} \\
\stackrel{d}{d}\end{array}$ & $\begin{array}{c}\widehat{a} \\
\substack{0 \\
\Xi}\end{array}$ & 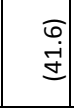 & 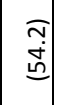 & $\begin{array}{l}\widehat{m} \\
\stackrel{5}{\Xi}\end{array}$ & $\begin{array}{l}\widehat{\infty} \\
\dot{+} \\
\Xi \\
\Xi\end{array}$ & $\begin{array}{l}\infty \\
\stackrel{\infty}{\Xi} \\
\end{array}$ \\
\hline & 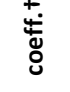 & ọ & 옥 & ô & \%̆ & ô & $\overbrace{\substack{n \\
0}}^{n}$ & గ్యీ & 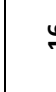 & e. & 0 & ר̂. & $\stackrel{\tilde{n}}{\text { T. }}$ & 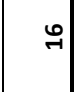 & 7 & $\stackrel{\infty}{\bullet}$ & $\vec{\varphi}$ & $\stackrel{\infty}{\rightarrow}$ & $\begin{array}{l}\hat{f} \\
\vdots\end{array}$ & $\stackrel{\infty}{\stackrel{\infty}{\imath}}$ \\
\hline \multirow{6}{*}{ 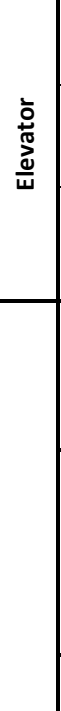 } & 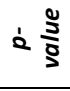 & $\begin{array}{l}\circ \\
\text { ¿. } \\
\end{array}$ & $\begin{array}{l}0 \\
0 \\
0 \\
\end{array}$ & $\begin{array}{l}: \\
\vdots \\
\vdots\end{array}$ & $\begin{array}{l}\text { ঃ } \\
\circ\end{array}$ & $\begin{array}{l}\text { : } \\
\circ\end{array}$ & $\stackrel{\mathcal{Z}}{\mathcal{J}}$ & 워 & $\xi$ & : & $\begin{array}{l}0 \\
\vdots \\
\vdots\end{array}$ & $\begin{array}{l}\vec{m} \\
0 \\
0 \\
0\end{array}$ & $\begin{array}{l}0 \\
0 \\
0 \\
0\end{array}$ & $\begin{array}{l}\text { : } \\
\vdots \\
0\end{array}$ & $\begin{array}{l}n \\
0 \\
0 \\
0 \\
0\end{array}$ & 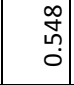 & $\mid \begin{array}{l}\tilde{T} \\
0 \\
0\end{array}$ & $\begin{array}{l}\circ \\
\circ \\
\circ\end{array}$ & $\begin{array}{l}\infty \\
\stackrel{o}{f} \\
0\end{array}$ & $\begin{array}{l}\text { O̊ } \\
0 \\
0\end{array}$ \\
\hline & 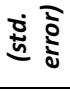 & $\begin{array}{l}\bar{a} \\
\dot{d}\end{array}$ & 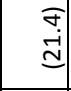 & I. & $\stackrel{\bar{\Xi}}{\stackrel{\Xi}{ \pm}}$ & $\stackrel{\vec{I}}{\stackrel{\Xi}{\Xi}}$ & $\begin{array}{l}\infty \\
\infty \\
\infty \\
0\end{array}$ & I & $\vdots$ & aे & $\begin{array}{l}\bar{\sigma} \\
\dot{m} \\
\dot{m}\end{array}$ & $\begin{array}{l}\widehat{\alpha} \\
\infty \\
\stackrel{0}{0}\end{array}$ & $\begin{array}{l}\widehat{T} \\
\stackrel{ \pm}{ \pm}\end{array}$ & $\begin{array}{l}\overrightarrow{0} \\
\stackrel{d}{d}\end{array}$ & $\begin{array}{l}\widehat{\sigma} \\
\dot{\sigma}\end{array}$ & $\begin{array}{l}\sqrt[n]{n} \\
\stackrel{\Xi}{\mathbf{D}}\end{array}$ & \begin{tabular}{|l|l}
$\overline{0}$ \\
0 \\
0 \\
\end{tabular} & $\begin{array}{l}\bar{f} \\
\dot{g} \\
\dot{y}\end{array}$ & $\begin{array}{l}\overline{0} \\
\dot{i} \\
\underline{\infty}\end{array}$ & $\begin{array}{l}\overrightarrow{7} \\
0 \\
0 \\
0\end{array}$ \\
\hline & $\underset{\stackrel{\Xi}{ \pm}}{+}$ & $\stackrel{\tilde{m}}{\rightarrow}$ & : & 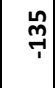 & $\stackrel{\mathscr{n}}{\rightarrow}$ & 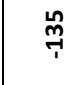 & $\stackrel{m}{\rightarrow}$ & $\underset{7}{\mathcal{7}}$ & 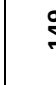 & t. & $\underset{7}{\stackrel{9}{7}}$ & $\hat{\mathbf{p}}$ & ฐิ & 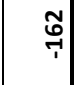 & $\wedge$ & $\stackrel{m}{7}$ & 0 & ఫ్ & น̊. & $\tilde{\mathrm{m}}$ \\
\hline & 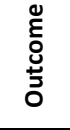 & ) & 离 & : & ๑) & ๑) & 吉 & ๑ & 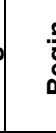 & छे & 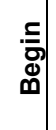 & $\begin{array}{l}\text { : } \\
\Phi \\
\Phi\end{array}$ & Фั) & $\begin{array}{l}\square \\
\varpi\end{array}$ & 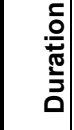 & 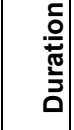 & 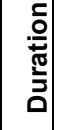 & 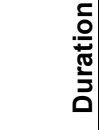 & 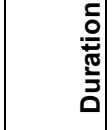 & 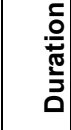 \\
\hline & 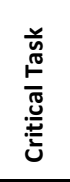 & 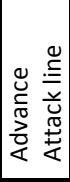 & 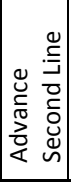 & 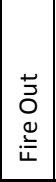 & 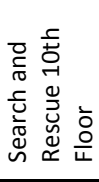 & 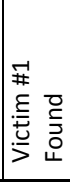 & 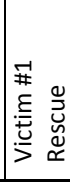 & 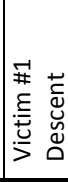 & 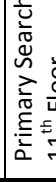 & & $\begin{array}{ll}0 \\
\vdots \\
\vdots\end{array}$ & 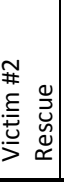 & 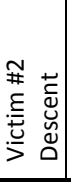 & 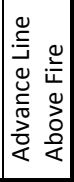 & 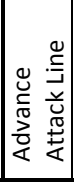 & 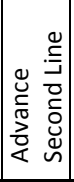 & 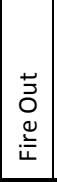 & 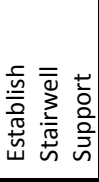 & 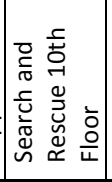 & 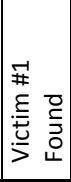 \\
\hline & $\#$ & $\rightarrow$ & $\sim$ & $m$ & in & 0 & $r$ & $\infty$ & . & б. & 인 & $\Rightarrow$ & $\approx$ & $m$ & $\rightarrow$ & $\sim$ & $m$ & $\nabla$ & n & 0 \\
\hline
\end{tabular}




\begin{tabular}{|c|c|c|c|c|c|c|c|c|c|c|c|c|c|c|c|c|c|c|c|c|c|c|c|c|}
\hline $\begin{array}{l}1 \\
0 \\
0 \\
0\end{array}$ & $\begin{array}{l}0 \\
\stackrel{0}{0} \\
0\end{array}$ & 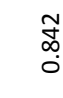 & $\begin{array}{l}\vec{m} \\
o \\
0\end{array}$ & & . & $\begin{array}{c}n \\
0 \\
0 \\
0\end{array}$ & 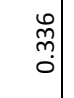 & $\begin{array}{l}f \\
\infty \\
0 \\
0\end{array}$ & 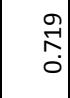 & $\begin{array}{l}\infty \\
: \\
: \\
0 \\
0\end{array}$ & 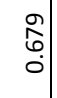 & $\begin{array}{c}\infty \\
\infty \\
\infty \\
0 \\
0\end{array}$ & 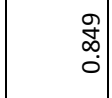 & $\begin{array}{l}\text { के } \\
\text { वे }\end{array}$ & $\begin{array}{c}0 \\
0 \\
\infty \\
0 \\
0\end{array}$ & & 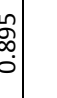 & $\begin{array}{c}0 \\
0 \\
0 \\
0 \\
0\end{array}$ & 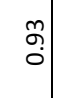 & $\begin{array}{l}\vec{\vdots} \\
\text {. } \\
0\end{array}$ & $\begin{array}{l}\text { هे } \\
\text { ১. }\end{array}$ & \begin{tabular}{l}
\multirow{J}{*}{} \\
$\dot{0}$ \\
0
\end{tabular} & & o. \\
\hline$\stackrel{\infty}{+}$ & $\stackrel{\infty}{+}$ & $\stackrel{\infty}{+}$ & $\stackrel{\infty}{+}$ & 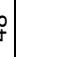 & $\stackrel{\infty}{+}$ & $\stackrel{\infty}{+}$ & $\stackrel{\infty}{+}$ & $\stackrel{\infty}{+}$ & $\stackrel{\infty}{+}$ & $\stackrel{\infty}{+}$ & $\stackrel{\infty}{+}$ & $\stackrel{\infty}{+}$ & $\stackrel{\infty}{+}$ & $\stackrel{\infty}{+}$ & $\stackrel{\infty}{+}$ & 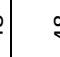 & $\infty$ & $\stackrel{\infty}{+}$ & $\stackrel{\infty}{+}$ & $\stackrel{\infty}{+}$ & $\stackrel{\infty}{+}$ & $\stackrel{\infty}{+}$ & $\stackrel{\infty}{+}$ & 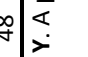 \\
\hline $\begin{array}{l}0 \\
\vdots \\
0\end{array}$ & $\begin{array}{l}0 \\
\vdots \\
0\end{array}$ & : & ঃ & & : & $\begin{array}{l}8 \\
\vdots \\
\dot{0}\end{array}$ & $\begin{array}{l}8 \\
\vdots \\
0\end{array}$ & $\begin{array}{l}\stackrel{8}{0} \\
\circ\end{array}$ & $\begin{array}{l}\stackrel{8}{0} \\
\circ \\
0\end{array}$ & $\begin{array}{l}8 \\
\vdots \\
0\end{array}$ & $\begin{array}{l}0 \\
\vdots \\
\vdots\end{array}$ & $\begin{array}{l}8 \\
\vdots \\
0\end{array}$ & $\begin{array}{l}0 \\
\vdots \\
\vdots\end{array}$ & $\stackrel{8}{\circ}$ & ঃั & & : & \begin{tabular}{l}
0 \\
\hdashline \\
0
\end{tabular} & $\begin{array}{l}8 \\
\vdots \\
\vdots\end{array}$ & \begin{tabular}{l}
8 \\
\hdashline \\
0
\end{tabular} & $\begin{array}{l}\circ \\
\vdots \\
\vdots\end{array}$ & $\begin{array}{l}0 \\
\vdots \\
0\end{array}$ & & b. \\
\hline $\begin{array}{l}\overline{0} \\
\dot{\varphi}\end{array}$ & 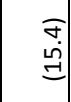 & 곰 & $\begin{array}{l}\text { 于. } \\
\dot{5}\end{array}$ & $\begin{array}{l}f \\
f \\
f\end{array}$ & $\begin{array}{l}\widehat{a} \\
\stackrel{d}{\Delta}\end{array}$ & $\begin{array}{l}\widehat{o} \\
\dot{d} \\
\mathbb{d}\end{array}$ & 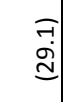 & $\begin{array}{l}\widehat{m} \\
\stackrel{\Xi}{\Xi}\end{array}$ & $\begin{array}{l}\widehat{\infty} \\
0 \\
i \\
0\end{array}$ & $\begin{array}{l}\widehat{\infty} \\
\stackrel{j}{\tilde{g}}\end{array}$ & $\begin{array}{l}\overline{0} \\
\stackrel{-}{ \pm} \\
\dot{\underbrace{}}\end{array}$ & $\begin{array}{l}0 \\
\hat{a} \\
\text { an }\end{array}$ & 㿣 & 8 & 孞 & & & $\stackrel{\bar{I}}{\stackrel{\Delta}{\Xi}}$ & $\begin{array}{l}\hat{\imath} \\
\hat{\vdots} \\
\vdots\end{array}$ & $\begin{array}{l}\overline{0} \\
\stackrel{-}{\sigma}\end{array}$ & $\begin{array}{c}\hat{N} \\
\stackrel{\infty}{\infty} \\
\underline{n}\end{array}$ & 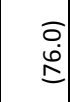 & & 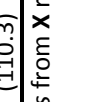 \\
\hline 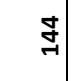 & $\vec{N}$ & $\underset{7}{\mathcal{H}}$ & : & 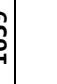 & J & $\stackrel{\infty}{\sim}$ & $\stackrel{\Xi}{\exists}$ & $\begin{array}{l}\overrightarrow{0} \\
\stackrel{0}{n}\end{array}$ & స్త్ర & ఫ్సి & $\begin{array}{l}\overrightarrow{\mathrm{N}} \\
\mathrm{f}\end{array}$ & $\vec{A}$ & 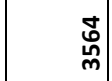 & $\stackrel{\vec{ః}}{\curvearrowright}$ & 憂 & & స్లి & 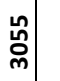 & 志 & $\stackrel{\infty}{\stackrel{m}{m}}$ & 虽 & 㟔 & & 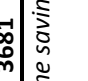 \\
\hline 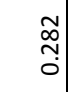 & $\begin{array}{l}m \\
\vdots \\
0 \\
0\end{array}$ & \begin{tabular}{l}
\multirow{2}{0}{} \\
0 \\
0
\end{tabular} & $\begin{array}{l}\stackrel{0}{\circ} \\
\stackrel{0}{0}\end{array}$ & & $\begin{array}{l}0 \\
\text { : } \\
0 \\
0\end{array}$ & 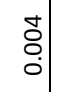 & $\begin{array}{l}\hat{\jmath} \\
0 \\
0\end{array}$ & $\begin{array}{l}\tilde{D} \\
\stackrel{0}{0} \\
0\end{array}$ & $\begin{array}{l}\stackrel{0}{n} \\
\text { D. }\end{array}$ & 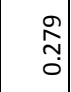 & $\begin{array}{l}0 \\
⿱ \\
0 \\
0\end{array}$ & $\begin{array}{c}\overrightarrow{0} \\
\stackrel{0}{0} \\
0\end{array}$ & $\begin{array}{c}\vec{n} \\
0 \\
0\end{array}$ & 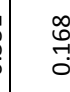 & $\underset{\sim}{\tilde{m}} \stackrel{0}{0}$ & & & 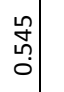 & \begin{tabular}{l}
0 \\
\multirow{7}{0}{} \\
0
\end{tabular} & $\begin{array}{l}0 \\
0 \\
0 \\
0\end{array}$ & 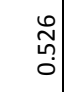 & \begin{tabular}{l}
0 \\
$m$ \\
\hdashline \\
0
\end{tabular} & & 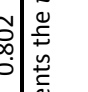 \\
\hline 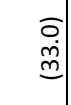 & $\begin{array}{l}\widehat{D} \\
\stackrel{0}{\beth}\end{array}$ & $\begin{array}{l}\overrightarrow{7} \\
\stackrel{0}{\infty}\end{array}$ & 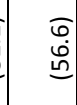 & & $\begin{array}{l}\widehat{\infty} \\
\stackrel{\Xi}{\Xi}\end{array}$ & $\begin{array}{l}\widehat{n} \\
\stackrel{\Xi}{\Xi}\end{array}$ & $\begin{array}{l}\overrightarrow{7} \\
\stackrel{\vec{n}}{=}\end{array}$ & $\begin{array}{l}\underset{T}{1} \\
\underset{\Xi}{=}\end{array}$ & 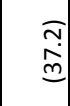 & 곰 & $\begin{array}{l}\widehat{N} \\
\stackrel{5}{5} \\
\vdots\end{array}$ & $\begin{array}{l}\widehat{\infty} \\
\hat{\infty} \\
\underline{\infty}\end{array}$ & 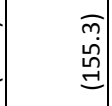 & 每 & $\begin{array}{l}\text { त्. } \\
\text { g. }\end{array}$ & & & $\begin{array}{l}\bar{a} \\
\stackrel{0}{\Delta}\end{array}$ & $\begin{array}{c}\widehat{\sim} \\
\infty \\
\triangleq\end{array} \mid$ & $\begin{array}{l}\widehat{\infty} \\
\infty \\
\Delta \\
=\end{array}$ & 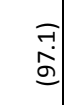 & $\begin{array}{l}\text { ન્} \\
\text { ஸ્ }\end{array}$ & & 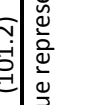 \\
\hline$\stackrel{0}{p}$ & $\eta$ & ํำ & ๆ & 9 & 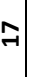 & : & 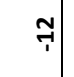 & $\stackrel{\mathscr{N}}{ }$ & \& & ़ְ & | & क़ & $\stackrel{f}{-}$ & $\stackrel{m}{\stackrel{m}{*}}$ & 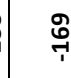 & $\xi$ & : & $\stackrel{\infty}{i}$ & $\stackrel{\mathscr{N}}{\stackrel{\Xi}{2}}$ & $\mathcal{F}$ & ธิ & $\vec{\infty}$ & & 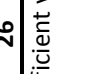 \\
\hline 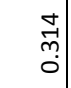 & $\begin{array}{l}\tilde{o} \\
0 \\
0\end{array}$ & 怘 & 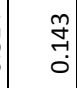 & & $\begin{array}{c}\underset{1}{\sim} \\
0 \\
\end{array}$ & 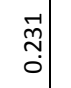 & 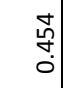 & $\begin{array}{l}7 \\
0 \\
0 \\
0\end{array}$ & $\begin{array}{l}\infty \\
\stackrel{-}{7} \\
0\end{array}$ & $\begin{array}{l}0 \\
0 \\
0 \\
0\end{array}$ & $\begin{array}{l}\hat{o} \\
\substack{0 \\
0}\end{array}$ & $\begin{array}{l}0 \\
\vec{t} \\
0 \\
0\end{array}$ & $\begin{array}{l}\overrightarrow{0} \\
0 \\
0\end{array}$ & $\stackrel{\text { ò }}{\circ}$ & ঃ̊ & & & 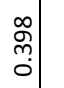 & $\begin{array}{l}0 \\
f \\
⿱ \\
0\end{array}$ & $\begin{array}{l}\overrightarrow{9} \\
\stackrel{9}{0} \\
0\end{array}$ & $\begin{array}{l}0 \\
\vdots \\
0 \\
0\end{array}$ & $\begin{array}{c}\vec{D} \\
0 \\
0\end{array}$ & & : \\
\hline $\begin{array}{l}1 \\
\dot{0} \\
\vdots\end{array}$ & 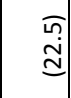 & $\begin{array}{l}\sqrt[1]{0} \\
\infty \\
\infty\end{array}$ & $\begin{array}{l}\bar{\sigma} \\
\dot{g} \\
\dot{g}\end{array}$ & & 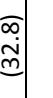 & $\begin{array}{l}\overline{\underline{G}} \\
\stackrel{d}{\mathfrak{d}}\end{array}$ & $\underset{\substack{f\\
}}{ }$ & $\begin{array}{l}\bar{\sigma} \\
\stackrel{\rho}{\Xi} \\
\stackrel{\Xi}{2}\end{array}$ & $\begin{array}{l}\widehat{\hat{n}} \\
\stackrel{0}{0}\end{array}$ & $\begin{array}{l}\widehat{T} \\
\dot{b} \\
\underline{G}\end{array}$ & $\begin{array}{l}\vec{A} \\
\stackrel{5}{S}\end{array}$ & $\begin{array}{c}\widehat{m} \\
\stackrel{S}{S}\end{array}$ & $\begin{array}{l}0 \\
\dot{d} \\
\dot{d}\end{array}$ & $\mid \begin{array}{l}n \\
\end{array}$ & $\begin{array}{l}\widehat{a} \\
\dot{0} \\
\stackrel{0}{3}\end{array}$ & & & 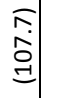 & $\begin{array}{l}\widehat{m} \\
\dot{m} \\
\mathbb{b}\end{array}$ & $\underset{\substack{\not \\
\infty}}{\stackrel{\Phi}{\infty}}$ & $\begin{array}{l}\mathbb{R} \\
\dot{f} \\
g\end{array}$ & $\begin{array}{l}\widehat{m} \\
\vec{b}\end{array}$ & & \\
\hline$\exists$ & 7 & ధุ & ధุ & ? & १ & $\vec{\varphi}$ & $\stackrel{\mathfrak{N}}{ }$ & ఫ̛ & $\stackrel{\infty}{\rightarrow}$ & $\frac{m}{7}$ & $\stackrel{\infty}{\uparrow}$ & ?ุ. & 占 & ด् & @̊ & $\begin{array}{l}0 \\
\vdots \\
\vdots\end{array}$ & î. & จุ & ํำ & ธุ. & $\stackrel{\infty}{q}$ & $\stackrel{\unlhd}{\neg}$ & & ט. \\
\hline 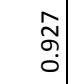 & 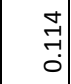 & 웅 & $\begin{array}{l}\text { : } \\
\text { : }\end{array}$ & : & ০. & $\begin{array}{l}\stackrel{0}{0} \\
\vdots \\
0\end{array}$ & $\begin{array}{l}\stackrel{0}{n} \\
\stackrel{0}{0}\end{array}$ & ठే. & $\begin{array}{l}\infty \\
\stackrel{0}{0} \\
0\end{array}$ & $\begin{array}{l}\infty \\
0 \\
0 \\
0 \\
0\end{array}$ & 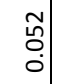 & $\begin{array}{c}\stackrel{1}{0} \\
0 \\
0 \\
0\end{array}$ & $\begin{array}{l}-1 \\
0 \\
0\end{array}$ & ঃั & ঃั & & : & \begin{tabular}{l}
$\circ$ \\
$\vdots$ \\
\hdashline
\end{tabular} & $\begin{array}{l}0 \\
\vdots \\
0\end{array}$ & $\begin{array}{l}\text { Dे } \\
\vdots \\
0\end{array}$ & $\begin{array}{l}\circ \\
\vdots \\
\vdots\end{array}$ & $\begin{array}{l}n \\
\hat{n} \\
0\end{array}$ & & 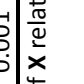 \\
\hline $\begin{array}{l}\widehat{\sigma} \\
\dot{\tilde{m}}\end{array}$ & $\begin{array}{l}\widehat{m} \\
\stackrel{\Xi}{\Xi}\end{array}$ & $\begin{array}{l}\widehat{\infty} \\
\stackrel{\rho}{\Lambda}\end{array}$ & $\begin{array}{l}\bar{\sigma} \\
\dot{\infty} \\
\Phi\end{array}$ & 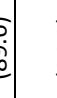 & 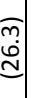 & 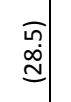 & $\begin{array}{l}\widehat{\Im} \\
\dot{J}\end{array}$ & $\begin{array}{l}\widehat{0} \\
\dot{j} \\
\stackrel{\Xi}{\Xi}\end{array}$ & 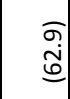 & $\begin{array}{l}\sigma \\
\dot{\sigma} \\
\stackrel{9}{0}\end{array}$ & $\begin{array}{l}\widehat{a} \\
\vdots \\
\vdots \\
\end{array}$ & $\begin{array}{c}\widehat{m} \\
\stackrel{ \pm}{ \pm}\end{array}$ & $\begin{array}{l}0 \\
\dot{d} \\
\stackrel{g}{\Xi} \\
=\end{array}$ & $\begin{array}{l}\infty \\
\infty \\
\infty \\
\stackrel{\infty}{\rightleftharpoons}\end{array}$ & 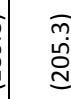 & & & 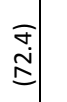 & $\begin{array}{l}\mathbb{N} \\
\mathbb{S}\end{array}$ & 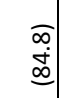 & $\begin{array}{l}\widehat{T} \\
0 \\
0 \\
0\end{array}$ & 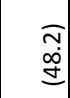 & & 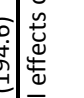 \\
\hline$m$ & $f$ & $\begin{array}{l}\text { \& } \\
\stackrel{8}{1}\end{array}$ & $\stackrel{m}{q}$ & $f$ & $?$ & $\vec{\varphi}$ & 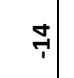 & $\stackrel{\mathbb{2}}{2}$ & ఫ్ & $\mathcal{Y}$ & ڤે| & $\stackrel{\infty}{\rightarrow}$ & 옴 & $\stackrel{n}{\infty}$ & $\stackrel{\infty}{\infty}_{\substack{\infty \\
i}}$ & & 영 & $\begin{array}{l}0 \\
q \\
y\end{array}$ & ר̂. & $\frac{9}{1}$ & กิ & $N$ & & \\
\hline 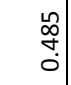 & 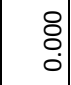 & $\begin{array}{l}\text { જ̆ } \\
\text { જ̆ }\end{array}$ & $\begin{array}{l}-1 \\
0 \\
0 \\
0\end{array}$ & s. & $\begin{array}{l}\hat{0} \\
0 \\
0 \\
0\end{array}$ & $\begin{array}{l}8 \\
\vdots \\
0\end{array}$ & $\begin{array}{l}0 \\
\vdots \\
\hat{0}\end{array}$ & oี & $\begin{array}{l}\stackrel{8}{0} \\
0 \\
0\end{array}$ & \begin{tabular}{l}
0 \\
\hdashline \\
0
\end{tabular} & $\begin{array}{l}0 \\
\grave{0} \\
\vdots\end{array}$ & 吕 & 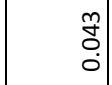 & $\stackrel{7}{7}$ & 웜 & & ठ & $\begin{array}{l}\tilde{z} \\
\text { ơ }\end{array}$ & $\begin{array}{l}\overrightarrow{1} \\
0 \\
0\end{array}$ & $\begin{array}{l}0 \\
\text { : } \\
0 \\
0\end{array}$ & $\begin{array}{l}\circ \\
\vdots \\
\vdots\end{array}$ & $\begin{array}{l}8 \\
0 \\
0\end{array}$ & oี & \\
\hline $\begin{array}{l}\sigma \\
\vec{g} \\
\vec{\Xi}\end{array}$ & $\begin{array}{l}0 \\
\stackrel{0}{0} \\
\stackrel{\Xi}{ \pm}\end{array}$ & 甭 & $\begin{array}{l}\overline{0} \\
\dot{+} \\
\stackrel{m}{N}\end{array}$ & 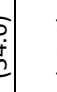 & 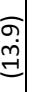 & 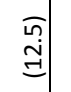 & $\begin{array}{l}\sigma \\
\dot{\Xi} \\
\stackrel{\Xi}{2}\end{array}$ & $\begin{array}{l}f \\
\dot{g} \\
\Xi\end{array}$ & 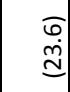 & $\begin{array}{l}\widehat{m} \\
\dot{m} \\
\stackrel{m}{m}\end{array}$ & $\begin{array}{l}\overline{0} \\
\stackrel{\rho}{\hat{m}}\end{array}$ & $\begin{array}{l}\mathcal{f} \\
\dot{g} \\
\dot{d}\end{array}$ & $\begin{array}{l}\vec{f} \\
\dot{\sigma} \\
\dot{a}\end{array}$ & $\begin{array}{l}\infty \\
\infty \\
\infty \\
0\end{array}$ & $\begin{array}{l}\stackrel{0}{ \pm} \\
\stackrel{+}{I}\end{array}$ & & $\begin{array}{c}\vec{s} \\
\stackrel{5}{s}\end{array}$ & $\underset{\vec{I}}{\stackrel{\vec{f}}{ \pm}}$ & $\begin{array}{l}\widehat{\infty} \\
\infty \\
\infty \\
\cong\end{array}$ & $\begin{array}{l}\vec{f} \\
\dot{\theta} \\
\dot{y}\end{array}$ & 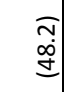 & 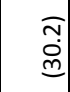 & $\begin{array}{l}\mathcal{F} \\
\dot{b} \\
\Xi\end{array}$ & ?. \\
\hline$\infty$ & : & 0 & $\tilde{N}$ & n. & 6 & ఫ్ & 0 & ట్ & $\stackrel{\text { กิ }}{7}$ & 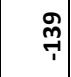 & | & : & ตี & $\stackrel{0}{1}$ & $\underset{7}{7}$ & & 岕 & 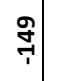 & $\hat{\phi}$ & $\vec{\Phi}$ & શ્̣̂ & $\stackrel{\hat{n}}{\rightarrow}$ & 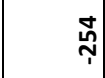 & \\
\hline 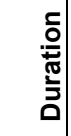 & 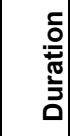 & 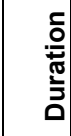 & 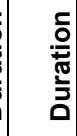 & 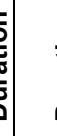 & בְ. & 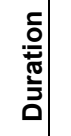 & 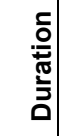 & 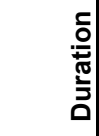 & 岾 & 咅 & 竧 & 胥 & 恿 & $\bar{\square}$ & 它 & & 岀 & 空 & 흔 & 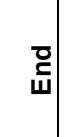 & 흔 & 홒 & 할 & $\frac{0}{4}$ \\
\hline 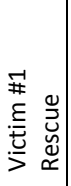 & 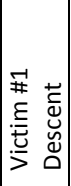 & 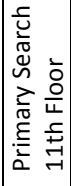 & 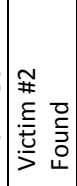 & 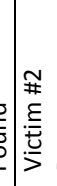 & & 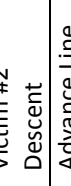 & 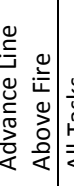 & 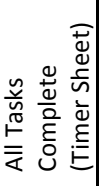 & 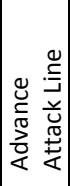 & 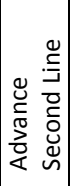 & 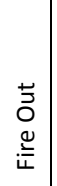 & 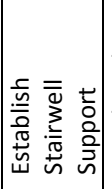 & 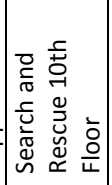 & 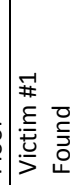 & 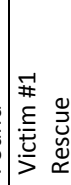 & $=$ & & 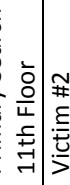 & 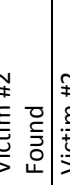 & 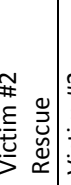 & 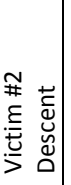 & 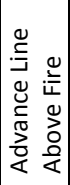 & 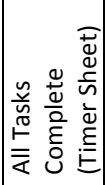 & 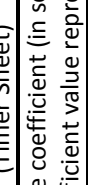 \\
\hline$\wedge$ & $\infty$ & $a$ & 우 & 4 & $\exists$ & $\approx$ & $\stackrel{m}{\rightarrow}$ & $\underset{\sim}{\Delta}$ & $\rightarrow$ & $\sim$ & $m$ & 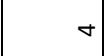 & n & $\bullet$ & r & & $\infty$ & $a$ & 이 & $\exists$ & $\approx$ & 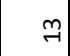 & $\underset{\sim}{\Delta}$ & 4 \\
\hline
\end{tabular}




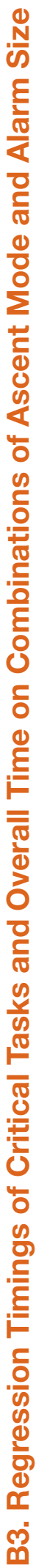

\begin{tabular}{|c|c|c|c|c|c|c|c|c|c|c|c|c|c|c|c|c|c|c|c|c|c|c|c|c|}
\hline \multirow{2}{*}{ 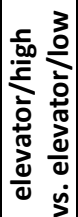 } & $2 \frac{1}{3}$ & 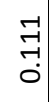 & \begin{tabular}{|c}
0 \\
0 \\
0 \\
0
\end{tabular} & $\begin{array}{l}\infty \\
0 \\
0\end{array}$ & $\begin{array}{l}\text { o } \\
0 \\
0\end{array}$ & $\begin{array}{l}\infty \\
0 \\
0\end{array}$ & $\begin{array}{l}\infty \\
\mathscr{o} \\
o\end{array}$ & $\begin{array}{l}\stackrel{m}{0} \\
0 \\
0\end{array}$ & $\begin{array}{l}\text { ஜ } \\
\circ \\
\circ\end{array}$ & $\begin{array}{l}\text { : } \\
\text { ठ } \\
\text {. }\end{array}$ & $\begin{array}{c}\stackrel{+}{~} \\
\stackrel{-}{0}\end{array}$ & 権 & $\begin{array}{l}\text { \%े } \\
0\end{array}$ & 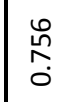 & $\begin{array}{l}\infty \\
\stackrel{\infty}{7} \\
0\end{array}$ & 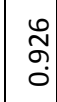 & 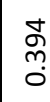 & 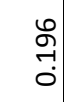 & $\begin{array}{l}m \\
\vdots \\
\vdots \\
0\end{array}$ & శ్ & $\mid \begin{array}{c}\tilde{N} \\
\stackrel{2}{0} \\
\mid\end{array}$ & $\begin{array}{l}\mathfrak{N} \\
\stackrel{2}{0} \\
\vdots\end{array}$ & $\begin{array}{l}\circ \\
\stackrel{2}{0} \\
0\end{array}$ & $c$ \\
\hline & 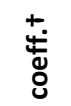 & q & $\stackrel{n}{\rightarrow}$ & ४ै & ケ & †ุ & $\nabla$ & $\stackrel{\infty}{\sim}$ & 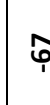 & I & $\stackrel{\text { n }}{\uparrow}$ & ळి & $\stackrel{\infty}{\wedge}$ & ? & $\vec{y}$ & † & g & $\mathcal{J}$ & g & $\Xi$ & $\stackrel{\infty}{\sim}$ & $\stackrel{\infty}{\rightarrow}$ & $N$ & $\bar{Y}$ \\
\hline
\end{tabular}

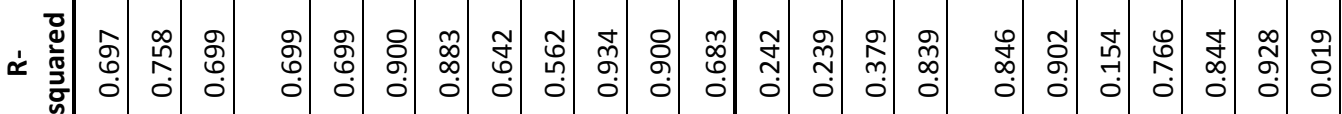

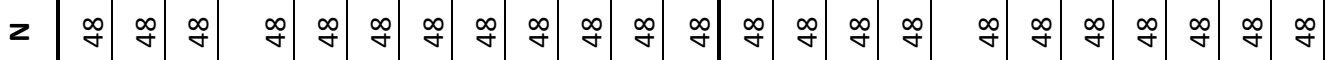

之 :

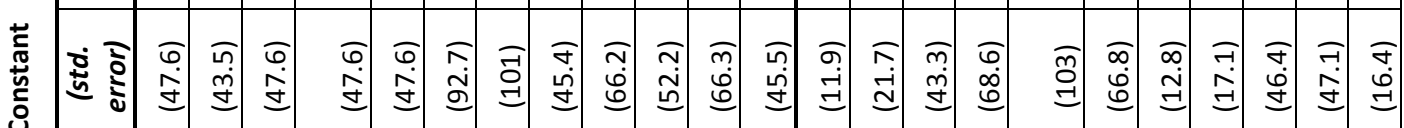

莣

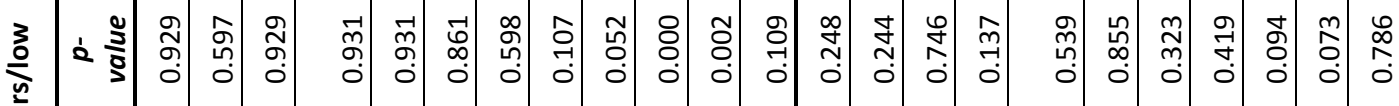

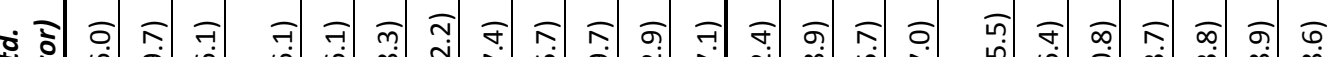

য

高

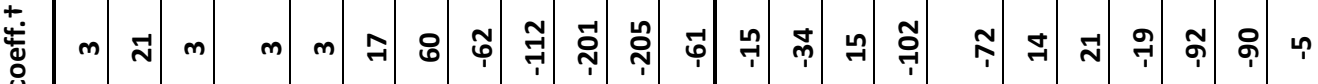

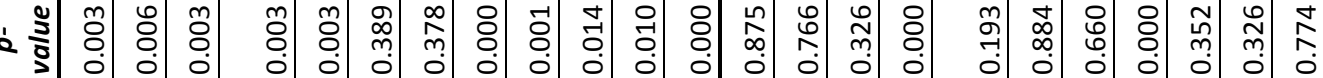

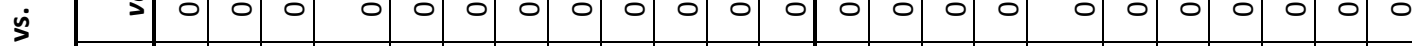

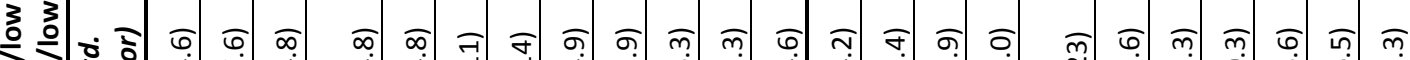
产 氨

蓆

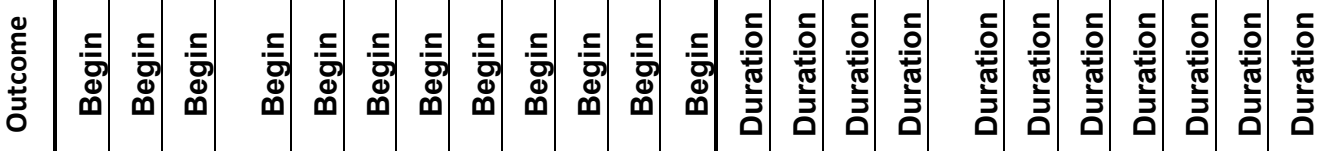

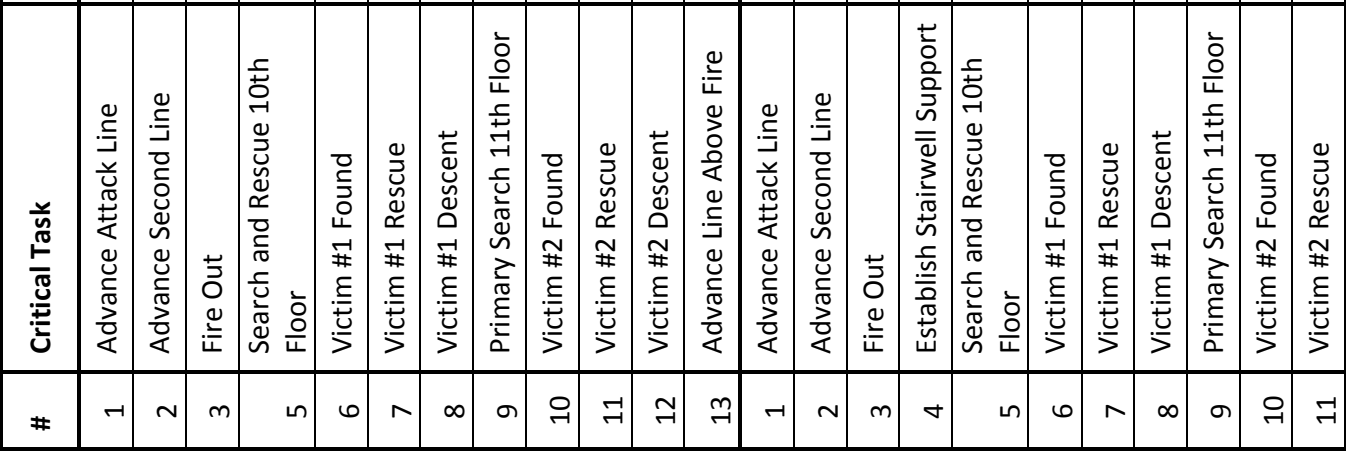




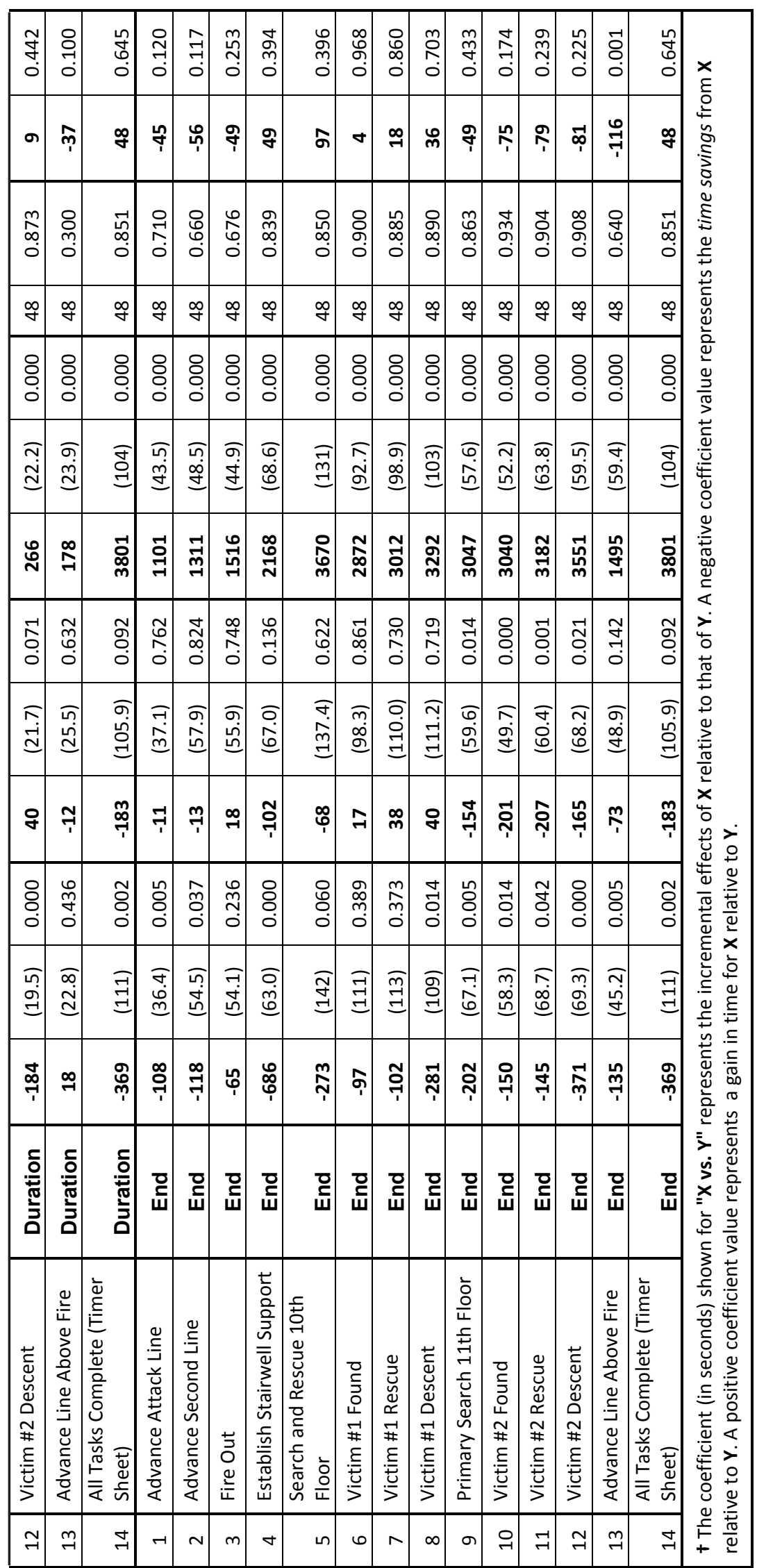




\section{Appendix C: Comparisons Using Regression Results (Refer to Figures 65-85)}

C1. Using Regression Results to Compare the Incremental Effects of Crew Si e on Critical Tasks

\begin{tabular}{|c|c|c|c|c|c|c|c|c|c|c|c|c|}
\hline \multirow[b]{3}{*}{$\#$} & \multirow[b]{3}{*}{$\begin{array}{l}\text { Critical } \\
\text { Task }\end{array}$} & \multirow[b]{3}{*}{ Outcome } & \multicolumn{10}{|c|}{ What is the effect of adding to crew size? } \\
\hline & & & \multicolumn{2}{|c|}{4 vs. 3} & \multicolumn{2}{|c|}{5 vs. 4} & \multicolumn{2}{|c|}{6 vs. 5} & \multicolumn{2}{|c|}{5 vs. 3} & \multicolumn{2}{|c|}{6 vs. 4} \\
\hline & & & coeff. $t$ & signif. $¥$ & coeff. $t$ & signif.‡ & coeff. $t$ & signif. $¥$ & coeff. $t$ & signif. $¥$ & coeff. $†$ & signif.‡ \\
\hline 1 & $\begin{array}{l}\text { Advance } \\
\text { Attack } \\
\text { Line }\end{array}$ & Begin & -83 & $* * *$ & -64 & $* *$ & -74 & $* * *$ & -147 & $* * *$ & -138 & $* * *$ \\
\hline 2 & $\begin{array}{l}\text { Advance } \\
\text { Second } \\
\text { Line } \\
\end{array}$ & Begin & -95 & $* *$ & -114 & $* * *$ & -60 & $* * *$ & -208 & $* * *$ & -174 & $* * *$ \\
\hline 3 & Fire Out & Begin & -83 & ** & -64 & $* *$ & -79 & $* * *$ & -147 & $* * *$ & -143 & $* * *$ \\
\hline 5 & $\begin{array}{l}\text { Search } \\
\text { and } \\
\text { Rescue } \\
\text { 10th } \\
\text { Floor }\end{array}$ & Begin & -83 & $* *$ & -64 & $* *$ & -79 & $* * *$ & -147 & $* * *$ & -143 & $* * *$ \\
\hline 6 & $\begin{array}{l}\text { Victim \#1 } \\
\text { Found }\end{array}$ & Begin & -83 & $* *$ & -64 & $* *$ & -79 & $* * *$ & -147 & $* * *$ & -143 & $* * *$ \\
\hline 7 & $\begin{array}{l}\text { Victim \#1 } \\
\text { Rescue }\end{array}$ & Begin & -792 & $* * *$ & -727 & $* * *$ & -194 & $* *$ & -1519 & $* * *$ & -921 & $* * *$ \\
\hline 8 & $\begin{array}{l}\text { Victim \#1 } \\
\text { Descent }\end{array}$ & Begin & -752 & $* * *$ & -709 & $* * *$ & -208 & $* *$ & -1461 & $* * *$ & -917 & $* * *$ \\
\hline 9 & $\begin{array}{l}\text { Primary } \\
\text { Search } \\
\text { 11th } \\
\text { Floor }\end{array}$ & Begin & -15 & & -94 & $* * *$ & 4 & & -109 & $* * *$ & -90 & $* * *$ \\
\hline 10 & $\begin{array}{l}\text { Victim \#2 } \\
\text { Found }\end{array}$ & Begin & -40 & & -74 & $*$ & -1 & & -114 & $* *$ & -75 & $* *$ \\
\hline 11 & $\begin{array}{l}\text { Victim \#2 } \\
\text { Rescue }\end{array}$ & Begin & -882 & $* * *$ & -161 & $* * *$ & -7 & & -1043 & $* * *$ & -169 & $* * *$ \\
\hline 12 & $\begin{array}{l}\text { Victim \#2 } \\
\text { Descent }\end{array}$ & Begin & -866 & $* * *$ & -134 & $* * *$ & -38 & & -1000 & $* * *$ & -172 & $* *$ \\
\hline 13 & $\begin{array}{l}\text { Advance } \\
\text { Line } \\
\text { Above } \\
\text { Fire }\end{array}$ & Begin & -15 & & -98 & $* * *$ & -16 & & -113 & $* * *$ & -113 & $* * *$ \\
\hline 1 & $\begin{array}{l}\text { Advance } \\
\text { Attack } \\
\text { Line }\end{array}$ & Duration & -20 & & -1 & & -26 & $*$ & -21 & $*$ & -27 & $*$ \\
\hline 2 & $\begin{array}{l}\text { Advance } \\
\text { Second } \\
\text { Line }\end{array}$ & Duration & 14 & & -50 & & -34 & & -35 & & -83 & $* * *$ \\
\hline 3 & Fire Out & Duration & -51 & & -11 & & -133 & $* * *$ & -63 & & -145 & $* * *$ \\
\hline 4 & $\begin{array}{l}\text { Establish } \\
\text { Stairwell } \\
\text { Support }\end{array}$ & Duration & -55 & & -68 & & -110 & $*$ & -123 & $*$ & -178 & $* * *$ \\
\hline 5 & $\begin{array}{l}\text { Search } \\
\text { and } \\
\text { Rescue } \\
\text { 10th } \\
\text { Floor }\end{array}$ & Duration & -598 & $* * *$ & -750 & $* * *$ & -97 & & -1348 & $* * *$ & -847 & $* * *$ \\
\hline 6 & $\begin{array}{l}\text { Victim \#1 } \\
\text { Found }\end{array}$ & Duration & -710 & $* * *$ & -663 & $* * *$ & -115 & $*$ & -1372 & $* * *$ & -778 & $* * *$ \\
\hline 7 & $\begin{array}{l}\text { Victim \#1 } \\
\text { Rescue }\end{array}$ & Duration & 1 & & 27 & $*$ & -5 & & 28 & & 22 & $*$ \\
\hline
\end{tabular}




\begin{tabular}{|c|c|c|c|c|c|c|c|c|c|c|c|c|}
\hline 8 & $\begin{array}{l}\text { Victim \#1 } \\
\text { Descent } \\
\end{array}$ & Duration & 33 & $* *$ & -14 & & 0 & & 20 & & -14 & \\
\hline 9 & $\begin{array}{l}\text { Primary } \\
\text { Search } \\
\text { 11th } \\
\text { Floor }\end{array}$ & Duration & -556 & $* * *$ & -63 & & -154 & $* *$ & -619 & $* * *$ & -217 & $* * *$ \\
\hline 10 & $\begin{array}{l}\text { Victim \#2 } \\
\text { Found }\end{array}$ & Duration & -841 & $* * *$ & -88 & $* *$ & -6 & & -929 & $* * *$ & -94 & $* *$ \\
\hline 11 & \begin{tabular}{|l|} 
Victim \#2 \\
Rescue
\end{tabular} & Duration & 9 & & 5 & & -4 & & 14 & & 1 & \\
\hline 12 & \begin{tabular}{|l|} 
Victim \#2 \\
Descent \\
\end{tabular} & Duration & -32 & $*$ & 11 & & -40 & $* *$ & -22 & & -29 & \\
\hline 13 & $\begin{array}{l}\text { Advance } \\
\text { Line } \\
\text { Above } \\
\text { Fire } \\
\end{array}$ & Duration & -51 & $*$ & -13 & & -24 & & -65 & $* * *$ & -37 & \\
\hline 14 & $\begin{array}{l}\text { All Tasks } \\
\text { Complete } \\
\text { (Timer } \\
\text { Sheet) } \\
\end{array}$ & Duration & -719 & $* * *$ & -538 & $* * *$ & -121 & & -1257 & $* * *$ & -659 & $* * *$ \\
\hline 1 & $\begin{array}{l}\text { Advance } \\
\text { Attack } \\
\text { Line }\end{array}$ & End & -103 & $* *$ & -65 & $* *$ & -101 & $* * *$ & -167 & $* * *$ & -165 & $* * *$ \\
\hline 2 & $\begin{array}{l}\text { Advance } \\
\text { Second } \\
\text { Line } \\
\end{array}$ & End & -80 & & -163 & $* * *$ & -94 & $* *$ & -244 & $* * *$ & -257 & $* * *$ \\
\hline 3 & Fire Out & End & -134 & $* * *$ & -75 & & -212 & $* * *$ & -209 & $* * *$ & -287 & $* * *$ \\
\hline 4 & $\begin{array}{l}\text { Establish } \\
\text { Stairwell } \\
\text { Support }\end{array}$ & End & -55 & & -69 & & -110 & $*$ & -124 & $*$ & -178 & $* * *$ \\
\hline 5 & $\begin{array}{l}\text { Search } \\
\text { and } \\
\text { Rescue } \\
\text { 10th } \\
\text { Floor }\end{array}$ & End & -681 & $* * *$ & -814 & $* * *$ & -177 & & -1495 & $* * *$ & -990 & $* * *$ \\
\hline 6 & \begin{tabular}{|l|} 
Victim \#1 \\
Found
\end{tabular} & End & -792 & $* * *$ & -727 & $* * *$ & -194 & $* *$ & -1519 & $* * *$ & -921 & $* * *$ \\
\hline 7 & \begin{tabular}{|l} 
Victim \#1 \\
Rescue
\end{tabular} & End & -791 & $* * *$ & -699 & $* * *$ & -199 & $* *$ & -1490 & $* * *$ & -898 & $* * *$ \\
\hline 8 & $\begin{array}{l}\text { Victim \#1 } \\
\text { Descent } \\
\end{array}$ & End & -719 & $* * *$ & -722 & $* * *$ & -208 & $* *$ & -1441 & $* * *$ & -931 & $* * *$ \\
\hline 9 & \begin{tabular}{|l} 
Primary \\
Search \\
11th \\
Floor
\end{tabular} & End & -571 & $* * *$ & -157 & $* *$ & -150 & $* *$ & -728 & $* * *$ & -308 & $* * *$ \\
\hline 10 & $\begin{array}{l}\text { Victim \#2 } \\
\text { Found }\end{array}$ & End & -882 & $* * *$ & -161 & $* * *$ & -7 & & -1043 & $* * *$ & -169 & $* * *$ \\
\hline 11 & \begin{tabular}{|l} 
Victim \#2 \\
Rescue
\end{tabular} & End & -873 & $* * *$ & -156 & $* * *$ & -11 & & -1029 & $* * *$ & -168 & $* *$ \\
\hline 12 & \begin{tabular}{|l|} 
Victim \#2 \\
Descent \\
\end{tabular} & End & -898 & $* * *$ & -124 & $* *$ & -78 & & -1021 & $* * *$ & -201 & $* * *$ \\
\hline 13 & \begin{tabular}{|l|} 
Advance \\
Line \\
Above \\
Fire \\
\end{tabular} & End & -67 & & -111 & $* * *$ & -39 & & -178 & $* * *$ & -151 & $* * *$ \\
\hline 14 & \begin{tabular}{|l|} 
All Tasks \\
Complete \\
\end{tabular} & End & -719 & $* * *$ & -538 & $* * *$ & -121 & & -1257 & $* * *$ & -659 & $* * *$ \\
\hline & $\begin{array}{l}\text { (Timer } \\
\text { Sheet) }\end{array}$ & & & & & & & & & & & \\
\hline $\begin{array}{l}\text { + Th } \\
\text { repr }\end{array}$ & $\begin{array}{l}\text { coefficier } \\
\text { sents the }\end{array}$ & $\begin{array}{l}\text { seconds } \\
\text { savings }\end{array}$ & $\begin{array}{l}\text { own f } \\
X \text { re }\end{array}$ & $\begin{array}{l}\text { s. } Y^{\prime} \\
\text { o } Y \text {. }\end{array}$ & $\begin{array}{l}\text { esents } \\
\text { itive c }\end{array}$ & ncre & $\begin{array}{l}\text { effe } \\
\text { repre }\end{array}$ & re & $\begin{array}{l}\text { to tha } \\
\text { time } f\end{array}$ & $\begin{array}{l}\text { A n } \\
\text { elati }\end{array}$ & coef & nt value \\
\hline
\end{tabular}


C2. Using Regression Results to Compare the Effects of Alarm Size and Ascent Mode
What are the effects of alarm response and ascent mode?

\begin{tabular}{|c|c|c|c|c|c|c|}
\hline & & & hig & low & elevat & stairs \\
\hline $\begin{array}{l}\text { Task } \\
\#\end{array}$ & Critical Task & Outcome & coeff.t & signif. $¥$ & coeff.t & signif. $¥$ \\
\hline 1 & Advance Attack Line & Begin & -18 & & -132 & $* * *$ \\
\hline 2 & Advance Second Line & Begin & 3 & & -126 & $* * *$ \\
\hline 3 & Fire Out & Begin & -21 & & -135 & $* * *$ \\
\hline 5 & Search and Rescue 10th Floor & Begin & -21 & & -135 & $* * *$ \\
\hline 6 & Victim \#1 Found & Begin & -21 & & -135 & $* * *$ \\
\hline 7 & Victim \#1 Rescue & Begin & 11 & & -103 & \\
\hline 8 & Victim \#1 Descent & Begin & 39 & & -122 & \\
\hline 9 & Primary Search 11th Floor & Begin & -64 & $* * *$ & -149 & $* * *$ \\
\hline 10 & Victim \#2 Found & Begin & -94 & $* * *$ & -179 & $* * *$ \\
\hline 11 & Victim \#2 Rescue & Begin & -138 & $* * *$ & -87 & $* *$ \\
\hline 12 & Victim \#2 Descent & Begin & -147 & $* * *$ & -129 & $* * *$ \\
\hline 13 & Advance Line Above Fire & Begin & -70 & $* * *$ & -162 & $* * *$ \\
\hline 1 & Advance Attack Line & Duration & -10 & & 7 & \\
\hline 2 & Advance Second Line & Duration & -38 & $*$ & -13 & \\
\hline 3 & Fire Out & Duration & 6 & & 36 & \\
\hline 4 & Establish Stairwell Support & Duration & -27 & & -610 & $* * *$ \\
\hline 5 & Search and Rescue 10th Floor & Duration & 35 & & -56 & \\
\hline 6 & Victim \#1 Found & Duration & 32 & & 32 & \\
\hline 7 & Victim \#1 Rescue & Duration & 17 & & -8 & \\
\hline 8 & Victim \#1 Descent & Duration & -1 & & -161 & $* * *$ \\
\hline 9 & Primary Search 11th Floor & Duration & -37 & & 0 & \\
\hline 10 & Victim \#2 Found & Duration & -44 & & 92 & $* *$ \\
\hline 11 & Victim \#2 Rescue & Duration & -4 & & 6 & \\
\hline 12 & Victim \#2 Descent & Duration & 25 & $*$ & -199 & $* * *$ \\
\hline 13 & Advance Line Above Fire & Duration & -25 & & 6 & \\
\hline 14 & All Tasks Complete (Timer Sheet) & Duration & -68 & & -254 & $* * *$ \\
\hline 1 & Advance Attack Line & End & -28 & & -125 & $* * *$ \\
\hline 2 & Advance Second Line & End & -35 & & -139 & $* * *$ \\
\hline 3 & Fire Out & End & -15 & & -98 & $* * *$ \\
\hline 4 & Establish Stairwell Support & End & -27 & & -610 & $* * *$ \\
\hline 5 & Search and Rescue 10th Floor & End & 14 & & -191 & $* *$ \\
\hline 6 & Victim \#1 Found & End & 11 & & -103 & \\
\hline 7 & Victim \#1 Rescue & End & 28 & & -112 & \\
\hline 8 & Victim \#1 Descent & End & 38 & & -283 & $* * *$ \\
\hline 9 & Primary Search 11th Floor & End & -101 & $* *$ & -149 & $* * *$ \\
\hline 10 & Victim \#2 Found & End & -138 & $* * *$ & -87 & $* *$ \\
\hline 11 & Victim \#2 Rescue & End & -143 & $* * *$ & -81 & $*$ \\
\hline 12 & Victim \#2 Descent & End & -123 & $* *$ & -329 & $* * *$ \\
\hline 13 & Advance Line Above Fire & End & -94 & $* * *$ & -157 & $* * *$ \\
\hline 14 & All Tasks Complete (Timer Sheet) & End & -68 & & -254 & $* * *$ \\
\hline $\begin{array}{l}+ \text { The } \\
\text { negat } \\
\text { gain ir }\end{array}$ & $\begin{array}{l}\text { efficient (in seconds) shown for "X vs } \\
\text { coefficient value represents the time } \\
\text { ime for } \mathbf{X} \text { relative to } \mathbf{Y} \text {. }\end{array}$ & $\begin{array}{l}\text { resents the } \\
\text { s from } \mathbf{X} \text { rel }\end{array}$ & $\begin{array}{l}\text { crement } \\
\text { ve to } \mathbf{Y} \text {. }\end{array}$ & $\begin{array}{l}\text { cts of } \mathbf{X} \\
\text { tive coef }\end{array}$ & $\begin{array}{l}\text { ve to tha } \\
\text { t value }\end{array}$ & $\begin{array}{l}\text { Y. A } \\
\text { sents a }\end{array}$ \\
\hline
\end{tabular}


C3. Using Regression Results to Compare the Effects of High Response with Lower Crew Size to Low Response with Higher Crew Size
What are the effects of a high response with lower crew size compared to a low response with higher crew size?

\begin{tabular}{|c|c|c|c|c|c|c|c|c|}
\hline $\begin{array}{l}\text { Task } \\
\# \\
\end{array}$ & Critical Task & Outcome & coeff. $t$ & signif. $¥$ & coeff. $t$ & signif.‡ & coeff. $t$ & signif.‡ \\
\hline 1 & Advance Attack Line & Begin & -93 & $*$ & -57 & $*$ & -28 & \\
\hline 2 & Advance Second Line & Begin & -110 & $*$ & -112 & $* * *$ & -51 & \\
\hline 3 & Fire Out & Begin & -93 & $*$ & -57 & $*$ & -28 & \\
\hline 5 & $\begin{array}{l}\text { Search and Rescue 10th } \\
\text { Floor }\end{array}$ & Begin & -93 & $*$ & -57 & $*$ & -28 & \\
\hline 6 & Victim \#1 Found & Begin & -93 & $*$ & -57 & $*$ & -28 & \\
\hline 7 & Victim \#1 Rescue & Begin & -875 & $* * *$ & -659 & $* * *$ & -133 & \\
\hline 8 & Victim \#1 Descent & Begin & -857 & $* * *$ & -694 & $* * *$ & -181 & \\
\hline 9 & Primary Search 11th Floor & Begin & 16 & & -34 & & 102 & $* *$ \\
\hline 10 & Victim \#2 Found & Begin & 6 & & 16 & & 142 & $* *$ \\
\hline 11 & Victim \#2 Rescue & Begin & -737 & $* * *$ & -50 & & 125 & \\
\hline 12 & Victim \#2 Descent & Begin & -732 & $* * *$ & -17 & & 123 & \\
\hline 13 & Advance Line Above Fire & Begin & 16 & & -39 & & 93 & $* *$ \\
\hline 1 & Advance Attack Line & Duration & -11 & & 9 & & -16 & \\
\hline 2 & Advance Second Line & Duration & 68 & & -1 & & -12 & \\
\hline 3 & Fire Out & Duration & -61 & & -21 & & -135 & $* *$ \\
\hline 4 & Establish Stairwell Support & Duration & -18 & & -59 & & -94 & \\
\hline 5 & $\begin{array}{l}\text { Search and Rescue 10th } \\
\text { Floor }\end{array}$ & Duration & -647 & $* * *$ & -718 & $* * *$ & -119 & \\
\hline 6 & Victim \#1 Found & Duration & -783 & $* * *$ & -602 & $* * *$ & -105 & \\
\hline 7 & Victim \#1 Rescue & Duration & -3 & & 11 & & -36 & \\
\hline 8 & Victim \#1 Descent & Duration & 47 & & -17 & & -13 & \\
\hline 9 & Primary Search 11th Floor & Duration & -486 & $* * *$ & -58 & & -150 & * \\
\hline 10 & Victim \#2 Found & Duration & -743 & $* * *$ & -65 & & -17 & \\
\hline 11 & Victim \#2 Rescue & Duration & -3 & & -8 & & 17 & \\
\hline 12 & Victim \#2 Descent & Duration & -61 & $* *$ & -31 & & -60 & $* * *$ \\
\hline 13 & Advance Line Above Fire & Duration & -14 & & 25 & & -12 & \\
\hline 14 & $\begin{array}{l}\text { All Tasks Complete (Timer } \\
\text { Sheet) }\end{array}$ & Duration & -729 & $* * *$ & -462 & $* *$ & 26 & \\
\hline 1 & Advance Attack Line & End & -104 & & -48 & & -44 & \\
\hline 2 & Advance Second Line & End & -42 & & -113 & $*$ & -63 & \\
\hline 3 & Fire Out & End & -154 & $*$ & -78 & & -162 & $* *$ \\
\hline 4 & Establish Stairwell Support & End & -18 & & -59 & & -94 & \\
\hline 5 & $\begin{array}{l}\text { Search and Rescue 10th } \\
\text { Floor }\end{array}$ & End & -740 & $* * *$ & -775 & $* * *$ & -147 & \\
\hline 6 & Victim \#1 Found & End & -875 & $* * *$ & -659 & $* * *$ & -133 & \\
\hline 7 & Victim \#1 Rescue & End & -878 & $* * *$ & -648 & $* * *$ & -169 & \\
\hline 8 & Victim \#1 Descent & End & -810 & $* * *$ & -711 & $* * *$ & -194 & \\
\hline 9 & Primary Search 11th Floor & End & -470 & $* * *$ & -92 & & -48 & \\
\hline 10 & Victim \#2 Found & End & -737 & $* * *$ & -50 & & 125 & \\
\hline 11 & Victim \#2 Rescue & End & -740 & $* * *$ & -57 & & 142 & $*$ \\
\hline 12 & Victim \#2 Descent & End & -792 & $* * *$ & -48 & & 62 & \\
\hline 13 & Advance Line Above Fire & End & 2 & & -14 & & 81 & \\
\hline 14 & $\begin{array}{l}\text { All Tasks Complete (Timer } \\
\text { Sheet) }\end{array}$ & End & -729 & $* * *$ & -462 & $* *$ & 26 & \\
\hline
\end{tabular}

† The coefficient (in seconds) shown for "X vs. $Y$ " represents the incremental effects of $\mathbf{X}$ relative to that of $\mathbf{Y}$. A negative coefficient value represents the time savings from $\mathbf{X}$ relative to $\mathbf{Y}$. A positive coefficient value represents a gain in time for $\mathbf{X}$ relative to $\mathbf{Y}$.

\# Legend: ${ }^{* * *}=$ significant at the 0.01 level; ${ }^{* *}=$ significant at the 0.05 level; ${ }^{*}$ significant at the 0.10 level 
C4. Using Regression Results to Compare the Effects of Combinations of Alarm Size and Ascent Mode

\begin{tabular}{|c|c|c|c|c|c|c|c|c|}
\hline & & & stail & low & stai & low & eleva & r/low \\
\hline$\#$ & Critical Task & Outcome & coeff. $t$ & signif. $¥$ & coeff. $†$ & signif. $¥$ & coeff. $t$ & signif.‡ \\
\hline 1 & Advance Attack Line & Begin & -111 & $* * *$ & 3 & & -40 & \\
\hline 2 & Advance Second Line & Begin & -108 & $* * *$ & 21 & & -15 & \\
\hline 3 & Fire Out & Begin & -111 & $* * *$ & 3 & & -45 & $*$ \\
\hline 5 & Search and Rescue 10th Floor & Begin & -111 & $* * *$ & 3 & & -45 & $*$ \\
\hline 6 & Victim \#1 Found & Begin & -111 & $* * *$ & 3 & & -45 & * \\
\hline 7 & Victim \#1 Rescue & Begin & -97 & & 17 & & 4 & \\
\hline 8 & Victim \#1 Descent & Begin & -101 & & 60 & & 18 & \\
\hline 9 & Primary Search 11th Floor & Begin & -146 & $* * *$ & -62 & & -67 & $* * *$ \\
\hline 10 & Victim \#2 Found & Begin & -196 & $* * *$ & -112 & $*$ & -77 & $* * *$ \\
\hline 11 & Victim \#2 Rescue & Begin & -150 & $* *$ & -201 & $* * *$ & -75 & \\
\hline 12 & Victim \#2 Descent & Begin & -187 & $* *$ & -205 & $* * *$ & -89 & \\
\hline 13 & Advance Line Above Fire & Begin & -153 & $* * *$ & -61 & & -78 & $* * *$ \\
\hline 1 & Advance Attack Line & Duration & 2 & & -15 & & -5 & \\
\hline 2 & Advance Second Line & Duration & -9 & & -34 & & -41 & \\
\hline 3 & Fire Out & Duration & 46 & & 15 & & -4 & \\
\hline 4 & Establish Stairwell Support & Duration & -686 & $* * *$ & -102 & & 49 & \\
\hline 5 & Search and Rescue 10th Floor & Duration & -163 & & -72 & & 142 & \\
\hline 6 & Victim \#1 Found & Duration & 14 & & 14 & & 49 & \\
\hline 7 & Victim \#1 Rescue & Duration & -5 & & 21 & & 14 & \\
\hline 8 & Victim \#1 Descent & Duration & -180 & $* * *$ & -19 & & 18 & \\
\hline 9 & Primary Search 11th Floor & Duration & -55 & & -92 & $*$ & 18 & \\
\hline 10 & Victim \#2 Found & Duration & 46 & & -90 & $*$ & 2 & \\
\hline 11 & Victim \#2 Rescue & Duration & 6 & & -5 & & -4 & \\
\hline 12 & Victim \#2 Descent & Duration & -184 & $* * *$ & 40 & $*$ & 9 & \\
\hline 13 & Advance Line Above Fire & Duration & 18 & & -12 & & -37 & * \\
\hline 14 & $\begin{array}{l}\text { All Tasks Complete (Timer } \\
\text { Sheet) }\end{array}$ & Duration & -369 & $* * *$ & -183 & $*$ & 48 & \\
\hline 1 & Advance Attack Line & End & -108 & $* * *$ & -11 & & -45 & \\
\hline 2 & Advance Second Line & End & -118 & $* *$ & -13 & & -56 & \\
\hline 3 & Fire Out & End & -65 & & 18 & & -49 & \\
\hline 4 & Establish Stairwell Support & End & -686 & $* * *$ & -102 & & 49 & \\
\hline 5 & Search and Rescue 10th Floor & End & -273 & $*$ & -68 & & 97 & \\
\hline 6 & Victim \#1 Found & End & -97 & & 17 & & 4 & \\
\hline 7 & Victim \#1 Rescue & End & -102 & & 38 & & 18 & \\
\hline 8 & Victim \#1 Descent & End & -281 & $* *$ & 40 & & 36 & \\
\hline 9 & Primary Search 11th Floor & End & -202 & $* * *$ & -154 & $* *$ & -49 & \\
\hline 10 & Victim \#2 Found & End & -150 & $* *$ & -201 & $* * *$ & -75 & \\
\hline 11 & Victim \#2 Rescue & End & -145 & $* *$ & -207 & $* * *$ & -79 & \\
\hline 12 & Victim \#2 Descent & End & -371 & $* * *$ & -165 & $* *$ & -81 & \\
\hline 13 & Advance Line Above Fire & End & -135 & $* * *$ & -73 & & -116 & $* * *$ \\
\hline 14 & $\begin{array}{l}\text { All Tasks Complete (Timer } \\
\text { Sheet) }\end{array}$ & End & -369 & $* * *$ & -183 & $*$ & 48 & \\
\hline
\end{tabular}

† The coefficient (in seconds) shown for "X vs. $Y$ " represents the incremental effects of $\mathbf{X}$ relative to that of $\mathbf{Y}$. A negative coefficient value represents the time savings from $\mathbf{X}$ relative to $\mathbf{Y}$. A positive coefficient value represents a gain in time for $\mathbf{X}$ relative to $\mathbf{Y}$. 


\begin{tabular}{|c|c|c|c|c|c|c|c|c|c|c|c|c|c|c|c|c|}
\hline \multirow{13}{*}{ 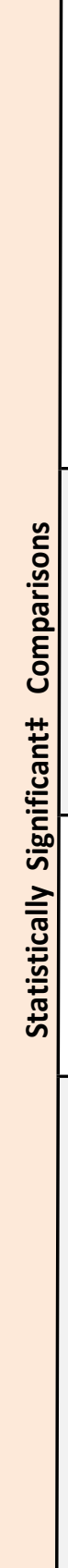 } & \multirow{3}{*}{ 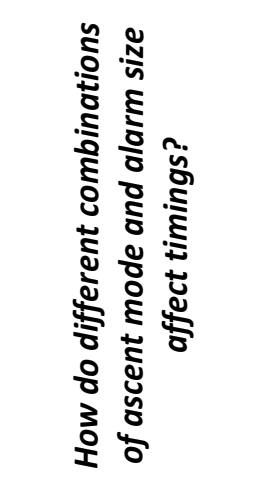 } & 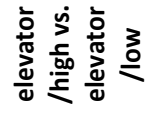 & & & $*$ & * & * & & & $\begin{array}{l}\stackrel{*}{*} \\
* \\
*\end{array}$ & $\begin{array}{l}* \\
* \\
*\end{array}$ & & & $\stackrel{*}{*}$ & & \\
\hline & & 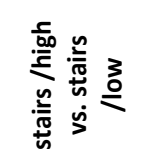 & & & & & & & & & $*$ & $\stackrel{*}{*} \underset{*}{*}$ & $\stackrel{*}{*}$ & & & \\
\hline & & 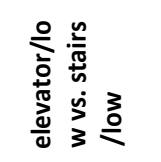 & $\begin{array}{l}\stackrel{*}{*} \\
* \\
*\end{array}$ & $\stackrel{*}{*}$ & $\begin{array}{l}* \\
* \\
*\end{array}$ & $\stackrel{*}{*}$ & $\stackrel{*}{*}$ & & & $\stackrel{*}{*} \underset{*}{*}$ & $\begin{array}{l}* \\
* \\
*\end{array}$ & $\stackrel{*}{*}$ & $\stackrel{*}{*}$ & $\stackrel{*}{*} \underset{*}{*}$ & & \\
\hline & \multirow{3}{*}{ 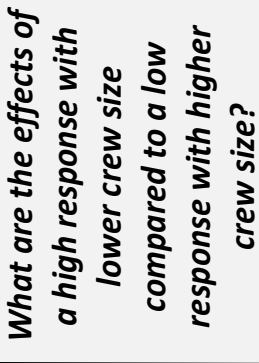 } & 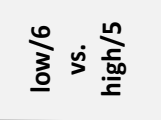 & & & & & & & & $\stackrel{*}{*}$ & $*$ & & & $\stackrel{*}{*}$ & & \\
\hline & & 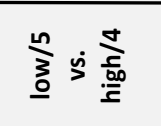 & * & $\stackrel{*}{*}$ & * & * & $*$ & $\stackrel{*}{*}$ & $\stackrel{*}{*}$ & & & & & & & \\
\hline & & 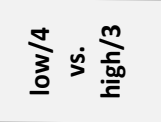 & * & $*$ & * & $*$ & * & $\stackrel{*}{*}$ & $\begin{array}{l}* \\
* \\
*\end{array}$ & & & $\begin{array}{l}\stackrel{*}{*} \\
*\end{array}$ & $\begin{array}{l}* \\
* \\
*\end{array}$ & & & \\
\hline & \multirow{2}{*}{ 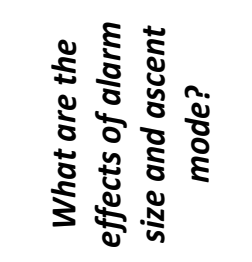 } & 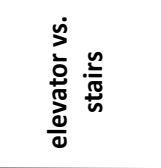 & $\stackrel{*}{*} \underset{*}{*}$ & $\stackrel{*}{*}$ & $\stackrel{*}{*}$ & $\stackrel{*}{*}$ & $\begin{array}{l}* \\
* \\
*\end{array}$ & & & $\begin{array}{l}* \\
* \\
* \\
*\end{array}$ & $\stackrel{*}{*}$ & $\stackrel{*}{*}$ & $\begin{array}{l}* * \\
* \\
*\end{array}$ & $\stackrel{*}{*}$ & & \\
\hline & & 点紫 & & & & & & & & $\stackrel{*}{*}$ & $\stackrel{*}{*}$ & $\stackrel{*}{*}$ & $\stackrel{*}{*}$ & $\stackrel{*}{*}$ & & * \\
\hline & \multirow{5}{*}{ 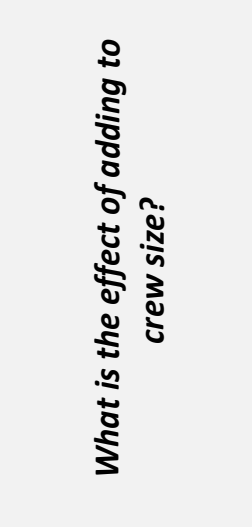 } & $\sum_{0}^{\infty}+$ & $\begin{array}{l}\stackrel{*}{*} \\
*\end{array}$ & $\stackrel{*}{*} \underset{*}{*}$ & $\begin{array}{l}\stackrel{*}{*} \\
*\end{array}$ & $\stackrel{*}{*}$ & $\stackrel{*}{*}$ & $\stackrel{*}{*}$ & $\begin{array}{l}* \\
* \\
*\end{array}$ & 菊 & $*$ & $\stackrel{*}{*}$ & $*$ & $\stackrel{*}{*}$ & * & $\stackrel{*}{*}$ \\
\hline & & $\sum_{\text {in }}^{\dot{s}} m$ & $\stackrel{*}{*}$ & $\stackrel{*}{*}$ & $\stackrel{*}{*}$ & $\stackrel{*}{*}$ & $\stackrel{*}{*}$ & $\stackrel{*}{*}$ & $\stackrel{*}{*}$ & $\stackrel{*}{*}$ & $*$ & $\stackrel{*}{*}$ & $\stackrel{*}{*}$ & $\stackrel{*}{*}$ & * & \\
\hline & & $\sum_{0}^{\infty}$ & $\begin{array}{l}* \\
* \\
*\end{array}$ & $\stackrel{*}{*}$ & $\stackrel{*}{*}$ & $\stackrel{*}{*}$ & $\stackrel{*}{*}$ & $\stackrel{*}{*}$ & $*$ & & & & & & * & \\
\hline & & $\dot{s}_{\text {in }}^{\dot{1}}$ & $*$ & $\stackrel{*}{*}$ & $*$ & $\stackrel{*}{*}$ & $*$ & $\stackrel{*}{*}$ & $\stackrel{*}{*}$ & $\begin{array}{l}* \\
* \\
*\end{array}$ & $*$ & $\stackrel{*}{*}$ & $\stackrel{*}{*}$ & $\stackrel{*}{*} \underset{*}{*}$ & & \\
\hline & & $\sum_{\vec{q}}^{\dot{s}} m$ & $*$ & $\stackrel{*}{*}$ & $\stackrel{*}{*}$ & $\stackrel{*}{*}$ & $\stackrel{*}{*}$ & $\stackrel{*}{*}$ & $\stackrel{*}{*}$ & & & $\underset{*}{*}$ & $\stackrel{*}{*}$ & & & \\
\hline & & 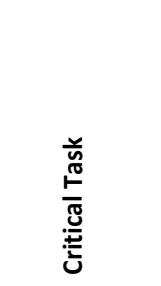 & 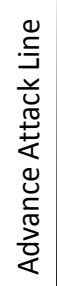 & 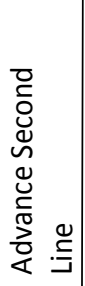 & 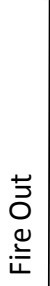 & 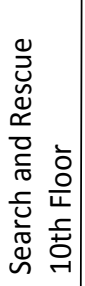 & 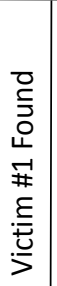 & 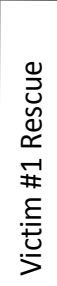 & 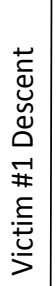 & 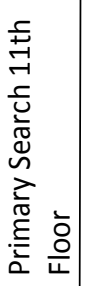 & 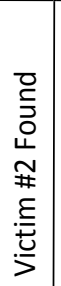 & 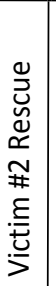 & 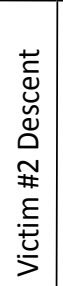 & 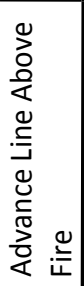 & 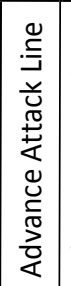 & 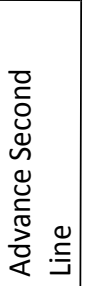 \\
\hline & & 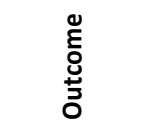 & & & & & & & & & & & & & uoụt & e.sna \\
\hline & & $\#$ & -1 & $\sim$ & $m$ & n & 0 & $r$ & $\infty$ & $a$ & 어 & $\exists$ & $\approx$ & $m$ & -1 & $\sim$ \\
\hline
\end{tabular}




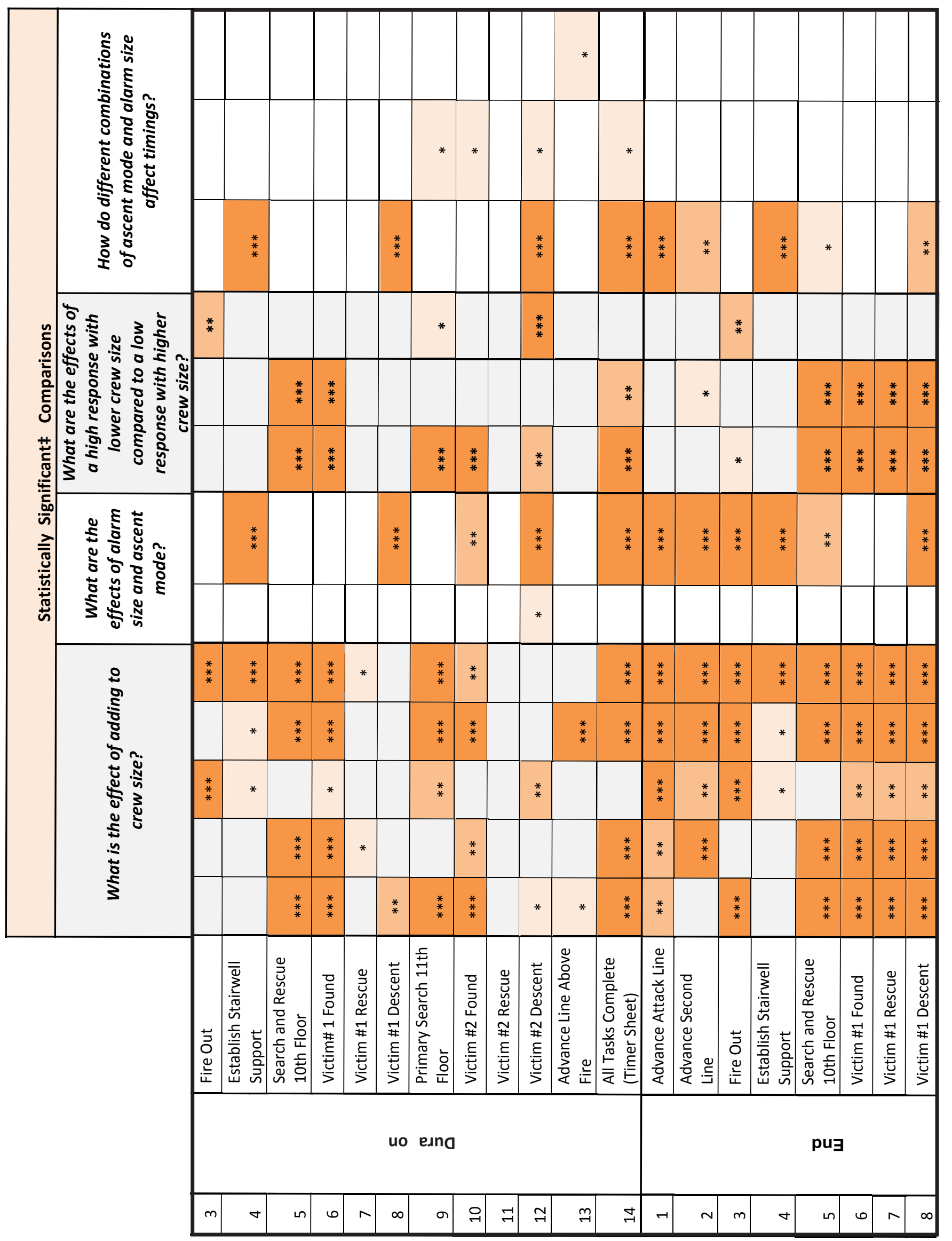




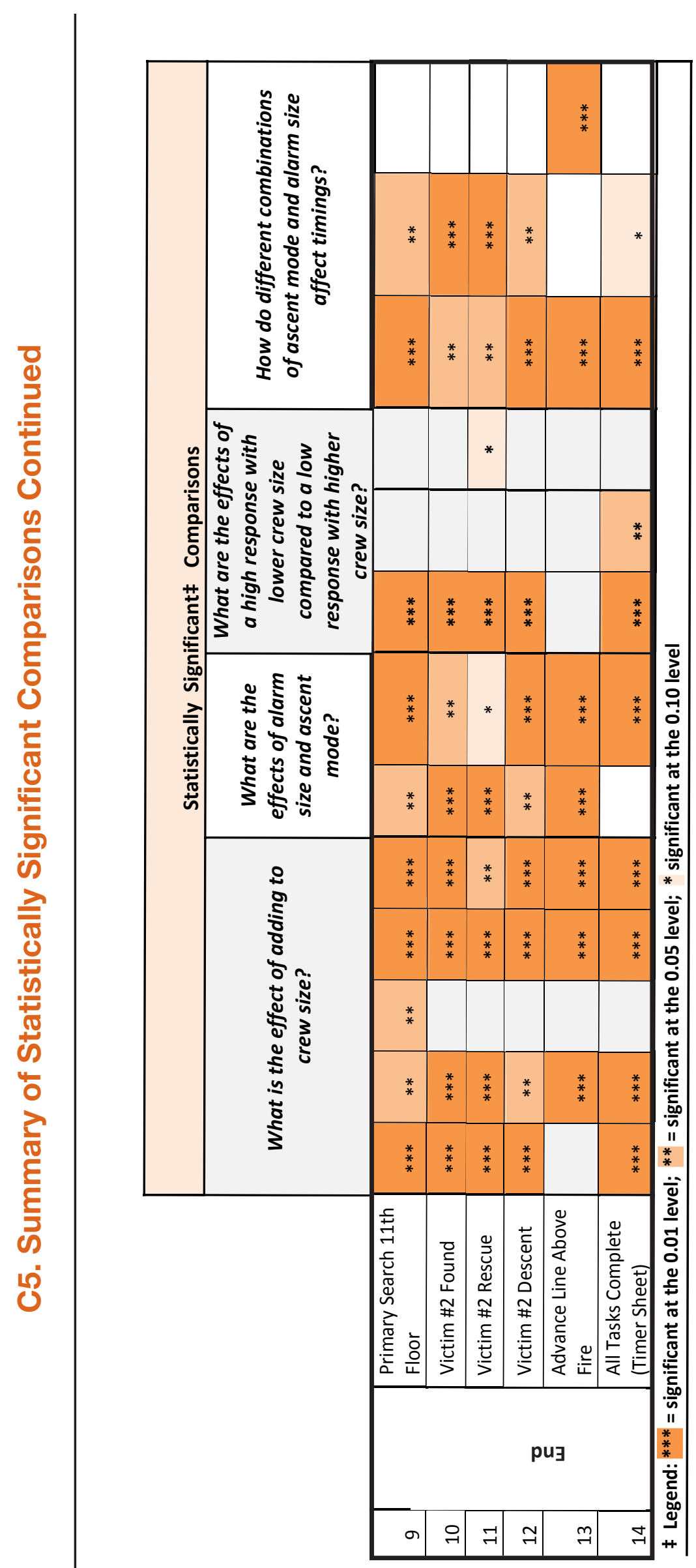




\section{Effects of Crew Size}

In this section, the results of the regression analyses are used to identify and discuss findings regarding the effect of crew size on critical tasks. Appendix C1 reconfigures the earlier regression results (Appendices B1-B3) for an analysis of the incremental effect of crew size on critical tasks. These results were assembled into separate tables reflecting the three timing outcomes in this study - begin time, duration, and end time. In this synthesis, three tables are provided, one for each outcome type (begin, duration, end). Table 17 presents the effect on time to begin a critical task when crew size is incremented by one or two firefighters. Note that these results control for (i.e., remove the effects of) ascent mode and alarm size, so the results indicate the net impact of crew size change. The leftmost three-column set shows the decrease in task begin times when a single firefighter is added to crews of 3, 4 and 5. Generally, going from a 3-person to a 4-person crew size has a large impact on Advancing the Attack Line, backing it up by Advancing a Second Line, and commencing Search and Rescue of the 10th Floor. Reductions are in the range of $1 \frac{1}{2} 2 \mathrm{~min}$. Increases in crew size from 4 to 5 see significant reductions in all of the critical task begin times, with reductions ranging between $1 \mathrm{~min}$ and $2 \mathrm{~min}$. Raising crew size from 5 to 6 shows significant time reductions of just over 1 min for Advance Attack Line, Advance Second Line, and Search and Rescue of the 10th Floor. As one might expect, increasing crew sizes by 2 firefighters from 3 to 5 or from 4 to 6 results in the largest improvements in begin times for critical tasks. Reductions range from a low of $1 \mathrm{~min}$ (for Advance Second Line 6 vs. 5 crew size) to $25 \mathrm{~min}$ (for Victim \#1 Rescue for 5 vs. 3 crew size). These findings suggest that meaningful improvements in critical task start times can be attained when adding a single firefighter to a crew regardless of the current crew size (under 6), and the benefits in time reduction continue when adding 2 firefighters to a crew.

Next, the effects of incrementing crew size on the duration of critical tasks are examined. The duration is the time it takes to complete a task once it commences. Table 18 presents the result of this analysis, controlling for ascent mode and alarm size. It is apparent that incrementing crew size by one firefighter (see the

\begin{tabular}{|c|c|c|c|c|c|c|c|c|c|c|c|}
\hline \multirow[b]{3}{*}{ Outcome } & \multirow[b]{3}{*}{ Critical Tasks } & \multicolumn{10}{|c|}{ What is the effect of adding to crew size on BEGIN time? } \\
\hline & & \multicolumn{2}{|c|}{4 vs. 3} & \multicolumn{2}{|c|}{5 vs. 4} & \multicolumn{2}{|c|}{6 vs. 5} & \multicolumn{2}{|c|}{5 vs. 3} & \multicolumn{2}{|c|}{6 vs. 4} \\
\hline & & $\begin{array}{c}\text { coeff. } \\
\dagger\end{array}$ & $\begin{array}{c}\text { Signif } \\
. \neq\end{array}$ & $\begin{array}{c}\text { coeff. } \\
+\end{array}$ & $\begin{array}{c}\text { Signif } \\
. \neq\end{array}$ & $\begin{array}{c}\text { coeff. } \\
+\end{array}$ & $\begin{array}{c}\text { Signif } \\
. \neq\end{array}$ & $\begin{array}{c}\text { coeff. } \\
+\end{array}$ & $\begin{array}{c}\text { Signif } \\
. \neq\end{array}$ & $\begin{array}{c}\text { coeff. } \\
\dagger\end{array}$ & $\begin{array}{l}\text { Signif. } \\
\ddagger\end{array}$ \\
\hline \multirow{12}{*}{ 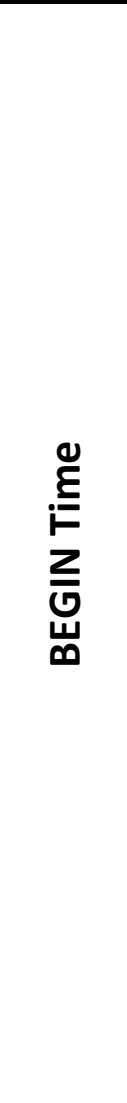 } & $\begin{array}{l}\text { Advance } \\
\text { Attack Line }\end{array}$ & -83 & $* *$ & -64 & $* *$ & -74 & $* * *$ & -147 & $* * *$ & -138 & $* * *$ \\
\hline & $\begin{array}{l}\text { Advance } \\
\text { Second Line }\end{array}$ & -95 & $* *$ & -114 & $* * *$ & -60 & $* * *$ & -208 & $* * *$ & -174 & $* * *$ \\
\hline & Fire Out & -83 & $* *$ & -64 & $* *$ & -79 & $* * *$ & -147 & $* * *$ & -143 & $* * *$ \\
\hline & $\begin{array}{l}\text { Search and } \\
\text { Rescue 10th } \\
\text { Floor }\end{array}$ & -83 & $* *$ & -64 & $* *$ & -79 & $* * *$ & -147 & $* * *$ & -143 & $* * *$ \\
\hline & $\begin{array}{l}\text { Victim \#1 } \\
\text { Found }\end{array}$ & -83 & $* *$ & -64 & $* *$ & -79 & $* * *$ & -147 & $* * *$ & -143 & $* * *$ \\
\hline & $\begin{array}{l}\text { Victim \#1 } \\
\text { Rescue } \\
\end{array}$ & -792 & $* * *$ & -727 & $* * *$ & -194 & $* *$ & $\begin{array}{c}- \\
1519\end{array}$ & $* * *$ & -921 & $* * *$ \\
\hline & $\begin{array}{l}\text { Victim \#1 } \\
\text { Descent } \\
\end{array}$ & -752 & $* * *$ & -709 & $* * *$ & -208 & $* *$ & $\begin{array}{c}- \\
1461 \\
\end{array}$ & $* * *$ & -917 & $* * *$ \\
\hline & $\begin{array}{l}\text { Primary } \\
\text { Search 11th } \\
\text { Floor }\end{array}$ & -15 & & -94 & $* * *$ & 4 & & -109 & $* * *$ & -90 & $* * *$ \\
\hline & $\begin{array}{l}\text { Victim \#2 } \\
\text { Found }\end{array}$ & -40 & & -74 & $*$ & -1 & & -114 & $* *$ & -75 & $* *$ \\
\hline & $\begin{array}{l}\text { Victim \#2 } \\
\text { Rescue } \\
\end{array}$ & -882 & $* * *$ & -161 & $* * *$ & -7 & & $\begin{array}{c}- \\
1043 \\
\end{array}$ & $* * *$ & -169 & $* * *$ \\
\hline & $\begin{array}{l}\text { Victim \#2 } \\
\text { Descent } \\
\end{array}$ & -866 & $* * *$ & -134 & $* * *$ & -38 & & $\begin{array}{c}- \\
1000\end{array}$ & $* * *$ & -172 & $* *$ \\
\hline & $\begin{array}{l}\text { Advance } \\
\text { Line Above } \\
\text { Fire }\end{array}$ & -15 & & -98 & $* * *$ & -16 & & -113 & $* * *$ & -113 & $* * *$ \\
\hline \multicolumn{12}{|c|}{$\begin{array}{l}\text { The coefficient represents the effect of adding a single crew member, i.e., } X+1 \text { vs. X. A negative coefficient represents the time } \\
\text { savings in seconds from adding a firefighter to the crew size. A positive coefficient represents a gain in time by adding a firefighter to } \\
\text { a crew. }\end{array}$} \\
\hline
\end{tabular}

Table 17: Effects of incrementing crew size on begin time, controlling for ascent mode and alarm size; coefficients are in seconds 
leftmost three row sets) has a negligible effect on duration for Advance Attack Line, Advance Second Line, Fire Out, and Establish Stairwell Support when going from 3 to 4 and from 4 to 5 crew size. Small improvements (roughly $2 \mathrm{~min}$ ) for these tasks occur when going from 5 to 6 crew size. In contrast, sizeable improvements of $10 \mathrm{~min}$ to $12.5 \mathrm{~min}$ are seen for Search and Rescue 10th Floor when going from 3 to 4 and from 4 to $5 \mathrm{crew}$ sizes. Similar-sized reductions in time occur for Primary Search of Floor 11 when going from 3 to 4 crew size. The cumulative effects of incrementing crew size by one firefighter are seen in the bottom row of the leftmost three-column set. Overall duration times decreased by an average of $12 \mathrm{~min}$ when increasing crew size from 3 to 4 , by 9 min when going from 4 to 5 crew size, and by 2 min when increasing crew size from 5 to 6 .

The rightmost two row sets present the effect of incrementing crew size by two firefighters when going from 3 to 5 or from 4 to 6 crew sizes. When going from 3 to 5 crew size, significant reductions in duration times occurred in the later tasks: Search and Rescue 10th Floor witnessed an average 22 1/2 min reduction, Search of Floor 11 saw roughly a 10 1/2 min reduction, and Overall Response Time saw an average 21 min decrease. The differences in task duration times between crew sizes of 4 and 6 were substantial but generally not nearly as large as between crew sizes of 3 and 5 .

Next the effect of incrementing crew size on the end times of critical tasks is examined. Results are presented in Table 19. Due most likely to the cumulative benefits of time savings over the course of the full task set, about three quarters of the observed end time reductions on critical task end times are statistically significant when incrementing crew size by a single firefighter (i.e., 3 to 4,4 to 5 , and 5 to 6 ). The largest time improvements are seen when going from crew size 3 to 4 (see the leftmost column set).

As firefighter crews navigate the later tasks, the improvements reach the $10 \mathrm{~min}$ to $15 \mathrm{~min}$ range (e.g., see the set of Victim \#2 tasks). Very large gains are seen for the 10th Floor Search and Victim \#1 Rescue

\begin{tabular}{|c|c|c|c|c|c|c|c|c|c|c|c|}
\hline \multirow[b]{3}{*}{ Outcome } & \multirow[b]{3}{*}{ Critical Task } & \multicolumn{10}{|c|}{ What is the effect of adding to crew size on task DURATION time? } \\
\hline & & \multicolumn{2}{|c|}{4 vs. 3} & \multicolumn{2}{|c|}{5 vs. 4} & \multicolumn{2}{|c|}{6 vs. 5} & \multicolumn{2}{|c|}{5 vs. 3} & \multicolumn{2}{|c|}{6 vs. 4} \\
\hline & & $\begin{array}{c}\text { coeff. } \\
+\end{array}$ & $\begin{array}{c}\text { Signi } \\
f . \mp\end{array}$ & $\begin{array}{c}\text { coeff. } \\
+\end{array}$ & $\begin{array}{c}\text { Signi } \\
f . \ddagger\end{array}$ & $\begin{array}{c}\text { coeff. } \\
+\end{array}$ & $\begin{array}{l}\text { Signi } \\
f . \ddagger\end{array}$ & coeff. $t$ & $\begin{array}{c}\text { Signi } \\
f . \ddagger\end{array}$ & $\begin{array}{c}\text { coeff. } \\
+\end{array}$ & $\begin{array}{c}\text { Signi } \\
\text { f. } \neq\end{array}$ \\
\hline \multirow{14}{*}{ 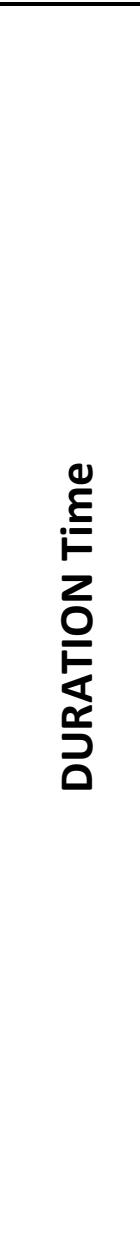 } & $\begin{array}{l}\text { Advance } \\
\text { Attack Line }\end{array}$ & -20 & & -1 & & -26 & * & -21 & $*$ & -27 & * \\
\hline & $\begin{array}{l}\text { Advance } \\
\text { Second Line }\end{array}$ & 14 & & -50 & & -34 & & -35 & & -83 & $* * *$ \\
\hline & Fire Out & -51 & & -11 & & -133 & $* * *$ & -63 & & -145 & $* * *$ \\
\hline & $\begin{array}{l}\text { Establish } \\
\text { Stairwell } \\
\text { Support }\end{array}$ & -55 & & -68 & & -110 & $*$ & -123 & $*$ & -178 & $* * *$ \\
\hline & $\begin{array}{l}\text { Search and } \\
\text { Rescue 10th } \\
\text { Floor }\end{array}$ & -598 & $* * *$ & -750 & $* * *$ & -97 & & -1348 & $* * *$ & -847 & $* * *$ \\
\hline & $\begin{array}{l}\text { Victim \#1 } \\
\text { Found }\end{array}$ & -710 & $* * *$ & -663 & $* * *$ & -115 & * & -1372 & $* * *$ & -778 & $* * *$ \\
\hline & $\begin{array}{l}\text { Victim \#1 } \\
\text { Rescue }\end{array}$ & 1 & & 27 & * & -5 & & 28 & & 22 & * \\
\hline & $\begin{array}{l}\text { Victim \#1 } \\
\text { Descent }\end{array}$ & 33 & $* *$ & -14 & & 0 & & 20 & & -14 & \\
\hline & $\begin{array}{l}\text { Primary } \\
\text { Search 11th } \\
\text { Floor }\end{array}$ & -556 & $* * *$ & -63 & & -154 & $* *$ & -619 & $* * *$ & -217 & $* * *$ \\
\hline & $\begin{array}{l}\text { Victim \#2 } \\
\text { Found }\end{array}$ & -841 & $* * *$ & -88 & $* *$ & -6 & & -929 & $* * *$ & -94 & $* *$ \\
\hline & $\begin{array}{l}\text { Victim \#2 } \\
\text { Rescue }\end{array}$ & 9 & & 5 & & -4 & & 14 & & 1 & \\
\hline & $\begin{array}{l}\text { Victim \#2 } \\
\text { Descent }\end{array}$ & -32 & $*$ & 11 & & -40 & $* *$ & -22 & & -29 & \\
\hline & $\begin{array}{l}\text { Advance Line } \\
\text { Above Fire }\end{array}$ & -51 & $*$ & -13 & & -24 & & -65 & $* * *$ & -37 & \\
\hline & $\begin{array}{l}\text { All Tasks } \\
\text { Complete } \\
\text { (Timer Sheet) }\end{array}$ & -719 & $* * *$ & -538 & $* * *$ & -121 & & -1257 & $* * *$ & -659 & $* * *$ \\
\hline \multicolumn{12}{|c|}{$\begin{array}{l}\text { The coefficient represents the effect of adding a single crew member, i.e., } X+1 \text { vs. } X \text {. A negative coefficient represents the time } \\
\text { savings in seconds from adding a firefighter to the crew size. A positive coefficient represents a gain in time by adding a firefighter to } \\
\text { a crew. }\end{array}$} \\
\hline & & 0.01 & $* *$ & ificar & the 0.0 & vel; * & 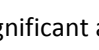 & the 0. & & & \\
\hline
\end{tabular}


tasks (over $11 \mathrm{~min}$ ) when incrementing crew size from 4 to 5 . The improvements in All Tasks Complete end times are substantial (9 1/2 $\mathrm{min}$ to $12 \mathrm{~min}$ ) when incrementing crew size from 3 to 4 or from 4 to 5. Reductions of end time All Tasks Complete are modest to minor when incrementing crew size from 5 to 6.

Turning to crew increments of 2 firefighters, Table 19 shows sizeable, significant improvements in end time for virtually every critical task (see rightmost two column sets). Modest

improvements in the 2 min to 4 min range are seen in the earlier critical tasks (Advance Attack Line through Establish Stairwell Support). For the remainder of the critical tasks, large reductions in end times (ranging from $12 \mathrm{~min}$ to $25 \mathrm{~min}$ ) are seen for all but one task (Advance Fire Line Above Fire) when incrementing crew size from 3 to 5 . Even incrementing from 4 to $6 \mathrm{crew}$ size shows significant albeit smaller improvements of $15 \mathrm{~min}$ for the Search and Victim \#1 Rescue collection of tasks, and roughly 3 min to 5 min reductions for the tasks related to Floor 11 and Victim \#2 Rescue. The overall reduction in end time is over $20 \mathrm{~min}$ when incrementing crew size from 3 to 5 , and over $10 \mathrm{~min}$ when increasing crew size from 4 to 6 .

The findings from the crew size analysis suggest that size does matter when it comes to the number of firefighters assigned to crews. Even the increment of a single firefighter can have a positive impact on the start, duration and completion of varied critical tasks. And incrementing crew size by two is also beneficial. The most sizeable improvements were seen when incrementing from a smaller crew size to a larger crew size, e.g., 3 to 4,4 to 5 , or 3 to 5 .

\section{Effects of Alarm Size and Ascent Mode}

Next the effects of alarm response (high, low) and ascent mode (elevator, stairs) on critical task timings are explored. Appendix C2 uses the results of regression to compare high and low alarm response times and to compare elevator to stair response times for all critical tasks and associated outcomes. Because alarm response and ascent mode are design factors built into the field experiment in order to examine crew size effects, a summary is provided in this section rather than a detailed assessment of the findings. With regard to alarm response, about one third of the 27 distinct

\begin{tabular}{|c|c|c|c|c|c|c|c|c|c|c|c|}
\hline \multirow[b]{3}{*}{ Outcome } & \multirow[b]{3}{*}{ Critical Tasks } & \multicolumn{10}{|c|}{ What is the effect of adding to crew size on END time? } \\
\hline & & \multicolumn{2}{|c|}{4 vs. 3} & \multicolumn{2}{|c|}{5 vs. 4} & \multicolumn{2}{|c|}{6 vs. 5} & \multicolumn{2}{|c|}{5 vs. 3} & \multicolumn{2}{|c|}{6 vs. 4} \\
\hline & & $\begin{array}{c}\text { coeff. } \\
+\end{array}$ & $\begin{array}{c}\text { Signi } \\
f . \ddagger\end{array}$ & $\begin{array}{c}\text { coeff. } \\
+\end{array}$ & $\begin{array}{c}\text { Signi } \\
f . \ddagger\end{array}$ & $\begin{array}{c}\text { coeff. } \\
+\end{array}$ & $\begin{array}{c}\text { Signi } \\
f . \pm\end{array}$ & coeff. + & $\begin{array}{c}\text { Signi } \\
f . \ddagger\end{array}$ & $\begin{array}{c}\text { coeff. } \\
+\end{array}$ & $\begin{array}{c}\text { Sign } \\
f . \ddagger\end{array}$ \\
\hline \multirow{14}{*}{ 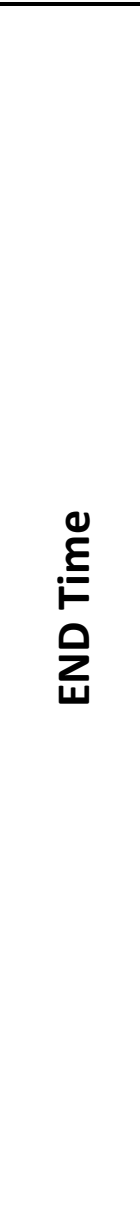 } & $\begin{array}{l}\text { Advance } \\
\text { Attack Line }\end{array}$ & -103 & $* *$ & -65 & $* *$ & -101 & $* * *$ & -167 & $* * *$ & -165 & $* * *$ \\
\hline & $\begin{array}{l}\text { Advance } \\
\text { Second Line }\end{array}$ & -80 & & -163 & $* * *$ & -94 & $* *$ & -244 & $* * *$ & -257 & $* * *$ \\
\hline & Fire Out & -134 & $* * *$ & -75 & & -212 & $* * *$ & -209 & $* * *$ & -287 & $* * *$ \\
\hline & $\begin{array}{l}\text { Establish } \\
\text { Stairwell } \\
\text { Support }\end{array}$ & -55 & & -69 & & -110 & $*$ & -124 & * & -178 & $* * *$ \\
\hline & $\begin{array}{l}\text { Search and } \\
\text { Rescue 10th } \\
\text { Floor }\end{array}$ & -681 & $* * *$ & -814 & $* * *$ & -177 & & -1495 & $* * *$ & -990 & $* * *$ \\
\hline & $\begin{array}{l}\text { Victim \#1 } \\
\text { Found }\end{array}$ & -792 & $* * *$ & -727 & $* * *$ & -194 & $* *$ & -1519 & $* * *$ & -921 & $* * *$ \\
\hline & $\begin{array}{l}\text { Victim \#1 } \\
\text { Rescue }\end{array}$ & -791 & $* * *$ & -699 & $* * *$ & -199 & $* *$ & -1490 & $* * *$ & -898 & $* * *$ \\
\hline & $\begin{array}{l}\text { Victim \#1 } \\
\text { Descent }\end{array}$ & -719 & $* * *$ & -722 & $* * *$ & -208 & $* *$ & -1441 & $* * *$ & -931 & $* * *$ \\
\hline & $\begin{array}{l}\text { Primary Search } \\
\text { 11th Floor }\end{array}$ & -571 & $* * *$ & -157 & $* *$ & -150 & $* *$ & -728 & $* * *$ & -308 & $* * *$ \\
\hline & $\begin{array}{l}\text { Victim \#2 } \\
\text { Found }\end{array}$ & -882 & $* * *$ & -161 & $* * *$ & -7 & & -1043 & $* * *$ & -169 & $* * *$ \\
\hline & $\begin{array}{l}\text { Victim \#2 } \\
\text { Rescue }\end{array}$ & -873 & $* * *$ & -156 & $* * *$ & -11 & & -1029 & $* * *$ & -168 & $* *$ \\
\hline & $\begin{array}{l}\text { Victim \#2 } \\
\text { Descent }\end{array}$ & -898 & $* * *$ & -124 & $* *$ & -78 & & -1021 & $* * *$ & -201 & $* * *$ \\
\hline & $\begin{array}{l}\text { Advance Line } \\
\text { Above Fire }\end{array}$ & -67 & & -111 & $* * *$ & -39 & & -178 & $* * *$ & -151 & $* * *$ \\
\hline & $\begin{array}{l}\text { All Tasks } \\
\text { Complete } \\
\text { (Timer Sheet) }\end{array}$ & -719 & $* * *$ & -538 & $* * *$ & -121 & & -1257 & $* * *$ & -659 & $* * *$ \\
\hline \multicolumn{12}{|c|}{$\begin{array}{l}\text { The coefficient represents the effect of adding a single crew member, i.e., } X+1 \text { vs. } X \text {. A negative coefficient represents the time } \\
\text { savings in seconds from adding a firefighter to the crew size. A positive coefficient represents a gain in time by adding a firefighter to a } \\
\text { crew. }\end{array}$} \\
\hline gen & $f_{1}$ & & $*=$ & an & 08 & 1. * & & 0 & & & \\
\hline
\end{tabular}

Table 19: Effects of incrementing crew size on task end time, controlling for ascent mode and alarm size; coefficients are in seconds 
outcome-task comparisons between high and low response were statistically significant. Six of the seven significant differences between high and low alarm sizes involved reductions of the end time of tasks, ranging from $1 \frac{1}{2} \mathrm{~min}$ to $2 \frac{1}{2} \mathrm{~min}$ smaller for the high response. These task end times involved Search of Floor 11, the three rescue tasks related to Victim \#2 (Find, Rescue, Descent) and Advancing Line Above Fire.

Comparing elevator to stair ascent, statistically significant reductions in time were seen in favor of elevator use for over two thirds of the critical task outcomes. The most sizeable time reduction for the elevator was for Establishing Stairwell Support (duration and end time reductions of about $10 \mathrm{~min}$ ). Other notable reductions in favor of elevator were for the end times of Descent for Victim \#1 (4 min $42 \mathrm{~s}$ ) and Victim \#2 (5 1/2 $\mathrm{min}$ ). When other significant reductions occurred they were smaller, in the range of about $1 \frac{1}{2} 2 \mathrm{~min}$ to $4 \mathrm{~min}$ (see Appendix C2 for details).

The assessment of alarm size and ascent mode confirmed the logical direction of differences when they were found to exist. Illogical timing patterns (i.e., smaller times for low alarm size than for high or smaller times for stairs than for elevators) were not observed.

\section{Combined Effects of Alarm Size and Crew Size}

Given the findings from the crew size analysis that adding one or two firefighters to a crew can generally achieve substantial task time decreases, a logical question is whether the meaningful benefits of a higher crew size could be realized by implementing a higher alarm response at a smaller crew size (e.g., 4/high compared to 5/low). The hope might be that a high response with a lower crew size might yield similar results in task timing to that of a low response with higher crew size. This issue is explored in the following analysis.

Appendix C3 presents regression results that compare the effect of high response with lower crew size compared to a low response with higher crew size. These results were used to compile separate summary tables for begin, duration, and end times. Table 20 provides a summary of findings for tasks deemed critical for begin times. The leftmost two column sets show that statistically significant begin times occurred for low alarm responses with 4 or 5 crew size compared to smaller sized counterparts - high response with crew sizes of 3 and 4, respectively. There are significant reductions in begin times for all critical tasks from Advance Attack Line through Victim \#1 Descent. Sizeable reductions of about 12 min are also seen for Victim \#2 Rescue and Descent. This suggests that a low alarm response with crews of size 4 or 5 outperforms a high alarm response with crew sizes smaller by one firefighter. When time decreases occur they are in the range of $1 \mathrm{~min}$ to just under $2 \mathrm{~min}$.

It is noteworthy that this pattern does not hold for a low response with crew size 6 compared to a high response with crew size 5 . The observed differences in task begin times were significantly higher for Primary Search of Floor 11 and Advance Line Above Fire.

\begin{tabular}{|c|c|c|c|c|c|c|c|}
\hline \multirow[b]{3}{*}{ Outcome } & \multirow[b]{3}{*}{ Critical Tasks } & \multicolumn{6}{|c|}{$\begin{array}{c}\text { What are the BEGIN time effects of a high response } \\
\text { with lower crew size compared to a low response with } \\
\text { higher crew size? }\end{array}$} \\
\hline & & \multicolumn{2}{|c|}{ low/4 vs. high/3 } & \multicolumn{2}{|c|}{ low/5 vs. high/4 } & \multicolumn{2}{|c|}{ low/6 vs. high/5 } \\
\hline & & coeff. $†$ & $\begin{array}{c}\text { Signif. } \\
\ddagger\end{array}$ & coeff. $t$ & Signif. $\ddagger$ & coeff. $t$ & $\begin{array}{c}\text { Signif. } \\
\ddagger\end{array}$ \\
\hline \multirow{12}{*}{ 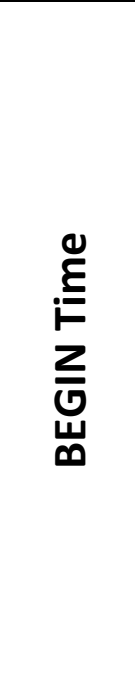 } & Advance Attack Line & -93 & $*$ & -57 & $*$ & -28 & \\
\hline & Advance Second Line & -110 & $*$ & -112 & $* * *$ & -51 & \\
\hline & Fire Out & -93 & $*$ & -57 & $*$ & -28 & \\
\hline & Search and Rescue 10th Floor & -93 & $*$ & -57 & $*$ & -28 & \\
\hline & Victim \#1 Found & -93 & $*$ & -57 & $*$ & -28 & \\
\hline & Victim \#1 Rescue & -875 & $* * *$ & -659 & $* * *$ & -133 & \\
\hline & Victim \#1 Descent & -857 & $* * *$ & -694 & $* * *$ & -181 & \\
\hline & Primary Search 11th Floor & 16 & & -34 & & 102 & $* *$ \\
\hline & Victim \#2 Found & 6 & & 16 & & 142 & $* *$ \\
\hline & Victim \#2 Rescue & -737 & $* * *$ & -50 & & 125 & \\
\hline & Victim \#2 Descent & -732 & $* * *$ & -17 & & 123 & \\
\hline & Advance Line Above Fire & 16 & & -39 & & 93 & $* *$ \\
\hline \multicolumn{8}{|c|}{$\begin{array}{l}\text { The coefficient represents the effect of a low alarm response with a larger crew (larger by } 1 \text { firefighter) relative to a higher alarm } \\
\text { response but with a crew size that is smaller by one firefighter. The contrast shown is the first scenario relative to the second: the } \\
\text { coefficient for "X vs. Y" is (X-Y). A negative coefficient represents the time savings in seconds from a larger crew with a lower alarm } \\
\text { response. A positive coefficient represents a gain in time from a larger crew with a lower alarm response. }\end{array}$} \\
\hline
\end{tabular}

Table 20: Contrasting high response to low response with smaller crew sizes for begin times; coefficients are in seconds 
Next duration is examined. Table 21 provides a summary of findings for tasks deemed critical for duration times. Although less than a third of these comparisons were statistically significant, they tended to be prominent when they occurred. For Search of the 10th Floor and Victim \#1 Rescue, a low response with crew size 4 showed about $11 \mathrm{~min}$ to $13 \mathrm{~min}$ reductions compared to that of a high response with crew size 3 . And for low response crew size 5 the duration time reductions were $10 \mathrm{~min}$ to $13 \mathrm{~min}$ compared to a high response with crew size 4 . The low/4 combination revealed an $8 \mathrm{~min}$ reduction in duration time for Search of Floor 11 and about a 12 min reduction for Victim \#2 Rescue compared to a high response with crew size 3 . Similarly large reductions in duration time appear for All Tasks Complete under these two response crew size scenarios. The low $/ 6$ combination shows three significant duration reductions compared to high/5: a $2.2 \mathrm{~min}$ reduction for Fire Out, a 2 1/2 min reduction for Search of Floor 11, and a $1 \mathrm{~min}$ reduction for Victim \#2 Descent.

Finally, the end time comparisons for this analysis are examined. Table 22 presents critical task end time contrasts of low alarm response to that of a high response with one less crew on staff. Over half of the task end time comparisons were statistically significant when testing the low/4 and low/5 combinations against their counterparts (i.e., high/3 and high/4, respectively). When significant, low response with crew sizes 4 and 5 displayed task end time reductions of $2.6 \mathrm{~min}$ to well over $14 \mathrm{~min}$. The low $/ 4$ combination showed significant end time reductions compared to high/3 for 11 of the 14 critical tasks in this analysis (see leftmost column set of Table 22). For the low/5 combination, 6 of 14 tasks showed significant reductions in end time compared to high/4 (see middle column set in figure). Only 2 of 14 tasks exhibited significantly lower end times for the low/6 combination relative to that of high/5 (as shown in the rightmost column set of the table). Generally, for critical task end times, reductions were most pronounced when comparing low/4 to high/3, followed by low/5 compared to high/4. The low/ 6 combination featured the smallest reductions compared to the high/5.

In summary, the analysis of the alarm response and crew size combinations suggests that the benefits of crew size increases (in terms of reduced begin, duration, and end times for critical tasks) are fairly robust. Low alarm response with a higher crew size tends to be more favorable in critical task timings than the

corresponding timings for a high alarm response with a crew size of one less firefighter.

\section{Combined Effects of Alarm Size and Ascent}

The effects of different combinations of alarm size (high, low) and ascent mode (stairs, elevator) are now examined. This combination of variables is important to understand the synergies that may exist

\begin{tabular}{|c|c|c|c|c|c|c|c|}
\hline \multirow[b]{3}{*}{ Outcome } & \multirow[b]{3}{*}{ Critical Tasks } & \multicolumn{6}{|c|}{$\begin{array}{l}\text { What are the effects on DURATION of a high response } \\
\text { with lower crew size compared to a low response with } \\
\text { higher crew size? }\end{array}$} \\
\hline & & \multicolumn{2}{|c|}{ low/4 vs. high/3 } & \multicolumn{2}{|c|}{ low/5 vs. high/4 } & \multicolumn{2}{|c|}{ low/6 vs. high/5 } \\
\hline & & coeff. $†$ & Signif. $¥$ & coeff. ${ }^{\dagger}$ & Signif. $\ddagger$ & coeff. $t$ & Signif. $\ddagger$ \\
\hline \multirow{14}{*}{ 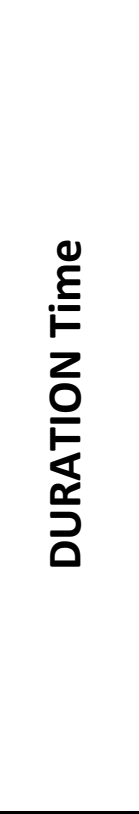 } & Advance Attack Line & -11 & & 9 & & -16 & \\
\hline & Advance Second Line & 68 & & -1 & & -12 & \\
\hline & Fire Out & -61 & & -21 & & -135 & $* *$ \\
\hline & Establish Stairwell Support & -18 & & -59 & & -94 & \\
\hline & Search and Rescue 10th Floor & -647 & $* * *$ & -718 & $* * *$ & -119 & \\
\hline & Victim \#1 Found & -783 & $* * *$ & -602 & $* * *$ & -105 & \\
\hline & Victim \#1 Rescue & -3 & & 11 & & -36 & \\
\hline & Victim \#1 Descent & 47 & & -17 & & -13 & \\
\hline & Primary Search 11th Floor & -486 & $* * *$ & -58 & & -150 & $*$ \\
\hline & Victim \#2 Found & -743 & $* * *$ & -65 & & -17 & \\
\hline & Victim \#2 Rescue & -3 & & -8 & & 17 & \\
\hline & Victim \#2 Descent & -61 & $* *$ & -31 & & -60 & $* * *$ \\
\hline & Advance Line Above Fire & -14 & & 25 & & -12 & \\
\hline & All Tasks Complete (Timer Sheet) & -729 & $* * *$ & -462 & $* *$ & 26 & \\
\hline \multicolumn{8}{|c|}{$\begin{array}{l}\text { The coefficient represents the effect of a low alarm response with a larger crew (larger by } 1 \text { firefighter) relative to a higher alarm } \\
\text { response but with a crew size that is smaller by one firefighter. The contrast shown is the first scenario relative to the second: the } \\
\text { coefficient for "X vs. } Y \text { " is }(X-Y) \text {. A negative coefficient represents the time savings in seconds from a larger crew with a lower alarm } \\
\text { response. A positive coefficient represents a gain in time from a larger crew with a lower alarm response. }\end{array}$} \\
\hline
\end{tabular}

Table 21: Contrasting high response to low response with smaller crew sizes for duration times; coefficients are in seconds 
on the fire ground. Appendix C4 presents regression results that contrast several of combinations of alarm size and ascent mode. Three scenarios were contrasted in the appendix:

- elevator/low vs. stairs/low - this comparison addresses the relative gains or losses in time when the alarm size is low and firefighters are faced with stairs or elevator ascent;

- stairs/high vs. stairs/low - this scenario focuses on stair ascent and examines the impact of high versus low alarm size;

elevator/high vs. elevator/low - this is the complement to the second scenario - it focuses on elevator ascent and explores the effects of high versus low alarm size.

Elevator/low vs. stairs/low. The leftmost column set of Appendix C4 contrasts the critical task timings for elevator ascent with a low alarm size to stair ascent with similarly low alarm size. Elevator ascent significantly reduces all begin times and most end times compared to stair ascent. Most of the reductions due to elevator ascent are in the 2 min to 4 min range, with a few obvious exceptions. Elevator ascent allows Establishment of Stairwell Support more than 11 min more quickly than stair ascent. Both Victim \#2 Descent and All Task Complete occur more than 6 min quicker for elevator ascent. Except for Establishment of Stairwell Support, there are no ascent mode differences for task duration. This also makes sense, since crew size is controlled in these comparisons.
Stairs/high vs. stairs/low. The middle column set of Appendix C4 presents the effects of high versus low alarm size when firefighters are faced with stair ascent. The alarm size is seen to have virtually no effect for critical task timings except for tasks related to Search of Floor 11 (duration and end) and Victim \#2 Found, Rescue and Descent. High alarm size realized a mean reduction in the range of $1 \frac{1 / 2}{\min }$ to $3 \mathrm{~min} 24 \mathrm{~s}$ for these tasks. All Tasks Complete was also significantly smaller for high alarm size by $3 \mathrm{~min}$. No other task timing comparisons were statistically different.

Elevator/high vs. elevator/low. The rightmost column set of Appendix C4 presents the effects of high versus low alarm size when firefighters employ elevator ascent. High alarm size led to only eight significantly lower timings than a low alarm size: $45 \mathrm{~s}$ reductions in begin time for Fire Out, Search of Floor 10, and Victim \#1 Found; small reductions of just over $1 \mathrm{~min}$ in begin times for Search of Floor 11 and Victim \#2 Found, and small reductions for begin, duration, and end times related to the Advancement of Line Above the Fire. Timing reductions were in the range of just over $30 \mathrm{~s}$ to just under $2 \mathrm{~min}$. No other task timing comparisons were statistically different.

\begin{tabular}{|c|c|c|c|c|c|c|c|}
\hline \multirow[b]{3}{*}{ Outcome } & \multirow[b]{3}{*}{ Critical Tasks } & \multicolumn{6}{|c|}{$\begin{array}{c}\text { What are the effects on END time of a high response } \\
\text { with lower crew size compared to a low response } \\
\text { with higher crew size? }\end{array}$} \\
\hline & & \multicolumn{2}{|c|}{ low/4 vs. high/3 } & \multicolumn{2}{|c|}{ low/5 vs. high/4 } & \multicolumn{2}{|c|}{ low/6 vs. high/5 } \\
\hline & & coeff. $t$ & Signif.‡ & coeff. $t$ & Signif.‡ & coeff. $t$ & Signif. $\ddagger$ \\
\hline \multirow{14}{*}{$\begin{array}{l}\stackrel{\text { E }}{E} \\
\text { : } \\
\sum_{\boldsymbol{U}}\end{array}$} & Advance Attack Line & -104 & & -48 & & -44 & \\
\hline & Advance Second Line & -42 & & -113 & $*$ & -63 & \\
\hline & Fire Out & -154 & $*$ & -78 & & -162 & $* *$ \\
\hline & Establish Stairwell Support & -18 & & -59 & & -94 & \\
\hline & Search and Rescue 10th Floor & -740 & $* * *$ & -775 & $* * *$ & -147 & \\
\hline & Victim \#1 Found & -875 & $* * *$ & -659 & $* * *$ & -133 & \\
\hline & Victim \#1 Rescue & -878 & $* * *$ & -648 & $* * *$ & -169 & \\
\hline & Victim \#1 Descent & -810 & $* * *$ & -711 & $* * *$ & -194 & \\
\hline & Primary Search 11th Floor & -470 & $* * *$ & -92 & & -48 & \\
\hline & Victim \#2 Found & -737 & $* * *$ & -50 & & 125 & \\
\hline & Victim \#2 Rescue & -740 & $* * *$ & -57 & & 142 & $*$ \\
\hline & Victim \#2 Descent & -792 & $* * *$ & -48 & & 62 & \\
\hline & Advance Line Above Fire & 2 & & -14 & & 81 & \\
\hline & All Tasks Complete (Timer Sheet) & -729 & $* * *$ & -462 & $* *$ & 26 & \\
\hline \multicolumn{8}{|c|}{$\begin{array}{l}\text { The coefficient represents the effect of a low alarm response with a larger crew (larger by } 1 \text { firefighter) relative to a higher alarm } \\
\text { response but with a crew size that is smaller by one firefighter. The contrast shown is the first scenario relative to the second: the } \\
\text { coefficient for "X vs. Y" is (X-Y). A negative coefficient represents the time savings in seconds from a larger crew with a lower alarm } \\
\text { response. A positive coefficient represents a gain in time from a larger crew with a lower alarm response. }\end{array}$} \\
\hline 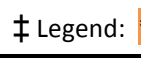 & $=$ signi & & 政 & & -0.07 & & \\
\hline
\end{tabular}

Table 22: Contrasting high response to low response with smaller crew sizes for end times; coefficients are in seconds 


\section{How to Interpret Percentage Tables}

The results from the time-to-task analyses can be displayed in a number of ways. Of particular interest in this study is the difference in the time it takes firefighters using different crew sizes (or alarm sizes or vertical response mode) to complete a given task or to reach a certain milestone. Table 23 represents the raw time data (minutes and seconds) to complete one of the tasks in the experiments.

\begin{tabular}{|c|c|}
\hline Crew Size & Time to complete task \\
\hline 3-person & A min A s \\
\hline 4-person & B min B s \\
\hline 5-person & C min C s \\
\hline 6-person & D min D s \\
\hline
\end{tabular}

Table 23: Times for each crew size to complete a task

A comparison between times for different crew sizes can be presented as an array of differences between the time it takes one crew size to complete a task and the time it takes another crew size to complete the same task. Table 24 displays a sample matrix in which the time taken by the crew size listed in each row has been subtracted from the time taken by the crew size listed in the column. So if a 3-person crew took A min A s and a 6-person crew took D min D s to complete the task, the value shown in row 6-person/column 3-person is equal to $(\mathrm{A} \min \mathrm{As})-(\mathrm{D} \min \mathrm{D} s)=(\mathrm{Z} \min \mathrm{Zs})$. The value in row 3-person/column 6-person is the negative of this value, (D min D s) $(\mathrm{A} \min \mathrm{A} s)=-(\mathrm{Z}$ min $\mathrm{Z} s)$. Since the task time tends to decrease as the number of persons on the crew increases, the numbers below the diagonal cells of the matrix are generally positive indicating a decrease in time-to-task or faster performance times. Likewise, the cells above the diagonal cells in the matrix are generally negative indicating slower performance times. The values in the cells along the diagonal are equal to zero.

Although the tasks carried out in this study are intended to be representative of tasks on a real fireground, the time required to complete a task will be dependent on the circumstances of the individual fire. Of greater meaning for comparing one crew size against another, or one mode of vertical response to another, is the change in time relative to the total time required for the task. This is most easily expressed as a percentage: $100 \times$ the difference in time divided by the total time required by the base scenario. The selection of the base scenario depends on the change in crew size being compared.

In Table 25, time-to-task percentages are calculated as the time differences in Table 24 divided by the total time taken to complete the task by the crew size in that column (from Table 23). Note that the values in corresponding cells above and below the diagonal (e.g., 3-person row/6-person column vs. 6-person row/3-person column) are no longer the same value with different sign. Their values have been changed by calculations using different denominators based on actual performance times in the study.

\begin{tabular}{|l|l|l|l|l|}
\hline Crew Size & 3-person & 4-person & 5-person & 6-person \\
\hline 3-person & & $-(X \min X \mathrm{~s})$ & $-(\mathrm{Y} \min \mathrm{Y} \mathrm{s})$ & $-(\mathrm{Z} \min \mathrm{Z} \mathrm{s})$ \\
\hline 4-person & $\mathrm{X} \min \mathrm{X} \mathrm{s}$ & & $-(\mathrm{Q} \min \mathrm{Q} \mathrm{s})$ & $-(\mathrm{R} \min \mathrm{R} \mathrm{s})$ \\
\hline 5-person & $\mathrm{Y} \min \mathrm{Y} \mathrm{s}$ & $\mathrm{Q} \min \mathrm{Q} \mathrm{s}$ & & $-(\mathrm{S} \min \mathrm{S} \mathrm{s})$ \\
\hline 6-person & $\mathrm{Z} \min \mathrm{Z} \mathrm{s}$ & $\mathrm{R} \min \mathrm{R} \mathrm{s}$ & $\mathrm{S} \min \mathrm{S} \mathrm{s}$ & \\
\hline
\end{tabular}

Table 24: Array of time differences.

\begin{tabular}{|c|c|c|c|c|}
\hline Crew Size & 3-person & 4-person & 5-person & 6-person \\
\hline 3-person & & $-100 \times \frac{(X \min X s)}{B \min B s}$ & $-100 \times \frac{(Y \min Y s)}{C \min C s}$ & $-100 \times \frac{(Z \min Z s)}{D \min D s}$ \\
\hline 4-person & $100 \times \frac{X \min X s}{A \min A s}$ & & $\frac{-100 \times \frac{(Q \min Q s)}{C \min C s}}{C}$ & $-100 \times \frac{(R \min R s)}{D \min D s}$ \\
\hline 5-person & $100 \times \frac{Y \min Y s}{A \min A s}$ & $100 \times \frac{Q \min Q s}{B \min B s}$ & & $-100 \times \frac{(S \min S s)}{D \min D s}$ \\
\hline 6-person & $100 \times \frac{Z \min Z s}{A \min A s}$ & $100 \times \frac{R \min R s}{B \min B s}$ & $100 \times \frac{S \min S s}{C \min C s}$ & \\
\hline
\end{tabular}

Table 25: Calculations of time-to-task percentages 
Table 26 shows an example from the time-to-task percentage tables presented in this appendix. Note that the values in cells above the diagonal are higher than their counterparts below the diagonal. This is because the total time-to-task completion decreases with larger crew size, making the denominator larger and thus the percentage smaller for cells below the diagonal.

The value in each cell can be described as the percentage that the crew size in the row differs from the crew size in the column (which is the base for the comparison).

The advantage of displaying the results in this manner is that it provides answers to questions about the impact of changing the crew size on the completion of fireground tasks. For example, if a fire department that staffs with 4-person crews is interested in the effect of going to a different crew size, they can look down the 4-person column (indicated by the arrow in Table 26) and see that, according to this study, a 5-person crew would complete this task $6.7 \%$ faster and a 6-person crew $14.5 \%$ faster than the 4-person crew. A 3-person crew would be $8.7 \%$ slower, as indicated by the negative sign in the table.

\begin{tabular}{|c|c|c|c|c|}
\hline Crew Size & 3-person & 4-person & 5-person & 6-person \\
\hline 3-person & & -8.7 & -16.5 & -27.1 \\
\hline 4-person & 8.0 & & -7.2 & -16.9 \\
\hline 5-person & 14.2 & 6.7 & & -9.1 \\
\hline 6-person & 21.3 & 14.5 & 8.3 & \\
\hline
\end{tabular}

Table 26: Sample time-to-task percentage table.

Time-to-Task: Percentage Tables by Task

The following tables present time-to-task percentages for the critical tasks in this study. The start times, durations, and end times used in calculations are the same as those shown in related

\begin{tabular}{|c|c|c|c|c|}
\hline Crew Size & 3-person & 4-person & 5-person & 6-person \\
\hline 3-person & & -23.7 & -50.4 & -58.0 \\
\hline 4-person & 19.2 & & -21.6 & -27.7 \\
\hline 5-person & 33.5 & 17.7 & & -5.1 \\
\hline 6-person & 36.7 & 21.7 & 4.8 & \\
\hline
\end{tabular}

Positive numbers indicate \% faster; negative numbers indicate $\%$ slower

Table 27: Percentage Table for Overall Time-to-Task Completion by Crew Size (see Figure 57)

figures in Section 9.4 Regression Analyses and Chapter 10 Time-to-Task Results. Positive percentage values in these tables indicate that the scenario given by the row is faster than the scenario given by the column and negative numbers indicate the percentage given by the row is slower than the scenario given by the column.

Most time-to-task percentage tables compare results due to various crew sizes only, with results for other effects included in the averaging. For Overall Time-to-Task Completion, percentage differences due to vertical ascent mode (stair or elevator) or to high or low alarm size are listed in addition to crew size.

\section{Overall Time-to-Task Completion}

The following two tables show results from multiple factors. Table 28 displays the Overall Time-to-Task Completion for crew size and vertical ascent model, either stair or elevator. The headings are abbreviated with the number in the crew and S or E for stairs or elevator, respectively. This table allows a quantitative comparison of the effects from using stairs or elevators for each crew size.

\begin{tabular}{|c|c|c|c|c|c|c|c|c|}
\hline $\begin{array}{c}\text { Crew } \\
\text { Size }\end{array}$ & $3 \mathrm{~S}$ & $3 \mathrm{E}$ & $4 \mathrm{~S}$ & $4 \mathrm{E}$ & $5 \mathrm{~S}$ & $5 \mathrm{E}$ & $6 \mathrm{~S}$ & $6 \mathrm{E}$ \\
\hline 3S & & -7.7 & -23.8 & -33.1 & -46.0 & -67.2 & -57.8 & -70.4 \\
\hline 3E & 7.1 & & -15.0 & -23.6 & -35.6 & -55.3 & -46.5 & -58.2 \\
\hline $4 \mathrm{~S}$ & 19.2 & 13.0 & & -7.5 & -18.0 & -35.1 & -27.4 & -37.6 \\
\hline $4 \mathrm{E}$ & 24.8 & 19.1 & 7.0 & & -9.8 & -25.7 & -18.6 & -28.1 \\
\hline $5 \mathrm{~S}$ & 31.5 & 26.3 & 15.2 & 8.9 & & -14.5 & -8.0 & -16.7 \\
\hline $5 \mathrm{E}$ & 40.2 & 35.6 & 26.0 & 20.4 & 12.7 & & 5.7 & -1.9 \\
\hline $6 \mathrm{~S}$ & 36.6 & 31.7 & 21.5 & 15.7 & 7.4 & -6.0 & & -8.0 \\
\hline $6 \mathrm{E}$ & 41.3 & 36.8 & 27.3 & 21.9 & 14.3 & 1.8 & 7.4 & \\
\hline
\end{tabular}

Table 28: Percentage Table for Overall Time-to-Task Completion by Crew Size and Stair/Elevator Ascent Mode (see Figure 58) 
Table 29 displays the Overall Time-to-Task for crew size and high or low alarm size. The headings are abbreviated with the number in the crew and $\mathrm{L}$ or $\mathrm{H}$ for low or high alarm size, respectively. This table allows a quantitative comparison of the effects from alarm size for each crew size.

\begin{tabular}{|c|c|c|c|c|c|c|c|c|}
\hline $\begin{array}{c}\text { Crew } \\
\text { Size }\end{array}$ & $3 \mathrm{~L}$ & $3 \mathrm{H}$ & $4 \mathrm{~L}$ & $4 \mathrm{H}$ & $5 \mathrm{~L}$ & $5 \mathrm{H}$ & $6 \mathrm{~L}$ & $6 \mathrm{H}$ \\
\hline $3 \mathrm{~L}$ & & 1.5 & -22.0 & -23.5 & -45.8 & -52.7 & -51.2 & -62.8 \\
\hline $3 \mathrm{H}$ & -1.6 & & -23.9 & -25.4 & -48.1 & -55.1 & -53.5 & -65.3 \\
\hline $4 \mathrm{~L}$ & 18.0 & 19.3 & & -1.2 & -19.5 & -25.2 & -23.9 & -33.4 \\
\hline $4 \mathrm{H}$ & 19.0 & 20.2 & 1.2 & & -18.1 & -23.7 & -22.4 & -31.8 \\
\hline $5 \mathrm{~L}$ & 31.4 & 32.5 & 16.3 & 15.3 & & -4.8 & -3.7 & -11.6 \\
\hline $5 \mathrm{H}$ & 34.5 & 35.5 & 20.1 & 19.2 & 4.5 & & 1.0 & -6.6 \\
\hline $6 \mathrm{~L}$ & 33.9 & 34.9 & 19.3 & 18.3 & 3.6 & -1.0 & & -7.6 \\
\hline $6 \mathrm{H}$ & 38.6 & 39.5 & 25.1 & 24.1 & 10.4 & 6.1 & 7.1 & \\
\hline
\end{tabular}

Table 29: Percentage Table for Overall Time-to-Task Completion by Crew Size and High/Low Alarm Size (see Figure 59)

\section{Advance Attack Line: Begin time, duration, and end time}

\begin{tabular}{|c|c|c|c|c|}
\hline Crew Size & 3-person & 4-person & 5-person & 6-person \\
\hline 3-person & & -8.7 & -16.52 & -27.08 \\
\hline 4-person & 8 & & -7.19 & -16.91 \\
\hline 5-person & 14.18 & 6.71 & & -9.07 \\
\hline 6-person & 21.31 & 14.47 & 8.31 & \\
\hline
\end{tabular}

Table 30: Percentage Table for Advance Attack Line begin time (See Figure 70)

\begin{tabular}{|c|c|c|c|c|}
\hline Crew Size & 3-person & 4-person & 5-person & 6-person \\
\hline 3-person & & -12.93 & -13.7 & -39.5 \\
\hline 4-person & 11.45 & & -0.68 & -23.53 \\
\hline 5-person & 12.05 & 0.68 & & -22.69 \\
\hline 6-person & 28.31 & 19.05 & 18.49 & \\
\hline
\end{tabular}

Table 31: Percentage Table for Advance Attack Line duration (See Figure 70)

\begin{tabular}{|c|c|c|c|c|}
\hline Crew Size & 3-person & 4-person & 5-person & 6-person \\
\hline 3-person & & -12.93 & -13.7 & -39.5 \\
\hline 4-person & 11.45 & & -0.68 & -23.53 \\
\hline 5-person & 12.05 & 0.68 & & -22.69 \\
\hline 6-person & 28.31 & 19.05 & 18.49 & \\
\hline
\end{tabular}

Table 32: Percentage Table for Advance Attack Line end time (See Figure 70) 
Advance Second Line: Begin time, duration, and end time

\begin{tabular}{|c|c|c|c|c|}
\hline Crew Size & 3-person & 4-person & 5-person & 6-person \\
\hline 3-person & & -8.9 & -22.0 & -30.2 \\
\hline 4-person & 8.1 & & -12.0 & -19.6 \\
\hline 5-person & 18.0 & 10.8 & & -6.8 \\
\hline 6-person & 23.2 & 16.4 & 6.3 & \\
\hline
\end{tabular}

Table 33: Percentage Table for Advance Second Line begin time (See Figure 71)

\begin{tabular}{|c|c|c|c|c|}
\hline Crew Size & 3-person & 4-person & 5-person & 6-person \\
\hline 3-person & & 5.3 & -16.3 & -38.1 \\
\hline 4-person & -5.6 & & -22.8 & -45.9 \\
\hline 5-person & 14.0 & 18.6 & & -18.8 \\
\hline 6-person & 27.6 & 31.4 & 15.8 & \\
\hline
\end{tabular}

Table 34: Percentage Table for Advance Second Line duration (See Figure 71)

\begin{tabular}{|c|c|c|c|c|}
\hline Crew Size & 3-person & 4-person & 5-person & 6-person \\
\hline 3-person & & -6.1 & -21.0 & -31.7 \\
\hline 4-person & 5.8 & & -14.0 & -24.1 \\
\hline 5-person & 17.4 & 12.3 & & -8.8 \\
\hline 6-person & 24.1 & 19.4 & 8.1 & \\
\hline
\end{tabular}

Table 35: Percentage Table for Advance Second Line end time (See Figure 71)
Fire Out: Begin time, duration, and end time

\begin{tabular}{|c|c|c|c|c|}
\hline Crew Size & 3-person & 4-person & 5-person & 6-person \\
\hline 3-person & & -8.7 & -16.5 & -27.9 \\
\hline 4-person & 8.0 & & -7.2 & -17.6 \\
\hline 5-person & 14.2 & 6.7 & & -9.7 \\
\hline 6-person & 21.8 & 15.0 & 8.9 & \\
\hline
\end{tabular}

Table 36: Percentage Table for Fire Out begin time (See Figure 72)

\begin{tabular}{|c|c|c|c|c|}
\hline Crew Size & 3-person & 4-person & 5-person & 6-person \\
\hline 3-person & & -9.1 & -11.5 & -47.5 \\
\hline 4-person & 8.4 & & -2.2 & -35.1 \\
\hline 5-person & 10.3 & 2.1 & & -32.2 \\
\hline 6-person & 32.2 & 26.0 & 24.4 & \\
\hline
\end{tabular}

Table 37: Percentage Table for Fire Out duration (See Figure 72)

\begin{tabular}{|c|c|c|c|c|}
\hline Crew Size & 3-person & 4-person & 5-person & 6-person \\
\hline 3-person & & -8.9 & -14.5 & -34.5 \\
\hline 4-person & 8.1 & & -5.2 & -23.5 \\
\hline 5-person & 12.7 & 5.0 & & -17.4 \\
\hline 6-person & 25.6 & 19.0 & 14.8 & \\
\hline
\end{tabular}

Table 38: Percentage Table for Fire Out end time (See Figure 72) 
Search and Rescue 10th Floor: Begin time, duration, and end time

\begin{tabular}{|c|c|c|c|c|}
\hline Crew Size & 3-person & 4-person & 5-person & 6-person \\
\hline 3-person & & -8.7 & -16.4 & -27.7 \\
\hline 4-person & 7.8 & & -7.1 & -17.5 \\
\hline 5-person & 14.1 & 6.7 & & -9.7 \\
\hline 6-person & 21.7 & 14.9 & 8.8 & \\
\hline
\end{tabular}

Table 39: Percentage Table for Search and Rescue 10th Floor begin time (See Figure 73)

\begin{tabular}{|c|c|c|c|c|}
\hline Crew Size & 3-person & 4-person & 5-person & 6-person \\
\hline 3-person & & -28.9 & -102.2 & -118.2 \\
\hline 4-person & 22.4 & & -56.8 & -69.3 \\
\hline 5-person & 50.5 & 36.2 & & -7.9 \\
\hline 6-person & 54.2 & 40.9 & 7.3 & \\
\hline
\end{tabular}

Table 40: Percentage Table for Search and Rescue 10th Floor duration (See Figure 73)

\begin{tabular}{|c|c|c|c|c|}
\hline Crew Size & 3-person & 4-person & 5-person & 6-person \\
\hline 3-person & & -22.5 & -67.4 & -82.0 \\
\hline 4-person & 18.4 & & -36.7 & -48.5 \\
\hline 5-person & 40.3 & 26.8 & & -8.7 \\
\hline 6-person & 45.0 & 32.7 & 8.0 & \\
\hline
\end{tabular}

Table 41: Percentage Table for Search and Rescue 10th Floor end time (See Figure 73)
Victim \#1 Found: Begin time, duration, and end time

\begin{tabular}{|c|c|c|c|c|}
\hline Crew Size & 3-person & 4-person & 5-person & 6-person \\
\hline 3-person & & -8.75 & -16.4 & -27.7 \\
\hline 4-person & 7.8 & & -7.1 & -17.5 \\
\hline 5-person & 14.1 & 6.7 & & -9.7 \\
\hline 6-person & 21.7 & 14.9 & 8.8 & \\
\hline
\end{tabular}

Table 42: Percentage Table for Victim \#1 Found begin time (See Figure 74)

\begin{tabular}{|l|c|c|c|c|}
\hline Crew Size & 3-person & 4-person & 5-person & 6-person \\
\hline 3-person & & -56.9 & -234.1 & -316.6 \\
\hline 4-person & 36.3 & & -113.0 & -165.5 \\
\hline 5-person & 70.1 & 53.0 & & -24.7 \\
\hline 6-person & 76.0 & 62.3 & 19.8 & \\
\hline
\end{tabular}

Table 43: Percentage Table for Victim \#1 Found duration (See Figure 74)

\begin{tabular}{|l|c|c|c|c|}
\hline Crew Size & 3-person & 4-person & 5-person & 6-person \\
\hline 3-person & & -35.9 & -102.5 & -133.2 \\
\hline 4-person & 26.4 & & -49.0 & -71.6 \\
\hline 5-person & 50.6 & 32.9 & & -15.2 \\
\hline 6-person & 57.1 & 41.7 & 13.2 & \\
\hline
\end{tabular}

Table 44: Percentage Table for Victim \#1 Found end time (See Figure 74) 
Victim \#1 Rescue: Begin time, duration, and end time

\begin{tabular}{|c|c|c|c|c|}
\hline Crew Size & 3-person & 4-person & 5-person & 6-person \\
\hline 3-person & & -35.9 & -102.5 & -133.2 \\
\hline 4-person & 26.4 & & -49.0 & -71.6 \\
\hline 5-person & 50.6 & 32.9 & & -15.2 \\
\hline 6-person & 57.1 & 41.7 & 13.2 & \\
\hline
\end{tabular}

Table 45: Percentage Table for Victim \# 1 Rescue begin time (See Figure 75)

\begin{tabular}{|c|c|c|c|c|}
\hline Crew Size & 3-person & 4-person & 5-person & 6-person \\
\hline 3-person & & 0.7 & 16.1 & 13.6 \\
\hline 4-person & -0.7 & & 15.5 & 13.0 \\
\hline 5-person & -19.2 & -18.4 & & -3.0 \\
\hline 6-person & -15.8 & -15.0 & 2.9 & \\
\hline
\end{tabular}

Table 46: Percentage Table for Victim \# 1 Rescue duration (See Figure 75)

\begin{tabular}{|c|c|c|c|c|}
\hline Crew Size & 3-person & 4-person & 5-person & 6-person \\
\hline 3-person & & -33.6 & -90.0 & -116.0 \\
\hline 4-person & 25.1 & & -42.2 & -61.7 \\
\hline 5-person & 47.4 & 29.7 & & -13.7 \\
\hline 6-person & 53.7 & 38.1 & 12.0 & \\
\hline
\end{tabular}

Table 47: Percentage Table for Victim \# 1 Rescue end time (See Figure 75)
Victim \#1 Descent: Begin time, duration, and end time

\begin{tabular}{|c|c|c|c|c|}
\hline \multirow{2}{*}{ Crew Size } & \multirow{2}{*}{ 3-person } & 4-person & 5-person & 6-person \\
\cline { 1 - 3 } 3-person & & -30.5 & -83.1 & -107.7 \\
\hline 4-person & 23.4 & & -40.4 & -59.2 \\
\hline 5-person & 45.4 & 28.8 & & -13.4 \\
\hline 6-person & 51.9 & 37.2 & 11.8 & \\
\hline
\end{tabular}

Table 48: Percentage Table for Victim \#1 Descent begin time (See Figure 76)

\begin{tabular}{|c|c|c|c|c|}
\hline Crew Size & 3-person & 4-person & 5-person & 6-person \\
\hline 3-person & & 21.1 & 13.4 & 13.4 \\
\hline 4-person & -26.8 & & -9.9 & -9.9 \\
\hline 5-person & -15.4 & 9.0 & & 0.0 \\
\hline 6-person & -15.4 & 9.0 & 0.0 & \\
\hline
\end{tabular}

Table 49: Percentage Table for Victim \#1 Descent duration (See Figure 76)

\begin{tabular}{|c|c|c|c|c|}
\hline Crew Size & 3-person & 4-person & 5-person & 6-person \\
\hline 3-person & & -27.4 & -75.8 & -97.68 \\
\hline 4-person & 21.5 & & -38.0 & -55.1 \\
\hline 5-person & 43.1 & 27.5 & & -12.4 \\
\hline 6-person & 49.4 & 35.5 & 11.0 & \\
\hline
\end{tabular}

Table 50: Percentage Table for Victim \#1 Descent end time (See Figure 76) 
Advance Line Above the Fire (11th Floor): Begin time, duration, and end time

\begin{tabular}{|c|c|c|c|c|}
\hline Crew Size & 3-person & 4-person & 5-person & 6-person \\
\hline 3-person & & -1.1 & -8.9 & -10.3 \\
\hline 4-person & 1.09 & & -7.8 & -9.1 \\
\hline 5-person & 8.21 & 7.2 & & -1.3 \\
\hline 6-person & 9.37 & 8.4 & 1.3 & \\
\hline
\end{tabular}

Table 51: Percentage Table for Advance Line Above the Fire begin time (See Figure 77)

\begin{tabular}{|l|c|c|c|c|}
\hline Crew Size & 3-person & 4-person & 5-person & 6-person \\
\hline 3-person & & -42.3 & -59.1 & -103.5 \\
\hline 4-person & 29.7 & & -11.8 & -43.0 \\
\hline 5-person & 37.1 & 10.6 & & -27.9 \\
\hline 6-person & 50.9 & 30.1 & 21.8 & \\
\hline
\end{tabular}

Table 52: Percentage Table for Advance Line Above the Fire duration (See Figure 77)

\begin{tabular}{|c|c|c|c|c|}
\hline Crew Size & 3-person & 4-person & 5-person & 6-person \\
\hline 3-person & & -4.4 & -13.0 & -16.3 \\
\hline 4-person & 4.3 & & -8.2 & -11.3 \\
\hline 5-person & 11.5 & 7.5 & & -2.9 \\
\hline 6-person & 14.0 & 10.2 & 2.8 & \\
\hline
\end{tabular}

Table 53: Percentage Table for Advance Line Above the Fire end time (See Figure 77)
Search and Rescue 11th Floor: Begin time, duration, and end time

\begin{tabular}{|l|c|c|c|c|}
\hline Crew Size & 3-person & 4-person & 5-person & 6-person \\
\hline 3-person & & -1.2 & -8.6 & -8.3 \\
\hline 4-person & 1.2 & & -7.3 & -7.1 \\
\hline 5-person & 7.9 & 6.8 & & 0.2 \\
\hline 6-person & 7.7 & 6.6 & -0.2 & \\
\hline
\end{tabular}

Table 54: Percentage Table for Search and Rescue 11th Floor begin time (See Figure 78)

\begin{tabular}{|c|c|c|c|c|}
\hline Crew Size & 3-person & 4-person & 5-person & 6-person \\
\hline 3-person & & -49.1 & -58.1 & -84.8 \\
\hline 4-person & 32.9 & & -6.0 & -23.9 \\
\hline 5-person & 36.7 & 5.7 & & -16.9 \\
\hline 6-person & 45.9 & 19.3 & 14.4 & \\
\hline
\end{tabular}

Table 55: Percentage Table for Search and Rescue 11th Floor duration (See Figure 78)

\begin{tabular}{|l|c|c|c|c|}
\hline Crew Size & 3-person & 4-person & 5-person & 6-person \\
\hline 3-person & & -22.9 & -31.1 & -40.1 \\
\hline 4-person & 18.6 & & -6.7 & -14.0 \\
\hline 5-person & 23.7 & 6.3 & & -6.7 \\
\hline 6-person & 28.6 & 12.3 & 6.4 & \\
\hline
\end{tabular}

Table 56: Percentage Table for Search and Rescue 11th Floor end time (See Figure 78) 
Victim \#2 Found: Begin time, duration, and end time

\begin{tabular}{|c|c|c|c|c|}
\hline Crew Size & 3-person & 4-person & 5-person & 6-person \\
\hline 3-person & & -2.9 & -8.6 & -9.0 \\
\hline 4-person & 2.8 & & -5.7 & -5.8 \\
\hline 5-person & 8.1 & 5.4 & & -0.1 \\
\hline 6-person & 8.2 & 5.5 & 0.1 & \\
\hline
\end{tabular}

Table 57: Percentage Table for Victim \#2 Found begin time (See Figure 79)

\begin{tabular}{|c|c|c|c|c|}
\hline Crew Size & 3-person & 4-person & 5-person & 6-person \\
\hline 3-person & & -103.3 & -127.7 & -129.5 \\
\hline 4-person & 50.8 & & -12.0 & -12.9 \\
\hline 5-person & 56.1 & 10.7 & & -0.8 \\
\hline 6-person & 56.4 & 11.4 & 0.8 & \\
\hline
\end{tabular}

Table 58: Percentage Table for Victim \#2 Found duration (See Figure 79)

\begin{tabular}{|l|c|c|c|c|}
\hline Crew Size & 3-person & 4-person & 5-person & 6-person \\
\hline 3-person & & -40.3 & -51.6 & -52.1 \\
\hline 4-person & 28.7 & & -8.0 & -8.4 \\
\hline 5-person & 34.0 & 7.4 & & -0.3 \\
\hline 6-person & 34.3 & 7.7 & 0.3 & \\
\hline
\end{tabular}

Table 59: Percentage Table for Victim \#2 Found end time (See Figure 79)
Victim \#2 Rescue: Begin time, duration, and end time

\begin{tabular}{|l|c|c|c|c|}
\hline Crew Size & 3-person & 4-person & 5-person & 6-person \\
\hline 3-person & & -40.3 & -51.6 & -52.1 \\
\hline 4-person & 28.7 & & -8.0 & -8.4 \\
\hline 5-person & 34.0 & 7.4 & & -0.3 \\
\hline 6-person & 34.2 & 7.7 & 0.3 & \\
\hline
\end{tabular}

Table 60: Percentage Table for Victim \#2 Rescue begin time (See Figure 80)

\begin{tabular}{|l|c|c|c|c|}
\hline Crew Size & 3-person & 4-person & 5-person & 6-person \\
\hline 3-person & & 6.0 & 9.0 & 6.7 \\
\hline 4-person & -6.3 & & 3.2 & 0.7 \\
\hline 5-person & -9.9 & -3.3 & & -2.6 \\
\hline 6-person & -7.0 & -0.7 & 2.6 & \\
\hline
\end{tabular}

Table 61: Percentage Table for Victim \#2 Rescue duration (See Figure 80)

\begin{tabular}{|c|c|c|c|c|}
\hline Crew Size & 3-person & 4-person & 5-person & 6-person \\
\hline 3-person & & -37.4 & -47.2 & -48.0 \\
\hline 4-person & 27.2 & & -7.2 & -7.7 \\
\hline 5-person & 32.1 & 6.7 & & -0.51 \\
\hline 6-person & 32.4 & 7.1 & 0.5 & \\
\hline
\end{tabular}

Table 62: Percentage Table for Victim \#2 Rescue end time (See Figure 80) 
Victim \#2 Descent: Begin time, duration, and end time

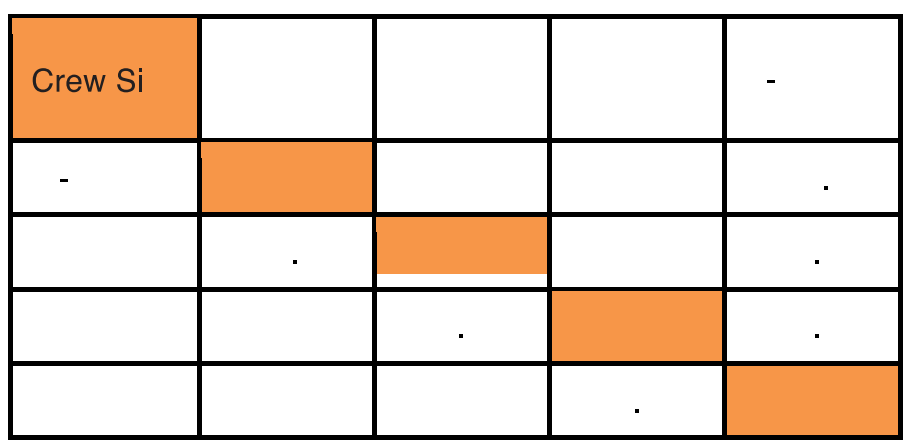

Table 63: Percentage Table for Victim \#2 Descent begin time (See Figure 81)

\begin{tabular}{|l|c|c|c|c|}
\hline Crew Size & 3-person & 4-person & 5-person & 6-person \\
\hline 3-person & & -35.7 & -43.6 & -46.1 \\
\hline 4-person & 26.3 & & -5.9 & -7.7 \\
\hline 5-person & 30.4 & 5.5 & & -1.7 \\
\hline 6-person & 31.6 & 7.1 & 1.7 & \\
\hline
\end{tabular}

Table 64: Percentage Table for Victim \#2 Descent duration (See Figure 81)

\begin{tabular}{|l|c|c|c|c|}
\hline Crew Size & 3-person & 4-person & 5-person & 6-person \\
\hline 3-person & & -34.8 & -41.6 & -46.3 \\
\hline 4-person & 25.8 & & -5.1 & -8.5 \\
\hline 5-person & 29.4 & 4.8 & & -3.3 \\
\hline 6-person & 31.6 & 7.8 & 3.2 & \\
\hline
\end{tabular}

Table 65: Percentage Table for Victim \#2 Descent end time (See Figure 81) 
T he measurements of length and time taken in these experiments have unique components of uncertainty that must be evaluated in order to determine the fidelity of the data. These components of uncertainty can be grouped into two categories: Type A and Type B. Type A uncertainties are those evaluated by statistical methods, such as calculating the standard deviation of the mean of a set of measurements. Type B uncertainties are based on scientific judgment using all available and relevant information. Using relevant information, the upper and lower limits of the expected value are estimated so that the probability that the measurement falls within these limits is essentially $100 \%$. After all the component uncertainties of a measurement have been identified and evaluated it is necessary to use them to compute the combined standard uncertainty using the law of propagation of uncertainty (the "root sum of squares"). Although this expresses the uncertainty of a given measurement to about $60 \%$ confidence, it is useful to define an interval for which the measurement will fall within a high level of statistical confidence. This is known as the expanded uncertainty. The current international practice is to multiply the combined standard uncertainty by a factor of two $(\mathrm{k}=2)$, giving a confidence of $95 \%$.

To construct a geometrically accurate computer model of the high-rise floor to use in the fire simulations, the structure dimensions needed to be accurately measured. Long measurements such as hallway and corridor lengths were taken using a HILTI PD20 laser range meter. The estimated accuracy of the laser is 0.19 in $(3 \mathrm{~mm})$ over an operating range of greater than $330 \mathrm{ft}(100 \mathrm{~m})$. The large-scale measurements were on the order of $100 \mathrm{ft}(30 \mathrm{~m})$ to $140 \mathrm{ft}(40 \mathrm{~m})$. This leads to an uncertainty of \pm $0.01 \%$. Shorter length measurements such as door openings, cubicle sizes, and button locations were taken using a measuring tape with a resolution of 0.02 in $(0.5 \mathrm{~mm})$. However, measurement error due to uneven and non-level surfaces resulted in an estimated uncertainty of $\pm 0.5 \%$ for length measurements taken on the scale of room dimensions. Taking a conservative approach, the small-scale measurement uncertainty was considered for all measurements. Therefore, the estimated total expanded uncertainty for length measurements was $\pm 1.0 \%$.
All timing staff were equipped with the same model of digital stopwatch with a resolution of $0.01 \mathrm{~s}$ and an uncertainty of $\pm 3 \mathrm{~s}$ per $24 \mathrm{hr}$; the uncertainty of the timing mechanism in the stopwatches was small enough over the duration of an experiment that it can be neglected. There are three components of uncertainty when using people to time firefighting tasks. First, timers may have a bias depending on whether they record the time in anticipation of, or reaction to an event. A second component exists because multiple timers were used to record all tasks. The third component is the mode of the stimulus to which the staff is reacting: audible (firefighters announcing task updates over the radio) or visual (timing staff sees a task start or stop).

Milestone events in these experiments were recorded both audibly and visually. A test series described in the NIST Recommended Practice Guide for Stopwatch and Timer Calibrations (Gust et al., 2009) found the reaction times for the two modes of stimulus to be approximately the same, so this component can be neglected. Because of the lack of knowledge regarding the mean bias of the timers, a rectangular distribution was assumed and the worst case reaction time bias of $120 \mathrm{~ms}$ was used, giving a standard deviation of $69 \mathrm{~ms}$. The standard deviation of the reaction time was assumed to be the worst case of $230 \mathrm{~ms}$. The estimated total expanded uncertainty of task times measured in these experiments was $240 \mathrm{~ms}$.

An additional component of uncertainty exists for the firefighters pressing the target buttons on the fire floor. The target buttons on the fire floor were located along one of the three interior walls of the cubicles. The location of the button within the cubicle was random to force firefighters to search the entire cubicle and not become conditioned to search just one wall. There was no experimental procedure set for searching the cubicles, just a directive to search the entire cubicle. While the individual cubicles were small, the search could have been conducted in a variety of manners depending on the individual firefighter. From experimental observations a reasonable estimate for the time it takes to search a cubicle and press the button, when applicable, is $6 \mathrm{~s}$ with a standard deviation of $\pm 2 \mathrm{~s}$. This results in an expanded uncertainty of $\pm 4 \mathrm{~s}$.

A summary of experimental measurement uncertainty is given in Table 66.

\begin{tabular}{|c|c|c|c|}
\hline Measurement & $\begin{array}{c}\text { Component Standard } \\
\text { Uncertainty }\end{array}$ & $\begin{array}{l}\text { Combined Standard } \\
\text { Uncertainty }\end{array}$ & $\begin{array}{l}\text { Total Expandec } \\
\text { Uncertainty }\end{array}$ \\
\hline \multicolumn{4}{|l|}{ Length Measurements } \\
\hline Cubicle Dimensions & $\pm 0.5 \%$ & \multirow{2}{*}{ $\pm 1 \%$} & \multirow{2}{*}{ $\pm 2 \%$} \\
\hline Building Dimensions & $\pm 0.5 \%$ & & \\
\hline \multicolumn{4}{|l|}{ Timer Data } \\
\hline Timer Bias & $\pm 0.069 \mathrm{~s}$ & \multirow{2}{*}{ $\pm 0.299 \mathrm{~s}$} & \multirow{2}{*}{ $\pm 0.598 \mathrm{~s}$} \\
\hline Reaction Time & $\pm 0.230 \mathrm{~s}$ & & \\
\hline \multicolumn{4}{|l|}{ Button Time Data } \\
\hline Finding/Pressing Buttons & Elevator & $\pm 2 \mathrm{~s}$ & $\pm 4 \mathrm{~s}$ \\
\hline
\end{tabular}

Table 66: Summary of Measurement Uncertainty 
In addition to experimental uncertainty, there exists uncertainty in the computational models. Models are used when the equations describing the governing physics of a problem cannot be solved directly. To test the ability of a model to correctly capture the chemistry and physics, the computational software must be validated by testing against experimental results. The FDS model used for the simulations in this report undergoes extensive and on-going validation (McGrattan et al. 2012c, USNRC 2007). For questions regarding air tenability, the parameter of interest is the fractional effective dose (FED) ${ }^{31}$. According to ISO 13571, the exact distribution of human response to toxic gas inhalation is not known. As a result, there is considerable uncertainty relating the computed FED value from the computation model to the percentage of the population likely to be incapacitated. According to the ISO standard for the gases considered in this study, the uncertainty can be as much as $\pm 20 \%$ to $35 \%$ (ISO 13571). 
$\mathrm{T}$

he first step in calculating a design fire is to determine what fuel or fuels could be used to represent a cubicle. Cubicles are generally composed of a wide range of combustibles, from natural materials (such as wood furniture and paper) to synthetics (such as foam padding and plastic computers). Instead of modeling each individual component, the composition of the cubicle was lumped into a natural or wood component and a synthetic or plastic (polyurethane) component, based on mass composition. This resulted in a source fire whose fuel was composed of $70 \%$ wood and $30 \%$ polyurethane by mass (Ohlemiller et al. 2005). Determination of the representative fuels for the fire is important because each fuel consumes oxygen at a different rate, releases different amounts of energy, produces different amounts of carbon monoxide and soot, and has different extinction criteria. Table 67 shows properties of interest for two fuels.

From Table 67, polyurethane has a larger heat of combustion than wood, which means that it releases more energy per kilogram of fuel consumed. However, wood makes up $70 \%$ of the fuel load in the cubicle, so wood is responsible for a larger portion of the total energy release. Polyurethane has a carbon monoxide yield that is approximately an order of magnitude larger than that of wood. The difference in carbon monoxide yields illustrates the need for a multi-fuel source fire, as carbon monoxide is one of the three contributing species to the FED analysis performed for this report.

To determine the remaining parameters for constructing the design fire curve, experiments performed as part of the NIST study of the Cook County Administration building fire were examined (Madrzykowski et al. 2004). Part of the incident reconstruction work conducted by NIST scientists included performing fire tests of a single workstation under a full-scale calorimeter. A calorimeter is an experimental apparatus used to determine the heat release rate of an object or set of objects. Heat release rate (HRR) is the amount of energy released per unit of time from the fire. Figure 104 shows the HRR as a function of time from ignition until burnout for a typical single workstation in the Cook County Administration building. To better understand the magnitude of the values in Figure 104, Table 68 shows common objects and their peak HRR values found from calorimeter experiments.

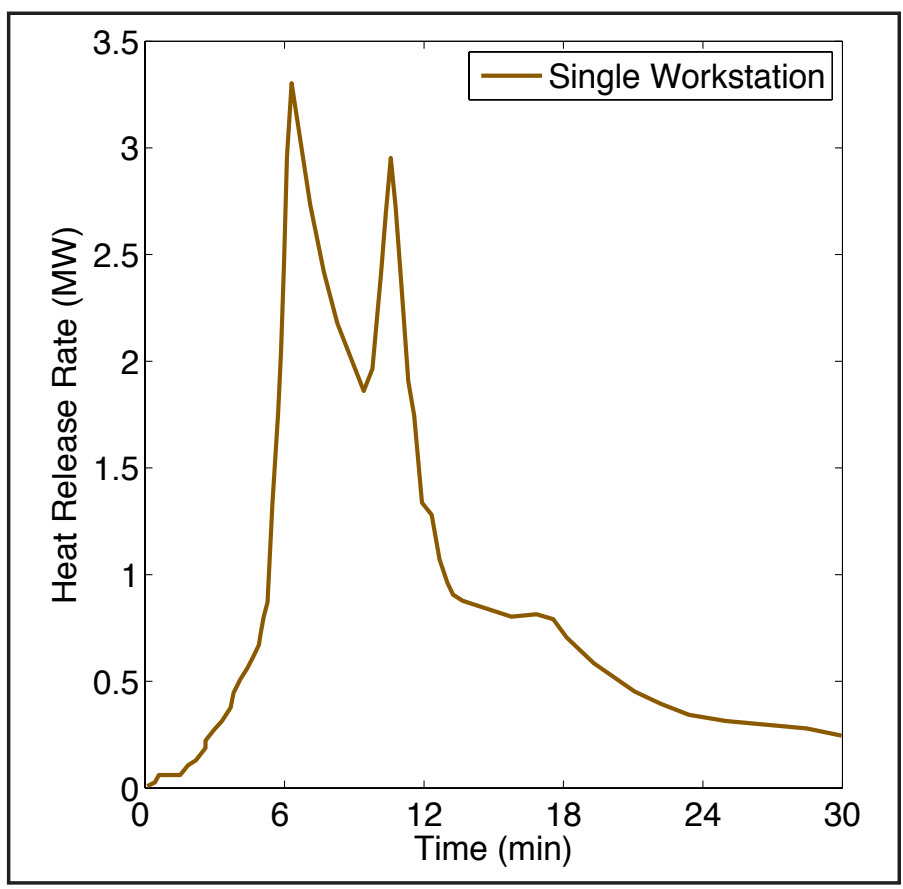

Figure 104: Heat release rate versus time of a typical single workstation fire from the Cook County Administration building (Madrzykowski et al. 2004)

\begin{tabular}{|c|c|c|c|c|}
\hline Fuel & $\begin{array}{c}\text { Chemical } \\
\text { Formula }\end{array}$ & $\begin{array}{c}\text { Heat of Combustion } \\
(\mathrm{kJ} / \mathrm{kg})\end{array}$ & CO Yield & Soot Yield \\
\hline Wood & $\mathrm{C}_{25} \mathrm{H}_{42} \mathrm{O}_{6} \mathrm{~N}_{2}$ & 16400 & 0.004 & 0.015 \\
\hline Polyurethane & $\mathrm{CH}_{1.7} \mathrm{~N}_{0.74} \mathrm{O}_{0.002}$ & 26200 & 0.01 & 0.131 \\
\hline
\end{tabular}

Table 67: Fuel properties and product yields for fuel components of design fire (Tewarson 2008).

\begin{tabular}{|c|c|c|}
\hline Peak Heat Release Rate & Object & Source \\
\hline $5 \mathrm{~W}$ & Burning cigarette & Babrauskas et al. 1985 \\
\hline $80 \mathrm{~W}$ & Burning match or candle & $\begin{array}{c}\text { Babrauskas et al. 1985, } \\
\text { Hamins et al. 2005 }\end{array}$ \\
\hline $30 \mathrm{~kW}$ to $300 \mathrm{~kW}$ & $\begin{array}{c}\text { Small Plastic Waste Container } \\
\text { to Large (30 gal) Waste } \\
\text { Container }\end{array}$ & $\begin{array}{c}\text { Stroup et al. 2003, } \\
\text { Madrzykowski et al. 2009 }\end{array}$ \\
\hline $80 \mathrm{~kW}$ to $2.5 \mathrm{MW}$ & $\begin{array}{c}\text { Burning Upholstered Chair } \\
\text { Stroup et al. 2001, DCA 1991 }\end{array}$ \\
\hline $2.5 \mathrm{MW}$ to $5 \mathrm{MW}$ & Burning Upholstered Sofa & Madrzykowski et al. 2009 \\
\hline $1.5 \mathrm{MW}$ to $5 \mathrm{MW}$ & Burning Christmas Tree & $\begin{array}{c}\text { Stroup et al. 1999, } \\
\text { Madryzkowski 2008 }\end{array}$ \\
\hline 30 MW & Rail Car & Babrauskas, 2008 \\
\hline
\end{tabular}

Table 68: Peak heat release rate values from common objects 
The next step in building the design fire curves was to determine the criteria for ignition of adjacent cubicles. In addition to conducting fire tests on single cubicles, NIST scientists also conducted calorimeter tests for multiple cubicles (Madrzykowski et al. 2004). The experiments noted the time at which an adjacent cubicle ignited. From this time, the total amount of energy that needed to be released for ignition was found, approximately 600 MJ (cf. Figure 105).

This critical total energy value was used as the criteria for fire spread to adjacent cubicles in the computer model. The vertical line in Figure 105 indicates when ignition occurred. In the model, fire spread meant invoking additional design fire curves. If more than one fire is burning at a given time, then the total HRR is the sum of the HRR values from each active cubicle fire. Since fire spread is based on total energy released, the faster growth rate design fires spread faster.

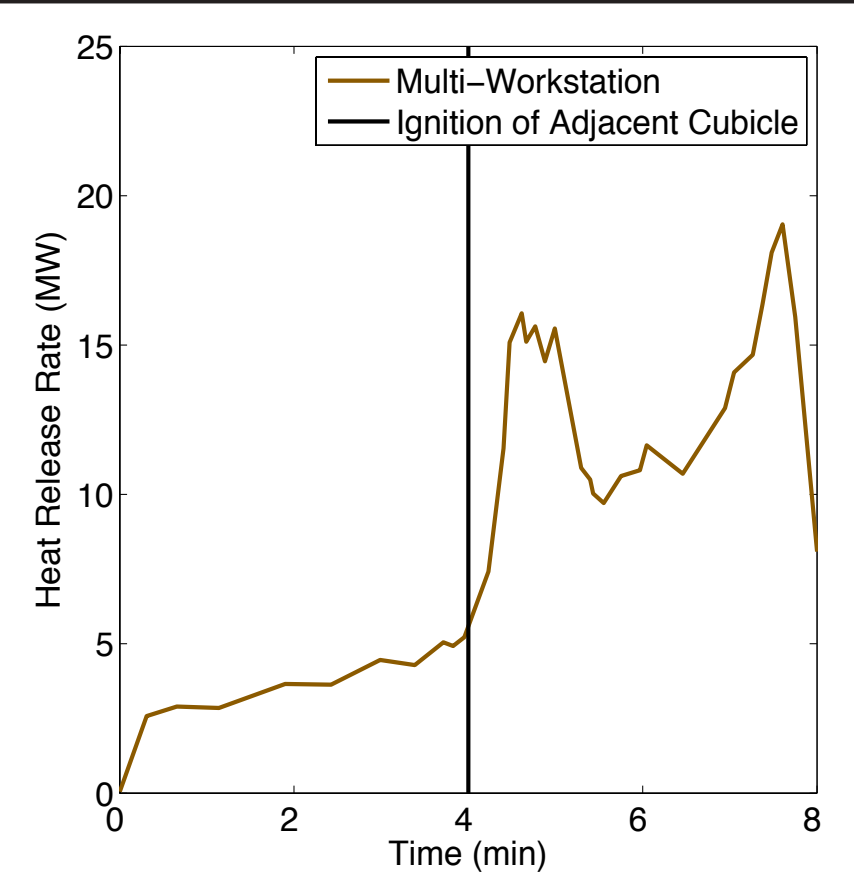

Figure 105: Heat release rate versus time curve showing ignition of an adjacent cubicle from the Cook County Administration building experiments (Madrzykowski et al. 2004) 
NOTES 
http://dx.doi.org/10.6028/NIST.TN.1797 